Cochrane Database of Systematic Reviews

\title{
Cognitive training for people with mild to moderate dementia
} (Review)

Bahar-Fuchs A, Martyr A, Goh AMY, Sabates J, Clare L

Bahar-Fuchs A, Martyr A, Goh AMY, Sabates J, Clare L.

Cognitive training for people with mild to moderate dementia.

Cochrane Database of Systematic Reviews 2019, Issue 3. Art. No.: CD013069.

DOI: 10.1002/14651858.CD013069.pub2.

www.cochranelibrary.com 
TABLE OF CONTENTS

HEADER

ABSTRACT

PLAIN LANGUAGE SUMMARY

SUMMARY OF FINDINGS

BACKGROUND

OBJECTIVES

METHODS

RESULTS

Figure 1.

Figure 2.

Figure 3.

Figure 4.

Figure 5.

Figure 6.

Figure 7.

Figure 8.

Figure 9.

Figure 10.

Figure 11.

Figure 12.

Figure 13.

Figure 14.

Figure 15.

DISCUSSION

AUTHORS' CONCLUSIONS

ACKNOWLEDGEMENTS

REFERENCES

CHARACTERISTICS OF STUDIES

DATA AND ANALYSES

Analysis 1.1. Comparison 1 Cognitive training vs control immediately post intervention, Outcome 1 Change in a global measure of cognition (composite).

Analysis 1.2. Comparison 1 Cognitive training vs control immediately post intervention, Outcome 2 Change in a global measure of cognition (composite)_zero correlation.

Analysis 1.3. Comparison 1 Cognitive training vs control immediately post intervention, Outcome 3 Change in a global measure of cognition.

Analysis 1.4. Comparison 1 Cognitive training vs control immediately post intervention, Outcome 4 Change in a global measure of cognition_zero correlation.

Analysis 1.5. Comparison 1 Cognitive training vs control immediately post intervention, Outcome 5 Change in disease progression.

Analysis 1.6. Comparison 1 Cognitive training vs control immediately post intervention, Outcome 6 Change in delayed memory.

Analysis 1.7. Comparison 1 Cognitive training vs control immediately post intervention, Outcome 7 Change in immediate memory.

Analysis 1.8. Comparison 1 Cognitive training vs control immediately post intervention, Outcome 8 Change in attention and working memory.

Analysis 1.9. Comparison 1 Cognitive training vs control immediately post intervention, Outcome 9 Change in language (naming).

Analysis 1.10. Comparison 1 Cognitive training vs control immediately post intervention, Outcome 10 Change in verbal letter fluency.

Analysis 1.11. Comparison 1 Cognitive training vs control immediately post intervention, Outcome 11 Change in verbal category fluency.

Analysis 1.12. Comparison 1 Cognitive training vs control immediately post intervention, Outcome 12 Change in executive function. 
Analysis 1.13. Comparison 1 Cognitive training vs control immediately post intervention, Outcome 13 Change in speed of information processing.

Analysis 1.14. Comparison 1 Cognitive training vs control immediately post intervention, Outcome 14 Change in meta cognition (self-reported).

Analysis 1.15. Comparison 1 Cognitive training vs control immediately post intervention, Outcome 15 Change in meta cognition (informant-reported).

Analysis 1.16. Comparison 1 Cognitive training vs control immediately post intervention, Outcome 16 Change in participants' mood.

Analysis 1.17. Comparison 1 Cognitive training vs control immediately post intervention, Outcome 17 Change in capacity for activities of daily living.

Analysis 1.18. Comparison 1 Cognitive training vs control immediately post intervention, Outcome 18 Change in general health and quality of life.

Analysis 1.19. Comparison 1 Cognitive training vs control immediately post intervention, Outcome 19 Change in behavioural and psychological symptoms of dementia (BPSD).

Analysis 1.20. Comparison 1 Cognitive training vs control immediately post intervention, Outcome 20 Participant burden (retention rates).

Analysis 1.21. Comparison 1 Cognitive training vs control immediately post intervention, Outcome 21 Change in burden of care (CAREGIVER).

Analysis 1.22. Comparison 1 Cognitive training vs control immediately post intervention, Outcome 22 Change in quality of life (CAREGIVER).

Analysis 1.23. Comparison 1 Cognitive training vs control immediately post intervention, Outcome 23 Change in mood and well-being (CAREGIVER).

Analysis 2.1. Comparison 2 Cognitive training vs control in the medium term ( 3 to 12 months post intervention), Outcome 1 Change in a global measure of cognition (composite).

Analysis 2.2. Comparison 2 Cognitive training vs control in the medium term ( 3 to 12 months post intervention), Outcome 2 Change in a global measure of cognition (composite)_zero correlation.

Analysis 2.3. Comparison 2 Cognitive training vs control in the medium term ( 3 to 12 months post intervention), Outcome 3 Change in a global measure of cognition.

Analysis 2.4. Comparison 2 Cognitive training vs control in the medium term ( 3 to 12 months post intervention), Outcome 4 Change in a global measure of cognition (zero correlation).

Analysis 2.5. Comparison 2 Cognitive training vs control in the medium term ( 3 to 12 months post intervention), Outcome 5 Change in disease progression.

Analysis 2.6. Comparison 2 Cognitive training vs control in the medium term ( 3 to 12 months post intervention), Outcome 6 Change in disease progression (zero correlation).

Analysis 2.7. Comparison 2 Cognitive training vs control in the medium term ( 3 to 12 months post intervention), Outcome 7 Change in delayed memory.

Analysis 2.8. Comparison 2 Cognitive training vs control in the medium term ( 3 to 12 months post intervention), Outcome 8 Change in immediate memory.

Analysis 2.9. Comparison 2 Cognitive training vs control in the medium term ( 3 to 12 months post intervention), Outcome 9 Change in attention and working memory.

Analysis 2.10. Comparison 2 Cognitive training vs control in the medium term ( 3 to 12 months post intervention), Outcome 10 Change in language (naming).

Analysis 2.11. Comparison 2 Cognitive training vs control in the medium term ( 3 to 12 months post intervention), Outcome 11 Change in verbal letter fluency.

Analysis 2.12. Comparison 2 Cognitive training vs control in the medium term ( 3 to 12 months post intervention), Outcome 12 Change in verbal category fluency.

Analysis 2.13. Comparison 2 Cognitive training vs control in the medium term ( 3 to 12 months post intervention), Outcome 13 Change in executive function.

Analysis 2.14. Comparison 2 Cognitive training vs control in the medium term ( 3 to 12 months post intervention), Outcome 14 Change in speed of information processing.

Analysis 2.15. Comparison 2 Cognitive training vs control in the medium term ( 3 to 12 months post intervention), Outcome 15 Change in meta cognition (self-reported).

Analysis 2.16. Comparison 2 Cognitive training vs control in the medium term ( 3 to 12 months post intervention), Outcome 16 Change in meta cognition (informant-reported).

Analysis 2.17. Comparison 2 Cognitive training vs control in the medium term ( 3 to 12 months post intervention), Outcome 17 Change in participants' mood. 
Analysis 2.18. Comparison 2 Cognitive training vs control in the medium term ( 3 to 12 months post intervention), Outcome 18 Change in capacity for activities of daily living.

Analysis 2.19. Comparison 2 Cognitive training vs control in the medium term (3 to 12 months post intervention), Outcome 19 Change in general health and quality of life.

Analysis 2.20. Comparison 2 Cognitive training vs control in the medium term ( 3 to 12 months post intervention), Outcome 20 Change in behavioural and psychological symptoms of dementia (BPSD).

Analysis 3.1. Comparison 3 Cognitive training vs alternative treatment immediately post intervention, Outcome 1 Change in a global measure of cognition (composite).

Analysis 3.2. Comparison 3 Cognitive training vs alternative treatment immediately post intervention, Outcome 2 Change in a global measure of cognition (composite)_zero correlation.

Analysis 3.3. Comparison 3 Cognitive training vs alternative treatment immediately post intervention, Outcome 3 Change in a global measure of cognition.

Analysis 3.4. Comparison 3 Cognitive training vs alternative treatment immediately post intervention, Outcome 4 Change in a global measure of cognition_zero correlation.

Analysis 3.5. Comparison 3 Cognitive training vs alternative treatment immediately post intervention, Outcome 5 Change in disease progression.

Analysis 3.6. Comparison 3 Cognitive training vs alternative treatment immediately post intervention, Outcome 6 Change in delayed memory.

Analysis 3.7. Comparison 3 Cognitive training vs alternative treatment immediately post intervention, Outcome 7 Change in immediate memory.

Analysis 3.8. Comparison 3 Cognitive training vs alternative treatment immediately post intervention, Outcome 8 Change in attention and working memory.

Analysis 3.9. Comparison 3 Cognitive training vs alternative treatment immediately post intervention, Outcome 9 Change in language (naming).

Analysis 3.10. Comparison 3 Cognitive training vs alternative treatment immediately post intervention, Outcome 10 Change in verbal letter fluency.

Analysis 3.11. Comparison 3 Cognitive training vs alternative treatment immediately post intervention, Outcome 11 Change in verbal category fluency.

Analysis 3.12. Comparison 3 Cognitive training vs alternative treatment immediately post intervention, Outcome 12 Change in executive function.

Analysis 3.13. Comparison 3 Cognitive training vs alternative treatment immediately post intervention, Outcome 13 Change in speed of information processing.

Analysis 3.16. Comparison 3 Cognitive training vs alternative treatment immediately post intervention, Outcome 16 Change in participants' mood.

Analysis 3.17. Comparison 3 Cognitive training vs alternative treatment immediately post intervention, Outcome 17 Change in capacity for activities of daily living.

Analysis 3.18. Comparison 3 Cognitive training vs alternative treatment immediately post intervention, Outcome 18 Change in general health and quality of life.

Analysis 3.19. Comparison 3 Cognitive training vs alternative treatment immediately post intervention, Outcome 19 Change in behavioural and psychological symptoms of dementia (BPSD).

Analysis 3.20. Comparison 3 Cognitive training vs alternative treatment immediately post intervention, Outcome 20 Participant burden (retention rates).

Analysis 3.21. Comparison 3 Cognitive training vs alternative treatment immediately post intervention, Outcome 21 Change in burden of care (CAREGIVER).

Analysis 3.22. Comparison 3 Cognitive training vs alternative treatment immediately post intervention, Outcome 22 Change in quality of life (CAREGIVER).

Analysis 3.23. Comparison 3 Cognitive training vs alternative treatment immediately post intervention, Outcome 23 Change in mood and well-being (CAREGIVER).

Analysis 4.1. Comparison 4 Cognitive training vs alternative treatment in the medium term ( 3 to 12 months post intervention), Outcome 1 Change in a global measure of cognition (composite).

Analysis 4.2. Comparison 4 Cognitive training vs alternative treatment in the medium term ( 3 to 12 months post intervention), Outcome 2 Change in a global measure of cognition (composite)_zero correlation.

Analysis 4.3. Comparison 4 Cognitive training vs alternative treatment in the medium term ( 3 to 12 months post intervention), Outcome 3 Change in a global measure of cognition.

Analysis 4.6. Comparison 4 Cognitive training vs alternative treatment in the medium term ( 3 to 12 months post intervention), Outcome 6 Change in delayed memory. 
Analysis 4.7. Comparison 4 Cognitive training vs alternative treatment in the medium term ( 3 to 12 months post intervention), Outcome 7 Change in immediate memory.

Analysis 4.8. Comparison 4 Cognitive training vs alternative treatment in the medium term ( 3 to 12 months post intervention), Outcome 8 Change in attention and working memory.

Analysis 4.9. Comparison 4 Cognitive training vs alternative treatment in the medium term ( 3 to 12 months post intervention), Outcome 9 Change in language (naming).

Analysis 4.10. Comparison 4 Cognitive training vs alternative treatment in the medium term ( 3 to 12 months post intervention), Outcome 10 Change in verbal letter fluency.

Analysis 4.11. Comparison 4 Cognitive training vs alternative treatment in the medium term ( 3 to 12 months post intervention), Outcome 11 Change in verbal category fluency.

Analysis 4.12. Comparison 4 Cognitive training vs alternative treatment in the medium term ( 3 to 12 months post intervention), Outcome 12 Change in executive function.

Analysis 4.13. Comparison 4 Cognitive training vs alternative treatment in the medium term ( 3 to 12 months post intervention), Outcome 13 Change in speed of information processing.

Analysis 4.16. Comparison 4 Cognitive training vs alternative treatment in the medium term ( 3 to 12 months post intervention), Outcome 16 Change in participants' mood.

Analysis 4.18. Comparison 4 Cognitive training vs alternative treatment in the medium term ( 3 to 12 months post intervention), Outcome 18 Change in general health and quality of life.

Analysis 5.1. Comparison 5 Cognitive training vs control immediately post intervention - risk of bias, Outcome 1 Change in a global measure of cognition.

Analysis 5.2. Comparison 5 Cognitive training vs control immediately post intervention - risk of bias, Outcome 2 Change in a global measure of cognition_zero correlation.

Analysis 5.3. Comparison 5 Cognitive training vs control immediately post intervention - risk of bias, Outcome 3 Change in a global measure of cognition (composite).

Analysis 5.4. Comparison 5 Cognitive training vs control immediately post intervention - risk of bias, Outcome 4 Change in a global measure of cognition (composite)_zero correlation.

Analysis 5.5. Comparison 5 Cognitive training vs control immediately post intervention - risk of bias, Outcome 5 Change in immediate memory.

Analysis 5.6. Comparison 5 Cognitive training vs control immediately post intervention - risk of bias, Outcome 6 Change in delayed memory.

Analysis 5.7. Comparison 5 Cognitive training vs control immediately post intervention - risk of bias, Outcome 7 Change in attention and working memory.

Analysis 5.8. Comparison 5 Cognitive training vs control immediately post intervention - risk of bias, Outcome 8 Change in language (naming).

Analysis 5.9. Comparison 5 Cognitive training vs control immediately post intervention - risk of bias, Outcome 9 Change in verbal letter fluency.

Analysis 5.10. Comparison 5 Cognitive training vs control immediately post intervention - risk of bias, Outcome 10 Change in speed of information processing.

Analysis 5.11. Comparison 5 Cognitive training vs control immediately post intervention - risk of bias, Outcome 11 Change in executive function.

Analysis 5.12. Comparison 5 Cognitive training vs control immediately post intervention - risk of bias, Outcome 12 Change in verbal category fluency.

Analysis 5.13. Comparison 5 Cognitive training vs control immediately post intervention - risk of bias, Outcome 13 Change in meta cognition (self-reported).

Analysis 5.14. Comparison 5 Cognitive training vs control immediately post intervention - risk of bias, Outcome 14 Change in meta cognition (informant-reported).

Analysis 5.15. Comparison 5 Cognitive training vs control immediately post intervention - risk of bias, Outcome 15 Change in participants' mood.

Analysis 5.16. Comparison 5 Cognitive training vs control immediately post intervention - risk of bias, Outcome 16 Change in capacity for activities of daily living.

Analysis 5.17. Comparison 5 Cognitive training vs control immediately post intervention - risk of bias, Outcome 17 Change in general health and quality of life.

Analysis 5.18. Comparison 5 Cognitive training vs control immediately post intervention - risk of bias, Outcome 18 Change in behavioural and psychological symptoms of dementia (BPSD).

Analysis 5.19. Comparison 5 Cognitive training vs control immediately post intervention - risk of bias, Outcome 19 Change in disease progression. 
Analysis 5.20. Comparison 5 Cognitive training vs control immediately post intervention - risk of bias, Outcome 20 Change in burden of care (CAREGIVER).

Analysis 5.21. Comparison 5 Cognitive training vs control immediately post intervention - risk of bias, Outcome 21 Change in quality of life (CAREGIVER).

Analysis 5.22. Comparison 5 Cognitive training vs control immediately post intervention - risk of bias, Outcome 22 Change in mood and well-being (CAREGIVER).

Analysis 5.23. Comparison 5 Cognitive training vs control immediately post intervention - risk of bias, Outcome 23 Participant burden (retention rates).

Analysis 5.24. Comparison 5 Cognitive training vs control immediately post intervention - risk of bias, Outcome 24 Change in general health and quality of life.

Analysis 6.1. Comparison 6 Cognitive training vs control immediately post intervention - intervention dose, Outcome 1 Change in a global measure of cognition.

Analysis 6.2. Comparison 6 Cognitive training vs control immediately post intervention - intervention dose, Outcome 2 Change in a global measure of cognition_zero correlation.

Analysis 6.3. Comparison 6 Cognitive training vs control immediately post intervention - intervention dose, Outcome 3 Change in a global measure of cognition (composite).

Analysis 6.4. Comparison 6 Cognitive training vs control immediately post intervention - intervention dose, Outcome 4 Change in a global measure of cognition (composite)_zero correlation.

Analysis 6.5. Comparison 6 Cognitive training vs control immediately post intervention - intervention dose, Outcome 5 Change in immediate memory.

Analysis 6.6. Comparison 6 Cognitive training vs control immediately post intervention - intervention dose, Outcome 6 Change in delayed memory.

Analysis 6.7. Comparison 6 Cognitive training vs control immediately post intervention - intervention dose, Outcome 7 Change in attention and working memory.

Analysis 6.8. Comparison 6 Cognitive training vs control immediately post intervention - intervention dose, Outcome 8 Change in language (naming).

Analysis 6.9. Comparison 6 Cognitive training vs control immediately post intervention - intervention dose, Outcome 9 Change in verbal letter fluency.

Analysis 6.10. Comparison 6 Cognitive training vs control immediately post intervention - intervention dose, Outcome 10 Change in speed of information processing.

Analysis 6.11. Comparison 6 Cognitive training vs control immediately post intervention - intervention dose, Outcome 11 Change in executive function.

Analysis 6.12. Comparison 6 Cognitive training vs control immediately post intervention - intervention dose, Outcome 12 Change in verbal category fluency.

Analysis 6.13. Comparison 6 Cognitive training vs control immediately post intervention - intervention dose, Outcome 13 Change in meta cognition (self-reported).

Analysis 6.14. Comparison 6 Cognitive training vs control immediately post intervention - intervention dose, Outcome 14 Change in meta cognition (informant-reported).

Analysis 6.15 . Comparison 6 Cognitive training vs control immediately post intervention - intervention dose, Outcome 15 Change in participants' mood.

Analysis 6.16. Comparison 6 Cognitive training vs control immediately post intervention - intervention dose, Outcome 16 Change in capacity for activities of daily living.

Analysis 6.17. Comparison 6 Cognitive training vs control immediately post intervention - intervention dose, Outcome 17 Change in disease progression.

Analysis 6.18. Comparison 6 Cognitive training vs control immediately post intervention - intervention dose, Outcome 18 Change in behavioural and psychological symptoms of dementia (BPSD).

Analysis 6.19. Comparison 6 Cognitive training vs control immediately post intervention - intervention dose, Outcome 19 Change in attention and working memory.

Analysis 6.20. Comparison 6 Cognitive training vs control immediately post intervention - intervention dose, Outcome 20 Change in burden of care (CAREGIVER).

Analysis 6.21. Comparison 6 Cognitive training vs control immediately post intervention - intervention dose, Outcome 21 Change in quality of life (CAREGIVER).

Analysis 6.22. Comparison 6 Cognitive training vs control immediately post intervention - intervention dose, Outcome 22 Change in mood and well-being (CAREGIVER).

Analysis 6.23. Comparison 6 Cognitive training vs control immediately post intervention - intervention dose, Outcome 23 Participant burden (retention rates). 
Analysis 6.24. Comparison 6 Cognitive training vs control immediately post intervention - intervention dose, Outcome 24 Change in general health and quality of life.

Analysis 7.1. Comparison 7 Cognitive training vs control immediately post intervention - intervention duration, Outcome 1 Change in a global measure of cognition.

Analysis 7.2. Comparison 7 Cognitive training vs control immediately post intervention - intervention duration, Outcome 2 Change in a global measure of cognition_zero correlation.

Analysis 7.3. Comparison 7 Cognitive training vs control immediately post intervention - intervention duration, Outcome 3 Change in a global measure of cognition (composite).

Analysis 7.4. Comparison 7 Cognitive training vs control immediately post intervention - intervention duration, Outcome 4 Change in a global measure of cognition (composite)_zero correlation.

Analysis 7.5. Comparison 7 Cognitive training vs control immediately post intervention - intervention duration, Outcome 5 Change in immediate memory.

Analysis 7.6. Comparison 7 Cognitive training vs control immediately post intervention - intervention duration, Outcome 6 Change in delayed memory.

Analysis 7.7. Comparison 7 Cognitive training vs control immediately post intervention - intervention duration, Outcome 7 Change in attention and working memory.

Analysis 7.8. Comparison 7 Cognitive training vs control immediately post intervention - intervention duration, Outcome 8 Change in language (naming).

Analysis 7.9. Comparison 7 Cognitive training vs control immediately post intervention - intervention duration, Outcome 9 Change in verbal letter fluency.

Analysis 7.10. Comparison 7 Cognitive training vs control immediately post intervention - intervention duration, Outcome 10 Change in speed of information processing.

Analysis 7.11. Comparison 7 Cognitive training vs control immediately post intervention - intervention duration, Outcome 11 Change in executive function.

Analysis 7.12. Comparison 7 Cognitive training vs control immediately post intervention - intervention duration, Outcome 12 Change in verbal category fluency.

Analysis 7.13. Comparison 7 Cognitive training vs control immediately post intervention - intervention duration, Outcome 13 Change in meta cognition (self-reported).

Analysis 7.14. Comparison 7 Cognitive training vs control immediately post intervention - intervention duration, Outcome 14 Change in meta cognition (informant-reported).

Analysis 7.15. Comparison 7 Cognitive training vs control immediately post intervention - intervention duration, Outcome 15 Change in participants' mood.

Analysis 7.16. Comparison 7 Cognitive training vs control immediately post intervention - intervention duration, Outcome 16 Change in capacity for activities of daily living.

Analysis 7.17. Comparison 7 Cognitive training vs control immediately post intervention - intervention duration, Outcome 17 Change in disease progression.

Analysis 7.18. Comparison 7 Cognitive training vs control immediately post intervention - intervention duration, Outcome 18 Change in behavioural and psychological symptoms of dementia (BPSD).

Analysis 7.19. Comparison 7 Cognitive training vs control immediately post intervention - intervention duration, Outcome 19 Participant burden (retention rates).

Analysis 7.20. Comparison 7 Cognitive training vs control immediately post intervention - intervention duration, Outcome 20 Change in burden of care (CAREGIVER).

Analysis 7.21. Comparison 7 Cognitive training vs control immediately post intervention - intervention duration, Outcome 21 Change in quality of life (CAREGIVER).

Analysis 7.22. Comparison 7 Cognitive training vs control immediately post intervention - intervention duration, Outcome 22 Change in mood and well-being (CAREGIVER).

Analysis 7.23. Comparison 7 Cognitive training vs control immediately post intervention - intervention duration, Outcome 23 Change in general health and quality of life.

Analysis 8.1. Comparison 8 Cognitive training vs control immediately post intervention - type of CT (traditional vs augmented), Outcome 1 Change in a global measure of cognition.

Analysis 8.2. Comparison 8 Cognitive training vs control immediately post intervention - type of CT (traditional vs augmented), Outcome 2 Change in a global measure of cognition_zero correlation.

Analysis 8.3. Comparison 8 Cognitive training vs control immediately post intervention - type of CT (traditional vs augmented), Outcome 3 Change in a global measure of cognition (composite).

Analysis 8.4. Comparison 8 Cognitive training vs control immediately post intervention - type of CT (traditional vs augmented), Outcome 4 Change in a global measure of cognition (composite)_zero correlation. 
Analysis 8.5. Comparison 8 Cognitive training vs control immediately post intervention - type of CT (traditional vs augmented), Outcome 5 Change in immediate memory.

Analysis 8.6. Comparison 8 Cognitive training vs control immediately post intervention - type of CT (traditional vs augmented), Outcome 6 Change in delayed memory.

Analysis 8.7. Comparison 8 Cognitive training vs control immediately post intervention - type of CT (traditional vs augmented), Outcome 7 Change in attention and working memory.

Analysis 8.8. Comparison 8 Cognitive training vs control immediately post intervention - type of CT (traditional vs augmented), Outcome 8 Change in language (naming).

Analysis 8.9. Comparison 8 Cognitive training vs control immediately post intervention - type of CT (traditional vs augmented), Outcome 9 Change in verbal letter fluency.

Analysis 8.10. Comparison 8 Cognitive training vs control immediately post intervention - type of CT (traditional vs augmented), Outcome 10 Change in speed of information processing.

Analysis 8.11. Comparison 8 Cognitive training vs control immediately post intervention - type of CT (traditional vs augmented), Outcome 11 Change in executive function.

Analysis 8.12. Comparison 8 Cognitive training vs control immediately post intervention - type of CT (traditional vs augmented), Outcome 12 Change in verbal category fluency.

Analysis 8.13. Comparison 8 Cognitive training vs control immediately post intervention - type of CT (traditional vs augmented), Outcome 13 Change in meta cognition (self-reported).

Analysis 8.14. Comparison 8 Cognitive training vs control immediately post intervention - type of CT (traditional vs augmented), Outcome 14 Change in meta cognition (informant-reported).

Analysis 8.15. Comparison 8 Cognitive training vs control immediately post intervention - type of CT (traditional vs augmented), Outcome 15 Change in participants' mood.

Analysis 8.16. Comparison 8 Cognitive training vs control immediately post intervention - type of CT (traditional vs augmented), Outcome 16 Change in capacity for activities of daily living.

Analysis 8.17. Comparison 8 Cognitive training vs control immediately post intervention - type of CT (traditional vs augmented), Outcome 17 Change in disease progression.

Analysis 8.18. Comparison 8 Cognitive training vs control immediately post intervention - type of CT (traditional vs augmented), Outcome 18 Change in behavioural and psychological symptoms of dementia (BPSD).

Analysis 8.19. Comparison 8 Cognitive training vs control immediately post intervention - type of CT (traditional vs augmented), Outcome 19 Participant burden (retention rates).

Analysis 8.20. Comparison 8 Cognitive training vs control immediately post intervention - type of CT (traditional vs augmented), Outcome 20 Change in burden of care (CAREGIVER).

Analysis 8.21. Comparison 8 Cognitive training vs control immediately post intervention - type of CT (traditional vs augmented), Outcome 21 Change in quality of life (CAREGIVER).

Analysis 8.22. Comparison 8 Cognitive training vs control immediately post intervention - type of CT (traditional vs augmented), Outcome 22 Change in mood and well-being (CAREGIVER).

Analysis 8.23. Comparison 8 Cognitive training vs control immediately post intervention - type of CT (traditional vs augmented), Outcome 23 Change in general health and quality of life.

Analysis 9.1. Comparison 9 Cognitive training vs control immediately post intervention - type of CT (multi-domain vs single domain), Outcome 1 Change in a global measure of cognition.

Analysis 9.2. Comparison 9 Cognitive training vs control immediately post intervention - type of CT (multi-domain vs single domain), Outcome 2 Change in a global measure of cognition_zero correlation.

Analysis 9.3. Comparison 9 Cognitive training vs control immediately post intervention - type of CT (multi-domain vs single domain), Outcome 3 Change in a global measure of cognition (composite).

Analysis 9.4. Comparison 9 Cognitive training vs control immediately post intervention - type of CT (multi-domain vs single domain), Outcome 4 Change in a global measure of cognition (composite)_zero correlation.

Analysis 9.5. Comparison 9 Cognitive training vs control immediately post intervention - type of CT (multi-domain vs single domain), Outcome 5 Change in immediate memory.

Analysis 9.6. Comparison 9 Cognitive training vs control immediately post intervention - type of CT (multi-domain vs single domain), Outcome 6 Change in delayed memory.

Analysis 9.7. Comparison 9 Cognitive training vs control immediately post intervention - type of CT (multi-domain vs single domain), Outcome 7 Change in attention and working memory.

Analysis 9.8. Comparison 9 Cognitive training vs control immediately post intervention - type of CT (multi-domain vs single domain), Outcome 8 Change in language (naming).

Analysis 9.9. Comparison 9 Cognitive training vs control immediately post intervention - type of CT (multi-domain vs single domain), Outcome 9 Change in verbal letter fluency. 
Analysis 9.10. Comparison 9 Cognitive training vs control immediately post intervention - type of CT (multi-domain vs single domain), Outcome 10 Change in speed of information processing.

Analysis 9.11. Comparison 9 Cognitive training vs control immediately post intervention - type of CT (multi-domain vs single domain), Outcome 11 Change in executive function.

Analysis 9.12. Comparison 9 Cognitive training vs control immediately post intervention - type of CT (multi-domain vs single domain), Outcome 12 Change in verbal category fluency.

Analysis 9.13. Comparison 9 Cognitive training vs control immediately post intervention - type of CT (multi-domain vs single domain), Outcome 13 Change in meta cognition (self-reported).

Analysis 9.14. Comparison 9 Cognitive training vs control immediately post intervention - type of CT (multi-domain vs single domain), Outcome 14 Change in meta cognition (informant-reported).

Analysis 9.15. Comparison 9 Cognitive training vs control immediately post intervention - type of CT (multi-domain vs single domain), Outcome 15 Change in participants' mood.

Analysis 9.16. Comparison 9 Cognitive training vs control immediately post intervention - type of CT (multi-domain vs single domain), Outcome 16 Change in capacity for activities of daily living.

Analysis 9.17. Comparison 9 Cognitive training vs control immediately post intervention - type of CT (multi-domain vs single domain), Outcome 17 Change in disease progression.

Analysis 9.18. Comparison 9 Cognitive training vs control immediately post intervention - type of CT (multi-domain vs single domain), Outcome 18 Change in behavioural and psychological symptoms of dementia (BPSD).

Analysis 9.19. Comparison 9 Cognitive training vs control immediately post intervention - type of CT (multi-domain vs single domain), Outcome 19 Participant burden (retention rates).

Analysis 9.20. Comparison 9 Cognitive training vs control immediately post intervention - type of CT (multi-domain vs single domain), Outcome 20 Change in burden of care (CAREGIVER).

Analysis 9.21. Comparison 9 Cognitive training vs control immediately post intervention - type of CT (multi-domain vs single domain), Outcome 21 Change in quality of life (CAREGIVER).

Analysis 9.22. Comparison 9 Cognitive training vs control immediately post intervention - type of CT (multi-domain vs single domain), Outcome 22 Change in mood and well-being (CAREGIVER).

Analysis 9.23. Comparison 9 Cognitive training vs control immediately post intervention - type of CT (multi-domain vs single domain), Outcome 23 Change in general health and quality of life.

Analysis 10.1. Comparison 10 Cognitive training vs control immediately post intervention - type of control (passive vs active), Outcome 1 Change in a global measure of cognition.

Analysis 10.2. Comparison 10 Cognitive training vs control immediately post intervention - type of control (passive vs active), Outcome 2 Change in a global measure of cognition_zero correlation.

Analysis 10.3. Comparison 10 Cognitive training vs control immediately post intervention - type of control (passive vs active), Outcome 3 Change in a global measure of cognition (composite).

Analysis 10.4. Comparison 10 Cognitive training vs control immediately post intervention - type of control (passive vs active), Outcome 4 Change in a global measure of cognition (composite)_zero correlation.

Analysis 10.5. Comparison 10 Cognitive training vs control immediately post intervention - type of control (passive vs active), Outcome 5 Change in immediate memory.

Analysis 10.6. Comparison 10 Cognitive training vs control immediately post intervention - type of control (passive vs active), Outcome 6 Change in delayed memory.

Analysis 10.7. Comparison 10 Cognitive training vs control immediately post intervention - type of control (passive vs active), Outcome 7 Change in attention and working memory.

Analysis 10.8. Comparison 10 Cognitive training vs control immediately post intervention - type of control (passive vs active), Outcome 8 Change in language (naming).

Analysis 10.9. Comparison 10 Cognitive training vs control immediately post intervention - type of control (passive vs active), Outcome 9 Change in verbal letter fluency.

Analysis 10.10. Comparison 10 Cognitive training vs control immediately post intervention - type of control (passive vs active), Outcome 10 Change in speed of information processing.

Analysis 10.11. Comparison 10 Cognitive training vs control immediately post intervention - type of control (passive vs active), Outcome 11 Change in executive function.

Analysis 10.12. Comparison 10 Cognitive training vs control immediately post intervention - type of control (passive vs active), Outcome 12 Change in verbal category fluency.

Analysis 10.13. Comparison 10 Cognitive training vs control immediately post intervention - type of control (passive vs active), Outcome 13 Change in meta cognition (self-reported).

Analysis 10.14. Comparison 10 Cognitive training vs control immediately post intervention - type of control (passive vs active), Outcome 14 Change in meta cognition (informant-reported). 
Analysis 10.15. Comparison 10 Cognitive training vs control immediately post intervention - type of control (passive vs active), Outcome 15 Change in participants' mood.

Analysis 10.16. Comparison 10 Cognitive training vs control immediately post intervention - type of control (passive vs active), Outcome 16 Change in capacity for activities of daily living.

Analysis 10.17. Comparison 10 Cognitive training vs control immediately post intervention - type of control (passive vs active), Outcome 17 Change in disease progression.

Analysis 10.18. Comparison 10 Cognitive training vs control immediately post intervention - type of control (passive vs active), Outcome 18 Change in behavioural and psychological symptoms of dementia (BPSD).

Analysis 10.19. Comparison 10 Cognitive training vs control immediately post intervention - type of control (passive vs active), Outcome 19 Participant burden (retention rates).

Analysis 10.20. Comparison 10 Cognitive training vs control immediately post intervention - type of control (passive vs active), Outcome 20 Change in burden of care (CAREGIVER).

Analysis 10.21. Comparison 10 Cognitive training vs control immediately post intervention - type of control (passive vs active), Outcome 21 Change in quality of life (CAREGIVER).

Analysis 10.22. Comparison 10 Cognitive training vs control immediately post intervention - type of control (passive vs active), Outcome 22 Change in mood and well-being (CAREGIVER).

Analysis 10.23. Comparison 10 Cognitive training vs control immediately post intervention - type of control (passive vs active), Outcome 23 Change in general health and quality of life.

Analysis 11.1. Comparison 11 Cognitive training vs alternative treatment immediately post intervention - intervention dose, Outcome 1 Change in a global measure of cognition.

Analysis 11.2. Comparison 11 Cognitive training vs alternative treatment immediately post intervention - intervention dose, Outcome 2 Change in a global measure of cognition_zero correlation.

Analysis 11.3. Comparison 11 Cognitive training vs alternative treatment immediately post intervention - intervention dose, Outcome 3 Change in a global measure of cognition (composite).

Analysis 11.4. Comparison 11 Cognitive training vs alternative treatment immediately post intervention - intervention dose, Outcome 4 Change in a global measure of cognition (composite)_zero correlation.

Analysis 11.5. Comparison 11 Cognitive training vs alternative treatment immediately post intervention - intervention dose, Outcome 5 Change in immediate memory.

Analysis 11.6. Comparison 11 Cognitive training vs alternative treatment immediately post intervention - intervention dose, Outcome 6 Change in delayed memory.

Analysis 11.7. Comparison 11 Cognitive training vs alternative treatment immediately post intervention - intervention dose, Outcome 7 Change in attention and working memory.

Analysis 11.8. Comparison 11 Cognitive training vs alternative treatment immediately post intervention - intervention dose, Outcome 8 Change in language (naming).

Analysis 11.9. Comparison 11 Cognitive training vs alternative treatment immediately post intervention - intervention dose, Outcome 9 Change in verbal letter fluency.

Analysis 11.10. Comparison 11 Cognitive training vs alternative treatment immediately post intervention - intervention dose, Outcome 10 Change in verbal category fluency.

Analysis 11.11. Comparison 11 Cognitive training vs alternative treatment immediately post intervention - intervention dose, Outcome 11 Change in executive function.

Analysis 11.14. Comparison 11 Cognitive training vs alternative treatment immediately post intervention - intervention dose, Outcome 14 Change in participants' mood.

Analysis 11.15. Comparison 11 Cognitive training vs alternative treatment immediately post intervention - intervention dose, Outcome 15 Change in capacity for activities of daily living.

Analysis 11.16. Comparison 11 Cognitive training vs alternative treatment immediately post intervention - intervention dose, Outcome 16 Change in behavioural and psychological symptoms of dementia (BPSD).

Analysis 11.17. Comparison 11 Cognitive training vs alternative treatment immediately post intervention - intervention dose, Outcome 17 Change in disease progression.

Analysis 11.18. Comparison 11 Cognitive training vs alternative treatment immediately post intervention - intervention dose, Outcome 18 Participant burden (retention rates).

Analysis 11.19. Comparison 11 Cognitive training vs alternative treatment immediately post intervention - intervention dose, Outcome 19 Change in mood and well-being (CAREGIVER).

Analysis 11.20. Comparison 11 Cognitive training vs alternative treatment immediately post intervention - intervention dose, Outcome 20 Change in burden of care (CAREGIVER).

Analysis 11.21. Comparison 11 Cognitive training vs alternative treatment immediately post intervention - intervention dose, Outcome 21 Change in quality of life (CAREGIVER). 
Analysis 11.22. Comparison 11 Cognitive training vs alternative treatment immediately post intervention - intervention dose, Outcome 22 Change in speed of information processing.

Analysis 11.23. Comparison 11 Cognitive training vs alternative treatment immediately post intervention - intervention dose, Outcome 23 Change in general health and quality of life.

Analysis 12.1. Comparison 12 Cognitive training vs alternative treatment immediately post intervention - type of CT (traditional vs augmented), Outcome 1 Change in a global measure of cognition.

Analysis 12.2. Comparison 12 Cognitive training vs alternative treatment immediately post intervention - type of CT (traditional vs augmented), Outcome 2 Change in a global measure of cognition_zero correlation.

Analysis 12.3. Comparison 12 Cognitive training vs alternative treatment immediately post intervention - type of CT (traditional vs augmented), Outcome 3 Change in a global measure of cognition (composite).

Analysis 12.4. Comparison 12 Cognitive training vs alternative treatment immediately post intervention - type of CT (traditional vs augmented), Outcome 4 Change in a global measure of cognition (composite)_zero correlation.

Analysis 12.5. Comparison 12 Cognitive training vs alternative treatment immediately post intervention - type of CT (traditional vs augmented), Outcome 5 Change in immediate memory.

Analysis 12.6. Comparison 12 Cognitive training vs alternative treatment immediately post intervention - type of CT (traditional vs augmented), Outcome 6 Change in delayed memory.

Analysis 12.7. Comparison 12 Cognitive training vs alternative treatment immediately post intervention - type of CT (traditional vs augmented), Outcome 7 Change in attention and working memory.

Analysis 12.8. Comparison 12 Cognitive training vs alternative treatment immediately post intervention - type of CT (traditional vs augmented), Outcome 8 Change in language (naming).

Analysis 12.9. Comparison 12 Cognitive training vs alternative treatment immediately post intervention - type of CT (traditional vs augmented), Outcome 9 Change in verbal letter fluency.

Analysis 12.10. Comparison 12 Cognitive training vs alternative treatment immediately post intervention - type of CT (traditional vs augmented), Outcome 10 Change in verbal category fluency.

Analysis 12.11. Comparison 12 Cognitive training vs alternative treatment immediately post intervention - type of CT (traditional vs augmented), Outcome 11 Change in executive function.

Analysis 12.14. Comparison 12 Cognitive training vs alternative treatment immediately post intervention - type of CT (traditional vs augmented), Outcome 14 Change in participants' mood.

Analysis 12.15. Comparison 12 Cognitive training vs alternative treatment immediately post intervention - type of CT (traditional vs augmented), Outcome 15 Change in capacity for activities of daily living.

Analysis 12.16. Comparison 12 Cognitive training vs alternative treatment immediately post intervention - type of CT (traditional vs augmented), Outcome 16 Change in behavioural and psychological symptoms of dementia (BPSD).

Analysis 12.17. Comparison 12 Cognitive training vs alternative treatment immediately post intervention - type of CT (traditional vs augmented), Outcome 17 Change in disease progression.

Analysis 12.18. Comparison 12 Cognitive training vs alternative treatment immediately post intervention - type of CT (traditional vs augmented), Outcome 18 Participant burden (retention rates).

Analysis 12.19. Comparison 12 Cognitive training vs alternative treatment immediately post intervention - type of CT (traditional vs augmented), Outcome 19 Change in mood and well-being (CAREGIVER).

Analysis 12.20. Comparison 12 Cognitive training vs alternative treatment immediately post intervention - type of CT (traditional vs augmented), Outcome 20 Change in burden of care (CAREGIVER).

Analysis 12.21. Comparison 12 Cognitive training vs alternative treatment immediately post intervention - type of CT (traditional vs augmented), Outcome 21 Change in quality of life (CAREGIVER).

Analysis 12.22. Comparison 12 Cognitive training vs alternative treatment immediately post intervention - type of CT (traditional vs augmented), Outcome 22 Change in speed of information processing. ............................................................ Analysis 12.23. Comparison 12 Cognitive training vs alternative treatment immediately post intervention - type of CT (traditional vs augmented), Outcome 23 Change in general health and quality of life.

Analysis 13.1. Comparison 13 Cognitive training vs control in the medium term ( 3 to 12 months post intervention) - follow-up period, Outcome 1 Change in a global measure of cognition.

Analysis 13.2. Comparison 13 Cognitive training vs control in the medium term ( 3 to 12 months post intervention) - follow-up period, Outcome 2 Change in a global measure of cognition (zero correlation).

Analysis 13.3. Comparison 13 Cognitive training vs control in the medium term ( 3 to 12 months post intervention) - follow-up period, Outcome 3 Change in a global measure of cognition (composite).

Analysis 13.4. Comparison 13 Cognitive training vs control in the medium term ( 3 to 12 months post intervention) - follow-up period, Outcome 4 Change in a global measure of cognition (composite)_zero correlation.

Analysis 13.5. Comparison 13 Cognitive training vs control in the medium term ( 3 to 12 months post intervention) - follow-up period, Outcome 5 Change in disease progression (zero correlation). 
Analysis 13.6. Comparison 13 Cognitive training vs control in the medium term ( 3 to 12 months post intervention) - follow-up period, Outcome 6 Change in disease progression.

Analysis 13.7. Comparison 13 Cognitive training vs control in the medium term ( 3 to 12 months post intervention) - follow-up period, Outcome 7 Change in immediate memory.

Analysis 13.8. Comparison 13 Cognitive training vs control in the medium term ( 3 to 12 months post intervention) - follow-up period, Outcome 8 Change in delayed memory.

Analysis 13.9. Comparison 13 Cognitive training vs control in the medium term ( 3 to 12 months post intervention) - follow-up period, Outcome 9 Change in language (naming).

Analysis 13.10. Comparison 13 Cognitive training vs control in the medium term ( 3 to 12 months post intervention) - follow-up period, Outcome 10 Change in verbal letter fluency.

Analysis 13.11. Comparison 13 Cognitive training vs control in the medium term ( 3 to 12 months post intervention) - follow-up period, Outcome 11 Change in verbal category fluency.

Analysis 13.12. Comparison 13 Cognitive training vs control in the medium term ( 3 to 12 months post intervention) - follow-up period, Outcome 12 Change in attention and working memory.

Analysis 13.13. Comparison 13 Cognitive training vs control in the medium term ( 3 to 12 months post intervention) - follow-up period, Outcome 13 Change in speed of information processing.

Analysis 13.14. Comparison 13 Cognitive training vs control in the medium term ( 3 to 12 months post intervention) - follow-up period, Outcome 14 Change in meta cognition (self-reported).

Analysis 13.15. Comparison 13 Cognitive training vs control in the medium term ( 3 to 12 months post intervention) - follow-up period, Outcome 15 Change in meta cognition (informant-reported).

Analysis 13.16. Comparison 13 Cognitive training vs control in the medium term ( 3 to 12 months post intervention) - follow-up period, Outcome 16 Change in capacity for activities of daily living.

Analysis 13.17. Comparison 13 Cognitive training vs control in the medium term ( 3 to 12 months post intervention) - follow-up period, Outcome 17 Change in behavioural and psychological symptoms of dementia (BPSD).

Analysis 13.18. Comparison 13 Cognitive training vs control in the medium term ( 3 to 12 months post intervention) - follow-up period, Outcome 18 Change in general health and quality of life.

Analysis 13.19. Comparison 13 Cognitive training vs control in the medium term ( 3 to 12 months post intervention) - follow-up period, Outcome 19 Change in participants' mood.

Analysis 13.23. Comparison 13 Cognitive training vs control in the medium term ( 3 to 12 months post intervention) - follow-up period, Outcome 23 Change in executive function.

ADDITIONAL TABLES

APPENDICES

CONTRIBUTIONS OF AUTHORS 
[Intervention Review]

\title{
Cognitive training for people with mild to moderate dementia
}

\author{
Alex Bahar-Fuchs ${ }^{1}$, Anthony Martyr ${ }^{2}$, Anita MY Goh ${ }^{1}$, Julieta Sabates ${ }^{1}$, Linda Clare 2 \\ ${ }^{1}$ Academic Unit for Psychiatry of Old Age, Department of Psychiatry, University of Melbourne, Melbourne, Australia. ${ }^{2} \mathrm{REACH}$ : The Centre \\ for Research in Ageing and Cognitive Health, University of Exeter, Exeter, UK
}

Contact address: Alex Bahar-Fuchs, Academic Unit for Psychiatry of Old Age, Department of Psychiatry, University of Melbourne, 34-54 Poplar Road, Parkville, Melbourne, Victoria, 3052, Australia. alex.bahar@unimelb.edu.au, alex.baharfuchs@anu.edu.au.

Editorial group: Cochrane Dementia and Cognitive Improvement Group

Publication status and date: New, published in Issue 3, 2019.

Citation: Bahar-Fuchs A, Martyr A, Goh AMY, Sabates J, Clare L. Cognitive training for people with mild to moderate dementia. Cochrane Database of Systematic Reviews 2019, Issue 3. Art. No.: CD013069. DOI: 10.1002/14651858.CD013069.pub2.

Copyright (c 2019 The Cochrane Collaboration. Published by John Wiley \& Sons, Ltd.

\section{A B S T R A C T}

\section{Background}

Cognitive impairment, a defining feature of dementia, plays an important role in the compromised functional independence that characterises the condition. Cognitive training (CT) is an approach that uses guided practice on structured tasks with the direct aim of improving or maintaining cognitive abilities.

\section{Objectives}

- To assess effects of CT on cognitive and non-cognitive outcomes for people with mild to moderate dementia and their caregivers.

- To compare effects of CT with those of other non-pharmacological interventions, including cognitive stimulation or rehabilitation, for people with mild to moderate dementia and their caregivers.

- To identify and explore factors related to intervention and trial design that may be associated with the efficacy of CT for people with mild to moderate dementia and their caregivers.

\section{Search methods}

We searched ALOIS, the Cochrane Dementia and Cognitive Improvement Group Specialised Register, on 5 July 2018. ALOIS contains records of clinical trials identified through monthly searches of several major healthcare databases and numerous trial registries and grey literature sources. In addition to this, we searched MEDLINE, Embase, PsycINFO, CINAHL, LILACS, Web of Science Core Collection, ClinicalTrials.gov, and the World Health Organization's trials portal, ICTRP, to ensure that searches were comprehensive and up-to-date.

\section{Selection criteria}

We included randomised controlled trials (RCTs) that described interventions for people with mild to moderate dementia and compared $\mathrm{CT}$ versus a control or alternative intervention.

\section{Data collection and analysis}

We extracted relevant data from published manuscripts and through contact with trial authors if required. We assessed risk of bias using the Cochrane 'Risk of bias' tool. We divided comparison conditions into active or passive control conditions and alternative treatments. We used a large number of measures and data to evaluate 19 outcomes at end of treatment, as well as 16 outcomes at follow-up in the medium term; we pooled this information in meta-analyses. We calculated pooled estimates of treatment effect using a random-effects model, and we estimated statistical heterogeneity using a standard $\mathrm{Chi}^{2}$ statistic. We graded the evidence using GradePro. 


\section{Main results}

The 33 included trials were published between 1988 and 2018 and were conducted in 12 countries; most were unregistered, parallel-group, single-site RCTs, with samples ranging from 12 to 653 participants. Interventions were between two and 104 weeks long. We classified most experimental interventions as 'straight CT', but we classified some as 'augmented CT', and about two-thirds as multi-domain interventions. Researchers investigated 18 passive and 13 active control conditions, along with 15 alternative treatment conditions, including occupational therapy, mindfulness, reminiscence therapy, and others.

The methodological quality of studies varied, but we rated nearly all studies as having high or unclear risk of selection bias due to lack of allocation concealment, and high or unclear risk of performance bias due to lack of blinding of participants and personnel.

We used data from 32 studies in the meta-analysis of at least one outcome. Relative to a control condition, we found moderate-quality evidence showing a small to moderate effect of CT on our first primary outcome, composite measure of global cognition at end of treatment (standardised mean difference (SMD) $0.42,95 \%$ confidence interval ( $\mathrm{Cl}) 0.23$ to 0.62 ), and high-quality evidence showing a moderate effect on the secondary outcome of verbal semantic fluency (SMD $0.52,95 \% \mathrm{Cl} 0.23$ to 0.81 ) at end of treatment, with these gains retained in the medium term ( 3 to 12 months post treatment). In relation to many other outcomes, including our second primary outcome of clinical disease severity in the medium term, the quality of evidence was very low, so we were unable to determine whether CT was associated with any meaningful gains.

When compared with an alternative treatment, we found that CT may have little to no effect on our first primary outcome of global cognition at end of treatment (SMD $0.21,95 \% \mathrm{Cl}-0.23$ to 0.64 ), but the quality of evidence was low. No evidence was available to assess our second primary outcome of clinical disease severity in the medium term. We found moderate-quality evidence showing that CT was associated with improved mood of the caregiver at end of treatment, but this was based on a single trial. The quality of evidence in relation to many other outcomes at end of treatment and in the medium term was too low for us to determine whether CT was associated with any gains, but we are moderately confident that CT did not lead to any gains in mood, behavioural and psychological symptoms, or capacity to perform activities of daily living.

\section{Authors' conclusions}

Relative to a control intervention, but not to a variety of alternative treatments, $\mathrm{CT}$ is probably associated with small to moderate positive effects on global cognition and verbal semantic fluency at end of treatment, and these benefits appear to be maintained in the medium term. Our certainty in relation to many of these findings is low or very low. Future studies should take stronger measures to mitigate wellestablished risks of bias, and should provide long-term follow-up to improve our understanding of the extent to which observed gains are retained. Future trials should also focus on direct comparison of CT versus alternative treatments rather than passive or active control conditions.

\section{PLAIN LANGUAGE SUMMARY}

\section{Cognitive training for people with mild to moderate dementia}

\section{Background}

Dementia due to Alzheimer's and other diseases is a leading cause of disability and an enormous health and societal problem. More than 40 million people in the world currently live with dementia, and this number is expected to increase to more than 115 million by the year 2050. Effective treatments to reduce the burden of dementia are urgently needed. Cognitive training (CT) is a non-pharmacological form of treatment that focuses on guided practice on tasks that target specific cognitive functions, such as memory, attention, or problem-solving. Whether CT can help people with mild to moderate dementia maintain or improve their thinking, well-being, and general functioning remains unclear.

\section{Main findings}

We analysed data from 33 studies of CT that included a total of approximately 2000 participants and were conducted in 12 countries. We found that, compared with receiving usual treatment or engaging in non-specific activities, people completing CT may show some benefits in overall cognition, as well as in more specific cognitive abilities such as verbal fluency, and that improvements may last for at least a few months. We did not find any evidence that participating in CT was associated with increased burden for participants. However, we also found no evidence that CT was better than participating in other active treatments.

\section{Limitations of this review}

The quality of the studies we reviewed varied but overall was not very high, so our certainty in some of these findings is low. Future studies should continue improving on quality, should continue comparing CT with other treatments, and should follow participants for a longer period to understand whether observed benefits for cognition last beyond the short or medium term. 
S U M MARY OF FINDINGS

Summary of findings for the main comparison. Cognitive training compared to control immediately post intervention for people with mild to moderate dementia

Cognitive training compared to control immediately post intervention for people with mild to moderate dementia

Patient or population: people with mild to moderate dementia

Setting: Community dwelling or in residential care

Intervention: cognitive training

Comparison: control immediately post intervention

\begin{tabular}{|c|c|c|c|c|c|c|}
\hline \multirow[t]{2}{*}{ Outcomes } & \multicolumn{2}{|c|}{ Anticipated absolute effects ${ }^{\star}(95 \% \mathrm{Cl})$} & \multirow{2}{*}{$\begin{array}{l}\text { Relative ef- } \\
\text { fect } \\
(95 \% \mathrm{CI})\end{array}$} & \multirow{2}{*}{$\begin{array}{l}\text { No. of partic- } \\
\text { ipants } \\
\text { (studies) }\end{array}$} & \multirow{2}{*}{$\begin{array}{l}\text { Certainty of } \\
\text { the evidence } \\
\text { (GRADE) }\end{array}$} & \multirow[t]{2}{*}{ Comments } \\
\hline & $\begin{array}{l}\text { Risk with control immedi- } \\
\text { ately post intervention }\end{array}$ & $\begin{array}{l}\text { Risk with cognitive train- } \\
\text { ing }\end{array}$ & & & & \\
\hline $\begin{array}{l}\text { Change in a global } \\
\text { measure of cognition } \\
\text { (composite) }\end{array}$ & $\begin{array}{l}\text { Mean change in a global mea- } \\
\text { sure of cognition (composite) } \\
\text { was } 0\end{array}$ & $\begin{array}{l}\text { SMD } 0.42 \text { higher } \\
(0.23 \text { higher to } 0.62 \text { high- } \\
\text { er) }\end{array}$ & - & $\begin{array}{l}1389 \\
\text { (27 RCTs) }\end{array}$ & $\begin{array}{l}\oplus \oplus \oplus \ominus \\
\text { MODERATE } a\end{array}$ & $\begin{array}{l}\text { Cognitive training probably has } \\
\text { a modest effect on global cog- } \\
\text { nition (based on a composite } \\
\text { score) }\end{array}$ \\
\hline $\begin{array}{l}\text { Change in a global } \\
\text { measure of cognition }\end{array}$ & $\begin{array}{l}\text { Mean change in a global mea- } \\
\text { sure of cognition was } 0\end{array}$ & $\begin{array}{l}\text { SMD } 0.65 \text { higher } \\
(0.26 \text { higher to } 1.05 \text { high- } \\
\text { er) }\end{array}$ & - & $\begin{array}{l}1288 \\
\text { (20 RCTs) }\end{array}$ & $\begin{array}{l}\oplus \oplus \ominus \ominus \\
\mathrm{LOW} b\end{array}$ & $\begin{array}{l}\text { Cognitive training may have } \\
\text { a moderate effect on perfor- } \\
\text { mance in global cognition } \\
\text { (based on a screening mea- } \\
\text { sure). }\end{array}$ \\
\hline $\begin{array}{l}\text { Change in delayed } \\
\text { memory }\end{array}$ & $\begin{array}{l}\text { Mean change in delayed } \\
\text { memory was } 0\end{array}$ & $\begin{array}{l}\text { SMD } 0.81 \text { higher } \\
\text { ( } 0.29 \text { higher to } 1.32 \text { high- } \\
\text { er) }\end{array}$ & - & $\begin{array}{l}543 \\
(11 \text { RCTs })\end{array}$ & $\begin{array}{l}\oplus \Theta \Theta \Theta \\
\text { VERY LOWb,c }\end{array}$ & $\begin{array}{l}\text { We are unable to determine } \\
\text { whether there is any effect on } \\
\text { delayed memory due to the } \\
\text { very low quality of evidence }\end{array}$ \\
\hline $\begin{array}{l}\text { Change in partici- } \\
\text { pants' mood }\end{array}$ & $\begin{array}{l}\text { Mean change in participants' } \\
\text { mood was } 0\end{array}$ & $\begin{array}{l}\text { SMD } 0.72 \text { higher } \\
\text { ( } 0.1 \text { lower to } 1.54 \text { higher) }\end{array}$ & - & $\begin{array}{l}577 \\
\text { (8 RCTs) }\end{array}$ & $\begin{array}{l}\oplus \odot \Theta \odot \\
\text { VERY LOWb,d }\end{array}$ & $\begin{array}{l}\text { We are unable to determine } \\
\text { whether there is any effect on } \\
\text { participants' mood due to the } \\
\text { very low quality of evidence }\end{array}$ \\
\hline $\begin{array}{l}\text { Change in capacity } \\
\text { for activities of daily } \\
\text { living }\end{array}$ & $\begin{array}{l}\text { Mean change in capacity for } \\
\text { activities of daily living was } 0 \\
\text { SD }\end{array}$ & $\begin{array}{l}\text { SMD } 0.12 \text { SD higher } \\
\text { ( } 0.11 \text { lower to } 0.35 \text { higher) }\end{array}$ & - & $\begin{array}{l}687 \\
(10 \mathrm{RCTs})\end{array}$ & $\begin{array}{l}\oplus \oplus \ominus \odot \\
\text { LOWd }\end{array}$ & $\begin{array}{l}\text { Cognitive training may not have } \\
\text { an effect on capacity for activi- } \\
\text { ties of daily living }\end{array}$ \\
\hline $\begin{array}{l}\text { Participant burden } \\
\text { (retention rates) }\end{array}$ & Study population & & $\begin{array}{l}\text { OR } 0.73 \\
\text { (0.37 to } 1.43 \text { ) }\end{array}$ & $\begin{array}{l}1282 \\
\text { (17 RCTs) }\end{array}$ & $\begin{array}{l}\oplus \oplus \ominus \ominus \\
\text { LOWe }\end{array}$ & $\begin{array}{l}\text { Cognitive training may not be } \\
\text { associated with an increase in }\end{array}$ \\
\hline
\end{tabular}

associated with an increase in 


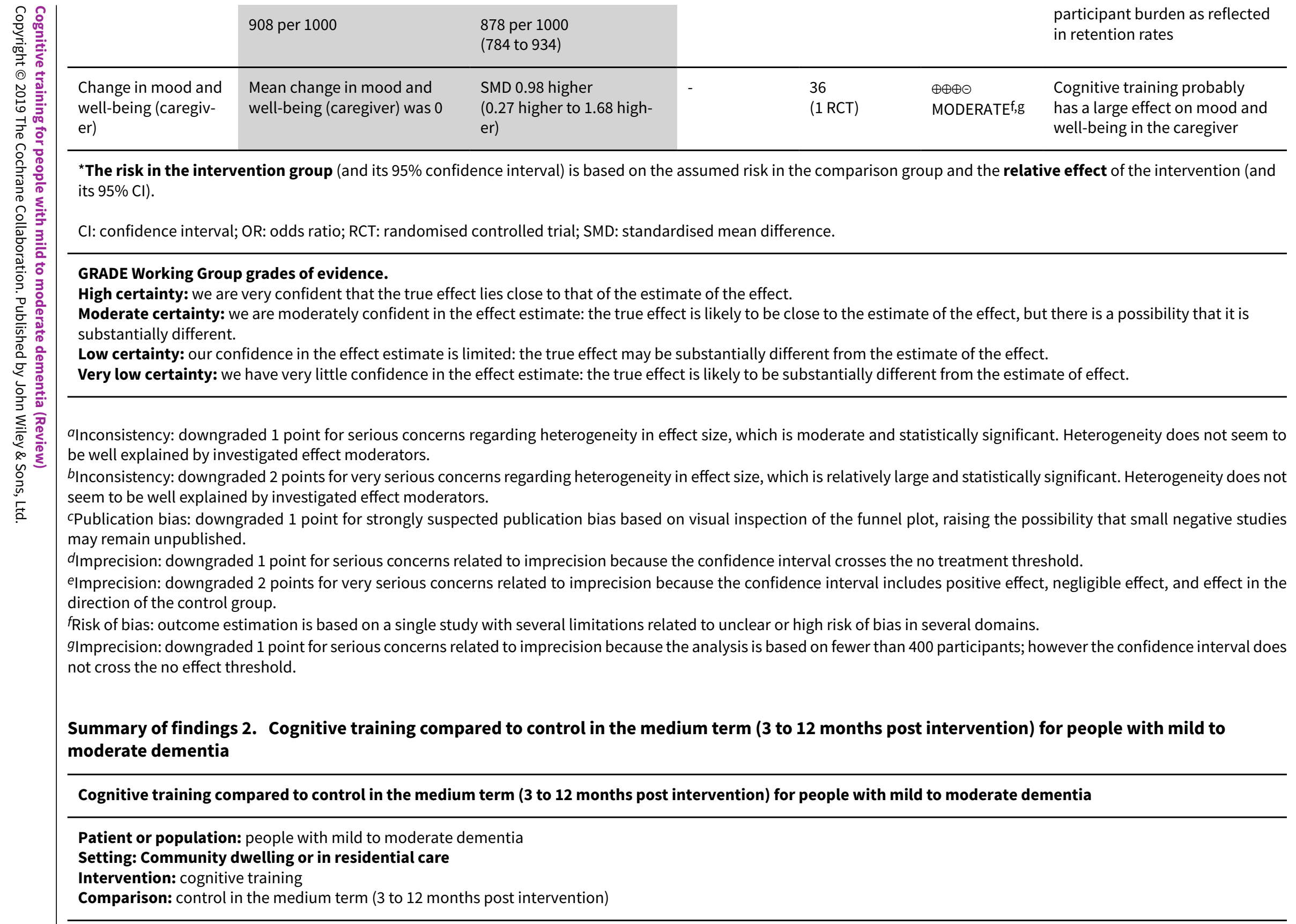

${ }^{*}$ The risk in the intervention group (and its 95\% confidence interval) is based on the assumed risk in the comparison group and the relative effect of the intervention (and its $95 \% \mathrm{Cl})$.

GRADE Working Group grades of evidence.

High certainty: we are very confident that the true effect lies close to that of the estimate of the effect.

substantially different.

Low certainty: our confidence in the effect estimate is limited: the true effect may be substantially different from the estimate of the effect.

Very low certainty: we have very little confidence in the effect estimate: the true effect is likely to be substantially different from the estimate of effect.

Inconsistency: downgraded 1 point for serious concerns regarding heterogeneity in effect size, which is moderate and statistically significant. Heterogeneity does not seem to bInconsisto seem to be well explained by investigated effect moderators.

may remain unpublished.

eImprecision: downgraded 2 points for very serious concerns related to imprecision because the confidence interval includes positive effect, negligible effect, and effect in the ction of the control group.

IImprecision: downgraded 1 point for serious concerns related to imprecision because the analysis is based on fewer than 400 participants; however the confidence interval does

Summary of findings 2. Cognitive training compared to control in the medium term ( 3 to 12 months post intervention) for people with mild to moderate dementia 


\begin{tabular}{|c|c|c|c|c|c|c|}
\hline \multirow[t]{2}{*}{ Outcomes } & \multicolumn{2}{|c|}{ Anticipated absolute effects ${ }^{\star}(95 \% \mathrm{Cl})$} & \multirow{2}{*}{$\begin{array}{l}\text { Relative ef- } \\
\text { fect } \\
(95 \% \mathrm{CI})\end{array}$} & \multirow{2}{*}{$\begin{array}{l}\text { No. of partic- } \\
\text { ipants } \\
\text { (studies) }\end{array}$} & \multirow{2}{*}{$\begin{array}{l}\text { Certainty of } \\
\text { the evidence } \\
\text { (GRADE) }\end{array}$} & \multirow[t]{2}{*}{ Comments } \\
\hline & $\begin{array}{l}\text { Risk with control in the medium } \\
\text { term ( } 3 \text { to } 12 \text { months post inter- } \\
\text { vention) }\end{array}$ & $\begin{array}{l}\text { Risk with cognitive } \\
\text { training }\end{array}$ & & & & \\
\hline $\begin{array}{l}\text { Change in a global } \\
\text { measure of cognition } \\
\text { (composite) }\end{array}$ & $\begin{array}{l}\text { Mean change in a global measure } \\
\text { of cognition (composite) was set at } \\
0 \text { SDs }\end{array}$ & $\begin{array}{l}\text { SMD } 0.65 \text { higher } \\
\text { (0.11 higher to } 1.2 \text { high- } \\
\text { er) }\end{array}$ & - & $\begin{array}{l}387 \\
(8 \mathrm{RCTs})\end{array}$ & $\begin{array}{l}\oplus \odot \Theta \odot \\
\text { VERY LOWa,b,c }\end{array}$ & $\begin{array}{l}\text { We are unable to determine } \\
\text { whether there is any effect } \\
\text { on global cognition (com- } \\
\text { posite) due to the very low } \\
\text { quality of evidence }\end{array}$ \\
\hline $\begin{array}{l}\text { Change on global } \\
\text { cognition (screening) } \\
\text { (Global cog) }\end{array}$ & $\begin{array}{l}\text { Mean change in global cognition } \\
\text { (screening) was set at } 0 \text { SDs }\end{array}$ & $\begin{array}{l}\text { SMD } 1.33 \text { higher } \\
\text { ( } 0.31 \text { higher to } 2.34 \text { high- } \\
\text { er) }\end{array}$ & - & $\begin{array}{l}387 \\
(6 \mathrm{RCTs})\end{array}$ & $\begin{array}{l}\oplus \odot \Theta \Theta \\
\text { VERY LOWa,d,e }\end{array}$ & $\begin{array}{l}\text { We are unable to determine } \\
\text { whether there is any effect } \\
\text { on performance in global } \\
\text { cognition due to the very } \\
\text { low quality of evidence }\end{array}$ \\
\hline $\begin{array}{l}\text { Change in disease } \\
\text { progression }\end{array}$ & $\begin{array}{l}\text { Mean change in disease progres- } \\
\text { sion was set at } 0 \text { SDs }\end{array}$ & $\begin{array}{l}\text { SMD } 0.55 \text { higher } \\
\text { ( } 0.12 \text { higher to } 0.98 \text { high- } \\
\text { er) }\end{array}$ & - & $\begin{array}{l}98 \\
\text { (3 RCTs) }\end{array}$ & $\begin{array}{l}\oplus \odot \Theta \odot \\
\text { VERY LOWa,c }\end{array}$ & $\begin{array}{l}\text { We are unable to determine } \\
\text { whether CT slows down dis- } \\
\text { ease progression due to the } \\
\text { very low quality of evidence }\end{array}$ \\
\hline $\begin{array}{l}\text { Change in delayed } \\
\text { memory }\end{array}$ & $\begin{array}{l}\text { Mean change in delayed memory } \\
\text { was set at } 0 \text { SDs }\end{array}$ & $\begin{array}{l}\text { SMD } 0.97 \text { SD higher } \\
\text { ( } 0.02 \text { higher to } 1.92 \text { high- } \\
\text { er) }\end{array}$ & - & $\begin{array}{l}253 \\
(4 \mathrm{RCTs})\end{array}$ & $\begin{array}{l}\oplus \Theta \Theta \Theta \\
\text { VERY LOWa,c,d }\end{array}$ & $\begin{array}{l}\text { We are unable to determine } \\
\text { whether there is any effect } \\
\text { on performance in delayed } \\
\text { memory due to the very low } \\
\text { quality of evidence }\end{array}$ \\
\hline $\begin{array}{l}\text { Change in capacity } \\
\text { for activities of daily } \\
\text { living }\end{array}$ & $\begin{array}{l}\text { Mean change in capacity for activi- } \\
\text { ties of daily living was set at } 0 \text { SDs }\end{array}$ & $\begin{array}{l}\text { SMD } 0.22 \text { higher } \\
\text { ( } 0.5 \text { lower to } 0.94 \text { higher) }\end{array}$ & - & $\begin{array}{l}64 \\
(3 \mathrm{RCTS})\end{array}$ & $\begin{array}{l}\oplus \oplus \ominus \ominus \\
\text { LOWC }\end{array}$ & $\begin{array}{l}\text { Cognitive training may not } \\
\text { have an effect on capacity } \\
\text { for activities of daily living }\end{array}$ \\
\hline $\begin{array}{l}\text { Change in partici- } \\
\text { pants' mood }\end{array}$ & $\begin{array}{l}\text { Mean change in participants' mood } \\
\text { was set at 0 SDs }\end{array}$ & $\begin{array}{l}\text { SMD } 0.21 \text { higher } \\
\text { (0.54 lower to } 0.96 \text { high- } \\
\text { er) }\end{array}$ & - & $\begin{array}{l}30 \\
(2 \mathrm{RCTs})\end{array}$ & $\begin{array}{l}\oplus \oplus \ominus \ominus \\
\text { LOWC }\end{array}$ & $\begin{array}{l}\text { Cognitive training may not } \\
\text { have an effect on partici- } \\
\text { pants' mood }\end{array}$ \\
\hline $\begin{array}{l}\text { Change in mood and } \\
\text { well-being (caregiv- } \\
\text { er) }\end{array}$ & - & See comment & - & (0 studies) & - & $\begin{array}{l}\text { No studies have evaluated } \\
\text { this outcome in the inter- } \\
\text { mediate term }\end{array}$ \\
\hline
\end{tabular}

${ }^{\star}$ The risk in the intervention group (and its $95 \%$ confidence interval) is based on the assumed risk in the comparison group and the relative effect of the intervention (and its $95 \% \mathrm{Cl})$. 
GRADE Working Group grades of evidence.

High certainty: we are very confident that the true effect lies close to that of the estimate of the effect.

Moderate certainty: we are moderately confident in the effect estimate: the true effect is likely to be close to the estimate of the effect, but there is a possibility that it is

substantially different.

Low certainty: our confidence in the effect estimate is limited: the true effect may be substantially different from the estimate of the effect.

Very low certainty: we have very little confidence in the effect estimate: the true effect is likely to be substantially different from the estimate of effect.

aRisk of bias: downgraded 2 points for very serious concerns related to risk of bias: removal of high-risk studies leads to reasonably large changes in the effect estimate.

$b$ Inconsistency: downgraded 1 point for serious concerns regarding heterogeneity in effect size, which is large and statistically significant. However, heterogeneity seems to be partially explained by investigated effect moderators.

Imprecision: downgraded 2 points for very serious concerns related to imprecision because the analysis is based on fewer than 400 participants, and the confidence interval crosses the no effect threshold.

$d$ Inconsistency: downgraded 2 points for very serious concerns regarding heterogeneity in effect size, which is relatively large and statistically significant. Heterogeneity does not seem to be well explained by investigated effect moderators.

elmprecision: downgraded 1 point for serious concerns related to imprecision because the analysis is based on fewer than 400 participants; however the confidence interval does not cross the no effect threshold.

\section{Summary of findings 3. Cognitive training compared to alternative treatment immediately post intervention for people with mild to moderate} dementia

\section{Cognitive training compared to alternative treatment immediately post intervention for people with mild to moderate dementia}

Patient or population: people with mild to moderate dementia

Setting: Community dwelling or in residential care

Intervention: cognitive training

Comparison: alternative treatment immediately post intervention

\begin{tabular}{|c|c|c|c|c|c|c|}
\hline \multirow[t]{2}{*}{ Outcomes } & \multicolumn{2}{|c|}{ Anticipated absolute effects ${ }^{\star}(95 \% \mathrm{Cl})$} & \multirow{2}{*}{$\begin{array}{l}\text { Relative ef- } \\
\text { fect } \\
(95 \% \mathrm{Cl})\end{array}$} & \multirow{2}{*}{$\begin{array}{l}\text { No. of partic- } \\
\text { ipants } \\
\text { (studies) }\end{array}$} & \multirow{2}{*}{$\begin{array}{l}\text { Certainty of } \\
\text { the evidence } \\
\text { (GRADE) }\end{array}$} & \multirow[t]{2}{*}{ Comments } \\
\hline & $\begin{array}{l}\text { Risk with alternative treat- } \\
\text { ment immediately post inter- } \\
\text { vention }\end{array}$ & $\begin{array}{l}\text { Risk with cognitive train- } \\
\text { ing }\end{array}$ & & & & \\
\hline $\begin{array}{l}\text { Change in a global } \\
\text { measure of cognition } \\
\text { (composite) }\end{array}$ & $\begin{array}{l}\text { Mean change in a global mea- } \\
\text { sure of cognition (composite) } \\
\text { was 0 SD }\end{array}$ & $\begin{array}{l}\text { SMD } 0.21 \text { SD higher } \\
\text { ( } 0.23 \text { lower to } 0.64 \text { higher) }\end{array}$ & - & $\begin{array}{l}769 \\
\text { (7 RCTs) }\end{array}$ & $\begin{array}{l}\oplus \oplus \ominus \ominus \\
\text { LOWa }\end{array}$ & $\begin{array}{l}\text { Cognitive training may not } \\
\text { have an effect on global cog- } \\
\text { nition }\end{array}$ \\
\hline $\begin{array}{l}\text { Change in a global } \\
\text { measure of cognition }\end{array}$ & $\begin{array}{l}\text { Mean change in a global mea- } \\
\text { sure of cognition was } 0\end{array}$ & $\begin{array}{l}\text { SMD } 0.16 \text { higher } \\
\text { ( } 0.28 \text { lower to } 0.6 \text { higher) }\end{array}$ & - & $\begin{array}{l}724 \\
(7 \mathrm{RCTs})\end{array}$ & $\begin{array}{l}\oplus \odot \Theta \odot \\
\text { VERY LOWa,b }\end{array}$ & $\begin{array}{l}\text { We are unable to determine } \\
\text { whether there is any effect } \\
\text { on global cognition (as mea- } \\
\text { sured by a screening tool) }\end{array}$ \\
\hline
\end{tabular}




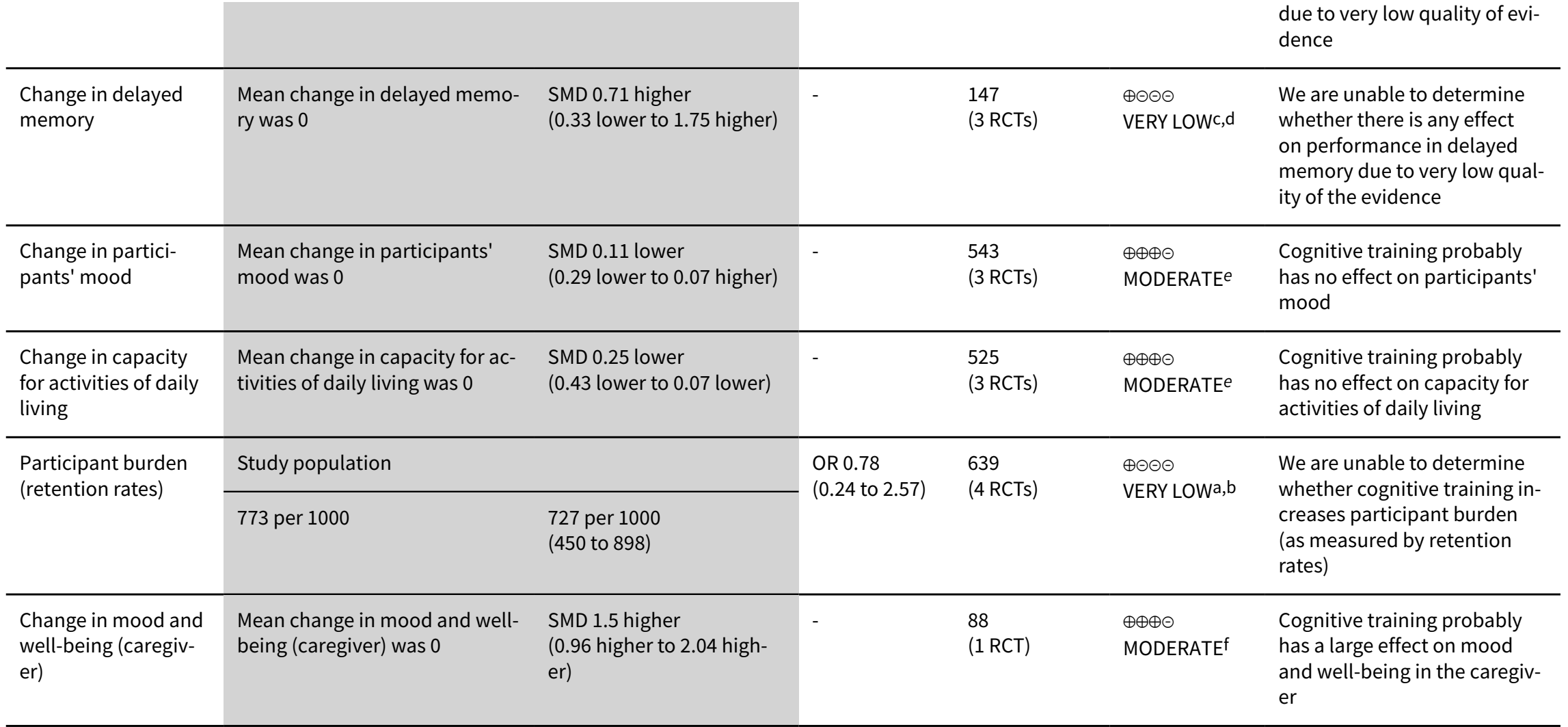

${ }^{\star}$ The risk in the intervention group (and its $95 \%$ confidence interval) is based on the assumed risk in the comparison group and the relative effect of the intervention (and its $95 \% \mathrm{Cl})$.

Cl: confidence interval; OR: odds ratio; RCT: randomised controlled trial; SD: standard deviation; SMD: standardised mean difference.

\section{GRADE Working Group grades of evidence.}

High certainty: we are very confident that the true effect lies close to that of the estimate of the effect.

Moderate certainty: we are moderately confident in the effect estimate: the true effect is likely to be close to the estimate of the effect, but there is a possibility that it is substantially different.

Low certainty: our confidence in the effect estimate is limited: the true effect may be substantially different from the estimate of the effect.

Very low certainty: we have very little confidence in the effect estimate: the true effect is likely to be substantially different from the estimate of effect.

almprecision: downgraded 2 points for very serious concerns related to imprecision because the confidence interval includes positive effect, negligible effect, and effect in the direction of the control group.

$b$ Inconsistency: downgraded 1 point for serious concerns regarding heterogeneity in effect size, which is moderate and statistically significant. Heterogeneity does not seem to be well explained by investigated effect moderators. 


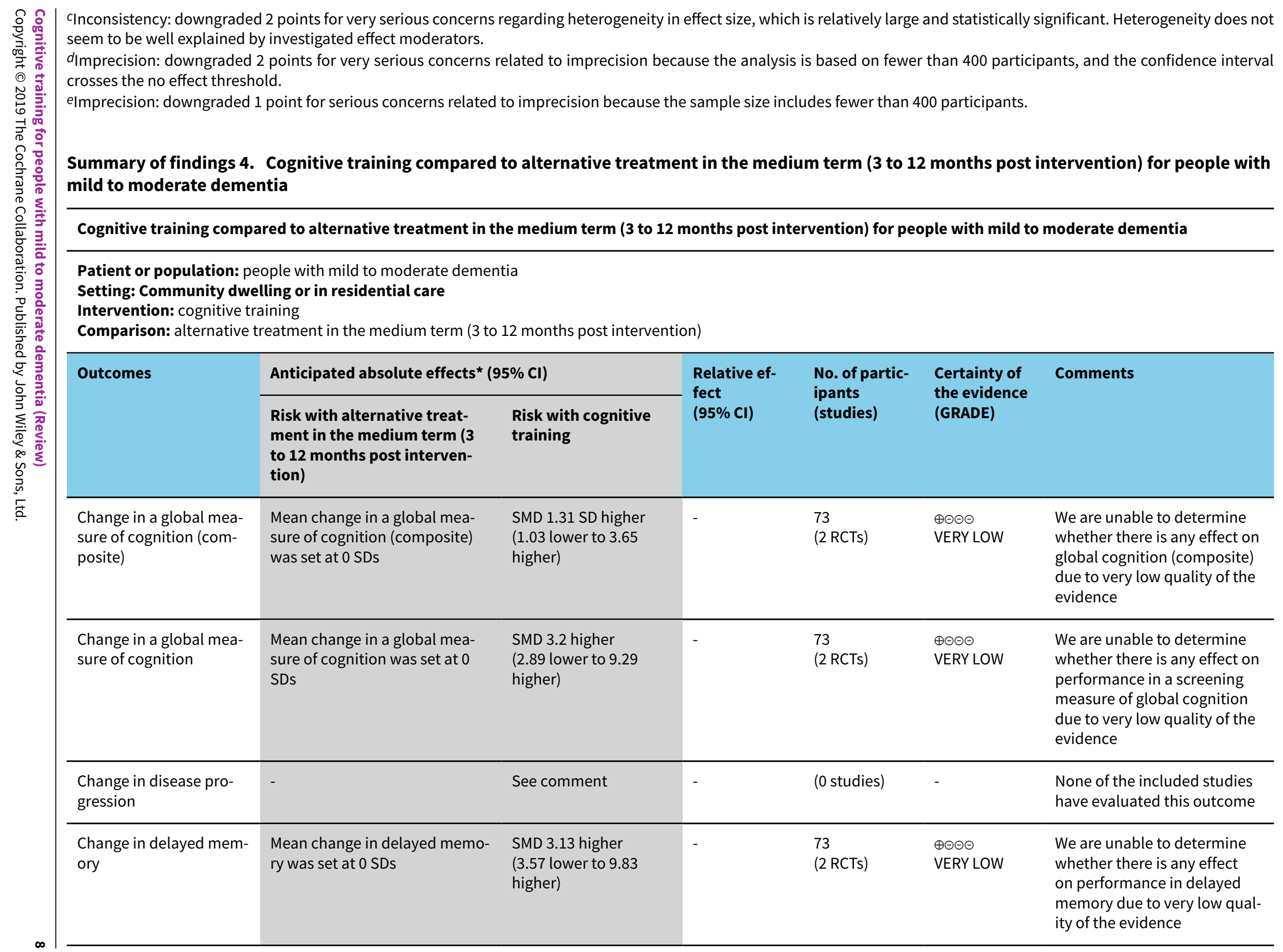




\begin{tabular}{|c|c|c|c|c|c|c|}
\hline 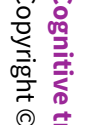 & $\begin{array}{l}\text { Change in participants' } \\
\text { mood }\end{array}$ & $\begin{array}{l}\text { Mean change in participants' } \\
\text { mood was set at } 0 \text { SDs }\end{array}$ & $\begin{array}{l}\text { SMD } 0.66 \text { lower } \\
\text { (1.35 lower to } 0.02 \\
\text { higher) }\end{array}$ & $\begin{array}{l}39 \\
(1 \mathrm{RCT})\end{array}$ & $\begin{array}{l}\oplus \oplus \ominus \ominus \\
\text { LOWa }\end{array}$ & $\begin{array}{l}\text { Cognitive training may not } \\
\text { have an effect on a partici- } \\
\text { pants' mood }\end{array}$ \\
\hline 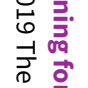 & $\begin{array}{l}\text { Change in capacity for } \\
\text { activities of daily living }\end{array}$ & - & See comment & (0 studies) & - & $\begin{array}{l}\text { None of the included studies } \\
\text { have evaluated this outcome }\end{array}$ \\
\hline 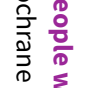 & $\begin{array}{l}\text { Change in mood and } \\
\text { well-being (caregiver) }\end{array}$ & - & See comment & (0 studies) & - & $\begin{array}{l}\text { None of the included studies } \\
\text { have evaluated this outcome }\end{array}$ \\
\hline
\end{tabular}

${ }^{\star}$ The risk in the intervention group (and its $95 \%$ confidence interval) is based on the assumed risk in the comparison group and the relative effect of the intervention (and its $95 \% \mathrm{Cl}$ ).

Cl: confidence interval; OR: odds ratio; RCT: randomised controlled trial; SD: standard deviation; SMD: standardised mean difference.

\section{GRADE Working Group grades of evidence.}

High certainty: we are very confident that the true effect lies close to that of the estimate of the effect.

Moderate certainty: we are moderately confident in the effect estimate: the true effect is likely to be close to the estimate of the effect, but there is a possibility that it is substantially different.

Low certainty: our confidence in the effect estimate is limited: the true effect may be substantially different from the estimate of the effect.

Very low certainty: we have very little confidence in the effect estimate: the true effect is likely to be substantially different from the estimate of effect.

almprecision: downgraded 2 points for very serious concerns related to imprecision because the confidence interval includes positive effect, negligible effect, and effect in the direction of the control group. 


\section{B A C K G R O U N D}

\section{Description of the condition}

Dementia is a clinical syndrome in which functional independence is compromised due to intellectual and cognitive impairment (mostly of gradual onset). Dementia typically is caused by agerelated pathophysiological processes. Alzheimer's disease (AD) and mixed $A D$ and cerebrovascular disease are the most common causes of dementia in older people (Alzheimer's Association 2018). Other common causes include Lewy body pathology (in dementia with Lewy bodies (DLB) and in Parkinson's disease dementia (PDD)) and frontotemporal lobar degeneration (in the frontotemporal dementias (FTDs), there are numerous other, rarer causes) (Alzheimer's Disease International 2009).

Dementia due to most neurodegenerative conditions is usually associated with aggregates of folded or misfolded proteins (Villemagne 2018). In the case of dementia due to AD, this includes aggregates of the $A \beta$ protein that form into plaques in the space between neurons, as well as aggregates of misfolded tau protein that form neurofibrillary tangles inside neurons. Other protein aggregates are implicated in other neurodegenerative diseases (e.g. TDP-43 in FTD, alpha-synuclein protein aggregates in dementia with Lewy bodies). Aggregated proteinopathies usually spread in a predictable and well-described manner through cortical and subcortical regions (Braak \& Braak 2012). In the case of most dementia aetiologies, the pathophysiological chain of events commences years or even decades before the onset of obvious clinical symptoms, at which stage individuals are increasingly brought to clinical attention (Alzheimer's Association 2018).

Regardless of cause, dementia usually has an insidious onset and a progressive course (although in some cases, e.g. vascular cognitive impairment, a more rapid onset may be seen) (Wilson 2012). Although the clinical presentation at early or mild stages may vary according to underlying disease aetiology, global cognitive impairment, changes in personality and behaviour, and compromised functional independence are common characteristics with clinical progression. Cognitive impairment (in the case of $A D$ and vascular disease) and behavioural, personality, or language changes (in the case of frontotemporal neurodegeneration) are typically present well before a clinical diagnosis is made, but at early stages, these can be difficult to differentiate from common age-related changes, or from symptoms associated with common psychiatric conditions (e.g. depression) - a factor that often leads to delays in bringing the situation to medical attention. During the pre-dementia phase, individuals usually present with mild cognitive impairment during a period in which cognitive impairment can be detected on formal examination (Albert 2011; Petersen 2004), but the individual usually shows no, or only minimal, impairment in ability to carry out most activities of daily living. In mild to moderate stages of dementia, cognitive impairment becomes more profound and widespread, functional disability becomes increasingly evident - particularly in relation to more complex activities - and caregiver burden tends to significantly increase (Berger 2005; Gaugler 2000). In more advanced stages of dementia, most cognitive and functional abilities are profoundly impaired, and behavioural changes such as apathy, depression, aggression, and agitation are frequently observed (Förstl 1999).
Despite some overlap, the cognitive symptom signature that characterises the different disease aetiologies that tend to develop into dementia can often be distinguished, at least at early stages. In the case of dementia due to $A D$, the earliest cognitive signs on formal neuropsychological examination are almost invariably related to episodic memory function. Within the memory domain, the most striking deficits are usually observed in measures of new learning and delayed recall - deficits that precede the diagnosis of $A D$ by several years (Weintraub 2012). Once deficits in measures of learning and memory have developed, individuals often show increasing difficulty performing tasks related to semantic memory, language, executive functions, and visuospatial/constructional abilities. In dementia with Lewy bodies, early cognitive impairments are more likely to involve striking visuospatial deficits, fluctuating attention, and reduced working memory capacity, along with the development of vivid hallucinations. In dementias related to frontotemporal lobar degeneration, early symptoms may be predominantly behavioural and may be related to social cognition in behavioural-variant FTD, or in temporal subtypes may involve predominantly language skills and verbal expression (Weintraub 2012). Although impaired performance on measures of episodic memory is central to vascular dementia, people with this condition typically display a more striking deficit on executive and attention tasks, as well as on measures of semantic knowledge and visuospatial function (Graham 2004).

Dementia is highly prevalent in older people, is a leading cause of disability worldwide, and is associated with enormous financial, emotional, and societal burden (Wimo 2017), making research in this area a global priority (World Health Organization 2012). Despite years of research and numerous clinical trials, no cure is yet available for any of the irreversible causes of dementia. Cholinesterase inhibitors remain the primary pharmacological treatment for cognitive symptoms in $A D$ and related dementias; however, the effects of these drugs are not universal and are always temporary (Birks 2006). A range of non-pharmacological interventions (NPIs) that target different aspects of the clinical syndrome, associated disability, and caregiver burden are available (for a comprehensive systematic review, see Olazaran 2010). NPIs generally are not disease-specific and do not directly engage underlying biological targets; they are therefore not 'diseasemodifying'. On the other hand, NPIs are more likely to target a broader spectrum of clinically meaningful outcomes and are less likely to cause adverse reactions. Within the broad category of NPIs, cognition-oriented treatments, particularly $\mathrm{CT}$, have been a topic of much interest among researchers, clinicians, and the general public.

\section{Description of the intervention}

'Cognition-oriented treatments' (COTs), referred to previously as 'cognition-focused interventions' (Clare 2002; Clare 2004), is an umbrella term referring to a group of NPIs in which a range of techniques are applied to engage thinking and cognition with various degrees of breadth and specificity. Unlike NPIs, which are primarily oriented towards outcomes that are behavioural (e.g. wandering), emotional (e.g. anxiety), or physical (e.g. sedentary lifestyle), in COTs, the goals include improving or maintaining cognitive processes or addressing the impact of impairment in cognitive processes on associated functional ability in daily life (Bahar-Fuchs 2013; Clare 2004). CT, sometimes described 
in the literature as 'brain training', 'retraining', or 'remediation', typically involves guided practice of a set of structured - usually standardised - tasks, designed to train individuals on relatively well-defined cognitive processes and abilities such as speed of information processing, attention, memory, or problem-solving (Bahar-Fuchs 2013; Mowszowski 2010). Other COTs described in the literature include cognitive stimulation therapy (CST) and cognitive rehabilitation (CR); these approaches are regarded as distinct in terms of their underlying theoretical assumptions and core elements, as well as the contexts or populations in which they have been traditionally applied, but it is acknowledged that some overlap exists, and that differentiating between these approaches is not always straightforward (Bahar-Fuchs 2013; Gates 2014). Indeed, these terms have been and continue to be applied somewhat interchangeably in the literature (e.g. Fernandez-Prado 2012; Giordano 2010), despite the availability of broad definitions and descriptions of these distinct forms of intervention (BaharFuchs 2013; Clare 2004; Woods 2012). Table 1 (below) summarises key defining features and common properties of these approaches. Cognitive stimulation is the focus of a separate Cochrane Review, which concluded that general cognitive stimulation consistently produces improvements in general cognition and, in some cases, in self-reported quality of life and well-being, primarily for people with mild to moderate dementia (Woods 2012). Cognitive rehabilitation, which is an inherently individualised approach emphasising collaborative goal-setting and a functional orientation (Bahar-Fuchs 2016; Clare 2001), has been considered alongside CT in previous versions of this Cochrane Review (Bahar-Fuchs 2013; Clare 2004); however, as the body of evidence for this approach has increased in recent years, and as it involves different methods and targets different outcomes, cognitive rehabilitation will be considered in a separate Cochrane Review, and the current review accordingly will focus only on CT.

\section{Cognitive training}

Cognitive training (CT) is historically couched within the broader field of neuropsychological rehabilitation of individuals with brain injury and neurological diseases, with efforts to systematically retrain specific cognitive functions originally described by clinical researchers such as Leonard Diller and Yehuda Ben-Yishay in their pioneering work with victims of stroke and head trauma throughout the 1970s (Ben-Yishay 1978; Diller 1974). In the early 1980s, the principles of CT began to be applied in cognitively healthy older adults with subjective cognitive complaints (e.g. Zarit, 1981); however it was not until the late 1980s that CT was first attempted with people with dementia (e.g. Beck 1988). A central assumption underlying CT is that practice has the potential to improve or at least maintain functioning in the given cognitive domain. A further important assumption is that any effects of practice will generalise beyond the immediate training context. In other words, improved performance on a given task should lead to improved performance on other, related tasks that depend on the same cognitive process or ability. Although this last assumption often has not been supported by the evidence (Owen 2010; Papp 2009), some have argued that failure to produce transferable benefits is related in part to problems with task design (Jaeggi 2010). As noted above, CT traditionally involves the repeated practice of a set of structured tasks designed to target particular cognitive processes and abilities. Some study authors have proposed that CT should be divided into subtypes of cognitive exercise and strategy training (Gates 2011), the latter of which involves instruction and practice in the use of specific cognitive strategies designed to further enhance performance, or minimise the impact of impaired cognition (e.g. method of loci, visual imagery) (Hampstead 2016). CT is different from the type of skill training often exercised by occupational therapists, in that the target is usually an underlying process or ability, rather than a specific skill. Early versions of CT tended to be delivered in an inflexible 'one size fits all' approach; however, in recent years, technological developments are leading to increased tailoring of training focus based on the individual cognitive profile and adaptive difficulty level (Bahar-Fuchs 2017; Peretz 2011). CT may be offered through individual sessions (Davis 2001; de Vreese 1998a; de Vreese 1998b; Farina 2002; Koltai 2001; Loewenstein 2004), it may be provided in group sessions (Cahn-Weiner 2003; Ermini Fuenfsch 1995; Kesslak 1997; Koltai 2001; Moore 2001), or it may be facilitated by family members with therapist support (Neely 2009; Quayhagen 1995a; Quayhagen 2000). Initially delivered mainly in paper-and-pencil format, computerised cognitive training (CCT) programmes have largely replaced more traditional methods over the past two decades (Davis 2001; de Vreese 1998; Quayhagen 1995; Quayhagen 2000). In some cases, the tasks or activities that form the focus of practice/training are analogues of actual daily activities, such as doing online shopping or setting up a dinner table (Farina 2002; Loewenstein 2004; Neely 2009; Zanetti 1994; Zanetti 1997; Zanetti 2001), and in these cases the distinction between $\mathrm{CT}$ and functional skills training becomes more difficult. Skills-oriented interventions in which the target task is well structured and is broken into relatively well-defined underlying cognitive performance elements, and where the outcomes of interest are cognitive processes rather than merely performance of the intervention task itself (e.g. Neely 2009), appear to fit the conceptual framework of CT. Conversely, when the focus of the intervention is a specific skill and there is no expectation to improve an underlying cognitive ability/process, and where cognitive underpinnings are unclear or are only vaguely addressed, the intervention might be best classified as 'functional skills training.' In accordance with the suggestion that CT may enhance effects of pharmacological therapy (Newhouse 1997), some studies have evaluated the efficacy of CT in combination with the use of cholinesterase inhibitors (Cahn-Weiner 2003; de Vreese 1998a; de Vreese 1998b; Loewenstein 2004), or given with other medications (Heiss 1993; Yesavage 1981).

Table 1. Selected characteristics of cognitive training, rehabilitation, and stimulation

Cognitive training

Cognitive rehabilitation

Cognitive stimulation

Target

Impairment

Participation restriction 


\section{Context Structured tasks and environ- The person's natural environment} ments
Usually a clinic/residential care or daycare setting

\begin{tabular}{ll}
\hline $\begin{array}{l}\text { Focus of in- } \\
\text { tervention }\end{array}$ & $\begin{array}{l}\text { Specific cognitive abilities and } \\
\text { processes; psychoeducation and } \\
\text { strategy training sometimes in- } \\
\text { cluded }\end{array}$
\end{tabular}

Focus of in- Specific cognitive abilities and strategy training sometimes in-
Groups of cognitive abilities and processes required to perform individually relevant everyday tasks; behaviour, environment, and everyday activity. Psychoeducation and strategy training sometimes included

\begin{tabular}{llll}
\hline Format & Individualised or group & Individualised & Typically group \\
\hline $\begin{array}{l}\text { Proposed } \\
\text { mechanism } \\
\text { of action }\end{array}$ & $\begin{array}{l}\text { Mainly restorative; mechanisms re- } \\
\text { lated to neuroplasticity }\end{array}$ & $\begin{array}{l}\text { Combination of restorative and compensatory } \\
\text { approaches; reduction of 'excess disability' }\end{array}$ & $\begin{array}{l}\text { Improved orientation; } \\
\text { general activation }\end{array}$ \\
\hline Goals & $\begin{array}{l}\text { Improved or maintained ability in } \\
\text { specific cognitive domains }\end{array}$ & $\begin{array}{l}\text { Performance and functioning in relation to collab- } \\
\text { oratively set behavioural or functional goals }\end{array}$ & $\begin{array}{l}\text { Improved overall orien- } \\
\text { tation and engagement } \\
\text { in pleasant abilities }\end{array}$ \\
\hline
\end{tabular}

\section{How the intervention might work}

Cognitive training (CT) aims to improve or maintain specific cognitive processes or global cognitive ability; when used as an intervention approach in clinical populations, it is expected that improvements in cognition will generalise to improvement in functional outcomes. Much has been written about the lack of unifying theories in the field of NPIs, including in relation to interventions aimed at changing behaviour (Michie 2008), in relation to cognition and function (Wilson 2002), and in relation to rehabilitation in general (Hart 2014). Indeed, no single theory exists that comprehensively explains such issues as why or how CT should lead to improved cognitive and functional outcomes, whether and why some cognitive domains are more likely to respond to training than others, whether training should target single or multiple cognitive domains, or whether training should focus on improving impaired functions or building on preserved ones. To various extents, CT interventions in healthy and in clinical populations draw instead on a range of theories and discoveries grounded in cognitive neuroscience (e.g. Jaeggi 2008; Sohlberg 1987), or in clinical practice and rehabilitation of patients with neurological injuries and diseases (Ponsford 2012; Stuss 1999); CT continues to be shaped in response to relevant technological developments including those reported in the gaming industry (Anguera 2015). Unfortunately, many CT interventions have been and continue to be developed without clear reference to any relevant theoretical work.

A central assumption held by many advocates of CT is that training in an underlying cognitive ability or process will lead to generalised improvements that go beyond the training context (Lampit 2014). In cognitively healthy younger and older adults, and to a lesser extent in individuals with mild cognitive impairment $(\mathrm{MCl})$, there is little doubt that CT leads to improvement in trained or 'criterion' tasks. However, in both healthy and clinical populations, the evidence concerning learning transfer remains mixed, and the issue is hotly debated, with much debate concerning identification of barriers and enablers of transfer of gains to untrained tasks that reflect the cognitive domain targeted by the training (near transfer) and other untrained cognitive domains, as well as non-cognitive outcomes (far transfer) (Jaeggi 2010). In a recent comprehensive review and critique of the commercial CT industry, Simons and colleagues pointed out that discussion concerning transfer of learning can be traced back to very early theoretical accounts (Simons 2016), such as the so-called formal discipline theory and the theory of transfer by identical elements proposed by Edward Thorndike in the early 20th century. It is beyond the scope of this review to cover these theories in detail, but a critical discussion of these accounts in relation to the $\mathrm{CT}$ literature and industry is included in the review by Simons and colleagues (Simons 2016). Contemporary empirical findings suggest that factors that appear to be implicated in CTrelated gain-transfer include degree of similarity or overlap among elements of trained and transfer tasks, extent of actual gain on trained tasks, baseline cognitive abilities, and age (Zinke 2014).

In addition to theories of learning and transfer, knowledge and expertise related to brain-behaviour relationships - as well as to mechanisms of injury, disease, and recovery - are critical in informing the development of COTs, including $\mathrm{CT}$, in the context of work with persons with acquired disorders of the central nervous system (including traumatic brain injury, stroke, and neurodegenerative conditions). Historically, such interventions have reflected two broad conceptual frameworks for recovery of function after brain illness or injury: a restorative approach, and a contextualised or compensatory approach (Ylvisaker 2002). Techniques usually associated with cognitive rehabilitation, such as optimising residual cognitive abilities in impaired domains and making the most of unimpaired cognitive abilities, lend themselves more to compensatory approaches (Clare 2001b). In contrast, techniques usually associated with $\mathrm{CT}$, such as the repeated exercise of standardised cognitive tests of increasing difficulty and the targeting of specific cognitive domains, tend to reflect restorative principles and "thrive on the lure of neuroplasticity" (Rabipour \& Raz 2012). Indeed, a range of neuroplasticity-related observations in animal and human studies, including changes at the molecular, synaptic, structural, and functional levels associated with enriched environments and a structured training programme, are routinely cited as the proposed 
mechanisms of action in CT (Valenzuela 2012). In recent years, growing evidence has shown that $\mathrm{CT}$ is associated with changes in patterns of neural activation in key brain regions among healthy older adults (Belleville 2014), as well as in people with $\mathrm{MCl}$ (Belleville 2011; Hampstead 2011). Such increased brain activation may be the result of processes of synaptic growth and repair triggered by repeated practice on standardised tests.

\section{Why it is important to do this review}

The Alzheimer's disease drug development pipeline is slow, and trials of disease-modifying treatments have generally failed to produce improvement in any clinically meaningful outcomes, although they have succeeded in disrupting targeted pathophysiological processes (Cummings 2014; Cummings 2016; Salomone 2012), leading some to question the relevance of the dominant amyloid cascade hypothesis when it comes to the development of an effective treatment for dementia as a clinical syndrome (D'Alton 2011). NPIs aimed at developing ways of living better with dementia, in part by targeting relevant clinical outcomes and caregiver burden, are assuming an increasingly central role in the management of dementia and are recognised as an important adjunct, and even alternative, to available pharmacological treatments. A recent Lancet Commission on Dementia Prevention, Intervention, and Care argued that some NPIs can already play an important role in managing some of the cognitive, behavioural, and neuropsychiatric symptoms of dementia, and pointed to positive findings for cognitive stimulation therapy and preliminary supportive evidence on cognitive rehabilitation (Livingston 2017).

In healthy older adults (Edwards 2017; Lampit 2014), and in persons with $\mathrm{MCl}$ (Chandler 2016; Hill 2017), systematic review findings on effects of $\mathrm{CT}$ on cognitive and several non-cognitive outcomes have been generally encouraging, and factors associated with increased intervention efficacy in $\mathrm{CT}$ are becoming better understood. Indeed, recently published clinical practice guidelines for $\mathrm{MCl}$ have classified CT as supported by Level C evidence, meaning that clinicians may recommend this form of intervention (Petersen 2018).

In contrast, most systematic reviews of $\mathrm{CT}$ for persons with dementia have to date produced largely negative findings (e.g. Bahar-Fuchs 2013; Hill 2017; but see Sitzer 2006). Our previous Cochrane Review on CT for persons with dementia included 11 randomised controlled trials but provided no evidence to support $\mathrm{CT}$ in relation to any of the examined outcomes. We noted, however, that the certainty of these findings may be reduced by the relatively small number of highly heterogenous studies, which often were of low methodological quality. Against the background of a heavily divided scientific community and an ever growing industry of commercial CT products that have at times made highly misleading claims, it is vital that clinicians, policy-makers, and the general public are presented with an up-to-date, rigorous, and unbiased review of the current literature on $\mathrm{CT}$ for persons with mild to moderate dementia.

\section{O B JE C T IVES}

- To assess effects of CT on cognitive and non-cognitive outcomes for people with mild to moderate dementia and their caregivers.

- To compare effects of CT with those of other nonpharmacological interventions, including cognitive stimulation or rehabilitation, for people with mild to moderate dementia and their caregivers.

- To identify and explore factors related to intervention and trial design that may be associated with the efficacy of CT for people with mild to moderate dementia and their caregivers.

\section{METHODS}

\section{Criteria for considering studies for this review \\ Types of studies}

In keeping with the previous version of this review, and to ensure the inclusion of unbiased estimates of treatment effects only (Reeves 2011), we considered only randomised controlled trials (RCTs) for inclusion. Wherever possible, we did not exclude studies published in a language other than English, and we made every effort to obtain an English translation from the study authors. In cases where we could not obtain a translation from study authors, we engaged in reasonable efforts to obtain a reliable translation, and we excluded a study only if these efforts were unsuccessful.

\section{Types of participants}

We included trials in which all participants had received a medical diagnosis of dementia, of any subtype, as long as the underlying aetiology was assumed to be non-reversible. It was expected that the diagnosis of dementia was generally made on the basis of established clinical or research diagnostic criteria, including criteria specified by the following.

- The Diagnostic and Statistical Manual of Mental Disorders, Fifth Edition (DSM-V; APA 2013) or earlier versions (APA 1995).

- The International Classification of Diseases, Tenth Revision (ICD-10) (WHO 1992).

- The National Institute of Neurological and Communicative Disorders and Stroke - Alzheimer's Disease and Related Disorders Association (NINCDS-ADRDA) (McKhann 1984).

- The National Institutes of Health - Alzheimer's Association (NIHAA) (McKhann 2011).

- The Association Internationale pour la Recherché et l'Enseignement en Neurosciences (NINDS-AIREN) (Roman 1993).

- Vascular Impairment of Cognition Classification Consensus Study (McKeith 1996; McKeith 2006; McKeith 2017).

- The International Behavioural Variant FTD Criteria Consortium (FTDC) (Skrobot 2017).

On average, participants in included studies were classified as having a mild to moderate level of severity. Dementia severity was usually determined in primary trials on the basis of group mean scores, ranges of scores, or individual scores on a standardised scale, such as scores over 12 on the Mini Mental State Examination (MMSE; Folstein 1975), or scores of 0.5 to 2 on the Clinical Dementia Rating scale (CDR 2; Hughes 1982).

- Studies in which it was clear that only a small proportion of participants (i.e. $<15 \%$ ) fell within the more severe range or the questionable dementia range were considered acceptable if this information was clearly indicated in the study.

- Qualifying participants generally resided at home, or in a residential care facility. We excluded studies in which recruited participants could be long-term residents of psychiatric 
hospitals, where pre-existing psychiatric conditions are likely to be present.

- We set no specific age restrictions, although it was expected that, with the exception of participants with younger-onset dementia (YOD), most participants would be 65 years of age or older.

- No restrictions were placed on current pharmacological treatment. When available, information about participants' use of cholinesterase inhibitors was noted.

- Primary studies that included a mixture of participants, only some of whom meet our inclusion criteria (e.g. dementia, $\mathrm{MCl}$ ), were eligible for inclusion as long as outcomes were reported separately for the group of interest, or as long as we could obtain that information from trial authors.

\section{Types of interventions}

\section{Experimental interventions}

Interventions meeting our definition of $\mathrm{CT}$ were eligible for inclusion. As the terms used to refer to $\mathrm{CT}$ vary considerably, interventions may be referred to as 'brain' or 'mental' training, and they may be described as 'retraining', 'exercise', 'stimulation', 'rehabilitation', 'therapy', 'remediation', 'support', etc.; our operational definition of eligible interventions included the following criteria.

- Participants were trained on tasks designed to target one or more cognitive processes either directly or indirectly. Training generally took the form of repeated practice. Trials in which the primary goal was to compare performances of participants who learned how to perform a task under different learning conditions (e.g. errorless vs errorful) in a single session (single trial training) were not eligible for inclusion.

- Tasks were completed in pen-and-paper format or through computerised exercises, or were structured analogues of everyday tasks in which the cognitive underpinnings are explicit, and the intervention targeted a cognitive ability or process rather than a specific skill. The nature of the intervention (i.e. computerised or pen-and-paper or analogues of daily activities) was noted.

- Interventions were delivered on commercially available platforms, or were designed specifically for the purposes of the study.

- Interventions could target single or multiple cognitive domains.

- Level of difficulty was expected to vary; however, this did not form part of the inclusion criteria.

- We excluded from this review interventions in which CT was combined with another distinct experimental intervention (e.g. physical activity, brain stimulation), but this did not apply to standard treatments, as participants were generally expected to remain on their standard (usually pharmacological) treatment.

- Modified/alternative CT: it was acknowledged that CT and other cognition-oriented treatment approaches (i.e. cognitive stimulation or rehabilitation) may share some features, some of which could not be distinguished in a straightforward manner. Hence, we will include trials of complex cognitionoriented treatments that also include elements of cognitive stimulation (e.g. orientation), rehabilitation (e.g. goal setting), or psychoeducation (e.g. using cognitive strategies), if it was determined by consensus that $\mathrm{CT}$ was clearly the predominant component. When relevant and indicated by statistical heterogeneity, we considered these interventions separately in subgroup analyses.

\section{Comparator interventions}

- Wait-list. In studies of this type, the experimental intervention was offered to the control group after the study had ended.

- No treatment/standard treatment. Unless otherwise specified, whenever groups were described as 'no treatment' in individual studies, we assumed that this referred to usual/standard treatment, and not to withholding of treatment. 'Usual or standard treatment' referred to what would normally be provided in the study locality to participants with mild dementia, and might include provision of medication, clinic consultations, and contact with a community mental health team or daycare, or support from voluntary organisations, but not a specific $\mathrm{CT}$ intervention.

- Active control. This referred to conditions in which participants engaged in some form of activity, typically for an equivalent number of sessions or visits, and received similar levels of contact with the researchers, but during which no structured intervention was offered.

- Alternative treatments. These were distinct, alternative treatments that might (e.g. cognitive stimulation) or might not have been cognition-focused (e.g. physical activity).

\section{All interventions}

- We did not include interventions conducted in individual or group format, with or without involvement of family caregivers.

- We did not impose restrictions regarding intervention dose-related parameters, including overall duration of the intervention or number of treatment sessions. However, as described above, we excluded single-session treatments.

\section{Types of outcome measures}

We considered outcomes within the following broad categories as relevant for this review.

- Clinical disease progression.

- Cognitive outcomes.

- Psychosocial outcomes for the person with dementia.

- Psychosocial outcomes for the primary caregiver.

- Surrogate/mechanism/biomarker outcomes.

- Economic outcomes.

Although it is acknowledged that surrogate and economic outcomes are important, we determined them to be beyond the scope of the current review; therefore we selected the main primary and secondary outcomes from the top four categories, as further outlined below.

\section{Primary outcomes}

\section{Outcomes for the person with dementia}

- Global cognitive status at end of treatment (i.e. immediately post intervention). We measured this by determining change in scores on a composite measure of global cognition derived from all cognitive measures included in each trial, with additional analyses focusing on global cognition, as reflected on screening measures of global cognition (e.g. MMSE) 
- Clinical disease severity in the medium term. We measured this by determining change in scores on measures of clinical disease progression (e.g. Clinical Dementia Rating Scale (CDR), Dementia Rating Scale (DRS)) in a follow-up assessment conducted between 3 and 12 months after treatment cessation

\section{Secondary outcomes}

\section{Outcomes for the person with dementia}

- Global cognitive status in the medium term. We measured this by determining change in scores on a composite measure of global cognition at the relevant follow-up assessment, with additional analyses focusing on global cognition as reflected on screening measures of global cognition (e.g. MMSE)

- Clinical disease severity at end of treatment. We measured this by determining change in scores on measures of clinical disease progression (e.g. CDR, DRS) in the immediate post-treatment assessment

- Domain-specific cognitive status at end of treatment. We measured this by determining change in scores on neuropsychological measures of speed of processing, immediate memory, delayed memory, attention and working memory, language (naming), verbal letter fluency, verbal category fluency, and executive function

- Domain-specific cognitive status in the medium term (3 and 12 months after treatment cessation). We measured this by determining change in scores on neuropsychological measures of speed of processing, immediate memory, delayed memory, attention and working memory, language (naming), verbal letter fluency, verbal category fluency, and executive function

- Meta-cognition (subjective beliefs regarding cognition - selfreported) at end of treatment and in the medium term

- Meta-cognition (subjective beliefs regarding cognition informant-reported) at end of treatment and in the medium term

- Mood (as reflected in change in self- or informant-reported measures of depression, anxiety, etc.) at end of treatment and in the medium term

- Capacity for activities of daily living at end of treatment and in the medium term

- Behavioural and psychological symptoms of dementia (BPSD) at end of treatment and in the medium term

- General health or quality of life at end of treatment and in the medium term

- Participant burden as reflected in rates of retention of trial participants at end of treatment

\section{Outcomes for the primary caregiver at end of treatment}

- Mood and well-being (as reflected in change in self-reported measures of depression, anxiety, etc.) at end of treatment and in the medium term

- Burden of care at end of treatment and in the medium term

- Quality of life at end of treatment and in the medium term

\section{Outcome measures}

Where possible, we used data from published and validated tests, questionnaires, or techniques for evaluation of a given outcome. In cases in which an outcome was evaluated by an unpublished or non-established measure, we made every effort to source information about statistical properties of the test or scale in question, before determining whether or not to accept the measure. We classified cognitive measures to specific cognitive domains according to established authoritative texts (Spreen 1998), wherever possible, and by consensus between study authors as required.

\section{Outcome evaluation}

We included trials if they included, at minimum, a baseline evaluation and one post-treatment evaluation.

\section{Search methods for identification of studies}

\section{Electronic searches}

We searched ALOIS (www.medicine.ox.ac.uk/alois), the Specialised Register of the Cochrane Dementia and Cognitive Improvement Group (CDCIG), on 5 July 2018.

ALOIS, which is maintained by the Information Specialists for CDCIG, contains studies that fall within the areas of dementia prevention, dementia treatment and management, and cognitive enhancement in healthy elderly populations. These studies are identified by:

- searching several major healthcare databases: MEDLINE, Embase, Cumulative Index to Nursing and Allied Health Literature (CINAHL), and PsycINFO;

- searching several trial registers: ClinicalTrials.gov and the International Clinical Trials Register Platform (ICTRP) of the World Health Organization (WHO), which includes International Standard Randomized Controlled Trials Number (ISRCTN); the Chinese Clinical Trials Register; the German Clinical Trials Register; the Iranian Registry of Clinical Trials; and the Netherlands National Trials Register, plus others;

- searching the Central Register of Controlled Trials, in the Cochrane Library (CENTRAL); and

- searching grey literature sources: Institute for Scientific Information (ISI) Web of Science Core Collection.

To view a list of all sources searched for ALOIS, please visit the ALOIS website (www.medicine.ox.ac.uk/alois).

Details of the search strategies run in healthcare bibliographic databases, used for retrieval of reports of dementia, cognitive improvement, and cognitive enhancement trials, can be viewed on the website of the Cochrane Dementia and Cognitive Improvement Group at http://dementia.cochrane.org/searches.

We ran additional searches in MEDLINE, Embase, PsycINFO, CINAHL, Latin American Caribbean Health Sciences Literature (LILACS), ClinicalTrials.gov, and the WHO Portal/ICTRP, to ensure that searches for this review were as comprehensive and as upto-date as possible. Search strategies used and the number of hits retrieved can be seen in Appendix 1.

\section{Searching other resources}

We screened reference lists from included trials, as well as reference lists of recent systematic reviews, and relevant recent guidelines. We contacted experts in the field to request additional randomised trial reports not identified by the search. 


\section{Data collection and analysis}

\section{Selection of studies}

One review author (AM) reviewed titles and abstracts from the complete de-duplicated list of search results, and we split the records for an independent screening by two additional review authors (ABF, AG), to identify all potentially relevant RCTs of CT for people with dementia and to remove obviously irrelevant studies. Whenever there was doubt regarding the eligibility of a trial, we selected it for full review of the methods. Following the initial screening, we applied the same approach for evaluation of full methods from short-listed articles. We identified and merged multiple reports from the same study, and we contacted study authors to clarify issues related to the eligibility of a trial for inclusion. We settled discrepancies in the classification of trials through discussion between two review authors and ruling of a senior review author who is a content area expert (LC). The study selection process was unblinded.

\section{Data extraction and management}

JS extracted data from study reports onto a standardised, structured data entry form under supervision of the lead review author (ABF), who also independently extracted data for variables requiring some judgement (e.g. intervention integrity/ fidelity), and we subsequently entered the data into Review Manager 5 software (Review Manager 5). We sought additional information from study authors as appropriate. Data extracted from each trial included detailed characteristics of trials (e.g. settings, outcomes), design features (e.g. delivery format, blinding), participant characteristics (e.g. diagnoses, age, gender, education, medications), and elements of experimental and control interventions (e.g. intensity, frequency, duration, key intervention features). We also extracted information about additional variables of interest for the investigation of effect moderators, including registration status, sources of funding, conflicts of interest, adherence and retention, type of control, whether intervention integrity/fidelity was addressed, and adverse events. For each outcome of interest, we extracted mean scores and standard deviations on relevant measures from all available evaluations.

\section{Assessment of risk of bias in included studies}

Pairs of review authors independently conducted assessment of risk of bias using Cochrane's 'Risk of bias' tool (Higgins 2017). We resolved disagreements by discussion with a third review author who is a subject matter expert (LC). Consistent with Cochrane's 'Risk of bias' tool, we assessed bias in the following domains: sequence generation, allocation concealment, blinding of participants and investigators, incomplete outcome data, and selective reporting of outcomes. We rated studies as 'low risk', 'high risk', or 'unclear risk' in each of these domains.

\section{Measures of treatment effect}

We generally calculated effect estimates in primary trials along with their 95\% confidence intervals (Cls) using change-from-baseline scores. Calculations of the standard deviation of change scores were based on the assumption that the correlation between measurements at baseline and those at subsequent time points is $r=0.8$, in keeping with other relevant reviews (e.g. Lampit 2014). However, for consistency with previous versions of this review, we also conducted sensitivity analyses of the primary outcome with a conservative $r=0$ assumption, which overestimates the standard deviation of the change. We treated outcome measures as measured on a continuous scale. In some cases, we derived outcomes from ordinal rating scales; provided these contained a reasonably large number of categories (more than 10), we treated data as continuous variables arising from a normal distribution. For dichotomous outcomes (e.g. participant retention), we expressed effects as risk ratios (RRs) along with $95 \% \mathrm{Cls}$.

\section{Unit of analysis issues}

We expected four types of unit of analysis issues: cross-over trial designs, multiple-armed trials (more than one treatment/control condition), repeated assessments, and availability of multiple measures of the same outcome in primary trials. Our approach to the management of these issues was as follows.

- Cross-over trials: we used only data from the first treatment period (before cross-over).

- Multiple conditions:

- experimental conditions: in trials that include at least three conditions, assuming that at least one condition satisfies our definition of a comparison condition (see above), we combined data from all conditions that we judged to fit our definition of $\mathrm{CT}$ into a single group using a relevant formula (Higgins 2017). We excluded from this review trials that include two relevant experimental conditions but no eligible control condition; and

- control conditions: we combined data from two control conditions of the same broad type (i.e. no treatment). In the event that a trial included different types of control comparisons that are not alternative treatments (e.g. it included both no treatment and active control groups), we used in the analysis data from both these control conditions by splitting the sample size of the experimental condition into two separate groups, according to the procedure described in Chapter 7 of the Cochrane Handbook for Systematic Reviews of Interventions (Higgins 2017).

- Repeated post-intervention assessments: we conducted separate comparisons to assess primary and secondary outcomes at end of treatment (i.e. immediately post intervention), and in the short to medium term (up to 12 months post intervention). Within this follow-up period, we used in the analysis data from the last available assessment. We did not use data from followup assessments conducted more than 12 months following the end of treatment assessment.

- Multiple measures of the same outcome: in primary trials in which multiple measures of the same outcome were used, the following principles guided the selection of measures for data extraction:

* general principles: we used a composite outcome measure if one was derived by the study authors. If no composite was available, we generally used data from a test that matched the most commonly used measure in other studies that contributed data to the particular outcome. Established/ published measures of the outcome were preferred over measures developed for the specific study. If more than one established measure of an outcome was used, and no measure was identified that was used by most trials contributing to the specific outcome, we created a simple 
composite score from standardised scores on the different measures and used it in the analysis;

* cognitive outcomes: for each trial, we computed a global composite cognitive score by calculating a standardised change-from-baseline score for each measure (change score divided by the standard deviation of the change score), and deriving a simple mean and standard deviation of the zscores associated with all cognitive measures from a trial. In addition, for evaluation of domain-specific cognitive scores, we used the following principles:

$\square$ Psychomotor information processing speed: we preferred visuospatial measures where available.

Attention, immediate and delayed memory: we preferred auditory-verbal measures for evaluation of attention and immediate and delayed memory. We preferred tasks that involve the learning of information over several trials (i.e. word lists) over tasks in which the information is presented only once (e.g. story or figure recall). We preferred measures of free recall over measures of cued/ recognition where available.

Executive functions: we preferred tasks that reflect planning, organisation, decision-making, regulation of performance, and set-shifting aspects of executive functions over tasks that are more strongly associated with volition or purposive action aspects of executive functions (Lezak 2004). In the event that several measures of executive function were used in a study, we computed a composite executive function score by taking the mean of standardised scores for each of these measures.

* Meta-cognitive outcomes: we generally preferred selfreported measures of contentment/satisfaction with one's cognitive ability over informant-reported measures;

* Mood outcomes: we generally preferred measures of depression over measures of anxiety or apathy, and selfreported measures over informant-reported measures; and

* Activities of daily living (ADLs): we preferred measures of instrumental ADLs over measures of basic ADLs, and informant-reported measures over self-reported measures. This is based on the finding that self- and informant-reported daily functions show significant discrepancy in people with dementia, and that informant reports of daily function are more closely associated with actual memory performance (Farias 2005).

\section{Dealing with missing data}

We extracted the number of participants who commenced and completed the intervention in each condition, and this contributed to our assessment of risk of bias due to incomplete outcome data. Wherever possible, we contacted trial authors in an effort to obtain relevant unreported data. In general, we assumed that data were missing at random, and that analyses in individual studies were generally performed on a per-protocol (PP) rather than on an intention-to-treat (ITT) basis. When a trial report included relevant data from both ITT and PP samples, we generally used the PP data for consistency with most of the trials. We evaluated the impact of missing data on pooled effect estimates by performing sensitivity analyses (see below).

\section{Assessment of heterogeneity}

In addition to a visual inspection of the forest plots, we assessed statistical heterogeneity using a standard $\mathrm{Chi}^{2}$ statistic and the associated $\mathrm{l}^{2}$ statistic. Consistent with recommendations (Deeks 2017), we deemed heterogeneity to be present when the $\mathrm{Chi}^{2}$ statistic is significant at the $P=0.1$ level, or when $\mathrm{I}^{2}$ suggests that more than $40 \%$ of the variability in the effect estimate is due to heterogeneity. Where substantial heterogeneity was detected, we explored the sources of heterogeneity by conducting subgroup analyses (see below).

\section{Assessment of reporting biases}

For primary outcomes, we first evaluated the presence of reporting bias through visual examination of funnel plots for small-study effects. We examined the significance of any apparent asymmetry by using Egger's test (Egger 1997), and by providing follow-up with the 'trim and fill' test (Duval 2000), if asymmetry of the plot was confirmed.

\section{Data synthesis}

We performed data synthesis using Review Manager 5 software. In relation to each of the main outcomes of interest, we undertook the following separate comparisons.

- CT versus control (no/standard treatment/wait-list or active control) at end of treatment (i.e. immediately post intervention).

- CT versus control (no/standard treatment/wait-list or active control) in the medium term ( 3 to 12 months following end of treatment).

- CT versus alternative treatment at end of treatment (i.e. immediately post intervention).

- CT versus alternative treatment in the medium term (3 to 12 months following end of treatment).

Within each of the planned comparisons, we pooled data in relation to each outcome of interest when data from at least two trials were available.

We performed inverse-variance, random-effects meta-analyses for all outcomes. We used mean differences (MDs) with 95\% Cls whenever studies used the same outcome measure, whereas we used standardised mean differences (SMDs), which show the absolute mean difference divided by the pooled standard deviation, when the same outcome was assessed by different measures.

\section{Subgroup analysis and investigation of heterogeneity}

In relation to each outcome, we carried out subgroup analyses to evaluate the potential impact of categorical treatment modifiers. We carried out subgroup analyses only when statistical heterogeneity was suggested by the relevant statistics $\left(1^{2} \geq 40 \%\right)$ (Deeks 2017), and when at least three studies were available for each subgroup. We examined the following categorical effect modifiers.

- Type of intervention 1: 'straight' CT versus 'augmented' CT - in which CT was combined with elements of cognitive rehabilitation or cognitive stimulation (or both).

- Type of intervention 2: multi-domain CT versus single-domain CT (e.g. working memory). 
- Intervention dose: more intense (i.e. more than three formal sessions per week) versus less intense interventions (i.e. up to three formal sessions per week).

- Intervention duration: longer interventions (i.e. more than three months) versus shorter interventions (i.e. three months or less).

- Follow-up period: we compared studies with follow-up in the short term (up to three months after treatment cessation) versus trials that included longer-term follow-up (up to 12 months after treatment cessation).

- Risk of bias: studies with high risk of bias in at least two critical domains versus other studies with lower risk of bias. For the purposes of these analyses, critical domains were sequence generation, blinding of outcome assessment, incomplete data, and selective reporting. Although we acknowledge that allocation concealment is increasingly regarded as a critical domain, this remains a relatively infrequent practice in these types of studies.

- Funding source: trials funded by commercial entities versus those based on competitive funding.

- Registration: registration status of the trial (prospective/ retrospective vs not registered/not reported).

\section{Sensitivity analysis}

To determine whether findings for the primary outcomes were affected by assumptions made regarding strength of the correlation between scores before and after the interventions, we repeated analyses of the primary outcomes after applying the zero correlation assumption, which overestimates the standard deviation of change scores. We repeated evaluation of primary outcomes by performing a further sensitivity analysis using postintervention scores only, thus avoiding the need to estimate the standard deviation of change scores.

\section{GRADE and 'Summary of findings' tables}

We expressed our overall confidence in the evidence for each outcome using GRADE, and we presented this in 'Summary of findings' tables and in the review text. We described the quality of evidence as 'high', 'moderate', 'low', or 'very low', using the GRADE framework, which we applied to all primary and secondary outcomes in each comparison. In relation to each outcome, we considered certainly in the estimates in relation to risk of bias, indirectness, inconsistency, imprecision, and publication bias for studies contributing data to estimation of the outcome. Two review authors (ABF and JS) worked together to grade the evidence. We considered estimates based on data from a single study against the same parameters, with the exception of inconsistency and publication bias dimensions. In relation to risk of bias, we generally downgraded by 1 point, reflecting serious concern, when sensitivity analysis in which we removed studies classified as at overall 'high risk' led to a difference in the estimate of effect of between 0.2 standard deviation (SD) and 0.3 SD. We downgraded by 2 points, reflecting very serious concern, when sensitivity analysis led to a difference in the estimate of effect that was greater than 0.3 SD. We generally downgraded by 1 point for serious concerns regarding inconsistency when moderate heterogeneity was observed $(40 \%$ $<\mathrm{I}^{2}<75 \%$ ) and when subgroup analyses (when relevant; see below) did not seem to explain heterogeneity in the estimates. We generally downgraded by 2 points when high heterogeneity $\left(l^{2}>75 \%\right)$ was observed and when subgroup analyses (when relevant) did not seem to explain the heterogeneity. Concerning imprecision, following the rule of thumb in the GRADE Handbook, we downgraded by 1 point when the sample size on which the estimate was based was smaller than 400 participants, or in the event that the confidence interval of the estimate included both a potentially important effect and a clinically unimportant effect (defined as an effect smaller than 0.2 in either direction for continuous outcomes). We downgraded by 2 points when the estimate was based on fewer than 400 participants (for continuous outcomes), and when the $\mathrm{Cl}$ of the estimate included both a potentially important effect and no effect, or in the event that the $\mathrm{Cl}$ included all relevant possibilities (positive effect, no effect, and effect in the opposite direction), irrespective of the sample size. Regarding publication bias, we indicated that it was 'strongly suspected' in cases where on visual inspection, asymmetry in the funnel plot for a relevant outcome was reasonably evident. We did not conduct formal tests of asymmetry, and we inspected funnel plots only when at least 10 studies contributed to the outcome. Hence, we could not evaluate this for many outcomes, including all outcomes in the comparison between cognitive training and use of an alternative treatment. Finally, we generally regarded the correspondence between findings in relation to various outcomes and the review question as specified in the PICO to be adequate, so we decided not to downgrade the evidence on the basis of indirectness.

We generated 'Summary of findings' tables using GRADEpro GDT software (GRADEpro GDT), and we imported these into the review. Summary of findings for the main comparison, Summary of findings 2, Summary of findings 3, and Summary of findings 4 include the following primary and secondary outcomes.

- Global cognition at end of intervention.

- Clinical disease severity at latest follow-up, up to 12 months following treatment cessation.

- Delayed memory ability at end of intervention.

- Capacity to perform activities of daily living.

- Mood and well-being of participant.

- Mood and well-being of informant/caregiver.

- Treatment burden (retention rates).

\section{RE S U L T S}

\section{Description of studies}

\section{Results of the search}

The flow of studies through the search and screening process can be seen in Figure 1 (review flow chart). After de-duplication, 1166 records underwent full title and abstract review, on the basis of which we deemed 157 titles to be potentially relevant; we then reviewed the full text of these studies (when available) to confirm eligibility. The full-text review revealed that 33 studies met our inclusion criteria and 32 studies contributed data for at least one meta-analysis. Of these, 10 studies were included in a previous Cochrane Review on CT and rehabilitation for people with mild to moderate dementia (Bahar-Fuchs 2013). 
Figure 1. Study flow diagram.

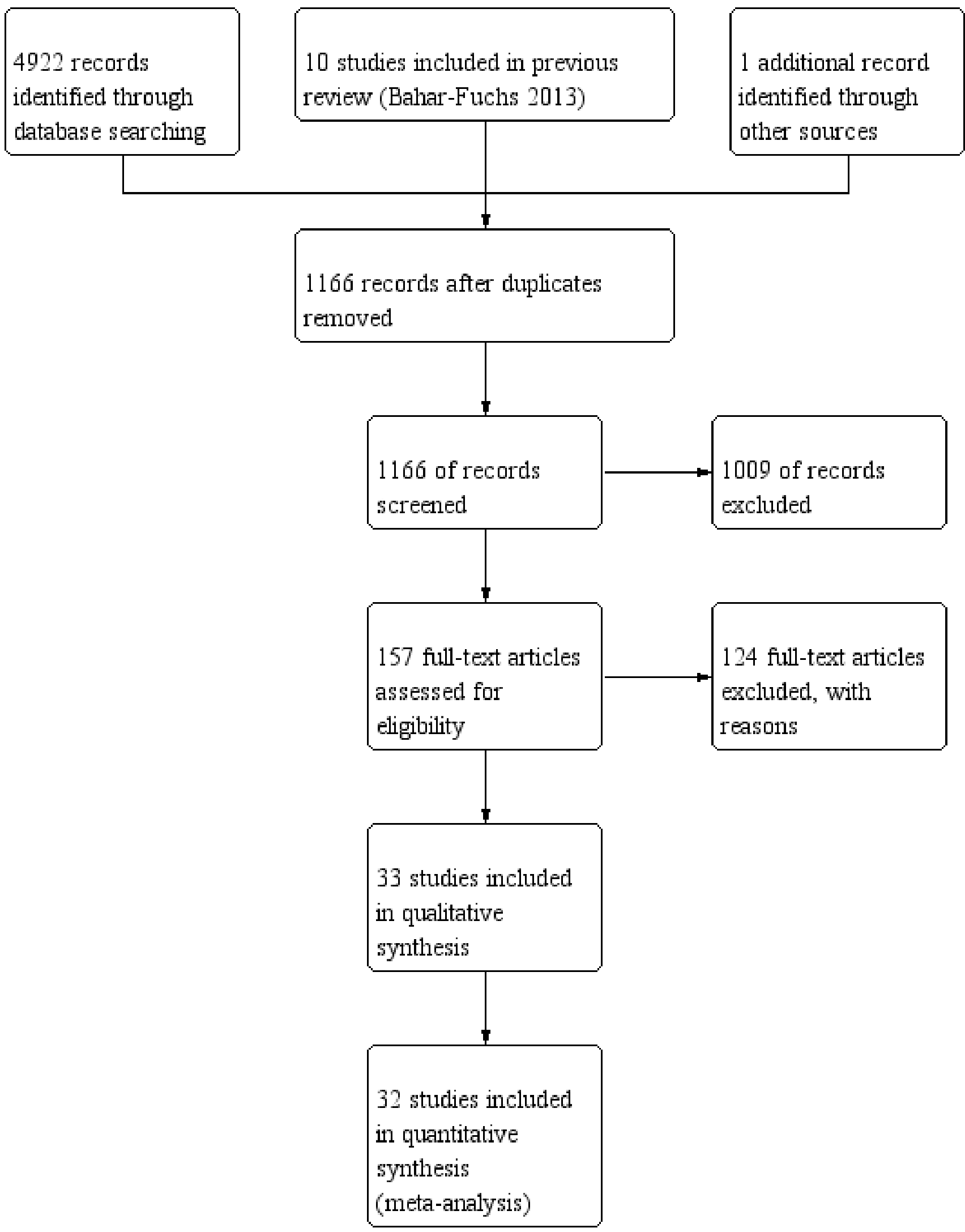




\section{Included studies}

Pertinent details of the included studies, extracted from the published manuscript and, where noted, provided by study authors, are presented in the Characteristics of included studies table. Further details concerning the characteristics of participants in included studies are presented in Table 1, and details concerning dose and duration of the interventions are shown in Table 2. The 33 studies selected for inclusion in the current review were published between 1988 and 2018. With the exception of Davis 2001 and Barban 2016, which did not use a cross-over design, all trials were parallel-group RCTs. Amieva 2016, Barban 2016, Beck 1988, Jelcic 2014, Kallio 2018, Kao 2016, Lee 2013, and Tsantali 2017 were described as multi-site trials; all others were assumed to be single-site trials. Only three of the included trials made reference to registration in a public trial registry; Brueggen 2017 and Kallio 2018 were prospectively registered, whereas Kao 2016 was retrospectively registered. We assumed that all other trials were unregistered. The included trials were conducted in 12 countries, with six conducted in the USA (Beck 1988; Cahn-Weiner 2003; Davis 2001; Koltai 2001; Quayhagen 1995; Quayhagen 2000), two in Germany (Brueggen 2017; Heiss 1993), 12 in Italy (Bergamaschi 2013; Cavallo 2016; de Vreese 1998; Galante 2007; Giovagnoli 2017; Giuli 2016; Jelcic 2012; Jelcic 2014; Mapelli 2013; Serino 2017; Trebbastoni 2018; Venturelli 2016 ), one in Sweden (Neely 2009), one in Japan (Kawashima 2005), three in France (Amieva 2016; Boller 2011; Goudour 2011), two in Spain (Fernández-Calvo 2011; Quintana Hernandez 2014), two in China (Kao 2016; Lee 2013), one in Korea (Kim 2015), one in Greece (Tsantali 2017), and one in Finland (Kallio 2018). Barban 2016 was a multi-country trial with recruitment in Italy, Greece, Norway, and Spain. It is worth noting that approximately one-third of all included studies (13) were conducted in Italy, 10 of these in the past six years. We further note that no eligible trials were found from the UK, Canada, or Australia, and that no eligible studies were found that were conducted in the USA since 2003 (these studies were all included in a previous review - Bahar-Fuchs 2013). Samples in the included studies ranged from 12 participants in Galante 2007 to 653 participants in Amieva 2016 , and 13 of the included studies had samples of more than 50 participants. Of the 33 included studies, 30 were published in English, two in Spanish, and one in French. A member of the review team (JS) translated essential information from Spanish and French studies to English.

\section{General characteristics of participants}

Participants in all trials had a diagnosis of mild to moderate dementia according to the published paper. Diagnosis was based exclusively on NINCDS-ADRDA criteria in 12 of the included trials, whereas six studies used either NINCDS-ADRDA or DSMIV criteria, and one study used either NINCDS-ADRDA or Milan Overall Dementia Scale (MODS) criteria. In four studies, diagnoses were supported by scores on the Mini Mental State Examination (MMSE) test alone, and one study used MMSE in combination with the Global Deterioration Scale (GDS). Two studies used DSMIV alone, three used the Clinical Dementia Rating Scale (CDR) alone, and one used the Chinese version of the Dementia Rating Scale (DRS) alone. Two studies used the DRS in combination with the GDS, and one study used physician judgement as the basis for diagnosis. In almost all trials, the presumed aetiology was $A D$, but other aetiologies were suspected in some studies: probable mixed dementia (Beck 1988); cardiovascular dementia or Parkinson's disease (Quayhagen 2000); vascular dementia (Neely
2009); and Parkinson's disease, vascular dementia, Lewy body dementia, or unknown dementia (Kallio 2018). With the exception of a small number of studies in which participants were recruited from hospital or nursing home facilities (Beck 1988; Cavallo 2016; Kawashima 2005; Mapelli 2013; Venturelli 2016), participants generally resided in the community. Severity of dementia among participants residing in a nursing home environment may have been somewhat greater, with MMSE scores ranging between 15 and 20 in Beck 1988, between 7 and 30 in Kawashima 2005, between 14 and 24 in Mapelli 2013, and between 10 and 15 in Venturelli 2016.

In two studies, the mean age of participants was greater than 65, but less than 70 years (Heiss 1993; Goudour 2011). In 19 of the included studies, the mean age of participants was between 70 and 80 years (Cavallo 2016; Quayhagen 1995; Tsantali 2017). In ten of the included trials, the mean age of participants was greater than 80 years (Boller 2011; Kawashima 2005; Kallio 2018). Two studies did not report the mean age of participants although, in both of them, they were 50 years and above (Brueggen 2017; Lee 2013)

\section{General characteristics of experimental interventions}

All studies included at least one condition that met our criteria for CT. In six studies, two conditions met our criteria for CT (Boller 2011; Fernández-Calvo 2011; Jelcic 2014; Koltai 2001; Lee 2013; Neely 2009), and for these studies, data from the two conditions were combined as specified in the protocol to form a single experimental condition. Of a total of $39 \mathrm{CT}$ interventions, 26 were classified as multi-domain interventions and 13 as single-domain interventions (Boller 2011; Cahn-Weiner 2003; Davis 2001; Goudour 2011; Jelcic 2012; Jelcic 2014; Kao 2016; Lee 2013; Neely 2009). We classified most experimental interventions as 'straight CT', but we classified 13 experimental interventions as 'augmented CT' due to the inclusion of additional elements, usually associated with reality orientation, cognitive stimulation, or cognitive rehabilitation, as was the case with de Vreese 1998 or Davis 2001, Mapelli 2013 or Kim 2015, for example. We classified 23 of the 39 experimental interventions as primarily individual training (although in some cases, participants could receive some assistance from their caregivers) and 11 as group training. Two experimental conditions involved a combination of group and individual training (Kallio 2018; Kim 2015), and one experimental treatment involved dyads (Neely 2009). The remaining studies provided insufficient detail to show whether participants in the experimental conditions were trained individually or in a group (Boller 2011; Kao 2016).

\section{General characteristics of comparison conditions}

In seven studies, two conditions met our criteria for a comparison condition (Brueggen 2017; de Vreese 1998; Giovagnoli 2017; Kao 2016; Mapelli 2013; Venturelli 2016; Tsantali 2017). In a further two studies (Amieva 2016; Quintana Hernandez 2014), three conditions met our criteria for comparison conditions, and in one study (Quayhagen 2000), four conditions could be classified as comparison conditions. We classified 17 of the comparison conditions as passive controls (involving a wait-list condition, a no-contact condition, placebo medication, or usual care (i.e. continuing with usual activities of the nursing home or hospital, or receiving conventional medical care)) and 14 as active controls (including social support groups, activities similar to those in the experimental condition but with a passive approach, unstructured conversation or discussion, educational information, semi-structured interviews, clinical support, unstructured or non- 
specific cognitive activity, and other non-specific activities). We considered that 15 interventions met our criteria for an alternative treatment. These included a new medication (de Vreese 1998); dyadic counselling, dual supportive seminar groups, and early-stage daycare programmes (Quayhagen 2000); occupational therapy (Mapelli 2013); mindfulness and muscular relaxation (Quintana Hernandez 2014); reminiscence therapy and cognitive rehabilitation (Amieva 2016; Brueggen 2017); and spaced retrieval combined with Montessori activities (Kao 2016), aerobic exercise (Venturelli 2016), cognitive stimulation (Tsantali 2017), and music therapy and neuroeducation (Giovagnoli 2017).

\section{Intervention dose and duration}

The duration of interventions ranged from two weeks in Boller 2011 to approximately 104 weeks in Quintana Hernandez 2014. In seven of the 33 studies, the intervention lasted three months or longer (Amieva 2016; Bergamaschi 2013; Heiss 1993; Kawashima 2005; Quintana Hernandez 2014; Trebbastoni 2018; Tsantali 2017). In nine studies, researchers delivered the intervention in more than three sessions per week (Bergamaschi 2013; Boller 2011; Brueggen 2017; Kao 2016; Kawashima 2005; Mapelli 2013; Quayhagen 1995; Quintana Hernandez 2014; Venturelli 2016).

\section{Excluded studies}

We have summarised the characteristics of excluded studies in the Characteristics of excluded studies table.

\section{Risk of bias in included studies}

We have summarised risk of bias for individual studies, along with a justification for our ratings, in the Characteristics of included studies tables. We have summarised risk of bias for specific domains across studies in Figure 2 and Figure 3.

\section{Figure 2. Risk of bias graph: review authors' judgements about each risk of bias item presented as percentages across all included studies.}

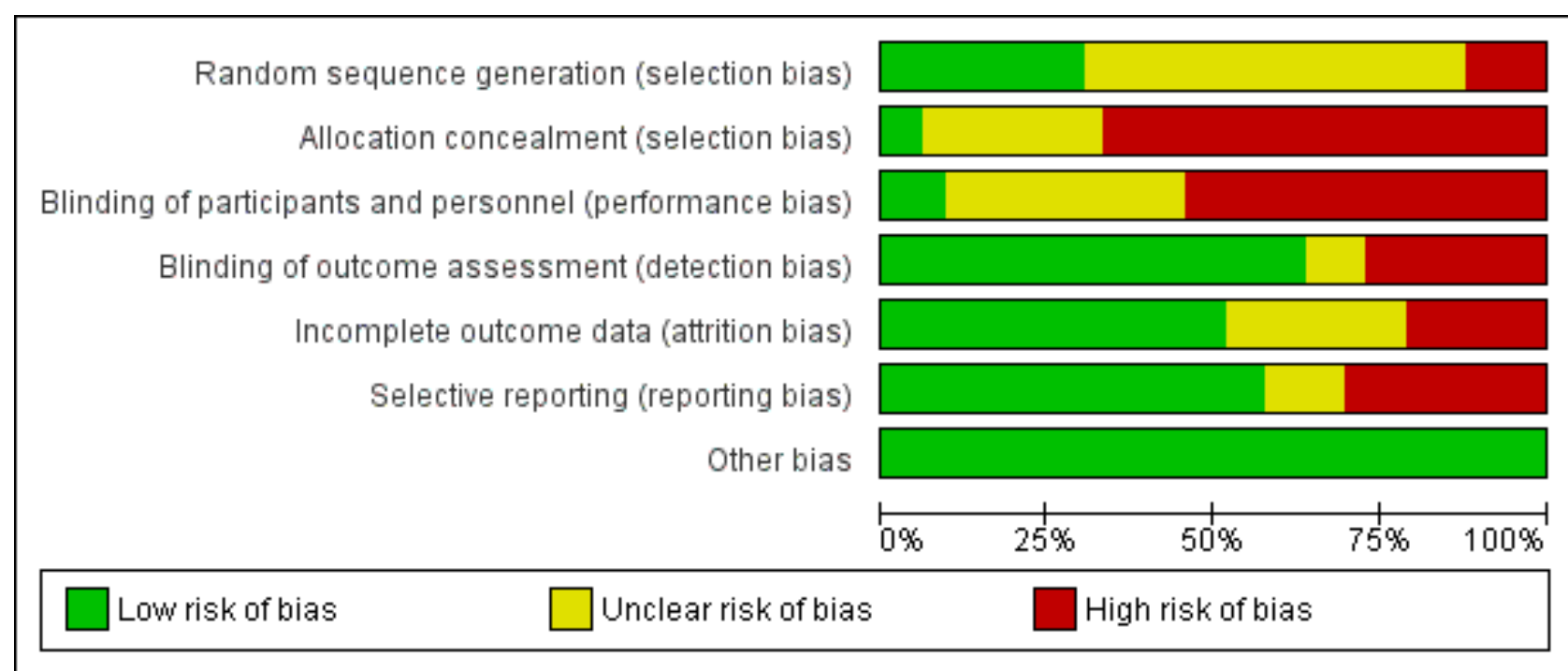


Figure 3. Risk of bias summary: review authors' judgements about each risk of bias item for each included study.

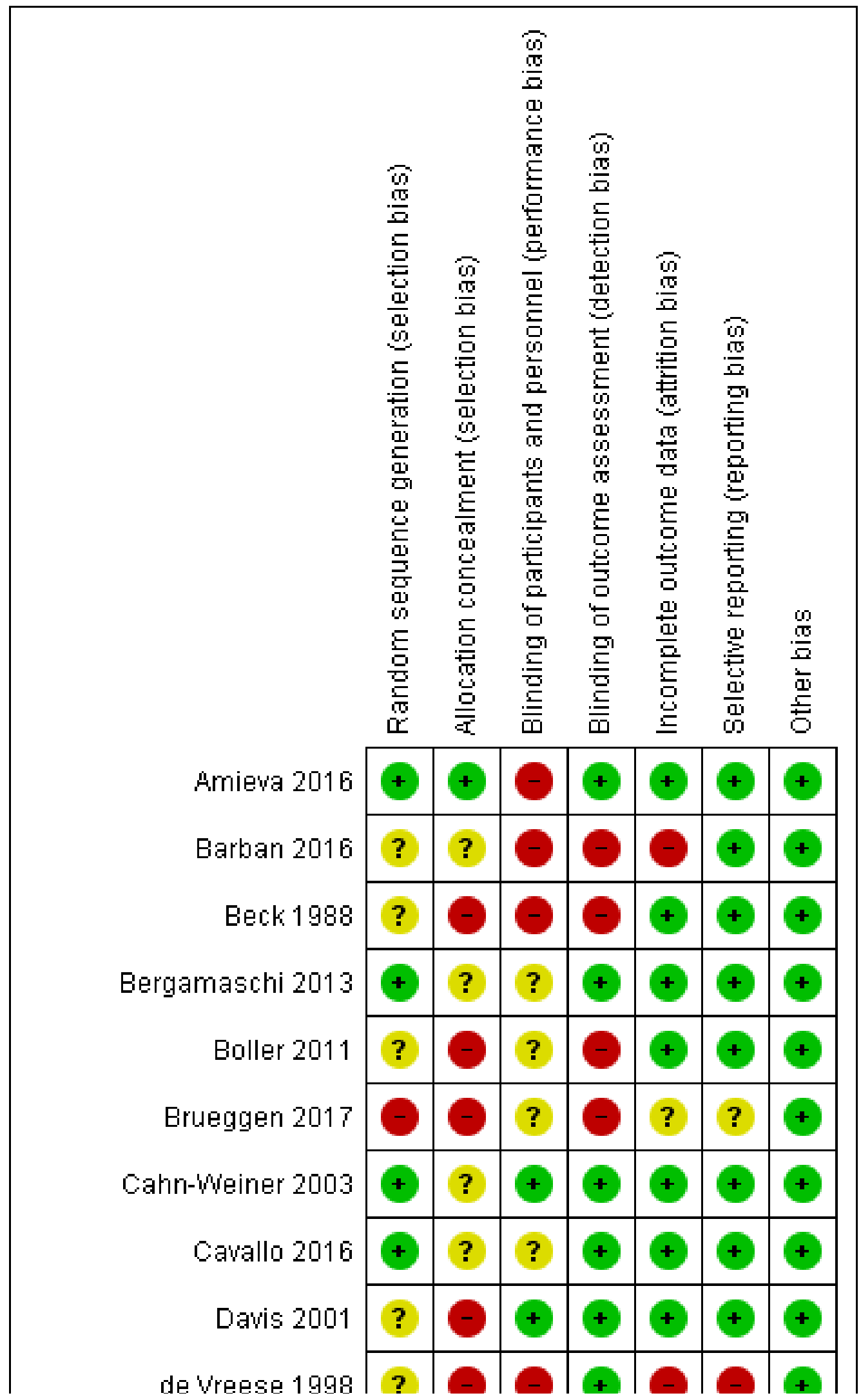


Figure 3. (Continued)

\begin{tabular}{|c|c|c|c|c|c|c|c|}
\hline de Vreese 1998 & ? & - & - & + & - & & + \\
\hline Fernández-Calvo 2011 & $?$ & & 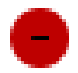 & $\oplus$ & $?$ & $\oplus$ & + \\
\hline Galante 2007 & - & & 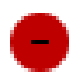 & + & + & + & + \\
\hline Giovagnoli 2017 & + & $?$ & $?$ & + & $?$ & $?$ & + \\
\hline Giuli 2016 & + & $?$ & $?$ & - & + & & + \\
\hline Goudour 2011 & ? & C & $?$ & + & $?$ & $?$ & + \\
\hline Heiss 1993 & ? & & ? & - & - & + & + \\
\hline Jelcic 2012 & + & ? & 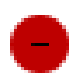 & + & + & 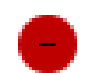 & + \\
\hline Jelcic 2014 & - & & $?$ & + & + & 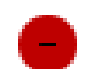 & + \\
\hline Kallio 2018 & + & + & - & + & $?$ & $?$ & + \\
\hline Kao 2016 & ? & & & + & + & 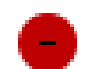 & + \\
\hline Kawashima 2005 & $?$ & & & $?$ & + & + & + \\
\hline Kim 2015 & ? & & $?$ & + & + & + & + \\
\hline Koltai 2001 & - & & & ( & ? & & + \\
\hline Lee 2013 & ? & 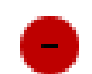 & ? & + & c & + & + \\
\hline Mapelli 2013 & + & ? & 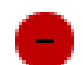 & + & + & 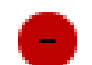 & + \\
\hline Neely 2009 & ? & & & & + & 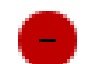 & + \\
\hline Quayhagen 1995 & ? & & & ? & - & 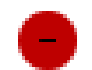 & + \\
\hline Quayhagen 2000 & ? & & - & + & ? & + & + \\
\hline Quintana Hernandez 2014 & ? & - & + & $?$ & + & + & + \\
\hline Serino 2 & $?$ & - & $?$ & - & $?$ & + & + \\
\hline
\end{tabular}


Figure 3. (Continued)

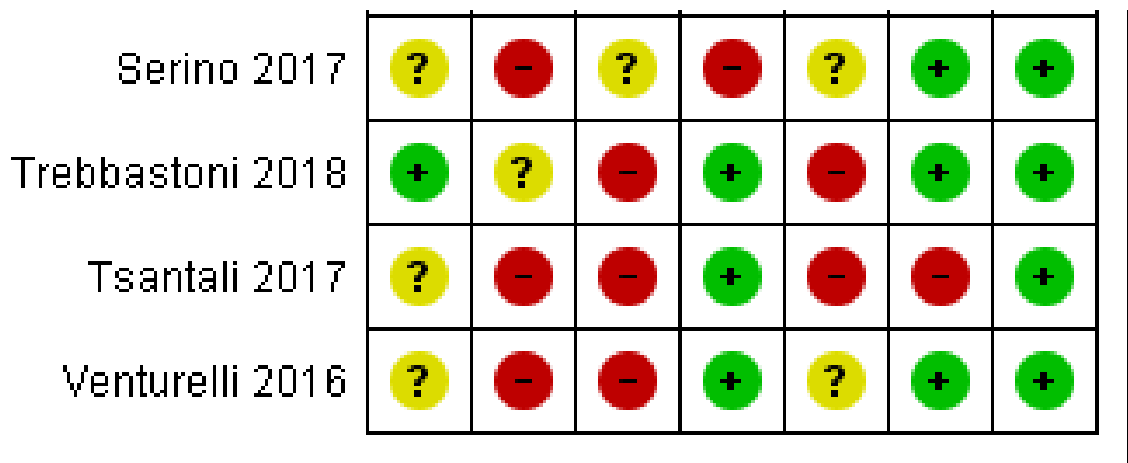

\section{Allocation}

Although all studies are described as randomised trials, many studies provided insufficient detail regarding the randomisation procedure to determine whether the sequence was indeed random; accordingly we rated these as having unclear risk of bias. We rated two studies as having high risk of selection bias, generating the sequence in a manner that was unlikely to be truly random (Galante 2007; Jelcic 2014). We rated studies in which a remote, computerised randomisation method was carried out as low risk in relation to allocation concealment, as this is intrinsic to the method (e.g. Amieva 2016; Kallio 2018). However, we assumed that allocation was not concealed in studies that generated the sequence in a manner that did not guarantee allocation concealment or that provided insufficient detail concerning the randomisation procedure, and did not state explicitly that allocation was concealed (e.g. Beck 1988; Kawashima 2005).

\section{Blinding}

In most studies, post-intervention assessments were performed by research staff, who were unaware of the condition to which participants were assigned, although a small number of studies provided insufficient detail to ensure that this was done (Kawashima 2005; Quayhagen 1995; Quintana Hernandez 2014). However, in approximately 25\% of trials (Barban 2016; Beck 1988; Boller 2011; Brueggen 2017; Giuli 2016; Heiss 1993; Koltai 2001; Neely 2009; Serino 2017), unmasked personnel completed outcome assessments, leading to increased risk of detection bias. In relation to performance bias, because blinding of those delivering the intervention typically is not possible in studies of CT, we focused our assessment of risk on the extent to which participants were blinded to whether they were assigned to an experimental or control intervention. This is not possible in studies that included only a passive (e.g. treatment as usual) control condition; we therefore rated these studies as having high risk of performance bias. We rated studies that used an active control or an alternative treatment as having unclear risk of performance bias if no mention was made of an attempt to mask whether the allocated condition was an experimental or control intervention. We rated approximately $90 \%$ of studies as having high or unclear risk of performance bias.

\section{Incomplete outcome data}

In approximately $50 \%$ of the included studies, we found no evidence of attrition bias; however, we judged about half of the remaining studies to have unclear risk (e.g. Giovagnoli 2017;
Quayhagen 2000), and we judged half to be at high risk of bias due to attrition (e.g. de Vreese 1998; Tsantali 2017).

\section{Selective reporting}

In most cases, studies seem to have reported all outcomes, or study authors provided them in the required format upon request. We sought information from trial registries to determine whether all pre-specified outcomes were reported, but we found no published protocols for any of the included studies.

\section{Effects of interventions}

See: Summary of findings for the main comparison Cognitive training compared to control immediately post intervention for people with mild to moderate dementia; Summary of findings 2 Cognitive training compared to control in the medium term ( 3 to 12 months post intervention) for people with mild to moderate dementia; Summary of findings 3 Cognitive training compared to alternative treatment immediately post intervention for people with mild to moderate dementia; Summary of findings 4 Cognitive training compared to alternative treatment in the medium term ( 3 to 12 months post intervention) for people with mild to moderate dementia

We summarised main outcomes when CT was compared with control interventions at end of treatment in Summary of findings for the main comparison.

We summarised main outcomes when CT was compared with control interventions in the medium term in Summary of findings 2.

We summarised main outcomes when CT was compared with alternative treatments at end of treatment in Summary of findings 3.

We summarised main outcomes when CT was compared with alternative treatments in the medium term in Summary of findings 4.

\section{Participant outcomes}

Global cognition (composite outcome measure) at end of treatment (primary outcome) and in the medium term (secondary outcome)

\section{Comparison with control}

We found a small to moderate effect favouring CT relative to a control condition on the primary outcome, namely, global 
cognition measured with a composite cognitive score at end of training (standardised mean difference (SMD) $0.42,95 \%$ confidence interval $(\mathrm{Cl}) 0.23$ to $0.61 ; 27$ trials; 1389 participants; Analysis 1.1; Figure 4). Our certainty in this finding is moderate due to heterogeneity in effect estimates, which was not explained by planned subgroup analyses. We did not detect clear evidence of publication bias when examining the funnel plot in Figure 5. We performed a more conservative sensitivity analysis in which we assumed no correlation between observations before and after the intervention and still found moderate-quality evidence of a small beneficial effect of CT relative to a control on global cognition at end of treatment (SMD 0.24, $95 \% \mathrm{Cl} 0.12$ to 0.36 ; Analysis 1.2).

Figure 4. Forest plot of comparison: 1 Cognitive training vs control immediately post intervention, outcome: 1.1 Change in a global measure of cognition (composite).

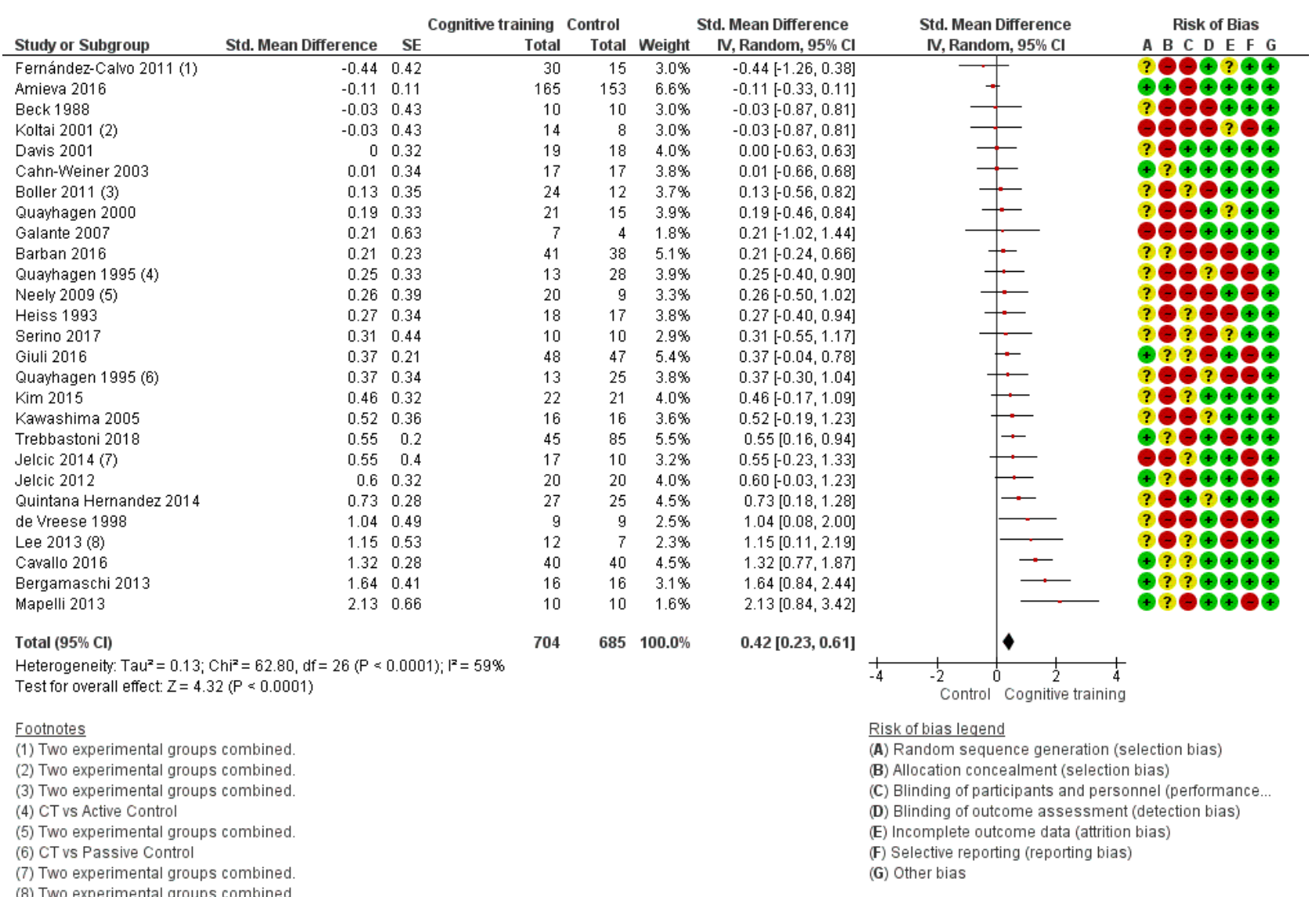


Figure 5. Funnel plot of comparison: 1 Cognitive training vs control immediately post intervention, outcome: 1.1 Change in a global measure of cognition (composite).

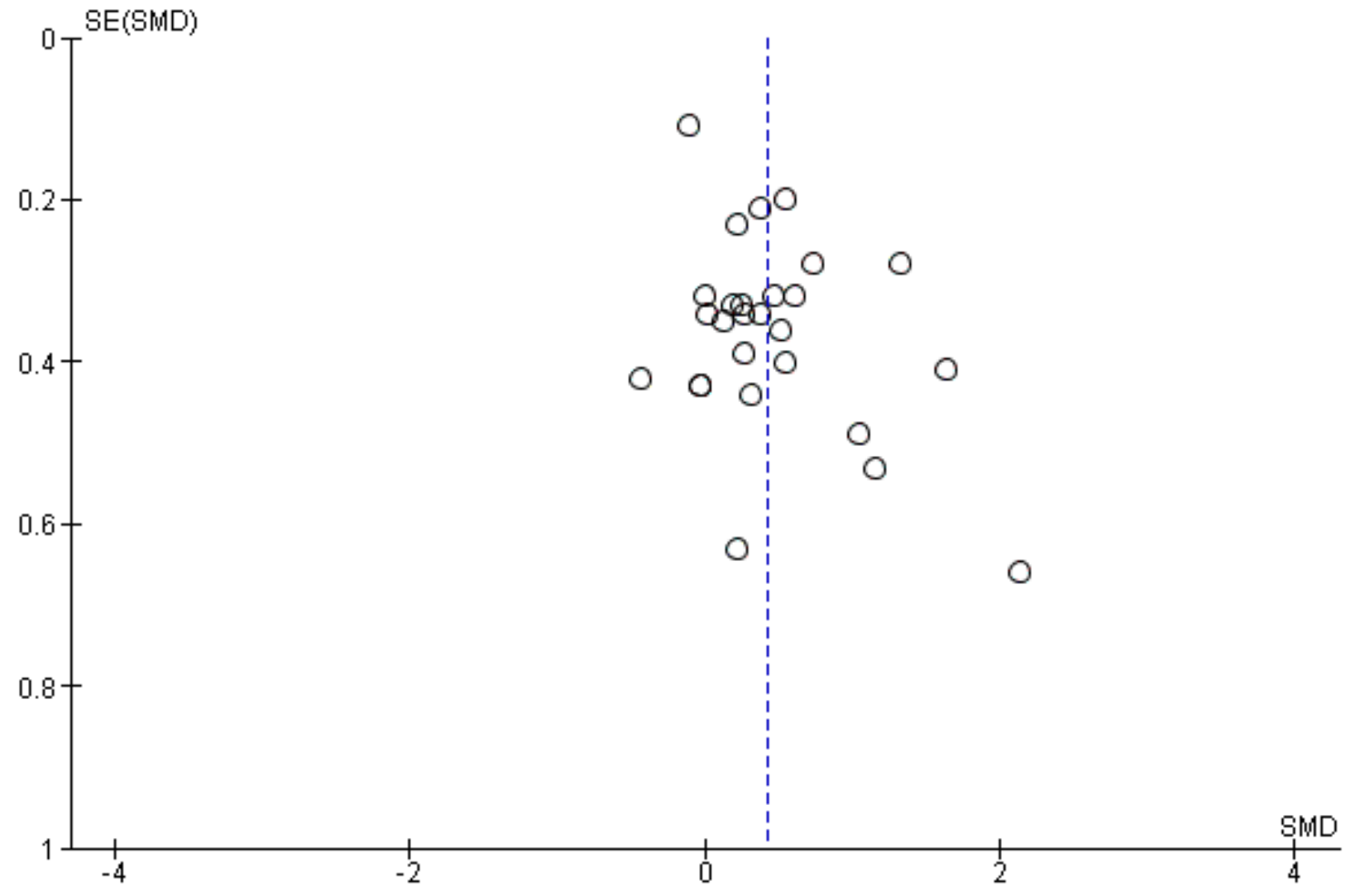

We were uncertain whether $\mathrm{CT}$ had an effect relative to a control condition on a composite measure of global cognition in the medium term (i.e. between 3 and 12 months post treatment) due to the very low quality of evidence, both in our main analysis (SMD $0.65,95 \% \mathrm{Cl} 0.11$ to $1.2,8$ trials; 387 participants; Analysis 2.1 ) and in a sensitivity analysis in which we assumed no correlation between observations before and after the intervention (SMD 0.40, 95\% Cl 0.09 to 0.71 ; Analysis 2.2). Quality concerns were related to risk of bias, heterogeneity, and imprecision.

\section{Comparison with an alternative treatment}

In comparison with an alternative treatment, we found no clear evidence of an effect of CT on a global measure of cognition at end of treatment, but the quality of evidence for this finding is low due to very serious imprecision (SMD $0.21,95 \% \mathrm{Cl}-0.23$ to $0.64 ; 7$ trials; 769 participants; Analysis 3.1 ; Figure 6$)$. In a more conservative sensitivity analysis, assuming zero correlation between observations before and after the intervention, we found there may be little or no effect of CT (SMD $-0.03,95 \% \mathrm{Cl}-0.23$ to 0.17 ; Analysis 3.2) on a composite global cognition score at end of training. The quality of evidence related to this outcome is also low. 
Figure 6. Forest plot of comparison: 3 Cognitive training vs alternative treatment immediately post intervention, outcome: 3.1 Change in a global measure of cognition (composite).

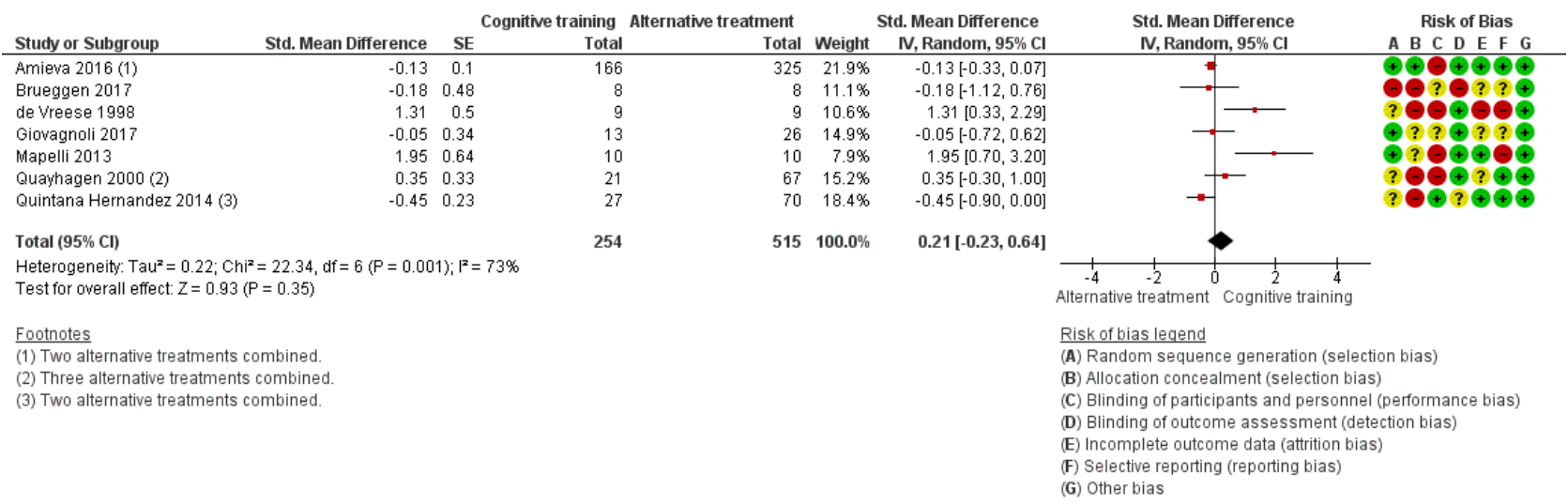

In the medium term, we were unable to determine whether $\mathrm{CT}$ was associated with any gains in global cognition relative to an alternative treatment because of the very low quality of evidence (SMD 1.31, $95 \% \mathrm{Cl}-1.03$ to $3.65 ; 2$ studies; 73 participants; Analysis 4.1 Analysis 4.2).

\section{Global cognition (screening measures) at end of treatment and in the medium term (secondary outcomes)}

\section{Comparison with control}

Findings were similar when global cognition was assessed using a screening measure, typically the MMSE (although nine studies used another measure as well as the MMSE, including the Alzheimer's Disease Assessment Scale - cognitive subscale (ADAS-Cog), the Milan Overall Dementia Scale (MODA), the Cambridge Cognitive Assessment (CAMCOG), and the complete neuropsychological battery (ENB-2), and one study used only the ADAS-Cog). We found low-quality evidence suggesting a moderate effect of $\mathrm{CT}$ on global cognition at end of training (SMD $0.65,95 \% \mathrm{Cl} 0.26$ to $1.05 ; 20$ trials; 1288 participants; Analysis 1.3; Figure 7) and a smaller but still beneficial effect in our more conservative sensitivity analysis (SMD $0.27,95 \% \mathrm{Cl} 0.04$ to 0.50 ; Analysis 1.4 ).

Figure 7. Forest plot of comparison: 1 Cognitive training vs control immediately post intervention, outcome: 1.3 Change in a global measure of cognition.

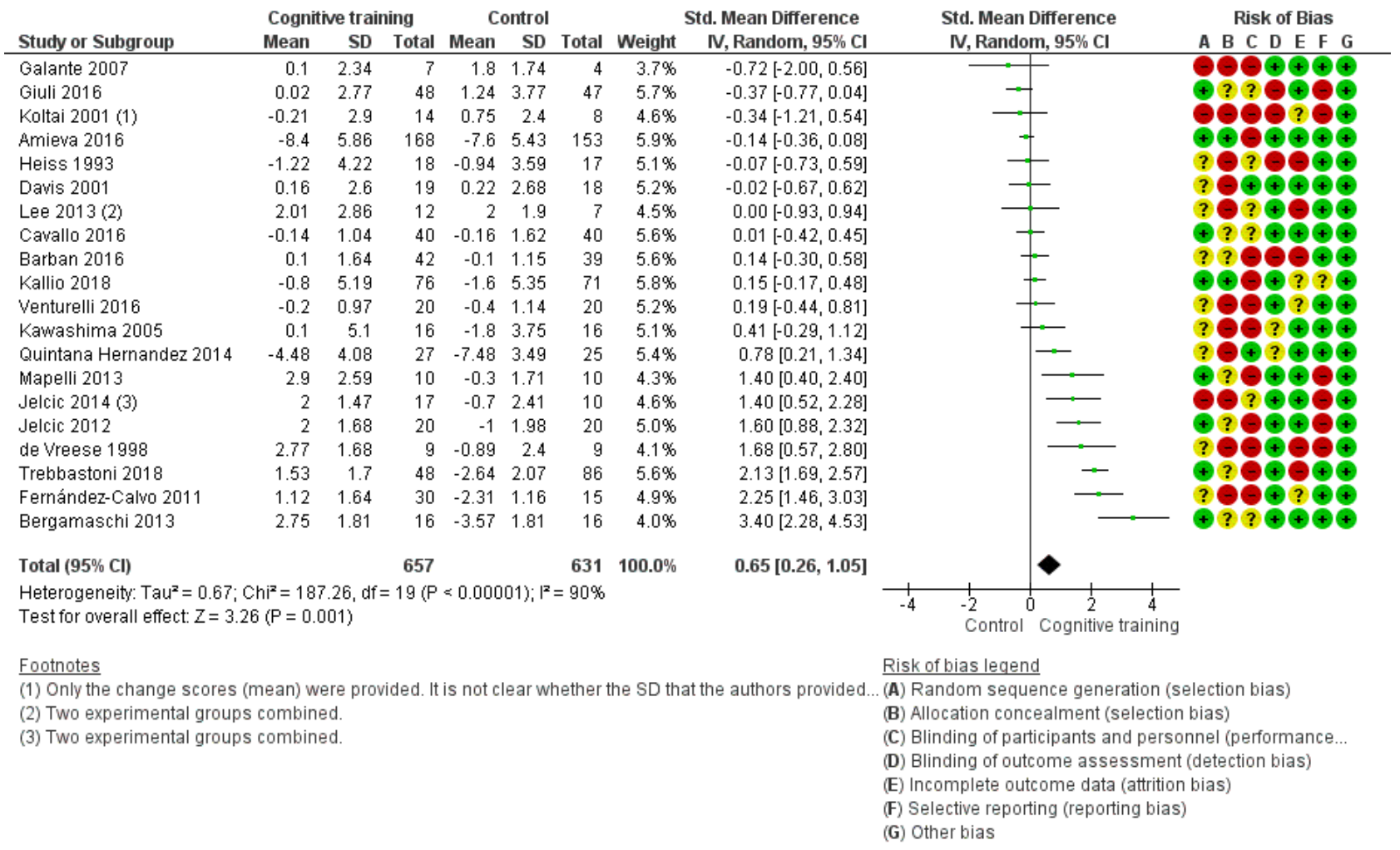


We are unable to determine whether there is any effect on global cognition assessed with screening measures in the medium term due to the very low quality of evidence, including following a sensitivity analysis (6 trials; 387 participants; Analysis 2.3 Analysis 2.4). Quality concerns were related to risk of bias, heterogeneity, and imprecision.

\section{Comparison with an alternative treatment}

We are uncertain of any effect of CT on a global measure of cognition when compared to an alternative treatment immediately after the intervention due to the very low quality of evidence (SMD 0.16, 95\% $\mathrm{Cl}-0.28$ to $0.60 ; 7$ trials; 724 participants; Analysis 3.3). This was also true in the medium term (SMD 3.20, 95\% Cl -2.89 to 9.29; 2 trials; 73 participants; Analysis 4.3).

\section{Subgroup analyses}

To explore the sources of heterogeneity in our main comparison for global cognition, we performed several pre-specified subgroup analyses, including type of control condition (see Analysis 10.3), type of CT (see Analysis 8.3; Analysis 9.3), dose delivered (frequency and duration; see Analysis 6.3; Analysis 7.3), and risk of bias (see Analysis 5.3). We found no significant differences between subgroups in any of the subgroup analyses in relation to global cognition, although we found non-significant trends suggesting that trials in which the intervention was delivered at a frequency greater than three times per week were associated with larger effects than trials in which the intervention was delivered up to three times per week (Analysis 6.3), and that traditional CT trials were associated with larger effect sizes than 'augmented' CT trials (Analysis 8.1).

\section{Clinical disease severity at end of treatment (secondary outcome)}

\section{Comparison with control}

We found a large effect of $\mathrm{CT}$ relative to a control condition on the secondary outcome of clinical disease severity at end of treatment (SMD 1.07, 95\% Cl 0.59 to 1.55 ; 6 trials; 215 participants; Analysis 1.5). However, owing to concerns regarding heterogeneity and imprecision, our certainty in the accuracy of the estimate is low.

\section{Comparison with an alternative treatment}

When compared with an alternative treatment, we found no evidence of an effect of CT on clinical disease severity, and the quality of the evidence was low due to very serious imprecision (SMD $0.15,95 \% \mathrm{Cl}-0.33$ to $0.63 ; 3$ trials; 131 participants; Analysis 3.5).

\section{Clinical disease severity in the medium term (primary outcome)}

\section{Comparison with control}

We were unable to determine whether there is an effect of CT relative to a control intervention on the primary outcome of clinical disease severity in the medium term (3 to 12 months post treatment), as the quality of the evidence is very low due to concerns regarding risk of bias and imprecision (SMD 0.55, $95 \% \mathrm{Cl} 0.12$ to $0.98 ; 2$ trials; 98 participants; Analysis 2.5 Figure 8). We performed a sensitivity analysis in which a conservative assumption of no correlation between observations before and after the intervention was applied to the data, and we were again unable to determine whether there was any effect of CT relative to a control condition on clinical disease severity in the medium term due to the very low quality of evidence (SMD $0.28,95 \% \mathrm{Cl}-0.14$ to 0.71 ; Analysis 2.6).

Figure 8. Forest plot of comparison: 2 Cognitive training vs control in the medium term ( 3 to 12 months post intervention), outcome: 2.5 Change in disease progression.

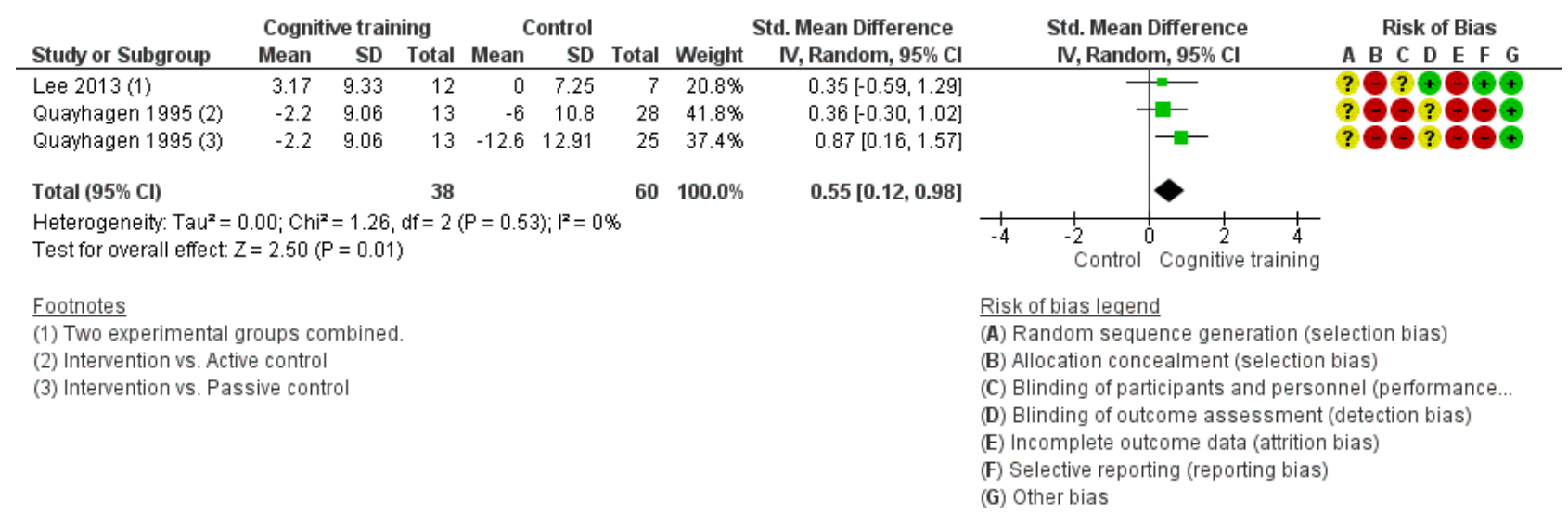

\section{Comparison with an alternative treatment}

None of the included studies measured this outcome in the medium term.

\section{Specific cognitive domains (secondary outcomes)}

\section{Comparison with control}

Results comparing effects CT versus a control condition at end of treatment on specific cognitive domains (secondary outcomes) are depicted in Analysis 1.6 Analysis 1.7 Analysis 1.8 Analysis 1.9 Analysis 1.10 Analysis 1.11 Analysis 1.12 Analysis 1.13 and Figure 9. CT showed a positive effect in immediate and delayed 
memory, attention and working memory, language (naming), executive functions, and verbal category fluency immediately after the intervention. However, with the exception of findings regarding category fluency (high certainty), our certainty in findings concerning specific cognitive domains was generally very low to low. As can also be seen in Analysis 2.7 Analysis 2.8 Analysis 2.9 Analysis 2.10 Analysis 2.11 Analysis 2.12 Analysis 2.13 and Analysis 2.14, gains in some of the specific cognitive domains (delayed memory, naming, executive functions, and verbal category fluency) were maintained in the medium term. However, again, with the exception of category fluency scores (high certainty), our certainty in these findings ranges from low to very low.

We performed pre-specified subgroup analyses to explore effects of sources of heterogeneity in the comparison of $\mathrm{CT}$ versus control interventions on scores in specific cognitive domains, including high risk of bias studies versus lower risk of bias studies, type of CT (traditional vs augmented), type of domain (multi-domain vs single domain), and type of control group (passive vs active). Subgroup analyses suggest that the intervention dose moderated effects of $\mathrm{CT}$ on verbal letter fluency $\left(\mathrm{Chi}^{2}=3.96, \mathrm{df}=1, \mathrm{P}=0.05\right)$, and larger effects were associated with interventions delivered more than three times per week (SMD 1.0, 95\% Cl 0.09 to 1.92; 3 trials; 84 participants) relative to interventions delivered up to three times per week (SMD $0.05,95 \% \mathrm{Cl}-0.13$ to $0.24 ; 9$ trials; 460 participants; Analysis 6.9; Figure 10). In addition, subgroup analyses suggest that type of CT moderated effects on verbal category fluency $\left(\mathrm{Chi}^{2}=\right.$ $4.81, \mathrm{df}=1, \mathrm{P}=0.03$ ), and multi-domain training was associated with larger effects (SMD 0.70, 95\% Cl 0.38 to 1.02; 6 trials; 371 participants) than single-domain training (SMD 0.14, 95\% Cl -0.25 to 0.52 ; 3 trials; 104 participants; Analysis 9.12; Figure 11). We found no other explanations of heterogeneity in other subgroup analyses.

Figure 9. Forest plot of comparison: 1 Cognitive training vs control immediately post intervention, outcome: 1.6 Change in delayed memory.

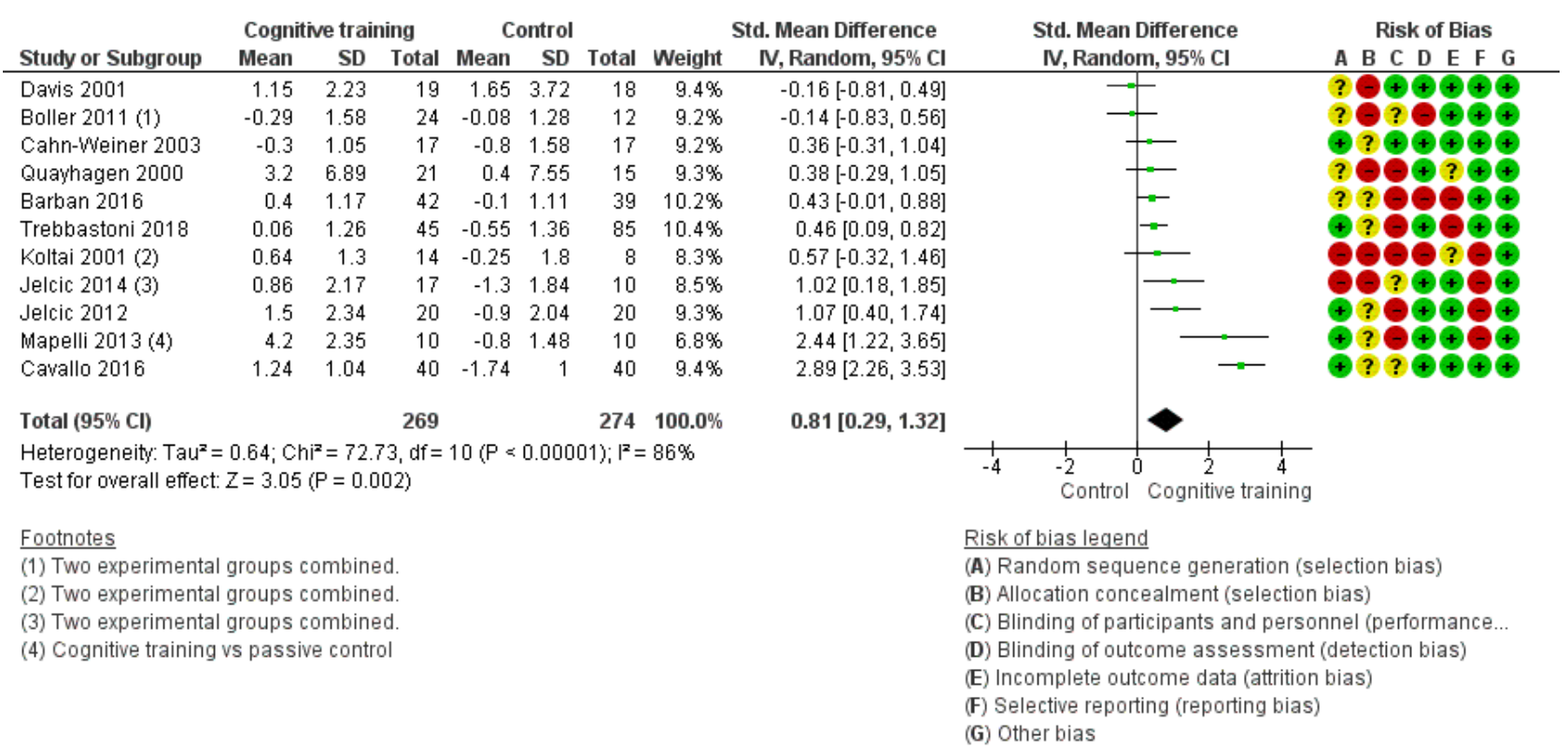


Figure 10. Forest plot of comparison: 6 Cognitive training vs control immediately post intervention - intervention dose, outcome: 6.9 Change in verbal letter fluency.

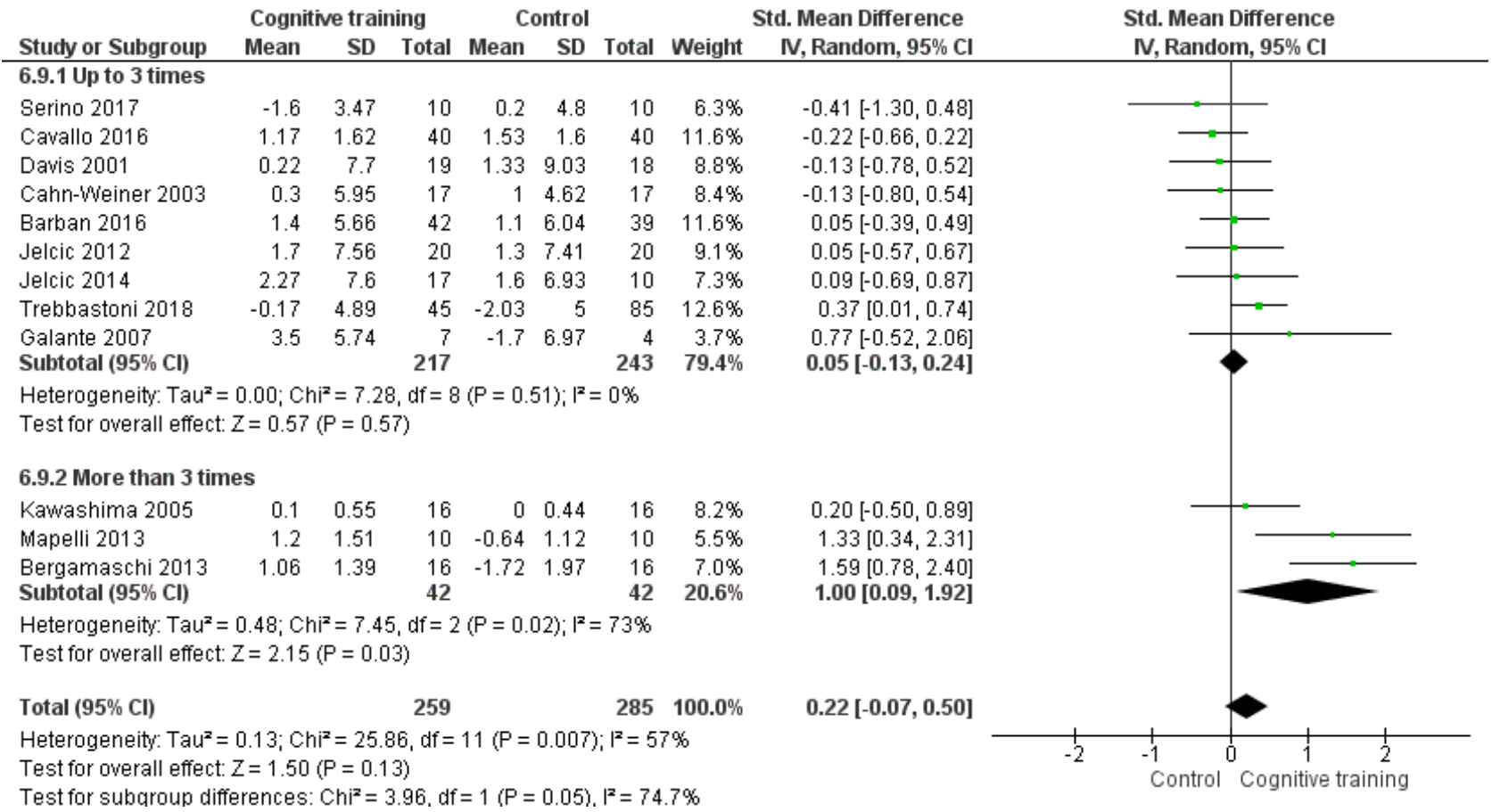

Figure 11. Forest plot of comparison: 9 Cognitive training vs control immediately post intervention - type of CT (multi-domain vs single domain), outcome: 9.12 Change in verbal category fluency.

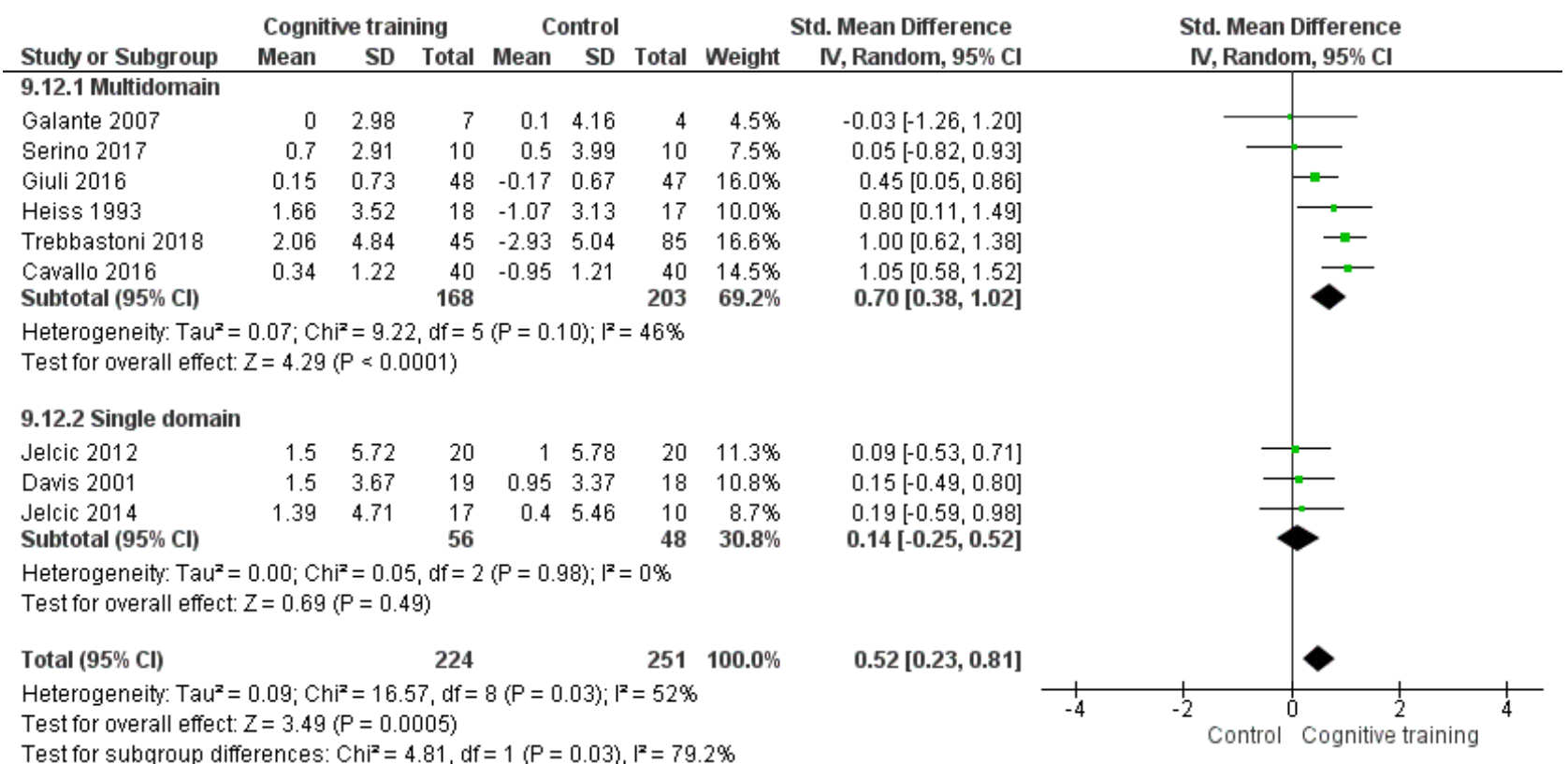

\section{Comparison with an alternative treatment}

Results comparing effects of CT versus an alternative treatment at end of treatment on specific cognitive domains are depicted in Analysis 3.6 Analysis 3.7 Analysis 3.8 Analysis 3.9 Analysis 3.10 Analysis 3.11 Analysis 3.12 and Analysis 3.13. Due to the very low quality of evidence, we could not determine whether $\mathrm{CT}$ is associated with any benefit for specific cognitive domains compared with an alternative treatment at the end of the intervention. Results for comparison of $\mathrm{CT}$ versus an alternative treatment in the medium term are depicted in Analysis 4.6 Analysis 4.7 Analysis 4.8 Analysis 4.9 Analysis 4.10 Analysis 4.11 Analysis 4.12 and Analysis 4.13. As can be seen, with the exception of immediate and delayed memory, other specific cognitive domains were evaluated by a single study; therefore we could not perform 
meta-analyses. The quality of evidence in relation to all of these outcomes was low to very low, so we are unable to determine whether, relative to an alternative treatment, $\mathrm{CT}$ is associated with any gains in specific cognitive domains in the medium term.

\section{Meta cognition - self-reported (secondary outcome)}

\section{Comparison with control}

We found no evidence that $\mathrm{CT}$ had an effect on self-rated cognitive ability immediately post treatment (SMD $0.12,95 \% \mathrm{Cl}-0.87$ to 1.12 ; 2 trials; 41 participants; Analysis 1.14; Analysis 2.15). However, the quality of evidence was low, and the findings were very imprecise. Data for this outcome in the medium term were available from only one trial (Lee 2013), in which participants in the experimental condition (which combined data from two experimental conditions - a computerised version and a non-computerised version of the training) reported fewer difficulties related to prospective memory following treatment relative to control. However, this is a very small trial $(n=19)$, which we rated to be at high risk of attrition bias due to incomplete outcome data and at high risk of selection bias due to lack of allocation concealment. Given the subjective nature of the outcome, and use of a 'no treatment' control condition, our certainty in this finding is very low. Therefore, we are unable to determine whether $\mathrm{CT}$ is associated with any gains in meta cognition in the medium term relative to a control treatment.

\section{Comparison with an alternative treatment}

Data concerning this outcome were not available in the comparison of $\mathrm{CT}$ versus an alternative treatment in the immediate or medium term.

\section{Meta cognition - informant-reported (secondary outcome)}

\section{Comparison with control}

We are unable to determine whether relative to a control condition, CT had an effect on informant-rated cognitive ability immediately post training due to very low quality of the evidence (SMD -0.01 , $95 \% \mathrm{Cl}-1.29$ to $1.26 ; 2$ trials; 56 participants; Analysis 1.15). In the medium term, a single study found no evidence of an effect of CT on informant-rated cognition in the medium term (SMD - $0.06,95 \%$ $\mathrm{Cl}-0.73$ to 0.62 ; Analysis 2.16 ), but the quality of the evidence was low (Cahn-Weiner 2003).

\section{Comparison with an alternative treatment}

No studies contributed to this outcome when comparing CT versus alternative treatments in the immediate or medium term.

\section{Mood (secondary outcome)}

\section{Comparison with control}

We are unable to determine whether relative to a control condition, CT had an effect on participants' mood, as reflected on measures of depression, because the quality of the evidence was very low due to concerns related to inconsistency and imprecision (SMD $0.72,95 \% \mathrm{Cl}-0.10$ to $1.54 ; 8$ trials; 577 participants; Analysis 1.16, Figure 12). We found no evidence of an effect of CT over a control condition in the medium term. The quality of evidence was low due to very serious imprecision; thus, our results were inconclusive (i.e. between 3 and 12 months post treatment; SMD $0.21,95 \% \mathrm{Cl}-0.54$ to $0.96 ; 2$ trials; 30 participants; Analysis 2.17). We performed pre-specified subgroup analyses to explore the sources of heterogeneity in participants' mood when CT was compare with control, including type of control condition and type of CT (traditional vs augmented). The test for subgroup differences could not explain heterogeneity in traditional $\mathrm{CT}$ versus augmented $\mathrm{CT}$ $(P=0.64)$, nor in passive control versus active control $(P=0.71)$ (Analysis 8.15 Analysis 10.15).

Figure 12. Forest plot of comparison: 1 Cognitive training vs control immediately post intervention, outcome: 1.16 Change in participants' mood.

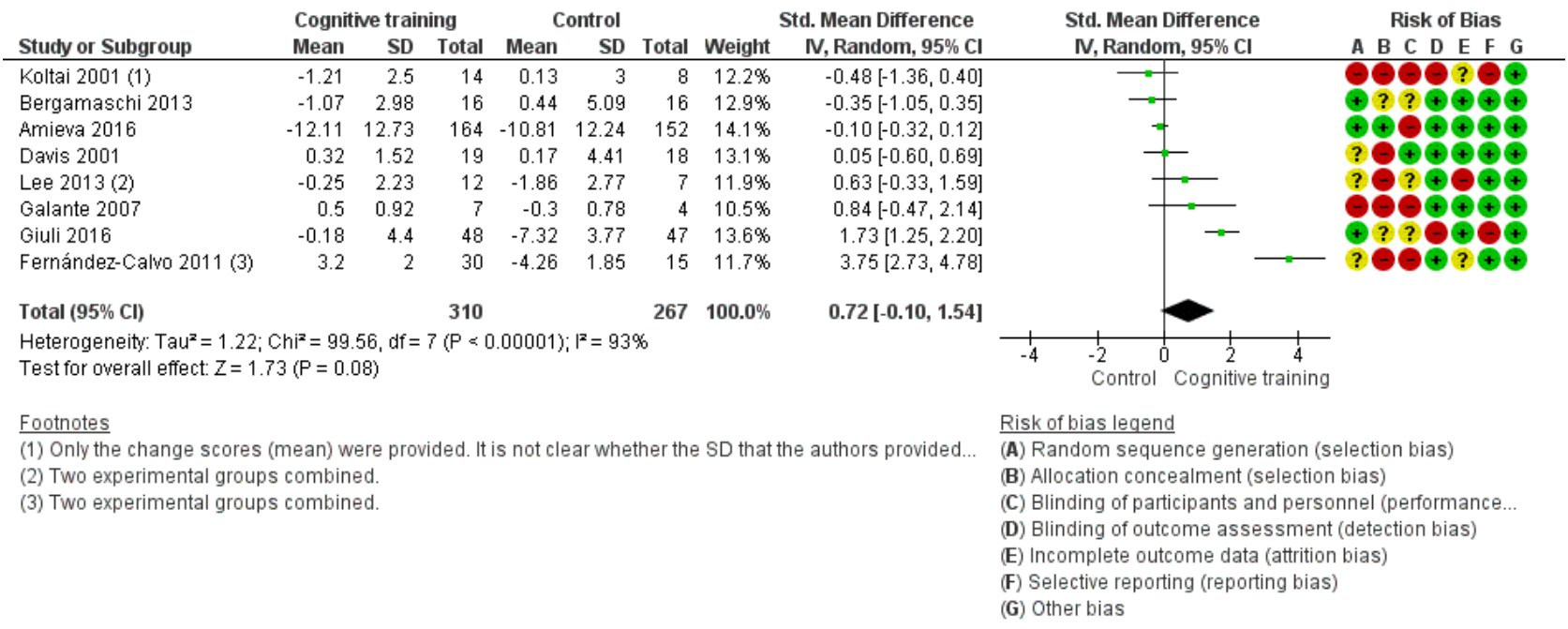




\section{Comparison with an alternative treatment}

When compared with an alternative treatment, we found that $\mathrm{CT}$ probably had little or no effect on participants' mood immediately post treatment; we are moderately certain of this finding (SMD $-0.11,95 \% \mathrm{Cl}-0.29$ to $0.07 ; 3$ trials; 543 participants; Analysis 3.16 ) Only one study contributed to this same outcome in the medium term (Giovagnoli 2017); therefore we could not perform a metaanalysis. Results of this study suggest that benefits in the medium term may favour an alternative treatment (SMD $-0.66,95 \% \mathrm{Cl}$ -1.35 to 0.02 ). However, due to very serious concerns related to imprecision, the quality of the evidence was low; therefore, the results are inconclusive.

\section{Capacity for activities of daily living (secondary outcome)}

\section{Comparison with control}

Relative to a control intervention, we found that CT may have little to no effect on capacity for activities of daily living immediately post treatment (SMD $0.12,95 \% \mathrm{Cl}-0.11$ to $0.35 ; 10$ trials; 687 participants; Analysis 1.17 Figure 13); however the quality of the evidence was low due to concerns related to risk of bias and imprecision. Therefore clear evidence is lacking.

Figure 13. Forest plot of comparison: 1 Cognitive training vs control immediately post intervention, outcome: 1.17 Change in capacity for activities of daily living.

\begin{tabular}{|c|c|c|c|c|c|c|c|c|c|c|c|}
\hline \multirow[b]{2}{*}{ Study or Subgroup } & \multicolumn{3}{|c|}{ Cognitive training } & \multicolumn{3}{|c|}{ Control } & \multicolumn{2}{|c|}{ Std. Mean Difference } & \multirow{2}{*}{\multicolumn{2}{|c|}{$\begin{array}{l}\text { Std. Mean Difference } \\
\text { IV, Random, } 95 \% \mathrm{Cl}\end{array}$}} & Risk of Bias \\
\hline & Mean & SD & Total & Mean & SD & Total & Weight & IV, Random, $95 \% \mathrm{Cl}$ & & & A B C D E F G \\
\hline Galante 2007 & -0.6 & 1.36 & 7 & 0.5 & 0.48 & 4 & $2.7 \%$ & $-0.88[-2.19,0.43]$ & & & \\
\hline de Vreese 1998 & -1.33 & 4.37 & 9 & 1.01 & 3.98 & 9 & $4.8 \%$ & $-0.53[-1.48,0.41]$ & & & \\
\hline Amieva 2016 & -1.76 & 8.46 & 167 & -1.22 & 8.27 & 153 & $24.0 \%$ & $-0.06[-0.28,0.16]$ & & & \\
\hline Cahn-Weiner 2003 & -0.2 & 3.16 & 17 & 0 & 3.07 & 17 & $8.3 \%$ & $-0.06[-0.74,0.61]$ & & & \\
\hline Kim 2015 & 0 & 0.86 & 21 & 0 & 1.05 & 21 & $9.6 \%$ & $0.00[-0.60,0.60]$ & & & \\
\hline Jelcic 2012 & 0.1 & 1.05 & 20 & 0 & 1.36 & 20 & $9.3 \%$ & $0.08[-0.54,0.70]$ & & & \\
\hline Barban 2016 & -0.1 & 0.96 & 38 & -0.3 & 1.08 & 38 & $13.9 \%$ & $0.19[-0.26,0.64]$ & & $=-$ & \\
\hline Giuli 2016 & 0.26 & 1.2 & 48 & -0.24 & 1.26 & 47 & $15.5 \%$ & $0.40[-0.00,0.81]$ & & - & \\
\hline Bergamaschi 2013 & -0.38 & 1.54 & 16 & -1.32 & 1.46 & 16 & $7.6 \%$ & $0.61[-0.10,1.32]$ & & 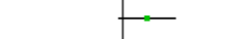 & \\
\hline Lee 2013 (1) & 2.24 & 3.33 & 12 & -2.14 & 4.54 & 7 & $4.3 \%$ & $1.10[0.09,2.11]$ & & & \\
\hline Total $(95 \% \mathrm{Cl})$ & & & 355 & & & 332 & $100.0 \%$ & $0.12[-0.11,0.35]$ & \multirow{2}{*}{\multicolumn{3}{|c|}{\begin{tabular}{ccccc}
1 & 1 & \multicolumn{1}{|c}{} & 1 & 1 \\
-4 & -2 & 0 & 2 & 4 \\
& Control & Cognitive training
\end{tabular}}} \\
\hline \multicolumn{7}{|c|}{$\begin{array}{l}\text { Heterogeneity: } \text { Tau }^{2}=0.04 ; \mathrm{Chi}^{2}=14.29, \mathrm{df}=9(\mathrm{P}=0.11) ; \mathrm{I}^{2}=37 \% \\
\text { Test for overall effect: } Z=1.04(P=0.30)\end{array}$} & & & & & \\
\hline \multirow{2}{*}{\multicolumn{7}{|c|}{$\frac{\text { Footnotes }}{\text { (1) Two experimental groups combined. }}$}} & & & \multirow{2}{*}{\multicolumn{3}{|c|}{$\begin{array}{l}\text { Risk of bias leqend } \\
\text { (A) Random sequence generation (selection bias) } \\
\text { (B) Allocation concealment (selection bias) } \\
\text { (C) Blinding of participants and personnel (performance... } \\
\text { (D) Blinding of outcome assessment (detection bias) } \\
\text { (E) Incomplete outcome data (attrition bias) } \\
\text { (F) Selective reporting (reporting bias) } \\
\text { (G) Other bias }\end{array}$}} \\
\hline & & & & & & & & & & & \\
\hline
\end{tabular}

We also found no evidence that CT has an effect on activities of daily living in the medium term (SMD $0.22,95 \% \mathrm{Cl}-0.5$ to $0.94 ; 3$ trials; 64 participants; Analysis 2.18); the quality of evidence was again low due to very serious concerns regarding imprecision. Therefore clear evidence is lacking.

\section{Comparison with an alternative treatment}

We are moderately certain that $\mathrm{CT}$, when compared with an alternative treatment, has little or no effect on participants' capacity for activities of daily living at end of treatment (SMD - 0.25 , $95 \% \mathrm{Cl}-0.43$ to $-0.07 ; 3$ trials; 525 participants; Analysis 3.17 ). No studies evaluated this outcome in the medium term.

\section{General health and quality of life (secondary outcome)}

\section{Comparison with control}

Because the quality of evidence was very low, we are unable to determine whether, relative to a control condition, $C T$ had an effect on general health and quality of life immediately post intervention (SMD $-0.04,95 \% \mathrm{Cl}-0.38$ to $0.29 ; 5$ trials; 630 participants; Analysis 1.18). In the medium term, only one study contributed to this outcome; therefore, we could not perform a meta-analysis (Kallio 2018). This study found no evidence of any CT relative to a control condition (SMD $-0.02,95 \% \mathrm{Cl}-0.39$ to 0.35 ; Analysis 2.19 ), but the quality of the evidence was low due to very serious imprecision.

\section{Comparison with an alternative treatment}

In comparison with an alternative treatment, the quality of the evidence was low. We found that an alternative treatment may be favoured, but these results were imprecise, so there could be little or no effect of CT immediately post intervention (SMD -0.49, $95 \% \mathrm{Cl}-1$ to $0.02 ; 4$ trials; 631 participants; Analysis 3.18). Only one study contributed to this outcome in the medium term, finding no evidence of a positive effect of $\mathrm{CT}$ relative to alternative treatment (SMD $0.33,95 \% \mathrm{Cl}-0.34$ to 1 ; Analysis 4.18 ), but the quality of evidence was low, so the results are inconclusive (Giovagnoli 2017).

\section{Behavioural and psychological symptoms of dementia (secondary outcome)}

\section{Comparison with control}

The quality of the evidence was very low due to serious concerns related to heterogeneity and imprecision, so we are unable to determine whether, relative to a control treatment, СT had an effect on behavioural and psychological symptoms of dementia immediately post intervention (SMD $0.44,95 \% \mathrm{Cl}-0.34$ to $1.22 ; 6$ trials; 493 participants). 
In the medium term, only one study contributed to this outcome and found no evidence of an effect of CT on general health and quality of life (SMD $-1.34,95 \% \mathrm{Cl}-2.75$ to $0.07 ; 1$ trial; 11 participants; Analysis 2.20) (Galante 2007). However, we have low certainty in this result.

\section{Comparison with an alternative treatment}

We found moderate-quality evidence showing that CT probably has no effect relative to an alternative treatment immediately post intervention (SMD $-0.11,95 \% \mathrm{Cl}-0.27$ to $0.06 ; 3$ studies; 672 participants; Analysis 3.19). No trials compared CT versus an alternative treatment on this outcome in the medium term.

\section{Participant burden (retention rates at end of treatment)} (secondary outcome)

Meta-analyses of participant retention rates at end of treatment showed that participants receiving CT were not more likely to discontinue participating in the trial relative to participants receiving a control (odds ratio (OR) $0.73,95 \% \mathrm{Cl} 0.37$ to 1.43 ; 17 trials; 1282 participants; Analysis 1.20 Figure 14) or an alternative treatment $(\mathrm{OR} 0.78,95 \% \mathrm{Cl} 0.24$ to $2.57 ; 4$ trials; 639 participants; Analysis 3.20), but our certainty in these findings is very low to low due to imprecision; therefore, our results are inconclusive.

Figure 14. Forest plot of comparison: 1 Cognitive training vs control immediately post intervention, outcome: 1.20 Participant burden (retention rates).

\begin{tabular}{|c|c|c|c|c|c|}
\hline \multirow[b]{2}{*}{ Study or Subgroup } & \multicolumn{2}{|c|}{ Cognitive training } & \multicolumn{2}{|c|}{ Control } & \multirow[b]{2}{*}{ Weight } \\
\hline & Events & Total & Events & Total & \\
\hline Kallio 2018 & 76 & 76 & 71 & 71 & \\
\hline Serino 2017 & 10 & 10 & 10 & 10 & \\
\hline Beck 1988 & 10 & 10 & 10 & 10 & \\
\hline Davis 2001 & 19 & 19 & 18 & 18 & \\
\hline Jelcic 2012 & 20 & 20 & 20 & 20 & \\
\hline Cavallo 2016 & 40 & 40 & 40 & 40 & \\
\hline Kim 2015 & 22 & 22 & 21 & 21 & \\
\hline Mapelli 2013 & 10 & 10 & 10 & 10 & \\
\hline Trebbastoni 2018 & 48 & 54 & 86 & 86 & $4.8 \%$ \\
\hline Quintana Hernandez 2014 & 27 & 32 & 25 & 25 & $4.7 \%$ \\
\hline Koltai 2001 (1) & 14 & 16 & 8 & 8 & $4.1 \%$ \\
\hline Kao 2016 & 95 & 110 & 45 & 48 & $17.3 \%$ \\
\hline Cahn-Weiner 2003 & 16 & 19 & 17 & 20 & $11.3 \%$ \\
\hline Giuli 2016 & 48 & 51 & 47 & 50 & $12.3 \%$ \\
\hline Amieva 2016 & 124 & 170 & 109 & 154 & $38.1 \%$ \\
\hline Neely 2009 (2) & 10 & 10 & 9 & 10 & $3.8 \%$ \\
\hline Galante 2007 & 7 & 7 & 4 & 5 & $3.6 \%$ \\
\hline Total $(95 \% \mathrm{Cl})$ & & 676 & & 606 & $100.0 \%$ \\
\hline Total events & 596 & & 550 & & \\
\hline \multicolumn{6}{|c|}{$\begin{array}{l}\text { Heterogeneity: } \text { Tau }^{2}=0.25 ; \mathrm{Chi}^{2}=10.82, \mathrm{df}=8(P=0.21) ;\left.\right|^{2}=26 \% \\
\text { Test for overall effect: } Z=0.92(P=0.36)\end{array}$} \\
\hline
\end{tabular}

Random, 95\% Cl

Notestimable

Not estimable

Not estimable

Not estimable

Not estimable

Not estimable

Not estimable

Not estimable

$0.04[0.00,0.78]$

$0.10[0.01,1.86]$

$0.34[0.01,7.98]$

$0.42[0.12,1.53]$

$0.94[0.17,5.36]$

$1.02[0.20,5.32]$

$1.11[0.69,1.81]$

$3.32[0.12,91.60]$

$5.00[0.17,150.92]$

$0.73[0.37,1.43]$

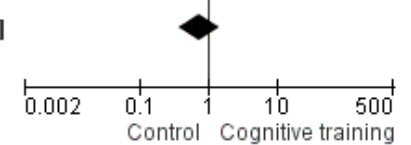

Risk of bias legend

(A) Random sequence generation (selection bias)

(B) Allocation concealment (selection bias)

(C) Blinding of participants and personnel (performance...

(D) Blinding of outcome assessment (detection bias)

(E) Incomplete outcome data (attrition bias)

F) Selective reporting (reporting bias)

(G) Other bias

\section{Caregiver outcomes}

\section{Comparison with control}

Results concerning caregiver outcomes are depicted in Analysis 1.21 Analysis 1.22 Analysis 1.23 Analysis 3.21 Analysis 3.22 Analysis 3.23 and Figure 15. The quality of evidence in relation to quality of life of the caregiver immediately at the end of treatment was low, and we found no evidence of any effects of CT relative to a control condition (SMD $0.16,95 \% \mathrm{Cl}-0.50$ to $0.83 ; 1$ trial; 36 participants;

Analysis 1.22). We also found moderate-quality evidence showing that $\mathrm{CT}$ was not associated with lower burden of care at end of treatment relative to a control treatment (SMD $-0.11,95 \% \mathrm{Cl}-0.36$ to $0.15 ; 2$ trials; 405 participants; Analysis 1.21). One study found a positive effect of CT on caregiver mood at end of treatment relative to control (SMD $0.98,95 \% \mathrm{Cl} 0.27$ to $1.68 ; 1$ trial; 36 participants; Analysis 1.23) (Quayhagen 2000). We have moderate certainty in this finding. 
Figure 15. Forest plot of comparison: 1 Cognitive training vs control immediately post intervention, outcome: 1.23 Change in mood and well-being (CAREGIVER).

\begin{tabular}{|c|c|c|c|c|c|c|c|c|c|c|c|}
\hline \multirow[b]{2}{*}{ Study or Subgroup } & \multicolumn{3}{|c|}{ Cognitive training } & \multicolumn{3}{|c|}{ Control } & \multicolumn{2}{|c|}{ Std. Mean Difference } & & \multirow{2}{*}{$\begin{array}{c}\text { Std. Mean Difference } \\
\text { IV, Random, } 95 \% \mathrm{Cl}\end{array}$} & Risk of Bias \\
\hline & Mean & SD & Total & Mean & SD & Total & Weight & IV, Random, $95 \% \mathrm{Cl}$ & & & A B C D E F G \\
\hline Quayhagen 2000 & 0.09 & 0.06 & 21 & 0.03 & 0.06 & 15 & $100.0 \%$ & $0.98[0.27,1.68]$ & & & 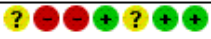 \\
\hline Total $(95 \% \mathrm{Cl})$ & & & 21 & & & 15 & $100.0 \%$ & $0.98[0.27,1.68]$ & & & \\
\hline $\begin{array}{l}\text { Heterogeneity: Not a } \\
\text { Test for overall effect }\end{array}$ & $\begin{array}{l}\text { plicable } \\
Z=2.72\end{array}$ & $P=0.0$ & & & & & & & $\frac{1}{-4}$ & $\begin{array}{ccc}-2 & 0 & 2 \\
\text { Control } & \text { Cognitive }\end{array}$ & \\
\hline
\end{tabular}

Risk of bias legend

(A) Random sequence generation (selection bias)

(B) Allocation concealment (selection bias)

(C) Blinding of participants and personnel (performance bias)

(D) Blinding of outcome assessment (detection bias)

(E) Incomplete outcome data (attrition bias)

(F) Selective reporting (reporting bias)

(G) Other bias

\section{Comparison with an alternative treatment}

Based on one study (Quayhagen 2000), we found moderate-quality evidence of a positive effect of CT on caregiver mood at end of treatment relative to alternative treatment (SMD 1.5, 95\% Cl 0.96 to 2.04 ; 1 trial; 88 participants; Analysis 3.23). No available studies evaluated caregiver outcomes in the medium term.

\section{DISCUSSION}

\section{Summary of main results}

The aim of the current review was to evaluate current evidence regarding effects of cognitive training (CT) interventions on several key outcomes for people with mild to moderate dementia; we found 33 studies that met our inclusion criteria. We carried out separate comparisons of $\mathrm{CT}$ versus a control intervention (passive or active) and CT versus an alternative treatment. Key findings of this review are that $\mathrm{CT}$ probably has small to moderate positive effects on global cognition, as well as on the specific cognitive domain of verbal semantic fluency immediately after treatment, and these gains may be maintained in the medium term when compared with a passive or active control intervention. Although improvements at the end of treatment were found in several other cognitive domains or processes, the quality of evidence was generally low, so our certainty in these findings is low. Beyond cognition, CT may be associated with slower clinical progression immediately following treatment and in the medium term, but again, we are very uncertain of this finding. We found no evidence of increased participant burden associated with CT (as reflected in discontinuation rates). We carried out several prespecified subgroup analyses to explore potential effect modifiers but found that none were significant.

In contrast, we found no strong evidence of any benefit associated with CT relative to other treatments in relation to our primary outcomes of global cognition at end of treatment and clinical disease severity in the medium term, but our certainty in many of these findings is low. We also found no benefit associated with $\mathrm{CT}$ in relation to any of the secondary outcomes included in this review, and in fact, alternative treatments may have been favoured in relation to participants' mood, behavioural and psychological symptoms, or capacity for activities of daily living, but findings were imprecise and our confidence in them is low.

\section{Overall completeness and applicability of evidence}

\section{Numbers and sources of studies that met review criteria}

The current review included 33 randomised controlled trials (RCTs) with a total of 1924 participants, making it the largest systematic review on this topic to date. Eighteen studies, i.e. more than half of the total included studies, were published since 2013, when a previous Cochrane Review that covered CT studies and included only 10 studies was published (Bahar-Fuchs 2013). The large number of studies published in recent years was somewhat unexpected, given that in recent years, the focus of many intervention trials has shifted to the pre-dementia phase of mild cognitive impairment $(\mathrm{MCl})$ and cognitively unimpaired older adults at risk of dementia. In addition, previous reviews, including Cochrane and other reviews (e.g. Hill 2017; Oltra-Cucarella 2016), generally found little evidence to support CT for people with dementia. Against this context, the increasing number of trials of CT for people with dementia was surprising, but the availability of a relatively large number of studies generally strengthens our confidence in the findings of this current review. The included studies were conducted in over 12 countries, and in the current review, we did not restrict inclusion to studies published in English. It is worth noting, however, that a vast majority of studies, particularly those published in recent years, were conducted in European countries, and 11 of those were conducted in Italy alone. We found no eligible studies that were conducted in Englishspeaking countries such as the UK, Canada, or Australia, and all included studies that were conducted in the USA were included in previous reviews preceding 2013. The reasons for this trend are not clear, but the extent to which findings of the current review are applicable to individuals in other countries is not completely clear.

\section{Issues related to definition and scope of interventions}

Although we classified all experimental interventions in the included studies as CT, interventions nevertheless were clinically heterogeneous, with some delivered through paper and pencil and others via computerised platforms, some targeting single cognitive domains and others multiple domains simultaneously, and some focusing primarily on drill and practice while others employed a range of learning and performance strategies. The settings and doses at which the interventions were delivered were also diverse, with some delivered at home and others in community settings including daycare and hospital settings, and some delivered one or two times per week and others as many as five times per 
week. It is therefore more appropriate to think of the interventions included in the current review as consisting of a class or family of interventions with some shared features and, accordingly, that the interventions do not measure the exact same effect; this is reflected in our decision to conduct a random-effects metaanalysis, which is more suitable under these circumstances (Deeks 2017). Although observed clinical heterogeneity is most likely responsible for some of the observed statistical heterogeneity in effect estimates from individual trials, our pre-specified subgroup analyses, carried out in cases where we found at least moderate heterogeneity and a sufficient number of available trials, did not reveal strong evidence of differences that would justify separate meta-analyses for different subgroups.

\section{Outcomes and measures}

Our review focused on a large number of primary and secondary outcomes for the person with dementia and for their caregivers, and we made a distinction between outcomes immediately post intervention and outcomes reported in the medium term ( 3 to 12 months post treatment). Across studies, outcomes were evaluated with over 200 measures; however, in many cases, a given measure was counted multiple times owing to minor differences in naming across countries or in versions of the instrument, or the provision of insufficient detail to determine which exact measure was used. When studies provided individual subtest scores from test batteries or global indices, we counted each subtest as a measure. In some cases, studies used unpublished tests developed for the purpose of that particular study. In many cases, it was difficult to classify cognitive measures into a single cognitive domain; many other studies used multiple measures of the same cognitive outcome domain, and we followed our pre-specified plan (see Unit of analysis issues under Methods) in selecting measures for meta-analysis. Although we acknowledge that this method has limitations and may have not always resulted in an optimal assessment of a given cognitive domain, we adopted the procedure to reduce the likelihood of bias introduced by selecting a measure on the basis of effect size.

Our first primary outcome - change in global (composite) cognitive ability immediately post treatment - was evaluated by 33 studies with a total of 1914 participants. In contrast, our second primary outcome - change in disease progression in the medium term, an outcome that may be of greater importance to people with dementia and decision-makers - was evaluated in only two studies with a total of 98 participants, using a dementia severity rating scale. Although researchers found a moderate effect of CT relative to control treatment in relation to change in clinical disease severity, our certainty in this finding is very low. Many other important outcomes (e.g. change in caregiver mood, burden, or quality of life in the medium term) were evaluated by a single study so that no meta-analysis could be performed; other outcomes (e.g. changes in behavioural and psychological symptoms in the medium term, change in caregiver mood post treatment) were evaluated by a very small number of studies, or were not evaluated at all. It is important to note that although we were able to perform a meta-analysis based on large numbers of studies and participants for the primary outcome of global cognition, and despite our findings suggesting at least a small to moderate effect of $\mathrm{CT}$ relative to control treatments, the extent to which observed cognitive benefits are clinically meaningful remains unclear. Indeed, we found no evidence that CT leads to changes in people's capacity to perform activities of daily living immediately post treatment or in the medium term, although the quality of the evidence was low and findings are therefore inconclusive.

\section{Intervention fidelity and participant adherence}

Adherence to a prescribed intervention protocol is frequently a barrier for lifestyle interventions, particularly unsupervised, selfdelivered, home-based interventions, and interpretation of the actual effects of interventions may be biased in the context of suboptimal adherence. However, reference to intervention adherence was made only by a small number of mostly recent studies (Amieva 2016; Brueggen 2017; Cahn-Weiner 2003; Giovagnoli 2017; Kallio 2018; Trebbastoni 2018), and none of these prospectively defined 'adherence'. It is therefore difficult to know how similar or dissimilar adherences rates in different studies were, and to what extent effect estimates from individual studies were affected by issues of adherence. Similarly, with the exception of a small number of studies (Amieva 2016; Brueggen 2017; CahnWeiner 2003; Giovagnoli 2017), no study made reference to taking steps associated with assessing or monitoring the fidelity with which an intervention was delivered, including whether or not any changes to the intervention protocol were made after recruitment commenced, whether a manualised intervention protocol was followed, or whether those delivering the intervention underwent specific training. Lack of adequate measures to ensure fidelity has implications for the replicability of behavioural intervention studies; it is therefore more difficult to know whether findings from a trial observed in one context are likely to be seen when the intervention is applied in another context.

\section{Quality of the evidence}

We used the GRADE approach to evaluate our confidence in study findings in relation to the main outcomes of this review, with grading incorporating the risk of bias in included studies, inconsistency and imprecision in the results, directness of the evidence, and publication bias (GRADE Handbook; GRADEpro GDT). In comparing CT with a control intervention, from the large number of outcomes assessed, we are highly confident only in our findings in relation to a single outcome immediately after treatment, namely, verbal category fluency. Our confidence in trial findings was moderate for several other outcomes immediately following the intervention, including our primary outcome of global cognition as measured by a composite score, as well as caregiver outcomes of burden of care and mood and well-being. Our certainty in the findings of other outcomes, including our second primary outcome of clinical progression in the medium term, was very low. When CT was compared with an alternative treatment, we have moderate confidence in relation to our findings on change in participant mood, behavioural and psychological symptoms, and capacity for activities of daily living, as well as caregiver mood and well-being at end of treatment. Our confidence in our findings on all other assessed outcomes at end of treatment and in the medium term is low or very low.

\section{Risk of bias}

Although we rated most studies as having high risk of bias in at least two domains, our approach to the classification of studies into 'high' and 'low' risk of bias for the purposes of subgroup analyses and grading of the evidence was relatively lenient and led to only seven studies rated as 'high-risk' studies. However, with the exception of 'immediate memory' in our primary comparison (CT vs control intervention immediately post intervention), in which 
studies classified as 'high risk' were associated with smaller pooled effect estimates relative to 'low-risk' studies, risk of bias did not seem to account for much of the heterogeneity in effect estimates. We pre-defined 'high-risk' studies in this review as studies that were rated as having 'high risk' on at least two critical domains. For our purposes, we defined critical domains as randomisation, blinding of outcome assessment, incomplete outcome data, and selective reporting. We acknowledge the importance of allocation concealment and blinding of participants and personnel. However, allocation concealment is rarely reported in CT trials to date, and most studies would be classified as having high risk if we classified this as a 'critical domain'. Similarly, blinding of those delivering the interventions is typically not possible in trials of this nature, and blinding of participants can be attempted only in trials with active control or alternative treatment.

\section{Inconsistency}

In relation to our primary outcomes, we downgraded 1 point evidence of our findings on global cognition for serious concerns regarding inconsistency when $\mathrm{CT}$ was compared to a control group immediately after the intervention, but we did not find these issues when $\mathrm{CT}$ was compared to an alternative treatment. For disease progression in the medium term, we again found no issues related to inconsistency. For most other outcomes, we rated inconsistency as not serious, typically due to relatively small heterogeneity or moderate heterogeneity, which was explained at least in part by at least one of the subgroup analyses. However, in many cases, we rated inconsistency as serious or very serious. For eight of the outcomes included in our primary comparison, we found very high heterogeneity in effect estimates $\left(1^{2}>80 \%\right)$ and heterogeneity that was not generally well explained by any of the prognostic features included in our subgroup analyses.

\section{Indirectness}

Overall, outcomes evaluated in these studies and the measures used to assess them seem to be well mapped to our PICO question, so we did not downgrade the evidence in relation to any assessed outcomes for indirectness. Although measures of cognition and cognitive test scores do not directly correspond with quality of performance of daily activities, cognitive scores based on psychometric evaluation are considered a true reflection of one's objective cognitive abilities. Similarly, in measuring clinical disease status, most studies that reported this outcome did so with widely used clinical staging measures such as the Clinical Dementia Rating Scale (e.g. Mapelli 2013) or the Rapid Disability Rating Scale (e.g. Quintana Hernandez 2014), while none of the included studies used possibly more direct indications of clinical progression, such as admission to residential care. Unlike changes in mood, cognition, or behavioural symptoms, clinical disease progression is a longerterm outcome, in the context of mild dementia in particular, and assessment of this outcome requires large, adequately powered trials providing long-term follow-up.

\section{Imprecision}

For our primary comparison (CT vs control at end of treatment), we had no major concerns related to imprecision in the effect estimate of our primary outcome, namely, global cognition. However, we had serious or very serious concerns in relation to imprecision regarding most secondary outcomes, including all non-cognitive outcomes and several specific cognitive domains. These concerns were mainly due to large confidence intervals of effect estimates that included potentially meaningful effects, no effects, and effects favouring the control condition. In our additional comparisons (CT vs control in the medium term, and CT vs alternative treatment at end of treatment and in the medium term), we found that imprecision was a serious or a very serious concern in relation to all outcomes, including our primary outcome of clinical progression in the medium term. For these additional comparisons, imprecision was a concern due to the much smaller number of participants on which many of the effect estimates were based, as well as the large confidence intervals of these estimates. Hence, in relation to many outcomes of interest, we found that primary studies sometimes disagreed not only on the size, but also on the direction, of effects of CT.

\section{Publication bias}

Our search results suggested that several trials registered in recent years and interventions undertaken as part of student research projects might never have been published, raising some concern about positive publication bias. However, with the exception of a few outcomes (e.g. change in immediate and delayed memory, verbal letter fluency, executive function at end of treatment), we could not ascertain the presence of publication bias with much confidence on the basis of visual inspection of funnel plots for each outcome. In addition, asymmetry in funnel plots may be caused by factors other than publication bias, so we decided to take a conservative approach; we downgraded the evidence for suspected publication bias only in cases in which asymmetry in the funnel plot was reasonably evident, and a minimum of 10 studies had assessed the relevant outcome. Our approach may have resulted in an underestimation of the true extent of concerns related to the presence of publication bias.

\section{Potential biases in the review process}

We conducted a thorough search for articles and performed a rigorous screening procedure and risk of bias assessments, conducted independently by two review authors, with disagreements resolved by a third reviewer. Similarly, grading of the evidence was also completed by two of the review authors, who resolved disagreements by discussion until consensus was reached. The review team includes researchers at all stages of their professional career and with various levels of dementia-specific expertise, further reducing the likelihood of systematic bias in the review process. None of the included studies were conducted by one of the review authors, and no conflicts of interest have been identified. Although unlikely, we cannot rule out the possibility that difficulties associated with forming an accurate judgement in relation to some areas of potential bias might have led to a systematic overestimation (e.g. risk of selection bias due to lack of allocation concealment) or underestimation (e.g. publication bias) of the actual risk of bias.

\section{Agreements and disagreements with other studies or reviews}

In recent years, numerous systematic reviews with and without meta-analyses of cognition-oriented treatments for older adults with and without dementia have been completed, and we have recently reviewed this large body of work (Malmberg Gavelin). Of particular relevance to the current review are several recent reviews focused on CT for persons with dementia, including Alves 2013, Bahar-Fuchs 2013, Huntley 2015, Folkerts 2017, and Hill 2017. Alves 
and colleagues conducted a systematic review with a meta-analysis on four studies that met their inclusion criteria (three of which were included in the present review; Cahn-Weiner 2003 Davis 2001 Heiss 1993). Closer inspection suggested that the fourth study included in that review was of cognitive stimulation therapy and therefore would not meet criteria for the current review. Alves found that the intervention was beneficial, relative to a control intervention, only in relation to global cognition at end of treatment, as reflected in the Mini Mental State Examination (MMSE). Although the effect was large, the meta-analysis was based on only three studies (one of which was the cognitive stimulation study) and 104 participants, so precision was likely low. The other estimates were generally based on one or two studies only, so the conclusions drawn were naturally very limited. More recently, the Huntley 2015 systematic review conducted a meta-analysis of cognition-oriented treatment trials for people with dementia and provided separate effect estimates for global cognition from four studies classified as CT (all of which were included in the present review) and seven studies classified as mixed CT and stimulation (three of which were included in the current review and coded as 'augmented (T'). Based on three studies, those review authors did not find strong evidence of an effect of CT relative to an active control condition on global cognition, as reflected on the MMSE (standardised mean difference (SMD) $0.22,95 \%$ confidence interval $(\mathrm{Cl})-0.74$ to 1.18 ). Findings also suggested there were no benefits of mixed CT and stimulation on global cognition, whether compared with a passive (SMD 0.44 , $95 \% \mathrm{Cl}-0.56$ to 1.46 ) or an active control treatment (SMD 0.25 , $95 \% \mathrm{Cl}-0.18$ to 0.68$)$. In contrast, in the current review, change in global cognition as reflected by a composite score and on the basis of a screening measure (such as the MMSE) was greater at end of treatment in the $\mathrm{CT}$ group relative to the control condition, based on 20 studies, and effect estimates were similar in magnitude when active ( $k=8$; SMD 0.61) and passive $(k=12$; SMD 0.69) control conditions were directly contrasted. It is interesting to note that results of the current review show a trend suggesting that although 'traditional' CT $(\mathrm{k}=13)$ is associated with large effect relative to control treatment (SMD $0.83,95 \% \mathrm{Cl} 0.30$ to 1.39 ), 'augmented' CT $(\mathrm{k}=7)$ is associated with a relatively small effect estimate (SMD $0.25,95 \% \mathrm{Cl}-0.21$ to 0.70 ), and differences between the two subgroups approached significance $\left(\mathrm{Chi}^{2}=2.68, \mathrm{df}=1, \mathrm{P}=0.10\right)$. Folkerts 2017 recently reported a systematic review with metaanalysis of cognition-oriented treatments for people with dementia living in residential care; six trials were classified as $\mathrm{CT}$, two of which contributed data for meta-analysis (both studies - Kawashima 2005; Mapelli 2013 - are included in the current review). On the basis of these two studies with 47 participants, these review authors found that CT was superior to passive control treatment in relation to global cognition (SMD 1.16, $95 \% \mathrm{Cl} 0.52$ to 1.79 ) - a finding that is likely to be very imprecise due to the small number of participants on which it is based, but that is nonetheless in agreement with the findings of the current review. Finally, Hill 2017, a comprehensive systematic review, performed meta-analysis of computerised CT for people with mild cognitive impairment $(\mathrm{MCl})$ and dementia, and reported effects separately for each population. Based on a metaanalysis of 12 studies with a total of 389 participants, that review found a small effect of $\mathrm{CT}$ relative to a control treatment on global cognition at end of treatment (SMD $0.26,95 \% \mathrm{Cl} 0.01$ to 0.52 ) but found no effects in other cognitive domains.

\section{AUTHORS' CONCLUSIONS}

\section{Implications for practice}

For people with mild to moderate dementia, relative to usual treatment or non-specific activities, standardised cognitive training (CT) may lead to at least small improvements in overall cognition at end of treatment, and these improvements may be sustained over the medium term after treatment cessation (between 3 and 12 months). Benefits in the short and medium term may also be observed in more specific areas of cognition, such as verbal fluency. The evidence regarding gains associated with $\mathrm{CT}$ in clinical disease progression, mood, activities of daily living, or caregiver burden relative to usual or non-specific activities is not clear. No evidence suggests that $\mathrm{CT}$ is associated with any harm to the person with dementia in terms of negative impact on important outcomes such as mood and well-being, accelerated cognitive or functional decline, or worsening caregiver burden. For many important outcomes, particularly in the medium term, the overall quality of evidence was low, so further publication of high-quality evidence may lead to changes in observed effects. It is important to note that no evidence suggests that CT provides any benefit when compared to alternative treatments, such as cognitive stimulation therapy or physical exercise. The decision of whether a person with dementia should commence a formal cognitive training intervention to improve his or her cognition should be made with consideration of the balance of potentially modest effects on cognition in the short to medium term and any possible contraindications related to personal values and preferences, available resources in the person's locality, and other possible trade-offs. Clinicians should work together with the person with dementia and his or her significant others to carefully balance the various considerations, including the specific context of the patient, in deciding whether or not a formal CT intervention should be started. Although it is beyond the scope of this review to discuss all relevant health policy-related considerations, evidence from the current review should be interpreted within the broader context of evidence for treatments, including pharmacological treatments, for people with mild to moderate dementia. In particular, observed effects of CT on global cognition at end of treatment may be comparable or stronger than effects on cognition associated with approved medications (Birks 2015; Birks 2018), but without some of the adverse effects associated with these medications. Further work is required to better understand cost-effectiveness associated with cognitive training for people with mild to moderate dementia, to better inform health-related policy.

\section{Implications for research}

A relatively large body of work on the effects of CT for various outcomes among people with dementia is now available; unfortunately, despite improvement in some areas, the quality of these studies often is still low, leading to low confidence in the accuracy of some of these review findings. To increase our confidence in the findings of the current review, it is important that any further studies of CT for people with dementia are conducted with rigorous methodological standards, to ensure that risks of bias are adequately mitigated. In particular, trial registration and separate publication of detailed trial protocols including plans for analyses and dissemination are critical for reducing risk of bias due to selective reporting, and for identifying issues related to trial fidelity. It is recommended that ethical review boards ensure trials are registered before they give final 
approval for recruitment to commence, and that publication of a trial protocol is specified as a milestone when funding applications are made, and during ethical approval of the application. It is also important that key design features are adequately implemented and clearly reported in published reports, particularly around methods of randomisation, allocation concealment, and masking of participants and personnel. Although masking is not possible in behavioural interventions involving a passive or 'treatment as usual' comparison condition, it can be achieved in studies using active control conditions or alternative treatments. Our findings do not provide strong support for the use of active ("placebo") control conditions as far as effects of the interventions are concerned. However, more studies in which CT is compared with other specific interventions are needed to build a stronger evidence base that would allow consumers and decision-makers to make more informed choices between alternative treatments that may offer some benefit to people with dementia. To reduce some of the statistical heterogeneity observed in these studies, it is important that, wherever possible, evaluation of outcomes is done on the basis of published measures with established psychometric properties. It is important that future studies are better designed to explore dose-response-related issues, as well as issues related to maintenance or waning of treatment effects. Understanding of the long-term impact of CT on clinically relevant outcomes, including admission to residential care, quality of life, and caregiver burden, remains and important goal of future research. Several recent publications have provided further important advice and minimum standards for the conduct of cognitive training research (e.g. Simons 2016), and an international working party including several experts in this area is currently working to develop research guidelines in this area (Bahar-Fuchs 2014).

\section{ACKN OWLEDGEMENTS}

We are grateful for the support of the Cochrane Dementia and Cognitive Improvement Group, particularly Ms. Anna Noel-Storr and Ms. Candida Fenton for conducting the literature searches and for updating relevant search results sections of the manuscript. The review authors are also very grateful to Dr. Amit Lampit for statistical advice and for assistance with computing the composite cognitive scores. Dr. Alex Bahar-Fuchs is supported by a Boosting Dementia Leadership Fellowship from the National Health and Medical Research Council of Australia. Ms. Julieta Sabates was partially supported by a Cochrane Incentive Award.

We would like to thank peer reviewer Charlie Cheung for his comments and feedback and one other peer reviewer, who wishes to remain anonymous. 


\section{R E F E R E N C E S}

\section{References to studies included in this review}

Amieva 2016 \{published and unpublished data\}

Amieva H, Dartigues JF. ETNA3, a clinical randomized study assessing 3 cognitive-oriented therapies in Alzheimer's disease. Journal of Nutrition, Health and Aging 2013;169:752-6.

* Amieva H, Robert PH, Grandoulier AS, Meillon C, De Rotrou J, Andrieu S, et al. Group and individual cognitive therapies in Alzheimer's disease: the ETNA3 randomized trial. International Psychogeriatrics 2016;28(5):707-17.

Barban 2016 \{published and unpublished data\}

* Barban F, Annicchiarico R, Pantelopoulos S, Federici A, Perri R, Fadda $L$, et al. Protecting cognition from aging and Alzheimer's disease: a computerized cognitive training combined with reminiscence therapy. International Journal of Geriatric Psychiatry 2016;31(4):340-8.

Barban F, Annicchiarico R, Perri R, Fadda L, Carlesimo GA, Pantelopoulos S, et al. Randomized clinical trial of a computerbased cognitive treatment for healthy elderly, clinical and preclincal Alzheimer's disease. The SOCIABLE project. Journal of Alzheimer's Disease. 2012; Vol. Conference: 7th Congresso Sindem: Italian Association for the Study of Dementia Linked to the Italian Neurological Society, SIN Naples Italy. Conference Start: 20120322 Conference End: 20120324. Conference Publication:

\section{Beck 1988 \{published data only\}}

* Beck C, Heacock P, Mercer S, Thatcher R, Sparkman C. The impact of cognitive skills remediation training on persons with Alzheimer's disease or mixed dementia. Journal of Geriatric Psychiatry 1988;21:73-88.

\section{Bergamaschi 2013 \{published data only\}}

* Bergamaschi S, Arcara G, Calza A, Villani D, Orgeta V, Mondini S. One-year repeated cycles of cognitive training (CT) for Alzheimer's disease. Aging Clinical and Experimental Research 2017;25:421-6.

\section{Boller 2011 \{published and unpublished data\}}

* Boller B, Jennings JM, Dieudonné Verny M, Ergis AM. Recollection training and transfer effects in Alzheimer's disease: effectiveness of the repetition-lag procedure. Brain and Cognition 2011;78:169-77.

\section{Brueggen 2017 \{published and unpublished data\}}

* Brueggen K, Kasper E, Ochmann S, Pfaff H, Webel S, Schneider W, et al. Cognitive rehabilitation in Alzheimer's disease: a controlled intervention trial. Journal of Alzheimer's Disease 2017;57(4):1315-24.

Ochmann S, Dyrba M, Grothe MJ, Kasper E, Webel S, Hauenstein $\mathrm{K}$, et al. Does functional connectivity provide a marker for cognitive rehabilitation effects in Alzheimer's disease? An interventional study. Journal of Alzheimer's Disease 2017;57(4):1303-13.
Cahn-Weiner 2003 \{published data only\}

* Cahn-Weiner DA, Malloy PF, Rebok GW, Ott BR. Results of a placebo-controlled study of memory training for mildlyimpaired Alzheimer's disease patients. Applied Neuropsychology 2003;10:215-23.

\section{Cavallo 2016 \{published and unpublished data\}}

Cavallo M, Angilletta C. Long-lasting neuropsychological effects of a computerized cognitive training in patients affected by early stage Alzheimer's disease: are they stable over time?. Journal of Applied Gerontology 2018;37:0733464817750276.

* Cavallo M, Hunter Edyta M, van der Hiele K, Angilletta C. Computerized structured cognitive training in patients affected by early-stage Alzheimer's disease is feasible and effective: a randomized controlled study. Archives of Clinical Neuropsychology 2016;31:868-76.

Davis 2001 \{published data only\}

* Davis RN, Massman PJ, Doody RS. Cognitive intervention in Alzheimer disease: a randomized placebo-controlled study. Alzheimer Disease and Associated Disorders 2001;15(1):1-9.

de Vreese 1998 \{published and unpublished data\}

de Vreese LP, Neri M. Ecological impact of combined cognitive training programs (CTP) and drug treatment (ChE-I) in AD. International Psychogeriatric 1999;11 Suppl:S187.

de Vreese LP, Neri M, Fioravanti M, Belloi L, Zanetti O. Memory rehabilitation in Alzheimer's disease: a review of progress. International Journal of Geriatric Psychiatry 2001;16:794-809.

* de Vreese LP, Verlato C, Emiliani S, Schioppa S, Belloi L, Salvioli G, et al. Effect size of a three-month drug treatment in $A D$ when combined with individual cognitive retraining: preliminary results of a pilot study. European Archives of Psychiatry and Clinical Neuroscience 1998;248:41-2.

Fernández-Calvo 2011 \{published and unpublished data\} * Fernández-Calvo B, Rodríguez-Pérez R, Contador I, RubioSantorum A, Ramos F. Efficacy of cognitive training programs based on new software technologies in patients with Alzheimertype dementia. Psicothema 2011;23(1):44-50.

Galante 2007 \{published data only (unpublished sought but not used)\}

* Galante E, Venturini G, Fiaccadori C. Computer-based cognitive intervention for dementia: preliminary results of a randomized clinical trial. Giornale Italiano di Medicina del Lavoro ed Ergonomia 2007;29(3):26-32.

Giovagnoli 2017 \{published data only\}

* Giovagnoli AR, Manfredi V, Parente A, Schifano L, Oliveri S, Avanzini G. Cognitive training in Alzheimer's disease: a controlled randomized study. Neurological Sciences 2017;38(8):1485-93. 
Giuli 2016 \{published data only (unpublished sought but not used)\} * Giuli C, Papa R, Lattanzio F, Postacchini D. The effects of cognitive training for elderly: results from My Mind project. Rejuvenation Research 2016;19:1549-684.

\section{Goudour 2011 \{published and unpublished data\}}

* Goudour A, Samson S, Bakchine S, Ehrlé N. Semantic memory training in Alzheimer's disease. Gériatrie et Psychologie Neuropsychiatrie du Vieillissement 2011;9(2):237-47.

Heiss 1993 \{published data only\}

* Heiss W-D, Kessler J, Mielke R, Szelies B, Herholz K. Long-term effects of phosphatidylserine, pyritinol and cognitive training in Alzheimer's disease: a neuropsychological, EEG, and PET investigation. Dementia 1994;5(2):88-98.

Heiss WD, Kessler J, Slansky I, Mielke R, Szelies B, Herholz K. Activation PET as an instrument to determine therapeutic efficacy in Alzheimer's disease. Annals of the New York Academy of Sciences 1993;695:327-31.

Jelcic 2012 \{published data only (unpublished sought but not used)\}

* Jelcic N, Cagnin A, Meneghello F, Turolla A, Ermani M, Dam M. Effects of lexical-semantic treatment on memory in early Alzheimer disease: an observer-blinded randomized controlled trial. Neurorehabilitation and Neural Repair 2012;26(8):949-56.

Jelcic 2014 \{published data only (unpublished sought but not used)\}

* Jelcic N, Agostini M, Meneghello F, Busse C, Parise S, Galano A, et al. Feasibility and efficacy of cognitive telerehabilitation in early Alzheimer's disease: a pilot study. Clinical Interventions in Aging 2014;9:1605.

\section{Kallio 2018 \{published and unpublished data\}}

Kallio E L, Ohman H, Carlson S, Kautiainen H, Hietanen M, Pitkala KH. Feasibility and baseline findings of a Finnish cognitive training (FINCOG) intervention in a randomised controlled trial among community-dwelling persons with dementia. European Geriatric Medicine 2017; Jan 31:245-9.

* Kallio EL, Öhman H, Hietanen M, Soini H, Strandberg TE, Kautiainen $\mathrm{H}$. Effects of cognitive training on cognition and quality of life of older persons with dementia. Journal of the American Geriatrics Society 2018;66(4):664-70.

Kao 2016 \{published data only (unpublished sought but not used)\}

* Kao CC, Lin LC, Wu, SC, Lin, KN, Liu, CK. Effectiveness of different memory training programs on improving hyperphagic behaviors of residents with dementia: a longitudinal singleblind study. Clinical Interventions in Aging 2016;11:707.

Kawashima 2005 \{published and unpublished data\}

Kawashima R. Cognitive training. 27th International Conference of Alzheimer's Disease International. 2012.

Kawashima R. Mental exercises for cognitive function: clinical evidence. Journal of Preventive Medicine and Public Health 2013;46 Suppl 1:S22.
* Kawashima R, Okita K, Yamazaki R, Tajima N, Yoshida H, Taira M, et al. Reading aloud and arithmetic calculation improve frontal function of people with dementia. Journals of Gerontology Series A-Biological Sciences \& Medical Sciences 2005;60(3):380-4.

Kim 2015 \{published data only\}

* Kim S. Cognitive rehabilitation for elderly people with earlystage Alzheimer's disease. Journal of Physical Therapy Science 2015;27(2):543-6.

\section{Koltai 2001 \{published data only\}}

* Koltai DC, Welsh-Bohmer KA, Smechel DE. Influence of anosognosia on treatment outcome among dementia patients. Neuropsychological Rehabilitation 2001;11(3/4):455-75.

Lee 2013 \{published data only\}

* Lee GY, Yip, CC, Yu EC, Man DW. Evaluation of a computerassisted errorless learning-based memory training program for patients with early Alzheimer's disease in Hong Kong: a pilot study. Clinical Interventions in Aging 2013;8:623-33.

Mapelli 2013 \{published data only (unpublished sought but not used)\}

* Mapelli D, Di Rosa E, Nocita R, Sava D. Cognitive stimulation in patients with dementia: randomized controlled trial. Dementia and Geriatric Cognitive Disorders Extra 2013;3:263-71.

\section{Neely 2009 \{published data only\}}

* Neely AS, Vikstrom S, Josephsson S. Collaborative memory intervention in dementia: caregiver participation matters. Neuropsychological Rehabilitation 2009;19(5):696-715.

\section{Quayhagen 1995 \{published data only\}}

Corbeil RR, Quayhagen MP, Quayhagen M. Intervention effects on dementia caregiving interaction: a stressadaptation modeling approach. Journal of Aging and Health 1999;11(1):79-95.

* Quayhagen MP, Quayhagen M, Corbeil RR, Roth PA, Rodgers JA. A dyadic remediation program for care recipients with dementia. Nursing Research 1995;44(3):153-9.

Quayhagen 2000 \{published data only\}

* Quayhagen MP, Quayhagen M, Corbeil RR, Hendrix RC, Jackson JE, Snyder L, et al. Coping with dementia: evaluation of four nonpharmacologic interventions. International Psychogeriatrics 2000;12(2):249-65.

Quintana Hernandez 2014 \{published data only\} * Hernández DJQ, Barrachina MTM, Fernández II, del Pino AS, Hernández JR. Effects of a neuropsychology program based on mindfulness on Alzheimer's disease: randomized doubleblind clinical study [Efectos de un programa de intervención neuropsicológica basado en mindfulness sobre la enfermedad de Alzheimer: ensayo clínico aleatorizado a doble ciego]. Revista Española de Geriatría y Gerontología 2014;49:165-72.

\section{Serino 2017 \{published and unpublished data\}}

* Serino S, Pedroli E, Tuena C, De Leo G, Stramba-Badiale M, Riva G, et al. A novel virtual reality-based training protocol for 
the enhancement of the "mental frame syncing" in individuals with Alzheimer's disease: a development-of-concept trial. Frontiers in Aging Neuroscience 2017;9:240.

Trebbastoni 2018 \{published and unpublished data\}

* Trebbastoni A, Imbriano L, Podda L, Rendace L, Sacchetti ML, de Lena $\mathrm{C}$, et al. Cognitive training in patients with Alzheimer's disease: findings of a 12-month randomized controlled trial. Current Alzheimer Research 2018;15(5):452-61.

Tsantali 2017 \{published data only (unpublished sought but not used)\}

* Tsantali E, Economidis D, Rigopoulou S. Testing the benefits of cognitive training vs cognitive stimulation in mild Alzheimer's disease: a randomised controlled trial. Brain Impairment 2017;18(2):188-96.

Venturelli 2016 \{published data only (unpublished sought but not used)\}

* Venturelli M, Sollima A, Cè E, Limonta E, Bisconti AV, Brasioli A, et al. Effectiveness of exercise- and cognitive-based treatments on salivary cortisol levels and sundowning syndrome symptoms in patients with Alzheimer's disease. Journal of Alzheimer's Disease 2016;53(4):1631-40.

\section{References to studies excluded from this review}

\section{Actrn12616000827437p 2016 \{published data only\}}

ACTRN12616000827437. Individual cognitive stimulation therapy for people with mild to moderate dementia. https:// www.anzctr.org.au/Trial/Registration/TrialReview.aspx? id=370912 2016

\section{Actrn 2015 \{published data only\}}

ACTRN. The effect of computerised cognitive training on memory outcomes in older adults with documented cognitive decline. Unknown 2015.

\section{Alimova 1990 \{published data only\}}

Alimova R. Drug-free stimulation of memory in senile patients with initial vascular dementia [Russian]. Zhurnal Nevropatologii i Psikhiatrii imeni S S Korsakova 1990;90(0044-4588):125-9.

\section{Alves 2014 \{published data only\}}

Alves J, Alves-Costa F, Magalhaes R, et al. Cognitive stimulation for Portuguese older adults with cognitive impairment: a randomized controlled trial of efficacy, comparative duration, feasibility, and experiential relevance. American Journal of Alzheimer's Disease \& Other Dementias 2014;29(6):503-12.

\section{Anderson 2001 \{published data only\}}

Anderson J, Arens K, Johnson R, et al. Spaced retrieval vs. memory tape therapy in memory rehabilitation for dementia of the Alzheimer's type. Clinical Gerontologist 2001;24(1-2):123-39.

\section{Angelucci 2015 \{published data only\}}

Angelucci F, Peppe A, Carlesimo Giovanni A, Serafini F, Zabberoni S, Barban F, et al. A pilot study on the effect of cognitive training on BDNF serum levels in individuals with Parkinson's disease. Frontiers in Human Neuroscience 2015;9:130.
Avila 2007 \{published data only\}

Avila R, Carvalho IAM, Bottino CMC, Miotto EC. Neuropsychological rehabilitation in mild and moderate Alzheimer's disease patients. Behavioural Neurology 2007;18(4):225-33.

\section{Baglio 2015 \{published data only\}}

Baglio F, Griffanti L, Saibene FL, Ricci C, Alberoni M, Critelli R, et al. Multistimulation group therapy in Alzheimer's disease promotes changes in brain functioning. Neurorehabilitation and Neural Repair Vol. 29, issue 1:13-24.

Baltes 1989 \{published data only\}

Baltes PB, Sowarka D, Kliegl R. Cognitive training research on fluid intelligence in old age: what can older adults achieve by themselves?. Psychology and Aging 1989;4(2):217.

Bamidis 2015 \{published data only\}

Bamidis PD, Fissler P, Papageorgiou SG, Zilidou V, Konstantinidis El, Billis AS, et al. Gains in cognition through combined cognitive and physical training: the role of training dosage and severity of neurocognitive disorder. Frontiers in Aging Neuroscience 2015;7:152.

Basak 2008 \{published data only\}

Basak C, Boot WR, Voss MW, Kramer AF. Can training in a realtime strategy video game attenuate cognitive decline in older adults?. Psychology and Aging 2008;23(4):765.

Bernhardt 2002 \{published data only\}

Bernhardt T, Maurer K, Froelich L. Influence of a memory training program on attention and memory performance of patients with dementia [German]. Zeitschrift fuer Gerontologie und Geriatrie 2001;35:821-31.

Biasutti 2018 \{published data only\}

Biasutti M, Mangiacotti A. Assessing a cognitive music training for older participants: a randomised controlled trial. International Journal of Geriatric Psychiatry 2018;33(2):271-8.

\section{Boron 2007 \{published data only\}}

Boron JB, Turiano NA, Willis SL, Schaie KW. Effects on cognitive training on change in accuracy in inductive reasoning ability. Journals of Gerontology Series B: Psychological Sciences and Social Sciences 2007;62(3):179-86.

\section{Brinkman 1982 \{published data only\}}

Brinkman SD, Smith RC, Meyer JS, Vroulis G, Shaw T, Gordom JR, et al. Lecithin and memory training in suspected Alzheimer's disease. Journal of Gerontology 1982;37:4-9.

Brunelle 2015 \{published data only\}

Brunelle-Hamannetal L. Impact of a cognitive rehabilitation intervention on neuropsychiatric symptoms in mild to moderate Alzheimer's disease. Neuropsychological Rehabilitation 2015; Vol. 25, issue 5:677-707.

Buettner 2011 \{published data only\}

Buettner LL, Fitzsimmons S, Atav S, Sink K. Cognitive stimulation for apathy in probable early-stage Alzheimer's. Journal of Aging Research 2011;2011:1-6. 
Burgener 2009 \{published data only\}

Burgener SC, Yang Y, Gilbert R, Marsh-Yant S. The effects of a multimodal intervention on outcomes of persons with earlystage dementia. American Journal of Alzheimer's Disease \& Other Dementias 2008;23(4):382-94.

Buschert 2011 \{published data only\}

Buschert VC, Friese U, Teipel SJ, Schneider P, Merensky W, Rujescu D, et al. Effects of a newly developed cognitive intervention in amnestic mild cognitive impairment and mild alzheimer's disease: a pilot study. Journal of Alzheimer's Disease 2011;25(4):679-94.

\section{Ceccato 2012 \{published data only\}}

Ceccato E, Vigato G, Bonetto C, Bevilacqua A, Pizziolo P, Crociani S, et al. STAM protocol in dementia: a multicenter, single-blind, randomized, and controlled trial. American Journal of Alzheimer's Disease \& Other Dementias 2012;27(5):301-10.

\section{Chapman 2004 \{published data only\}}

Chapman SB, Weiner MF, Rackley A, Hynan LS, Zientz J. Effects of cognitive-communication stimulation for Alzheimer's disease patients treated with donepezil. Journal of Speech, Language, and Hearing Research 2004;47(5):1149-63.

\section{Cheng 2015 \{published data only\}}

Cheng CPW, Chan SSM, Mak ADP, Chan WC, Cheng ST. Would transcranial direct current stimulation (tDCS) enhance the effects of working memory training in older adults with mild neurocognitive disorder due to Alzheimer's disease: study protocol for a randomized controlled trial. Trials 2015;16(1):479.

\section{Chew 2015 \{published data only\}}

Chew J, Chong MS, Fong YL, Tay L. Outcomes of a multimodal cognitive and physical rehabilitation program for persons with mild dementia and their caregivers: a goal-oriented approach. Clinical Interventions in Aging 2015;10:1687.

\section{Choi 2012 \{published data only\}}

Choi J, Fiszdon J. Self-efficacy for cognitive remediation in Alzheimer's disease [abstract]. Alzheimer's \& Dementia: Journal of the Alzheimer's Association 2012;8(4):P235

\section{Cipriani 2006 \{published data only\}}

Cipriani G, Bianchetti A, Trabucchi M. Outcomes of a computerbased cognitive rehabilitation program on Alzheimer's disease patients compared with those on patients affected by mild cognitive impairment. Archives of Gerontology and Geriatrics 2006;43(3):327-35.

\section{Clare 2013 \{published data only\}}

Clare L, Bayer A, Burns A, Corbett A, Jones R, Knapp M. Goaloriented cognitive rehabilitation in early-stage dementia: study protocol for a multi-centre single-blind randomised controlled trial (GREAT). Trials 2013;14(1):152.

\section{Contador 2016 \{published data only\}}

Contador I, Fernandez-Calvo B, Ramos F, Olazarán J. Influence of educational attainment on cognition-based intervention programs for persons with mild Alzheimer's disease. Journal of the International Neuropsychological Society 2016;22(5):577-82.

\section{Costa 2014 \{published data only\}}

Costa A, Peppe A, Serafini F, Zabberoni S, Barban F, Caltagirone $\mathrm{C}$, et al. Prospective memory performance of patients with Parkinson's disease depends on shifting aptitude: evidence from cognitive rehabilitation. Journal of the International Neuropsychological Society 2014;20(7):717-26.

Costa 2015 \{published data only\}

Costa NB, Aramaki F, Cecato J, Stella B, Araujo I, Aprahamian I, et al. Benefits of a computer-based cognitive training program for elderly subjects with mild Alzheimer's disease. International Psychogeriatrics 2015;27:S119.

\section{Danassi 2015 \{published data only\}}

Danassi E. SOCIABLE: a surface computing platform empowering effective cognitive training for healthy and cognitively impaired elderly. In: Vlamos P, Alexiou A editor(s). Advances in Experimental Medicine and Biology. 821. Danassi E, 2015:129-130.

\section{De Luca 2016 \{published data only\}}

De Luca R, Bramanti A, De Cola MC, Leonardi S, Torrisi M, Aragona $B$, et al. Cognitive training for patients with dementia living in a Sicilian nursing home: a novel web-based approach. Neurological Sciences 2016;37(10):1685-91.

\section{De Paula 2013 \{published data only\}}

De Paula E, Coutinho A, Nunes R, Saran L, Prando S, Ono C, et al. Evaluation of cerebral perfusion changes through spect in people with mild Alzheimer's disease after a cognitive rehabilitation program. Alzheimer's \& Dementia: Journal of the Alzheimer's Association 2013;9(4):P73.

\section{Dwolatzky 2014 \{published data only\}}

Dwolatzky T, Tractinsky N, Sarne-Fleischmann V. Computersupported personal interventions for older people with cognitive impairment and dementia [abstract]. (SarneFleischmann V. Doctoral dissertation, Ben-Gurion University of the Negev). 2014

\section{Eckroth-Bucher 2009 \{published data only\}}

Eckroth-Bucher M, Siberski J. Preserving cognition through an integrated cognitive stimulation and training program. American Journal of Alzheimer's Disease \& Other Dementias 2009;24(3):234-45.

\section{Edwards 2013 \{published data only\}}

Edwards JD, Hauser RA, O'Connor ML, Valdés EG, Zesiewicz TA, UC EY. Randomized trial of cognitive speed of processing training in Parkinson disease. Neurology 2013;81(15):1284-90.

\section{Faggian 2007 \{published data only\}}

Faggian S. An intervention protocol on cognitive abilities for subjects with severe cognitive impairment [Protocollo di stimolazione cognitiva per soggetti con deterioramento cognitivo severo]. Giornale Di 2007;55:134-43.

\section{Fane 2013 \{published data only\}}

Fane M, Lysaker P, Fiszdon J, Twamley E, Gooding A, Baginski C, et al. Cognitive behavioral therapy to enhance cognitive rehabilitation efficacy in Alzheimer's disease [abstract]. 
Alzheimer's \& Dementia: Journal of the Alzheimer's Association 2013;9(4):P495.

\section{Farina 2002 \{published data only\}}

Farina E, Fioravanti R, Chiavari L, Imbornone E, Alberoni M, Pomati S, et al. Comparing two programs of cognitive training in Alzheimer's disease: a pilot study. Acta Neurologica Scandinavica 2002;105(5):365-71.

\section{Farina 2006 \{published data only\}}

Farina E, Mantovani F, Fioravanti R, Pignatti R, Chiavari L, Imbornone $\mathrm{E}$, et al. Evaluating two group programmes of cognitive training in mild-to-moderate AD: is there any difference between a 'global' stimulation and a 'cognitivespecific' one?. Aging and Mental Health 2006;10(3):211-8.

\section{Fernandez-Calvo 2010 \{published data only\}}

Fernandez-Calvo B, Contador I, Serna A, Menezes de Lucena V, Ramos $F$. The effect of an individual or group intervention format in cognitive stimulation of patients with Alzheimer's disease. Revista de Psicopatología y Psicología Clínica 2010;15(2):115-23.

\section{Fernández-Calvo 2015 \{published data only\}}

Fernández-Calvo B, Contador I, Ramos F, Olazarán J, Mograbi DC, Morris RG. Effect of unawareness on rehabilitation outcome in a randomised controlled trial of multicomponent intervention for patients with mild Alzheimer's disease. Neuropsychological Rehabilitation 2015;25(3):448-77.

\section{Fisher 2016 \{published data only\}}

Fisher BC, Garges DM. Cognitive training/therapuetic program for memory/neuropsychological deficits: ongoing research in a dementia population. Alzheimer's \& Dementia: The Journal of the Alzheimer's Association. 2016; Vol. 12 (7):P787.

\section{Gaitan 2013 \{published data only\}}

Gaitan A, Garolera M, Cerulla N, Chico G, Rodriguez-Querol, Canela-Soler J. Efficacy of an adjunctive computer-based cognitive training program in amnestic mild cognitive impairment and Alzheimer's disease: a single-blind, randomized clinical trial. International Journal of Geriatric Psychiatry 2013;28(1):91-9.

\section{Giordano 2010 \{published data only\}}

Giordano M, Dominguez LJ, Vitrano T, Curatolo M, Ferlisi A, Di Prima A, et al. Combination of intensive cognitive rehabilitation and donepezil therapy in Alzheimer's disease (AD). Archives of Gerontology and Geriatrics 2010;51(3):245-9.

\section{Graessel 2011 \{published data only\}}

Graessel E, Stemmer R, Eichenseer B, Pickel S, Donath C, Kornhuber J, Luttenberg K. Non-pharmacological, multicomponent group therapy in patients with degenerative dementia: a 12-month randomzied, controlled trial. BMC Medicine 2011;9(1):129.

\section{Grohman 2006 \{published data only\}}

Grohman K, Fals-Stewart W, Donnelly K. Improving treatment response of cognitively impaired veterans with neuropsychological rehabilitation. Brain and Cognition 2006;60(2):203-4.

Günther 2003 \{published data only\}

Günther VK, Schäfer P, Holzner BJ, Kemmler GW. Long-term improvements in cognitive performance through computerassisted cognitive training: a pilot study in a residential home for older people. Aging \& Mental Health 2003;7(3):200-6.

Han 2017 \{published data only\}

Han JW, Lee H, Hong JW, Kim J, Kim T, Byun HJ, et al. Multimodal cognitive enhancement therapy for patients with mild cognitive impairment and mild dementia: a multi-center, randomized, controlled, double-blind, crossover trial. Journal of Alzheimer's Disease 2017;55(2):787-96.

\section{Hayashi 2009 \{published data only\}}

Hayashi N, Kazui H, Morihara T, Yokokoji K, Kono A, Hata Y, et al. Cognitive training on elderly Japanese in Osaka: major outcome (ADAS) from prospective, randomized, open, blinded-endpoint trial. Alzheimer's \& Dementia 2009;1(1552-60):P407-8.

\section{Helcer 2012 \{published data only\}}

Helcer J, Santorelli G, Choi J. Cognitive behavioral therapy to combat hopelessness and low selfefficacy in Alzheimer's disease [abstract]. Alzheimer's \& Dementia. 2012; Vol. 1), issue 1552-60:P376.

\section{Hochhalter 2004 \{published data only\}}

Hochhalter AK. Effectiveness of adjusted rehearsal training as a memory intervention for older adults with dementia. Unknown 2004.

\section{Hofmann 2003 \{published data only\}}

Hofmann M, Rosler A, Schwarz W, Müller-Spahn F, Kräuchi K, Hock $C$, et al. Interactive computer-training as a therapeutic tool in Alzheimer's disease. Comprehensive Psychiatry 2003;44(3):213-9.

\section{Hopman-Rock 1999 \{published data only\}}

Hopman-Rock M, Staats PG, Tak EC, Dröes RM. The effects of a psychomotor activation programme for use in groups of cognitively impaired people in homes for the elderly. International Journal of Geriatric Psychiatry 1999;14(8):633-42.

\section{Huntley 2017 \{published data only\}}

Huntley JD, Hampshire A, Bor D, et al. Adaptive working memory strategy training in early Alzheimer's disease: randomised controlled trial. British Journal of Psychiatry 2017;210(1):61-6.

\section{Hwang 2012 \{published data only\}}

Hwang HR, Choi SH, Yoon DH, Yoon BN, Suh YJ, Lee D, et al. The effect of cognitive training in patients with mild cognitive impairment and early Alzheimer's disease: a preliminary study. Journal of Clinical Neurology 2012;8(3):190-7.

\section{Hwang 2015 \{published data only\}}

Hwang JH, Cha HG, Cho HS. The effects of cognitive rehabilitation on Alzheimer's dementia patients' cognitive assessment reference diagnosis system performance based 
on level of cognitive functioning. Journal of Physical Therapy Science 2015;27(9):2875-7.

\section{Hyer 2014 \{published data only\}}

Hyer L, Scott C, Lyles J, Dhabliwala J, McKenzie L. Memory intervention: the value of a clinical holistic program for older adults with memory impairments. Aging \& Mental Health 2014;18(2):169-78.

\section{Israel 1987 \{published data only\}}

Israel L, Dell'Accia E, Martin G, Hugonot R. Ginkgo biloba extract and memory training programs - comparative assessment on elderly outpatients. Psychologie Médicale 1987;19:1431-9.

\section{Jang 2015 \{published data only\}}

Jang JS, Lee JS, Yoo DH. Effects of spaced retrieval training with errorless learning in the rehabilitation of patients with dementia. Journal of Physical Therapy Science 2015;27(9):2735-8.

\section{Jin 2015 \{published data only\}}

Lee J, Lee B, Park Y, Kim Y. Effects of combined fine motor skill and cognitive therapy to cognition, degree of dementia, depression, and activities of daily living in the elderly with Alzheimer's disease. Journal of Physical Therapy Science 2015;27(10):3151-4

\section{Kanaan 2014 \{published data only\}}

Kanaan SF, McDowd JM, Colgrove Y, Burns JM, Gajewski B, Pohl PS. Feasibility and efficacy of intensive cognitive training in early-stage Alzheimer's disease. American Journal of Alzheimer's Disease \& Other Dementias 2014;29(2):150-8.

\section{Kang 2010 \{published data only\}}

Kang HY, Bae YS, Kim EH, Lee KS, Chae MJ, Ju RA. An integrated dementia intervention for Korean older adults. Journal of Psychosocial Nursing and Mental Health Services 2010;48(12):42-50

\section{Kawashima 2015 \{published data only\}}

Kawashima R, Hiller DL, Sereda SL, Antonczak M, Serger K, Gannon D, et al. SAIDO learning as a cognitive intervention for dementia care: a preliminary study. Journal of the American Medical Directors Association 2015;16(1):56-62.

\section{Kessels 2009 \{published data only\}}

Kessels RP, Hensken LM. Effects of errorless skill learning in people with mild-to-moderate or severe dementia: a randomized controlled pilot study. NeuroRehabilitation 2009;25(4):307-12

\section{Khurandy 2016 \{published data only\}}

Mahmoudi KZ, Talepasand S, Rahimian BI. The impact of computer-based cognitive rehabilitation program on Alzheimer's patients with mild cognitive deficits [The impact of computer-based cognitive rehabilitation program on Alzheimer's patients with mild cognitive deficits]. Advances in Cognitive Science 2016;17:22-32.
Kim, 2016 \{published data only\}

Kim MJ, Han CW, Min, KY, Cho CY, Lee CW, Ogawa Y, et al. Physical exercise with multicomponent cognitive intervention for older adults with Alzheimer's disease: a 6-month randomized controlled trial. Dementia and Geriatric Cognitive Disorders Extra 2016;6(2):222-32.

Kim 2005 \{published data only\}

Kim MY, Lee KS, Choi JS, Ki, HB, Park Cl. Effectiveness of cognitive training based on virtual reality for the elderly [Korean]. Journal of Korean Academy of Rehabilitation Medicine 2005;29(4):424-33.

Kovach 2018 \{published data only\}

Kovach CR, Evans CR, Satteli L, Rosenau K, Gopalakrishnan S. Feasibility and pilot testing of a mindfulness intervention for frail older adults and individuals with dementia. Research in Gerontological Nursing 2018;11(3):137-50.

\section{Latorre 2010 \{published data only\}}

Latorre Postigo JM, Hernandez-Viadel JV, Trives JJR. Efficacy of a group memory training method for older adults based on visualization and association techniques: a randomized, controlled trial with a placebo group. Applied Cognitive Psychology 2010;24(7):956-68.

\section{Lee, 2016 \{published data only\}}

Lee J, Choi BH, Oh E, Sohn EH, Lee AY. Treatment of Alzheimer's disease with repetitive transcranial magnetic stimulation combined with cognitive training: a prospective, randomized, double-blind, placebo-controlled study. Journal of Clinical Neurology 2016;12(1):57-64.

Lee, 2016a \{published data only\}

Lee $\mathrm{GH}$. Effects of virtual reality exercise program on balance, emotion and quality of life in patients with cognitive decline. Journal of Korean Physical Therapy 2016;28(6):355-63.

\section{Maci, a \{published data only\}}

Maci T, Quattrocchi G, Cirmi M, et al. The GAIA project: a pilot program for Alzheimer's disease. A follow-up study [abstract]. Unknown.

\section{Maci 2012 \{published data only}

Maci T, Pira FL, Quattrocchi G, Nuovo SD, Perciavalle V, Zappia M. Physical and cognitive stimulation in Alzheimer Disease. The GAIA Project: a pilot study. American Journal of Alzheimer's Disease \& Other Dementias 2012;27(2):107-13.

\section{Meguro 2008 \{published data only\}}

Meguro M, Kasai M, Akanuma K, Ishii H, Yamaguchi S, Meguro K. Comprehensive approach of donepezil and psychosocial interventions on cognitive function and quality of life for Alzheimer's disease: the Osaki-Tajiri project. Age and Ageing 2008;37(4):469-73.

\section{Middelstadt 2016 \{published data only\}}

Middelstadt J, Folkerts AK, Blawath S, Kalbe E. Cognitive stimulation for people with dementia in long-term care facilities: baseline cognitive level predicts cognitive gains, 
moderated by depression. Journal of Alzheimer's Disease 2016;54(1):253-68.

\section{NCT 2005 \{published data only\}}

NCT00146263. The effect of computerized cognitive training on neuropsychological measures of cognitive function in the elderly. https://protect-au.mimecast.com/s/ AX1UC5QZOxCIDlzytjpm1us?domain=clinicaltrials.gov 2005.

NCT 2011 \{published data only\}

NCT01329484. Computerized personal interventions for Alzheimer's patients. https://protect-au.mimecast.com/s/ rQWJC71ZQzSRJROrurJl1eV?domain=clinicaltrials.gov 2011.

NCT 2012 \{published data only\}

NCT01688128. Efficacy of ubiquitous SR-based memory advancement and rehabilitation training (U-SMART). https:// protect-au.mimecast.com/s/b_9ICxnMJ5sX3Xgps08h0Si? domain=clinicaltrials.gov 2012.

NCT 2013 \{published data only\}

NCT01825317. Effect of NeuroAD on Alzheimer patients. https:// protect-au.mimecast.com/s/GKuNCMwvygsJAJLpf99K3Ke? domain=clinicaltrials.gov 2013.

\section{NCT 2016a \{published data only\}}

NCT02671630. Computerized cognitive training program for older persons with mild dementia. https://clinicaltrials.gov/ct2/ show/NCT02671630 2016.

\section{Ochmann 2015 \{published data only\}}

Ochmann SL, Kasper E, Hoffmann W, Schneider W, Teipel SJ. Feasibility of a cognitive rehabilitation group program for patients with mild dementia in Alzheimer's disease: a randomized, controlled, single blinded pilot study. Alzheimer's \& Dementia: Journal of the Alzheimer's Association 2015;11(7):P96-7.

\section{Olazaran 2004 \{published data only\}}

Olazaran J, Muñiz R, Reisberg B, Peña-Casanova J, Del Ser T, Cruz-Jentoft AJ, et al. Benefits of cognitive-motor intervention in $\mathrm{MCl}$ and mild to moderate Alzheimer disease. Neurology 2004;63(12):2348-53.

\section{Optale 2010 \{published data only\}}

Optale G, Urgesi C, Busato V, Marin S, Piron L, Priftis K, et al. Controlling memory impairment in elderly adults using virtual reality memory training: a randomized controlled pilot study. Neurorehabilitation and Neural Repair 2010;24(4):348-57.

\section{Oswald 1996 \{published data only\}}

Oswald WD, Rupprecht R, Gunzelmann T, Tritt K. The SIMAproject: effects of 1 year cognitive and psychomotor training on cognitive abilities of the elderly. Behavioural Brain Research 1996;78(1):67-72.

\section{Park 2009 \{published data only\}}

Park MH, Kwon DY, Seo WK, Lim KS, Song MS. The effects of cognitive training on community-dwelling elderly Koreans. Journal of Psychiatric and Mental Health Nursing 2009;16(10):904-9.

\section{Pietila 2017 \{published data only\}}

Pietila P, Poutiainen $\mathrm{E}$, Nukari J, Launiainen $\mathrm{H}$, ArifullenHamalainen U, Sarajuuri J, et al. Multi-methodological rehabilitation with emphasis on cognitive training alleviates Alzheimer patients' mood symptoms and supports coping among family members. Psykologia 2017;52(5):356-75.

\section{Poon 2005 \{published data only\}}

Poon P, Hui E, Dai D, Kwok T, Woo J. Cognitive intervention for community-dwelling older persons with memory problems: telemedicine versus face-to-face treatment. International Journal of Geriatric Psychiatry 2005;20(3):285-6.

\section{Poptsi 2017 \{published data only\}}

Poptsi E, Kounti F, Samakouri M, Vorvolakos T, Tsolaki M. Longitudinal cognitive training program in people with mild Alzheimer's disease: a randomized controlled trial. Hellenic Journal of Nuclear Medicine 2017;20(2):233-43.

\section{Quintana-Hernandez 2015 \{published data only\}}

Quintana Hernandez DJ, Miró Barrachina MT, Ibanez Fernandez I, Santana del Pino A, Rojas Hernandez J, Rodríguez García, et al. Mindfulness-based stimulation in advanced Alzheimer's disease: a comparative, non-inferiority, clinical pilot study [Estimulación basada en mindfulness en la enfermedad de Alzheimer avanzada: ensayo clínico piloto comparativo de equivalencia.]. Revista Española de Geriatría y Gerontología 2015;50(4):168-73.

\section{Quintana-Hernandez 2016 \{published data only\}}

Quintana-Hernandez DJ, Miró-Barrachina MT, IbánezFernández IJ, Pino ASD, Quintana-Montesdeoca MP, Rodríguezde Vera $B$, et al. Mindfulness in the maintenance of cognitive capacities in Alzheimer's disease: a randomized clinical trial. Journal of Alzheimer's Diseas 2016;50:217-32.

\section{SeungHyun 2017 \{published data only\}}

SeungHyun Cho, Young-ae Yang. The effect of occupational therapy based multimodal cognitive rehabilitation therapy on cognitive function in elderly people with mild dementia: a randomized controlled trial. Korean Journal of Occupational Therapy 2017;25(3):71-86.

\section{Umin 2015 \{published data only\}}

Umin. The effects of cognitive rehabilitation for vascular dementia: a randomized controlled trial. https://upload.umin.ac.jp/cgi-open-bin/ctr/ctr.cgi? function=brows\&action=brows\&recptno=R000022227\&type=summary\&lan 2015.

\section{van Zon 2016 \{published data only\}}

van Zon L, Kirby JR, Anderson N. The efficacy of a volunteeradministered cognitive stimulation program in long-term care homes. International Psychogeriatrics 2016;28(6):995-1004.

Viola 2011 \{published data only\}

Viola LF, Nunes PV, Yassuda MS, Aprahamian I, Santos FS, Santos GD, et al. Effects of a multidisciplinary cognitive rehabilitation program for patients with mild Alzheimer's disease. Clinics 2011;66((8)):1395-400. 
Voigt-Radloff 2017 \{published data only\}

Voigt-Radloff S, de Werd MM, Leonhart R, Boelen DH, Rikkert MGO, Fliessbach K, et al. Structured relearning of activities of daily living in dementia: the randomized controlled REDALI-DEM trial on errorless learning. Alzheimer's Research \& Therapy 2017;9(1):22.

\section{Yun 2008 \{published data only\}}

Yun Young-Ji. Effects of playing musical instruments for the purpose of cognitive training on cognitive functioning of dementia patients [Korean]. Korean Journal of Music Therapy 2008;10:1598-916X.

\section{Zanetti 1997 \{published data only\}}

Zanetti O, Binetti G, Magni E, Rozzini L, Bianchetti A, Trabucchi M. Procedural memory stimulation in Alzheimer's disease: impact of a training programme. Acta Neurologica Scandinavica 1997;95(3):152-7.

\section{Zanetti 2001 \{published data only\}}

Zanetti O, Zanieri G, Giovanni GD, de Vreese LP, Pezzini A, Metitieri T, et al. Effectiveness of procedural memory stimulation in mild Alzheimer's disease patients: a controlled study. Neuropsychological Rehabilitation 2001;11(3-4):263-72.

\section{Zarit 1982 \{published data only\}}

Zarit SH, Zarit JM, Reever KE. Memory training for severe memory loss: effects on senile dementia patients and their families. The Gerontologist 1982;22(4):373-7.

\section{Zhuang 2013 \{published data only\}}

Zhuang JP, Fang R, Feng X, Xu XH, Liu LH, Bai QK, et al. The impact of human-computer interaction-based comprehensive training on the cognitive functions of cognitive impairment elderly individuals in a nursing home. Journal of Alzheimer's Disease 2013;36(2):245-51.

\section{이효정 2010 \{published data only\}}

이효정 (Name in Korean. English name not found). The effect of the cognitive rehabilitation upon EEG and cognition in the Alzheimer's dementia [Korean]. Korean Society for Sports Science 2010;19(2):1217-26.

\section{References to ongoing studies}

\section{ACTRN12618000600246 \{published data only\}}

Optimising functional independence of older persons with dementia: evaluation of the Interdisciplinary Home-bAsed Reablement Program (I-HARP). Unknown 18/0.

\section{Leung 2017 \{published data only\}}

Leung IHK, Lampit A, Mowszowski L, broadhouse KM, Naismith SL, Chung HK. Modifying cognitive trajectories in insidious cognitive decline using computerised cognitive training: methods and current progress of a two-phase randomised controlled trial. Alzheimer's \& Dementia:Journal of the Alzheimer's Association 2017;13(7):P526.
NCT 2016 \{published data only\}

NCT02772185. Computerized cognitive training program for older persons with mild dementia. https://clinicaltrials.gov/ct2/ show/NCT027721852016.

\section{Additional references}

\section{Albert 2011}

Albert MS, DeKosky ST, Dickson D, Dubois B, Feldman HH, Fox NC, et al. The diagnosis of mild cognitive impairment due to Alzheimer's disease: recommendations from the National Institute on Aging and Alzheimer's Association workgroup. Alzheimer's and Dementia 2011;7(3):270-9.

\section{Alves 2013}

Alves J, Magalhães R, Machado Á, Gonçalves ÓF, Sampaio A Petrosyan A. Non-pharmacological cognitive intervention for aging and dementia: current perspectives. World Journal of Clinical Cases 2013;1(8):233.

\section{Alzheimer's Association 2018}

Alzheimer's Association. 2018 Alzheimer's Disease facts and figures. Alzheimer's and Dementia 2018;14(3):367.

\section{Alzheimer's Disease International 2009}

Alzheimer's Disease International. World Alzheimer's Report. www.alz.co.uk 2009. [http://www.alz.co.uk/research/files/ WorldAlzheimerReport-ExecutiveSummary.pdf (Accessed 29th June 2018)]

\section{Anguera 2015}

Anguera JA, Gazzaley A. Video games, cognitive exercises, and the enhancement of cognitive abilities. Current Opinion in Behavioral Sciences 2015;4:160-5.

\section{APA 1995}

American Psychiatric Association. Diagnostic and Statistical Manual of Mental Disorders. Fourth. Washington, DC: American Psychiatric Association, 1995.

\section{APA 2013}

Association American Psychiatric. Diagnostic and Statistical Manual of Mental Disorders (DSM-5). Washington, DC: American Psychiatric Association, 2013.

\section{Bahar-Fuchs 2013}

Bahar-Fuchs A, Clare L, Woods B. Cognitive training and cognitive rehabilitation for mild to moderate Alzheimer's disease and vascular dementia. Cochrane Database of Systematic Reviews 2013, Issue 6. [DOI: 10.1002/14651858.CD003260.pub2]

\section{Bahar-Fuchs 2014}

Bahar-Fuchs A, Hampstead BM, Clare L. Cognitive training for older adults with $\mathrm{MCl}$ and mild dementia: state of the science, central challenges, and possible solutions. Alzheimer's \& Dementia: Journal of the Alzheimer's Association 2014;10(4):P157. 


\section{Bahar-Fuchs 2016}

Bahar-Fuchs A, Kudlicka A, Clare L. Cognitive rehabilitation for people with dementia: what is it and does it work?. Australian Journal of Dementia Care 2016;5(5):37.

\section{Bahar-Fuchs 2017}

Bahar-Fuchs A, Webb S, Bartsch L, Clare L, Rebok G, Cherbuin N, et al. Tailored and adaptive computerized cognitive training in older adults at risk for dementia: a randomized controlled trial. Journal of Alzheimer's Disease 2017;60(3):889-911.

\section{Belleville 2011}

Belleville S, Clement F, Mellah S, Gilbert B, Fontaine F, Gauthier S. Training-related brain plasticity in subjects at risk of developing Alzheimer's disease. Brain 2011; Vol. 134, issue Pt 6:1623-34.

\section{Belleville 2014}

Belleville S, Mellah S, de Boysson C, Demonet J-F, Bier B. The pattern and loci of training-induced brain changes in healthy older adults are predicted by the nature of the intervention. PLoS One 2014;9(8):e102710.

\section{Ben-Yishay 1978}

Ben-Yishay Y, Diller L, Rattok J. A modular approach to optimizing orientation, psychomotor alertness, and purposive behavior in severe head trauma patients. Rehabilitation Monograph 1978;59:63-7.

\section{Berger 2005}

Berger G, Bernhardt T, Weimer E, Peters J, Kratzsch T, Frolich L. Longitudinal study on the relationship between symptomatology of dementia and levels of subjective burden and depression among family caregivers in memory clinic patients. Journal of Geriatric Psychiatry and Neurology 2005;18(3):119-28.

\section{Birks 2006}

Birks JS. Cholinesterase inhibitors for Alzheimer's disease. Cochrane Database of Systematic Reviews 2006, Issue 1. [DOI: 10.1002/14651858.CD005593]

\section{Birks 2015}

Birks JS, Chong LY, Grimley Evans J. Rivastigmine for Alzheimer's disease. Cochrane Database of Systematic Reviews 2015, (9).

\section{Birks 2018}

Birks JS, Harvey RJ. Donepezil for dementia due to Alzheimer's disease. Cochrane Database of Systematic Reviews 2018;6.

\section{Braak \& Braak 2012}

Braak $\mathrm{H}$, Braak E. Evolution of the neuropathology of Alzheimer's disease. Acta Neurologica Scandinavica 2012;94(S165):3-12.

\section{Chandler 2016}

Chandler MJ, Parks AC, Marsiske M, Rotblatt LJ, Smith GE. Everyday impact of cognitive interventions in mild cognitive impairment: a systematic review and meta-analysis. Neuropsychology Review 2016;26(3):225-51.

\section{Clare 2001}

Clare L, Wilson BA, Carter G, Hodges JR, Adams M. Longterm maintenance of treatment gains following a cognitive rehabilitation intervention in early dementia of Alzheimer type: a single case study. Neuropsychological Rehabilitation 2001;11:477-94.

\section{Clare 2001b}

Clare L, Woods RT, Moniz-Cook ED, Orrell M, Spector A. Cognitive rehabilitation interventions to improve memory functioning in early-stage Alzheimer'sdisease and vascular dementia. Cochrane Database of Systematic Reviews 2001, Issue 4. [DOI: 10.1002/14651858.CD003260.pub2]

\section{Clare 2002}

Clare L, Wilson BA, Carter G, Roth I, Hodges JR. Relearning of face-name associations in early-stage Alzheimer's disease. Neuropsychology 2002;16(4):538-47. [3288528]

\section{Clare 2004}

Clare L, Wilson BA, Carter G, Roth I, Hodges JR. Awareness in early-stage dementia: relationship to outcome of cognitive rehabilitation intervention. Journal of Clinical and Experimental Neuropsychology 2004;26:215-26.

\section{Cummings 2014}

Cummings JL, Morstorf T, Zhong K. Alzheimer's disease drugdevelopment pipeline: few candidates, frequent failures. Alzheimer's Research \& Therapy 2014;6(4):37.

\section{Cummings 2016}

Cummings J, Morstorf T, Lee G. Alzheimer's drug-development pipeline: 2016. Alzheimer's and Dementia: Translational Research and Clinical Interventions 2016;2(4):222-32.

\section{D'Alton 2011}

D'Alton S, George DR. Changing perspectives on Alzheimer's disease: thinking outside the amyloid box. Journal of Alzheimer's Disease 2011;25:571-81.

\section{de Vreese 1998a}

de Vreese LP, Verlato C, Emiliani S, Schioppa S, Belloi L, Salvioli G, et al. Effect size of a three-month drug treatment in $A D$ when combined with individual cognitive retraining: preliminary results of a pilot study. European Archives of Psychiatry and Clinical Neuroscience 1998;248:41-2. [3288486]

\section{de Vreese 1998b}

de Vreese LP, Neri M. Ecological impact of combined cognitive training programs (CTP) and drug treatment (ChE-I) in AD. International Psychogeriatrics 1999;11 Suppl:S91. [3288485]

\section{Deeks 2017}

Deeks JJ, Higgins JPT, Altman DG (editors), on behalf of the Cochrane Statistical Methods Group. Chapter 9. Analysing data and undertaking meta-analysis. In: Higgins JPT, Churchill R, Chandler J, Cumpston MS editor(s). Cochrane Handbook for Systematic Reviews of Interventions version 5.2.0 (updated June 2017). London, UK: Cochrane, 2017. Available from www.training.cochrane.org/handbook. 


\section{Diller 1974}

Diller L, Weinberg J, Gordon W, Goodkin R, Gerstman LJ, BenYishay Y. Studies in Cognition and Rehabilitation in Hemiplegia [Studies in Cognition and Rehabilitation in Hemiplegia]. Studies in Cognition and Rehabilitation in Hemiplegia. Behavioral Science, Institute of Rehabilitation Medicine, New York University Medical Center, 1974.

\section{Duval 2000}

Duval S, Tweedie R. Trim and fill: a simple funnel-plot-based method of testing and adjusting for publication bias in metaanalysis. Biometrics 2000;56(2):455-63.

\section{Edwards 2017}

Edwards JD, Fausto BA, Tetlow AM, Corona RT, Valdés EG. Systematic review and meta-analyses of useful field of view cognitive training. Neuroscience \& Biobehavioral Reviews 2017;84:72-91.

\section{Egger 1997}

Egger M, Davey Smith G, Schneider M, Minder C. Bias in meta-analysis detected by a simple, graphical test. BMJ 1997;315(7109):629-34.

\section{Ermini Fuenfsch 1995}

Ermini Fuenfschilling D, Meier D. Memory training: an important part of a "milieu therapy" for patients with senile dementia [Gedaechtnistraining: Wichtiger Bestandteil der Milieutherapie bei seniler Demenz]. Zeitschrift fuer Gerontologie und Geriatrie 1995; Vol. 28, issue 3:190-4. [3288534]

\section{Farias 2005}

Farias ST, Mungas D, Jagust W. Degree of discrepancy between self and other-reported everyday functioning by cognitive status: dementia, mild cognitive impairment, and healthy elders. International Journal of Geriatric Psychiatry 2005;20(9):827-34.

\section{Fernandez-Prado 2012}

Fernandez-Prado S, Conlon S, Mayan-Santos JM, GandoyCrego M. The influence of a cognitive stimulation program on the quality of life perception among the elderly. Archives of Gerontology and Geriatrics 2012; Vol. 54, issue 1:181-4.

\section{Folkerts 2017}

Folkerts AK, Roheger M, Franklin J, Middelstaedt J, Kalbe E. Cognitive interventions in patients with dementia living in long-term care facilities: systematic review and meta-analysis. Archives of Gerontology and Geriatrics 2017;73:204-21.

\section{Folstein 1975}

Folstein MF, Folstein SE, McHugh PR. 'Mini-mental state': a practical method for grading the cognitive state of patients for the clinician. Journal of Psychiatric Research 1975;12:189-98.

\section{Förstl 1999}

Förstl H, Kurz A. Clinical features of Alzheimer's disease. European Archives of Psychiatry and Clinical Neuroscience 1999;249(6):288-90.

\section{Gates 2011}

Gates N, Sachdev P, Singh MF, Valenzuela M. Cognitive and memory training in adults at risk of dementia: a systematic review. BMC Geriatrics 2011;11(1):55.

\section{Gates 2014}

Gates NJ, Sachdev P. Is cognitive training an effective treatment for preclinical and early Alzheimer's Disease?. Journal of Alzheimer's Disease 2014;42 (s4):S551-9.

\section{Gaugler 2000}

Gaugler JE, Davey A, Pearlin LI, Zarit SH. Modeling caregiver adaptation over time: the longitudinal impact of behavior problems. Psychology and Aging 2000;15(3):437.

\section{GRADE Handbook}

Akl E, Mustafa R, Wojtek Wiercioch NS. Handbook for grading the quality of evidence and the strength of recommendations using the GRADE approach. Updated October 2013.. Handbook for Grading the Quality of Evidence and the Strength of Recommendations Using the GRADE Approach. GRADE Working Group, Updated October 2013.

\section{GRADEpro GDT [Computer program]}

McMaster University (developed by Evidence Prime). GRADEpro GDT. Version Accessed 29 June 2018. Hamilton (ON): McMaster University (developed by Evidence Prime), 2015.

\section{Graham 2004}

Graham NL, Emery T, Hodges JR. Distinctive cognitive profiles in Alzheimer's disease and subcortical vascular dementia. Journal of Neurololgy Neurosurgery and Psychiatry 2004;75(1):61-71.

\section{Hampstead 2011}

Hampstead BM, Stringer AY, Stilla RF, Deshpande G, Hu X, Moore $A B$, et al. Activation and effective connectivity changes following explicit-memory training for face-name pairs in patients with mild cognitive impairment: a pilot study. Neurorehabilitation \& Neural Repair 2011; Vol. 25, issue 3:210-22.

\section{Hampstead 2016}

Hampstead BM, Khoshnoodi M, Yan W, Deshpande G, Sathian K. Patterns of effective connectivity during memory encoding and retrieval differ between patients with mild cognitive impairment and healthy older adults. Neuroimage 2016;124(Pt A):997-1008.

\section{Hart 2014}

Hart T, Tsaousides T, Zanca JM, Whyte J, Packel A, Ferraro M, et al. Toward a theory-driven classification of rehabilitation treatments. Archives of Physical Medicine and Rehabilitation 2014;95(1):S33-S44. e2.

\section{Higgins 2017}

Higgins JPT, Altman DG, Sterne JA (editors). Chapter 8. Assessing risk of bias in included studies. Available from www.training.cochrane.org/handbook.. Cochrane Collaboration, 2017. 


\section{Hill 2017}

Hill NT, Mowszowski L, Naismith SL, Chadwick VL, Valenzuela M, Lampit A. Computerized cognitive training in older adults with mild cognitive impairment or dementia: a systematic review and meta-analysis. American Journal of Psychiatry 2017;174(4):329-40.

\section{Hughes 1982}

Hughes CP, Berg L, Danziger WL, Coben LA, Martin RL. A new clinical scale for the staging of dementia. British Journal of Psychiatry 1982;140(6):566-72.

\section{Huntley 2015}

Huntley JD, Gould RL, Liu K, Smith M, Howard RJ. Do cognitive interventions improve general cognition in dementia? A metaanalysis and meta-regression. BMJ Open 2015;5(4):e005247.

\section{Jaeggi 2008}

Jaeggi SM, Buschkuehl M, Jonides J, Perrig WJ. Improving fluid intelligence with training on working memory. Proceedings of the National Academy of Sciences 2008;105(19):6829-33.

\section{Jaeggi 2010}

Jaeggi SM, Studer-Luethi B, Buschkuehl M, Su YF, Jonides J, Perrig WJ. The relationship between $n$-back performance and matrix reasoning - implications for training and transfer. Intelligence 2010;38(6):625-35.

\section{Kesslak 1997}

Kesslak JP, Nackoul K, Sandman CA. Memory training for individuals with Alzheimer's disease improves name recall. Behavioural Neurology 1997;10:137-42.

\section{Lampit 2014}

Lampit A, Hallock H, Valenzuela M. Computerized cognitive training in cognitively healthy older adults: a systematic review and meta-analysis of effect modifiers. PLoS Med 2014;11(11):e1001756.

Lezak 2004

Lezak MD. Neuropsychological Assessment. New York, NY: Oxford University Press, USA, 2004.

\section{Livingston 2017}

Livingston G, Sommerlad A, Orgeta V, Costafreda SG, Huntley J, Ames $\mathrm{D}$, et al. Dementia prevention, intervention, and care. The Lancet 2017;390(10113):2673-734.

\section{Loewenstein 2004}

Loewenstein DA, Acevedo A, Czaja SJ, Duara R. Cognitive rehabilitation of mildly impaired Alzheimer's disease patients on cholinesterase inhibitors. American Journal of Geriatric Psychiatry 2004;12:395-402. [3288495]

\section{Malmberg Gavelin}

Malmberg Gavelin H, Lampit A, Hallock H, Sabates J, BaharFuchs A. Cognition-oriented treatments for older adults - a systematic overview of systematic reviews. Alzheimer's \& Dementia: The Journal of the Alzheimer's Association. 2018; Vol. 14, issue 7:P1627-P1628.

\section{McKeith 1996}

McKeith I G, Galasko D, Kosaka K, Perry EK, Dickson DW, Hansen LA, et al. Consensus guidelines for the clinical and pathologic diagnosis of dementia with Lewy bodies (DLB): report of the consortium on DLB international workshop. Neurology 1996;47(5):1113-24.

\section{McKeith 2006}

McKeith IG. Consensus guidelines for the clinical and pathologic diagnosis of dementia with Lewy bodies (DLB): report of the Consortium on DLB International Workshop. Journal of Alzheimer's Disease 2006;9(3 Suppl):417-23.

\section{McKeith 2017}

McKeith IG, Boeve BF, Dickson DW, Halliday G, Taylor J$P$, Weintraub $D$, et al. Diagnosis and management of dementia with Lewy bodies. Fourth consensus report of the DLB Consortium. Neurology 2017;89(1):10.1212/WNL. 0000000000004058.

\section{McKhann 1984}

McKhann G, Drachman D, Folstein M, Katzman R, Price D, Stadlan EM. Clinical diagnosis of Alzheimer's disease: report of the NINCDS-ADRDA Work Group under the auspices of Department of Health and Human Services task force on Alzheimer's disease. Neurology 1984;34:939-44.

\section{McKhann 2011}

McKhann GM, Knopman DS, Chertkow H, Hyman BT, Jack Jr CR, Kawas $\mathrm{CH}$, et al. The diagnosis of dementia due to Alzheimer's disease: recommendations from the National Institute on Aging and the Alzheimer's Association Workgroup. Alzheimer's and Dementia 2011;7(3):263-9.

\section{Michie 2008}

Michie S, Johnston M, Francis J, Hardeman W, Eccles M. From theory to intervention: mapping theoretically derived behavioural determinants to behaviour change techniques. Applied Psychology 2008;57(4):660-80.

\section{Moore 2001}

Moore S, Sandman CA, McGrady K, Kesslak JP. Memory training improves cognitive ability in patients with dementia. Neuropsychological Rehabilitation 2001;11(3/4):245-61.

\section{Mowszowski 2010}

Mowszowski L, Batchelor J, Naismith SL. Early intervention for cognitive decline: can cognitive training be used as a selective prevention technique?. International Psychogeriatrics 2010;22(4):537-48.

\section{Newhouse 1997}

Newhouse PA, Potter A, Levin ED. Nicotinic system involvement in Alzheimer's and Parkinson's diseases: implications for therapeutics. Drugs and Aging 1997;11:206-28.

\section{Olazaran 2010}

Olazarán J, Reisberg B, Clare L, Cruz I, Peña-Casanova J, Del Ser T, et al. Nonpharmacological therapies in Alzheimer's disease: a systematic review of efficacy. Dementia and Geriatric Cognitive Disorders 2012;30(2):161-78. 


\section{Oltra-Cucarella 2016}

Oltra-Cucarella J, Pérez-Elvira R, Espert R, Sohn McCormick A. Are cognitive interventions effective in Alzheimer's disease? A controlled meta-analysis of the effects of bias.. Neuropsychology 2016;30(5):631.

\section{Owen 2010}

Owen AM, Hampshire A, Grahn JA, Stenton R, Dajani S, Burns AS, et al. Putting brain training to the test. Nature 2010;465(7299):775-8.

\section{Papp 2009}

Papp KV, Walsh SJ, Snyder PJ. Immediate and delayed effects of cognitive interventions in healthy elderly: a review of current literature and future directions. Alzheimer's and Dementia 2009;5(1):50-60.

\section{Peretz 2011}

Peretz C, Korczyn AD, Shatil E, Aharonson V, Birnboim S, Giladi N. Computer-based, personalized cognitive training versus classical computer games: a randomized double-blind prospective trial of cognitive stimulation. Neuroepidemiology 2011;36(2):91-9.

\section{Petersen 2004}

Petersen RC. Mild cognitive impairment as a diagnostic entity. Journal of Internal Medicine 2004;256(3):183-94.

\section{Petersen 2018}

Petersen RC, Lopez O, Armstrong MJ, Getchius TS, Ganguli M, Gloss D, et al. Practice guideline update summary: mild cognitive impairment. Report of the Guideline Development, Dissemination, and Implementation Subcommittee of the American Academy of Neurology. Neurology 2018;90(3):126-35.

\section{Ponsford 2012}

Ponsford J, Sloan S, Snow P. Traumatic Brain Injury: Rehabilitation for Everyday Adaptive Living. Routledge, UK: Psychology Press, 2012.

\section{Quayhagen 1995a}

Corbeil RR, Quayhagen MP, Quayhagen M. Intervention effects on dementia caregiving interaction: a stressadaptation modeling approach. Journal of Aging and Health 1999;11(1):79-95. [3288499]

\section{Rabipour \& Raz 2012}

Rabipour S, Raz A. Training the brain: fact and fad in cognitive and behavioral remediation. Brain and Cognition 2012;79(2):159-79.

\section{Reeves 2011}

Reeves BC, Deeks JJ, Higgins JPT, Wells GA. Chapter 13. Including non-randomized studies. In: Higgins JPT, Green S editor(s). Cochrane Handbook for Systematic Reviews of Interventions Version 5.1.0 (updated March 2011). London, UK: The Cochrane Collaboration, 2011.

\section{Review Manager 5 [Computer program]}

Nordic Cochrane Centre, The Cochrane Collaboration. Review Manager 5 (RevMan 5). Version 5.3. Copenhagen: Nordic Cochrane Centre, The Cochrane Collaboration, 2014.

\section{Roman 1993}

Roman GC, Tatemichi MD, Erkinjuntti MD, et al. Vascular dementia: diagnostic criteria for research studies. Report of the NINDS-AIREN International Workshop. Neurology 1993;43:250-60.

\section{Salomone 2012}

Salomone S, Caraci F, Leggio GM, Fedotova J, Drago F. New pharmacological strategies for treatment of Alzheimer's disease: focus on disease modifying drugs. British Journal of Clinical Pharmacology 2012;73(4):504-17.

\section{Simons 2016}

Simons DJ, Boot WR, Charness N, Gathercole SE, Chabris CF, Hambrick DZ, et al. Do "brain-training" programs work? Psychological Science in the Public Interest 2016;17(3):103-86.

\section{Sitzer 2006}

Sitzer D, Twamley E, Jeste D. Cognitive training in Alzheimer's disease: a meta-analysis of the literature. Acta Psychiatrica Scandinavica 2006;114(2):75-90.

\section{Skrobot 2017}

Skrobot OA, Black SE, Chen C, DeCarli C, Erkinjuntti T, Ford GA, et al. Progress toward standardized diagnosis of vascular cognitive impairment: guidelines from the Vascular Impairment of Cognition Classification Consensus Study. Alzheimer's \& Dementia 2017;14(3):280-92.

\section{Sohlberg 1987}

Sohlberg McKay M, Mateer CA. Effectiveness of an attentiontraining program. Journal of Clinical and Experimental Neuropsychology 1987;9(2):117-30.

\section{Spreen 1998}

Spreen O, Strauss E. A compendium of neuropsychologial tests. Administration, Norms, and Commentary. New York, NY: Oxford University Press, 1998.

\section{Stuss 1999}

Stuss DT, Winocur G, Robertson IH. Cognitive Neurorehabilitation. Cambridge, UK: Cambridge University Press, 1999

\section{Valenzuela 2012}

Valenzuela MJ, Matthews FE, Brayne C, Ince P, Halliday G, Kril JJ, et al. Multiple biological pathways link cognitive lifestyle to protection from dementia. Biological Psychiatry 2012;71(9):783-91.

\section{Villemagne 2018}

Villemagne VL, Doré V, Burnham SC, Masters CL, Rowe CC. Imaging tau and amyloid- $\beta$ proteinopathies in Alzheimer disease and other conditions. Nature Reviews Neurology 2018;14(4):225. 


\section{Weintraub 2012}

Weintraub S, Wicklund AH, Salmon DP. The neuropsychological profile of Alzheimer disease. Cold Spring Harbor Perspectives in Medicine 2012;2(4):a006171.

\section{WHO 1992}

World Health Organization. The ICD-10 Classification of Mental and Behavioural Disorders: Clinical Descriptions and Diagnostic Guidelines. Geneva: World Health Organization, Division of Mental Health, 1992.

\section{Wilson 2002}

Wilson BA. Towards a comprehensive model of cognitive rehabilitation. Neuropsychological Rehabilitation 2002;12(2):97-110.

\section{Wilson 2012}

Wilson RS, Segawa E, Boyle PA, Anagnos SE, Hizel LP, Bennett DA. The natural history of cognitive decline in Alzheimer's disease. Psychology and Aging 2012;27(4):1008.

\section{Wimo 2017}

Wimo A, Guerchet, M, Ali G-C, Wu YT, Prina M. The worldwide costs of dementia 2015 and comparisons with 2010. Alzheimer's \& Dementia 2017; Vol. 13, issue 1:1-7.

\section{Woods 2012}

Woods B, Aguirre E, Spector A, Orrell M. Cognitive stimulation to improve cognitive functioning in people with dementia. Cochrane Database of Systematic Reviews 2012, Issue 2. [DOI: 10.1002/14651858.CD005562.pub2]

\section{World Health Organization 2012}

World Health Organization. Dementia: A Public Health Priority. Geneva, Switzerland: World Health Organization, 2012.

\section{Yesavage 1981}

Yesavage JA, Westphal J, Rush L. Senile dementia: combined pharmacologic and psychologic treatment. Journal of the

\section{CHARACTERISTICS OF STUDIES}

Characteristics of included studies [ordered by study ID]
American Geriatrics Society 1981; Vol. 29, issue 4:164-71. [3288606]

\section{Ylvisaker 2002}

Ylvisaker M, Hanks R, Johnson-Greene D. Perspectives on rehabilitation of individuals with cognitive impairment after brain injury: rationale for reconsideration of theoretical paradigms. Journal of Head Trauma Rehabilitation 2002;17(3):191-209.

\section{Zanetti 1994}

Zanetti O, Magni E, Binetti G, Bianchetti A, Trabucchi M. Is procedural memory stimulation effective in Alzheimer's disease?. International Journal of Geriatric Psychiatry 1994;9:1006-7.

\section{Zarit, 1981}

Zarit SH, Cole KD, Guider Rl. Memory training strategies and subjective complaints of memory in the aged. Gerontologist 1981;21:158-64.

\section{Zinke 2014}

Zinke K, Zeintl M, Rose NS, Putzmann J, Pydde A, Kliegel M. Working memory training and transfer in older adults: effects of age, baseline performance, and training gains. Developmental Psychology 2014;50(1):304.

\section{References to other published versions of this review \\ Bahar-Fuchs 2018}

Bahar-Fuchs A, Martyr A, Goh AMY, Sabates J, Clare L. Cognitive training for people with mild to moderate dementia. Cochrane Database of Systematic Reviews 2018, Issue 7. [DOI: 10.1002/14651858.CD013069]

* Indicates the major publication for the study

Amieva 2016

Methods A multi-centre randomised parallel-group trial comparing effects of CT, reminiscence therapy, and an individualised cognitive rehabilitation programme in AD vs usual care

Participants 653 community-dwelling participants aged 50 and above with mild to moderate AD (according to MMSE, score range 16 to 26 , and to the Global Deterioration Scale, score range 2 to 5) were recruited at 40 French clinical sites

Interventions

Participants in the CT condition $(n=170)$ were trained on a programme consisting of a set of standard tasks that covered different cognitive functions, as well as activities of daily life. The intervention was delivered to groups of 5 to 8 participants. Separate sessions were provided for the caregivers

Participants in the reminiscence therapy condition $(n=172)$ also received training in small groups, and each sessions focused on a different personal theme (e.g. schooldays, weddings, holidays) 
Amieva 2016 (Continued)

Participants in the individualised cognitive rehabilitation therapy condition $(n=157)$ received a tailored programme that focused on activities that were meaningful for both participant and caregiver

The 3 interventions lasted for 24 months, with 3 months of weekly 1.5-hours-long sessions, followed by maintenance sessions every 6 weeks for the next 21 months. Psychologists with at least 3 years' experience in the field of dementia delivered the interventions

Participants in the reference condition $(n=154)$ received usual medical care

\begin{tabular}{ll}
\hline Outcomes & $\begin{array}{l}\text { Primary outcome was rate of participants alive and without moderately severe to severe dementia at } 2 \\
\text { years, as measured with the MMSE } \\
\text { Secondary outcomes were institutionalisation, cognitive function, functional disability, behavioural } \\
\text { disturbance, apathy, quality of life, depression, caregiver burden, and resource utilisation } \\
\text { All assessments were conducted at } 3 \text { and } 24 \text { months after initiation of the interventions; they were per- } \\
\text { formed by physicians and psychologists blinded to allocation status }\end{array}$ \\
\hline Country & France \\
\hline Registration status\&\#160; & No information provided. Presumed to be unregistered \\
\hline Conflict of Interests\&\#160; & No \\
\hline Notes & Study authors sent a table with all scores on all assessment occasions
\end{tabular}

\section{Risk of bias}

Bias Authors' judgement Support for judgement

Random sequence genera- Low risk tion (selection bias)

"Participants were randomised through an independent and remote telephone randomisation service provided by the clinical trial unit"

"Balanced randomisation (1:1:1:1) was used"

\begin{tabular}{|c|c|c|}
\hline $\begin{array}{l}\text { Allocation concealment } \\
\text { (selection bias) }\end{array}$ & Low risk & $\begin{array}{l}\text { Study authors stated that they used a remote computerised randomisation } \\
\text { system. Allocation concealment is intrinsic to this method }\end{array}$ \\
\hline
\end{tabular}

\begin{tabular}{|c|c|c|}
\hline $\begin{array}{l}\text { Blinding of participants } \\
\text { and personnel (perfor- }\end{array}$ & High risk & $\begin{array}{l}\text { "Participants and clinical staff were aware of the trial arm to which the study } \\
\text { participants were allocated" }\end{array}$ \\
\hline
\end{tabular}

mance bias)

All outcomes

\begin{tabular}{lll}
\hline $\begin{array}{l}\text { Blinding of outcome as- } \\
\text { sessment (detection bias) } \\
\text { All outcomes }\end{array}$ & Low risk & Assessors were blinded to allocation status \\
\hline $\begin{array}{l}\text { Incomplete outcome data } \\
\text { (attrition bias) } \\
\text { All outcomes }\end{array}$ & Low risk & A flow chart shows attrition \\
\hline $\begin{array}{l}\text { Selective reporting (re- } \\
\text { porting bias) }\end{array}$ & Low risk & $\begin{array}{l}\text { All tests mentioned in the "Methods" section were reported in the "Results" } \\
\text { section }\end{array}$ \\
\hline Other bias & Low risk & We did not detect any other major sources of bias \\
\hline
\end{tabular}


Barban 2016

\begin{tabular}{ll}
\hline Methods & A multi-site RCT targeting cognitively healthy older adults, persons with $\mathrm{MCl}$, and persons with mild AD \\
to assess the efficacy of process-based CT combined with reminiscence therapy compared to a control
\end{tabular}

Participants

348 older adult (over 65 years of age) participants from Italy, Greece, Spain, and Norway were cognitively unimpaired, had MCI (MMSE 25 to 30), or had mild AD (MMSE 20 to 24). Participants had a minimum of 5 years of education

Interventions

Participants in the experimental condition $(n=42)$ were trained on a programme (SOCIABLE) that was provided on a touch-screen computer, and that delivered multi-component process-based CT combined with reminiscence training

Participants were trained twice a week, for 12 weeks, individually or in small groups; training was supervised by a trained cognitive therapist

Participants in the control condition received no treatment other than usual care

Outcomes
$\begin{aligned} & \text { Pecondary outcome was the effect of training on functional abilities, as reflected in instrumental activ- } \\ & \text { ities of daily living. Assessments were performed at baseline, at } 3 \text { months, and at } 6 \text { months (after the } \\ & \text { study had concluded) }\end{aligned}$

\begin{tabular}{ll}
\hline Country & Italy, Greece, Norway, and Spain \\
\hline Registration status\&\#160; & No information provided; presumed to be unregistered \\
\hline Conflict of Interests\&\#160; & No \\
\hline Notes & $\begin{array}{l}\text { Study authors sent the data for Arm A and Arm B at T0 and T1 (before the cross-over) for MMSE, RAVLT, } \\
\text { ROCF, PF, ADL, and Trail Making Test A. They reported that only a scant minority of individuals with mild } \\
\text { AD were able to execute the TMT B, so they did not send these scores }\end{array}$ \\
\hline
\end{tabular}

\section{Risk of bias}

\begin{tabular}{|c|c|c|}
\hline Bias & Authors' judgement & Support for judgement \\
\hline $\begin{array}{l}\text { Random sequence genera- } \\
\text { tion (selection bias) }\end{array}$ & Unclear risk & $\begin{array}{l}\text { Although the study was described as a randomised controlled trial (cross-over } \\
\text { design), no information on the method of randomisation was provided }\end{array}$ \\
\hline $\begin{array}{l}\text { Allocation concealment } \\
\text { (selection bias) }\end{array}$ & Unclear risk & $\begin{array}{l}\text { Study authors stated that the allocation procedure was concealed from the } \\
\text { raters but provided no details regarding the method of achieving this and how } \\
\text { effective it was }\end{array}$ \\
\hline $\begin{array}{l}\text { Blinding of participants } \\
\text { and personnel (perfor- } \\
\text { mance bias) } \\
\text { All outcomes }\end{array}$ & High risk & $\begin{array}{l}\text { Study authors did not mention blinding-related procedures of participants or } \\
\text { research personnel; this could not in fact be done in this type of cross-over trial } \\
\text { - 'no treatment' RCT. Study authors did not mention blinding of participants. } \\
\text { The study included a passive control condition (cross-over design), so blinding } \\
\text { was not possible }\end{array}$ \\
\hline $\begin{array}{l}\text { Incomplete outcome data } \\
\text { (attrition bias) } \\
\text { All outcomes }\end{array}$ & High risk & $\begin{array}{l}\text { Outcomes were reported only for those who completed post-intervention as- } \\
\text { sessments. The number of participants with dementia who were randomised } \\
\text { is unknown, and researchers did not report discontinuation rates within each } \\
\text { group. Overall dropout was greater in the control condition, and this could } \\
\text { have been the case in the dementia group, introducing risk of attrition bias }\end{array}$ \\
\hline
\end{tabular}


Barban 2016 (Continued)

\begin{tabular}{|c|c|c|}
\hline $\begin{array}{l}\text { Selective reporting (re- } \\
\text { porting bias) }\end{array}$ & Low risk & $\begin{array}{l}\text { The published manuscript presented complete outcome data only for out- } \\
\text { comes that were found to show an interaction between time and condition. } \\
\text { We could obtain remaining scores from study authors }\end{array}$ \\
\hline
\end{tabular}

Other bias Low risk We did not detect any other major sources of bias

Beck 1988

\begin{tabular}{ll}
\hline Methods & $\begin{array}{l}\text { An RCT targeting persons with AD or mixed dementia and comparing a cognitive skills remediation } \\
\text { training programme vs a control }\end{array}$ \\
\hline Participants & $\begin{array}{l}\text { Twenty participants (12 females, } 8 \text { males) who ranged in age between } 68 \text { and } 93 \text { (mean } 75) \text { years, and } \\
\text { who had clinical findings compatible with AD or probable mixed dementia (MMSE 15 to 20) were re- } \\
\text { cruited from the geriatric unit of a hospital and from } 4 \text { nursing homes in the USA. All participants com- } \\
\text { pleted at least grade school }\end{array}$
\end{tabular}

Interventions Participants in the experimental condition $(n=10)$ were trained on a cognitive skills remediation training programme 3 times a week for 6 weeks. Training was focused on paying attention and reading, concentrating on details, and remembering. The difficulty of tasks increased gradually

Participants in the control condition $(n=10)$ received no intervention but continued with all conventional treatments

Treatments were administered by research assistants

\begin{tabular}{ll}
\hline Outcomes & $\begin{array}{l}\text { Outcomes included paying attention and reading and remembering and concentrating on details. As- } \\
\text { sessments were conducted before and immediately after the training period }\end{array}$ \\
\hline Country & United States of America \\
\hline Registration status\&\#160; & No information provided; presumed to be unregistered \\
\hline Conflict of Interests\&\#160; & Not stated \\
\hline Notes
\end{tabular}

\section{Risk of bias}

\begin{tabular}{|c|c|c|}
\hline Bias & Authors' judgement & Support for judgement \\
\hline $\begin{array}{l}\text { Random sequence genera- } \\
\text { tion (selection bias) }\end{array}$ & Unclear risk & $\begin{array}{l}\text { Although the study was described as a randomised controlled trial, no infor- } \\
\text { mation on the method of randomisation was provided }\end{array}$ \\
\hline $\begin{array}{l}\text { Allocation concealment } \\
\text { (selection bias) }\end{array}$ & High risk & $\begin{array}{l}\text { Study authors did not mention allocation concealment. For this reason, we as- } \\
\text { sumed this was not done }\end{array}$ \\
\hline $\begin{array}{l}\text { Blinding of participants } \\
\text { and personnel (perfor- } \\
\text { mance bias) } \\
\text { All outcomes }\end{array}$ & High risk & $\begin{array}{l}\text { Study authors did not mention blinding of participants. The study included a } \\
\text { passive control condition, so blinding was not possible }\end{array}$ \\
\hline $\begin{array}{l}\text { Blinding of outcome as- } \\
\text { sessment (detection bias) } \\
\text { All outcomes }\end{array}$ & High risk & $\begin{array}{l}\text { Study authors did not mention blinding of outcome assessments. Probably } \\
\text { this was not done }\end{array}$ \\
\hline
\end{tabular}


Beck 1988 (Continued)

Incomplete outcome data Low risk Outcome data are available for the 20 participants in the study
(attrition bias)

All outcomes

\begin{tabular}{lll}
\hline $\begin{array}{l}\text { Selective reporting (re- } \\
\text { porting bias) }\end{array}$ & Low risk & $\begin{array}{l}\text { All tests mentioned in the "Methods" section were reported in the "Results" } \\
\text { section }\end{array}$ \\
\hline Other bias & Low risk & We did not detect any other major sources of bias \\
\hline
\end{tabular}

Bergamaschi 2013

\begin{tabular}{ll}
\hline Methods & $\begin{array}{l}\text { An RCT targeting persons with AD taking cholinesterase inhibitors (ChEIs) and comparing effects of CT } \\
\text { and ChEls with a non-specific cognitive treatment and ChEls alone (control group) on cognitive perfor- } \\
\text { mance }\end{array}$
\end{tabular}

\begin{tabular}{ll}
\hline Participants & 32 participants with mild to moderate AD (according to DSM-IV and NINCDS-ADRDA) with an MMSE \\
score range of 18 to $24 / 30$ were recruited from the Alzheimer's Evaluation Unit of Cremona, Italy. Mean \\
age across groups was 77.95 , and mean education level across groups was 6.43 years
\end{tabular}

Interventions

Participants in the experimental condition (combined treatment; $n=16$ ) received an intervention of combined CT+ChEls in five 1-month cycles ( 20 sessions per cycle, with a break of 4 weeks in between each cycle). Treatment aimed to stimulate spatial orientation, memory, attention, perception, visual analysis, and recognition of emotional expressions. Treatment was administered by an expert neuropsychologist

Participants in the control condition $(n=16)$ received non-specific cognitive treatment + ChEls at a daily centre

Participants in both conditions attended the sessions in groups of 4

\begin{tabular}{ll}
\hline Outcomes & $\begin{array}{l}\text { Outcomes included global cognition, as well as measures of memory, spatial reasoning, language, and } \\
\text { executive function. Non-cognitive outcomes included mood and activities of daily living. Assessments } \\
\text { were carried out at baseline and after the intervention had been completed (12 months) }\end{array}$ \\
\hline Country & Italy \\
\hline Registration status\&\#160; & No information provided; presumed to be unregistered \\
\hline Conflict of Interests\&\#160; & Not stated \\
\hline
\end{tabular}

Notes

\section{Risk of bias}

\begin{tabular}{lll}
\hline Bias & Authors' judgement & Support for judgement \\
\hline $\begin{array}{l}\text { Random sequence genera- } \\
\text { tion (selection bias) }\end{array}$ & Low risk & $\begin{array}{l}\text { Study authors stated: "patients were assigned to two groups using a computer } \\
\text { randomisation program" }\end{array}$ \\
\hline $\begin{array}{l}\text { Allocation concealment } \\
\text { (selection bias) }\end{array}$ & Unclear risk & $\begin{array}{l}\text { Study authors stated that they used a computerised randomisation system. It } \\
\text { is likely that allocation concealment was done, but this is not specified }\end{array}$ \\
\hline $\begin{array}{l}\text { Blinding of participants } \\
\text { and personnel (perfor- } \\
\text { mance bias) }\end{array}$ & Unclear risk & $\begin{array}{l}\text { Researchers referred to the study as a single-blind study, but it isn't clear } \\
\text { whether they meant that assessors or participants were blinded. Most likely, } \\
\text { they were referring to assessors only. Participants probably were not blinded, }\end{array}$
\end{tabular}




\begin{tabular}{lll}
\hline $\begin{array}{l}\text { Blinding of outcome as- } \\
\text { sessment (detection bias) } \\
\text { All outcomes }\end{array}$ & Low risk & The examiner was unaware of which group participants were in \\
\hline $\begin{array}{l}\text { Incomplete outcome data } \\
\text { (attrition bias) }\end{array}$ & Low risk \\
$\begin{array}{ll}\text { All outcomes } \\
\text { Selective reporting (re- }\end{array}$ & Low risk & All tests mentioned in the "Methods" section were reported in the "Results" \\
porting bias) & Low risk & We did not detect any other major sources of bias \\
\hline \begin{tabular}{l} 
Other bias \\
\hline
\end{tabular}
\end{tabular}

Boller 2011

$\begin{array}{ll}\text { Methods } & \text { An RCT targeting community-dwelling older adults with AD and comparing effects of the repetition lag } \\ \text { procedure, recognition practice, and a no-contact condition on memory recall }\end{array}$

\begin{tabular}{ll}
\hline Participants & $\begin{array}{l}36 \text { participants with a diagnosis of probable AD dementia according to NINCDS-ADRDA and DSM-IV cri- } \\
\text { teria. Dementia had to be considered mild to moderate with MMSE scores of } 19 \text { or above. Mean age of } \\
\text { participants was } 81.1 \text {, and mean education level was } 11.3\end{array}$ \\
\hline
\end{tabular}

Interventions Participants in the recollection training condition $(n=12)$ engaged in an activity adapted from the repetition-lag procedure developed by Jennings and Jacoby (2003), in which they had to remember a series of nouns and then recognise them from longer lists of words

Participants in the recognition practice condition $(n=12)$ received training on a cognitive training procedure, which shared some characteristics with the one designed for the other experimental condition

Participants in both training conditions received 4 training sessions per day 3 days a week for 2 weeks

Remaining participants $(n=12)$ were randomised to a no-contact control condition

\begin{tabular}{|c|c|c|}
\hline Outcomes & \multicolumn{2}{|c|}{ Outcomes included working memory, immediate and delayed memory, and recognition } \\
\hline Country & \multicolumn{2}{|l|}{ France } \\
\hline Registration status\&\#160; & \multicolumn{2}{|c|}{ No information provided; presumed to be unregistered } \\
\hline Conflict of Interests\&\#160; & \multicolumn{2}{|l|}{ Not stated } \\
\hline Notes & \multicolumn{2}{|c|}{ Study authors provided clarification on one of the scores, as it was not clear due to a typing error } \\
\hline \multicolumn{3}{|l|}{ Risk of bias } \\
\hline Bias & Authors' judgement & Support for judgement \\
\hline $\begin{array}{l}\text { Random sequence genera- } \\
\text { tion (selection bias) }\end{array}$ & Unclear risk & $\begin{array}{l}\text { Although the study was described as a randomised controlled trial, no infor- } \\
\text { mation on the method of randomisation was provided }\end{array}$ \\
\hline $\begin{array}{l}\text { Allocation concealment } \\
\text { (selection bias) }\end{array}$ & High risk & $\begin{array}{l}\text { Study authors did not mention allocation concealment. For this reason, we as- } \\
\text { sumed this was not done }\end{array}$ \\
\hline
\end{tabular}


Boller 2011 (Continued)

Blinding of participants Unclear risk Study authors did not mention blinding of participants. The study included a and personnel (perforpassive control condition, so blinding was not possible mance bias)

All outcomes

\begin{tabular}{|c|c|c|}
\hline $\begin{array}{l}\text { Blinding of outcome as- } \\
\text { sessment (detection bias) } \\
\text { All outcomes }\end{array}$ & High risk & $\begin{array}{l}\text { This is usually stated. As study authors did not mention it, it is unlikely that } \\
\text { this has been done }\end{array}$ \\
\hline
\end{tabular}

All outcomes

Incomplete outcome data Low risk

(attrition bias)

Data are available for 36 participants in the study

All outcomes

\begin{tabular}{lll}
\hline $\begin{array}{l}\text { Selective reporting (re- } \\
\text { porting bias) }\end{array}$ & Low risk & $\begin{array}{l}\text { All tests mentioned in the "Methods" section were reported in the "Results" } \\
\text { section }\end{array}$ \\
\hline Other bias & Low risk & We did not detect any other major sources of bias \\
\hline
\end{tabular}

\section{Brueggen 2017}

\begin{tabular}{ll}
\hline Methods & $\begin{array}{l}\text { A controlled partial-randomised design trial comparing CT with a group cognitive rehabilitation inter- } \\
\text { vention (active control group) in persons with AD dementia }\end{array}$
\end{tabular}

\begin{tabular}{ll}
\hline Participants & 20 community-dwelling participants with probable or possible AD according to NINCDS-ADRDA criteria \\
who were living in Germany. Age range of participants was 53 to 83 , and all had received over 10 years \\
of education
\end{tabular}

Interventions Participants in the intervention condition $(n=8)$ received standardised $\mathrm{CT}$ in the form of a single daily task that participants had to complete by themselves in the form of homework. Participants met with researchers every 4 weeks to evaluate the homework

Participants in the control condition $(n=10)$ were trained on a cognitive rehabilitation programme that was based on a manual-guided approach combining neuropsychological and psychotherapeutic elements (CORDIAL). A psychologist and an occupational therapist delivered the intervention

Both interventions lasted 3 months

Study authors stated that the change from baseline in capacity to perform activities of daily living was
their primary outcome
Secondary outcomes included cognitive abilities related to daily living (such as everyday memory abili-
ties and planning and organizational skills), functional cognitive state, and non-cognitive outcomes in-
cluding depression, consciousness, neurobehavioural disturbance, and caregiver burden
Assessments were conducted at baseline and after the interventions were finished

\begin{tabular}{ll}
\hline Country & Germany \\
\hline Registration status\&\#160; & Prospectively registered \\
\hline Conflict of Interests\&\#160; & Not stated \\
\hline
\end{tabular}

Notes Study authors designed the CT intervention group as the comparison group. For the purpose of this review, the CT group was selected as the experimental group

Two participants cancelled their participation before the intervention was started (CT group)

Study authors advised that the trial had been prospectively registered and provided a table with follow-up scores upon request 


\section{Risk of bias}

\begin{tabular}{lll}
\hline Bias & Authors' judgement & Support for judgement \\
\hline $\begin{array}{l}\text { Random sequence genera- } \\
\text { tion (selection bias) }\end{array}$ & High risk & $\begin{array}{l}\text { "Five subjects originated from a pilot trial waiting group and were already pre- } \\
\text { determined for the intervention group" }\end{array}$ \\
& $\begin{array}{l}\text { "We conducted a partial-randomization to assign the remaining subjects using } \\
\text { a computer-based balanced randomisation" }\end{array}$
\end{tabular}

\begin{tabular}{lll}
\hline $\begin{array}{l}\text { Allocation concealment } \\
\text { (selection bias) }\end{array}$ & High risk & $\begin{array}{l}\text { Some participants already knew they were going to participate in the interven- } \\
\text { tion group }\end{array}$ \\
\hline $\begin{array}{l}\text { Blinding of participants } \\
\text { and personnel (perfor- } \\
\text { mance bias) }\end{array}$ & Unclear risk & $\begin{array}{l}\text { Study authors did not mention blinding of participants. CT was compared to } \\
\text { an alternative treatment, so blinding may have been possible }\end{array}$ \\
All outcomes &
\end{tabular}

\begin{tabular}{lll}
\hline $\begin{array}{l}\text { Blinding of outcome as- } \\
\text { sessment (detection bias) } \\
\text { All outcomes }\end{array}$ & High risk & $\begin{array}{l}\text { Study authors provided no details to suggest that outcome assessment was } \\
\text { blind. It is likely that this was not the case }\end{array}$ \\
\hline $\begin{array}{l}\text { Incomplete outcome data } \\
\text { (attrition bias) }\end{array}$ & Unclear risk & $\begin{array}{l}\text { Two participants allocated to the intervention dropped out before commenc- } \\
\text { ing treatment, and } 2 \text { participants allocated to the control condition dropped } \\
\text { out during the intervention period; reasons seem to be unrelated. However, } \\
\text { analyses were carried out without their baseline data; the impact of this is un- } \\
\text { known, given the small sample size }\end{array}$ \\
\hline
\end{tabular}

Selective reporting (re- Unclear risk We were able to obtain all scores with the exception of the NPI upon request
porting bias)

Other bias Low risk We did not detect any other major sources of bias

Cahn-Weiner 2003

Methods An RCT targeting people with probable AD that aimed to assess efficacy for word-list recall and recogni-
tion relative to a control condition
Participants 39 participants who were referred from a university-based AD and memory disorder clinic in the USA who had a diagnosis of probable AD (according to NINCDS-ADRDA). Mean age across groups was 76.9. All participants were taking donepezil

Interventions Intervention condition: memory training programme of 6 weeks' duration to improve word list recall
and recognition

Active control: participants in the control condition received didactic presentations but no formal memory training

A clinical neuropsychologist delivered the interventions

Outcomes Cognitive performance in several domains, including verbal learning, verbal and visual memory, and
reasoning, were assessed, as were everyday memory functioning and activities of daily living
Assessments were conducted at baseline, post intervention, and 8 weeks after completion of the inter-
vention 
Cahn-Weiner 2003 (Continued)

\begin{tabular}{ll} 
Country & United States of America \\
\hline Registration status\&\#160; & No information provided; presumed to be unregistered \\
\hline Conflict of Interests\&\#160; & Not stated \\
\hline Notes & $\begin{array}{l}39 \text { persons enrolled in the study. } 5 \text { of them withdrew. Only } 1 \text { attended a session. The rest withdrew af- } \\
\text { ter baseline assessment }\end{array}$ \\
\hline
\end{tabular}

\section{Risk of bias}

\begin{tabular}{|c|c|c|}
\hline Bias & Authors' judgement & Support for judgement \\
\hline $\begin{array}{l}\text { Random sequence genera- } \\
\text { tion (selection bias) }\end{array}$ & Low risk & $\begin{array}{l}\text { "All participants were randomly assigned to one of two groups by a coin toss } \\
\text { performed at the time consent was obtained" }\end{array}$ \\
\hline $\begin{array}{l}\text { Allocation concealment } \\
\text { (selection bias) }\end{array}$ & Unclear risk & $\begin{array}{l}\text { Study authors stated that they used a coin for randomisation of participants. It } \\
\text { is not clear whether allocation was concealed }\end{array}$ \\
\hline $\begin{array}{l}\text { Blinding of participants } \\
\text { and personnel (perfor- } \\
\text { mance bias) } \\
\text { All outcomes }\end{array}$ & Low risk & $\begin{array}{l}\text { "Neither the patients nor their caregivers were informed as to which group } \\
\text { (training vs. control) they had been assigned)" }\end{array}$ \\
\hline $\begin{array}{l}\text { Blinding of outcome as- } \\
\text { sessment (detection bias) } \\
\text { All outcomes }\end{array}$ & Low risk & $\begin{array}{l}\text { Neuropsychological assessment was carried out by a trained psychometrist } \\
\text { who was blinded to group assignment ( } 1 \text { exception) }\end{array}$ \\
\hline $\begin{array}{l}\text { Incomplete outcome data } \\
\text { (attrition bias) } \\
\text { All outcomes }\end{array}$ & Low risk & $\begin{array}{l}\text { Missing data were balanced in numbers across groups; reasons for missing da- } \\
\text { ta were similar across groups }\end{array}$ \\
\hline $\begin{array}{l}\text { Selective reporting (re- } \\
\text { porting bias) }\end{array}$ & Low risk & $\begin{array}{l}\text { All tests mentioned in the "Methods" section were reported in the "Results" } \\
\text { section }\end{array}$ \\
\hline Other bias & Low risk & The study appears to be free of other sources of bias \\
\hline
\end{tabular}

Cavallo 2016

Methods An RCT targeting persons with early-stage AD conducted to assess effects at a neuropsychological level of computerised CT compared to a control intervention

Participants
NINCDS-ADRDA) were recruited in an Assisted Health Residence in Italy. Mean age of participants was
76.41 , and mean years of formal education was 8.32

Interventions Participants in the experimental condition $(n=40)$ received individual computerised CT, delivered by the rehabilitative software Brainer1 (https://www.brainer.it/), which includes over 100 exercises targeting different cognitive domains

Control intervention was delivered 1 -on-1 by a neuropsychologist. Participants in this condition $(n=40)$ could choose between reading newspaper articles online and discussing them with the neuropsychologist, playing online games and solving puzzles, or visiting websites suiting their interests 
Cavallo 2016 (Continued)

Both interventions conditions were delivered by a neuropsychologist over a 12-month period, with 30-

minute sessions held 3 times per week

Outcomes

Outcomes included cognitive performance in the domains of memory, semantic knowledge, language, visuospatial abilities, and executive functions, as well as anxiety and depression

All participants were evaluated before and after training, and at 6-month follow-up assessment

\begin{tabular}{ll}
\hline Country & Italy \\
\hline Registration status\&\#160; & No information provided; presumed to be unregistered \\
\hline Conflict of Interests\&\#160; & Not stated \\
\hline Notes & Study authors sent us the scores for the post-intervention assessment \\
\hline
\end{tabular}

Risk of bias

\begin{tabular}{|c|c|c|}
\hline Bias & Authors' judgement & Support for judgement \\
\hline $\begin{array}{l}\text { Random sequence genera- } \\
\text { tion (selection bias) }\end{array}$ & Low risk & $\begin{array}{l}\text { Study authors stated that participants were "randomised into two different } \\
\text { groups by means of a random number generator with mixed block sizes" }\end{array}$ \\
\hline $\begin{array}{l}\text { Allocation concealment } \\
\text { (selection bias) }\end{array}$ & Unclear risk & $\begin{array}{l}\text { Study authors stated that they used a computerised randomisation system. It } \\
\text { is likely that allocation concealment was done, but this is not specified }\end{array}$ \\
\hline $\begin{array}{l}\text { Blinding of participants } \\
\text { and personnel (perfor- } \\
\text { mance bias) } \\
\text { All outcomes }\end{array}$ & Unclear risk & $\begin{array}{l}\text { Study authors did not mention blinding of participants. They compared CT vs } \\
\text { an active condition, so blinding may have been possible }\end{array}$ \\
\hline $\begin{array}{l}\text { Blinding of outcome as- } \\
\text { sessment (detection bias) } \\
\text { All outcomes }\end{array}$ & Low risk & $\begin{array}{l}\text { Assessors were blind to the purpose of the study and to the group to which } \\
\text { each participant belonged }\end{array}$ \\
\hline $\begin{array}{l}\text { Incomplete outcome data } \\
\text { (attrition bias) } \\
\text { All outcomes }\end{array}$ & Low risk & $\begin{array}{l}\text { Missing outcome data were balanced in numbers across intervention groups, } \\
\text { and reasons for missing data were similar }\end{array}$ \\
\hline $\begin{array}{l}\text { Selective reporting (re- } \\
\text { porting bias) }\end{array}$ & Low risk & $\begin{array}{l}\text { Findings regarding the various outcomes were not presented in a consistent } \\
\text { way, but we were able to obtain the relevant data from study authors }\end{array}$ \\
\hline Other bias & Low risk & The study appears to be free of other sources of bias \\
\hline
\end{tabular}

Davis 2001

\begin{tabular}{ll}
\hline Methods & $\begin{array}{l}\text { An RCT with a cross-over design comparing a cognitive intervention vs a placebo condition in people } \\
\text { with AD }\end{array}$ \\
\hline Participants & $\begin{array}{l}37 \text { participants ( } 16 \text { men, } 21 \text { women) with probable AD (according to NINCDS-ADRDA). Mean MMSE Score } \\
\text { of the sample was } 22.31 \text {, and mean age was } 70.62 . \text { Average years of education for participants was } \\
14.01\end{array}$ \\
\hline Interventions & $\begin{array}{l}\text { Participants in the intervention condition ( } \mathrm{n}=19) \text { were engaged in individual 1-hour sessions of face- } \\
\text { name association and recall using spaced retrieval once weekly, in addition to } 30 \text {-minute home prac- } \\
\text { tice with the caregiver } 6 \text { days a week over } 5 \text { weeks }\end{array}$
\end{tabular}


Davis 2001 (Continued)

Participants in the "placebo condition" ( $n=18)$ were engaged in five 1-hour weekly sessions of unstructured conversation and questioning by an examiner; they also watched videotapes related to health issues

Outcomes Outcomes included cognitive performance on measures of global cognition, delayed memory recall, working memory and attention, language, and psychomotor abilities. Depression and quality of life were also assessed

All evaluations were conducted at baseline and after 5 weeks of treatment. A third assessment was carried out on participants originally in the placebo condition but who commenced the intervention after the cross-over

\begin{tabular}{ll}
\hline Country & United States of America \\
\hline Registration status\&\#160; & No information provided; presumed to be unregistered \\
\hline Conflict of Interests\&\#160; & Not stated
\end{tabular}

\section{Notes}

\section{Risk of bias}

\begin{tabular}{lll}
\hline Bias & Authors' judgement & Support for judgement \\
\hline $\begin{array}{l}\text { Random sequence genera- } \\
\text { tion (selection bias) }\end{array}$ & Unclear risk & $\begin{array}{l}\text { Although the study was described as a randomised controlled trial, no infor- } \\
\text { mation on the method of randomisation was provided }\end{array}$ \\
\hline $\begin{array}{l}\text { Allocation concealment } \\
\text { (selection bias) }\end{array}$ & High risk & $\begin{array}{l}\text { Study authors did not mention allocation concealment. For this reason, we as- } \\
\text { sumed that this was not done }\end{array}$ \\
\hline $\begin{array}{l}\text { Blinding of participants } \\
\text { and personnel (perfor- } \\
\text { mance bias) }\end{array}$ & Low risk & $\begin{array}{l}\text { "Patients and their caregivers were not informed of which condition (placebo } \\
\text { All outcomes }\end{array}$ \\
\hline
\end{tabular}

\begin{tabular}{|c|c|c|}
\hline $\begin{array}{l}\text { Blinding of outcome as- } \\
\text { sessment (detection bias) } \\
\text { All outcomes }\end{array}$ & Low risk & $\begin{array}{l}\text { Assessments were conducted by examiners who were blinded to the treatment } \\
\text { condition }\end{array}$ \\
\hline
\end{tabular}

\begin{tabular}{|c|c|c|}
\hline $\begin{array}{l}\text { Incomplete outcome data } \\
\text { (attrition bias) } \\
\text { All outcomes }\end{array}$ & Low risk & $\begin{array}{l}\text { No information was provided about attrition. However, it appears that all ran- } \\
\text { domised participants were included in the post-treatment assessment }\end{array}$ \\
\hline $\begin{array}{l}\text { Selective reporting (re- } \\
\text { porting bias) }\end{array}$ & Low risk & $\begin{array}{l}\text { All tests mentioned in the "Methods" section were reported in the "Results" } \\
\text { section }\end{array}$ \\
\hline Other bias & Low risk & The study appears to be free of other sources of bias \\
\hline
\end{tabular}

\begin{tabular}{ll} 
de Vreese 1998 & \\
\hline Methods & $\begin{array}{l}\text { An RCT comparing "cognitive retraining"+AChE-I condition vs AChE-I alone and a placebo drug condi- } \\
\text { tion in persons with mild-to-moderate AD. Initially, the trial also included a cognitive retraining only } \\
\text { condition (without a drug), but this condition was removed after the study commenced, and data from } \\
\text { this group }(n=6) \text { were not reported }\end{array}$ \\
\hline
\end{tabular}


de Vreese 1998 (Continued)

Participants

27 participants who had a clinical diagnosis of AD (NINCDS-ADRDA, DSM-IV). All were between 61 and 83 years old, with a mean age of 72.6

Interventions

Participants in the "cognitive retraining" (CR) condition received two 45-minute sessions of CR per week over a 3-month period, with focus on memory, language, and executive abilities

Those in the AChE-I condition received treatment with acetylcholinesterase inhibitors

Participants in the $C R+A C h E-I$ condition received 3 months of $A C h E-I$, then began to receive $C R$ in addition to AChE-I

Participants in the placebo condition received a placebo medication

\begin{tabular}{ll}
\hline Outcomes & $\begin{array}{l}\text { Outcomes included global cognitive functioning as measured by MMSE and ADAS-Cog. Non-cognitive } \\
\text { outcomes included instrumental activities of daily living and behavioural symptoms }\end{array}$ \\
\hline Country & Italy \\
\hline Registration status\&\#160; & No information provided; presumed to be unregistered \\
\hline Conflict of Interests\&\#160; & Not stated \\
\hline Notes & $\begin{array}{l}\text { Study authors provided a table with scores and stated that the condition did not differ in demographic } \\
\text { and baseline characteristics. However, group level data were not available }\end{array}$
\end{tabular}

\section{Risk of bias}

\begin{tabular}{lll}
\hline Bias & Authors' judgement & Support for judgement \\
\hline $\begin{array}{l}\text { Random sequence genera- } \\
\text { tion (selection bias) }\end{array}$ & Unclear risk & $\begin{array}{l}\text { Although the study was described as a randomised controlled trial, no infor- } \\
\text { mation on the method of randomisation was provided }\end{array}$ \\
\hline $\begin{array}{l}\text { Allocation concealment } \\
\text { (selection bias) }\end{array}$ & High risk & $\begin{array}{l}\text { Study authors did not mention allocation concealment. For this reason, we as- } \\
\text { sumed this was not done }\end{array}$ \\
\hline $\begin{array}{l}\text { Blinding of participants } \\
\text { and personnel (perfor- } \\
\text { mance bias) }\end{array}$ & High risk & $\begin{array}{l}\text { Study authors did not mention blinding of participants. The study included a } \\
\text { passive control condition, so blinding was not possible }\end{array}$ \\
\hline $\begin{array}{l}\text { Bll outcomes } \\
\text { sessment (detection bias) } \\
\text { All outcomes }\end{array}$ & Low risk & $\begin{array}{l}\text { The study was described as a 'single-blind' study. It appears assessments were } \\
\text { conducted by blinded personnel }\end{array}$ \\
\hline $\begin{array}{l}\text { Incomplete outcome data } \\
\text { (attrition bias) } \\
\text { All outcomes }\end{array}$ & High risk & $\begin{array}{l}\text { It appears that changes to the study protocol were made after the trial com- } \\
\text { menced, and that the CT-only arm of the study has been discontinued. Data } \\
\text { from participants who took part in this arm (n = 6) were not reported }\end{array}$ \\
\hline $\begin{array}{l}\text { Selective reporting (re- } \\
\text { porting bias) }\end{array}$ & High risk & $\begin{array}{l}\text { The trial was not registered and no protocol was available. It appears the study } \\
\text { included cognitive measures for ADLs and behavioural measures. However, } \\
\text { the data table sent by study authors included means and SDs for cognitive } \\
\text { measures (ADAS, MMSE) and the IADL scale only }\end{array}$ \\
\hline
\end{tabular}

Other bias Low risk The study appears to be free of other sources of bias


Fernández-Calvo 2011

\begin{tabular}{ll}
\hline Methods & $\begin{array}{l}\text { An RCT comparing in persons with AD the effects of a computerised CT programme, Big Brain Acade- } \\
\text { my (BBA), vs the Integrated Psychostimulation Program (IPP), a classical CT tool, and a wait-list control } \\
\text { condition on cognitive and functional measures and on psychological and behavioural symptoms }\end{array}$ \\
\hline Participants & $\begin{array}{l}45 \text { participants with a diagnosis of probable AD (according to NINCDS-ADRDA) who were receiving an- } \\
\text { ti-dementia drug therapy. Mean age of the sample was 75.75, and educational attainment was on aver- } \\
\text { age } 7.71 \text { years }\end{array}$
\end{tabular}

Interventions

Participants in the BBA condition $(n=15)$ were trained on a programme that involved various games at different levels of difficulty. Exercises were classified under 5 areas of stimulation: perception, memory, calculation, analysis, and acuity. Participants received continuous encouragement from researchers

Participants in the IPP condition $(n=15)$ received an intervention programme that targeted several cognitive areas: reasoning, attention, and concentration; verbal and written language; praxis; gnosis; arithmetic and calculation, and association-order

Participants in both conditions received treatment for 12 weeks, with three 60-minute sessions per week (total = 36 individual sessions); each session was delivered by an occupational therapist and a psychologist

Participants in the control condition $(n=15)$ did not receive treatment until the trial had been completed

Outcomes included global cognitive ability as reflected in MMSE scores, neuropsychiatric symptoms,
and clinical disease severity
Participants were assessed before and after the intervention

\begin{tabular}{ll}
\hline Country & Spain \\
\hline Registration status\&\#160; & No information provided; presumed to be unregistered \\
\hline Conflict of Interests\&\#160; & Not stated \\
\hline Notes & $\begin{array}{l}\text { Study authors provided the number of participants in each condition and scores on the MMSE upon re- } \\
\text { quest (although these were not analysed in the results) }\end{array}$
\end{tabular}

\section{Risk of bias}

\begin{tabular}{|c|c|c|}
\hline Bias & Authors' judgement & Support for judgement \\
\hline $\begin{array}{l}\text { Random sequence genera- } \\
\text { tion (selection bias) }\end{array}$ & Unclear risk & $\begin{array}{l}\text { Although the study was described as a randomised controlled trial, no infor- } \\
\text { mation on the method of randomisation was provided }\end{array}$ \\
\hline $\begin{array}{l}\text { Allocation concealment } \\
\text { (selection bias) }\end{array}$ & High risk & $\begin{array}{l}\text { Study authors did not mention allocation concealment. For this reason, we as- } \\
\text { sumed this was not done }\end{array}$ \\
\hline $\begin{array}{l}\text { Blinding of participants } \\
\text { and personnel (perfor- } \\
\text { mance bias) } \\
\text { All outcomes }\end{array}$ & High risk & $\begin{array}{l}\text { Study authors did not mention blinding of participants. The study included a } \\
\text { passive control condition, so blinding was not possible }\end{array}$ \\
\hline $\begin{array}{l}\text { Blinding of outcome as- } \\
\text { sessment (detection bias) } \\
\text { All outcomes }\end{array}$ & Low risk & $\begin{array}{l}\text { Study authors stated that all assessments were conducted by blinded asses- } \\
\text { sors }\end{array}$ \\
\hline $\begin{array}{l}\text { Incomplete outcome data } \\
\text { (attrition bias) } \\
\text { All outcomes }\end{array}$ & Unclear risk & $\begin{array}{l}\text { The published manuscript provided no information on the number of partic- } \\
\text { ipants allocated to each condition and no dropout rates. Study authors sup- } \\
\text { plied information on request suggesting that each condition included } 15 \text { par- }\end{array}$ \\
\hline
\end{tabular}


Fernández-Calvo 2011 (Continued)

ticipants, but it is not clear whether this represents the number of participants recruited or the number retained. It is relatively uncommon for studies in this area to have no attrition at all

\begin{tabular}{|c|c|c|}
\hline $\begin{array}{l}\text { Selective reporting (re- } \\
\text { porting bias) }\end{array}$ & Low risk & $\begin{array}{l}\text { The published manuscript did not report outcome data for MMSE, even though } \\
\text { it was administered on both occasions. However, study authors provided } \\
\text { these data upon request }\end{array}$ \\
\hline
\end{tabular}

Other bias Low risk The study appears to be free of other sources of bias

Galante 2007

\begin{tabular}{ll}
\hline Methods & $\begin{array}{l}\text { A single-blind RCT comparing a computer-based cognitive intervention to an active control in persons } \\
\text { with AD }\end{array}$ \\
\hline Participants & $\begin{array}{l}\text { Participants }(n=12) \text { had a diagnosis of probable AD (according to NINCDS-ADRDA criteria). Mean age of } \\
\text { participants was } 76.0 \text { years }(6.0) \text {, and mean education level was } 6.3(2.2) \text { years }\end{array}$ \\
\hline
\end{tabular}

Interventions Participants in the experimental condition $(n=7)$ were trained on a computerised programme comprising tasks selected from Software TNP (Training Neuropsicologico, by Tonetta 1998)

Participants in the control condition $(n=5)$ engaged in a non-specific treatment that included a semi-structured interview on current affairs and events relevant to participants' lives

Both interventions lasted for 4 weeks with 3 individual 1-hour sessions per week and were delivered by a neuropsychologist

Outcomes included global cognition and tasks measuring specific cognitive domains including atten-
tion and working memory, learning and memory, language and executive function, and visuospatial
abilities. In addition, behavioural and neuropsychiatric outcomes and capacity for activities of daily liv-
ing were assessed. Evaluations were performed at baseline, immediately after 12-week treatment, and
3 months later. Global cognition was also assessed at 9-month follow-up

\begin{tabular}{ll} 
Country & Italy \\
\hline Registration status\&\#160; & No information provided; presumed to be unregistered \\
\hline Conflict of Interests\&\#160; & Not stated \\
\hline Notes & $\begin{array}{l}\text { No information was provided on the extent to which groups were matched on relevant variables before } \\
\text { the intervention } \\
\text { Mean age and years of education for } 11 \text { participants were analysed, and } 1 \text { participant was excluded for } \\
\text { poor compliance }\end{array}$ \\
\hline
\end{tabular}

\section{Risk of bias}

\begin{tabular}{lll}
\hline Bias & Authors' judgement & Support for judgement \\
\hline $\begin{array}{l}\text { Random sequence genera- } \\
\text { tion (selection bias) }\end{array}$ & High risk & Participants were assigned to groups in order of recruiting \\
\hline $\begin{array}{l}\text { Allocation concealment } \\
\text { (selection bias) }\end{array}$ & High risk & $\begin{array}{l}\text { Participants were assigned to groups in order of recruiting. For this reason, al- } \\
\text { location concealment was not possible in this case }\end{array}$ \\
\hline
\end{tabular}


Galante 2007 (Continued)

Blinding of participants High risk Study authors described the study as a single-blind RCT (the person in charge and personnel (perforof conducting the assessments was blinded)

mance bias)

All outcomes

\begin{tabular}{|c|c|c|}
\hline $\begin{array}{l}\text { Blinding of outcome as- } \\
\text { sessment (detection bias) }\end{array}$ & Low risk & $\begin{array}{l}\text { Each participant was evaluated by a neuropsychologist who was blinded to } \\
\text { the participants' group allocation }\end{array}$ \\
\hline
\end{tabular}

Incomplete outcome data Low risk (attrition bias)

All outcomes

Selective reporting (re- Low risk
porting bias)
Low risk

Other bias Low risk The study appears to be free of other sources of bias

The sample was small, and 1 person was excluded due to poor compliance. No missing data were recorded, implying that the remaining 11 persons completed the study

Giovagnoli 2017

\begin{tabular}{ll}
\hline Methods & An RCT (single-blind) comparing CT with active music therapy and neuroeducation in people with mild \\
to moderate dementia
\end{tabular}

Participants 39 participants ( 24 female) with diagnosis of probable AD were included in the study. Mean age was 73.64 (SD 7.11) years, and mean years of education was 8.23 (SD 4.29)

Participants were recruited from "one centre"; however, no details were provided about the nature of this centre nor about the living situation of participants processing, executive function, and memory. A neuropsychologist delivered the intervention

Active music therapy $(n=13)$ was provided by a music therapist; participants selected and played an instrument of their choice

Neuroeducation ( $n=13$ ) was co-ordinated by a neurologist; it comprised 3 sessions focused on brain anatomy and function, symptoms of cognitive decline, coping with dementia, nutrition and eating, physical exercise, relaxation, coping, and leisure

\begin{tabular}{ll}
\hline Outcomes & $\begin{array}{l}\text { Primary outcome was initiation, as assessed by the Word Fluency test on phonemic cue score. Se- } \\
\text { condary outcome was episodic memory, as assessed by the Short Story test. Numerous other cognitive } \\
\text { measures were included, covering several cognitive domains including memory, attention, language, } \\
\text { and executive function. Additional outcomes included mood (depression and anxiety) and social net- } \\
\text { works }\end{array}$ \\
\hline Country & Italy \\
\hline Registration status\&\#160; & No information provided; presumed to be unregistered \\
\hline Conflict of Interests\&\#160; & No \\
\hline
\end{tabular}

\section{Notes}


Giovagnoli 2017 (Continued)

Risk of bias

\begin{tabular}{|c|c|c|}
\hline Bias & Authors' judgement & Support for judgement \\
\hline $\begin{array}{l}\text { Random sequence genera- } \\
\text { tion (selection bias) }\end{array}$ & Low risk & "A computer-generated list of random numbers was used" \\
\hline $\begin{array}{l}\text { Allocation concealment } \\
\text { (selection bias) }\end{array}$ & Unclear risk & $\begin{array}{l}\text { Study authors state they used a computerised randomisation system. It is like- } \\
\text { ly that allocation concealment was done, but this is not specified }\end{array}$ \\
\hline $\begin{array}{l}\text { Blinding of participants } \\
\text { and personnel (perfor- } \\
\text { mance bias) } \\
\text { All outcomes }\end{array}$ & Unclear risk & $\begin{array}{l}\text { Study authors describe the study as a single-blind RCT (the person in charge of } \\
\text { conducting the assessments was blinded). Participants were not blinded, how- } \\
\text { ever, as all conditions were forms of intervention (including the education con- } \\
\text { dition) and therefore may have had treatment-related expectations; whether } \\
\text { lack of blinding increased performance bias remains unclear }\end{array}$ \\
\hline $\begin{array}{l}\text { Blinding of outcome as- } \\
\text { sessment (detection bias) } \\
\text { All outcomes }\end{array}$ & Low risk & Study authors state that all assessments were conducted by blinded assessors \\
\hline $\begin{array}{l}\text { Incomplete outcome data } \\
\text { (attrition bias) } \\
\text { All outcomes }\end{array}$ & Unclear risk & $\begin{array}{l}\text { Eleven participants dropped out after randomisation and, according to study } \\
\text { authors, at an early stage of treatment. Numbers were more or less equally dis- } \\
\text { tributed across conditions. Analysis was per-protocol rather than by intent-to- } \\
\text { treat, but the reasons for this are not explained. Whether dropout had an effect } \\
\text { on outcomes remains unclear }\end{array}$ \\
\hline $\begin{array}{l}\text { Selective reporting (re- } \\
\text { porting bias) }\end{array}$ & Unclear risk & $\begin{array}{l}\text { The token test was reported in the "Results" section but not in the "Methods" } \\
\text { section. Whether all relevant measures given were reported is unknown, and } \\
\text { whether risk of bias due to selective reporting was elevated remains unclear }\end{array}$ \\
\hline Other bias & Low risk & Study appears to be free of other sources of bias \\
\hline
\end{tabular}

Giuli 2016

Methods An RCT comparing a comprehensive CT intervention vs a control condition in cognitively unimpaired older adults, people with mild cognitive impairment, and people with mild to moderate AD

Participants $\begin{aligned} & 321 \text { community-dwelling participants living in the Marche Region (Italy) who were } 65 \text { and over with } \\ & \text { a diagnosis of mild to moderate AD (according to DSM-IV or NINCDS-ADRDA). Most participants were } \\ & \text { women }\end{aligned}$
women

Interventions

Participants in the experimental condition received treatment with comprehensive $\mathrm{CT}$, which was adapted depending on the diagnostic group of the participant. The AD group $(n=51)$ was trained on a programme that targeted several cognitive functions including attention, orientation, planning, and episodic and prospective memory. Participants were asked to complete homework exercises every day with the support of a carer

AD participants assigned to the control condition $(n=50)$ received psychoeducation (including suggestions and simple strategies to improve their memory and health)

All participants with AD attended 10 individual sessions once a week at the INRCA Hospital in Fermo (Italy)

\section{Outcomes}

Outcomes included global cognition and performance on tasks measuring several cognitive domains, including attention, learning and memory, and language. In addition, mood, stress, capacity for activities of daily living, and clinical disease severity outcomes were assessed 
Giuli 2016 (Continued)

\begin{tabular}{ll} 
Country & Italy \\
\hline Registration status\&\#160; & No information provided; presumed to be unregistered \\
\hline Conflict of Interests\&\#160; & No \\
\hline Notes & $\begin{array}{l}\text { We tried to contact study authors to ask about assessment occasions (in weeks and months) and to re- } \\
\text { quest test scores that were missing (Phonemic Word Fluency test and CDR), but we were not able to } \\
\text { reach them }\end{array}$
\end{tabular}

\section{Risk of bias}

\begin{tabular}{lll} 
Bias & Authors' judgement & Support for judgement \\
\hline $\begin{array}{l}\text { Random sequence genera- } \\
\text { tion (selection bias) }\end{array}$ & Low risk & "The subjects were randomly assigned 1:1 to..." \\
& $\begin{array}{l}\text { "Randomization was performed separately for each group, by using a comput- } \\
\text { erized random number generator" }\end{array}$
\end{tabular}

\begin{tabular}{lll}
\hline $\begin{array}{l}\text { Allocation concealment } \\
\text { (selection bias) }\end{array}$ & Unclear risk & $\begin{array}{l}\text { Study authors stated that they used a computerised randomisation system. It } \\
\text { is likely that allocation concealment was done, but this is not specified }\end{array}$ \\
\hline $\begin{array}{l}\text { Blinding of participants } \\
\text { and personnel (perfor- } \\
\text { mance bias) }\end{array}$ & Unclear risk & $\begin{array}{l}\text { Study authors did not mention blinding of participants. They compared CT vs } \\
\text { an active condition, so blinding may have been possible }\end{array}$ \\
All outcomes &
\end{tabular}

\begin{tabular}{|c|c|c|}
\hline $\begin{array}{l}\text { Blinding of outcome as- } \\
\text { sessment (detection bias) } \\
\text { All outcomes }\end{array}$ & High risk & $\begin{array}{l}\text { Study authors provided no details to suggest that outcome assessment was } \\
\text { blind; it is likely that this was not the case }\end{array}$ \\
\hline
\end{tabular}

\begin{tabular}{ll}
\hline $\begin{array}{l}\text { Incomplete outcome data } \\
\text { (attrition bias) }\end{array}$ & $\begin{array}{l}\text { Study authors stated that they considered only participants who completed } \\
\text { both baseline and the follow-up assessments. However, among } 101 \text { partici- } \\
\text { All outcomes }\end{array}$ \\
& $\begin{array}{l}\text { pants in the dementia group, only } 6 \text { discontinued ( } 5 \% \text {; } 3 \text { in each condition), } \\
\text { so impact on outcomes is unlikely to be significant and risk of attrition bias is } \\
\text { likely to be low }\end{array}$
\end{tabular}

\begin{tabular}{|c|c|c|}
\hline $\begin{array}{l}\text { Selective reporting (re- } \\
\text { porting bias) }\end{array}$ & High risk & $\begin{array}{l}\text { Study authors did not report data related to } 2 \text { outcome measures (CDR and } \\
\text { phonemic fluency), and we received no response from study authors when we } \\
\text { contacted them }\end{array}$ \\
\hline
\end{tabular}

\begin{tabular}{ll}
\hline Other bias $\quad$ Low risk $\quad$ Study appears to be free of other sources of bias \\
\hline
\end{tabular}

Goudour 2011

\begin{tabular}{ll}
\hline Methods & $\begin{array}{l}\text { An RCT comparing effects of semantic stimulation vs psychological support in relation to semantic } \\
\text { memory in people with Alzheimer's disease }\end{array}$ \\
\hline Participants & $\begin{array}{l}\text { Ten participants, between } 57 \text { and } 78 \text { years of age, who were followed by CMRR of the White House } \\
\text { Hospital of Reims (France). All participants had a diagnosis of probable AD (according to DSM-IV and } \\
\text { NINCDS-ADRDA) with MMSE scores of } 17 \text { and above }\end{array}$ \\
\hline Interventions & $\begin{array}{l}\text { Participants in the experimental condition }(n=5) \text { were trained on a CT programme that focused on se- } \\
\text { mantic abilities related to music and human actions }\end{array}$
\end{tabular}


Participants in the control condition $(n=5)$ received clinical psychological support, which aimed to maintain self-esteem and favour exchanges

Both interventions were conducted by a neuropsychologist and lasted for 12 weeks, with 1 weekly individual session of approximately 50 minutes' duration

Outcomes

Outcomes included global cognition and measures of semantic memory, as well as mood (depression), capacity for activities of daily living, and carer burden

These outcomes were assessed before and after the intervention period. Two other assessments (only on semantic cluster) were conducted at week 6 and at week 11

\begin{tabular}{ll}
\hline Country & France \\
\hline Registration status\&\#160; & No information provided; presumed to be unregistered \\
\hline Conflict of Interests\&\#160; & No \\
\hline Notes & $\begin{array}{l}\text { Study authors answered some questions about study design. We then asked for a table with scores but } \\
\text { received no reply }\end{array}$
\end{tabular}

\section{Risk of bias}

\begin{tabular}{|c|c|c|}
\hline Bias & Authors' judgement & Support for judgement \\
\hline $\begin{array}{l}\text { Random sequence genera- } \\
\text { tion (selection bias) }\end{array}$ & Unclear risk & $\begin{array}{l}\text { Study authors responded to an email in which they described the study as ran- } \\
\text { domised; however, they provided no details about the randomisation process }\end{array}$ \\
\hline $\begin{array}{l}\text { Allocation concealment } \\
\text { (selection bias) }\end{array}$ & High risk & $\begin{array}{l}\text { Study authors did not mention allocation concealment; for this reason, we as- } \\
\text { sumed this was not done }\end{array}$ \\
\hline $\begin{array}{l}\text { Blinding of participants } \\
\text { and personnel (perfor- } \\
\text { mance bias) } \\
\text { All outcomes }\end{array}$ & Unclear risk & $\begin{array}{l}\text { Study authors did not mention blinding of participants. They compared CT vs } \\
\text { an active condition, so blinding may have been possible }\end{array}$ \\
\hline $\begin{array}{l}\text { Blinding of outcome as- } \\
\text { sessment (detection bias) } \\
\text { All outcomes }\end{array}$ & Low risk & $\begin{array}{l}\text { All visits were conducted by a neuropsychologist (blind to the type of interven- } \\
\text { tion given to participants and blind to the aims of the study) }\end{array}$ \\
\hline $\begin{array}{l}\text { Incomplete outcome data } \\
\text { (attrition bias) } \\
\text { All outcomes }\end{array}$ & Unclear risk & $\begin{array}{l}\text { No specific details could be ascertained about recruitment and retention of } \\
\text { participants. It seems that each condition pertained to } 5 \text { participants, but it is } \\
\text { unclear whether numbers recruited, retained, and analysed were the same }\end{array}$ \\
\hline $\begin{array}{l}\text { Selective reporting (re- } \\
\text { porting bias) }\end{array}$ & Unclear risk & $\begin{array}{l}\text { Study was published in French; whether all specified outcomes were reported } \\
\text { remains unclear }\end{array}$ \\
\hline Other bias & Low risk & Study appears to be free of other sources of bias \\
\hline
\end{tabular}

\section{Heiss 1993}

\begin{tabular}{ll} 
Methods & $\begin{array}{l}\text { An RCT comparing effects of a CT programme provided alone or in combination with pyritinol or phos- } \\
\text { phatidylserine, as well as effects a social support programme for people with AD }\end{array}$ \\
\hline Participants & $\begin{array}{l}80 \text { community-dwelling participants between } 48 \text { and } 79 \text { years of age who met the criteria for AD (ac- } \\
\text { cording to NINCDS-ADRDA) and had an MMSE score between } 13 \text { and } 26 \text { were recruited in Cologne, Ger- } \\
\text { many. Data were available for } 70 \text { of these }\end{array}$
\end{tabular}


Heiss 1993 (Continued)

Interventions
Participants in the CT condition ( $n=18$ ) were engaged in a computerised training programme that focused on memory and perceptual and motor tasks, with varying degrees of difficulty. Paritcipants attended 1-hour sessions twice a week

Participants in the $C T+$ pyritinol condition $(n=17)$ received the same intervention, in combination with oral pyritinol $600 \mathrm{mg}$ twice a day

Participants in the $C T+$ phosphatidylserine condition $(n=18)$ also received $C T$, but in combination with oral phosphatidylserine $200 \mathrm{mg}$ twice a day

Participants in the social support condition $(n=17)$ discussed their personal difficulties and daily life during 1-hour sessions, once per week

For the purpose of this review, social support served as the comparison condition

Outcomes evaluated included clinical dementia severity, as well as cognitive domains such as orientation and praxis, psychomotor speed, reaction time, attention, memory, and language capacity Functional imaging outcomes (PET and EEG) were also evaluated

Assessments were conducted before and after the treatment period. Some domains were assessed at weeks 8 and 16 of the intervention

\begin{tabular}{ll}
\hline Country & Germany \\
\hline Registration status\&\#160; & No information provided; presumed to be unregistered \\
\hline Conflict of Interests\&\#160; & Not stated \\
\hline Notes & \\
\hline
\end{tabular}

\section{Risk of bias}

\begin{tabular}{|c|c|c|}
\hline Bias & Authors' judgement & Support for judgement \\
\hline $\begin{array}{l}\text { Random sequence genera- } \\
\text { tion (selection bias) }\end{array}$ & Unclear risk & $\begin{array}{l}\text { Although the study was described as a randomised controlled trial, no infor- } \\
\text { mation on the method of randomisation was provided }\end{array}$ \\
\hline $\begin{array}{l}\text { Allocation concealment } \\
\text { (selection bias) }\end{array}$ & High risk & $\begin{array}{l}\text { Study authors did not mention allocation concealment; for this reason, we as- } \\
\text { sumed this was not done }\end{array}$ \\
\hline $\begin{array}{l}\text { Blinding of participants } \\
\text { and personnel (perfor- } \\
\text { mance bias) } \\
\text { All outcomes }\end{array}$ & Unclear risk & $\begin{array}{l}\text { Study authors did not mention blinding of participants. They compared CT vs } \\
\text { an active condition, so blinding may have been possible }\end{array}$ \\
\hline $\begin{array}{l}\text { Blinding of outcome as- } \\
\text { sessment (detection bias) } \\
\text { All outcomes }\end{array}$ & High risk & $\begin{array}{l}\text { Study authors did not mention blinding of outcome assessments; this proba- } \\
\text { bly was not done }\end{array}$ \\
\hline $\begin{array}{l}\text { Incomplete outcome data } \\
\text { (attrition bias) } \\
\text { All outcomes }\end{array}$ & High risk & $\begin{array}{l}\text { On balance, it appears that inconsistent reporting in the } 2 \text { reports from this tri- } \\
\text { al, changes made to the protocol, and lack of detail regarding reasons for (and } \\
\text { distribution of) missing data suggest that elevated risk of bias is significant }\end{array}$ \\
\hline $\begin{array}{l}\text { Selective reporting (re- } \\
\text { porting bias) }\end{array}$ & Low risk & $\begin{array}{l}\text { The } 1994 \text { paper seems to be the more complete report from this trial. Over- } \\
\text { all, and by acceptable standards at the time, it seems that study authors have } \\
\text { generally described relevant outcomes in the "Methods" section and reported } \\
\text { them all more or less consistently in the "Results" section }\end{array}$ \\
\hline
\end{tabular}


Heiss 1993 (Continued)

Other bias Low risk Study appears to be free of other sources of bias

Jelcic 2012

Methods An RCT comparing effects on memory of a focused lexical-semantic stimulation (LSS) intervention vs an unstructured cognitive stimulation treatment in persons with early $A D$

Participants

40 participants with a diagnosis of probable AD (according to NINCDS-ADRDA) who were not on anti-dementia drug therapy and had an MMSE score of 26 and above. Seven participants were male and 33 were female. Mean age of the sample was 82.3 , and mean educational attainment was 7.5 years. All participants were presumed to be community-dwelling persons (not stated)

Interventions

Participants in the LSS condition $(n=20)$ were trained on a programme that included focused lexical-semantic rehabilitation exercises, which aimed to enhance semantic verbal processing and focused on interpretation of written words, sentences, and stories

Participants in the UCS condition $(n=20)$ were involved in exercises consisting of creative work (practising manual skills, manufacturing external memory aids, stimulating fantasy and creativeness, reading the newspaper, and improving verbal communication)

Treatments for both conditions were delivered by the same neuropsychologist in two 60-minute smallgroup sessions per week over a 3-month period

Outcomes

Primary outcomes were global cognition, lexical-semantic ability, and verbal memory. Secondary outcomes included cognitive performance on measures of working memory, visuospatial memory, attention, and executive functions as well as capacity for instrumental activities of daily living. Assessments were conducted at baseline and after 3 months of treatment. Participants in the experimental condition were also assessed at 6-month follow-up

\begin{tabular}{ll}
\hline Country & Italy \\
\hline Registration status\&\#160; & No information provided; presumed to be unregistered \\
\hline Conflict of Interests\&\#160; & No \\
\hline Notes & $\begin{array}{l}\text { We tried to contact study authors to ask for all follow-up scores and Trail Making Test B scores at post- } \\
\text { intervention assessment, but we received no reply }\end{array}$
\end{tabular}

\section{Risk of bias}

\begin{tabular}{lll}
\hline Bias & Authors' judgement & Support for judgement \\
\hline $\begin{array}{l}\text { Random sequence genera- } \\
\text { tion (selection bias) }\end{array}$ & Low risk & $\begin{array}{l}\text { Study authors stated that participants were allocated to groups "by using a } \\
\text { simple computerized technique" }\end{array}$ \\
\hline $\begin{array}{l}\text { Allocation concealment } \\
\text { (selection bias) }\end{array}$ & Unclear risk & $\begin{array}{l}\text { Study authors stated that they used a computerised randomisation system. } \\
\text { It is likely that allocation concealment was done, but it is not intrinsic to this } \\
\text { method }\end{array}$
\end{tabular}

\begin{tabular}{|c|c|c|}
\hline $\begin{array}{l}\text { Blinding of participants } \\
\text { and personnel (perfor- } \\
\text { mance bias) }\end{array}$ & High risk & $\begin{array}{l}\text { The same therapist delivered both experimental and control interventions, } \\
\text { including blinding. This may have led to increased risk of performance bias. } \\
\text { Study authors did not mention blinding of participants }\end{array}$ \\
\hline
\end{tabular}

\begin{tabular}{ll}
\hline $\begin{array}{l}\text { Blinding of outcome as- } \\
\text { sessment (detection bias) }\end{array} \quad$ Low risk & $\begin{array}{l}\text { The assessor was blinded to the treatment group to which each participant } \\
\text { was allocated }\end{array}$
\end{tabular}


Jelcic 2012 (Continued)

All outcomes

$\begin{array}{ll}\begin{array}{l}\text { Incomplete outcome data } \\ \text { (attrition bias) }\end{array} & \text { Low risk } \\ \text { Noutcome data were missing. A flow chart shows that no participants } \\ \text { dropped out of the study. All were included in the analysis }\end{array}$

All outcomes

No outcome data were missing. A flow chart shows that no participants

\begin{tabular}{lll}
\hline $\begin{array}{l}\text { Selective reporting (re- } \\
\text { porting bias) }\end{array}$ & High risk & Results from Trail Making Test B are missing \\
\hline Other bias & Low risk & Study appears to be free of other sources of bias \\
\hline
\end{tabular}

Jelcic 2014

\begin{tabular}{ll}
\hline Methods & An RCT comparing LSS delivered through telecommunication technology vs the same training deliv- \\
ered face to face and with unstructured cognitive treatment in persons with early AD
\end{tabular}

Participants
AD, according to NINCDS-ADRDA, who were not on anti-dementia drug therapy. Mean age of partici-
pants was 83.7 , and they had received a minimum of 6 years of formal education. Twenty-one were fe-
male and 6 were male. Participants are presumed to be community-dwelling persons

Interventions Participants in the LSS-direct intervention condition $(n=10)$ received face-to-face training on lexical
tasks that aimed to enhance semantic verbal processing

Participants in the LSS-telecondition $(n=7)$ received the LSS intervention through telecommunication

Participants in the control condition $(n=10)$ completed face-to-face exercises, such as practising manual skills or reading the newspaper and engaging in discussion

The same therapist delivered all interventions, which lasted for 3 months and included two 1-hour small-group sessions per week. Between sessions, caregivers were encouraged to deliver non-specific cognitive reinforcement

Outcomes Primary outcomes were global cognitive performance, lexical-semantic abilities, and episodic verbal memory

Secondary outcomes were changes in attention, working memory, executive functions, and visual-spatial abilities

These domains were assessed by a neuropsychologist at baseline and after 3 months of treatment

\begin{tabular}{ll}
\hline Country & Italy \\
\hline Registration status\&\#160; & No information provided; presumed to be unregistered \\
\hline Conflict of Interests\&\#160; & No \\
\hline Notes & $\begin{array}{l}\text { We tried to contact study authors to ask for mean, SD, and sample size for Trail Making Test (A and B), } \\
\text { NPI, and ROCF Copy Test at baseline and at post-intervention assessment, but we received no reply }\end{array}$ \\
\hline Risk of bias &
\end{tabular}

\begin{tabular}{lll}
\hline Bias & Authors' judgement & Support for judgement \\
\hline $\begin{array}{ll}\text { Random sequence genera- } \\
\text { tion (selection bias) }\end{array}$ & High risk & $\begin{array}{l}\text { No information is provided regarding the method of randomisation. Howev- } \\
\text { er, study authors state, "The unequal distribution among the three treatment } \\
\text { groups was due to the preference of two patients, initially enrolled in the LSS- }\end{array}$
\end{tabular}


Jelcic 2014 (Continued)

tele group, to not be involved with computer technology and who were shifted into the other two treatment arms"

\begin{tabular}{|c|c|c|}
\hline $\begin{array}{l}\text { Allocation concealment } \\
\text { (selection bias) }\end{array}$ & High risk & $\begin{array}{l}\text { Two participants who were initially enrolled in the LSS-tele group were then } \\
\text { allocated to the other } 2 \text { conditions, with their preferences considered. For this } \\
\text { reason, allocation concealment was not possible in these cases }\end{array}$ \\
\hline $\begin{array}{l}\text { Blinding of participants } \\
\text { and personnel (perfor- } \\
\text { mance bias) } \\
\text { All outcomes }\end{array}$ & Unclear risk & $\begin{array}{l}\text { Study authors did not mention blinding of participants. They compared CT vs } \\
\text { an active condition, so blinding may have been possible }\end{array}$ \\
\hline $\begin{array}{l}\text { Blinding of outcome as- } \\
\text { sessment (detection bias) } \\
\text { All outcomes }\end{array}$ & Low risk & $\begin{array}{l}\text { All assessments were carried out by an experienced neuropsychologist who } \\
\text { was blinded to the treatment group to which each participant was allocated }\end{array}$ \\
\hline $\begin{array}{l}\text { Incomplete outcome data } \\
\text { (attrition bias) } \\
\text { All outcomes }\end{array}$ & Low risk & $\begin{array}{l}\text { No outcome data were missing. No participants dropped out of the study; all } \\
\text { were included in the analysis }\end{array}$ \\
\hline $\begin{array}{l}\text { Selective reporting (re- } \\
\text { porting bias) }\end{array}$ & High risk & $\begin{array}{l}\text { Results of Trail Making Tests A and B (secondary outcome measures) were not } \\
\text { reported }\end{array}$ \\
\hline Other bias & Low risk & Study appears to be free of other sources of bias \\
\hline
\end{tabular}

Kallio 2018

\begin{tabular}{ll}
\hline Methods & $\begin{array}{l}\text { A single-blind RCT comparing effect of CTs vs a control intervention on cognition and health-related } \\
\text { quality of life ( } \mathrm{HRQOL}) \text { in community-dwelling persons with dementia }\end{array}$
\end{tabular}

Participants

147 community-dwelling participants attending daycare (106 female). Education was split around 8 years, with 68 having more than 8 years of education. Most participants had AD (122), but 11 had received a diagnosis of vascular dementia, 4 had Parkinson's disease or Lewy body dementia, and 10 had other or unknown dementia

Interventions

Participants in the intervention condition $(n=76)$ received CT treatment sessions that involved paper-and-pencil tasks in small groups of 2 to 4 participants, or individually when needed

Participants in both intervention and control conditions received routine treatment at the daycare centre twice a week for 6 hours each day in groups of 12 to 16 persons

The intervention was delivered by trained psychology students under the supervision of an experienced neuropsychologist

Outcomes Primary outcomes were global cognition and health-related quality of life

Evaluations were carried out at baseline, at 3 months, and at 9-month follow-up

\begin{tabular}{ll}
\hline Country & Finland \\
\hline Registration status\&\#160; & $\begin{array}{l}\text { This trial was prospectively registered at the Australian and New Zealand Clinical Trials Registry; identi- } \\
\text { fier ACTRN12614000976684 }\end{array}$ \\
\hline Conflict of Interests\&\#160; & No \\
\hline Notes & $\begin{array}{l}\text { The CT programme reflected a relevant modification of cognitive remediation therapy, which is a train- } \\
\text { ing-based intervention aimed at improving executive functioning of chronic psychiatric patients. Study }\end{array}$
\end{tabular}


Kallio 2018 (Continued)

authors noted that this cognition-focused treatment was adapted in the trial by decreasing the difficulty level of the tasks, reducing the number of sessions (from 44 to 24), and increasing font size in the tasks. Techniques of repeated practice, errorless learning (reducing the opportunity to make errors), immediate feedback, scaffolding (44tpro';rviding strategies when needed, and gradually increasing task complexity), and facilitated planning and self-monitoring were used during training. Study authors noted that 2-year follow-up would be completed by the end of 2018.

Study authors provided mean and SD for each measure at all assessment occasions for each group separately

\section{Risk of bias}

\begin{tabular}{|c|c|c|}
\hline Bias & Authors' judgement & Support for judgement \\
\hline $\begin{array}{l}\text { Random sequence genera- } \\
\text { tion (selection bias) }\end{array}$ & Low risk & $\begin{array}{l}\text { Participants were assigned to groups via "computer-generated randomly allo- } \\
\text { cated numbers received by telephone from a randomisation center" }\end{array}$ \\
\hline $\begin{array}{l}\text { Allocation concealment } \\
\text { (selection bias) }\end{array}$ & Low risk & $\begin{array}{l}\text { Study authors stated that they used a remote, computerised randomisation } \\
\text { system. Allocation concealment is intrinsic to this method }\end{array}$ \\
\hline $\begin{array}{l}\text { Blinding of participants } \\
\text { and personnel (perfor- } \\
\text { mance bias) } \\
\text { All outcomes }\end{array}$ & High risk & $\begin{array}{l}\text { Study authors described a single-blind RCT (the neuropsychologist in charge } \\
\text { of conducting the assessments was blinded) }\end{array}$ \\
\hline $\begin{array}{l}\text { Blinding of outcome as- } \\
\text { sessment (detection bias) } \\
\text { All outcomes }\end{array}$ & Low risk & $\begin{array}{l}\text { Study authors stated that all assessments were conducted by blinded asses- } \\
\text { sors }\end{array}$ \\
\hline $\begin{array}{l}\text { Incomplete outcome data } \\
\text { (attrition bias) } \\
\text { All outcomes }\end{array}$ & Unclear risk & $\begin{array}{l}\text { By 9-month follow-up, } 10 \% \text { of experimental participants ( } 8 \text { ) and } 30 \% \text { of control } \\
\text { participants ( } 22) \text { had dropped out - reasons seem to be similar, but proportion } \\
\text { was much greater in the control condition; no details on baseline characteris- } \\
\text { tics of those who dropped out were provided to ensure that they are not differ- } \\
\text { ent from those who were retained }\end{array}$ \\
\hline $\begin{array}{l}\text { Selective reporting (re- } \\
\text { porting bias) }\end{array}$ & Unclear risk & $\begin{array}{l}\text { Several outcomes that were specified in the published protocol are not men- } \\
\text { tioned in the outcome paper. We were able to obtain some but not all of these } \\
\text { from the study authors, who stated that the article was still under review }\end{array}$ \\
\hline Other bias & Low risk & Study appears to be free of other sources of bias \\
\hline
\end{tabular}

\section{Kao 2016}

Methods

A 2-step cluster-randomised trial to compare the long-term effects of spaced retrieval (SR) training and $\mathrm{SR}$ training combined with Montessori activities $(\mathrm{SR}+\mathrm{M})$ and a control condition in persons with dementia suffering from hyperphagia

Participants

148 participants, recruited from 8 dementia special care units in China, who had received a diagnosis of dementia and hyperphagia-related behaviours from a physician

Interventions

Participants in the SR condition $(n=48)$ and in the SR+M condition $(n=52)$ participated in thirty 40 minute sessions over 6 weeks, which were held in dementia special care units. The SR sessions involved learning and reviewing a memory message. SR+M sessions involved the same activity, as well as practice-based structured Montessori activities 
Kao 2016 (Continued)

Interventions were delivered by 2 memory trainers who had received training in SR and Montessori activity methods

Participants in the control condition $(n=48)$ participated in the usual activities of the institution, which did not involve any particular memory training activities

\begin{tabular}{ll}
\hline Outcomes & $\begin{array}{l}\text { Outcomes included hyperphagic behaviours, measured by a 19-item scale of hyperphagia in residents } \\
\text { with dementia, and associated caregiver distress, measured by a scale of distress of the caregiver in re- } \\
\text { sponse to hyperphagic behaviours } \\
\text { Participants were assessed at baseline, immediately after the training period, and at months 1, 3, and } 6 \\
\text { after completion of the training }\end{array}$ \\
\hline Country & China \\
\hline Registration status\&\#160; & Retrospectively registered \\
\hline Conflict of Interests\&\#160; & No \\
\hline Notes & $\begin{array}{l}\text { We contacted study authors to ask for relevant scores (at all assessment occasions) and to find out } \\
\text { more details about the scales used ("scale for hyperphagia in residents with dementia", and "scale of } \\
\text { distress of the caregiver to hyperphagic behaviour"). No response was received }\end{array}$
\end{tabular}

\section{Risk of bias}

\begin{tabular}{lll}
\hline Bias & Authors' judgement & Support for judgement \\
\hline $\begin{array}{l}\text { Random sequence genera- } \\
\text { tion (selection bias) }\end{array}$ & Unclear risk & $\begin{array}{l}\text { Although the study was described as a randomised controlled trial, no infor- } \\
\text { mation on the method of randomisation was provided }\end{array}$ \\
\hline $\begin{array}{l}\text { Allocation concealment } \\
\text { (selection bias) }\end{array}$ & High risk & $\begin{array}{l}\text { Study authors did not mention allocation concealment; for this reason, we as- } \\
\text { sumed this was not done }\end{array}$ \\
\hline $\begin{array}{l}\text { Blinding of participants } \\
\text { and personnel (perfor- } \\
\text { mance bias) }\end{array}$ & High risk & $\begin{array}{l}\text { Study authors described the study as a single-blind RCT; however, this refers to } \\
\text { the outcome assessment }\end{array}$ \\
All outcomes & &
\end{tabular}

\begin{tabular}{|c|c|c|}
\hline $\begin{array}{l}\text { Blinding of outcome as- } \\
\text { sessment (detection bias) } \\
\text { All outcomes }\end{array}$ & Low risk & "The research assistants were blinded to the randomisation of subjects" \\
\hline $\begin{array}{l}\text { Incomplete outcome data } \\
\text { (attrition bias) } \\
\text { All outcomes }\end{array}$ & Low risk & $\begin{array}{l}\text { No outcome data were missing. Study authors reported the results of those } \\
\text { who completed the study and explained the reasons why some participants } \\
\text { dropped out. They provided a flow diagram of the study that is adherent to } \\
\text { CONSORT }\end{array}$ \\
\hline $\begin{array}{l}\text { Selective reporting (re- } \\
\text { porting bias) }\end{array}$ & High risk & $\begin{array}{l}\text { Study authors did not present findings for some of the outcomes mentioned in } \\
\text { the "Methods" section }\end{array}$ \\
\hline Other bias & Low risk & Study appears to be free of other sources of bias \\
\hline
\end{tabular}

\section{Kawashima 2005}

\begin{tabular}{ll}
\hline Methods & $\begin{array}{l}\text { An RCT comparing a training programme in reading and arithmetic problems (learning therapy) vs a } \\
\text { control condition in older adults with AD }\end{array}$
\end{tabular}


Kawashima 2005 (Continued)

Participants $\quad 32$ participants living in a nursing home in Japan (mean age 85.7, range 76 to 96) with a diagnosis of Alzheimer's-type dementia according to DSM-IV (mean MMSE score 19.8, range 7 to 30)

Interventions

Participants in the experimental condition $(n=16)$ were trained on a programme (Learning Therapy) that focused on reading aloud and solving arithmetic problems, provided at learning centres in Euju-no-Sato, with 2 to 6 sessions per week for about 20 minutes per day for longer than 6 months

Participants in the control condition $(n=16)$ were presumed to have had a no-contact condition

\begin{tabular}{ll}
\hline Outcomes & $\begin{array}{l}\text { Outcomes included change in global cognition and measures of executive functioning, verbal commu- } \\
\text { nication capacity, and functional independence. Assessments were conducted at baseline and after } \\
\text { completion of the intervention (6 months) }\end{array}$ \\
\hline Country & Japan \\
\hline Registration status\&\#160; & No information provided; presumed to be unregistered \\
\hline Conflict of Interests\&\#160; & Not stated
\end{tabular}

$\begin{array}{ll}\text { Notes } & \text { Study authors sent MMSE scores at post-intervention assessment, which were missing from the pub- } \\ \text { lished report, as well as overall scores for the Frontal Assessment Battery (FAB) }\end{array}$

\section{Risk of bias}

\begin{tabular}{|c|c|c|}
\hline Bias & Authors' judgement & Support for judgement \\
\hline $\begin{array}{l}\text { Random sequence genera- } \\
\text { tion (selection bias) }\end{array}$ & Unclear risk & $\begin{array}{l}\text { Although study authors described the study as an RCT, they provided no infor- } \\
\text { mation on the method of randomisation }\end{array}$ \\
\hline $\begin{array}{l}\text { Allocation concealment } \\
\text { (selection bias) }\end{array}$ & High risk & $\begin{array}{l}\text { Study authors did not mention allocation concealment; for this reason, we as- } \\
\text { sumed this was not done }\end{array}$ \\
\hline $\begin{array}{l}\text { Blinding of participants } \\
\text { and personnel (perfor- } \\
\text { mance bias) } \\
\text { All outcomes }\end{array}$ & High risk & $\begin{array}{l}\text { Study authors described the study as a single-blind RCT (research assistants in } \\
\text { charge of conducting the observation were blinded) }\end{array}$ \\
\hline $\begin{array}{l}\text { Blinding of outcome as- } \\
\text { sessment (detection bias) } \\
\text { All outcomes }\end{array}$ & Unclear risk & No blinding; outcomes could have been influenced by lack of blinding \\
\hline $\begin{array}{l}\text { Incomplete outcome data } \\
\text { (attrition bias) } \\
\text { All outcomes }\end{array}$ & Low risk & No outcome data were missing \\
\hline $\begin{array}{l}\text { Selective reporting (re- } \\
\text { porting bias) }\end{array}$ & Low risk & $\begin{array}{l}\text { Study authors did not report scores for MMSE at post-intervention assessment, } \\
\text { but we obtained them upon request }\end{array}$ \\
\hline Other bias & Low risk & Study appears to be free of other sources of bias \\
\hline
\end{tabular}

Kim 2015

Methods An RCT comparing the effect of "cognitive rehabilitation" (including CT tasks) relative to a control condition in relation to performance of everyday activities in older adults with early-stage AD 
Kim 2015 (Continued)

Participants 43 participants ( 15 males, 28 females) with early-stage AD (as indicated by MMSE) who were recruited from a daycare centre in South Korea. Mean age and educational level of the sample were 70.9 and 8.6, respectively

Interventions Participants in the experimental condition $(\mathrm{n}=22)$ were trained on a CR programme that included individual sessions (focused on a personally meaningful goal) and group sessions, which involved CT tasks and focused on practicing time-and-place orientation and memory, and sustaining attention. Participants in this condition attended a total of 8 sessions, each lasting 1 hour (30 minutes individually and 30 minutes in a group), once a week for 8 weeks. A researcher delivered the intervention

Participants in the control condition $(n=21)$ also participated in 8 sessions, each lasting 1 hour, once a week. This was an active control condition that involved participating in unstructured conversation and questioning with the examiner and watching health-related videos

Outcomes Outcomes included ratings of goal performance and satisfaction, self-care, mobility-related activities of daily living, quality of life, orientation, and memory. Assessments were undertaken before the intervention and once it was completed

\begin{tabular}{ll}
\hline Country & Korea \\
\hline Registration status\&\#160; & No information provided; presumed to be unregistered \\
\hline Conflict of Interests\&\#160; & Not stated \\
\hline Notes & \\
\hline
\end{tabular}

\section{Risk of bias}

\begin{tabular}{|c|c|c|}
\hline Bias & Authors' judgement & Support for judgement \\
\hline $\begin{array}{l}\text { Random sequence genera- } \\
\text { tion (selection bias) }\end{array}$ & Unclear risk & $\begin{array}{l}\text { Although study authors described the study as an RCT, the provided no infor- } \\
\text { mation on the method of randomisation }\end{array}$ \\
\hline $\begin{array}{l}\text { Allocation concealment } \\
\text { (selection bias) }\end{array}$ & High risk & $\begin{array}{l}\text { Study authors did not mention allocation concealment; for this reason, we as- } \\
\text { sumed this was not done }\end{array}$ \\
\hline $\begin{array}{l}\text { Blinding of participants } \\
\text { and personnel (perfor- } \\
\text { mance bias) } \\
\text { All outcomes }\end{array}$ & Unclear risk & $\begin{array}{l}\text { Study authors did not mention blinding of participants. They compared CT vs } \\
\text { an active condition, so blinding may have been possible }\end{array}$ \\
\hline $\begin{array}{l}\text { Blinding of outcome as- } \\
\text { sessment (detection bias) } \\
\text { All outcomes }\end{array}$ & Low risk & Blinded assessors administered assessments \\
\hline $\begin{array}{l}\text { Incomplete outcome data } \\
\text { (attrition bias) } \\
\text { All outcomes }\end{array}$ & Low risk & Study authors made no mention of attrition among participants in this trial \\
\hline $\begin{array}{l}\text { Selective reporting (re- } \\
\text { porting bias) }\end{array}$ & Low risk & $\begin{array}{l}\text { All tests mentioned in the "Methods" section were reported in the "Results" } \\
\text { section }\end{array}$ \\
\hline Other bias & Low risk & Study appears to be free of other sources of bias \\
\hline
\end{tabular}


Koltai 2001

\begin{tabular}{ll}
\hline Methods & $\begin{array}{l}\text { An RCT comparing effects of a Memory and Coping Program (MCP), in both individual and group for- } \\
\text { mats, vs those of a control condition on measures of cognition and emotional adjustment in people } \\
\text { with mild to moderate dementia who had difficulty adjusting to their cognitive losses }\end{array}$ \\
\hline Participants & $\begin{array}{l}24 \text { participants who were over } 60 \text { years old (mean age } 73.5) \text { and had received a diagnosis of mild to } \\
\text { moderate dementia, according to the Clinical Dementia Rating (CDR) scale. Average years of education } \\
\text { for the sample was } 15\end{array}$
\end{tabular}

Interventions

Participants in the individual MCP condition $(n=8)$ attended an average of 6 weekly intervention sessions focused on cognitive and affective functioning. When available, caregivers joined the sessions for the last 10 to 15 minutes

Participants assigned to the group MCP condition $(n=8)$ attended 5 one-hour weekly sessions in groups of 2 to 4 persons

Participants in the wait-list control condition $(n=8)$ received small-group MCP after the intervention and assessments had been completed

\section{Outcomes}

Outcomes included performance on measures of general cognition (including global cognition, memory, language, and construction), mood (depression), subjective memory ability (meta-memory), and awareness of cognitive deficits

Assessments were conducted before and after the intervention

\begin{tabular}{ll}
\hline Country & United States of America \\
\hline Registration status\&\#160; & Study authors provided no information; trial presumed to be unregistered \\
\hline Conflict of Interests\&\#160; & Not stated \\
\hline
\end{tabular}

Notes

\section{Risk of bias}

\begin{tabular}{|c|c|c|}
\hline Bias & Authors' judgement & Support for judgement \\
\hline $\begin{array}{l}\text { Random sequence genera- } \\
\text { tion (selection bias) }\end{array}$ & High risk & $\begin{array}{l}\text { Randomisation process not described, but interventions were divided (by or- } \\
\text { der of enrolment) into } 2 \text { groups of } 4\end{array}$ \\
\hline $\begin{array}{l}\text { Allocation concealment } \\
\text { (selection bias) }\end{array}$ & High risk & $\begin{array}{l}\text { Study authors did not mention allocation; for this reason, we assumed this } \\
\text { was not done }\end{array}$ \\
\hline $\begin{array}{l}\text { Blinding of participants } \\
\text { and personnel (perfor- } \\
\text { mance bias) } \\
\text { All outcomes }\end{array}$ & High risk & $\begin{array}{l}\text { Study authors did not mention blinding of participants, and the study included } \\
\text { a passive control condition (wait-list), so blinding was not possible }\end{array}$ \\
\hline $\begin{array}{l}\text { Blinding of outcome as- } \\
\text { sessment (detection bias) } \\
\text { All outcomes }\end{array}$ & High risk & No blinding; outcomes could have been influenced by lack of blinding \\
\hline $\begin{array}{l}\text { Incomplete outcome data } \\
\text { (attrition bias) } \\
\text { All outcomes }\end{array}$ & Unclear risk & $\begin{array}{l}\text { Two participants from the treatment condition withdrew from the study due } \\
\text { to serious illness. No participants withdrew from the control condition. No } \\
\text { details were provided regarding the } 2 \text { participants who withdrew, and analy- } \\
\text { sis was based only on participants who completed treatment, so it is unclear } \\
\text { whether bias may have been due to incomplete data }\end{array}$ \\
\hline
\end{tabular}


Lee 2013 (Continued)

All outcomes

Incomplete outcome data High risk (attrition bias)

All outcomes
Five participants who were found eligible for the study dropped out due to 'deterioration in their medical condition', but study authors provided no details as to the stage at which this occurred, whether baseline characteristics were different, or to which conditions participants had been allocated. Non-inclusion of their data is likely to have introduced bias due to incomplete reporting

All tests mentioned in the "Methods" section were reported in the "Results" section

Selective reporting (re- Low risk porting bias)

Other bias Low risk Study appears to be free of other sources of bias

Mapelli 2013

Methods An RCT comparing effects of a structured cognitive stimulation treatment, an occupational therapy in tervention, and a usual care control condition on cognition and behavioural symptoms in people with dementia

\section{Participants}

30 persons with mild to moderate dementia (mean MMSE score 19.5, range 14 to 24 ) living in a nursing home in Italy. Mean age of the sample was 83.93

Interventions

Participants in the experimental condition $(n=10)$ were trained on a cognitive stimulation programme that started with personal, spatial, and temporal orientation sessions and proceeded with individual exercises to stimulate specific cognitive domains. A therapist administered the intervention

Participants in the occupational therapy "placebo condition" $(n=10)$ received occupational therapy involving a series of activities, such as reading and debating the newspaper, playing bingo, or singing

Both interventions lasted 8 weeks, with 5 one-hour sessions per week

Participants assigned to the control condition $(n=10)$ continued with usual activities of the nursing home

\begin{tabular}{ll}
\hline Outcomes & $\begin{array}{l}\text { Outcomes included clinical dementia severity, cognitive abilities in several domains, mood (depres- } \\
\text { sion), functional status, behavioural symptoms, and caregiver burden } \\
\text { Assessments were conducted at baseline and after } 8 \text { weeks of intervention }\end{array}$ \\
\hline Country & Italy \\
\hline Registration status\&\#160; & No information provided; presumed to be unregistered \\
\hline Conflict of Interests\&\#160; & Not stated \\
\hline Notes & $\begin{array}{l}\text { We contacted study authors to ask for additional scores not presented in Table 1 in the published re- } \\
\text { port, for each group, at baseline and at post-intervention assessment (Digit Span tasks, Trail Making } \\
\text { Tests A and B, Token Test, Cognitive Estimation Test, Intricate Figures Test, House Figure Copy, Daisy } \\
\text { Drawing Test, Ideomotor Apraxia Test, Activity of Daily Living Scale, and Geriatric Depression Scale); } \\
\text { however, we received no reply }\end{array}$ \\
\hline
\end{tabular}

\section{Risk of bias}

\section{Bias}

\section{Authors' judgement Support for judgement}


Mapelli 2013 (Continued)

Random sequence genera- Low risk $\quad$ "Participants were randomized into three groups, i.e. experimental, placetion (selection bias) bo, and control groups, following a simple computerized randomisation technique"

\begin{tabular}{|c|c|c|}
\hline $\begin{array}{l}\text { Allocation concealment } \\
\text { (selection bias) }\end{array}$ & Unclear risk & $\begin{array}{l}\text { Study authors stated that they used a computerised randomisation system. It } \\
\text { is likely that allocation concealment was done, but this is not specified }\end{array}$ \\
\hline $\begin{array}{l}\text { Blinding of participants } \\
\text { and personnel (perfor- } \\
\text { mance bias) } \\
\text { All outcomes }\end{array}$ & High risk & $\begin{array}{l}\text { Study authors did not mention blinding of participants; the study included a } \\
\text { passive control condition, so blinding was not possible }\end{array}$ \\
\hline $\begin{array}{l}\text { Blinding of outcome as- } \\
\text { sessment (detection bias) } \\
\text { All outcomes }\end{array}$ & Low risk & Participants were evaluated by a blinded rater \\
\hline $\begin{array}{l}\text { Incomplete outcome data } \\
\text { (attrition bias) } \\
\text { All outcomes }\end{array}$ & Low risk & $\begin{array}{l}\text { No outcome data were missing. A flow chart shows that all participants com- } \\
\text { pleted the final assessment and all were included in the analysis }\end{array}$ \\
\hline $\begin{array}{l}\text { Selective reporting (re- } \\
\text { porting bias) }\end{array}$ & High risk & $\begin{array}{l}\text { Study authors did not present findings for some of the outcomes mentioned in } \\
\text { the "Methods" section }\end{array}$ \\
\hline Other bias & Low risk & Study appears to be free of other sources of bias \\
\hline
\end{tabular}

Neely 2009

An RCT comparing a collaborative memory intervention vs an identical intervention delivered individ-
ually, and vs a control condition, in people with mild to moderate AD and vascular dementia and their
caregiving spouses

\begin{tabular}{|c|c|}
\hline Participants & $\begin{array}{l}30 \text { dyads, including a community dwelling person with mild to moderate AD or vascular dementia, ac- } \\
\text { cording to DSM-IV, who had received the diagnosis within } 8 \text { months before the intervention, and their } \\
\text { caregiving spouse } \\
\text { Mean age of participants with dementia was } 75.4 \text { years }\end{array}$ \\
\hline
\end{tabular}

Interventions In the collaborative intervention condition ( $P w D ; n=10)$, participant dyads practised together strategies to enhance everyday mnemonic and occupational performance, with focus on spaced retrieval and hierarchical cueing

In the individual programme $(\mathrm{PwD} ; \mathrm{n}=10)$, participants received the same training but without involvement of the caregiver

Both programmes involved 1-hour weekly sessions over a period of 8 weeks and were delivered by a research assistant

Dyads in the control condition $(\mathrm{PwD} ; \mathrm{n}=10)$ received no intervention

\begin{tabular}{ll}
\hline Outcomes & $\begin{array}{l}\text { Outcomes included individual and collaborative recall. Burden and depressive symptoms among care- } \\
\text { givers were also assessed }\end{array}$ \\
\hline Country & Sweden \\
\hline Registration status\&\#160; & No information provided; presumed to be unregistered \\
\hline Conflict of Interests\&\#160; & Not stated
\end{tabular}


Neely 2009 (Continued)

Notes

\section{Risk of bias}

\begin{tabular}{lll}
\hline Bias & Authors' judgement & Support for judgement \\
\hline $\begin{array}{l}\text { Random sequence genera- } \\
\text { tion (selection bias) }\end{array}$ & Unclear risk & $\begin{array}{l}\text { Although study authors described an RCT, they provided no information on the } \\
\text { method of randomisation }\end{array}$ \\
\hline
\end{tabular}

\begin{tabular}{|c|c|c|}
\hline $\begin{array}{l}\text { Allocation concealment } \\
\text { (selection bias) }\end{array}$ & High risk & $\begin{array}{l}\text { Study authors did not mention allocation concealment; for this reason, we as- } \\
\text { sumed this was not done }\end{array}$ \\
\hline $\begin{array}{l}\text { Blinding of participants } \\
\text { and personnel (perfor- } \\
\text { mance bias) } \\
\text { All outcomes }\end{array}$ & High risk & $\begin{array}{l}\text { Study authors did not mention blinding of participants; study included a pas- } \\
\text { sive control condition, so blinding was not possible }\end{array}$ \\
\hline $\begin{array}{l}\text { Blinding of outcome as- } \\
\text { sessment (detection bias) } \\
\text { All outcomes }\end{array}$ & High risk & No blinding; the outcome could have been influenced by lack of blinding \\
\hline $\begin{array}{l}\text { Incomplete outcome data } \\
\text { (attrition bias) } \\
\text { All outcomes }\end{array}$ & Low risk & $\begin{array}{l}\text { No data were missing, other than memory test performance for } 1 \text { participant; } \\
\text { no reason was given for this }\end{array}$ \\
\hline $\begin{array}{l}\text { Selective reporting (re- } \\
\text { porting bias) }\end{array}$ & High risk & $\begin{array}{l}\text { Study authors did not present results for all outcomes mentioned in the } \\
\text { "Methods" section }\end{array}$ \\
\hline Other bias & Low risk & Study appears to be free of other sources of bias \\
\hline
\end{tabular}

Quayhagen 1995

$\begin{array}{ll}\text { Methods } & \begin{array}{l}\text { AnCT comparing effects of a cognitive remediation intervention and a placebo and a wait-list control } \\ \text { on functional outcomes in persons with AD }\end{array}\end{array}$

Participants 95 dyads (care recipient+carer) participated in the study. Care recipients were community-dwelling persons with a diagnosis of possible or probable Alzheimer's disease, with mild to moderate decline. Mean age of the sample was $73.6(8.0)$ years, and average education level was 12.6 (4.1) years. 51 participants were male and 27 were female. Participants were white (85\%), African-American (3\%), and Hispanic $(11 \%)$

Interventions Participants in the experimental condition $(n=25)$ trained on a cognitive remediation intervention,
which consisted of active cognitive stimulation focusing on memory, problem solving, and conversa-
tion activities, and was executed by the family caregiver 1 hour daily, 6 days a week, in the home

Participants in the "placebo condition" $(n=28)$ participated in similar activities to those in the experimental condition, but using a passive approach

The care recipient and caregivers from both treatment conditions attended 12 consecutive weekly inhome sessions, where they were trained in programme implementation techniques

Participants in the wait-list control condition $(n=25)$ did not receive any intervention until the trial had been completed 
Quayhagen 1995 (Continued)

Outcomes

Outcomes included global cognition, as well performance in specific cognitive domains such as memory, fluency, problem-solving, attention, and behavioural function. Assessments occurred at baseline, immediately after the intervention, and 6 months later

\begin{tabular}{ll}
\hline Country & United States of America \\
\hline Registration status\&\#160; & No information provided; presumed to be unregistered \\
\hline Conflict of Interests\&\#160; & Not stated \\
\hline Notes & $\begin{array}{l}\text { Comparison of the training programme vs a shortened version used in subsequent work is covered in } \\
\text { Quayhagen \& Quayhagen } 2001\end{array}$
\end{tabular}

\section{Risk of bias}

\begin{tabular}{|c|c|c|}
\hline Bias & Authors' judgement & Support for judgement \\
\hline $\begin{array}{l}\text { Random sequence genera- } \\
\text { tion (selection bias) }\end{array}$ & Unclear risk & $\begin{array}{l}\text { Although study authors described an RCT, they provided no information on the } \\
\text { method of randomisation }\end{array}$ \\
\hline $\begin{array}{l}\text { Allocation concealment } \\
\text { (selection bias) }\end{array}$ & High risk & $\begin{array}{l}\text { Study authors did not mention allocation concealment; for this reason, we as- } \\
\text { sumed this was not done }\end{array}$ \\
\hline $\begin{array}{l}\text { Blinding of participants } \\
\text { and personnel (perfor- } \\
\text { mance bias) } \\
\text { All outcomes }\end{array}$ & High risk & $\begin{array}{l}\text { Study authors did not mention blinding of participants; the study included a } \\
\text { passive control condition, so blinding was not possible }\end{array}$ \\
\hline $\begin{array}{l}\text { Blinding of outcome as- } \\
\text { sessment (detection bias) } \\
\text { All outcomes }\end{array}$ & Unclear risk & $\begin{array}{l}\text { Given the numerous subjective outcomes and the lack of detail regarding the } \\
\text { number of situations in which blinding was not kept, it is unclear whether risk } \\
\text { of bias was increased }\end{array}$ \\
\hline $\begin{array}{l}\text { Incomplete outcome data } \\
\text { (attrition bias) } \\
\text { All outcomes }\end{array}$ & High risk & $\begin{array}{l}\text { "Data is available only for the participants who completed the study" } \\
\text { Insufficient information is provided regarding reasons for attrition (only } 79 \text { of } \\
\text { the } 95 \text { initially included families completed the study) }\end{array}$ \\
\hline $\begin{array}{l}\text { Selective reporting (re- } \\
\text { porting bias) }\end{array}$ & High risk & $\begin{array}{l}\text { Results were not presented for all outcomes mentioned in the "Methods" sec- } \\
\text { tion }\end{array}$ \\
\hline Other bias & Low risk & \\
\hline
\end{tabular}

\section{Quayhagen 2000}

\begin{tabular}{ll} 
Methods & $\begin{array}{l}\text { An RCT comparing } 4 \text { treatment conditions (cognitive stimulation, dyadic counselling, dual supportive } \\
\text { seminar, and early-stage daycare) and a wait-list control condition in people with dementia and their } \\
\text { caregivers (spouses) }\end{array}$ \\
\hline Participants & $\begin{array}{l}103 \text { participant dyads (caregiver and dementia-diagnosed spouse) were recruited for the study. Partic- } \\
\text { ipants (65 men/38 women, mean age } 74.5, \text { mean education level } 14.5 \text { years) had to receive a diagnosis } \\
\text { of possible or probable AD, cardiovascular dementia, or Parkinson's dementia, all at mild to moderate } \\
\text { stages }\end{array}$ \\
\hline Interventions & $\begin{array}{l}\text { Participants in the cognitive stimulation condition }(n=21) \text { completed a home-based remediation pro- } \\
\text { gramme in which the caregiver was the intervening agent who helped to cognitively stimulate the per- } \\
\text { son with dementia }\end{array}$ \\
\hline
\end{tabular}


Participants in the dyadic counselling condition $(n=29)$ also completed a home-based intervention involving take-home tasks used to enhance the interaction and to improve learning of problem-solving skills. The intervention had an affective orientation

Participants in the dual supportive seminar intervention condition $(n=22)$ participated in meetings that aimed to enhance communication between persons with dementia and their caregivers

Participants in the early-stage daycare programme condition $(n=16)$ took part in group-based activities. Persons with dementia met for 4 hours per week and engaged in structured activities that aimed to enhance their remaining strengths and abilities. Caregivers met once a month in a support group

Each of the interventions was delivered over 8 weeks. The first 3 interventions were delivered over a total of 1.5 hours per week, whereas the early-stage daycare programme was delivered for a total of 4 weekly hours for patients, and in 2 sessions for caregivers

Trained graduate students and licensed clinical personnel from psychology, social work, and nursing delivered the interventions and assessments

Participants in the wait-list control condition $(n=15)$ received no treatment but were instead randomised to 1 of the 4 treatment conditions at the end of the study

Outcomes

Both patient and caregiver outcomes were measured. Patient outcomes included immediate and delayed memory, verbal fluency, problem-solving, and behavioural symptoms. Caregiver outcomes included marital satisfaction, emotional status, morale, physical health status, stress, coping, and social support

\begin{tabular}{ll}
\hline Country & United States of America \\
\hline Registration status\&\#160; & No information provided; presumed to be unregistered \\
\hline Conflict of Interests\&\#160; & Not stated \\
\hline Notes & $\begin{array}{l}\text { Although study authors reported in the article that treatment groups did not differ in terms of age, edu- } \\
\text { cation, or racial distribution, they did not provide demographic data at the group level }\end{array}$ \\
\hline
\end{tabular}

\section{Risk of bias}

\begin{tabular}{|c|c|c|}
\hline Bias & Authors' judgement & Support for judgement \\
\hline $\begin{array}{l}\text { Random sequence genera- } \\
\text { tion (selection bias) }\end{array}$ & Unclear risk & $\begin{array}{l}\text { Although study authors described an RCT, they provided no information on the } \\
\text { method of randomisation }\end{array}$ \\
\hline $\begin{array}{l}\text { Allocation concealment } \\
\text { (selection bias) }\end{array}$ & High risk & $\begin{array}{l}\text { Study authors did not mention allocation concealment; for this reason, we as- } \\
\text { sumed this was not done }\end{array}$ \\
\hline $\begin{array}{l}\text { Blinding of participants } \\
\text { and personnel (perfor- } \\
\text { mance bias) } \\
\text { All outcomes }\end{array}$ & High risk & $\begin{array}{l}\text { Study authors did not mention blinding of participants; the study included a } \\
\text { passive control condition, so blinding was not possible }\end{array}$ \\
\hline $\begin{array}{l}\text { Blinding of outcome as- } \\
\text { sessment (detection bias) } \\
\text { All outcomes }\end{array}$ & Low risk & $\begin{array}{l}\text { "The assessment team was blinded to the condition to which the unit was ran- } \\
\text { domised" }\end{array}$ \\
\hline $\begin{array}{l}\text { Incomplete outcome data } \\
\text { (attrition bias) } \\
\text { All outcomes }\end{array}$ & Unclear risk & $\begin{array}{l}\text { Study authors provided no information on whether all randomised dyads had } \\
\text { completed the post-treatment evaluation }\end{array}$ \\
\hline
\end{tabular}


Quayhagen 2000 (Continued)

$\begin{array}{ll}\begin{array}{l}\text { Selective reporting (re- } \\ \text { porting bias) }\end{array} \quad \text { Low risk } & \begin{array}{l}\text { All tests mentioned in the "Methods" section were reported in the "Results" } \\ \text { section }\end{array}\end{array}$

Other bias Low risk Study appears to be free of other sources of bias

\section{Quintana Hernandez 2014}

\begin{tabular}{|c|c|}
\hline Methods & $\begin{array}{l}\text { An RCT comparing effects of } 3 \text { treatments (mindfulness, progressive muscular relaxation, and cognitive } \\
\text { stimulation) and a control intervention on } A D\end{array}$ \\
\hline Participants & $\begin{array}{l}127 \text { participants over } 65 \text { years of age with a diagnosis of probable AD, according to NINCDS-ADRDA } \\
\text { (MMSE scores over 18). All were community dwellers and were taking donepezil. Most had completed } \\
\text { primary studies. Mean age across groups was } 80.11(6.74)\end{array}$ \\
\hline \multirow[t]{4}{*}{ Interventions } & $\begin{array}{l}\text { Participants in the IPP condition }(n=32) \text { were trained on an integral psychostimulation programme } 3 \\
\text { times a week over } 2 \text { years, in } 90 \text {-minute sessions }\end{array}$ \\
\hline & $\begin{array}{l}\text { Participants in both mindfulness }(n=36) \text { and muscular relaxation }(n=34) \text { conditions were trained with } \\
\text { the same frequency, for the same length of time }\end{array}$ \\
\hline & Three independent clinical psychologists delivered the interventions \\
\hline & Participants in the control condition $(n=25)$ received no intervention \\
\hline Outcomes & $\begin{array}{l}\text { Outomes included global cognition, neuropsychiatric symptoms, and clinical dementia severity. Partic- } \\
\text { ipants were assessed before and after the intervention period, and } 3 \text { additional evaluations were con- } \\
\text { ducted at } 6,12 \text {, and } 18 \text { months during the intervention years }\end{array}$ \\
\hline Country & Spain \\
\hline Registration status\&\#160; & No information provided; presumed to be unregistered \\
\hline Conflict of Interests\&\#160; & No \\
\hline Notes & \\
\hline
\end{tabular}

\section{Risk of bias}

\begin{tabular}{lll}
\hline Bias & Authors' judgement & Support for judgement \\
\hline $\begin{array}{l}\text { Random sequence genera- } \\
\text { tion (selection bias) }\end{array}$ & Unclear risk & $\begin{array}{l}\text { Although study authors described a randomised controlled trial, they provided } \\
\text { no information on the method of randomisation }\end{array}$ \\
\hline $\begin{array}{l}\text { Allocation concealment } \\
\text { (selection bias) }\end{array}$ & High risk & $\begin{array}{l}\text { Study authors did not mention allocation concealment; for this reason, we as- } \\
\text { sumed this was not done }\end{array}$ \\
\hline $\begin{array}{l}\text { Blinding of participants } \\
\begin{array}{l}\text { and personnel (perfor- } \\
\text { mance bias) } \\
\text { All outcomes }\end{array}\end{array}$ & Low risk & Study is described as using a double-blind design \\
\hline $\begin{array}{l}\text { Blinding of outcome as- } \\
\text { sessment (detection bias) } \\
\begin{array}{l}\text { All outcomes } \\
\hline\end{array}\end{array}$ & Unclear risk & $\begin{array}{l}\text { Unclear; study authors stated, "assessments were carried out by the neuropsy- } \\
\text { chologist, who was not involved in the delivery of the treatments" }\end{array}$ \\
\hline
\end{tabular}


Quintana Hernandez 2014 (Continued)

$\begin{array}{ll}\begin{array}{l}\text { Incomplete outcome data } \\ \text { (attrition bias) }\end{array} & \begin{array}{l}\text { The study flow chart shows that } 7 \text { participants died while the study was be- } \\ \text { ing conducted. Remaining participants completed the interventions and were } \\ \text { all outcomes }\end{array} \\ \text { analysed }\end{array}$

(attrition bias)

All outcomes ing conducted. Remaining participants completed the interventions and were nalysed
Low risk porting bias)
All tests mentioned in the "Methods" section were reported in the "Results" section

Other bias Low risk Study appears to be free of other sources of bias

Serino 2017

$\begin{array}{ll}\text { Methods } & \begin{array}{l}\text { A single-centre RCT evaluating whether a novel VR-based training protocol can improve general spatial } \\ \text { abilities in patients with } A D\end{array}\end{array}$

Participants 20 participants aged 65 years old were recruited from an Italian social senior centre. All met NINCDS-
ARDRA criteria, and all met criteria for probable dementia on the Milan Overall Dementia Scale
Mean age of participants in the VR condition ( 9 women, 1 man) was 86.60 (SD 6.13), and average years
of education was 9.80 (SD 3.97)
Participants in the control condition ( 8 women, 2 men) had a mean age of 88.70 (SD 3.59), and on aver-
age they received 7.00 years of education (SD 5.00)
8 cognitively unimpaired age-matched participants (4 women, 4 men) also received VR-based training.
Mean age for this group was 86.62 (SD 6.19); mean education level was 9.12 years (SD 5.05)

Interventions

Participants in the virtual reality $(V R)$-based condition $(A D ; n=10)$ underwent a VR programme developed to train their ability to sync between allocentric viewpoint-dependent and allocentric viewpoint-independent representations. The training programme consisted of 10 sessions for 3 to 4 consecutive weeks, with approximately 3 sessions each week. Each session contained an "encoding phase" and a "retrieval phase" and was based on (virtually) navigating a virtual city to find hidden objects

Participants in the control condition $(n=10)$ participated in "traditional cognitive rehabilitative activities" (i.e. cognitive stimulation programs, such as card games, naming, fluency, and music listening)

Eight cognitively healthy age-matched participants also participated in VR-based training ("VR Group Normal Aging"). This group was not taken into consideration for this review

Both interventions were delivered by neuropsychologists

$\begin{array}{ll}\text { Outcomes } & \text { Outcomes included global cognitive function, as well as performance in specific cognitive domains } \\ \text { such as executive functions, selective attention, short-term memory abilities, and short and long-term } \\ \text { spatial memory abilities. The battery was given at baseline and then after the intervention, } 3 \text { to } 4 \text { weeks } \\ \text { later }\end{array}$

Country Italy

\begin{tabular}{ll}
\hline Registration status\&\#160; & No information provided; presumed to be unregistered \\
\hline Conflict of Interests\&\#160; & No
\end{tabular}

\begin{tabular}{ll}
\hline Notes & Study authors sent a table with post-intervention scores upon request; they clarified that participants \\
had mild to moderate dementia
\end{tabular}

\section{Risk of bias}


Serino 2017 (Continued)

Random sequence genera- Unclear risk Although study authors described an RCT, they provided no information on the tion (selection bias) method of randomisation

Allocation concealment High risk (selection bias)

\section{Blinding of participants} and personnel (perfor-

mance bias)

All outcomes
Unclear risk
Study authors did not mention allocation concealment; for this reason, we assumed this was not done

Study authors did not mention blinding of participants; they compared CT vs an active condition, so blinding may have been possible

$\begin{array}{lll}\text { Blinding of outcome as- } & \text { High risk } & \text { Study authors provided no details to suggest that outcome assessment was } \\ \text { sessment (detection bias) } & \text { blind; it is likely that this was not the case }\end{array}$

All outcomes

\section{Incomplete outcome data Unclear risk}

(attrition bias)

Study authors provided no details regarding any discontinuation, but equally, All outcomes whether all participants were assessed post intervention remains unclear

\begin{tabular}{lll}
\hline $\begin{array}{l}\text { Selective reporting (re- } \\
\text { porting bias) }\end{array}$ & Low risk & $\begin{array}{l}\text { Some scores for the post-intervention assessment are missing, but we were } \\
\text { able to obtain them from study authors }\end{array}$ \\
\hline Other bias & Low risk & Study appears to be free of other sources of bias \\
\hline
\end{tabular}

\section{Trebbastoni 2018}

\begin{tabular}{ll}
\hline Methods & A single-blind RCT comparing effects on cognition of a group CT programme vs a no intervention con- \\
trol condition for persons with mild to moderate AD
\end{tabular}

\section{Participants}

140 community-dwelling participants ( 78 women) between 50 and 85 years of age with a diagnosis of Alzheimer-type dementia, according to NINCDS-ADRDA, were recruited in Rome, Italy

Interventions

Participants in the intervention condition $(n=54)$ participated in group CT sessions that involved paper-and-pencil tasks, as well as verbal-learning exercises. Participants were trained twice a week over 24 weeks

Participants assigned to the control condition $(n=86)$ received usual care at the hospital

\begin{tabular}{ll}
\hline Outcomes & $\begin{array}{l}\text { Oucomes included performance on several cognitive domains, including memory, attention, language, } \\
\text { visuospatial functions, frontal functions, and praxis } \\
\text { Participants were assessed at baseline, post intervention, and } 6 \text { months later }\end{array}$
\end{tabular}

\begin{tabular}{|c|c|}
\hline Country & Italy \\
\hline Registration status\&\#160; & No information provided; presumed to be unregistered \\
\hline Conflict of Interests\&\#160; & No \\
\hline Notes & $\begin{array}{l}\text { Six participants from the experimental group }(n=54) \text { were included only in the first part of the study } \\
\text { (from T0 to T1), but not from T1 to T2. They were excluded because they had attended less than } 80 \% \text { of } \\
\text { the sessions. Between T1 and T2, } 3 \text { participants were excluded from this group ( } 1 \text { loss to follow-up; } 1 \\
\text { decision to withdraw; } 1 \text { death). Therefore, } 54 \text { participants in the experimental group were assessed at } \\
\text { T1, but only } 45 \text { were assessed at T2 ( } 6 \text { months' follow-up) }\end{array}$ \\
\hline & $\begin{array}{l}\text { Study authors provided the original paper along with clarification on a discrepancy between the same } \\
\text { score in } 2 \text { different tables }\end{array}$ \\
\hline
\end{tabular}


Trebbastoni 2018 (Continued)

Risk of bias

\begin{tabular}{lll}
\hline Bias & Authors' judgement & Support for judgement \\
\hline $\begin{array}{l}\text { Random sequence genera- } \\
\text { tion (selection bias) }\end{array}$ & Low risk & "The ratio of intended numbers of participants in each of the comparison \\
& & $\begin{array}{l}\text { groups depended on the resources available at our site. Hence, we used a ran- } \\
\text { dom numbering with an unequal allocation ratio of } 1: 2(1 \text { treated:2 untreated). } \\
\text { Ten days prior to the commencement of the training, we computer-generat- } \\
\text { ed a randomisation list that assigned the patients belonging to the TG to nine } \\
\text { treatment sub-groups" }\end{array}$ \\
& \\
&
\end{tabular}

\begin{tabular}{|c|c|c|}
\hline $\begin{array}{l}\text { Allocation concealment } \\
\text { (selection bias) }\end{array}$ & Unclear risk & $\begin{array}{l}\text { Study authors stated that they used a computerised randomisation system. It } \\
\text { is likely that allocation concealment was done, but this is not specified }\end{array}$ \\
\hline
\end{tabular}

\begin{tabular}{lll}
\hline $\begin{array}{l}\text { Blinding of participants } \\
\text { and personnel (perfor- }\end{array}$ & High risk & $\begin{array}{l}\text { Study authors did not mention blinding of participants; the study included a } \\
\text { passive control condition, so blinding was not possible }\end{array}$
\end{tabular}
mance bias)

All outcomes

\begin{tabular}{|c|c|c|}
\hline $\begin{array}{l}\text { Blinding of outcome as- } \\
\text { sessment (detection bias) } \\
\text { All outcomes }\end{array}$ & Low risk & $\begin{array}{l}\text { "Independent expert evaluators, who were blinded to the treated or untreated } \\
\text { status of the patients, recorded the outcome measures at T0, T1 and T2" }\end{array}$ \\
\hline \multirow{2}{*}{$\begin{array}{l}\text { Incomplete outcome data } \\
\text { (attrition bias) } \\
\text { All outcomes }\end{array}$} & High risk & $\begin{array}{l}\text { Researchers stated, "We analysed the data of all the participants who conclud- } \\
\text { ed the study and were tested at T2" }\end{array}$ \\
\hline & & $\begin{array}{l}\text { Nine participants in the experimental condition and one in the control condi- } \\
\text { tion dropped out during the study; their data were not reported, which is likely } \\
\text { to introduce attrition bias }\end{array}$ \\
\hline $\begin{array}{l}\text { Selective reporting (re- } \\
\text { porting bias) }\end{array}$ & Low risk & $\begin{array}{l}\text { All tests mentioned in the "Methods" section were reported in the "Results" } \\
\text { section }\end{array}$ \\
\hline Other bias & Low risk & Study appears to be free of other sources of bias \\
\hline
\end{tabular}

\section{Tsantali 2017}

Methods $\quad$ An RCT comparing effects of CT vs cognitive stimulation (CS) on general cognitive function and memory
in mild Alzheimer's disease (mAD)

Participants $\begin{aligned} & 63 \text { mild AD outpatients (according to NINCDS-ADRDA and DSM-IV criteria) were recruited from } 2 \text { mem- } \\ & \text { ory clinics in Greece. Mean age of the sample was } 73.7 \text { (5.3), and age range was } 67 \text { to } 82 \text { years. Partici- } \\ & \text { pants had an average of 9.8 years of education (4.1), with a range of } 6 \text { to } 16 \text { years }\end{aligned}$

Interventions

Participants in the CT condition $(n=17)$ and in the CS condition $(n=17)$ received a 4-month individual intervention programme consisting of 3 individual sessions of 90 minutes per week

Participants in the CS condition engaged in non-complicated cognitive tasks that were not focused on a specific cognitively impaired ability (e.g. drawing, painting, copying figures, listening to music)

Programmes were administered by 4 licensed psychologists

Participants in the control condition $(C D)(n=21)$ received no treatment

Outcomes

Outcomes included global cognition, as well as performance on specific domains such as memory, attention, and language 
Tsantali 2017 (Continued)

Evaluations were conducted immediately post intervention and 8 months after treatment had been completed

\begin{tabular}{|c|c|c|}
\hline Country & \multicolumn{2}{|l|}{ Greece } \\
\hline Registration status\&\#160; & \multicolumn{2}{|c|}{ No information provided; presumed to be unregistered } \\
\hline Conflict of Interests\&\#160; & \multicolumn{2}{|l|}{ No } \\
\hline Notes & \multicolumn{2}{|c|}{$\begin{array}{l}\text { We contacted study authors to request missing scores (mean and SD for each group for general cogni- } \\
\text { tive state measures immediately post intervention), but we did not obtain a reply }\end{array}$} \\
\hline \multicolumn{3}{|l|}{ Risk of bias } \\
\hline Bias & Authors' judgement & Support for judgement \\
\hline $\begin{array}{l}\text { Random sequence genera- } \\
\text { tion (selection bias) }\end{array}$ & Unclear risk & Study authors stated that participants were allocated by lot \\
\hline $\begin{array}{l}\text { Allocation concealment } \\
\text { (selection bias) }\end{array}$ & High risk & $\begin{array}{l}\text { Study authors did not mention allocation concealment; for this reason, we as- } \\
\text { sumed this was not done }\end{array}$ \\
\hline $\begin{array}{l}\text { Blinding of participants } \\
\text { and personnel (perfor- } \\
\text { mance bias) } \\
\text { All outcomes }\end{array}$ & High risk & $\begin{array}{l}\text { Study authors described a single-blind RCT; however, blinding referred to the } \\
\text { outcome assessment }\end{array}$ \\
\hline $\begin{array}{l}\text { Blinding of outcome as- } \\
\text { sessment (detection bias) } \\
\text { All outcomes }\end{array}$ & Low risk & $\begin{array}{l}\text { Study authors stated that all post-baseline assessments were conducted by } \\
\text { blinded assessors }\end{array}$ \\
\hline $\begin{array}{l}\text { Incomplete outcome data } \\
\text { (attrition bias) } \\
\text { All outcomes }\end{array}$ & High risk & $\begin{array}{l}8 \text { participants ( } 4 \text { from each treatment condition) were not seen for post-in- } \\
\text { tervention assessment because they did not complete the intervention for } \\
\text { "health reasons". All participants from the control condition were seen post } \\
\text { treatment. No details were provided about participants who dropped out }\end{array}$ \\
\hline $\begin{array}{l}\text { Selective reporting (re- } \\
\text { porting bias) }\end{array}$ & High risk & Some scores for the post-intervention assessment are missing \\
\hline Other bias & Low risk & Study appears to be free of other sources of bias \\
\hline
\end{tabular}

Venturelli 2016

Methods An RCT aiming to determine whether aerobic exercise (AE) and CT treatments were effective in reducing sundowning symptoms via downregulation of cortisol levels in AD patients. Possible additive effects of combined $A E+C T$ were also assessed

\begin{tabular}{ll}
\hline Participants & 80 AD participants residing in nursing homes who were between 65 and 75 years old, had an MMSE \\
score between 10 and 15 , and had a clinical diagnosis of dementia and neurobehavioural symptoms of \\
sundown syndrome. 58 participants were female and only 22 were male
\end{tabular}

Interventions

Participants in the CT condition $(n=20)$ engaged in a cognition-oriented psychosocial intervention that was based on the reality orientation (RO) method

Participants in the AE intervention condition $(n=20)$ were engaged in walking at moderate intensity 
Venturelli 2016 (Continued)

Participants in the $A E+C T$ intervention condition $(n=20)$ were engaged in the same activity as those in the AE group, but with the caregiver providing cognitive stimulation via the RO method during walking

20 participants in the control condition received no specific treatment beyond standard therapy

Outcomes

Outcomes included global cognition, neuropsychiatric symptoms, agitation, and saliva cortisol as a measure of stress

Assessments were conducted at baseline and immediately post intervention

\begin{tabular}{ll}
\hline Country & Italy \\
\hline Registration status\&\#160; & No information provided; presumed to be unregistered \\
\hline Conflict of Interests\&\#160; & No \\
\hline Notes & $\begin{array}{l}\text { We contacted study authors to request data in the format required (means and SDs for each measure at } \\
\text { all assessment occasions, for each group separately), but we received no reply }\end{array}$ \\
& $\begin{array}{l}\text { For the purposes of this review, we used CT as our experimental condition, AE as an alternative treat- } \\
\text { ment comparison, and no-treatment control as a passive control. We did not take the AE+CT group into } \\
\text { consideration for our analysis }\end{array}$ \\
\hline
\end{tabular}

\section{Risk of bias}

\begin{tabular}{|c|c|c|}
\hline Bias & Authors' judgement & Support for judgement \\
\hline $\begin{array}{l}\text { Random sequence genera- } \\
\text { tion (selection bias) }\end{array}$ & Unclear risk & $\begin{array}{l}\text { Although study authors described an RCT, they provided no information on the } \\
\text { method of randomisation }\end{array}$ \\
\hline $\begin{array}{l}\text { Allocation concealment } \\
\text { (selection bias) }\end{array}$ & High risk & $\begin{array}{l}\text { Study authors did not mention allocation concealment; for this reason, we as- } \\
\text { sumed this was not done }\end{array}$ \\
\hline $\begin{array}{l}\text { Blinding of participants } \\
\text { and personnel (perfor- } \\
\text { mance bias) } \\
\text { All outcomes }\end{array}$ & High risk & $\begin{array}{l}\text { Study authors described a single-blind RCT; however, blinding referred to the } \\
\text { outcome assessment }\end{array}$ \\
\hline $\begin{array}{l}\text { Blinding of outcome as- } \\
\text { sessment (detection bias) } \\
\text { All outcomes }\end{array}$ & Low risk & $\begin{array}{l}\text { Although it is not entirely clear, it appears that evaluations were conducted by } \\
\text { neuropsychologists blinded to group assignment }\end{array}$ \\
\hline $\begin{array}{l}\text { Incomplete outcome data } \\
\text { (attrition bias) } \\
\text { All outcomes }\end{array}$ & Unclear risk & $\begin{array}{l}\text { Whether or not participants dropped out and were not seen during the post-in- } \\
\text { tervention evaluation is not stated and remains unclear }\end{array}$ \\
\hline $\begin{array}{l}\text { Selective reporting (re- } \\
\text { porting bias) }\end{array}$ & Low risk & $\begin{array}{l}\text { All tests mentioned in the "Methods" section were reported in the "Results" } \\
\text { section }\end{array}$ \\
\hline Other bias & Low risk & Study appears to be free of other sources of bias \\
\hline
\end{tabular}

AD: Alzheimer's disease.

ADL: activity of daily living.

AE: adverse event.

CD: control condition.

CDR: Clinical Dementia Rating Scale.

CELP: computerised errorless learning-based memory training programme.

ChEls: cholinesterase inhibitors. 
CS: cognitive stimulation.

$\mathrm{CT}$ : cognitive training.

DSM-IV: Diagnostical and Statistical Manual of Mental Disorders, Fourth Edition.

EEG: electroencephalography.

HRQoL: health-related quality of life.

IADLs: instrumental activities of daily living.

IPP: integrated psychostimulation programme.

LSS: lexical-semantic stimulation.

mAD: mild Alzheimer's disease.

$\mathrm{MCl}$ : mild cognitive impairment.

MCP: memory and coping programme.

MMSE: Mini Mental Status Examination.

NINCDS-ADRDA: National Institute of Neurological and Communicative Disorders and Stroke - Alzheimer's Disease and Related Disorders Association.

NPI: non-pharmacological intervention.

OT: occupational therapist.

PET: positron emission tomography.

PF: Phonetic Fluency

PwD: Person with Dementia.

RAVLT: Rey Auditory Verbal Learning Test.

$\mathrm{RCT}$ : randomised controlled trial.

RO: reality orientation.

ROCF: Rey-Osterrieth Complex Figure Test.

SD: standard deviation.

SR: spaced retrieval.

SR+M: spaced retrieval with Montessori activities.

TELP: therapist-led errorless programme.

TG: Treatment Group.

TMT: Trail Making Test.

VR: virtual reality.

\section{Characteristics of excluded studies [ordered by study ID]}

\begin{tabular}{ll}
\hline Study & Reason for exclusion \\
\hline Actrn 2015 & Does not meet intervention criteria \\
\hline Actrn12616000827437p 2016 & Does not meet intervention criteria \\
\hline Alimova 1990 & Article cannot be found \\
\hline Alves 2014 & Does not meet participant criteria \\
\hline Anderson 2001 & Includes no suitable control \\
\hline Angelucci 2015 & Does not meet participant criteria. \\
\hline Avila 2007 & Not an RCT \\
\hline Baglio 2015 & Does not meet intervention criteria \\
\hline Baltes 1989 & Does not meet participant criteria \\
\hline Bamidis 2015 & Not an RCT \\
\hline Basak 2008 & Does not meet participant criteria \\
\hline
\end{tabular}




\begin{tabular}{|c|c|}
\hline Study & Reason for exclusion \\
\hline Bernhardt 2002 & Article cannot be found \\
\hline Biasutti 2018 & Does not meet participant criteria \\
\hline Boron 2007 & Does not meet participant criteria \\
\hline Brinkman 1982 & Not an RCT \\
\hline Brunelle 2015 & Does not meet intervention criteria \\
\hline Buettner 2011 & Does not meet participant criteria \\
\hline Burgener 2009 & Does not meet intervention criteria \\
\hline Buschert 2011 & Does not meet intervention criteria \\
\hline Ceccato 2012 & Does not meet intervention criteria \\
\hline Chapman 2004 & Does not meet intervention criteria \\
\hline Cheng 2015 & Never published \\
\hline Chew 2015 & Not an RCT \\
\hline Choi 2012 & Never published \\
\hline Cipriani 2006 & Not an RCT \\
\hline Clare 2013 & Does not meet intervention criteria \\
\hline Contador 2016 & Not an RCT \\
\hline Costa 2014 & Does not meet participant criteria \\
\hline Costa 2015 & Never published \\
\hline Danassi 2015 & Never published \\
\hline De Luca 2016 & Includes no suitable control \\
\hline De Paula 2013 & Not enough information provided \\
\hline Dwolatzky 2014 & Not enough information provided \\
\hline Eckroth-Bucher 2009 & Does not meet participant criteria \\
\hline Edwards 2013 & Does not meet participant criteria \\
\hline Faggian 2007 & Does not meet participant criteria \\
\hline Fane 2013 & Never published \\
\hline Farina 2002 & Not an RCT \\
\hline Farina 2006 & Not an RCT \\
\hline
\end{tabular}




\begin{tabular}{|c|c|}
\hline Study & Reason for exclusion \\
\hline Fernandez-Calvo 2010 & Does not meet intervention criteria \\
\hline Fernández-Calvo 2015 & Does not meet intervention criteria \\
\hline Fisher 2016 & Does not meet participant criteria \\
\hline Gaitan 2013 & Does not meet participant criteria \\
\hline Giordano 2010 & Not an RCT \\
\hline Graessel 2011 & Does not meet intervention criteria \\
\hline Grohman 2006 & Does not meet participant criteria \\
\hline Günther 2003 & Does not meet participant criteria \\
\hline Han 2017 & Does not meet intervention criteria \\
\hline Hayashi 2009 & Never published \\
\hline Helcer 2012 & Never published \\
\hline Hochhalter 2004 & Not an RCT \\
\hline Hofmann 2003 & Not an RCT \\
\hline Hopman-Rock 1999 & Does not meet intervention criteria \\
\hline Huntley 2017 & Includes no suitable control \\
\hline Hwang 2012 & Not an RCT \\
\hline Hwang 2015 & Not an RCT \\
\hline Hyer 2014 & Not an RCT \\
\hline Israel 1987 & Does not meet participant criteria \\
\hline Jang 2015 & Not an RCT \\
\hline Jin 2015 & Not an RCT \\
\hline Kanaan 2014 & Not an RCT \\
\hline Kang 2010 & Not an RCT \\
\hline Kawashima 2015 & Not an RCT \\
\hline Kessels 2009 & Does not meet intervention criteria \\
\hline Khurandy 2016 & Article cannot be found \\
\hline Kim 2005 & Does not meet participant criteria \\
\hline Kim, 2016 & Does not meet intervention criteria \\
\hline
\end{tabular}




\begin{tabular}{|c|c|}
\hline Study & Reason for exclusion \\
\hline Kovach 2018 & Does not meet participant criteria \\
\hline Latorre 2010 & Does not meet participant criteria \\
\hline Lee, 2016 & Does not meet intervention criteria \\
\hline Lee, 2016a & Does not meet participant criteria \\
\hline Maci 2012 & Does not meet intervention criteria \\
\hline Maci, a & Does not meet intervention criteria \\
\hline Meguro 2008 & Does not meet intervention criteria \\
\hline Middelstadt 2016 & Does not meet intervention criteria \\
\hline NCT 2005 & Article cannot be found \\
\hline NCT 2011 & Article cannot be found \\
\hline NCT 2012 & Article cannot be found \\
\hline NCT 2013 & Article cannot be found \\
\hline NCT 2016a & Never published \\
\hline Ochmann 2015 & Does not meet intervention criteria \\
\hline Olazaran 2004 & Does not meet participant criteria \\
\hline Optale 2010 & Does not meet participant criteria \\
\hline Oswald 1996 & Not an RCT \\
\hline Park 2009 & Does not meet participant criteria \\
\hline Pietila 2017 & Article cannot be found \\
\hline Poon 2005 & Not enough information provided \\
\hline Poptsi 2017 & Does not meet intervention criteria \\
\hline Quintana-Hernandez 2015 & Does not meet participant criteria \\
\hline Quintana-Hernandez 2016 & Does not meet intervention criteria \\
\hline SeungHyun 2017 & Article cannot be found \\
\hline Umin 2015 & Article cannot be found \\
\hline van Zon 2016 & Not an RCT \\
\hline Viola 2011 & Not an RCT \\
\hline Voigt-Radloff 2017 & Does not meet intervention criteria \\
\hline
\end{tabular}




\begin{tabular}{ll}
\hline Study & Reason for exclusion \\
\hline Yun 2008 & Article cannot be found \\
\hline Zanetti 1997 & Not an RCT \\
\hline Zanetti 2001 & Not an RCT \\
\hline Zarit 1982 & Does not meet participant criteria \\
\hline Zhuang 2013 & Does not meet participant criteria \\
\hline 이효정 2010 & Does not meet intervention criteria. \\
\hline
\end{tabular}

RCT: randomised controlled trial.

Characteristics of ongoing studies [ordered by study ID]

\section{ACTRN12618000600246}

Trial name or title

plinary Home-bAsed Reablement Program (I-HARP)

\section{Methods}

A multi-centre pragmatic parallel-arm stratified randomised trial aiming to determine the effectiveness of I-HARP on functional independence, mobility, quality of life, and depression among people with dementia, their home environmental safety, carer burden and quality of life, and IHARP cost-effectiveness

Participants

176 participants (person with dementia and his/her carer) will be recruited across 3 public hospitals and 2 aged care services. Participants should be over 60 years old, have mild to moderate dementia according to FDRS, stage 4 to 5, have conversational English language ability, and have a cognitively able carer who has at least 4 days or 7 hours per week of contact

Interventions

I-HARP (an adaptation and expansion of a US reablement programme, which addresses common challenges that frail older people commonly experience, including environmental risks for disability, functional decline, and multi-morbidities), which consists of (1) up to 12 home visits of 1.5 hours ( 5 to $6 \times$ occupational therapy (OT), 3 to $4 \times$ registered nurse (RN), plus 2 to 4 additional options of allied health support), tailored to individual needs, (2) minor home modification/home repairs and/or provision of assistive devices (up to value $\$ 1000$ ) to improve home safety, (3) 3 individual carer support sessions of 1.5 hours at beginning, middle, and end of home visits by a case co-ordinator

Control group will be allowed to receive usual care under their hospital or community-based aged care services, which may involve ad hoc nursing and allied health services, and home modifications, without the components of structured cognitive rehabilitation

Outcomes

Primary outcome is mean functional independence score. Secondary outcomes include quality of life, mobility, depressive symptoms, health-related quality of life, carer burden, home environment safety, costs of delivery, costs to participants (including healthcare/aged care/community services, medications, and any other costs associated with falls and minor injuries with dementia and carer workforce participation), incidents of unplanned hospital admission, primary care (GP) visit events, events of residential aged care home admission, incidents of falls and other minor injuries, events of aged care service use, hours of carer paid workforce participation, and other system costs incurred by the healthcare system

Starting date 23 April 2018 (first participant enrolment)

Contact information

Prof Yun-Hee Jeon; yun-hee.jeon@sydney.edu.au 
Notes

Leung 2017

Trial name or title Modifying cognitive trajectories in insidious cognitive decline using computerised CT: methods and current progress of a two-phase randomised controlled trial

$\begin{array}{ll}\text { Methods } & 86 \text { memory clinic patients with documented cognitive decline (at least } 0.5 \text { SD decline in memory } \\ \text { performance between } 2 \text { follow-ups) are currently recruited to a randomised, active controlled tri- } \\ \text { al. Participants are randomly assigned to supervised, multi-domain, intensive CCT for } 3 \text { months } \\ \text { (phase A) followed by } 15 \text { months of monthly booster training (phase B), or } 3 \text { months of active con- } \\ \text { trol intervention (phase A) followed by } 3 \text { months of CCTand } 12 \text { months of no-contact follow-up } \\ \text { (phase B) }\end{array}$

Participants Memory clinic patients with documented cognitive decline (at least 0.5 SD decline in memory per-
formance between 2 follow-ups)

Interventions Supervised, multi-domain, intensive CCT for 3 months (phase A) followed by 15 months of monthly booster training (phase $B$ ), or 3 months of active control intervention (phase A) followed by 3 months of CCT and 12 months of no-contact follow-up (phase B)

Primary outcome is change in a memory composite. Neuropsychological assessments, daily func-
tioning measures, and sleep quality are assessed at baseline and at the end of each phase. Addi-
tional secondary measures include multi-modal neuroimaging, neuroeconomic decision-making,
and genetic predictors of response to training

Starting date

Contact information

Notes

NCT 2016

\begin{tabular}{ll}
\hline Trial name or title & Neurostimulation and cognitive intervention in Alzheimer's disease \\
\hline Methods & Randomised controlled trial; factorial assignment \\
\hline Participants & $\begin{array}{l}\text { Adults } 60 \text { to } 90 \text { years with diagnosis of Alzheimer's disease (according to DSM-IV and NINCDS-ADR- } \\
\text { DA), with a score between } 18 \text { and } 26 \text { on MMSE and CDR of } 1.0\end{array}$ \\
\hline
\end{tabular}

Interventions

Experimental: active tDCS plus real CT

- Participants will receive active transcranial direct current stimulation and real CT

Experimental: sham tDCS plus real CT

- Participants will receive sham transcranial direct current stimulation and real CT

Experimental: active tDCS plus placebo CT

- Participants will receive active transcranial direct current stimulation and placebo CT

Placebo comparator: sham tDCS plus placebo CT 
NCT 2016 (Continued)

- Participants will receive sham transcranial direct current stimulation and placebo CT

Outcomes Primary outcome is change in cognitive function assessed on the Alzheimer's Disease Assessment Scale - cognitive subscale (ADAS-Cog). As secondary outcomes, study authors will measure different cognitive domains, as well as functional ability, behavioural and psychological disturbances, subjective burden among caregivers, electrical activity of the brain, and side effects

\begin{tabular}{ll}
\hline Starting date & May 2016 \\
\hline Contact information & Dr. Suellen M Andrade; suellenandrade@gmail.com \\
\hline
\end{tabular}

Notes

ADAS-Cog: Alzheimer's Disease Assessment Scale - cognitive subscale.

CCT: computerised cognitive training.

CDR: Clinical Dementia Rating Scale.

$\mathrm{CT}$ : cognitive training.

DSM-IV: Diagnostical and Statistical Manual of Mental Disorders, Fourth Edition.

DRS: Dementia Rating Scale

GP: general practitioner.

MMSE: Mini Mental Status Examination.

NINCDS-ADRDA: National Institute of Neurological and Communicative Disorders and Stroke - Alzheimer's Disease and Related Disorders Association.

OT: occupational therapist.

RN: registered nurse.

SD: standard deviation.

tDCS: transcranial direct current stimulation.

\section{DATA AND ANALYSES}

\section{Comparison 1. Cognitive training vs control immediately post intervention}

\begin{tabular}{lllll}
\hline Outcome or subgroup title & $\begin{array}{l}\text { No. of } \\
\text { studies }\end{array}$ & $\begin{array}{l}\text { No. of } \\
\text { partici- } \\
\text { pants }\end{array}$ & Statistical method & Effect size \\
\hline $\begin{array}{l}1 \text { Change in a global measure } \\
\text { of cognition (composite) }\end{array}$ & 26 & 1389 & Std. Mean Difference (Random, 95\% Cl) & $0.42[0.23,0.61]$ \\
\hline $\begin{array}{l}2 \text { Change in a global measure } \\
\text { of cognition (composite)_zero } \\
\text { correlation }\end{array}$ & 26 & 1389 & Std. Mean Difference (Random, 95\% Cl) & $0.24[0.12,0.36]$ \\
\hline $\begin{array}{l}3 \text { Change in a global measure } \\
\text { of cognition }\end{array}$ & 20 & 1288 & Std. Mean Difference (IV, Random, 95\% Cl) & $0.65[0.26,1.05]$ \\
\hline $\begin{array}{l}4 \text { Change in a global measure } \\
\text { of cognition_zero correlation }\end{array}$ & 20 & 1287 & Std. Mean Difference (IV, Random, 95\% Cl) & $0.27[0.04,0.50]$ \\
\hline $\begin{array}{l}5 \text { Change in disease progres- } \\
\text { sion }\end{array}$ & 5 & 215 & Std. Mean Difference (IV, Random, 95\% Cl) & 1.07 [0.59, 1.55] \\
\hline \begin{tabular}{l}
6 Change in delayed memory \\
\hline
\end{tabular} & 11 & 543 & Std. Mean Difference (IV, Random, 95\% Cl) & $0.81[0.29,1.32]$ \\
\hline
\end{tabular}




\begin{tabular}{|c|c|c|c|c|}
\hline Outcome or subgroup title & $\begin{array}{l}\text { No. of } \\
\text { studies }\end{array}$ & $\begin{array}{l}\text { No. of } \\
\text { partici- } \\
\text { pants }\end{array}$ & Statistical method & Effect size \\
\hline $\begin{array}{l}7 \text { Change in immediate memo- } \\
\text { ry }\end{array}$ & 17 & 762 & Std. Mean Difference (IV, Random, 95\% CI) & $0.74[0.37,1.12]$ \\
\hline $\begin{array}{l}8 \text { Change in attention and } \\
\text { working memory }\end{array}$ & 12 & 551 & Std. Mean Difference (IV, Random, 95\% CI) & $0.56[0.08,1.05]$ \\
\hline 9 Change in language (naming) & 5 & 311 & Std. Mean Difference (IV, Random, 95\% CI) & $0.62[0.11,1.12]$ \\
\hline $\begin{array}{l}10 \text { Change in verbal letter flu- } \\
\text { ency }\end{array}$ & 12 & 544 & Std. Mean Difference (IV, Random, 95\% CI) & $0.22[-0.07,0.50]$ \\
\hline $\begin{array}{l}11 \text { Change in verbal category } \\
\text { fluency }\end{array}$ & 9 & 475 & Std. Mean Difference (IV, Random, 95\% CI) & $0.52[0.23,0.81]$ \\
\hline $\begin{array}{l}12 \text { Change in executive func- } \\
\text { tion }\end{array}$ & 11 & 511 & Std. Mean Difference (IV, Random, 95\% CI) & $0.75[0.28,1.22]$ \\
\hline $\begin{array}{l}13 \text { Change in speed of informa- } \\
\text { tion processing }\end{array}$ & 6 & 201 & Std. Mean Difference (IV, Random, 95\% CI) & $0.22[-0.11,0.54]$ \\
\hline $\begin{array}{l}14 \text { Change in meta cognition } \\
\text { (self-reported) }\end{array}$ & 2 & 41 & Std. Mean Difference (IV, Random, 95\% CI) & $0.50[-0.15,1.14]$ \\
\hline $\begin{array}{l}15 \text { Change in meta cognition } \\
\text { (informant-reported) }\end{array}$ & 2 & 56 & Std. Mean Difference (IV, Random, 95\% CI) & $-0.01[-1.29,1.26]$ \\
\hline $\begin{array}{l}16 \text { Change in participants' } \\
\text { mood }\end{array}$ & 8 & 577 & Std. Mean Difference (IV, Random, 95\% CI) & $0.72[-0.10,1.54]$ \\
\hline $\begin{array}{l}17 \text { Change in capacity for ac- } \\
\text { tivities of daily living }\end{array}$ & 10 & 687 & Std. Mean Difference (IV, Random, 95\% CI) & $0.12[-0.11,0.35]$ \\
\hline $\begin{array}{l}18 \text { Change in general health } \\
\text { and quality of life }\end{array}$ & 5 & 630 & Std. Mean Difference (IV, Random, 95\% CI) & $-0.04[-0.38,0.29]$ \\
\hline $\begin{array}{l}19 \text { Change in behavioural and } \\
\text { psychological symptoms of } \\
\text { dementia (BPSD) }\end{array}$ & 6 & 493 & Std. Mean Difference (IV, Random, 95\% CI) & $0.44[-0.34,1.22]$ \\
\hline $\begin{array}{l}20 \text { Participant burden (reten- } \\
\text { tion rates) }\end{array}$ & 17 & 1282 & Odds Ratio (M-H, Random, 95\% Cl) & $0.73[0.37,1.43]$ \\
\hline $\begin{array}{l}21 \text { Change in burden of care } \\
\text { (CAREGIVER) }\end{array}$ & 2 & 405 & Std. Mean Difference (IV, Random, 95\% CI) & $-0.11[-0.36,0.15]$ \\
\hline $\begin{array}{l}22 \text { Change in quality of life } \\
\text { (CAREGIVER) }\end{array}$ & 1 & 36 & Std. Mean Difference (IV, Random, 95\% CI) & $0.16[-0.50,0.83]$ \\
\hline $\begin{array}{l}23 \text { Change in mood and well- } \\
\text { being (CAREGIVER) }\end{array}$ & 1 & 36 & Std. Mean Difference (IV, Random, 95\% CI) & $0.98[0.27,1.68]$ \\
\hline
\end{tabular}


Analysis 1.1. Comparison 1 Cognitive training vs control immediately post intervention, Outcome 1 Change in a global measure of cognition (composite).

\begin{tabular}{|c|c|c|c|c|c|c|}
\hline \multirow{2}{*}{$\begin{array}{l}\text { Study or subgroup } \\
\text { Fernández-Calvo } 2011\end{array}$} & \multirow{2}{*}{$\begin{array}{l}\begin{array}{c}\text { Cognitive } \\
\text { training } \\
\mathbf{N}\end{array} \\
30\end{array}$} & \multirow{2}{*}{$\begin{array}{l}\text { Control } \\
\begin{array}{rr}\text { N } \\
15\end{array}\end{array}$} & \multirow{2}{*}{$\begin{array}{c}\begin{array}{c}\text { Std. Mean } \\
\text { Difference } \\
\text { (SE) }\end{array} \\
-0.4(0.42)\end{array}$} & $\begin{array}{l}\text { Std. Mean Difference } \\
\text { IV, Random, } 95 \% \mathrm{CI}\end{array}$ & \multirow{2}{*}{$\begin{array}{r}\text { Weight } \\
3.05 \%\end{array}$} & \multirow{2}{*}{$\begin{array}{l}\text { Std. Mean Difference } \\
\begin{array}{r}\text { IV, Random, 95\% Cl } \\
-0.44[-1.26,0.38]\end{array}\end{array}$} \\
\hline & & & & \begin{tabular}{l|l}
1 & -
\end{tabular} & & \\
\hline Amieva 2016 & 165 & 153 & $-0.1(0.11)$ & + & $6.61 \%$ & $-0.11[-0.33,0.11]$ \\
\hline Beck 1988 & 10 & 10 & $-0(0.43)$ & - & $2.97 \%$ & $-0.03[-0.87,0.81]$ \\
\hline Koltai 2001 & 14 & 8 & $-0(0.43)$ & 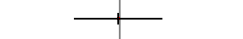 & $2.97 \%$ & $-0.03[-0.87,0.81]$ \\
\hline Cahn-Weiner 2003 & 17 & 17 & $0(0.34)$ & 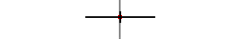 & $3.81 \%$ & $0.01[-0.66,0.68]$ \\
\hline Boller 2011 & 24 & 12 & $0.1(0.35)$ & 1 & $3.7 \%$ & $0.13[-0.56,0.82]$ \\
\hline Quayhagen 2000 & 21 & 15 & $0.2(0.33)$ & 1 & $3.92 \%$ & $0.19[-0.46,0.84]$ \\
\hline Galante 2007 & 7 & 4 & $0.2(0.63)$ & + & $1.77 \%$ & $0.21[-1.02,1.44]$ \\
\hline Barban 2016 & 41 & 38 & $0.2(0.23)$ & + & $5.12 \%$ & $0.21[-0.24,0.66]$ \\
\hline Quayhagen 1995 & 13 & 28 & $0.3(0.33)$ & + & $3.92 \%$ & $0.25[-0.4,0.9]$ \\
\hline Heiss 1993 & 18 & 17 & $0.3(0.34)$ & 1 & $3.81 \%$ & $0.27[-0.4,0.94]$ \\
\hline Serino 2017 & 10 & 10 & $0.3(0.44)$ & + & $2.89 \%$ & $0.31[-0.55,1.17]$ \\
\hline Giuli 2016 & 48 & 47 & $0.4(0.21)$ & + & $5.39 \%$ & $0.37[-0.04,0.78]$ \\
\hline Quayhagen 1995 & 13 & 25 & $0.4(0.34)$ & 1 & $3.81 \%$ & $0.37[-0.3,1.04]$ \\
\hline Kim 2015 & 22 & 21 & $0.5(0.32)$ & - & $4.03 \%$ & $0.46[-0.17,1.09]$ \\
\hline Kawashima 2005 & 16 & 16 & $0.5(0.36)$ & 1 & $3.6 \%$ & $0.52[-0.19,1.23]$ \\
\hline Trebbastoni 2018 & 45 & 85 & $0.6(0.2)$ & $\rightarrow$ & $5.52 \%$ & $0.55[0.16,0.94]$ \\
\hline Jelcic 2014 & 17 & 10 & $0.6(0.4)$ & $\longrightarrow$ & $3.22 \%$ & $0.55[-0.23,1.33]$ \\
\hline Jelcic 2012 & 20 & 20 & $0.6(0.32)$ & 1 & $4.03 \%$ & $0.6[-0.03,1.23]$ \\
\hline Quintana Hernandez 2014 & 27 & 25 & $0.7(0.28)$ & 1 & $4.49 \%$ & $0.73[0.18,1.28]$ \\
\hline de Vreese 1998 & 9 & 9 & $1(0.49)$ & 1 & $2.52 \%$ & $1.04[0.08,2]$ \\
\hline Lee 2013 & 12 & 7 & $1.2(0.53)$ & $\longrightarrow$ & $2.27 \%$ & $1.15[0.11,2.19]$ \\
\hline Cavallo 2016 & 40 & 40 & $1.3(0.28)$ & 1 & $4.49 \%$ & $1.32[0.77,1.87]$ \\
\hline \multicolumn{4}{|c|}{ Total $(95 \% \mathrm{Cl})$} & $\diamond$ & $100 \%$ & $0.42[0.23,0.61]$ \\
\hline \multicolumn{7}{|c|}{ Heterogeneity: $\mathrm{Tau}^{2}=0.13 ; \mathrm{Chi}^{2}=62.8, \mathrm{df}=26(\mathrm{P}<0.0001) ; \mathrm{I}^{2}=58.6 \%$} \\
\hline \multicolumn{4}{|c|}{ Test for overall effect: $Z=4.32(P<0.0001)$} & & & \\
\hline
\end{tabular}

Analysis 1.2. Comparison 1 Cognitive training vs control immediately post intervention, Outcome 2 Change in a global measure of cognition (composite)_zero correlation.

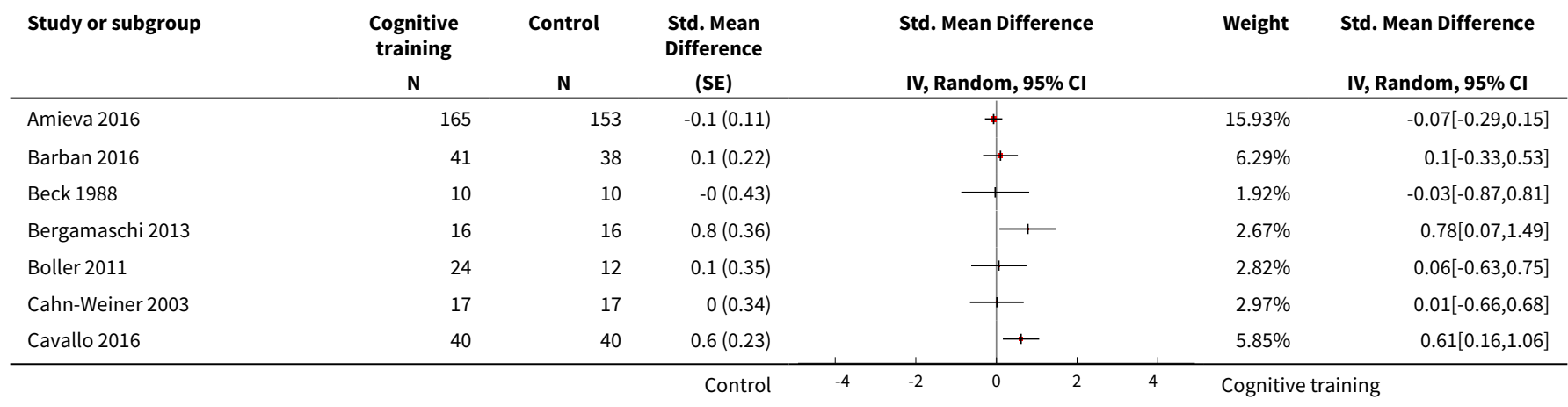




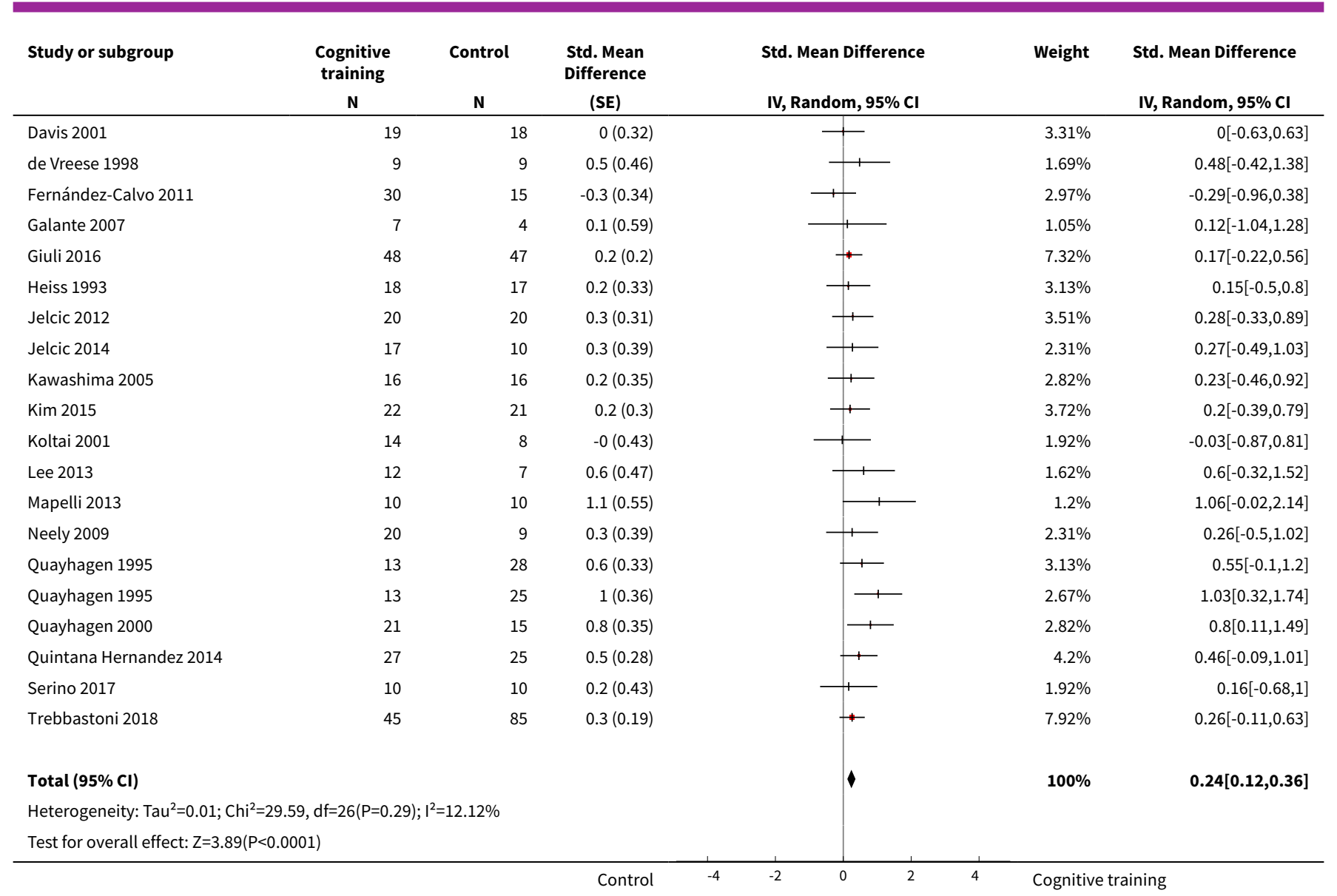

Analysis 1.3. Comparison 1 Cognitive training vs control immediately post intervention, Outcome 3 Change in a global measure of cognition.

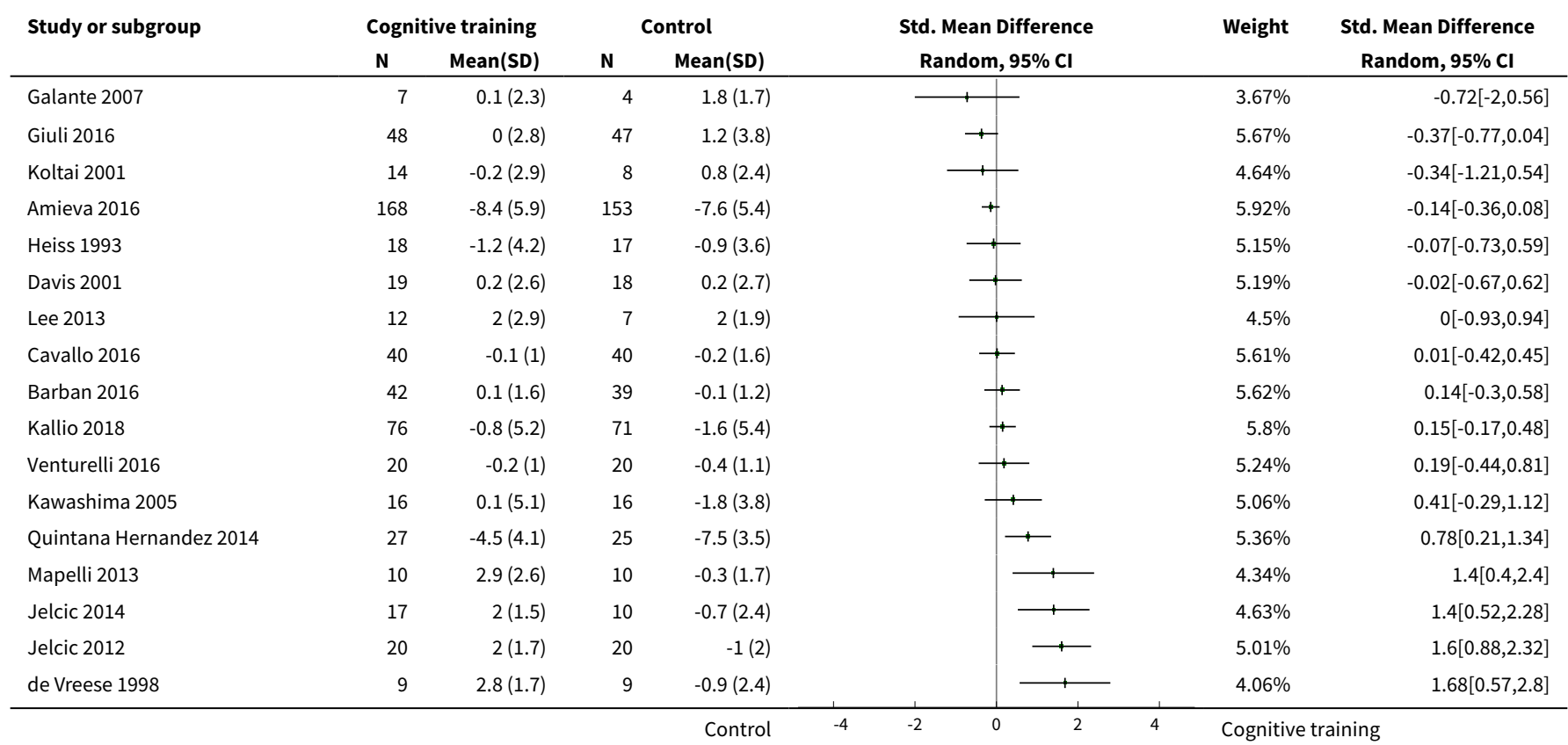




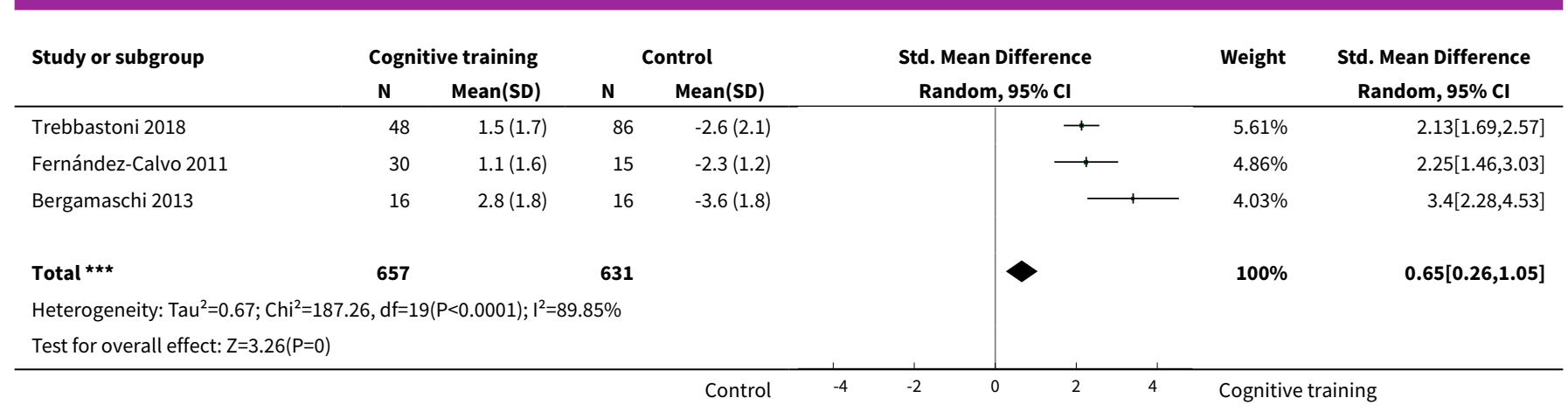

Analysis 1.4. Comparison 1 Cognitive training vs control immediately post intervention, Outcome 4 Change in a global measure of cognition_zero correlation.

\begin{tabular}{|c|c|c|c|c|c|c|c|}
\hline \multirow{3}{*}{$\begin{array}{l}\text { Study or subgroup } \\
\text { Venturelli } 2016\end{array}$} & \multicolumn{2}{|c|}{ Cognitive training } & \multicolumn{2}{|c|}{ Control } & \multirow{2}{*}{$\begin{array}{c}\text { Std. Mean Difference } \\
\text { Random, } 95 \% \mathrm{Cl}\end{array}$} & \multirow{3}{*}{$\begin{array}{r}\text { Weight } \\
5 \%\end{array}$} & \multirow{3}{*}{$\begin{array}{c}\begin{array}{c}\text { Std. Mean Difference } \\
\text { Random, 95\% Cl }\end{array} \\
-0.69[-1.33,-0.05]\end{array}$} \\
\hline & \multirow{2}{*}{$\frac{\mathbf{N}}{20}$} & \multirow{2}{*}{$\frac{\operatorname{Mean}(\mathbf{S D})}{-2(2.1)}$} & \multirow{2}{*}{$\frac{\mathbf{N}}{20}$} & \multirow{2}{*}{$\frac{\operatorname{Mean}(\text { SD) }}{-0.4(2.4)}$} & & & \\
\hline & & & & & 1 & & \\
\hline Galante 2007 & 7 & $0.1(5)$ & 4 & $1.8(3.3)$ & & $2.43 \%$ & $-0.35[-1.59,0.9]$ \\
\hline Koltai 2001 & 14 & $-0.2(5.8)$ & 8 & $0.8(4.8)$ & 1 & $3.77 \%$ & $-0.17[-1.04,0.7]$ \\
\hline Giuli 2016 & 48 & $0(6.2)$ & 47 & $1.2(8.4)$ & $\rightarrow$ & $6.5 \%$ & $-0.16[-0.57,0.24]$ \\
\hline Amieva 2016 & 168 & $-8.4(8.7)$ & 152 & $-7.6(5.4)$ & + & $7.55 \%$ & $-0.11[-0.33,0.11]$ \\
\hline Davis 2001 & 19 & $0.2(5.8)$ & 18 & $0.2(5.9)$ & -1 & $4.97 \%$ & $-0.01[-0.65,0.63]$ \\
\hline Lee 2013 & 12 & $2(6.2)$ & 7 & $2(4.2)$ & & $3.5 \%$ & $0[-0.93,0.93]$ \\
\hline Cavallo 2016 & 40 & $-0.1(2.3)$ & 40 & $-0.2(2.8)$ & + & $6.28 \%$ & $0.01[-0.43,0.45]$ \\
\hline Barban 2016 & 42 & $0.1(3.3)$ & 39 & $-0.1(2.6)$ & & $6.29 \%$ & $0.07[-0.37,0.5]$ \\
\hline Kallio 2018 & 76 & $-0.8(11.6)$ & 71 & $-1.6(12)$ & $\rightarrow$ & $6.99 \%$ & $0.07[-0.26,0.39]$ \\
\hline Kawashima 2005 & 16 & $0.1(11)$ & 16 & $-1.8(8.2)$ & +1 & $4.68 \%$ & $0.19[-0.5,0.89]$ \\
\hline Mapelli 2013 & 10 & $1.1(1.8)$ & 10 & $0.3(0.4)$ & & $3.65 \%$ & $0.56[-0.34,1.45]$ \\
\hline Jelcic 2014 & 17 & $2(3.2)$ & 10 & $-0.7(5)$ & 1 & $4.09 \%$ & $0.67[-0.14,1.47]$ \\
\hline Jelcic 2012 & 20 & $2(3.6)$ & 20 & $-1(4.2)$ & 1 & $4.98 \%$ & $0.75[0.11,1.39]$ \\
\hline de Vreese 1998 & 9 & $2.8(3.5)$ & 9 & $-0.9(5.2)$ & 1 & $3.35 \%$ & $0.79[-0.18,1.75]$ \\
\hline Trebbastoni 2018 & 48 & $1.5(3.7)$ & 86 & $-2.6(4.4)$ & $\rightarrow$ & $6.69 \%$ & $1[0.63,1.37]$ \\
\hline Fernández-Calvo 2011 & 30 & $1.1(3.6)$ & 15 & $-2.3(2.6)$ & 1 & $4.9 \%$ & $1.01[0.35,1.67]$ \\
\hline Bergamaschi 2013 & 16 & $2.8(3.6)$ & 16 & $-3.6(3.6)$ & $\longrightarrow$ & $3.98 \%$ & $1.73[0.9,2.55]$ \\
\hline 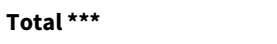 & 657 & & 630 & & $\gamma$ & $100 \%$ & $0.27[0.04,0.5]$ \\
\hline \multicolumn{8}{|c|}{ Heterogeneity: $\operatorname{Tau}^{2}=0.17 ; \mathrm{Chi}^{2}=65.7, \mathrm{df}=19(\mathrm{P}<0.0001) ; \mathrm{I}^{2}=71.08 \%$} \\
\hline \multicolumn{8}{|c|}{ Test for overall effect: $Z=2.29(P=0.02)$} \\
\hline & & & & Control & -2 & Cognitiv & ning \\
\hline
\end{tabular}

Analysis 1.5. Comparison 1 Cognitive training vs control immediately post intervention, Outcome 5 Change in disease progression.

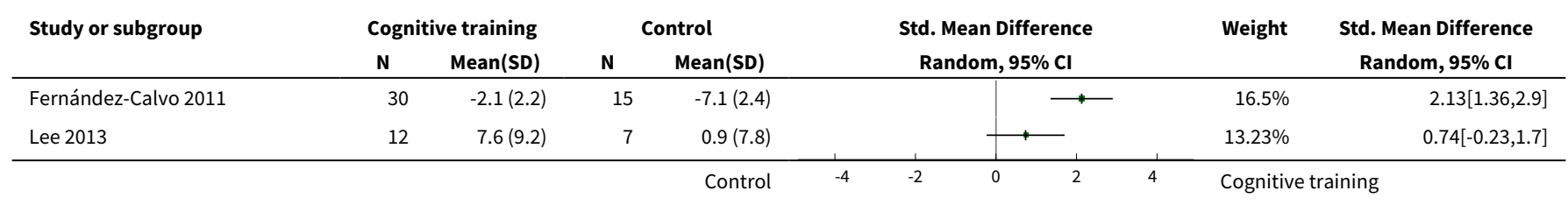




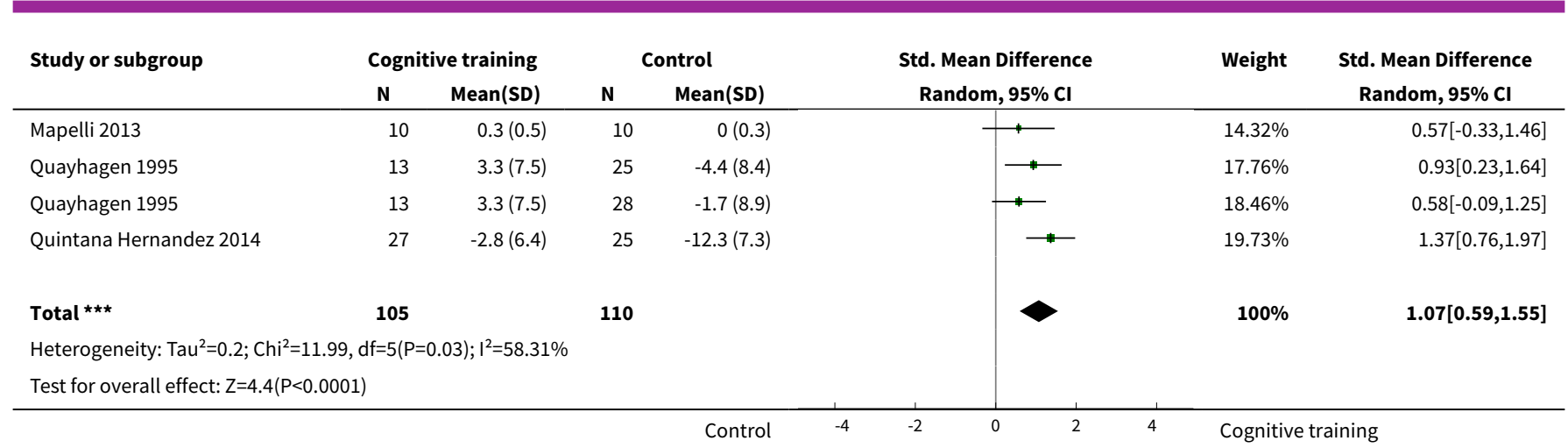

\section{Analysis 1.6. Comparison 1 Cognitive training vs control immediately post intervention, Outcome 6 Change in delayed memory.}

\begin{tabular}{|c|c|c|c|c|c|c|c|}
\hline \multirow[t]{2}{*}{ Study or subgroup } & \multicolumn{2}{|c|}{ Cognitive training } & \multicolumn{2}{|c|}{ Control } & \multirow{2}{*}{$\begin{array}{c}\text { Std. Mean Difference } \\
\text { Random, } 95 \% \mathrm{Cl}\end{array}$} & \multirow[t]{2}{*}{ Weight } & \multirow{2}{*}{$\begin{array}{c}\text { Std. Mean Difference } \\
\text { Random, } 95 \% \mathrm{CI}\end{array}$} \\
\hline & $\mathbf{N}$ & Mean(SD) & $\mathbf{N}$ & $\operatorname{Mean}(S D)$ & & & \\
\hline Davis 2001 & 19 & $1.2(2.2)$ & 18 & $1.7(3.7)$ & $\longrightarrow$ & $9.37 \%$ & $-0.16[-0.81,0.49]$ \\
\hline Boller 2011 & 24 & $-0.3(1.6)$ & 12 & $-0.1(1.3)$ & $\longrightarrow$ & $9.17 \%$ & $-0.14[-0.83,0.56]$ \\
\hline Cahn-Weiner 2003 & 17 & $-0.3(1.1)$ & 17 & $-0.8(1.6)$ & + & $9.23 \%$ & $0.36[-0.31,1.04]$ \\
\hline Quayhagen 2000 & 21 & $3.2(6.9)$ & 15 & $0.4(7.6)$ & —- & $9.28 \%$ & $0.38[-0.29,1.05]$ \\
\hline Barban 2016 & 42 & $0.4(1.2)$ & 39 & $-0.1(1.1)$ & - & $10.16 \%$ & $0.43[-0.01,0.88]$ \\
\hline Koltai 2001 & 14 & $0.6(1.3)$ & 8 & $-0.2(1.8)$ & * & $8.3 \%$ & $0.57[-0.32,1.46]$ \\
\hline Jelcic 2014 & 17 & $0.9(2.2)$ & 10 & $-1.3(1.8)$ & $\longrightarrow$ & $8.54 \%$ & $1.02[0.18,1.85]$ \\
\hline Jelcic 2012 & 20 & $1.5(2.3)$ & 20 & $-0.9(2)$ & $\longrightarrow$ & $9.28 \%$ & $1.07[0.4,1.74]$ \\
\hline Mapelli 2013 & 10 & $4.2(2.4)$ & 10 & $-0.8(1.5)$ & + & $6.84 \%$ & $2.44[1.22,3.65]$ \\
\hline Cavallo 2016 & 40 & $1.2(1)$ & 40 & $-1.7(1)$ & $\longrightarrow$ & $9.42 \%$ & $2.89[2.26,3.53]$ \\
\hline \multicolumn{8}{|c|}{ Heterogeneity: $\mathrm{Tau}^{2}=0.64 ; \mathrm{Chi}^{2}=72.73, \mathrm{df}=10(\mathrm{P}<0.0001) ; \mathrm{I}^{2}=86.25 \%$} \\
\hline \multicolumn{8}{|c|}{ Test for overall effect: $Z=3.05(P=0)$} \\
\hline
\end{tabular}

\section{Analysis 1.7. Comparison 1 Cognitive training vs control immediately} post intervention, Outcome 7 Change in immediate memory.

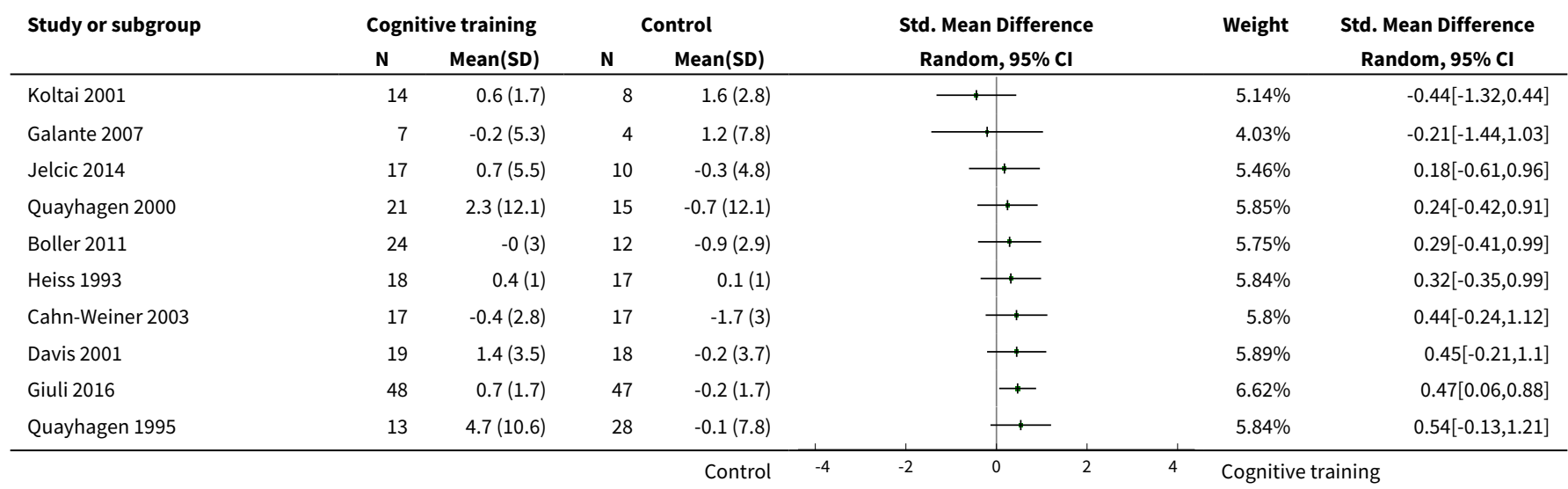




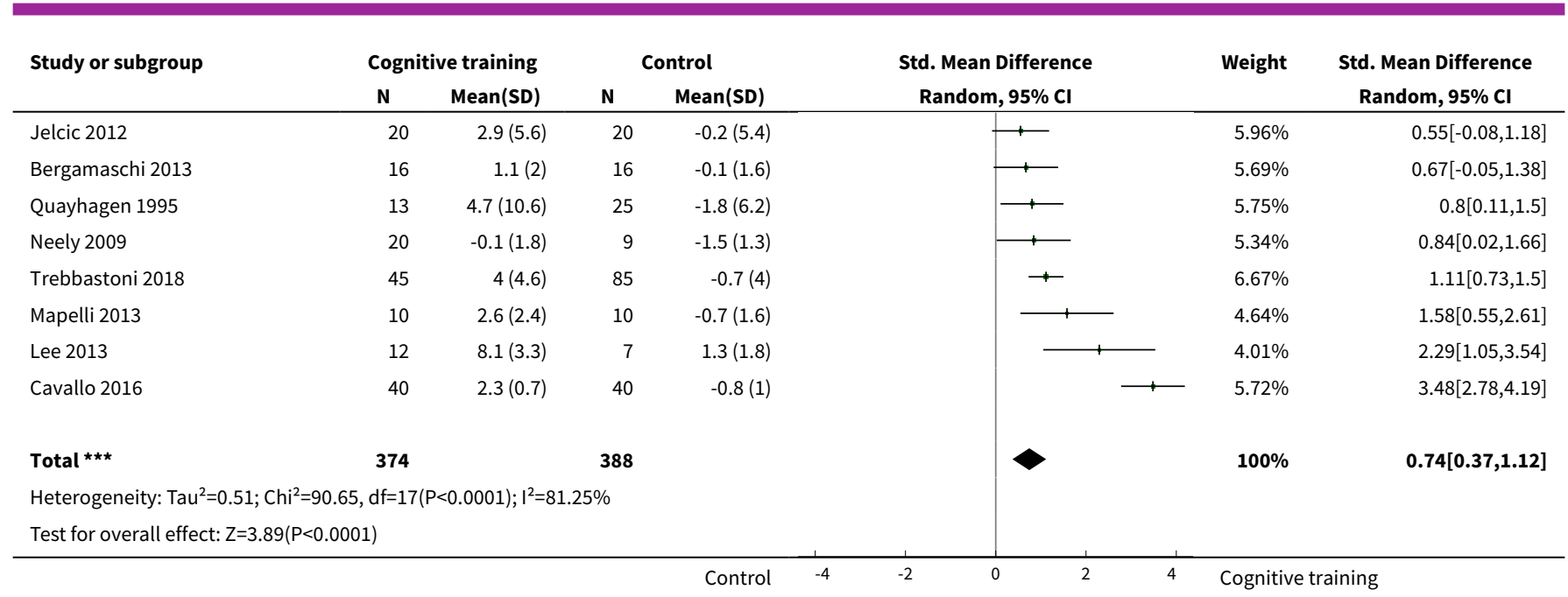

Analysis 1.8. Comparison 1 Cognitive training vs control immediately post intervention, Outcome 8 Change in attention and working memory.

\begin{tabular}{|c|c|c|c|c|c|c|c|}
\hline \multirow[t]{2}{*}{ Study or subgroup } & \multicolumn{2}{|c|}{ Cognitive training } & \multicolumn{2}{|c|}{ Control } & \multirow{2}{*}{$\begin{array}{c}\text { Std. Mean Difference } \\
\text { Random, } 95 \% \mathrm{Cl} \\
\end{array}$} & \multirow[t]{2}{*}{ Weight } & \multirow{2}{*}{$\begin{array}{c}\text { Std. Mean Difference } \\
\text { Random, } 95 \% \mathrm{CI}\end{array}$} \\
\hline & $\mathbf{N}$ & Mean(SD) & $\mathbf{N}$ & Mean(SD) & & & \\
\hline Heiss 1993 & 18 & $-0.6(1)$ & 17 & $-0(1)$ & $\longrightarrow$ & $8.68 \%$ & $-0.6[-1.28,0.08]$ \\
\hline Galante 2007 & 7 & $0.1(0.7)$ & 4 & $0.7(1.5)$ & \begin{tabular}{l|l}
$\longrightarrow$ &
\end{tabular} & $6.15 \%$ & $-0.53[-1.79,0.73]$ \\
\hline Trebbastoni 2018 & 45 & $-0.3(0.6)$ & 85 & $-0.2(0.7)$ & $\leftarrow$ & $9.88 \%$ & $-0.1[-0.46,0.27]$ \\
\hline Boller 2011 & 24 & $0(1.1)$ & 12 & $0.1(0.9)$ & & $8.62 \%$ & $-0.07[-0.77,0.62]$ \\
\hline Davis 2001 & 19 & $-0.4(1.6)$ & 18 & $-0.5(1.6)$ & $\longrightarrow$ & $8.83 \%$ & $0.04[-0.61,0.68]$ \\
\hline Serino 2017 & 10 & $0(0.9)$ & 10 & $-0.3(0.6)$ & $\rightarrow$ & $7.75 \%$ & $0.41[-0.47,1.3]$ \\
\hline Giuli 2016 & 48 & $0.3(0.9)$ & 47 & $-0.2(1)$ & $\rightarrow$ & $9.73 \%$ & $0.55[0.14,0.96]$ \\
\hline Beck 1988 & 10 & $0.7(1)$ & 10 & $-0.2(1.3)$ & & $7.63 \%$ & $0.75[-0.16,1.66]$ \\
\hline Jelcic 2012 & 20 & $0.2(0.6)$ & 20 & $-0.4(0.6)$ & $\longrightarrow$ & $8.73 \%$ & $1.07[0.4,1.74]$ \\
\hline Mapelli 2013 & 10 & $1.7(1.2)$ & 10 & $-0.1(1.3)$ & $\longrightarrow$ & $7.27 \%$ & $1.36[0.37,2.36]$ \\
\hline Jelcic 2014 & 17 & $0.3(1)$ & 10 & $-1.3(0.6)$ & $\longrightarrow$ & $7.49 \%$ & $1.85[0.9,2.8]$ \\
\hline Cavallo 2016 & 40 & $1.9(1)$ & 40 & $-0(0.9)$ & $\longrightarrow$ & $9.24 \%$ & $2.03[1.49,2.57]$ \\
\hline 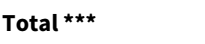 & 268 & & 283 & & & $100 \%$ & $0.56[0.08,1.05]$ \\
\hline \multicolumn{8}{|c|}{ Heterogeneity: $\mathrm{Tau}^{2}=0.59 ; \mathrm{Chi}^{2}=71.95, \mathrm{df}=11(\mathrm{P}<0.0001) ; \mathrm{I}^{2}=84.71 \%$} \\
\hline \multicolumn{8}{|c|}{ Test for overall effect: $Z=2.27(P=0.02)$} \\
\hline
\end{tabular}

Analysis 1.9. Comparison 1 Cognitive training vs control immediately post intervention, Outcome 9 Change in language (naming).

\begin{tabular}{|c|c|c|c|c|c|c|c|}
\hline \multirow[t]{2}{*}{ Study or subgroup } & \multicolumn{2}{|c|}{ Cognitive training } & \multicolumn{2}{|c|}{ Control } & \multirow{2}{*}{$\begin{array}{c}\text { Std. Mean Difference } \\
\text { Random, } 95 \% \mathrm{Cl}\end{array}$} & \multirow[t]{2}{*}{ Weight } & \multirow{2}{*}{$\begin{array}{c}\text { Std. Mean Difference } \\
\text { Random, } 95 \% \mathrm{Cl}\end{array}$} \\
\hline & $\mathbf{N}$ & Mean(SD) & $\mathbf{N}$ & Mean(SD) & & & \\
\hline Cavallo 2016 & 40 & $0.2(1.6)$ & 40 & $0.1(1.3)$ & $\rightarrow$ & $22.87 \%$ & $0.06[-0.38,0.5]$ \\
\hline Cahn-Weiner 2003 & 17 & $0.3(3.6)$ & 17 & $-0.1(3.5)$ & 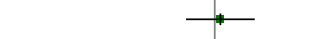 & $18.56 \%$ & $0.11[-0.56,0.78]$ \\
\hline Jelcic 2012 & 20 & $3.5(5.6)$ & 20 & $-0.8(4.6)$ & $\longrightarrow$ & $19.01 \%$ & $0.82[0.17,1.47]$ \\
\hline Jelcic 2014 & 17 & $2.2(2.6)$ & 10 & $-0.4(2.4)$ & $\longrightarrow$ & $15.81 \%$ & $1[0.17,1.83]$ \\
\hline Trebbastoni 2018 & 45 & $2(3.4)$ & 85 & $-1.8(3.3)$ & \# & $23.76 \%$ & $1.13[0.75,1.52]$ \\
\hline
\end{tabular}




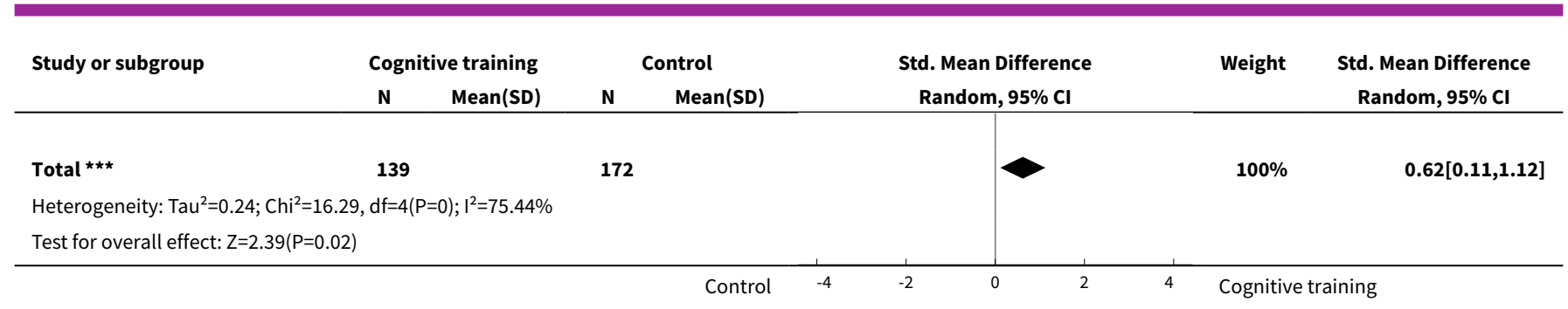

Analysis 1.10. Comparison 1 Cognitive training vs control immediately post intervention, Outcome 10 Change in verbal letter fluency.

\begin{tabular}{|c|c|c|c|c|c|c|c|}
\hline \multirow{3}{*}{$\begin{array}{l}\text { Study or subgroup } \\
\text { Serino } 2017\end{array}$} & \multicolumn{2}{|c|}{ Cognitive training } & \multicolumn{2}{|c|}{ Control } & \multirow{2}{*}{$\begin{array}{c}\text { Std. Mean Difference } \\
\text { Random, } 95 \% \mathrm{Cl}\end{array}$} & \multirow[t]{2}{*}{ Weight } & \multirow{2}{*}{$\begin{array}{c}\text { Std. Mean Difference } \\
\text { Random, } 95 \% \mathrm{Cl}\end{array}$} \\
\hline & $\mathbf{N}$ & $\operatorname{Mean}(\mathrm{SD})$ & $\mathbf{N}$ & $\operatorname{Mean}(\mathrm{SD})$ & & & \\
\hline & 10 & $-1.6(3.5)$ & 10 & $0.2(4.8)$ & $\longrightarrow \mid$ & $6.26 \%$ & $-0.41[-1.3,0.48]$ \\
\hline Cavallo 2016 & 40 & $1.2(1.6)$ & 40 & $1.5(1.6)$ & $\rightarrow$ & $11.55 \%$ & $-0.22[-0.66,0.22]$ \\
\hline Davis 2001 & 19 & $0.2(7.7)$ & 18 & $1.3(9)$ & $\longrightarrow$ & $8.77 \%$ & $-0.13[-0.78,0.52]$ \\
\hline Cahn-Weiner 2003 & 17 & $0.3(6)$ & 17 & $1(4.6)$ & & $8.44 \%$ & $-0.13[-0.8,0.54]$ \\
\hline Barban 2016 & 42 & $1.4(5.7)$ & 39 & $1.1(6)$ & + & $11.61 \%$ & $0.05[-0.39,0.49]$ \\
\hline Jelcic 2014 & 17 & $2.3(7.6)$ & 10 & $1.6(6.9)$ & & $7.25 \%$ & $0.09[-0.69,0.87]$ \\
\hline Kawashima 2005 & 16 & $0.1(0.6)$ & 16 & $0(0.4)$ & + & $8.19 \%$ & $0.2[-0.5,0.89]$ \\
\hline Trebbastoni 2018 & 45 & $-0.2(4.9)$ & 85 & $-2(5)$ & + & $12.64 \%$ & $0.37[0.01,0.74]$ \\
\hline Galante 2007 & 7 & $3.5(5.7)$ & 4 & $-1.7(7)$ & 1 & $3.73 \%$ & $0.77[-0.52,2.06]$ \\
\hline Mapelli 2013 & 10 & $1.2(1.5)$ & 10 & $-0.6(1.1)$ & $\longrightarrow$ & $5.46 \%$ & $1.33[0.34,2.31]$ \\
\hline Bergamaschi 2013 & 16 & $1.1(1.4)$ & 16 & $-1.7(2)$ & 7 & $6.99 \%$ & $1.59[0.78,2.4]$ \\
\hline 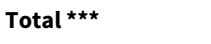 & 259 & & 285 & & 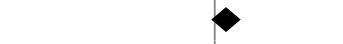 & $100 \%$ & $0.22[-0.07,0.5]$ \\
\hline \multicolumn{8}{|c|}{ Heterogeneity: Tau $^{2}=0.13 ; \mathrm{Chi}^{2}=25.86, \mathrm{df}=11(\mathrm{P}=0.01) ; \mathrm{I}^{2}=57.46 \%$} \\
\hline \multicolumn{8}{|c|}{ Test for overall effect: $Z=1.5(P=0.13)$} \\
\hline & & & & Control & -2 & Cognitiv & ning \\
\hline
\end{tabular}

Analysis 1.11. Comparison 1 Cognitive training vs control immediately post intervention, Outcome 11 Change in verbal category fluency.

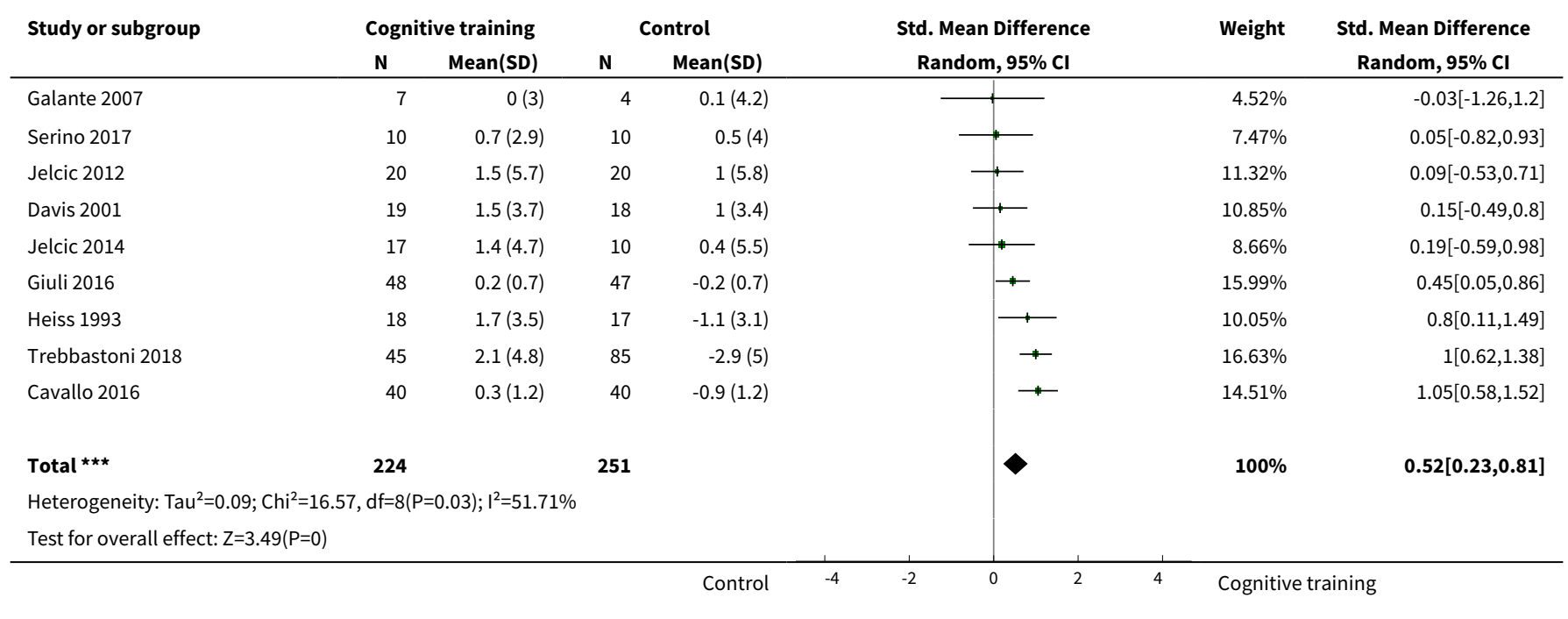


Analysis 1.12. Comparison 1 Cognitive training vs control immediately post intervention, Outcome 12 Change in executive function.

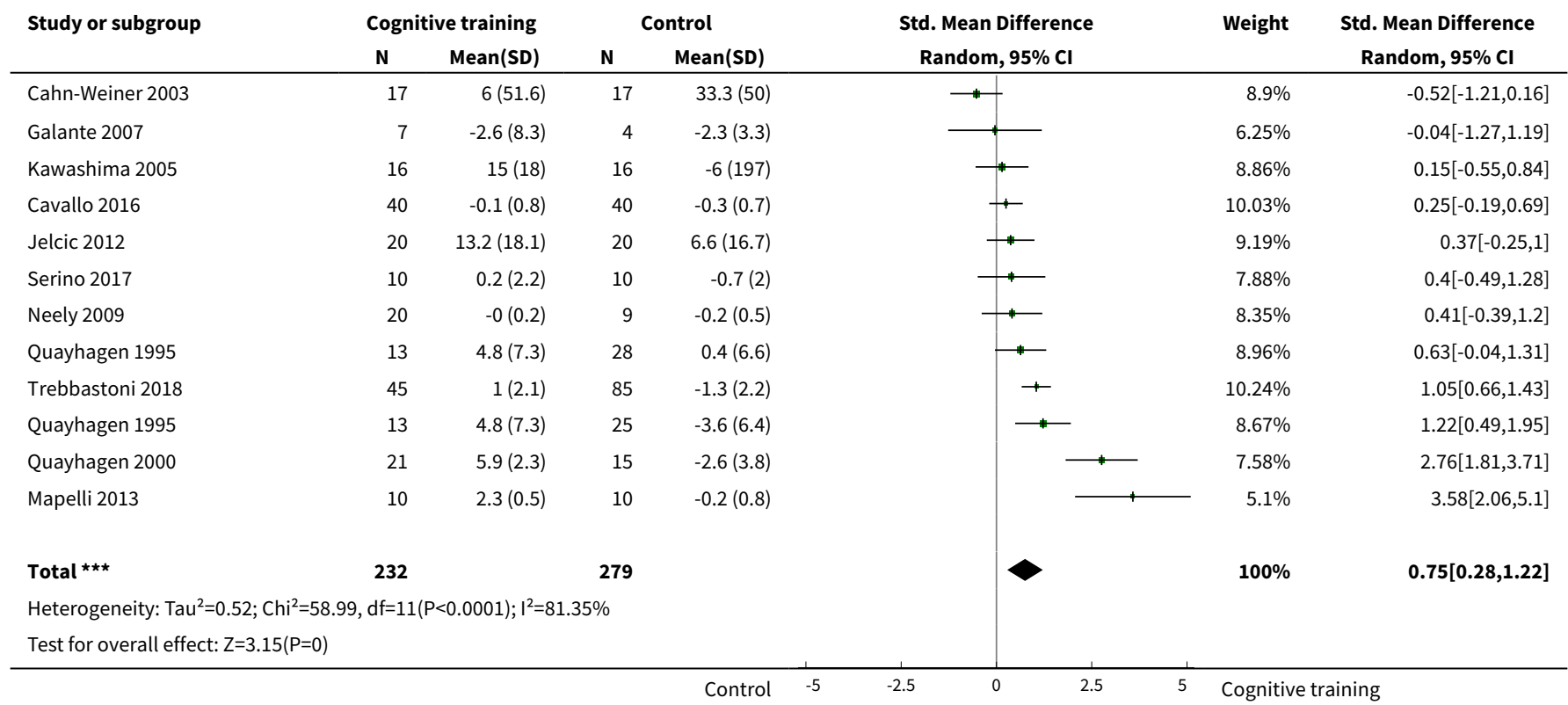

Analysis 1.13. Comparison 1 Cognitive training vs control immediately post intervention, Outcome 13 Change in speed of information processing.

\begin{tabular}{|c|c|c|c|c|c|c|c|}
\hline \multirow[t]{2}{*}{ Study or subgroup } & \multicolumn{2}{|c|}{ Cognitive training } & \multicolumn{2}{|c|}{ Control } & \multirow{2}{*}{$\begin{array}{c}\text { Std. Mean Difference } \\
\text { Random, } 95 \% \mathrm{Cl}\end{array}$} & \multirow[t]{2}{*}{ Weight } & \multirow{2}{*}{$\begin{array}{c}\text { Std. Mean Difference } \\
\text { Random, } 95 \% \mathrm{Cl}\end{array}$} \\
\hline & $\mathbf{N}$ & Mean(SD) & $\mathbf{N}$ & Mean(SD) & & & \\
\hline Beck 1988 & 10 & $-0.3(1.3)$ & 10 & $1.1(2.9)$ & $1+$ & $11.31 \%$ & $-0.6[-1.51,0.3]$ \\
\hline Galante 2007 & 7 & $-1.3(10.3)$ & 4 & $3.3(5.6)$ & $\longrightarrow$ & $6.26 \%$ & $-0.47[-1.72,0.79]$ \\
\hline Jelcic 2014 & 17 & $0.5(6.9)$ & 10 & $-0.4(8.2)$ & $\longrightarrow$ & $14.38 \%$ & $0.11[-0.67,0.9]$ \\
\hline Barban 2016 & 36 & $7.7(27.4)$ & 33 & $-1.9(27.7)$ & \pm- & $30.03 \%$ & $0.35[-0.13,0.82]$ \\
\hline Jelcic 2012 & 20 & $19.3(56.5)$ & 20 & $-2(20.6)$ & —- & $20.26 \%$ & $0.49[-0.14,1.12]$ \\
\hline 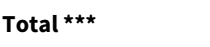 & 107 & & 94 & & & $100 \%$ & $0.22[-0.11,0.54]$ \\
\hline \multicolumn{8}{|c|}{ Heterogeneity: $\mathrm{Tau}^{2}=0.03 ; \mathrm{Chi}^{2}=6.24, \mathrm{df}=5(\mathrm{P}=0.28) ; \mathrm{I}^{2}=19.89 \%$} \\
\hline \multicolumn{8}{|c|}{ Test for overall effect: $Z=1.32(P=0.19)$} \\
\hline
\end{tabular}

Analysis 1.14. Comparison 1 Cognitive training vs control immediately post intervention, Outcome 14 Change in meta cognition (self-reported).

\begin{tabular}{|c|c|c|c|c|c|c|c|}
\hline \multirow[t]{2}{*}{ Study or subgroup } & \multicolumn{2}{|c|}{ Cognitive training } & \multicolumn{2}{|c|}{ Control } & \multirow{2}{*}{$\begin{array}{c}\text { Std. Mean Difference } \\
\text { Random, } 95 \% \mathrm{Cl} \\
\end{array}$} & \multirow[t]{2}{*}{ Weight } & \multirow{2}{*}{$\begin{array}{c}\text { Std. Mean Difference } \\
\text { Random, } 95 \% \mathrm{CI}\end{array}$} \\
\hline & $\mathbf{N}$ & Mean(SD) & $\mathbf{N}$ & Mean(SD) & & & \\
\hline Koltai 2001 & 14 & $6.4(14.3)$ & 8 & $1(14.1)$ & -1 & $54.54 \%$ & $0.37[-0.51,1.24]$ \\
\hline Lee 2013 & 12 & $0.3(0.3)$ & 7 & $0.1(0.3)$ & +1 & $45.46 \%$ & $0.65[-0.31,1.61]$ \\
\hline
\end{tabular}




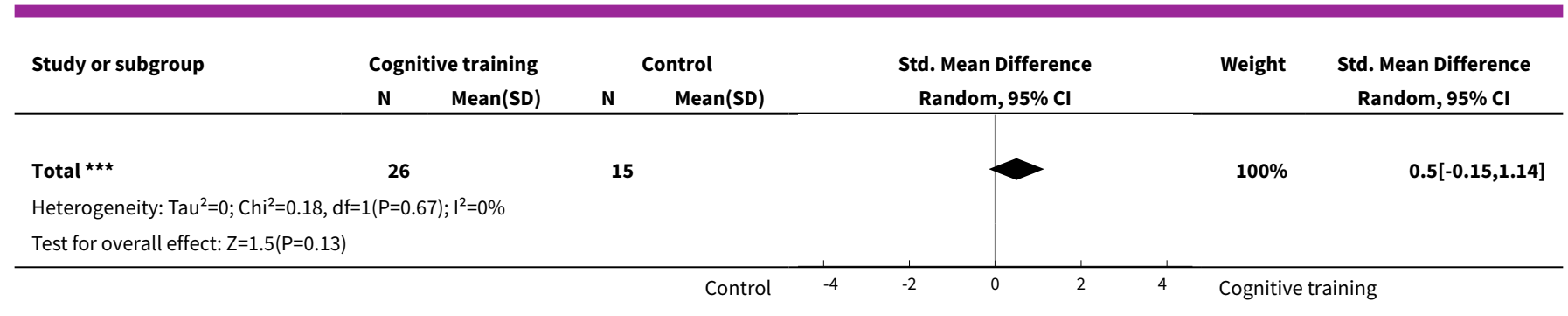

Analysis 1.15. Comparison 1 Cognitive training vs control immediately post intervention, Outcome 15 Change in meta cognition (informant-reported).

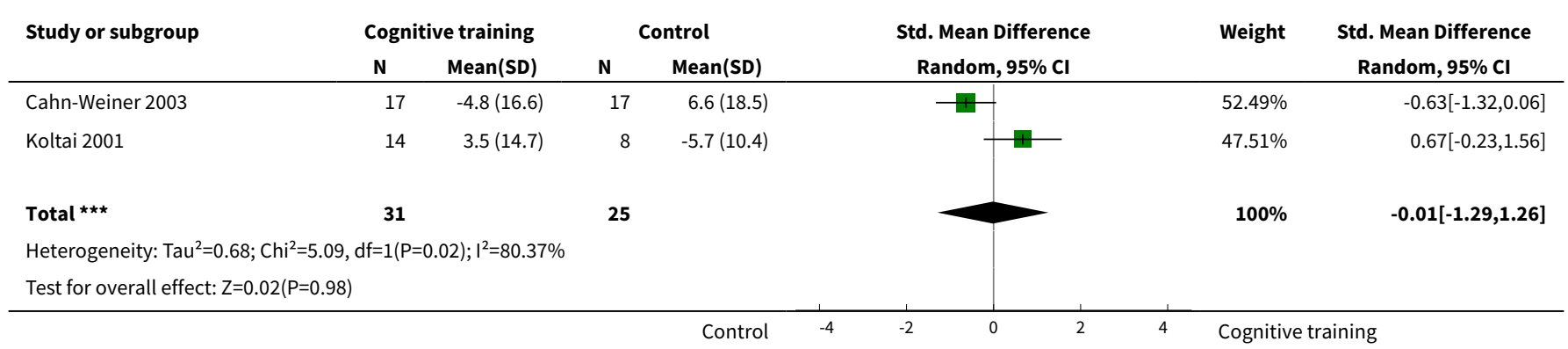

Analysis 1.16. Comparison 1 Cognitive training vs control immediately post intervention, Outcome 16 Change in participants' mood.

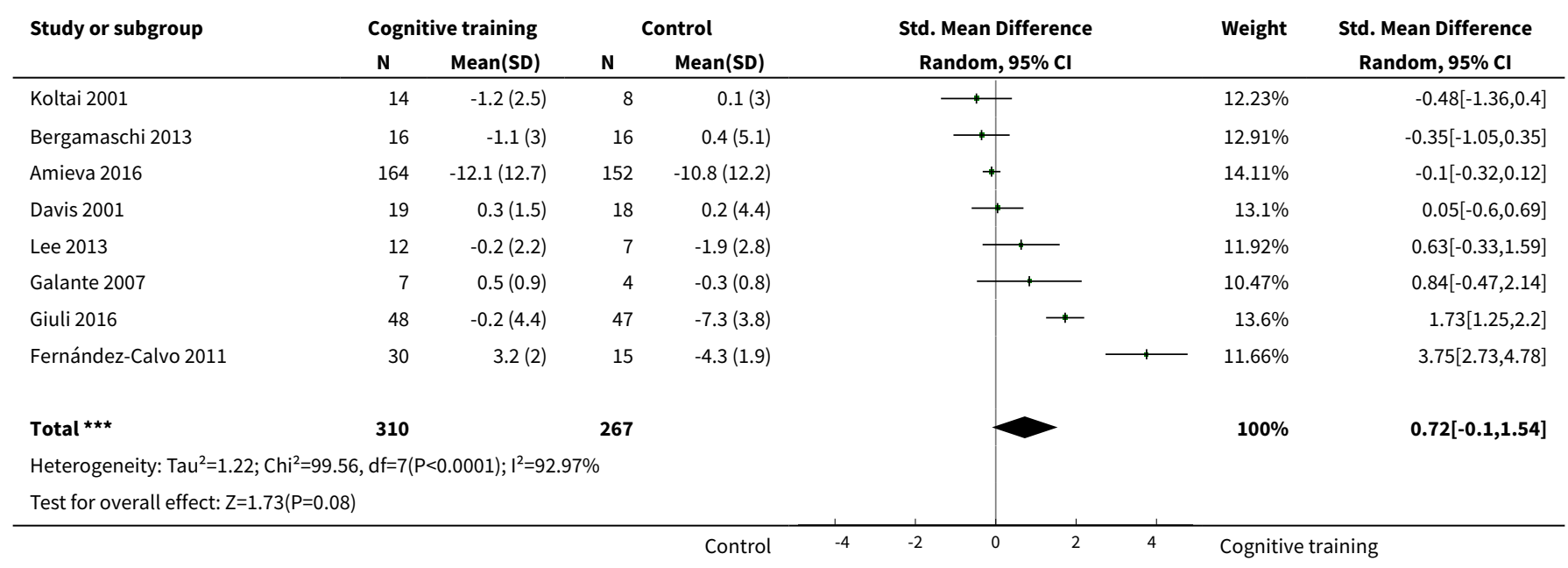

Analysis 1.17. Comparison 1 Cognitive training vs control immediately post intervention, Outcome 17 Change in capacity for activities of daily living.

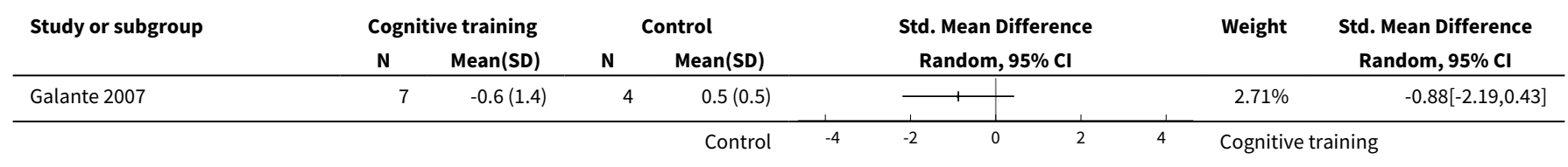




\begin{tabular}{|c|c|c|c|c|c|c|c|}
\hline \multirow[t]{2}{*}{ Study or subgroup } & \multicolumn{2}{|c|}{ Cognitive training } & \multicolumn{2}{|c|}{ Control } & \multirow{2}{*}{$\begin{array}{c}\text { Std. Mean Difference } \\
\text { Random, } 95 \% \mathrm{Cl}\end{array}$} & \multirow[t]{2}{*}{ Weight } & \multirow{2}{*}{$\begin{array}{c}\text { Std. Mean Difference } \\
\text { Random, } 95 \% \mathrm{Cl}\end{array}$} \\
\hline & $\mathbf{N}$ & Mean(SD) & $\mathbf{N}$ & Mean(SD) & & & \\
\hline de Vreese 1998 & 9 & $-1.3(4.4)$ & 9 & $1(4)$ & \begin{tabular}{l|l}
1 \\
\end{tabular} & $4.83 \%$ & $-0.53[-1.48,0.41]$ \\
\hline Amieva 2016 & 167 & $-1.8(8.5)$ & 153 & $-1.2(8.3)$ & + & $23.99 \%$ & $-0.06[-0.28,0.16]$ \\
\hline Cahn-Weiner 2003 & 17 & $-0.2(3.2)$ & 17 & $0(3.1)$ & $\longrightarrow$ & $8.28 \%$ & $-0.06[-0.74,0.61]$ \\
\hline Kim 2015 & 21 & $0(0.9)$ & 21 & $0(1.1)$ & $\longrightarrow$ & $9.62 \%$ & $0[-0.6,0.6]$ \\
\hline Jelcic 2012 & 20 & $0.1(1.1)$ & 20 & $0(1.4)$ & 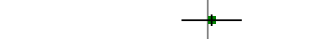 & $9.3 \%$ & $0.08[-0.54,0.7]$ \\
\hline Barban 2016 & 38 & $-0.1(1)$ & 38 & $-0.3(1.1)$ & + & $13.88 \%$ & $0.19[-0.26,0.64]$ \\
\hline Giuli 2016 & 48 & $0.3(1.2)$ & 47 & $-0.2(1.3)$ & $\rightarrow$ & $15.48 \%$ & $0.4[-0,0.81]$ \\
\hline Bergamaschi 2013 & 16 & $-0.4(1.5)$ & 16 & $-1.3(1.5)$ & 世 & $7.62 \%$ & $0.61[-0.1,1.32]$ \\
\hline Lee 2013 & 12 & $2.2(3.3)$ & 7 & $-2.1(4.5)$ & 1 & $4.3 \%$ & $1.1[0.09,2.11]$ \\
\hline Total $\star \star \star$ & 355 & & 332 & & 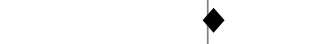 & $100 \%$ & $0.12[-0.11,0.35]$ \\
\hline \multicolumn{8}{|c|}{ Heterogeneity: $\mathrm{Tau}^{2}=0.04 ; \mathrm{Chi}^{2}=14.29, \mathrm{df}=9(\mathrm{P}=0.11) ; \mathrm{I}^{2}=37.04 \%$} \\
\hline \multicolumn{8}{|c|}{ Test for overall effect: $Z=1.04(P=0.3)$} \\
\hline & & & & Control & -4 & Cognitiv & ning \\
\hline
\end{tabular}

Analysis 1.18. Comparison 1 Cognitive training vs control immediately post intervention, Outcome 18 Change in general health and quality of life.

\begin{tabular}{|c|c|c|c|c|c|c|c|}
\hline \multirow{3}{*}{$\begin{array}{l}\text { Study or subgroup } \\
\text { Davis } 2001\end{array}$} & \multicolumn{2}{|c|}{ Cognitive training } & \multicolumn{2}{|c|}{ Control } & \multirow{2}{*}{$\begin{array}{c}\text { Std. Mean Difference } \\
\text { Random, } 95 \% \mathrm{Cl}\end{array}$} & \multirow[t]{2}{*}{ Weight } & \multirow{2}{*}{$\begin{array}{c}\text { Std. Mean Difference } \\
\text { Random, } 95 \% \mathrm{CI}\end{array}$} \\
\hline & $\mathbf{N}$ & $\operatorname{Mean}(S D)$ & $\mathbf{N}$ & Mean(SD) & & & \\
\hline & 19 & $-24.8(37.3)$ & 18 & $4.8(40.9)$ & $\longrightarrow$ & $14 \%$ & $-0.74[-1.41,-0.07]$ \\
\hline Quayhagen 2000 & 21 & $-0.4(2.8)$ & 67 & $0.1(2.6)$ & $\rightarrow$ & $18.83 \%$ & $-0.19[-0.68,0.3]$ \\
\hline Amieva 2016 & 164 & $-6.3(6.1)$ & 151 & $-5.6(6.4)$ & + & $27.77 \%$ & $-0.11[-0.33,0.11]$ \\
\hline Kallio 2018 & 76 & $-0(0.1)$ & 71 & $-0(0.1)$ & $\rightarrow$ & $24.41 \%$ & $0[-0.32,0.32]$ \\
\hline Kim 2015 & 22 & $3.4(3.3)$ & 21 & $0(4.4)$ & $\longrightarrow$ & $14.99 \%$ & $0.87[0.24,1.49]$ \\
\hline Total $\star \star \star ~$ & 302 & & 328 & & & $100 \%$ & $-0.04[-0.38,0.29]$ \\
\hline \multicolumn{8}{|c|}{ Heterogeneity: $\operatorname{Tau}^{2}=0.09 ; \mathrm{Chi}^{2}=12.93, \mathrm{df}=4(\mathrm{P}=0.01) ; \mathrm{I}^{2}=69.08 \%$} \\
\hline \multicolumn{8}{|c|}{ Test for overall effect: $Z=0.24(P=0.81)$} \\
\hline
\end{tabular}

\section{Analysis 1.19. Comparison 1 Cognitive training vs control immediately post intervention, Outcome 19 Change in behavioural and psychological symptoms of dementia (BPSD).}

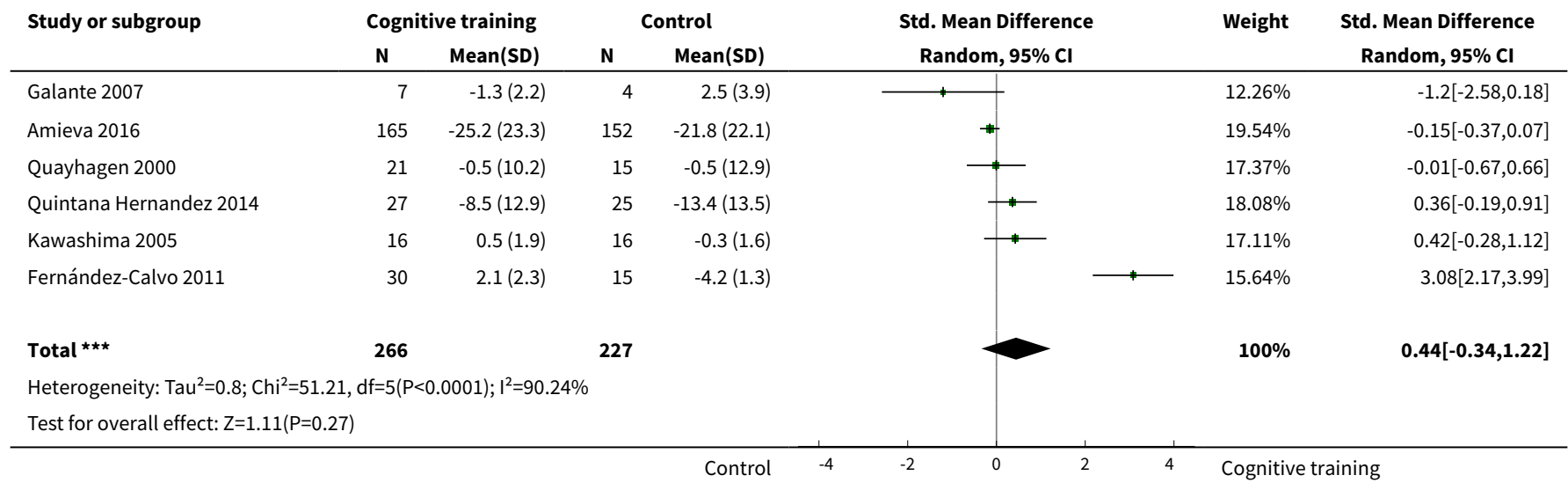


Analysis 1.20. Comparison 1 Cognitive training vs control immediately post intervention, Outcome 20 Participant burden (retention rates).

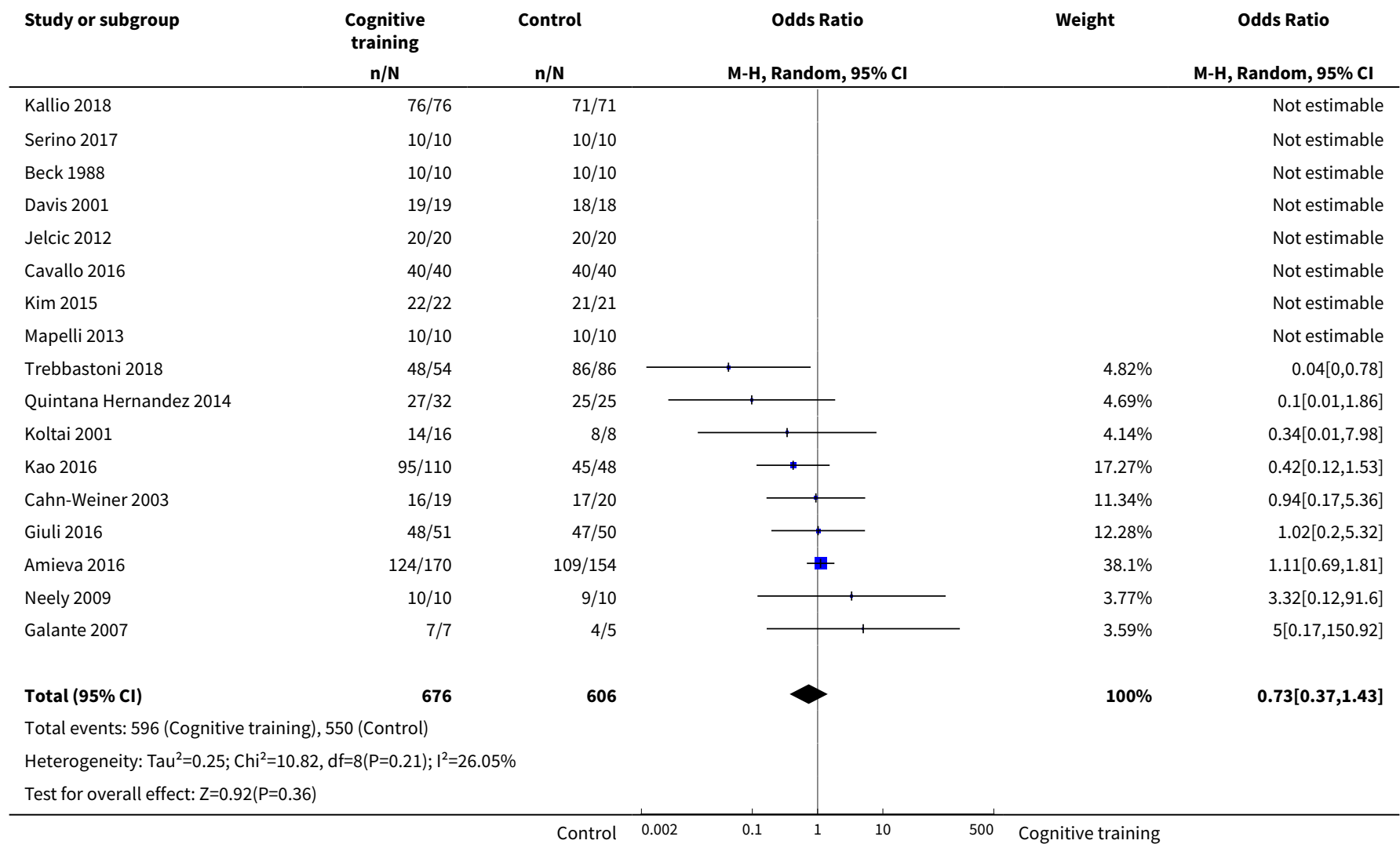

Analysis 1.21. Comparison 1 Cognitive training vs control immediately post intervention, Outcome 21 Change in burden of care (CAREGIVER).

\begin{tabular}{|c|c|c|c|c|c|c|c|}
\hline \multirow[t]{2}{*}{ Study or subgroup } & \multicolumn{2}{|c|}{ Cognitive training } & \multicolumn{2}{|c|}{ Control } & \multirow{2}{*}{$\begin{array}{c}\text { Std. Mean Difference } \\
\text { Random, } 95 \% \mathrm{Cl}\end{array}$} & \multirow[t]{2}{*}{ Weight } & \multirow{2}{*}{$\begin{array}{c}\text { Std. Mean Difference } \\
\text { Random, } 95 \% \mathrm{CI}\end{array}$} \\
\hline & $\mathbf{N}$ & Mean(SD) & $\mathbf{N}$ & $\operatorname{Mean}(S D)$ & & & \\
\hline Amieva 2016 & 165 & $-21.3(19.1)$ & 152 & $-17.9(19)$ & + & $76.98 \%$ & $-0.18[-0.4,0.04]$ \\
\hline Quayhagen 2000 & 21 & $-0.8(4.1)$ & 67 & $-1.3(3.7)$ & 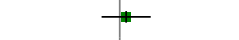 & $23.02 \%$ & $0.13[-0.36,0.62]$ \\
\hline 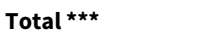 & 186 & & 219 & & & $100 \%$ & $-0.11[-0.36,0.15]$ \\
\hline \multicolumn{8}{|c|}{ Heterogeneity: $\mathrm{Tau}^{2}=0.01 ; \mathrm{Chi}^{2}=1.23, \mathrm{df}=1(\mathrm{P}=0.27) ; \mathrm{I}^{2}=18.62 \%$} \\
\hline
\end{tabular}


Analysis 1.22. Comparison 1 Cognitive training vs control immediately post intervention, Outcome 22 Change in quality of life (CAREGIVER).

\begin{tabular}{|c|c|c|c|c|c|c|c|}
\hline \multirow[t]{2}{*}{ Study or subgroup } & \multicolumn{2}{|c|}{ Cognitive training } & \multicolumn{2}{|c|}{ Control } & \multirow{2}{*}{$\begin{array}{c}\text { Std. Mean Difference } \\
\text { Random, } 95 \% \mathrm{Cl}\end{array}$} & \multirow[t]{2}{*}{ Weight } & \multirow{2}{*}{$\begin{array}{c}\text { Std. Mean Difference } \\
\text { Random, } 95 \% \mathrm{Cl}\end{array}$} \\
\hline & $\mathbf{N}$ & Mean(SD) & $\mathbf{N}$ & Mean(SD) & & & \\
\hline Quayhagen 2000 & 21 & $-0.6(9.8)$ & 15 & $-2.8(16.5)$ & & $100 \%$ & $0.16[-0.5,0.83]$ \\
\hline Total $* \star \star$ & 21 & & 15 & & & $100 \%$ & $0.16[-0.5,0.83]$ \\
\hline \multicolumn{8}{|c|}{ Heterogeneity: Not applicable } \\
\hline \multicolumn{8}{|c|}{ Test for overall effect: $Z=0.48(P=0.63)$} \\
\hline & & & & Control & -2.5 & Cognitiv & aining \\
\hline
\end{tabular}

Analysis 1.23. Comparison 1 Cognitive training vs control immediately post intervention, Outcome 23 Change in mood and well-being (CAREGIVER).

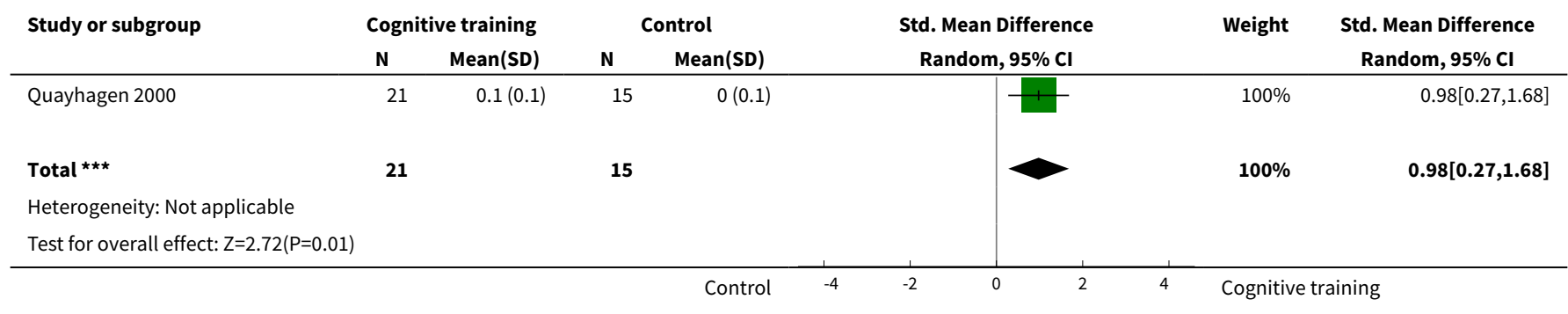

\section{Comparison 2. Cognitive training vs control in the medium term ( 3 to 12 months post intervention)}

\begin{tabular}{|c|c|c|c|c|}
\hline Outcome or subgroup title & $\begin{array}{l}\text { No. of } \\
\text { studies }\end{array}$ & $\begin{array}{l}\text { No. of } \\
\text { partici- } \\
\text { pants }\end{array}$ & Statistical method & Effect size \\
\hline $\begin{array}{l}1 \text { Change in a global measure of } \\
\text { cognition (composite) }\end{array}$ & 7 & 387 & Std. Mean Difference (Random, 95\% Cl) & $0.65[0.11,1.20]$ \\
\hline $\begin{array}{l}2 \text { Change in a global measure of } \\
\text { cognition (composite)_zero cor- } \\
\text { relation }\end{array}$ & 7 & 387 & Std. Mean Difference (Random, 95\% Cl) & $0.40[0.09,0.71]$ \\
\hline $\begin{array}{l}3 \text { Change in a global measure of } \\
\text { cognition }\end{array}$ & 6 & 387 & Std. Mean Difference (IV, Random, 95\% Cl) & $1.33[0.31,2.34]$ \\
\hline $\begin{array}{l}4 \text { Change in a global measure of } \\
\text { cognition (zero correlation) }\end{array}$ & 6 & 387 & Std. Mean Difference (IV, Random, 95\% CI) & $0.68[0.06,1.30]$ \\
\hline 5 Change in disease progression & 2 & 98 & Std. Mean Difference (IV, Random, 95\% CI) & $0.55[0.12,0.98]$ \\
\hline $\begin{array}{l}6 \text { Change in disease progression } \\
\text { (zero correlation) }\end{array}$ & 2 & 98 & Std. Mean Difference (IV, Random, 95\% CI) & $0.28[-0.14,0.71]$ \\
\hline 7 Change in delayed memory & 4 & 274 & Std. Mean Difference (IV, Random, 95\% Cl) & $0.97[0.02,1.92]$ \\
\hline 8 Change in immediate memory & 7 & 383 & Std. Mean Difference (IV, Random, 95\% CI) & $0.62[0.00,1.24]$ \\
\hline
\end{tabular}




\begin{tabular}{|c|c|c|c|c|}
\hline Outcome or subgroup title & $\begin{array}{l}\text { No. of } \\
\text { studies }\end{array}$ & $\begin{array}{l}\text { No. of } \\
\text { partici- } \\
\text { pants }\end{array}$ & Statistical method & Effect size \\
\hline $\begin{array}{l}9 \text { Change in attention and work- } \\
\text { ing memory }\end{array}$ & 3 & 215 & Std. Mean Difference (IV, Random, 95\% CI) & $0.50[-0.43,1.43]$ \\
\hline 10 Change in language (naming) & 4 & 274 & Std. Mean Difference (IV, Random, 95\% CI) & $0.71[0.07,1.34]$ \\
\hline $\begin{array}{l}11 \text { Change in verbal letter fluen- } \\
\text { cy }\end{array}$ & 4 & 247 & Std. Mean Difference (IV, Random, 95\% Cl) & $0.47[-0.28,1.23]$ \\
\hline $\begin{array}{l}12 \text { Change in verbal category } \\
\text { fluency }\end{array}$ & 3 & 213 & Std. Mean Difference (IV, Random, 95\% Cl) & $0.78[0.38,1.18]$ \\
\hline 13 Change in executive function & 5 & 330 & Std. Mean Difference (IV, Random, 95\% CI) & $0.56[0.02,1.10]$ \\
\hline $\begin{array}{l}14 \text { Change in speed of informa- } \\
\text { tion processing }\end{array}$ & 2 & 45 & Std. Mean Difference (IV, Random, 95\% CI) & $0.30[-0.44,1.04]$ \\
\hline $\begin{array}{l}15 \text { Change in meta cognition } \\
\text { (self-reported) }\end{array}$ & 1 & 19 & Std. Mean Difference (IV, Random, 95\% CI) & $0.99[-0.01,1.99]$ \\
\hline $\begin{array}{l}16 \text { Change in meta cognition (in- } \\
\text { formant-reported) }\end{array}$ & 1 & 34 & Std. Mean Difference (IV, Random, 95\% Cl) & $-0.06[-0.73,0.62]$ \\
\hline $\begin{array}{l}17 \text { Change in participants' } \\
\text { mood }\end{array}$ & 2 & 30 & Std. Mean Difference (IV, Random, 95\% Cl) & $0.21[-0.54,0.96]$ \\
\hline $\begin{array}{l}18 \text { Change in capacity for activi- } \\
\text { ties of daily living }\end{array}$ & 3 & 64 & Std. Mean Difference (IV, Random, 95\% CI) & $0.22[-0.50,0.94]$ \\
\hline $\begin{array}{l}19 \text { Change in general health and } \\
\text { quality of life }\end{array}$ & 1 & 117 & Std. Mean Difference (IV, Random, 95\% Cl) & $-0.02[-0.39,0.35]$ \\
\hline $\begin{array}{l}20 \text { Change in behavioural and } \\
\text { psychological symptoms of de- } \\
\text { mentia (BPSD) }\end{array}$ & 1 & 11 & Std. Mean Difference (IV, Random, 95\% Cl) & $-1.34[-2.75,0.07]$ \\
\hline $\begin{array}{l}21 \text { Change in burden of care } \\
\text { (CAREGIVER) }\end{array}$ & 0 & 0 & Std. Mean Difference (IV, Random, 95\% Cl) & $0.0[0.0,0.0]$ \\
\hline $\begin{array}{l}22 \text { Change in quality of life } \\
\text { (CAREGIVER) }\end{array}$ & 0 & 0 & Std. Mean Difference (IV, Random, 95\% Cl) & $0.0[0.0,0.0]$ \\
\hline $\begin{array}{l}23 \text { Change in mood and well-be- } \\
\text { ing (CAREGIVER) }\end{array}$ & 0 & 0 & Std. Mean Difference (IV, Random, 95\% CI) & $0.0[0.0,0.0]$ \\
\hline
\end{tabular}


Analysis 2.1. Comparison 2 Cognitive training vs control in the medium term ( 3 to 12 months post intervention), Outcome 1 Change in a global measure of cognition (composite).

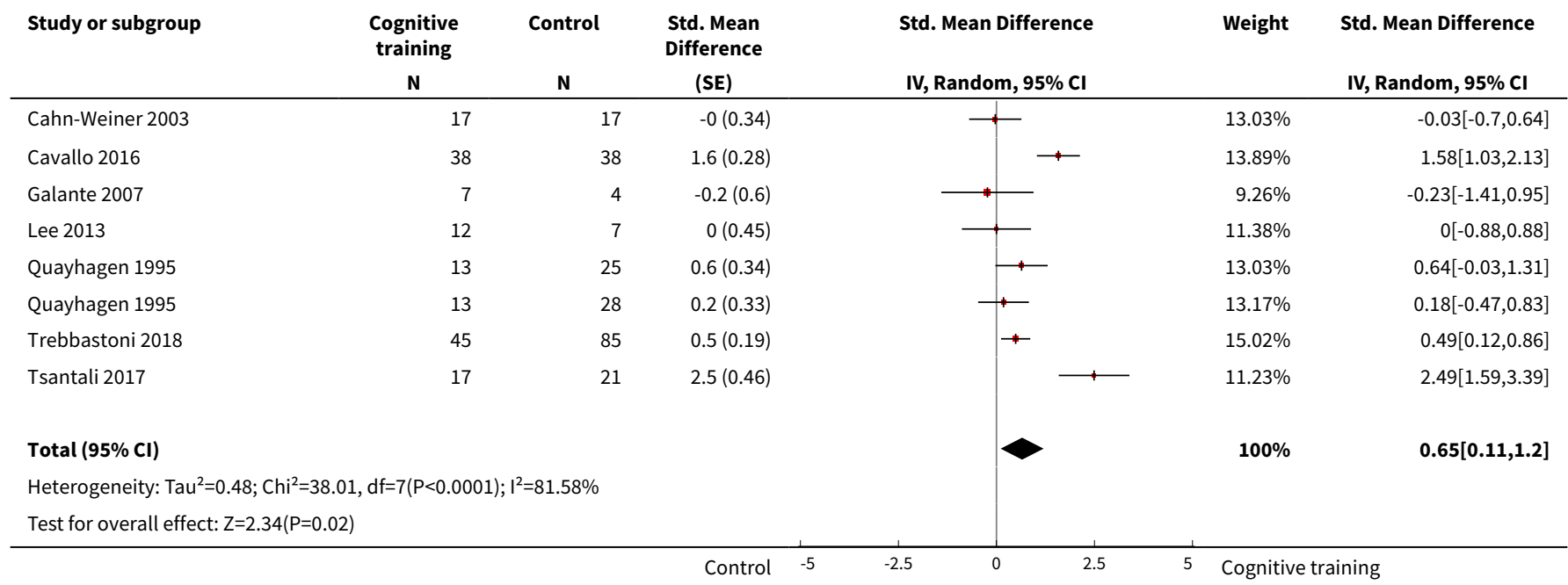

Analysis 2.2. Comparison 2 Cognitive training vs control in the medium term ( 3 to 12 months post intervention), Outcome 2 Change in a global measure of cognition (composite)_zero correlation.

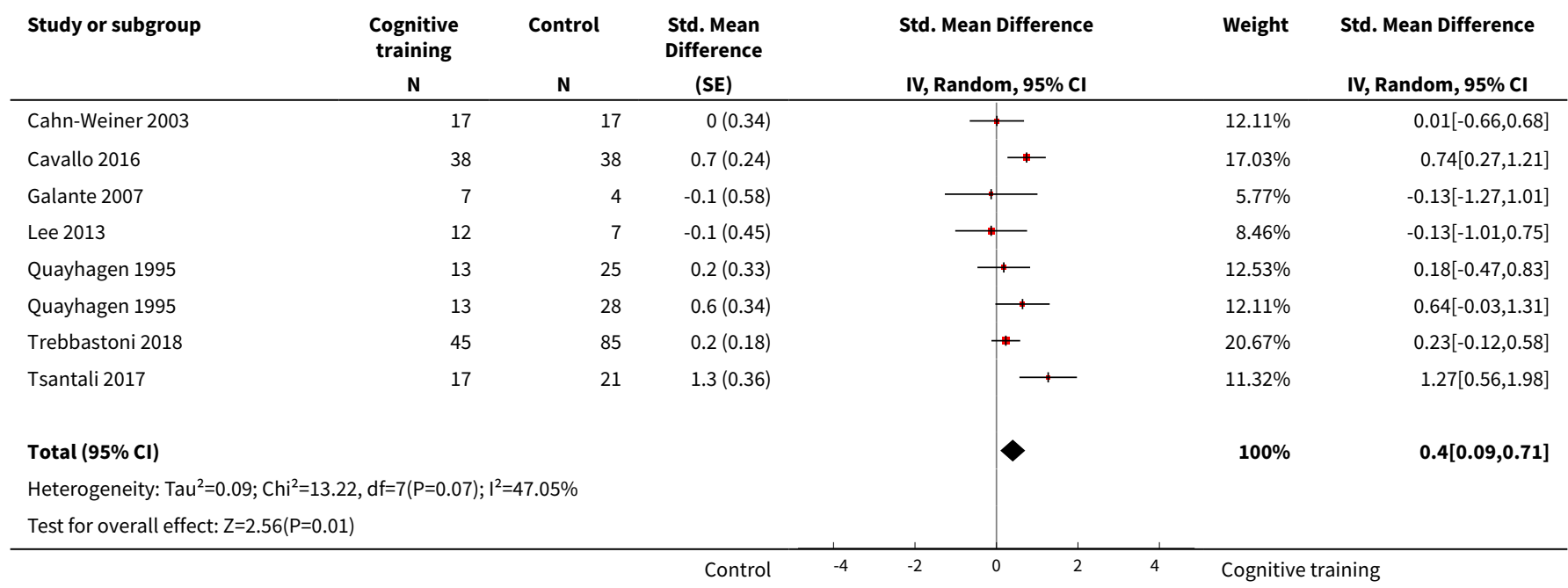

Analysis 2.3. Comparison 2 Cognitive training vs control in the medium term ( 3 to 12 months post intervention), Outcome 3 Change in a global measure of cognition.

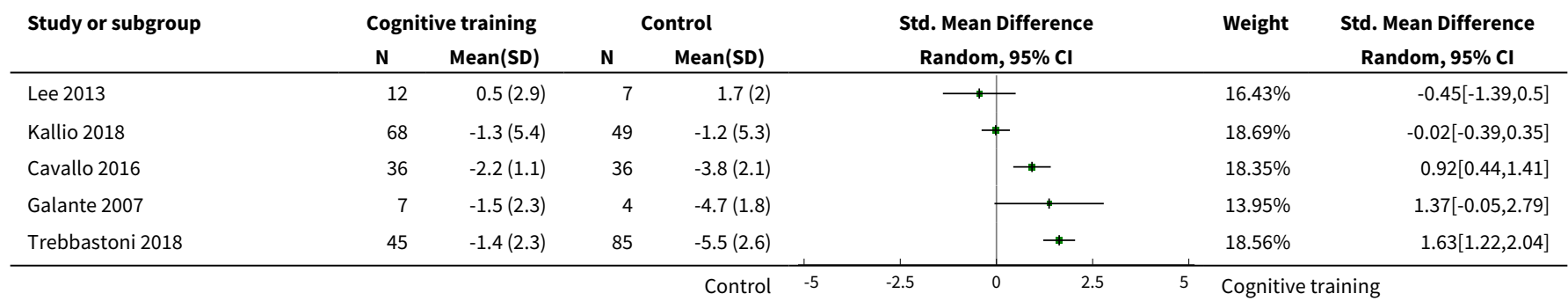




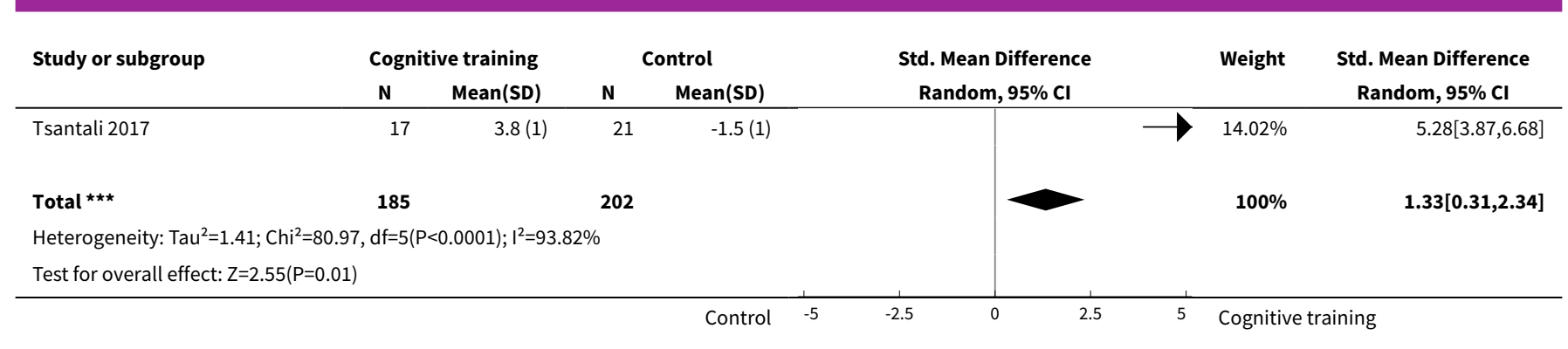

\section{Analysis 2.4. Comparison 2 Cognitive training vs control in the medium term ( 3 to 12 months post intervention), Outcome 4 Change in a global measure of cognition (zero correlation).}

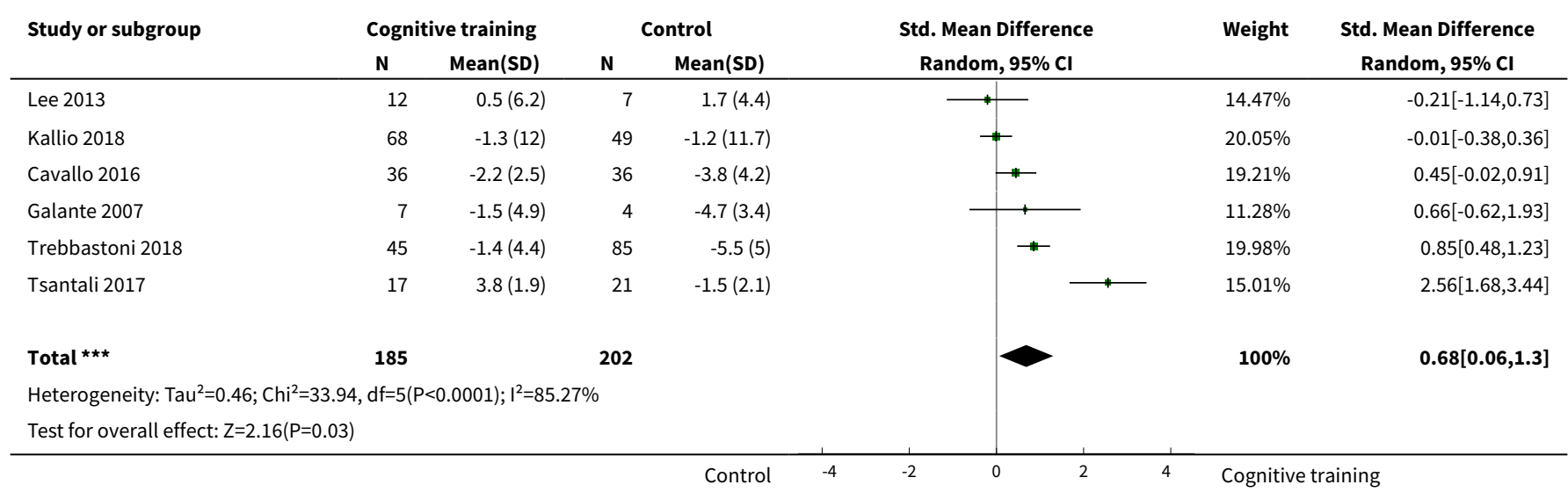

Analysis 2.5. Comparison 2 Cognitive training vs control in the medium term ( 3 to 12 months post intervention), Outcome 5 Change in disease progression.

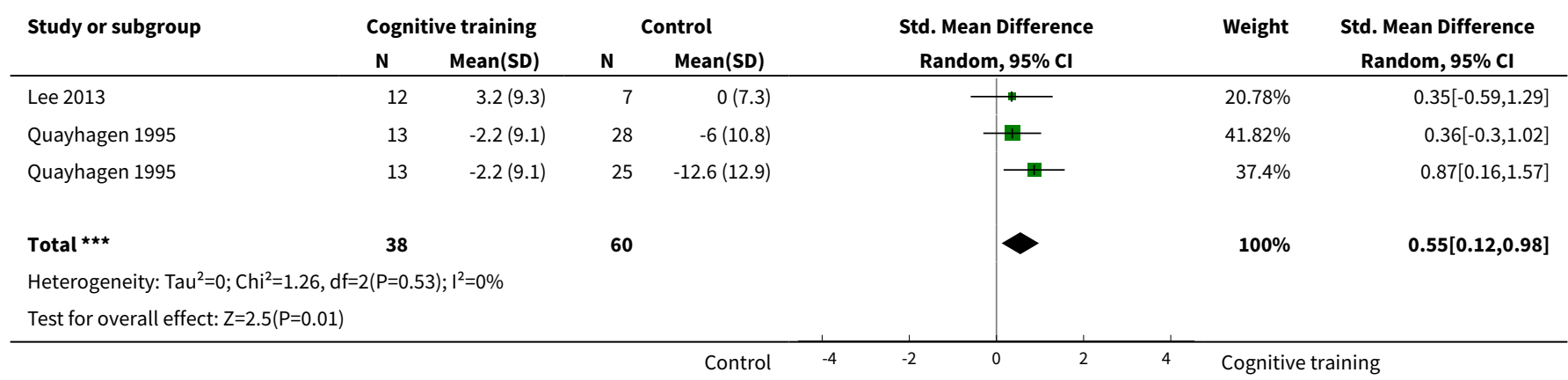

Analysis 2.6. Comparison 2 Cognitive training vs control in the medium term ( 3 to 12 months post intervention), Outcome 6 Change in disease progression (zero correlation).

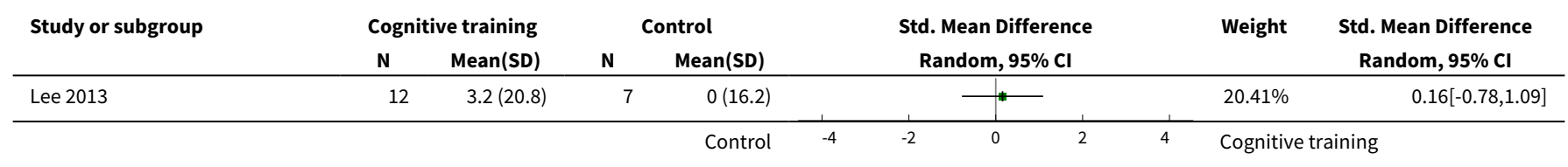




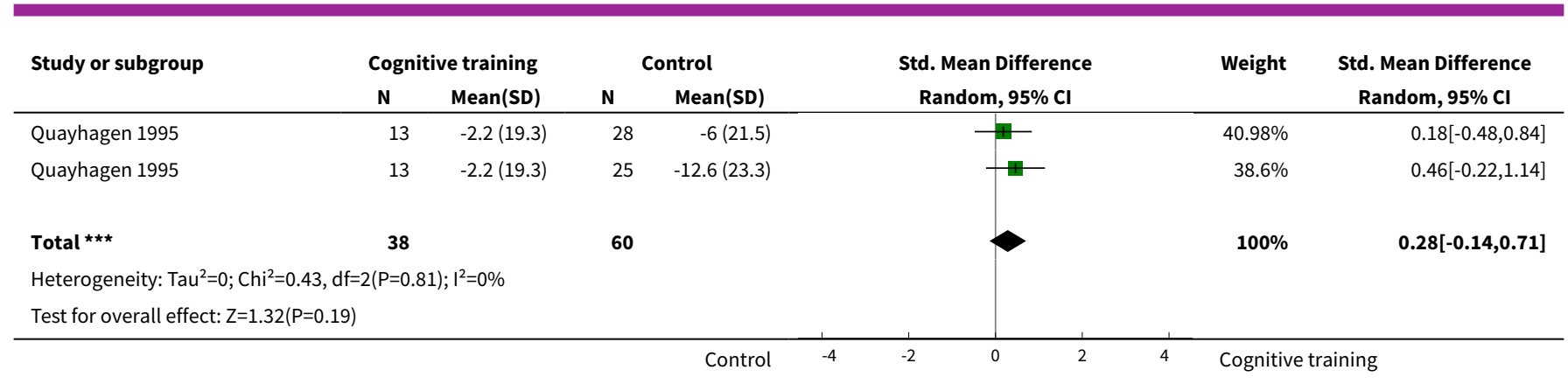

Analysis 2.7. Comparison 2 Cognitive training vs control in the medium term ( 3 to 12 months post intervention), Outcome 7 Change in delayed memory.

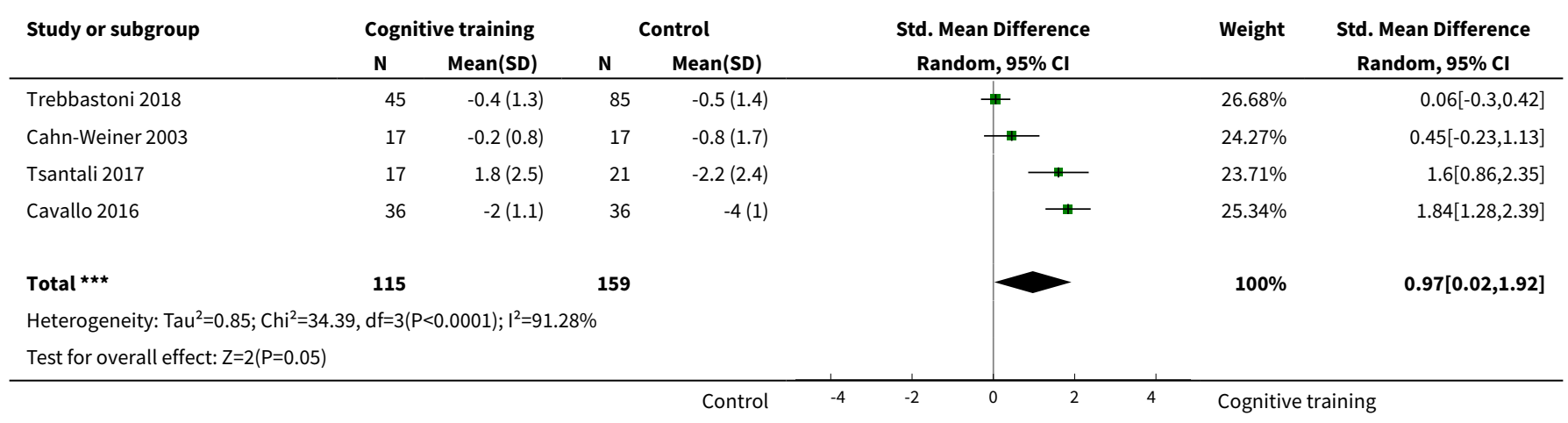

Analysis 2.8. Comparison 2 Cognitive training vs control in the medium term ( 3 to 12 months post intervention), Outcome 8 Change in immediate memory.

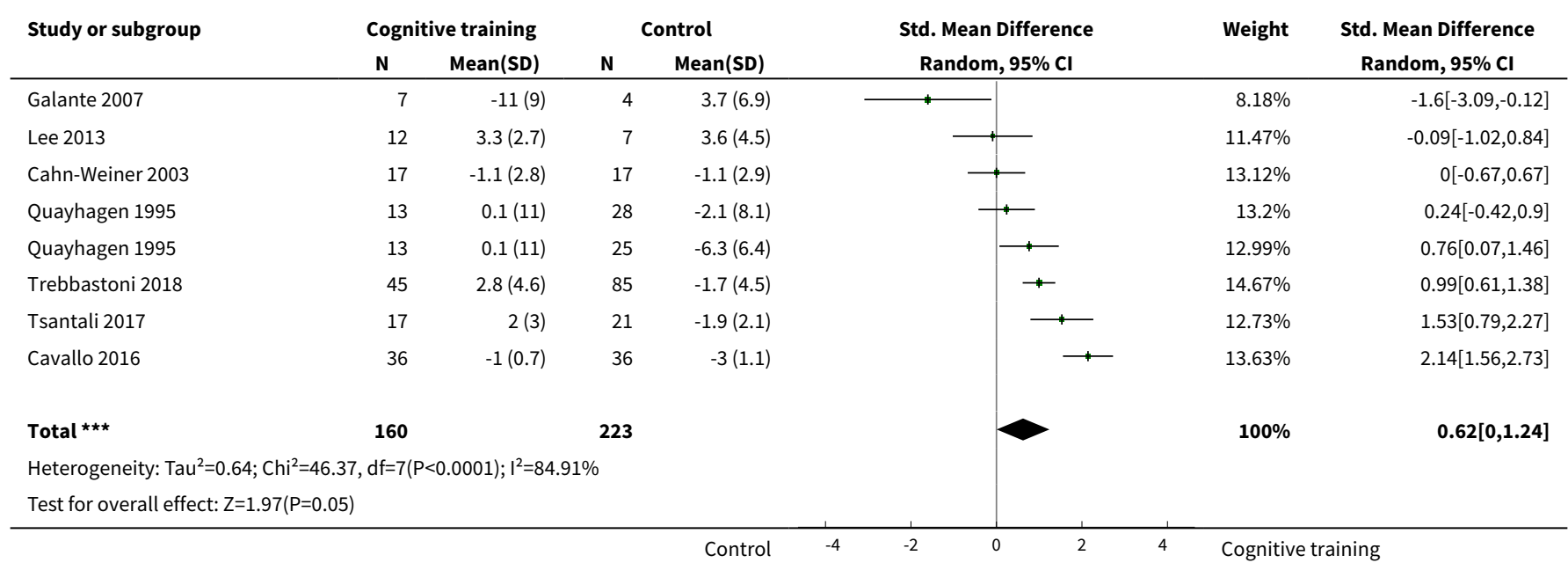


Analysis 2.9. Comparison 2 Cognitive training vs control in the medium term ( 3 to 12 months post intervention), Outcome 9 Change in attention and working memory.

\begin{tabular}{|c|c|c|c|c|c|c|c|}
\hline \multirow[t]{2}{*}{ Study or subgroup } & \multicolumn{2}{|c|}{ Cognitive training } & \multicolumn{2}{|c|}{ Control } & \multirow{2}{*}{$\begin{array}{c}\text { Std. Mean Difference } \\
\text { Random, } 95 \% \mathrm{Cl} \\
\end{array}$} & \multirow[t]{2}{*}{ Weight } & \multirow{2}{*}{$\begin{array}{c}\text { Std. Mean Difference } \\
\text { Random, } 95 \% \mathrm{Cl}\end{array}$} \\
\hline & $\mathbf{N}$ & Mean(SD) & $\mathbf{N}$ & Mean(SD) & & & \\
\hline Galante 2007 & 7 & $-0.3(0.7)$ & 4 & $1(1.6)$ & - & $22.32 \%$ & $-1.12[-2.48,0.24]$ \\
\hline Trebbastoni 2018 & 45 & $-0.4(0.5)$ & 85 & $-0.7(0.7)$ & \# & $39.92 \%$ & $0.58[0.21,0.95]$ \\
\hline Cavallo 2016 & 36 & $-0.1(1)$ & 38 & $-1.5(1.1)$ & -1 & $37.76 \%$ & $1.38[0.87,1.89]$ \\
\hline 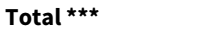 & 88 & & 127 & & & $100 \%$ & $0.5[-0.43,1.43]$ \\
\hline \multicolumn{3}{|c|}{ Test for overall effect: $Z=1.05(P=0.29)$} & & & & & \\
\hline
\end{tabular}

Analysis 2.10. Comparison 2 Cognitive training vs control in the medium term ( 3 to 12 months post intervention), Outcome 10 Change in language (naming).

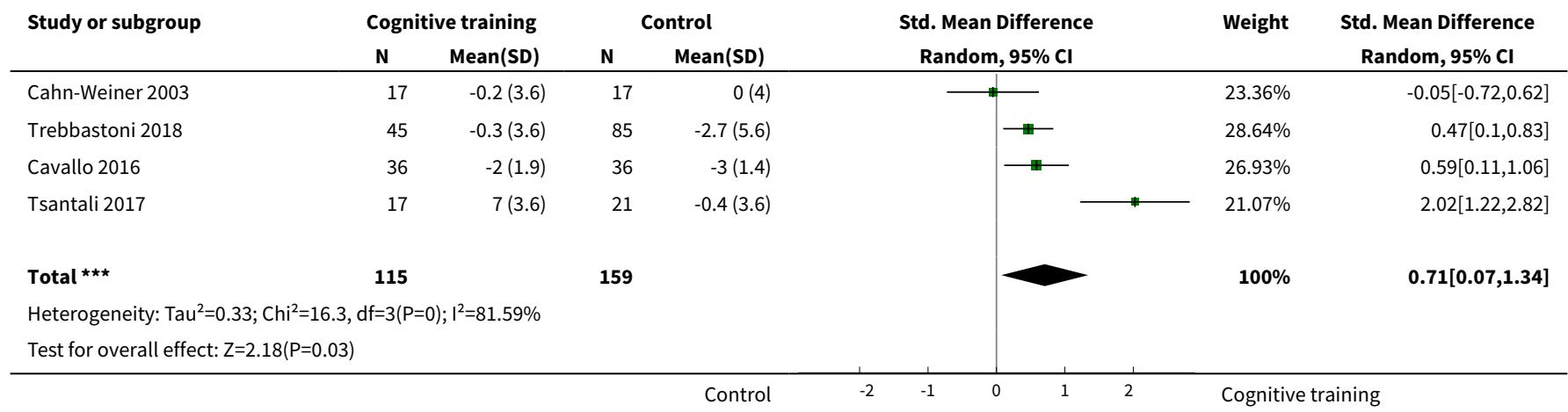

Analysis 2.11. Comparison 2 Cognitive training vs control in the medium term (3 to 12 months post intervention), Outcome 11 Change in verbal letter fluency.

\begin{tabular}{|c|c|c|c|c|c|c|c|}
\hline \multirow[t]{2}{*}{ Study or subgroup } & \multicolumn{2}{|c|}{ Cognitive training } & \multicolumn{2}{|c|}{ Control } & \multirow{2}{*}{$\begin{array}{c}\text { Std. Mean Difference } \\
\text { Random, } 95 \% \mathrm{Cl}\end{array}$} & \multirow[t]{2}{*}{ Weight } & \multirow{2}{*}{$\begin{array}{c}\text { Std. Mean Difference } \\
\text { Random, } 95 \% \mathrm{Cl}\end{array}$} \\
\hline & $\mathbf{N}$ & Mean(SD) & $\mathbf{N}$ & Mean(SD) & & & \\
\hline Cahn-Weiner 2003 & 17 & $-1.1(4.1)$ & 17 & $0.9(5.4)$ & $\longrightarrow-$ & $25.34 \%$ & $-0.41[-1.09,0.27]$ \\
\hline Galante 2007 & 7 & $1(3.9)$ & 4 & $0.3(7.6)$ & 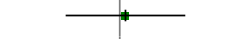 & $17.25 \%$ & $0.12[-1.11,1.35]$ \\
\hline Trebbastoni 2018 & 45 & $-2.4(4.9)$ & 85 & $-5.3(5.2)$ & - & $29.68 \%$ & $0.56[0.19,0.93]$ \\
\hline Cavallo 2016 & 36 & $-3.1(1.7)$ & 36 & $-5.4(1.6)$ & $ד$ & $27.73 \%$ & $1.41[0.89,1.93]$ \\
\hline \multicolumn{8}{|c|}{ Heterogeneity: $\mathrm{Tau}^{2}=0.46 ; \mathrm{Chi}^{2}=18.36, \mathrm{df}=3(\mathrm{P}=0) ; \mathrm{I}^{2}=83.66 \%$} \\
\hline \multicolumn{8}{|c|}{ Test for overall effect: $Z=1.23(P=0.22)$} \\
\hline
\end{tabular}


Analysis 2.12. Comparison 2 Cognitive training vs control in the medium term ( 3 to 12 months post intervention), Outcome 12 Change in verbal category fluency.

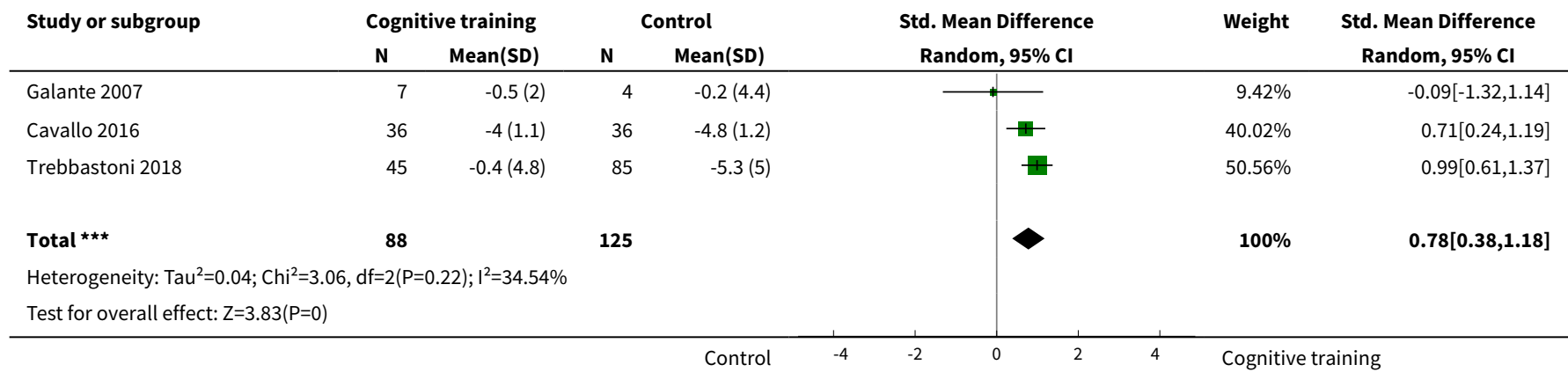

Analysis 2.13. Comparison 2 Cognitive training vs control in the medium term (3 to 12 months post intervention), Outcome 13 Change in executive function.

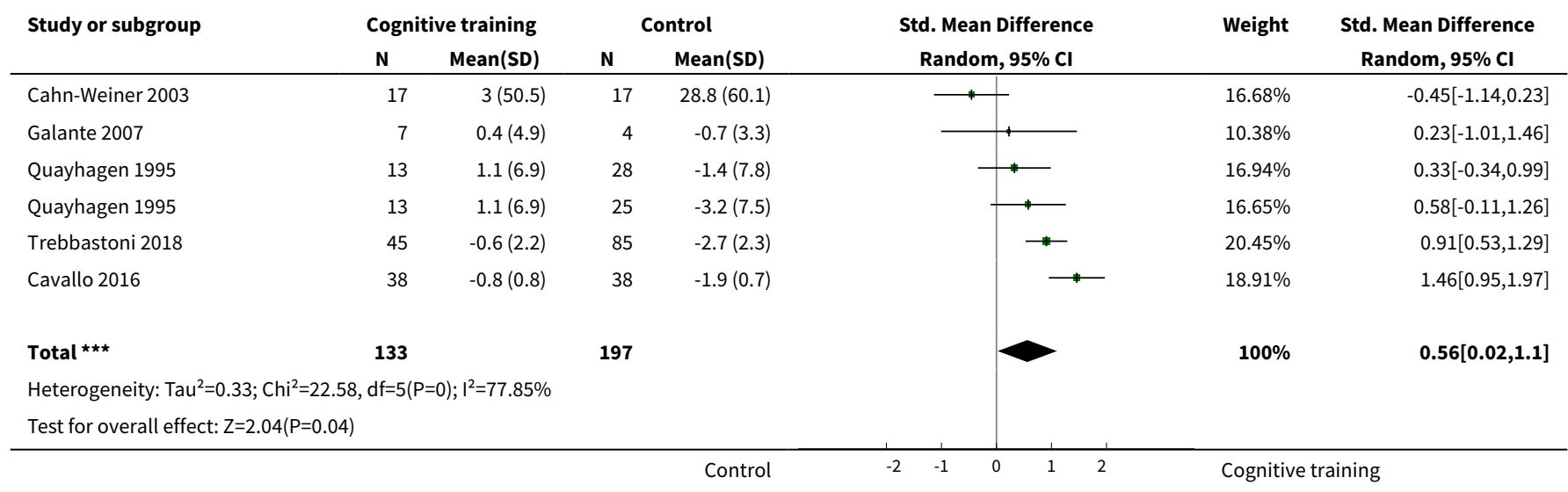

Analysis 2.14. Comparison 2 Cognitive training vs control in the medium term ( 3 to 12 months post intervention), Outcome 14 Change in speed of information processing.

\begin{tabular}{|c|c|c|c|c|c|c|c|}
\hline \multirow[t]{2}{*}{ Study or subgroup } & \multicolumn{2}{|c|}{ Cognitive training } & \multicolumn{2}{|c|}{ Control } & \multirow{2}{*}{$\begin{array}{c}\text { Std. Mean Difference } \\
\text { Random, } 95 \% \mathrm{Cl}\end{array}$} & \multirow[t]{2}{*}{ Weight } & \multirow{2}{*}{$\begin{array}{c}\text { Std. Mean Difference } \\
\text { Random, } 95 \% \mathrm{CI}\end{array}$} \\
\hline & $\mathbf{N}$ & Mean(SD) & $\mathbf{N}$ & Mean(SD) & & & \\
\hline Galante 2007 & 7 & $0(8.9)$ & 4 & $2.5(6.7)$ & $\longrightarrow$ & $29.86 \%$ & $-0.28[-1.51,0.96]$ \\
\hline Cahn-Weiner 2003 & 17 & $11.7(21.1)$ & 17 & $-1.4(25.3)$ & + & $70.14 \%$ & $0.55[-0.14,1.24]$ \\
\hline 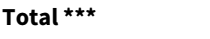 & 24 & & 21 & & & $100 \%$ & $0.3[-0.44,1.04]$ \\
\hline \multicolumn{8}{|c|}{ Heterogeneity: $\mathrm{Tau}^{2}=0.08 ; \mathrm{Chi}^{2}=1.31, \mathrm{df}=1(\mathrm{P}=0.25) ; \mathrm{I}^{2}=23.86 \%$} \\
\hline \multicolumn{8}{|c|}{ Test for overall effect: $Z=0.8(P=0.42)$} \\
\hline
\end{tabular}


Analysis 2.15. Comparison 2 Cognitive training vs control in the medium term ( 3 to 12 months post intervention), Outcome 15 Change in meta cognition (self-reported).

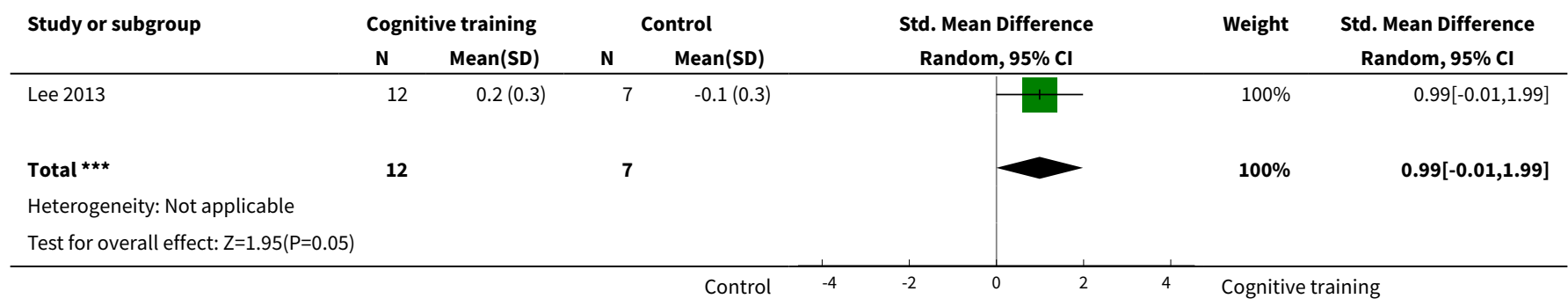

Analysis 2.16. Comparison 2 Cognitive training vs control in the medium term ( 3 to 12 months post intervention), Outcome 16 Change in meta cognition (informant-reported).

\begin{tabular}{|c|c|c|c|c|c|c|c|}
\hline \multirow[t]{2}{*}{ Study or subgroup } & \multicolumn{2}{|c|}{ Cognitive training } & \multicolumn{2}{|c|}{ Control } & \multirow{2}{*}{$\begin{array}{c}\text { Std. Mean Difference } \\
\text { Random, } 95 \% \mathrm{Cl}\end{array}$} & \multirow[t]{2}{*}{ Weight } & \multirow{2}{*}{$\begin{array}{c}\text { Std. Mean Difference } \\
\text { Random, } 95 \% \mathrm{Cl}\end{array}$} \\
\hline & $\mathbf{N}$ & Mean(SD) & $\mathbf{N}$ & Mean(SD) & & & \\
\hline Cahn-Weiner 2003 & 17 & $-2(16.1)$ & 17 & $-1(19.3)$ & - & $100 \%$ & $-0.06[-0.73,0.62]$ \\
\hline Total *** & 17 & & 17 & & & $100 \%$ & $-0.06[-0.73,0.62]$ \\
\hline \multicolumn{8}{|c|}{ Heterogeneity: Not applicable } \\
\hline \multicolumn{8}{|c|}{ Test for overall effect: $\mathrm{Z}=0.16(\mathrm{P}=0.87)$} \\
\hline & & & & Control & -2 & Cognitiv & ining \\
\hline
\end{tabular}

Analysis 2.17. Comparison 2 Cognitive training vs control in the medium term ( 3 to 12 months post intervention), Outcome 17 Change in participants' mood.

\begin{tabular}{|c|c|c|c|c|c|c|c|}
\hline \multirow[t]{2}{*}{ Study or subgroup } & \multicolumn{2}{|c|}{ Cognitive training } & \multicolumn{2}{|c|}{ Control } & \multirow{2}{*}{$\begin{array}{c}\text { Std. Mean Difference } \\
\text { Random, } 95 \% \mathrm{CI}\end{array}$} & \multirow[t]{2}{*}{ Weight } & \multirow{2}{*}{$\begin{array}{c}\text { Std. Mean Difference } \\
\text { Random, } 95 \% \mathrm{CI}\end{array}$} \\
\hline & $\mathbf{N}$ & Mean(SD) & $\mathbf{N}$ & Mean(SD) & & & \\
\hline Galante 2007 & 7 & $-0.1(1.6)$ & 4 & $0.2(0.7)$ & $\mp$ & $37.06 \%$ & $-0.2[-1.43,1.03]$ \\
\hline Lee 2013 & 12 & $-0.7(2.4)$ & 7 & $-1.9(2.8)$ & +1 & $62.94 \%$ & $0.45[-0.49,1.4]$ \\
\hline 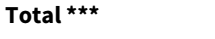 & 19 & & 11 & & & $100 \%$ & $0.21[-0.54,0.96]$ \\
\hline \multicolumn{8}{|c|}{ Heterogeneity: $\mathrm{Tau}^{2}=0 ; \mathrm{Chi}^{2}=0.68, \mathrm{df}=1(\mathrm{P}=0.41) ; \mathrm{I}^{2}=0 \%$} \\
\hline \multicolumn{8}{|c|}{ Test for overall effect: $Z=0.55(P=0.58)$} \\
\hline
\end{tabular}

Analysis 2.18. Comparison 2 Cognitive training vs control in the medium term ( 3 to 12 months post intervention), Outcome 18 Change in capacity for activities of daily living.

\begin{tabular}{|c|c|c|c|c|c|c|c|}
\hline \multirow[t]{2}{*}{ Study or subgroup } & \multicolumn{2}{|c|}{ Cognitive training } & \multicolumn{2}{|c|}{ Control } & \multirow{2}{*}{$\begin{array}{c}\text { Std. Mean Difference } \\
\text { Random, } 95 \% \mathrm{Cl}\end{array}$} & \multirow[t]{2}{*}{ Weight } & \multirow{2}{*}{$\begin{array}{c}\text { Std. Mean Difference } \\
\text { Random, } 95 \% \mathrm{Cl}\end{array}$} \\
\hline & $\mathbf{N}$ & Mean(SD) & $\mathbf{N}$ & Mean(SD) & & & \\
\hline Galante 2007 & 7 & $-0.7(1.4)$ & 4 & $0.3(0.4)$ & $\because \mid$ & $22.07 \%$ & $-0.77[-2.06,0.52]$ \\
\hline Cahn-Weiner 2003 & 17 & $0.6(3.1)$ & 17 & $-0.5(3.4)$ & $1-$ & $45.85 \%$ & $0.33[-0.35,1.01]$ \\
\hline Lee 2013 & 12 & $1.1(3.4)$ & 7 & $-1.7(3.8)$ & $\mp$ & $32.08 \%$ & $0.75[-0.22,1.72]$ \\
\hline 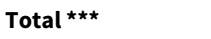 & 36 & & 28 & & & $100 \%$ & $0.22[-0.5,0.94]$ \\
\hline
\end{tabular}




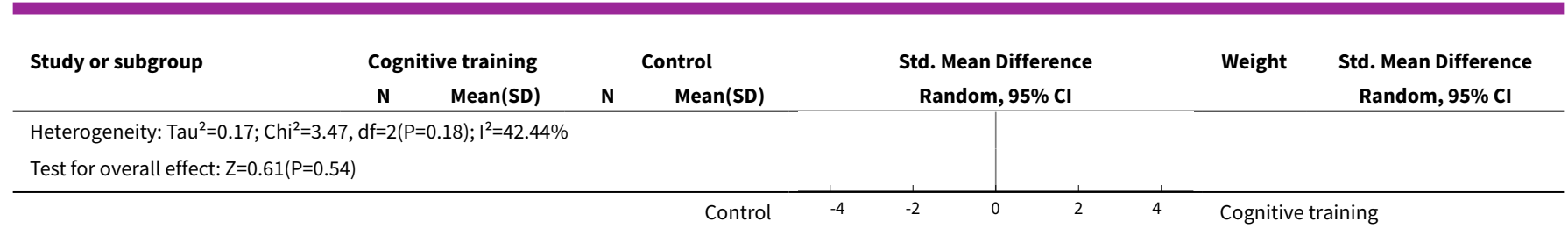

Analysis 2.19. Comparison 2 Cognitive training vs control in the medium term ( 3 to 12 months post intervention), Outcome 19 Change in general health and quality of life.

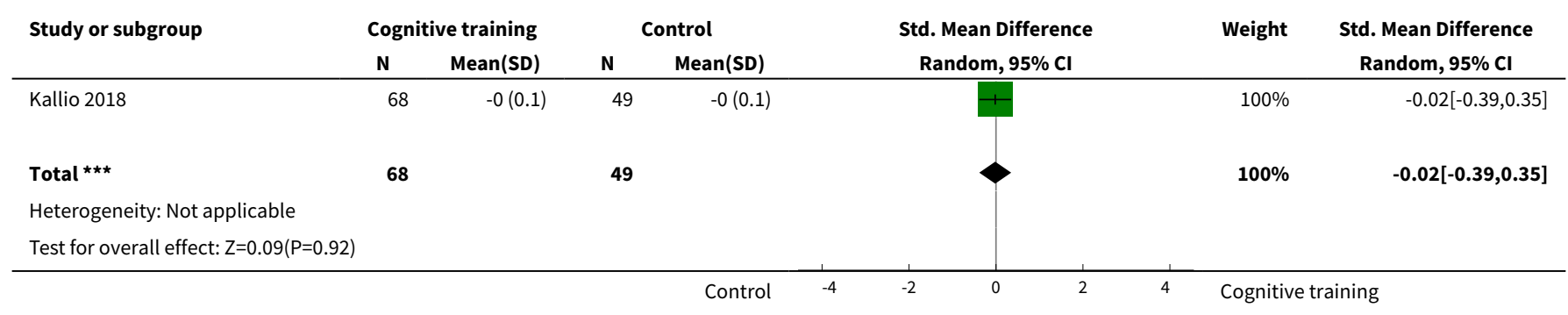

Analysis 2.20. Comparison 2 Cognitive training vs control in the medium term ( 3 to 12 months post intervention), Outcome 20 Change in behavioural and psychological symptoms of dementia (BPSD).

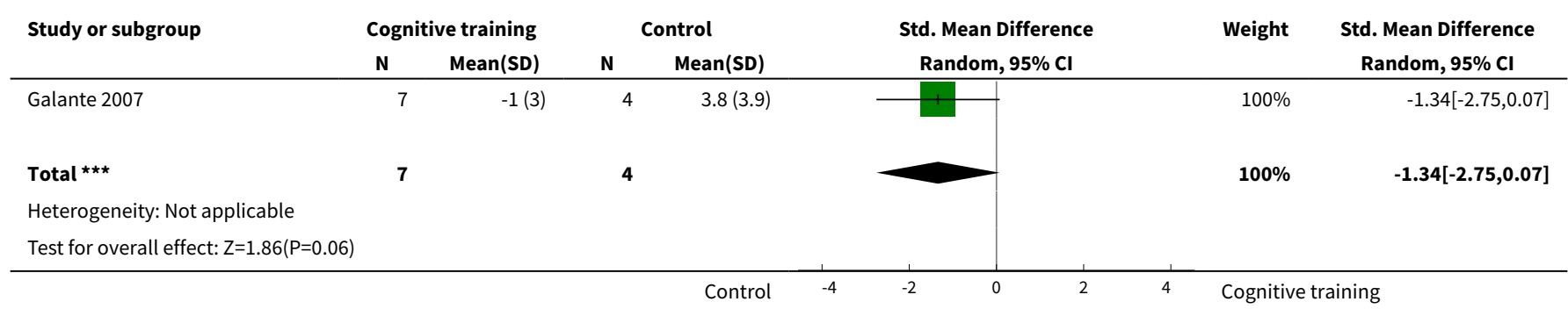

Comparison 3. Cognitive training vs alternative treatment immediately post intervention

\begin{tabular}{llllll}
\hline Outcome or subgroup title & $\begin{array}{l}\text { No. of } \\
\text { studies }\end{array}$ & $\begin{array}{l}\text { No. of } \\
\text { partici- } \\
\text { pants }\end{array}$ & Statistical method & Effect size \\
\hline $\begin{array}{l}1 \text { Change in a global measure } \\
\text { of cognition (composite) }\end{array}$ & 7 & 769 & Std. Mean Difference (Random, 95\% Cl) & $0.21[-0.23,0.64]$ \\
\hline $\begin{array}{l}2 \text { Change in a global measure } \\
\text { of cognition (composite)_zero } \\
\text { correlation }\end{array}$ & 7 & 769 & Std. Mean Difference (Random, 95\% Cl) & $-0.03[-0.23,0.17]$ \\
\hline $\begin{array}{l}3 \text { Change in a global measure } \\
\text { of cognition }\end{array}$ & 7 & 724 & Std. Mean Difference (IV, Random, 95\% CI) & $0.16[-0.28,0.60]$ \\
\hline $\begin{array}{l}4 \text { Change in a global measure } \\
\text { of cognition_zero correlation }\end{array}$ & 7 & 724 & Std. Mean Difference (IV, Random, 95\% CI) & $-0.02[-0.24,0.20]$ \\
\hline
\end{tabular}




\begin{tabular}{|c|c|c|c|c|}
\hline Outcome or subgroup title & $\begin{array}{l}\text { No. of } \\
\text { studies }\end{array}$ & $\begin{array}{l}\text { No. of } \\
\text { partici- } \\
\text { pants }\end{array}$ & Statistical method & Effect size \\
\hline $\begin{array}{l}5 \text { Change in disease progres- } \\
\text { sion }\end{array}$ & 3 & 131 & Std. Mean Difference (IV, Random, 95\% CI) & $0.15[-0.33,0.63]$ \\
\hline 6 Change in delayed memory & 3 & 147 & Std. Mean Difference (IV, Random, 95\% CI) & $0.71[-0.33,1.75]$ \\
\hline $\begin{array}{l}7 \text { Change in immediate memo- } \\
\text { ry }\end{array}$ & 3 & 147 & Std. Mean Difference (IV, Random, 95\% CI) & $0.51[-0.19,1.21]$ \\
\hline $\begin{array}{l}8 \text { Change in attention and } \\
\text { working memory }\end{array}$ & 2 & 69 & Std. Mean Difference (IV, Random, 95\% CI) & $0.91[-0.46,2.27]$ \\
\hline 9 Change in language (naming) & 1 & 16 & Std. Mean Difference (IV, Random, 95\% CI) & $-0.63[-1.65,0.38]$ \\
\hline $\begin{array}{l}10 \text { Change in verbal letter flu- } \\
\text { ency }\end{array}$ & 3 & 75 & Std. Mean Difference (IV, Random, 95\% CI) & $0.34[-0.38,1.05]$ \\
\hline $\begin{array}{l}11 \text { Change in verbal category } \\
\text { fluency }\end{array}$ & 2 & 55 & Std. Mean Difference (IV, Random, 95\% CI) & $-0.28[-1.46,0.89]$ \\
\hline $\begin{array}{l}12 \text { Change in executive func- } \\
\text { tion }\end{array}$ & 4 & 163 & Std. Mean Difference (IV, Random, 95\% CI) & $1.44[-0.26,3.14]$ \\
\hline $\begin{array}{l}13 \text { Change in speed of informa- } \\
\text { tion processing }\end{array}$ & 2 & 55 & Std. Mean Difference (IV, Random, 95\% CI) & $0.00[-0.55,0.55]$ \\
\hline $\begin{array}{l}14 \text { Change in meta cognition } \\
\text { (self-reported) }\end{array}$ & 0 & 0 & Std. Mean Difference (IV, Random, 95\% CI) & $0.0[0.0,0.0]$ \\
\hline $\begin{array}{l}15 \text { Change in meta cognition } \\
\text { (informant-reported) }\end{array}$ & 0 & 0 & Std. Mean Difference (IV, Random, 95\% CI) & $0.0[0.0,0.0]$ \\
\hline $\begin{array}{l}16 \text { Change in participants' } \\
\text { mood }\end{array}$ & 3 & 543 & Std. Mean Difference (IV, Random, 95\% CI) & $-0.11[-0.29,0.07]$ \\
\hline $\begin{array}{l}17 \text { Change in capacity for ac- } \\
\text { tivities of daily living }\end{array}$ & 3 & 525 & Std. Mean Difference (IV, Random, 95\% CI) & $-0.25[-0.43,-0.07]$ \\
\hline $\begin{array}{l}18 \text { Change in general health } \\
\text { and quality of life }\end{array}$ & 4 & 631 & Std. Mean Difference (IV, Random, 95\% CI) & $-0.49[1.00,0.02]$ \\
\hline $\begin{array}{l}19 \text { Change in behavioural and } \\
\text { psychological symptoms of } \\
\text { dementia (BPSD) }\end{array}$ & 3 & 672 & Std. Mean Difference (IV, Random, 95\% CI) & $-0.11[-0.27,0.06]$ \\
\hline $\begin{array}{l}20 \text { Participant burden (reten- } \\
\text { tion rates) }\end{array}$ & 4 & 639 & Odds Ratio (M-H, Random, 95\% Cl) & $0.78[0.24,2.57]$ \\
\hline $\begin{array}{l}21 \text { Change in burden of care } \\
\text { (CAREGIVER) }\end{array}$ & 3 & 591 & Std. Mean Difference (IV, Random, 95\% CI) & $-0.15[-0.47,0.17]$ \\
\hline $\begin{array}{l}22 \text { Change in quality of life } \\
\text { (CAREGIVER) }\end{array}$ & 1 & 88 & Std. Mean Difference (IV, Random, 95\% CI) & $-0.25[-0.74,0.24]$ \\
\hline
\end{tabular}




\begin{tabular}{lllll}
\hline Outcome or subgroup title & $\begin{array}{l}\text { No. of } \\
\text { studies }\end{array}$ & $\begin{array}{l}\text { No. of } \\
\text { partici- } \\
\text { pants }\end{array}$ & Statistical method & Effect size \\
\hline $\begin{array}{l}23 \text { Change in mood and well- } \\
\text { being (CAREGIVER) }\end{array}$ & 1 & 88 & Std. Mean Difference (IV, Random, 95\% CI) & $1.50[0.96,2.04]$ \\
\hline
\end{tabular}

Analysis 3.1. Comparison 3 Cognitive training vs alternative treatment immediately post intervention, Outcome 1 Change in a global measure of cognition (composite).

\begin{tabular}{|c|c|c|c|c|c|c|}
\hline Study or subgroup & $\begin{array}{c}\text { Cognitive } \\
\text { training } \\
\mathbf{N} \\
\end{array}$ & $\begin{array}{c}\text { Alternative } \\
\text { treatment } \\
\mathbf{N}\end{array}$ & $\begin{array}{c}\text { Std. Mean } \\
\text { Difference } \\
(\mathrm{SE}) \\
\end{array}$ & IV, Random, 95\% CI & Weight & IV, Random, 95\% CI \\
\hline Amieva 2016 & 166 & 325 & $-0.1(0.1)$ & $*$ & $21.9 \%$ & $-0.13[-0.33,0.07]$ \\
\hline Brueggen 2017 & 8 & 8 & $-0.2(0.48)$ & $\longrightarrow$ & $11.07 \%$ & $-0.18[-1.12,0.76]$ \\
\hline de Vreese 1998 & 9 & 9 & $1.3(0.5)$ & $\longrightarrow$ & $10.6 \%$ & $1.31[0.33,2.29]$ \\
\hline Giovagnoli 2017 & 13 & 26 & $-0(0.34)$ & $\longrightarrow$ & $14.91 \%$ & $-0.05[-0.72,0.62]$ \\
\hline Quayhagen 2000 & 21 & 67 & $0.4(0.33)$ & + & $15.22 \%$ & $0.35[-0.3,1]$ \\
\hline Quintana Hernandez 2014 & 27 & 70 & $-0.4(0.23)$ & $\rightarrow$ & $18.4 \%$ & $-0.45[-0.9,0]$ \\
\hline Total $(95 \% \mathrm{Cl})$ & & & & & $100 \%$ & $0.21[-0.23,0.64]$ \\
\hline \multicolumn{7}{|c|}{ Heterogeneity: $\mathrm{Tau}^{2}=0.22 ; \mathrm{Chi}^{2}=22.34, \mathrm{df}=6(\mathrm{P}=0) ; \mathrm{I}^{2}=73.14 \%$} \\
\hline \multicolumn{7}{|c|}{ Test for overall effect: $Z=0.93(P=0.35)$} \\
\hline
\end{tabular}

Analysis 3.2. Comparison 3 Cognitive training vs alternative treatment immediately post intervention, Outcome 2 Change in a global measure of cognition (composite)_zero correlation.

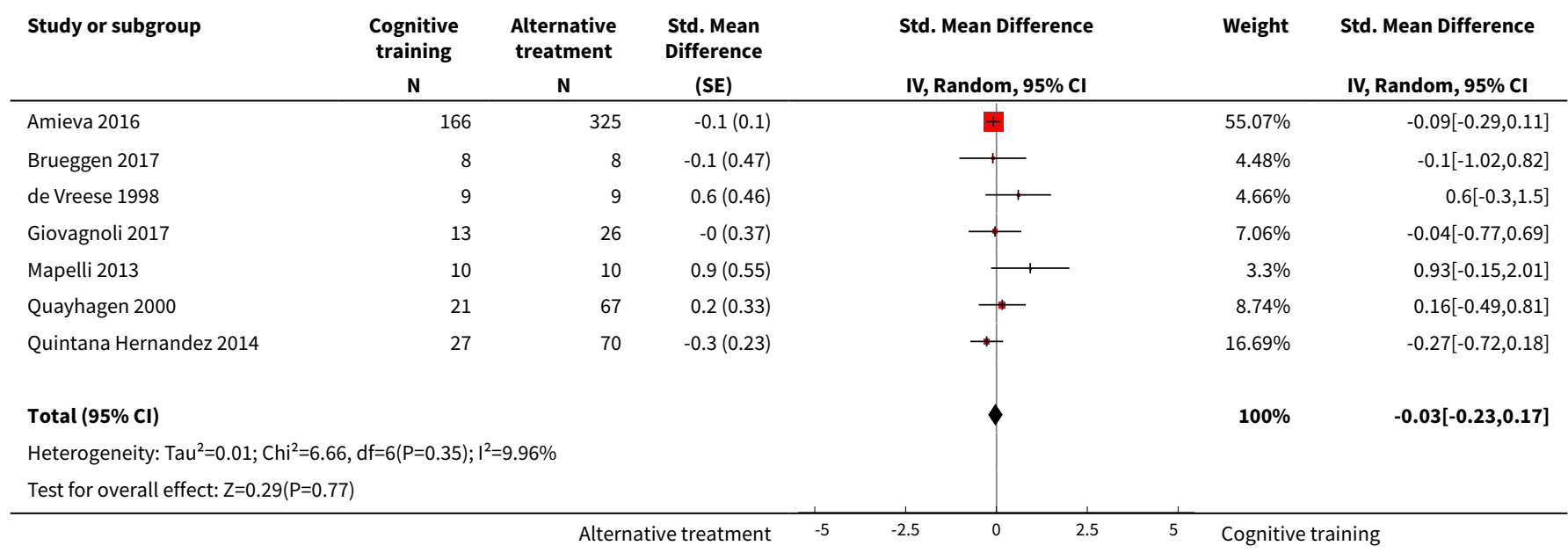


Analysis 3.3. Comparison 3 Cognitive training vs alternative treatment immediately post intervention, Outcome 3 Change in a global measure of cognition.

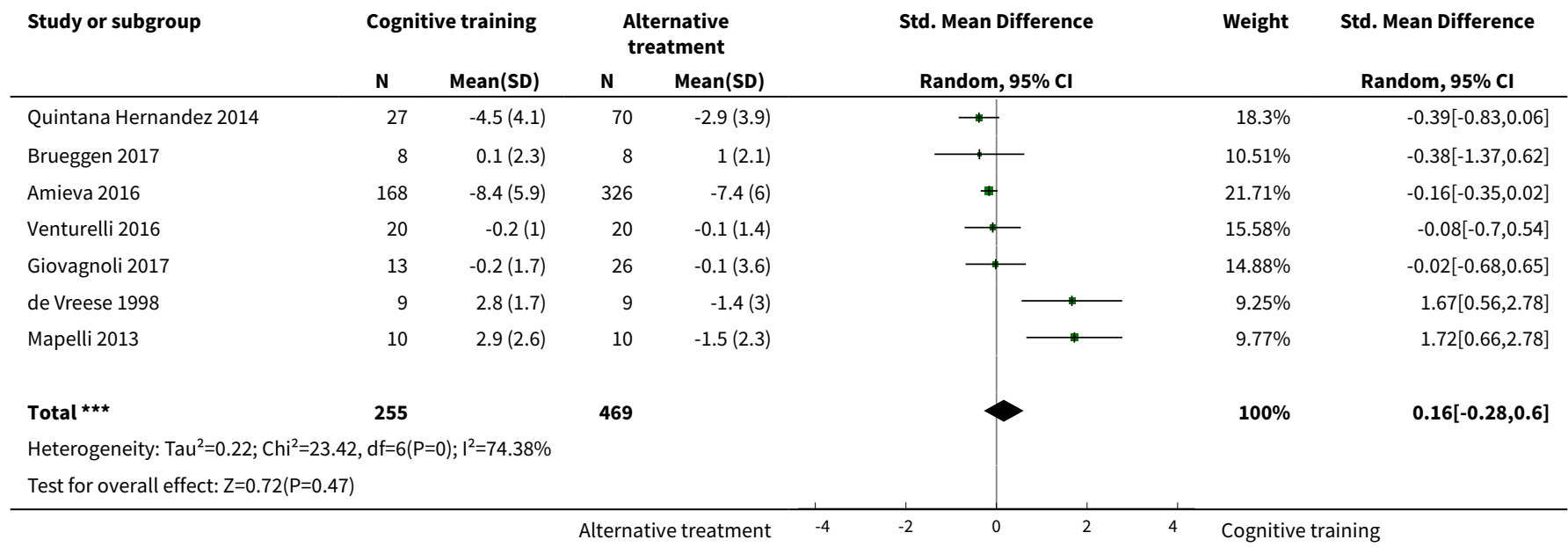

Analysis 3.4. Comparison 3 Cognitive training vs alternative treatment immediately post intervention, Outcome 4 Change in a global measure of cognition_zero correlation.

\begin{tabular}{|c|c|c|c|c|c|c|c|}
\hline \multirow[t]{2}{*}{ Study or subgroup } & \multicolumn{2}{|c|}{ Cognitive training } & \multicolumn{2}{|c|}{$\begin{array}{l}\text { Alternative } \\
\text { treatment }\end{array}$} & \multirow{2}{*}{$\begin{array}{l}\text { Std. Mean Difference } \\
\text { Random, } 95 \% \mathrm{Cl}\end{array}$} & \multirow[t]{2}{*}{ Weight } & \multirow{2}{*}{$\begin{array}{l}\text { Std. Mean Difference } \\
\text { Random, } 95 \% \mathrm{Cl}\end{array}$} \\
\hline & $\mathbf{N}$ & Mean(SD) & $\mathbf{N}$ & Mean(SD) & & & \\
\hline Quintana Hernandez 2014 & 27 & $-4.5(6.9)$ & 70 & $-2.9(6.7)$ & $\rightarrow$ & $18.36 \%$ & $-0.23[-0.67,0.22]$ \\
\hline Brueggen 2017 & 8 & $0.1(5.1)$ & 8 & $1(4.6)$ & 1 & $4.81 \%$ & $-0.17[-1.15,0.81]$ \\
\hline Amieva 2016 & 168 & $-8.4(8.7)$ & 326 & $-7.4(8.7)$ & $\Psi$ & $45.93 \%$ & $-0.11[-0.3,0.08]$ \\
\hline Venturelli 2016 & 20 & $-0.2(2.1)$ & 20 & $-0.1(3)$ & $\longrightarrow$ & $10.91 \%$ & $-0.04[-0.66,0.58]$ \\
\hline de Vreese 1998 & 9 & $2.8(3.5)$ & 9 & $-1.4(6.5)$ & & $4.96 \%$ & $0.77[-0.2,1.73]$ \\
\hline Mapelli 2013 & 10 & $2.9(5)$ & 10 & $-1.5(4.7)$ & 1 & $5.36 \%$ & $0.87[-0.06,1.79]$ \\
\hline 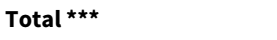 & 255 & & 469 & & & $100 \%$ & $-0.02[-0.24,0.2]$ \\
\hline \multicolumn{8}{|c|}{ Heterogeneity: $\mathrm{Tau}^{2}=0.02 ; \mathrm{Chi}^{2}=7.58, \mathrm{df}=6(\mathrm{P}=0.27) ; \mathrm{I}^{2}=20.8 \%$} \\
\hline \multicolumn{3}{|c|}{ Test for overall effect: $Z=0.18(P=0.85)$} & & & & & \\
\hline
\end{tabular}

Analysis 3.5. Comparison 3 Cognitive training vs alternative treatment immediately post intervention, Outcome 5 Change in disease progression.

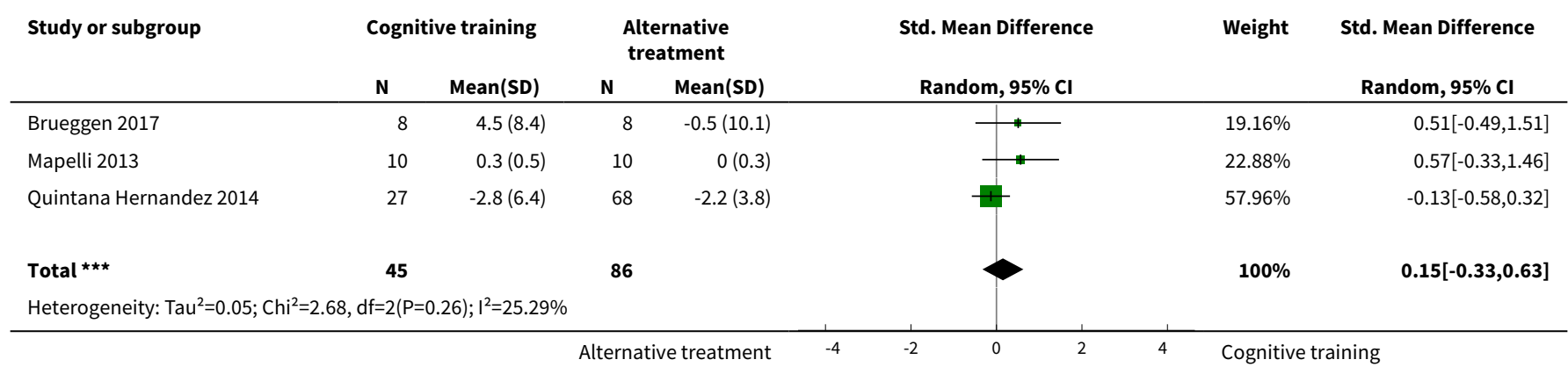




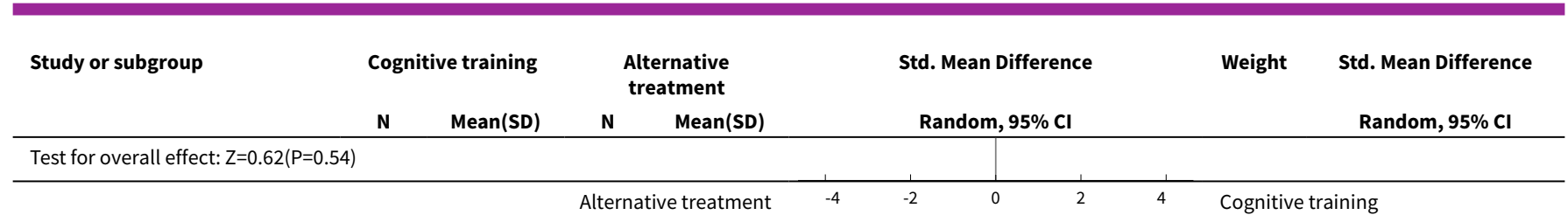

Analysis 3.6. Comparison 3 Cognitive training vs alternative treatment immediately post intervention, Outcome 6 Change in delayed memory.

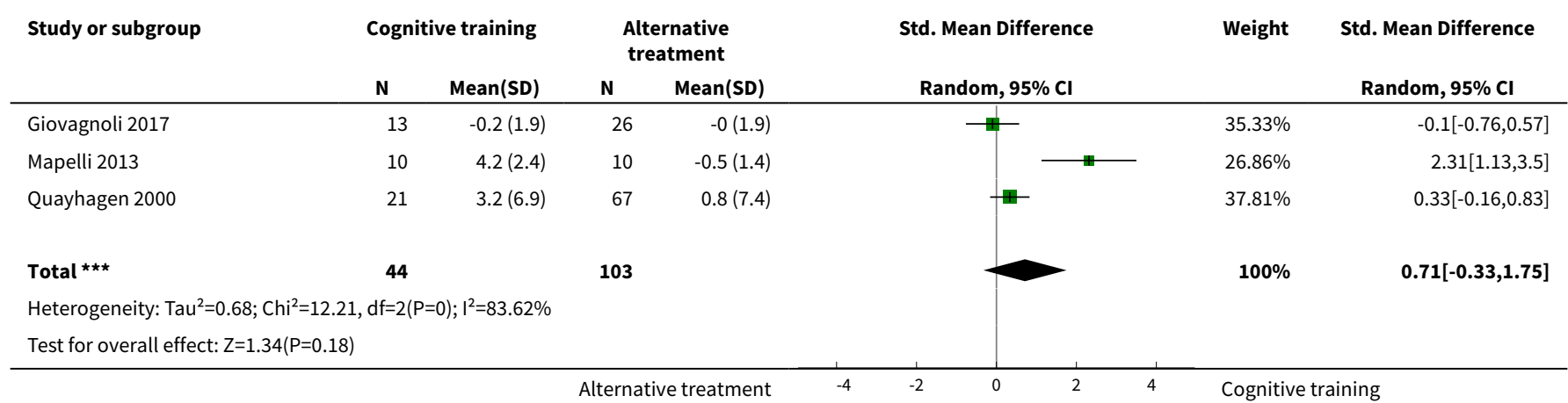

Analysis 3.7. Comparison 3 Cognitive training vs alternative treatment immediately post intervention, Outcome 7 Change in immediate memory.

\begin{tabular}{|c|c|c|c|c|c|c|c|}
\hline \multirow[t]{2}{*}{ Study or subgroup } & \multicolumn{2}{|c|}{ Cognitive training } & \multicolumn{2}{|c|}{$\begin{array}{c}\text { Alternative } \\
\text { treatment }\end{array}$} & \multirow{2}{*}{$\begin{array}{l}\text { Std. Mean Difference } \\
\text { Random, } 95 \% \mathrm{Cl}\end{array}$} & \multirow[t]{2}{*}{ Weight } & \multirow{2}{*}{$\begin{array}{l}\text { Std. Mean Difference } \\
\text { Random, } 95 \% \mathrm{Cl}\end{array}$} \\
\hline & $\mathbf{N}$ & $\operatorname{Mean}(S D)$ & $\mathbf{N}$ & Mean(SD) & & & \\
\hline Giovagnoli 2017 & 13 & $1(4.4)$ & 26 & $0.3(6.2)$ & 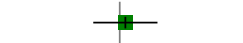 & $34.91 \%$ & $0.12[-0.55,0.78]$ \\
\hline Quayhagen 2000 & 21 & $2.3(12.1)$ & 67 & $0(9.3)$ & 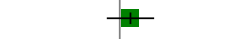 & $40.81 \%$ & $0.22[-0.27,0.71]$ \\
\hline Mapelli 2013 & 10 & $2.6(2.4)$ & 10 & $-0.5(1.3)$ & $\longrightarrow$ & $24.28 \%$ & $1.56[0.53,2.59]$ \\
\hline \multicolumn{8}{|c|}{ Heterogeneity: $\mathrm{Tau}^{2}=0.25 ; \mathrm{Chi}^{2}=6.02, \mathrm{df}=2(\mathrm{P}=0.05) ; \mathrm{I}^{2}=66.76 \%$} \\
\hline \multicolumn{8}{|c|}{ Test for overall effect: $Z=1.43(P=0.15)$} \\
\hline
\end{tabular}

Analysis 3.8. Comparison 3 Cognitive training vs alternative treatment immediately post intervention, Outcome 8 Change in attention and working memory.

\begin{tabular}{|c|c|c|c|c|c|c|c|}
\hline \multirow[t]{2}{*}{ Study or subgroup } & \multicolumn{2}{|c|}{ Cognitive training } & \multicolumn{2}{|c|}{$\begin{array}{l}\text { Alternative } \\
\text { treatment }\end{array}$} & \multirow{2}{*}{$\begin{array}{c}\text { Std. Mean Difference } \\
\text { Random, } 95 \% \mathrm{Cl} \\
\end{array}$} & \multirow[t]{2}{*}{ Weight } & \multirow{2}{*}{$\begin{array}{l}\text { Std. Mean Difference } \\
\text { Random, } 95 \% \mathrm{Cl} \\
\end{array}$} \\
\hline & $\mathbf{N}$ & Mean(SD) & $\mathbf{N}$ & Mean(SD) & & & \\
\hline Giovagnoli 2017 & 13 & $-0.1(0.6)$ & 26 & $-0.3(0.9)$ & $\frac{1}{1-}$ & $52.18 \%$ & $0.24[-0.43,0.91]$ \\
\hline Mapelli 2013 & 10 & $1.7(1.2)$ & 20 & $0.1(0.8)$ & $\longrightarrow$ & $47.82 \%$ & $1.63[0.76,2.51]$ \\
\hline 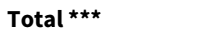 & 23 & & 46 & & & $100 \%$ & $0.91[-0.46,2.27]$ \\
\hline \multicolumn{8}{|c|}{ Heterogeneity: $\mathrm{Tau}^{2}=0.82 ; \mathrm{Chi}^{2}=6.15, \mathrm{df}=1(\mathrm{P}=0.01) ; \mathrm{I}^{2}=83.73 \%$} \\
\hline
\end{tabular}




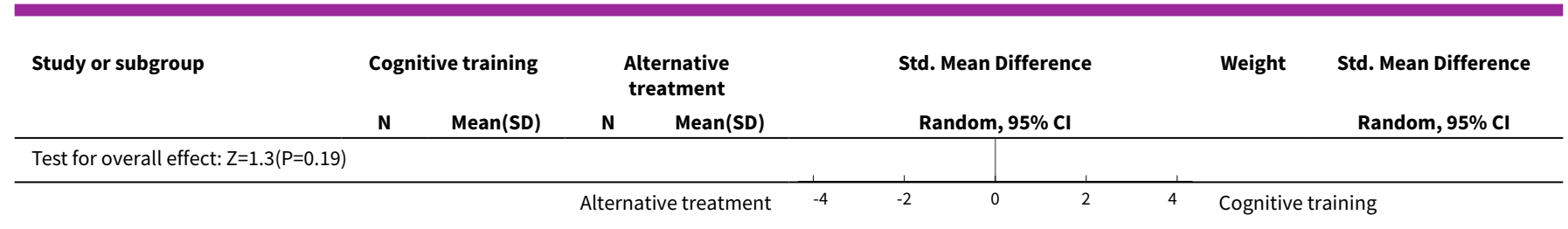

Analysis 3.9. Comparison 3 Cognitive training vs alternative treatment immediately post intervention, Outcome 9 Change in language (naming).

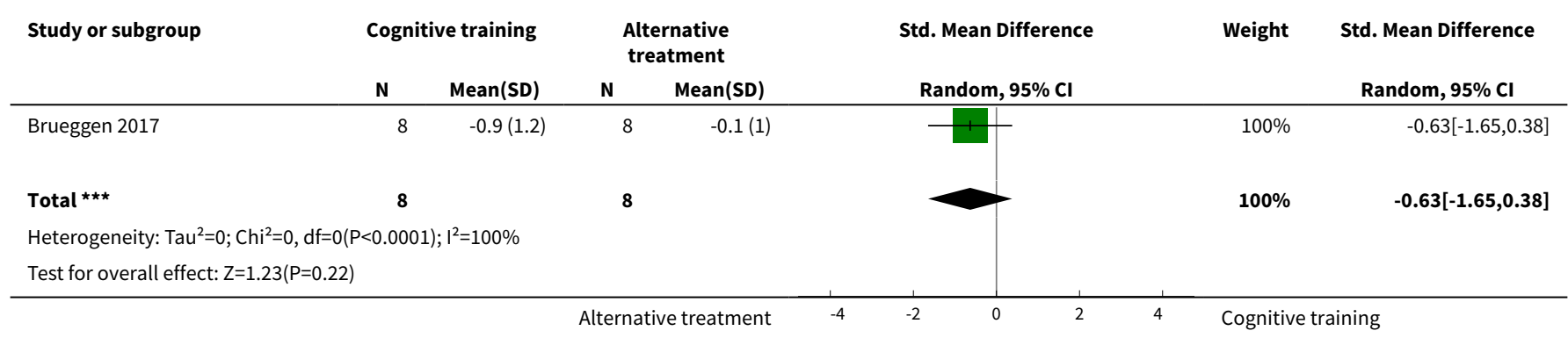

Analysis 3.10. Comparison 3 Cognitive training vs alternative treatment immediately post intervention, Outcome 10 Change in verbal letter fluency.

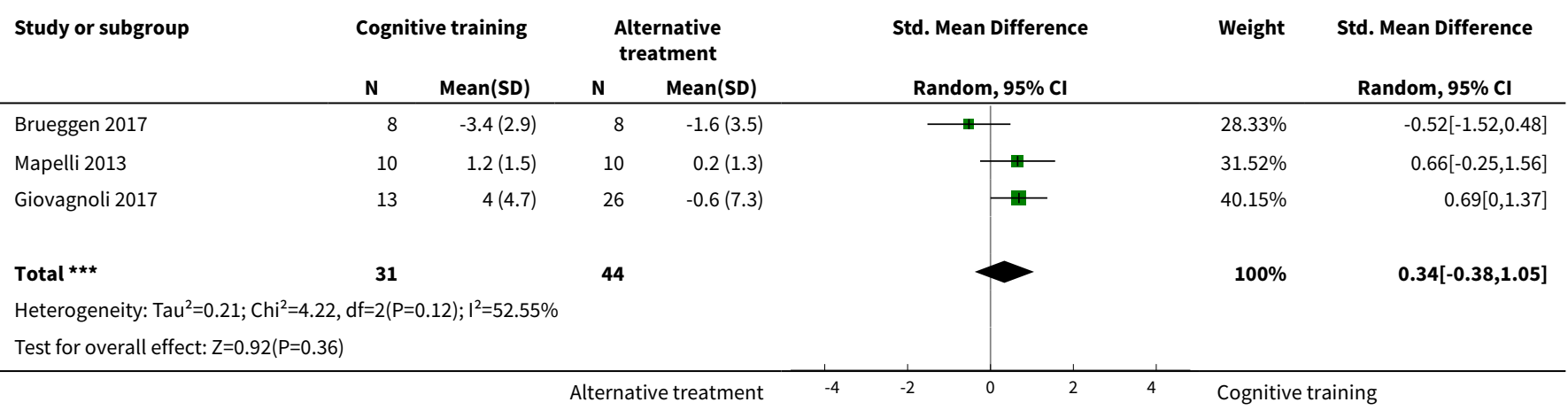

Analysis 3.11. Comparison 3 Cognitive training vs alternative treatment immediately post intervention, Outcome 11 Change in verbal category fluency.

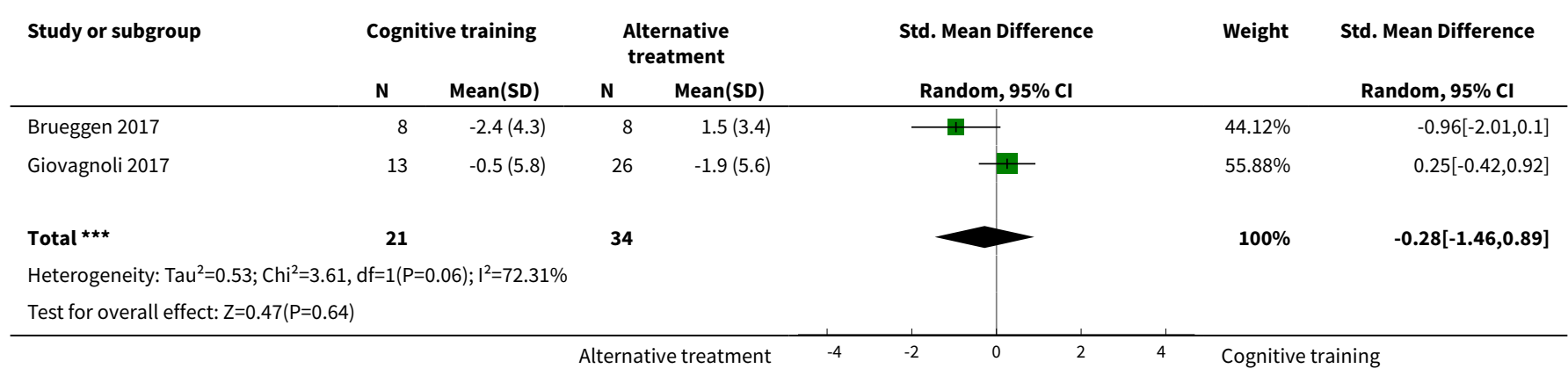


Analysis 3.12. Comparison 3 Cognitive training vs alternative treatment immediately post intervention, Outcome 12 Change in executive function.

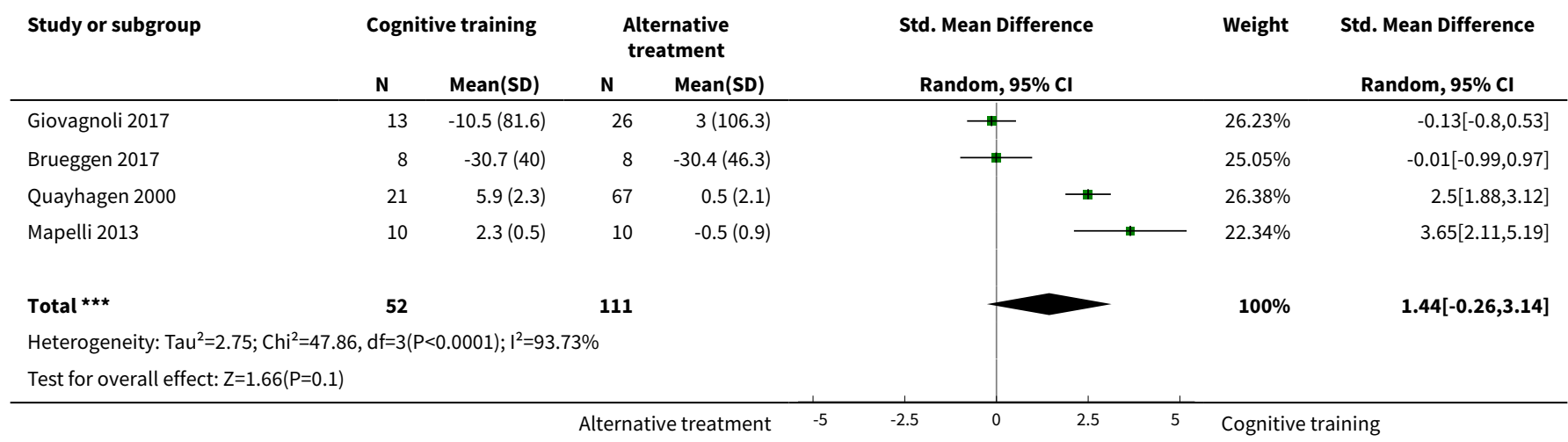

Analysis 3.13. Comparison 3 Cognitive training vs alternative treatment immediately post intervention, Outcome 13 Change in speed of information processing.

\begin{tabular}{|c|c|c|c|c|c|c|c|}
\hline \multirow[t]{2}{*}{ Study or subgroup } & \multicolumn{2}{|c|}{ Cognitive training } & \multicolumn{2}{|c|}{$\begin{array}{l}\text { Alternative } \\
\text { treatment }\end{array}$} & \multirow{2}{*}{$\begin{array}{l}\text { Std. Mean Difference } \\
\text { Random, } 95 \% \mathrm{Cl}\end{array}$} & \multirow[t]{2}{*}{ Weight } & \multirow{2}{*}{$\begin{array}{l}\text { Std. Mean Difference } \\
\text { Random, } 95 \% \mathrm{Cl}\end{array}$} \\
\hline & $\mathbf{N}$ & Mean(SD) & $\mathbf{N}$ & Mean(SD) & & & \\
\hline Giovagnoli 2017 & 13 & $-12.5(21.2)$ & 26 & $-7.6(51.3)$ & 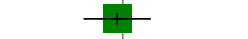 & $68.6 \%$ & $-0.11[-0.78,0.56]$ \\
\hline Brueggen 2017 & 8 & $-15.6(33.6)$ & 8 & $-24.2(33.5)$ & +1 & $31.4 \%$ & $0.24[-0.74,1.23]$ \\
\hline Total $\star \star \star$ & 21 & & 34 & & & $100 \%$ & $0[-0.55,0.55]$ \\
\hline \multicolumn{8}{|c|}{ Heterogeneity: $\mathrm{Tau}^{2}=0 ; \mathrm{Chi}^{2}=0.34, \mathrm{df}=1(\mathrm{P}=0.56) ; \mathrm{I}^{2}=0 \%$} \\
\hline \multicolumn{8}{|c|}{ Test for overall effect: $Z=0(P=1)$} \\
\hline
\end{tabular}

Analysis 3.16. Comparison 3 Cognitive training vs alternative treatment immediately post intervention, Outcome 16 Change in participants' mood.

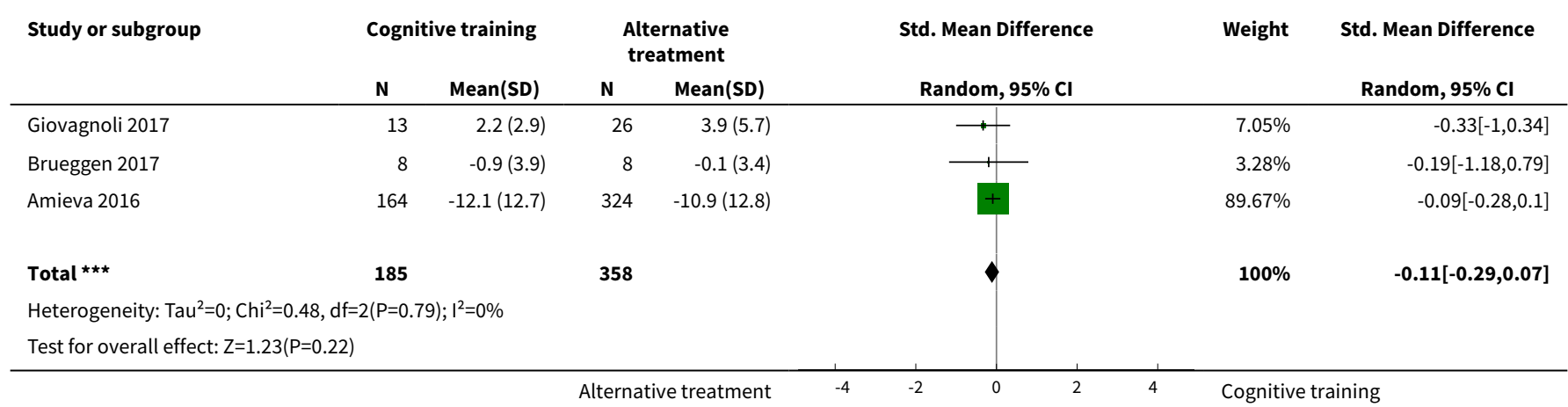


Analysis 3.17. Comparison 3 Cognitive training vs alternative treatment immediately post intervention, Outcome 17 Change in capacity for activities of daily living.

\begin{tabular}{|c|c|c|c|c|c|c|c|}
\hline \multirow[t]{2}{*}{ Study or subgroup } & \multicolumn{2}{|c|}{ Cognitive training } & \multicolumn{2}{|c|}{$\begin{array}{c}\text { Alternative } \\
\text { treatment }\end{array}$} & \multirow{2}{*}{$\begin{array}{c}\text { Std. Mean Difference } \\
\text { Random, } 95 \% \mathrm{Cl} \\
\end{array}$} & \multirow[t]{2}{*}{ Weight } & \multirow{2}{*}{$\begin{array}{c}\text { Std. Mean Difference } \\
\text { Random, 95\% Cl } \\
\end{array}$} \\
\hline & $\mathbf{N}$ & Mean(SD) & $\mathbf{N}$ & Mean(SD) & & & \\
\hline de Vreese 1998 & 9 & $-1.3(4.4)$ & 9 & $1.1(3.4)$ & $1-$ & $3.62 \%$ & $-0.59[-1.54,0.36]$ \\
\hline Brueggen 2017 & 8 & $-0.3(1.2)$ & 8 & $0.5(1.5)$ & 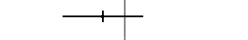 & $3.24 \%$ & $-0.54[-1.55,0.46]$ \\
\hline Amieva 2016 & 167 & $-1.8(8.5)$ & 324 & $0.1(7.6)$ & + & $93.14 \%$ & $-0.23[-0.42,-0.04]$ \\
\hline Total $\star \star \star ~$ & 184 & & 341 & & $\checkmark$ & $100 \%$ & $-0.25[-0.43,-0.07]$ \\
\hline \multicolumn{8}{|c|}{ Heterogeneity: $\operatorname{Tau}^{2}=0 ; \mathrm{Chi}^{2}=0.88, \mathrm{df}=2(\mathrm{P}=0.65) ; \mathrm{I}^{2}=0 \%$} \\
\hline \multicolumn{3}{|c|}{ Test for overall effect: $Z=2.74(P=0.01)$} & & & & & \\
\hline
\end{tabular}

Analysis 3.18. Comparison 3 Cognitive training vs alternative treatment immediately post intervention, Outcome 18 Change in general health and quality of life.

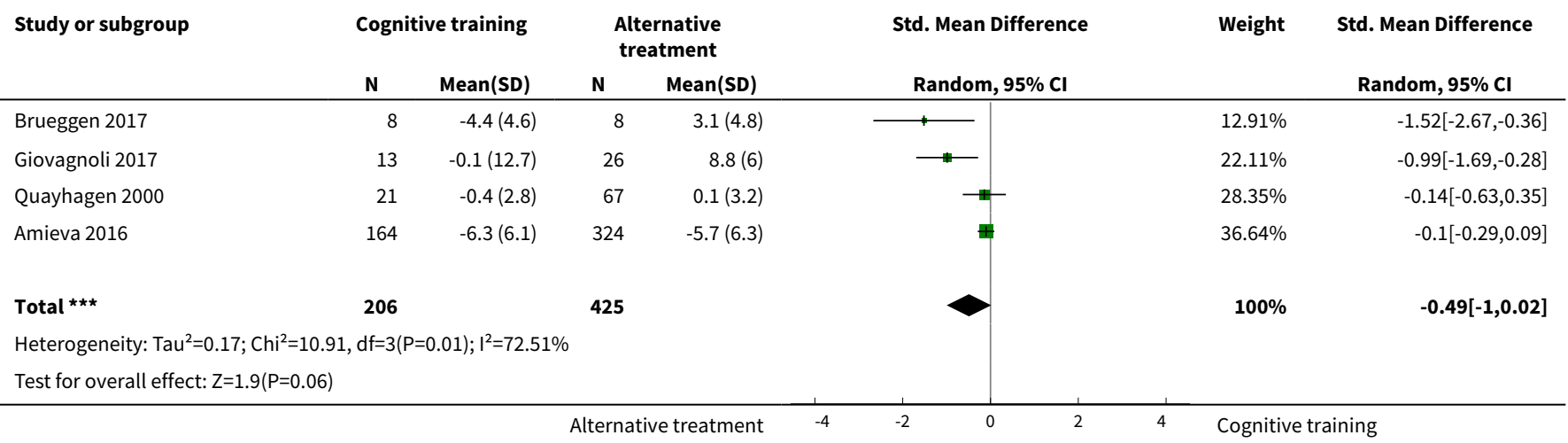

Analysis 3.19. Comparison 3 Cognitive training vs alternative treatment immediately post intervention, Outcome 19 Change in behavioural and psychological symptoms of dementia (BPSD).

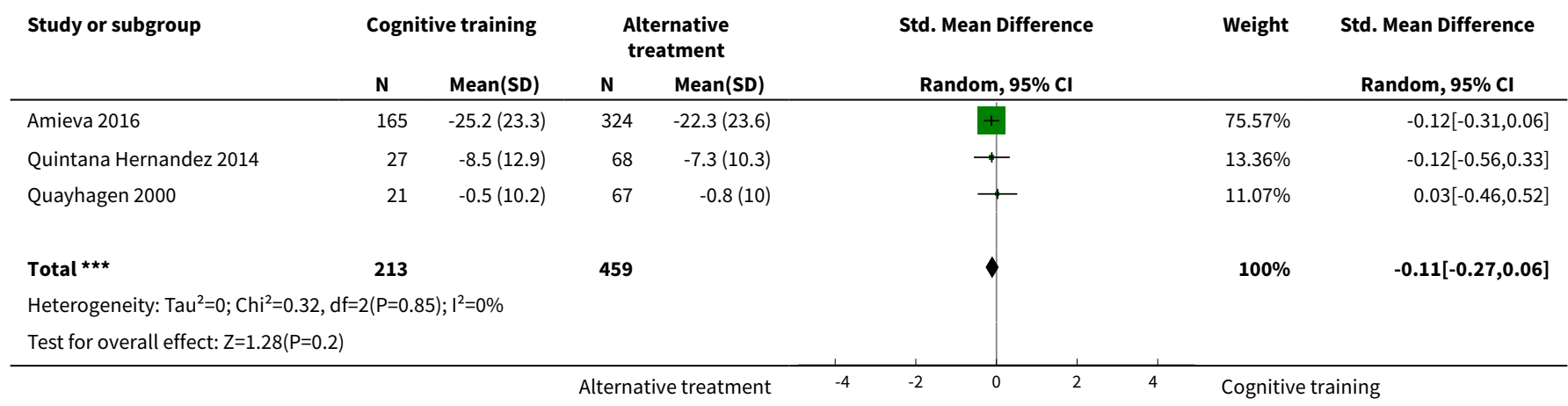


Analysis 3.20. Comparison 3 Cognitive training vs alternative treatment immediately post intervention, Outcome 20 Participant burden (retention rates).

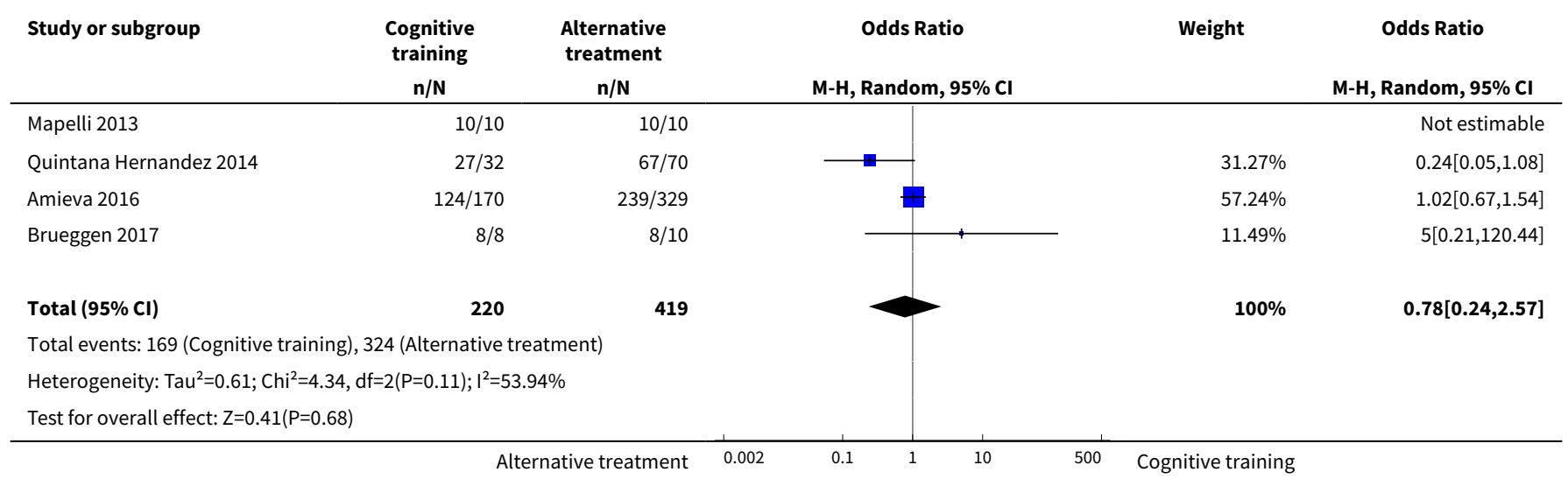

Analysis 3.21. Comparison 3 Cognitive training vs alternative treatment immediately post intervention, Outcome 21 Change in burden of care (CAREGIVER).

\begin{tabular}{|c|c|c|c|c|c|c|c|}
\hline \multirow[t]{2}{*}{ Study or subgroup } & \multicolumn{2}{|c|}{ Cognitive training } & \multicolumn{2}{|c|}{$\begin{array}{c}\text { Alternative } \\
\text { treatment }\end{array}$} & \multirow{2}{*}{$\begin{array}{l}\text { Std. Mean Difference } \\
\text { Random, } 95 \% \mathrm{Cl} \\
\end{array}$} & \multirow[t]{2}{*}{ Weight } & \multirow{2}{*}{$\begin{array}{l}\text { Std. Mean Difference } \\
\text { Random, 95\% Cl } \\
\end{array}$} \\
\hline & $\mathbf{N}$ & Mean(SD) & $\mathbf{N}$ & $\operatorname{Mean}(S D)$ & & & \\
\hline Amieva 2016 & 165 & $-21.3(19.1)$ & 322 & $-15.2(20.8)$ & + & $62.73 \%$ & $-0.3[-0.49,-0.11]$ \\
\hline Quayhagen 2000 & 21 & $-0.8(4.1)$ & 67 & $-0.6(4.4)$ & $\rightarrow$ & $28.08 \%$ & $-0.04[-0.53,0.45]$ \\
\hline Brueggen 2017 & 8 & $-0.7(9.2)$ & 8 & $-5.2(7.4)$ & \begin{tabular}{l|l}
$*$ \\
+
\end{tabular} & $9.19 \%$ & $0.51[-0.49,1.51]$ \\
\hline Total $\star \star \star$ & 194 & & 397 & & & $100 \%$ & $-0.15[-0.47,0.17]$ \\
\hline \multicolumn{8}{|c|}{ Heterogeneity: $\mathrm{Tau}^{2}=0.03 ; \mathrm{Chi}^{2}=3.17, \mathrm{df}=2(\mathrm{P}=0.21) ; \mathrm{I}^{2}=36.89 \%$} \\
\hline \multicolumn{3}{|c|}{ Test for overall effect: $Z=0.92(P=0.35)$} & & & & & \\
\hline
\end{tabular}

Analysis 3.22. Comparison 3 Cognitive training vs alternative treatment immediately post intervention, Outcome 22 Change in quality of life (CAREGIVER).

\begin{tabular}{|c|c|c|c|c|c|c|c|}
\hline \multirow[t]{2}{*}{ Study or subgroup } & \multicolumn{2}{|c|}{ Cognitive training } & \multicolumn{2}{|c|}{$\begin{array}{c}\text { Alternative } \\
\text { treatment }\end{array}$} & \multirow{2}{*}{$\begin{array}{l}\text { Std. Mean Difference } \\
\text { Random, } 95 \% \mathrm{Cl}\end{array}$} & \multirow[t]{2}{*}{ Weight } & \multirow{2}{*}{$\begin{array}{l}\text { Std. Mean Difference } \\
\text { Random, } 95 \% \mathrm{Cl} \\
\end{array}$} \\
\hline & $\mathbf{N}$ & Mean(SD) & $\mathbf{N}$ & Mean(SD) & & & \\
\hline Quayhagen 2000 & 21 & $-0.6(9.8)$ & 67 & $2.4(12.5)$ & & $100 \%$ & $-0.25[-0.74,0.24]$ \\
\hline 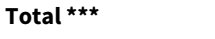 & 21 & & 67 & & & $100 \%$ & $-0.25[-0.74,0.24]$ \\
\hline \multicolumn{8}{|c|}{ Heterogeneity: Not applicable } \\
\hline
\end{tabular}


Analysis 3.23. Comparison 3 Cognitive training vs alternative treatment immediately post intervention, Outcome 23 Change in mood and well-being (CAREGIVER).

\begin{tabular}{|c|c|c|c|c|c|c|c|}
\hline \multirow{3}{*}{$\begin{array}{l}\text { Study or subgroup } \\
\text { Quayhagen } 2000\end{array}$} & \multicolumn{2}{|c|}{ Cognitive training } & \multicolumn{2}{|c|}{$\begin{array}{c}\text { Alternative } \\
\text { treatment }\end{array}$} & \multirow{2}{*}{$\begin{array}{c}\text { Std. Mean Difference } \\
\text { Random, 95\% Cl }\end{array}$} & \multirow{3}{*}{$\begin{array}{r}\text { Weight } \\
100 \%\end{array}$} & \multirow{3}{*}{$\begin{array}{l}\text { Std. Mean Difference } \\
\text { Random, 95\% Cl } \\
1.5[0.96,2.04]\end{array}$} \\
\hline & \multirow{2}{*}{$\frac{\mathbf{N}}{21}$} & \multirow{2}{*}{$\frac{\text { Mean(SD) }}{0.1(0.1)}$} & \multirow{2}{*}{$\frac{\mathbf{N}}{67}$} & \multirow{2}{*}{$\begin{array}{r}\text { Mean(SD) } \\
-0(0.1)\end{array}$} & & & \\
\hline & & & & & & & \\
\hline Total $\star \star \star ~$ & 21 & & 67 & & & $100 \%$ & $1.5[0.96,2.04]$ \\
\hline \multicolumn{8}{|c|}{ Heterogeneity: Not applicable } \\
\hline & & & Iterna & treatment -5 & -2.5 & Cognitiv & ining \\
\hline
\end{tabular}

\section{Comparison 4. Cognitive training vs alternative treatment in the medium term (3 to 12 months post intervention)}

\begin{tabular}{|c|c|c|c|c|}
\hline Outcome or subgroup title & $\begin{array}{l}\text { No. of } \\
\text { studies }\end{array}$ & $\begin{array}{l}\text { No. of } \\
\text { partici- } \\
\text { pants }\end{array}$ & Statistical method & Effect size \\
\hline $\begin{array}{l}1 \text { Change in a global measure } \\
\text { of cognition (composite) }\end{array}$ & 2 & 73 & Std. Mean Difference (Random, 95\% Cl) & $1.31[-1.03,3.65]$ \\
\hline $\begin{array}{l}2 \text { Change in a global measure } \\
\text { of cognition (composite)_zero } \\
\text { correlation }\end{array}$ & 2 & 73 & Std. Mean Difference (Random, 95\% Cl) & $0.62[-0.52,1.75]$ \\
\hline $\begin{array}{l}3 \text { Change in a global measure } \\
\text { of cognition }\end{array}$ & 2 & 73 & Std. Mean Difference (IV, Random, 95\% CI) & $3.20[-2.89,9.29]$ \\
\hline $\begin{array}{l}4 \text { Change in disease progres- } \\
\text { sion (zero correlation) }\end{array}$ & 0 & 0 & Std. Mean Difference (IV, Random, 95\% CI) & $0.0[0.0,0.0]$ \\
\hline $\begin{array}{l}5 \text { Change in disease progres- } \\
\text { sion }\end{array}$ & 0 & 0 & Std. Mean Difference (IV, Random, 95\% CI) & $0.0[0.0,0.0]$ \\
\hline 6 Change in delayed memory & 2 & 73 & Std. Mean Difference (IV, Random, 95\% CI) & $0.61[-1.07,2.30]$ \\
\hline $\begin{array}{l}7 \text { Change in immediate memo- } \\
\text { ry }\end{array}$ & 2 & 73 & Std. Mean Difference (IV, Random, 95\% CI) & $0.75[-0.61,2.10]$ \\
\hline $\begin{array}{l}8 \text { Change in attention and } \\
\text { working memory }\end{array}$ & 1 & 39 & Std. Mean Difference (IV, Random, 95\% CI) & $-0.05[-0.72,0.61]$ \\
\hline 9 Change in language (naming) & 1 & 34 & Std. Mean Difference (IV, Random, 95\% CI) & $1.98[1.14,2.82]$ \\
\hline $\begin{array}{l}10 \text { Change in verbal letter flu- } \\
\text { ency }\end{array}$ & 1 & 39 & Std. Mean Difference (IV, Random, 95\% CI) & $0.29[-0.38,0.96]$ \\
\hline $\begin{array}{l}11 \text { Change in verbal category } \\
\text { fluency }\end{array}$ & 1 & 39 & Std. Mean Difference (IV, Random, 95\% CI) & $-0.08[-0.74,0.59]$ \\
\hline $\begin{array}{l}12 \text { Change in executive func- } \\
\text { tion }\end{array}$ & 1 & 39 & Std. Mean Difference (IV, Random, 95\% CI) & $-0.30[-0.97,0.37]$ \\
\hline
\end{tabular}




\begin{tabular}{|c|c|c|c|c|}
\hline Outcome or subgroup title & $\begin{array}{l}\text { No. of } \\
\text { studies }\end{array}$ & $\begin{array}{l}\text { No. of } \\
\text { partici- } \\
\text { pants }\end{array}$ & Statistical method & Effect size \\
\hline $\begin{array}{l}13 \text { Change in speed of informa- } \\
\text { tion processing }\end{array}$ & 1 & 39 & Std. Mean Difference (IV, Random, 95\% CI) & $-0.11[-0.77,0.56]$ \\
\hline $\begin{array}{l}14 \text { Change in meta cognition } \\
\text { (self-reported) }\end{array}$ & 0 & 0 & Std. Mean Difference (IV, Random, 95\% CI) & $0.0[0.0,0.0]$ \\
\hline $\begin{array}{l}15 \text { Change in meta cognition } \\
\text { (informant-reported) }\end{array}$ & 0 & 0 & Std. Mean Difference (IV, Random, 95\% CI) & $0.0[0.0,0.0]$ \\
\hline $\begin{array}{l}16 \text { Change in participants' } \\
\text { mood }\end{array}$ & 1 & 39 & Std. Mean Difference (IV, Random, 95\% CI) & $-0.66[-1.35,0.02]$ \\
\hline $\begin{array}{l}17 \text { Change in capacity for ac- } \\
\text { tivities of daily living }\end{array}$ & 0 & 0 & Std. Mean Difference (IV, Random, 95\% CI) & $0.0[0.0,0.0]$ \\
\hline $\begin{array}{l}18 \text { Change in general health } \\
\text { and quality of life }\end{array}$ & 1 & 39 & Std. Mean Difference (IV, Random, 95\% CI) & $0.33[-0.34,1.00]$ \\
\hline $\begin{array}{l}19 \text { Change in behavioural and } \\
\text { psychological symptoms of } \\
\text { dementia (BPSD) }\end{array}$ & 0 & 0 & Std. Mean Difference (IV, Random, 95\% CI) & $0.0[0.0,0.0]$ \\
\hline $\begin{array}{l}20 \text { Change in burden of care } \\
\text { (CAREGIVER) }\end{array}$ & 0 & 0 & Std. Mean Difference (IV, Random, 95\% CI) & $0.0[0.0,0.0]$ \\
\hline $\begin{array}{l}21 \text { Change in quality of life } \\
\text { (CAREGIVER) }\end{array}$ & 0 & 0 & Std. Mean Difference (IV, Random, 95\% CI) & $0.0[0.0,0.0]$ \\
\hline $\begin{array}{l}22 \text { Change in mood and well- } \\
\text { being (CAREGIVER) }\end{array}$ & 0 & 0 & Std. Mean Difference (IV, Random, 95\% CI) & $0.0[0.0,0.0]$ \\
\hline
\end{tabular}

\section{Analysis 4.1. Comparison 4 Cognitive training vs alternative treatment in the medium term ( 3 to 12 months post intervention), Outcome 1 Change in a global measure of cognition (composite).}

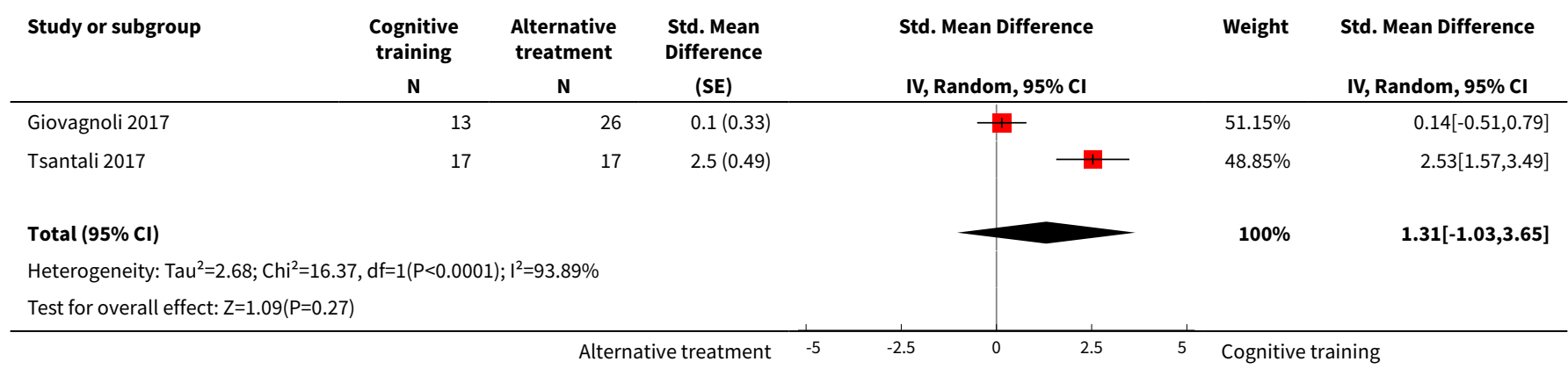


Analysis 4.2. Comparison 4 Cognitive training vs alternative treatment in the medium term ( 3 to 12 months post intervention), Outcome 2 Change in a global measure of cognition (composite)_zero correlation.

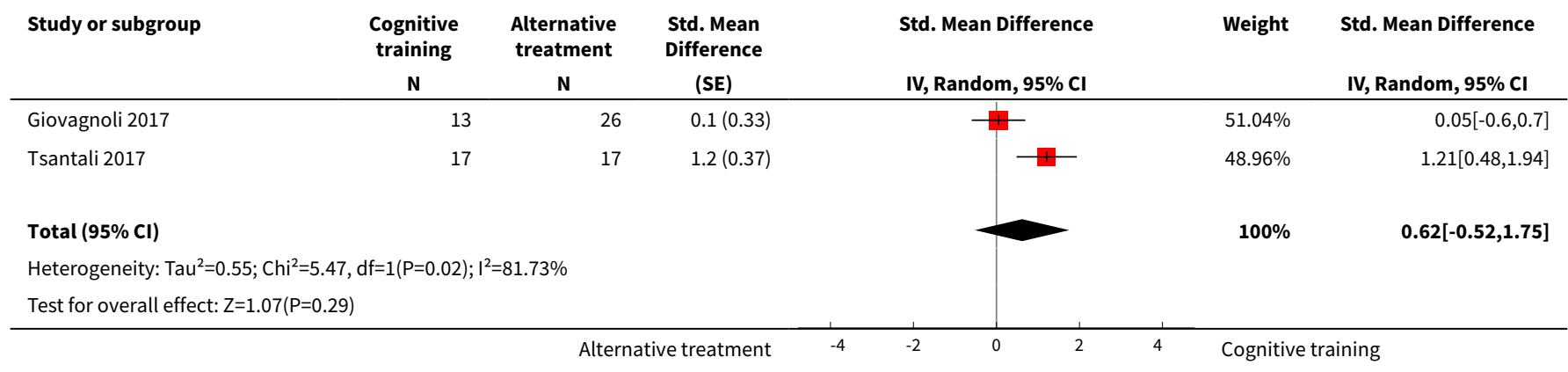

Analysis 4.3. Comparison 4 Cognitive training vs alternative treatment in the medium term ( 3 to 12 months post intervention), Outcome 3 Change in a global measure of cognition.

\begin{tabular}{|c|c|c|c|c|c|c|c|c|}
\hline \multirow[t]{2}{*}{ Study or subgroup } & \multicolumn{2}{|c|}{ Cognitive training } & \multicolumn{2}{|c|}{$\begin{array}{c}\text { Alternative } \\
\text { treatment }\end{array}$} & \multirow{2}{*}{$\begin{array}{c}\text { Std. Mean Difference } \\
\text { Random, } 95 \% \mathrm{Cl} \\
\end{array}$} & & \multirow[t]{2}{*}{ Weight } & \multirow{2}{*}{$\begin{array}{c}\text { Std. Mean Difference } \\
\text { Random, } 95 \% \mathrm{Cl} \\
\end{array}$} \\
\hline & $\mathbf{N}$ & Mean(SD) & $\mathbf{N}$ & Mean(SD) & & & & \\
\hline Giovagnoli 2017 & 13 & $0.4(2.1)$ & 26 & $-0.2(4.3)$ & + & & $50.87 \%$ & $0.15[-0.52,0.82]$ \\
\hline Tsantali 2017 & 17 & $3.8(1)$ & 17 & $-1.6(0.6)$ & & -1 & $49.13 \%$ & $6.37[4.62,8.11]$ \\
\hline Total $\star \star \star ~$ & 30 & & 43 & & & & $100 \%$ & $3.2[-2.89,9.29]$ \\
\hline \multicolumn{9}{|c|}{ Test for overall effect: $Z=1.03(P=0.3)$} \\
\hline
\end{tabular}

Analysis 4.6. Comparison 4 Cognitive training vs alternative treatment in the medium term (3 to 12 months post intervention), Outcome 6 Change in delayed memory.

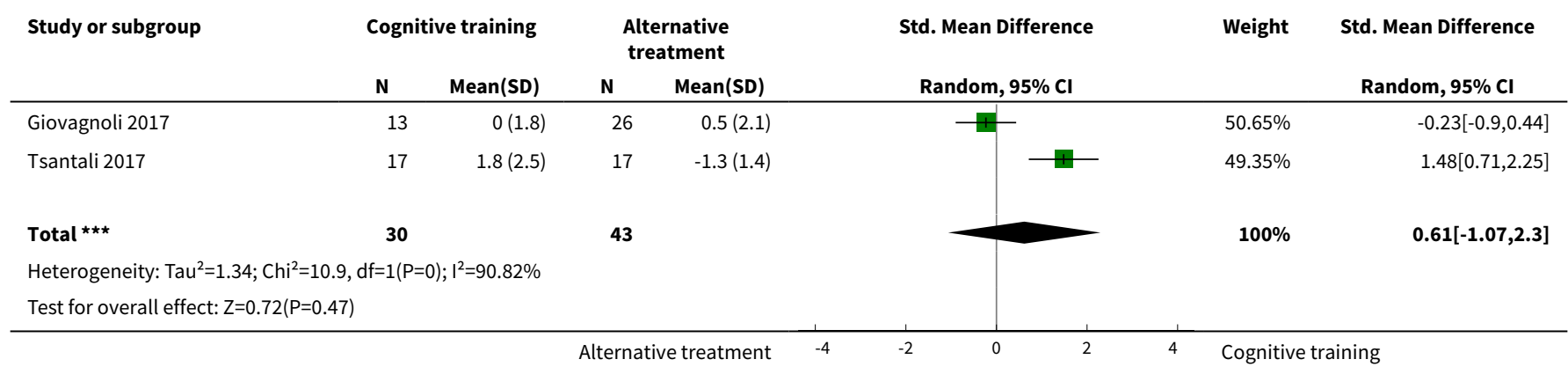


Analysis 4.7. Comparison 4 Cognitive training vs alternative treatment in the medium term ( 3 to 12 months post intervention), Outcome 7 Change in immediate memory.

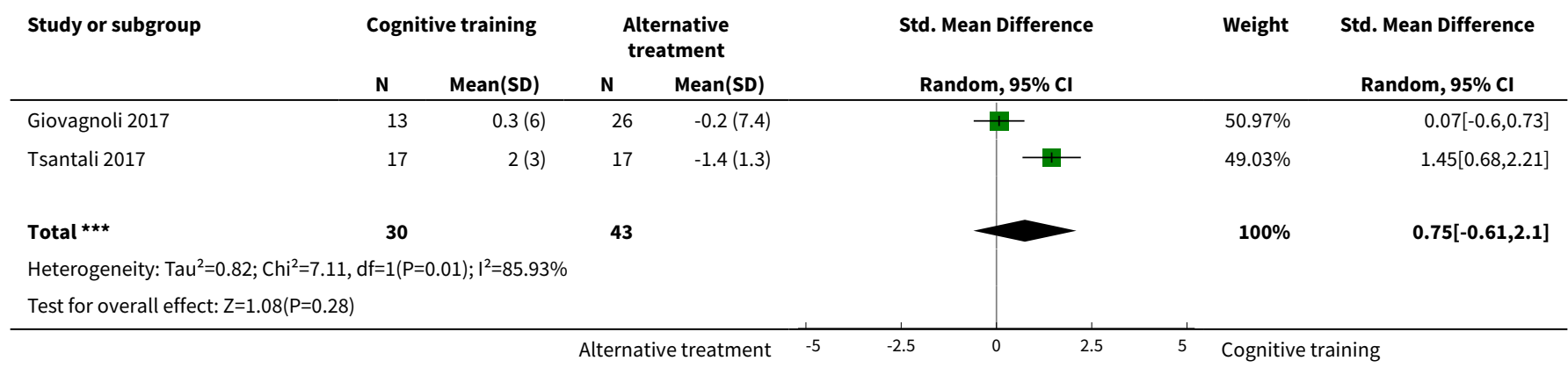

Analysis 4.8. Comparison 4 Cognitive training vs alternative treatment in the medium term ( 3 to 12 months post intervention), Outcome 8 Change in attention and working memory.

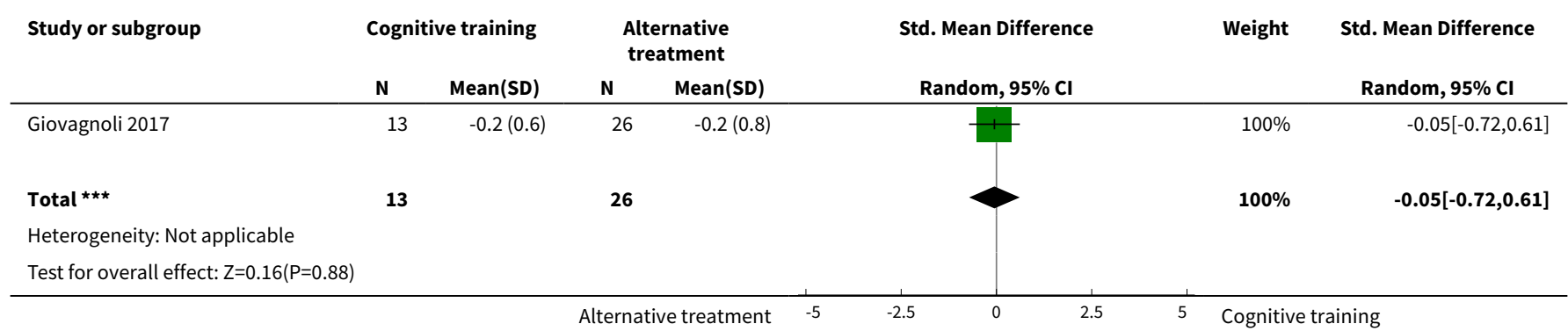

Analysis 4.9. Comparison 4 Cognitive training vs alternative treatment in the medium term (3 to 12 months post intervention), Outcome 9 Change in language (naming).

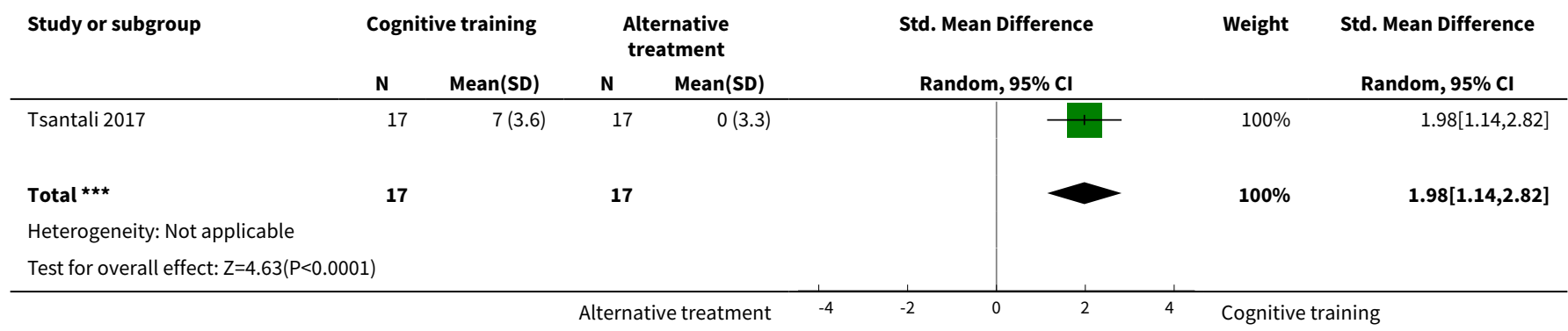

Analysis 4.10. Comparison 4 Cognitive training vs alternative treatment in the medium term ( 3 to 12 months post intervention), Outcome 10 Change in verbal letter fluency.

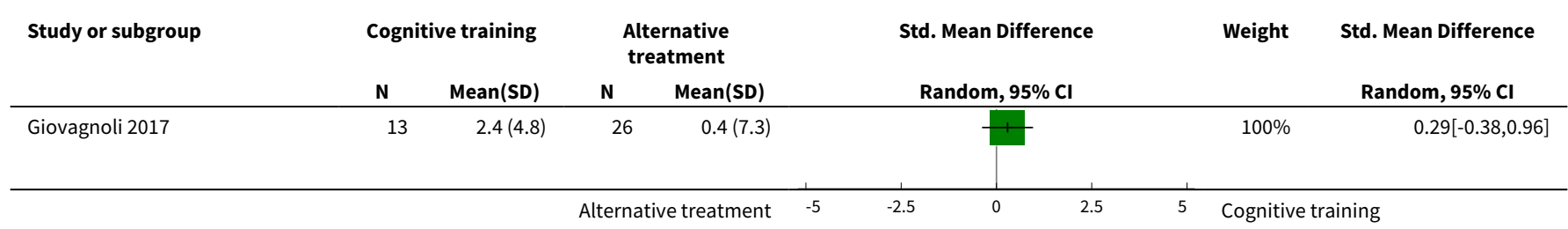




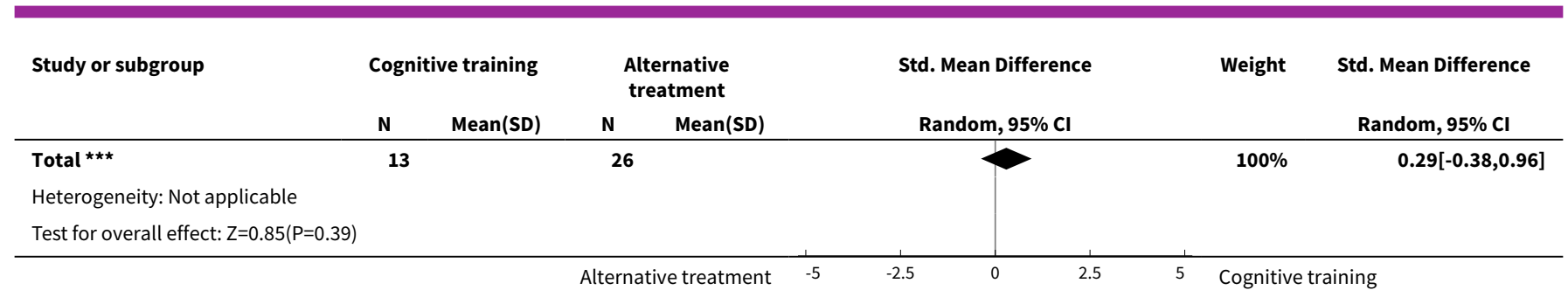

Analysis 4.11. Comparison 4 Cognitive training vs alternative treatment in the medium term (3 to 12 months post intervention), Outcome 11 Change in verbal category fluency.

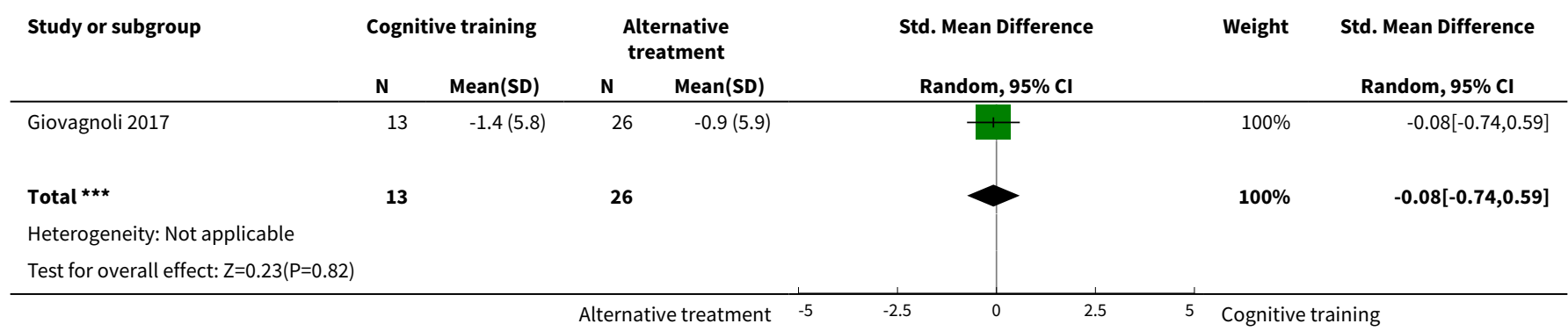

Analysis 4.12. Comparison 4 Cognitive training vs alternative treatment in the medium term ( 3 to 12 months post intervention), Outcome 12 Change in executive function.

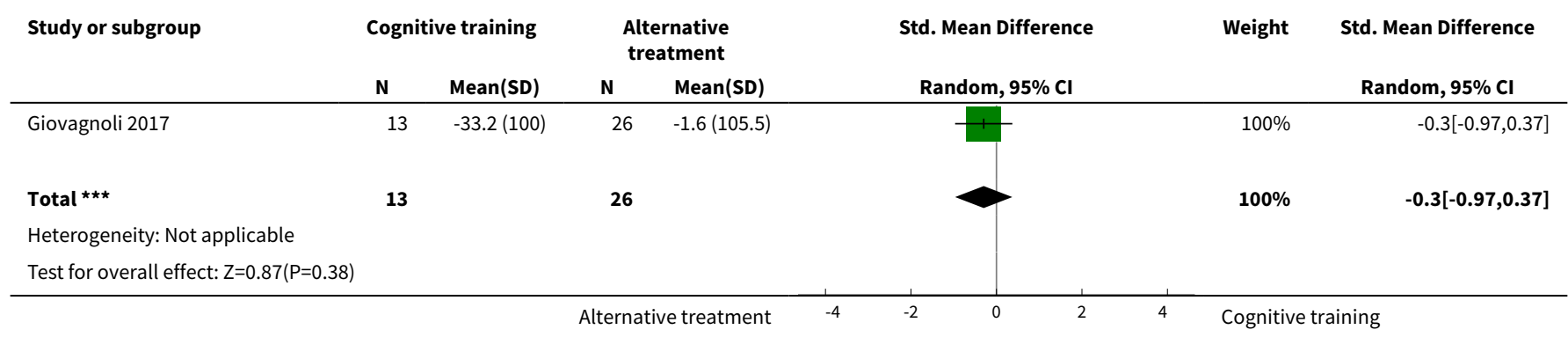

Analysis 4.13. Comparison 4 Cognitive training vs alternative treatment in the medium term

(3 to 12 months post intervention), Outcome 13 Change in speed of information processing.

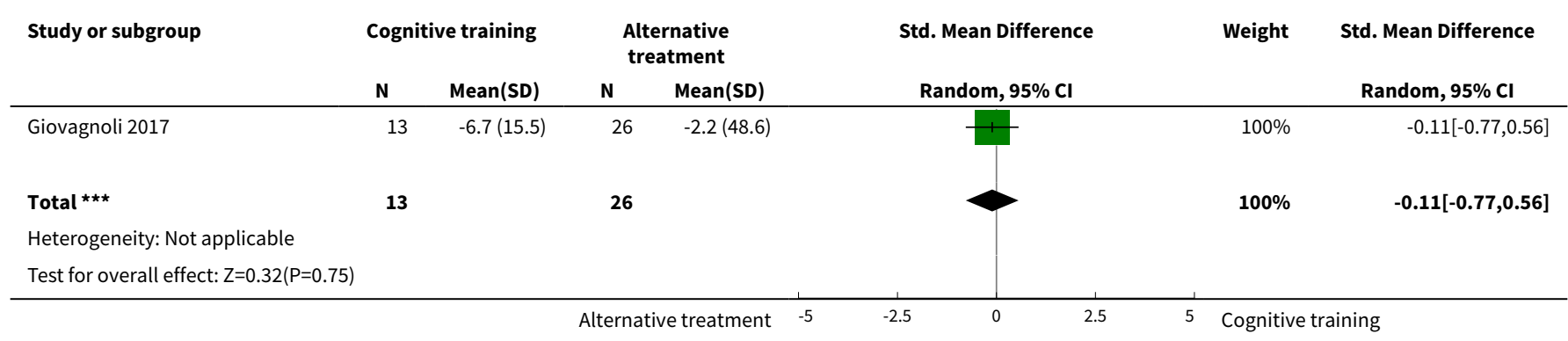


Analysis 4.16. Comparison 4 Cognitive training vs alternative treatment in the medium term (3 to 12 months post intervention), Outcome 16 Change in participants' mood.

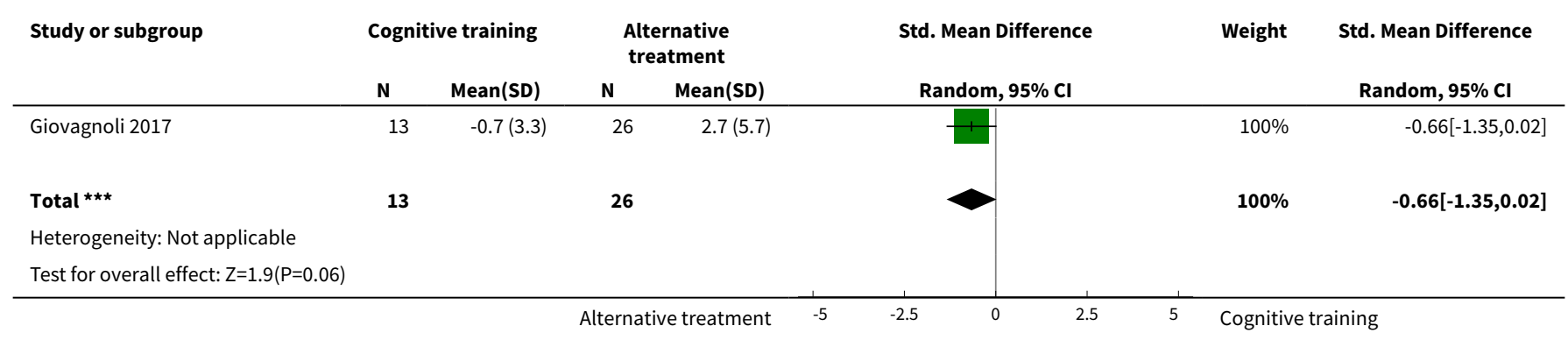

Analysis 4.18. Comparison 4 Cognitive training vs alternative treatment in the medium term (3 to 12 months post intervention), Outcome 18 Change in general health and quality of life.

\begin{tabular}{|c|c|c|c|c|c|c|c|}
\hline \multirow[t]{2}{*}{ Study or subgroup } & \multicolumn{2}{|c|}{ Cognitive training } & \multicolumn{2}{|c|}{$\begin{array}{c}\text { Alternative } \\
\text { treatment }\end{array}$} & \multirow{2}{*}{$\begin{array}{l}\text { Std. Mean Difference } \\
\text { Random, } 95 \% \mathrm{Cl} \\
\end{array}$} & \multirow[t]{2}{*}{ Weight } & \multirow{2}{*}{$\begin{array}{l}\text { Std. Mean Difference } \\
\text { Random, } 95 \% \mathrm{Cl} \\
\end{array}$} \\
\hline & $\mathbf{N}$ & Mean(SD) & $\mathbf{N}$ & Mean(SD) & & & \\
\hline Giovagnoli 2017 & 13 & $8.7(11.9)$ & 26 & $5.9(6)$ & & $100 \%$ & $0.33[-0.34,1]$ \\
\hline Total $\star \star \star ~$ & 13 & & 26 & & & $100 \%$ & $0.33[-0.34,1]$ \\
\hline \multicolumn{8}{|c|}{ Heterogeneity: Not applicable } \\
\hline \multicolumn{8}{|c|}{ Test for overall effect: $Z=0.95(P=0.34)$} \\
\hline
\end{tabular}

Comparison 5. Cognitive training vs control immediately post intervention - risk of bias

\begin{tabular}{|c|c|c|c|c|}
\hline Outcome or subgroup title & $\begin{array}{l}\text { No. of } \\
\text { studies }\end{array}$ & $\begin{array}{l}\text { No. of } \\
\text { partici- } \\
\text { pants }\end{array}$ & Statistical method & Effect size \\
\hline 1 Change in a global measure of cognition & 20 & 1288 & $\begin{array}{l}\text { Std. Mean Difference (IV, Random, } \\
95 \% \mathrm{CI})\end{array}$ & $0.65[0.26,1.05]$ \\
\hline 1.1 Lower risk & 14 & 1010 & $\begin{array}{l}\text { Std. Mean Difference (IV, Random, } \\
95 \% \mathrm{Cl} \text { ) }\end{array}$ & $0.79[0.28,1.30]$ \\
\hline 1.2 Higher risk & 6 & 278 & $\begin{array}{l}\text { Std. Mean Difference (IV, Random, } \\
95 \% \mathrm{CI} \text { ) }\end{array}$ & $0.30[-0.26,0.87]$ \\
\hline $\begin{array}{l}2 \text { Change in a global measure of cogni- } \\
\text { tion_zero correlation }\end{array}$ & 20 & 1287 & $\begin{array}{l}\text { Std. Mean Difference (IV, Random, } \\
95 \% \mathrm{CI})\end{array}$ & $0.27[0.04,0.50]$ \\
\hline 2.1 Lower risk & 14 & 1009 & $\begin{array}{l}\text { Std. Mean Difference (IV, Random, } \\
95 \% \mathrm{CI})\end{array}$ & $0.33[0.03,0.63]$ \\
\hline 2.2 Higher risk & 6 & 278 & $\begin{array}{l}\text { Std. Mean Difference (IV, Random, } \\
95 \% \mathrm{CI})\end{array}$ & $0.07[-0.20,0.34]$ \\
\hline
\end{tabular}




\begin{tabular}{|c|c|c|c|c|}
\hline Outcome or subgroup title & $\begin{array}{l}\text { No. of } \\
\text { studies }\end{array}$ & $\begin{array}{l}\text { No. of } \\
\text { partici- } \\
\text { pants }\end{array}$ & Statistical method & Effect size \\
\hline $\begin{array}{l}3 \text { Change in a global measure of cognition } \\
\text { (composite) }\end{array}$ & 26 & 1389 & $\begin{array}{l}\text { Std. Mean Difference (Random, } \\
95 \% \mathrm{Cl} \text { ) }\end{array}$ & $0.42[0.23,0.61]$ \\
\hline 3.1 Lower risk & 19 & 1034 & $\begin{array}{l}\text { Std. Mean Difference (Random, } \\
95 \% \mathrm{Cl} \text { ) }\end{array}$ & $0.46[0.19,0.72]$ \\
\hline 3.2 Higher risk & 7 & 355 & $\begin{array}{l}\text { Std. Mean Difference (Random, } \\
95 \% \mathrm{Cl} \text { ) }\end{array}$ & $0.33[0.12,0.55]$ \\
\hline $\begin{array}{l}4 \text { Change in a global measure of cognition } \\
\text { (composite)_zero correlation }\end{array}$ & 26 & 1390 & $\begin{array}{l}\text { Std. Mean Difference (Random, } \\
95 \% \mathrm{Cl} \text { ) }\end{array}$ & $0.24[0.12,0.36]$ \\
\hline 4.1 Lower risk & 19 & 1035 & $\begin{array}{l}\text { Std. Mean Difference (Random, } \\
95 \% \mathrm{Cl} \text { ) }\end{array}$ & $0.23[0.08,0.38]$ \\
\hline 4.2 Higher risk & 7 & 355 & $\begin{array}{l}\text { Std. Mean Difference (Random, } \\
95 \% \mathrm{Cl} \text { ) }\end{array}$ & $0.28[0.07,0.49]$ \\
\hline 5 Change in immediate memory & 17 & 762 & $\begin{array}{l}\text { Std. Mean Difference (IV, Random, } \\
95 \% \mathrm{Cl})\end{array}$ & $0.74[0.37,1.12]$ \\
\hline 5.1 Lower risk & 13 & 542 & $\begin{array}{l}\text { Std. Mean Difference (IV, Random, } \\
95 \% \mathrm{Cl} \text { ) }\end{array}$ & $0.95[0.46,1.44]$ \\
\hline 5.2 Higher risk & 5 & 220 & $\begin{array}{l}\text { Std. Mean Difference (IV, Random, } \\
95 \% \mathrm{CI} \text { ) }\end{array}$ & $0.33[0.06,0.60]$ \\
\hline 6 Change in delayed memory & 11 & 543 & $\begin{array}{l}\text { Std. Mean Difference (IV, Random, } \\
95 \% \mathrm{Cl})\end{array}$ & $0.81[0.29,1.32]$ \\
\hline 6.1 Lower risk & 8 & 413 & $\begin{array}{l}\text { Std. Mean Difference (IV, Random, } \\
95 \% \mathrm{Cl} \text { ) }\end{array}$ & $0.87[0.16,1.59]$ \\
\hline 6.2 Higher risk & 3 & 130 & $\begin{array}{l}\text { Std. Mean Difference (IV, Random, } \\
95 \% \mathrm{Cl})\end{array}$ & $0.56[0.21,0.92]$ \\
\hline $\begin{array}{l}7 \text { Change in attention and working mem- } \\
\text { ory }\end{array}$ & 12 & 551 & $\begin{array}{l}\text { Std. Mean Difference (IV, Random, } \\
95 \% \mathrm{Cl})\end{array}$ & $0.56[0.08,1.05]$ \\
\hline 7.1 Lower risk & 9 & 394 & $\begin{array}{l}\text { Std. Mean Difference (IV, Random, } \\
95 \% \mathrm{Cl} \text { ) }\end{array}$ & $0.57[-0.03,1.17]$ \\
\hline 7.2 Higher risk & 3 & 157 & $\begin{array}{l}\text { Std. Mean Difference (IV, Random, } \\
95 \% \mathrm{Cl} \text { ) }\end{array}$ & $0.56[-0.57,1.69]$ \\
\hline 8 Change in language (naming) & 5 & 311 & $\begin{array}{l}\text { Std. Mean Difference (IV, Random, } \\
95 \% \mathrm{CI} \text { ) }\end{array}$ & $0.62[0.11,1.12]$ \\
\hline 9 Change in verbal letter fluency & 12 & 544 & $\begin{array}{l}\text { Std. Mean Difference (IV, Random, } \\
95 \% \mathrm{CI} \text { ) }\end{array}$ & $0.22[-0.07,0.50]$ \\
\hline $\begin{array}{l}10 \text { Change in speed of information pro- } \\
\text { cessing }\end{array}$ & 6 & 201 & $\begin{array}{l}\text { Std. Mean Difference (IV, Random, } \\
95 \% \mathrm{CI} \text { ) }\end{array}$ & $0.22[-0.11,0.54]$ \\
\hline
\end{tabular}




\begin{tabular}{|c|c|c|c|c|}
\hline Outcome or subgroup title & $\begin{array}{l}\text { No. of } \\
\text { studies }\end{array}$ & $\begin{array}{l}\text { No. of } \\
\text { partici- } \\
\text { pants }\end{array}$ & Statistical method & Effect size \\
\hline 11 Change in executive function & 11 & 511 & $\begin{array}{l}\text { Std. Mean Difference (IV, Random, } \\
95 \% \mathrm{CI} \text { ) }\end{array}$ & $0.75[0.28,1.22]$ \\
\hline 12 Change in verbal category fluency & 9 & 475 & $\begin{array}{l}\text { Std. Mean Difference (IV, Random, } \\
95 \% \mathrm{CI} \text { ) }\end{array}$ & $0.52[0.23,0.81]$ \\
\hline 12.1 Lower risk & 6 & 318 & $\begin{array}{l}\text { Std. Mean Difference (IV, Random, } \\
95 \% \mathrm{CI} \text { ) }\end{array}$ & $0.49[0.06,0.93]$ \\
\hline 12.2 Higher risk & 3 & 157 & $\begin{array}{l}\text { Std. Mean Difference (IV, Random, } \\
95 \% \mathrm{CI} \text { ) }\end{array}$ & $0.48[0.16,0.80]$ \\
\hline $\begin{array}{l}13 \text { Change in meta cognition (self-report- } \\
\text { ed) }\end{array}$ & 2 & 41 & $\begin{array}{l}\text { Std. Mean Difference (IV, Random, } \\
95 \% \mathrm{Cl} \text { ) }\end{array}$ & $0.12[-0.87,1.12]$ \\
\hline $\begin{array}{l}14 \text { Change in meta cognition (infor- } \\
\text { mant-reported) }\end{array}$ & 2 & 56 & $\begin{array}{l}\text { Std. Mean Difference (IV, Random, } \\
95 \% \mathrm{CI} \text { ) }\end{array}$ & $-0.65[-1.19,-0.10]$ \\
\hline 15 Change in participants' mood & 8 & 576 & $\begin{array}{l}\text { Std. Mean Difference (IV, Random, } \\
95 \% \mathrm{CI} \text { ) }\end{array}$ & $0.72[-0.10,1.54]$ \\
\hline $\begin{array}{l}16 \text { Change in capacity for activities of dai- } \\
\text { ly living }\end{array}$ & 10 & 705 & $\begin{array}{l}\text { Std. Mean Difference (IV, Random, } \\
95 \% \mathrm{CI} \text { ) }\end{array}$ & $0.12[-0.10,0.34]$ \\
\hline 16.1 Lower risk & 7 & 516 & $\begin{array}{l}\text { Std. Mean Difference (IV, Random, } \\
95 \% \mathrm{CI} \text { ) }\end{array}$ & $0.09[-0.19,0.37]$ \\
\hline 16.2 Higher risk & 3 & 189 & $\begin{array}{l}\text { Std. Mean Difference (IV, Random, } \\
95 \% \mathrm{Cl} \text { ) }\end{array}$ & $0.18[-0.21,0.58]$ \\
\hline $\begin{array}{l}17 \text { Change in general health and quality } \\
\text { of life }\end{array}$ & 4 & 542 & $\begin{array}{l}\text { Std. Mean Difference (IV, Random, } \\
95 \% \mathrm{CI} \text { ) }\end{array}$ & $-0.00[-0.42,0.41]$ \\
\hline $\begin{array}{l}18 \text { Change in behavioural and psychologi- } \\
\text { cal symptoms of dementia (BPSD) }\end{array}$ & 6 & 493 & $\begin{array}{l}\text { Std. Mean Difference (IV, Random, } \\
95 \% \mathrm{CI} \text { ) }\end{array}$ & $0.44[-0.34,1.22]$ \\
\hline 19 Change in disease progression & 5 & 215 & $\begin{array}{l}\text { Std. Mean Difference (IV, Random, } \\
95 \% \mathrm{Cl} \text { ) }\end{array}$ & $1.07[0.59,1.55]$ \\
\hline 20 Change in burden of care (CAREGIVER) & 2 & 405 & $\begin{array}{l}\text { Std. Mean Difference (IV, Random, } \\
95 \% \mathrm{Cl} \text { ) }\end{array}$ & $-0.11[-0.36,0.15]$ \\
\hline 21 Change in quality of life (CAREGIVER) & 1 & 36 & $\begin{array}{l}\text { Std. Mean Difference (IV, Random, } \\
95 \% \mathrm{CI} \text { ) }\end{array}$ & $0.16[-0.50,0.83]$ \\
\hline $\begin{array}{l}22 \text { Change in mood and well-being } \\
\text { (CAREGIVER) }\end{array}$ & 1 & 36 & $\begin{array}{l}\text { Std. Mean Difference (IV, Random, } \\
95 \% \mathrm{Cl} \text { ) }\end{array}$ & $0.98[0.27,1.68]$ \\
\hline 23 Participant burden (retention rates) & 17 & 1282 & Odds Ratio (M-H, Random, 95\% Cl) & $0.73[0.37,1.43]$ \\
\hline $\begin{array}{l}24 \text { Change in general health and quality } \\
\text { of life }\end{array}$ & 5 & 630 & $\begin{array}{l}\text { Std. Mean Difference (IV, Random, } \\
95 \% \mathrm{Cl} \text { ) }\end{array}$ & $-0.04[-0.38,0.29]$ \\
\hline
\end{tabular}


Analysis 5.1. Comparison 5 Cognitive training vs control immediately post intervention - risk of bias, Outcome 1 Change in a global measure of cognition.

\begin{tabular}{|c|c|c|c|c|c|c|c|}
\hline \multirow{3}{*}{$\begin{array}{l}\text { Study or subgroup } \\
\text { 5.1.1 Lower risk }\end{array}$} & \multicolumn{2}{|c|}{ Cognitive training } & \multicolumn{2}{|c|}{ Control } & \multirow{2}{*}{$\begin{array}{c}\text { Std. Mean Difference } \\
\text { Random, } 95 \% \mathrm{Cl}\end{array}$} & \multirow[t]{2}{*}{ Weight } & \multirow{2}{*}{$\begin{array}{c}\text { Std. Mean Difference } \\
\text { Random, } 95 \% \mathrm{Cl}\end{array}$} \\
\hline & \multirow[t]{2}{*}{$\mathbf{N}$} & \multirow[t]{2}{*}{ Mean(SD) } & \multirow[t]{2}{*}{$\mathbf{N}$} & \multirow[t]{2}{*}{ Mean(SD) } & & & \\
\hline & & & & & & & \\
\hline Galante 2007 & 7 & $0.1(2.3)$ & 4 & $1.8(1.7)$ & \begin{tabular}{c|c}
1 & \\
\end{tabular} & $3.67 \%$ & $-0.72[-2,0.56]$ \\
\hline Amieva 2016 & 168 & $-8.4(5.9)$ & 153 & $-7.6(5.4)$ & + & $5.92 \%$ & $-0.14[-0.36,0.08]$ \\
\hline Davis 2001 & 19 & $0.2(2.6)$ & 18 & $0.2(2.7)$ & 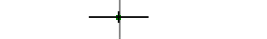 & $5.19 \%$ & $-0.02[-0.67,0.62]$ \\
\hline Lee 2013 & 12 & $2(2.9)$ & 7 & $2(1.9)$ & & $4.5 \%$ & $0[-0.93,0.94]$ \\
\hline Kallio 2018 & 76 & $-0.8(5.2)$ & 71 & $-1.6(5.4)$ & + & $5.8 \%$ & $0.15[-0.17,0.48]$ \\
\hline Venturelli 2016 & 20 & $-0.2(1)$ & 20 & $-0.4(1.1)$ & 1 & $5.24 \%$ & $0.19[-0.44,0.81]$ \\
\hline Kawashima 2005 & 16 & $0.1(5.1)$ & 16 & $-1.8(3.8)$ & 1 & $5.06 \%$ & $0.41[-0.29,1.12]$ \\
\hline Quintana Hernandez 2014 & 27 & $-4.5(4.1)$ & 25 & $-7.5(3.5)$ & $\longrightarrow$ & $5.36 \%$ & $0.78[0.21,1.34]$ \\
\hline Mapelli 2013 & 10 & $2.9(2.6)$ & 10 & $-0.3(1.7)$ & 1 & $4.34 \%$ & $1.4[0.4,2.4]$ \\
\hline Jelcic 2012 & 20 & $2(1.7)$ & 20 & $-1(2)$ & 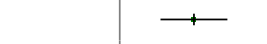 & $5.01 \%$ & $1.6[0.88,2.32]$ \\
\hline Fernández-Calvo 2011 & 30 & $1.1(1.6)$ & 15 & $-2.3(1.2)$ & 1 & $4.86 \%$ & $2.25[1.46,3.03]$ \\
\hline Bergamaschi 2013 & 16 & $2.8(1.8)$ & 16 & $-3.6(1.8)$ & $\longrightarrow$ & $4.03 \%$ & $3.4[2.28,4.53]$ \\
\hline Subtotal $* \star \star$ & 509 & & 501 & & & $70.22 \%$ & $0.79[0.28,1.3]$ \\
\hline \multicolumn{8}{|c|}{ Heterogeneity: $\operatorname{Tau}^{2}=0.8 ; \mathrm{Chi}^{2}=158.79, \mathrm{df}=13(\mathrm{P}<0.0001) ; \mathrm{I}^{2}=91.81 \%$} \\
\hline \multicolumn{8}{|c|}{ Test for overall effect: $Z=3.06(P=0)$} \\
\hline \multicolumn{8}{|l|}{ 5.1.2 Higher risk } \\
\hline Giuli 2016 & 48 & $0(2.8)$ & 47 & $1.2(3.8)$ & $\rightarrow$ & $5.67 \%$ & $-0.37[-0.77,0.04]$ \\
\hline Koltai 2001 & 14 & $-0.2(2.9)$ & 8 & $0.8(2.4)$ & $\longrightarrow$ & $4.64 \%$ & $-0.34[-1.21,0.54]$ \\
\hline Heiss 1993 & 18 & $-1.2(4.2)$ & 17 & $-0.9(3.6)$ & - & $5.15 \%$ & $-0.07[-0.73,0.59]$ \\
\hline Barban 2016 & 42 & $0.1(1.6)$ & 39 & $-0.1(1.2)$ & & $5.62 \%$ & $0.14[-0.3,0.58]$ \\
\hline Jelcic 2014 & 17 & $2(1.5)$ & 10 & $-0.7(2.4)$ & $\longrightarrow$ & $4.63 \%$ & $1.4[0.52,2.28]$ \\
\hline de Vreese 1998 & 9 & $2.8(1.7)$ & 9 & $-0.9(2.4)$ & 1 & $4.06 \%$ & $1.68[0.57,2.8]$ \\
\hline \multicolumn{8}{|c|}{ Test for overall effect: $Z=1.05(P=0.29)$} \\
\hline Total ${ }^{\star \star \star}$ & 657 & & 631 & & & $100 \%$ & $0.65[0.26,1.05]$ \\
\hline \multicolumn{8}{|c|}{ Heterogeneity: $\mathrm{Tau}^{2}=0.67 ; \mathrm{Chi}^{2}=187.26, \mathrm{df}=19(\mathrm{P}<0.0001) ; \mathrm{I}^{2}=89.85 \%$} \\
\hline \multicolumn{8}{|c|}{ Test for overall effect: $Z=3.26(P=0)$} \\
\hline Test for subgroup differenc & $61, \mathrm{df}=$ & $P=0.21), I^{2}=37$ & & & & & \\
\hline
\end{tabular}

\section{Analysis 5.2. Comparison 5 Cognitive training vs control immediately post intervention - risk of bias, Outcome 2 Change in a global measure of cognition_zero correlation.}

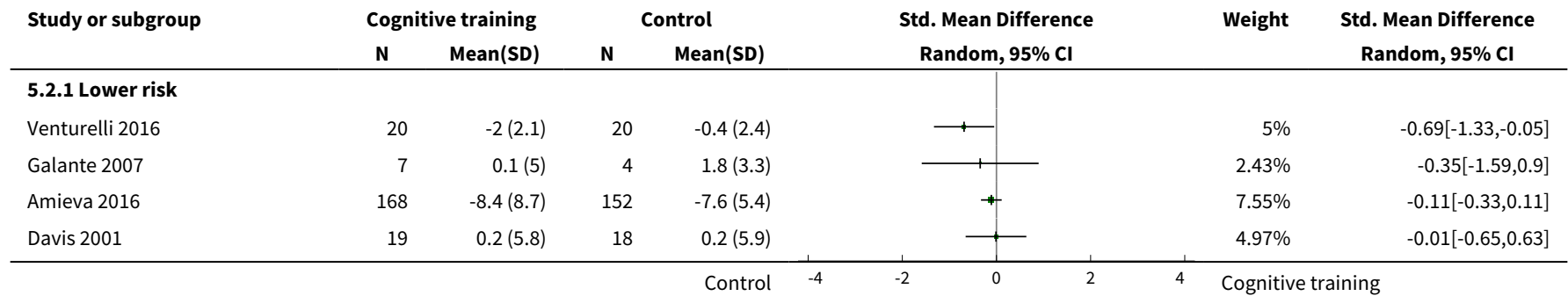




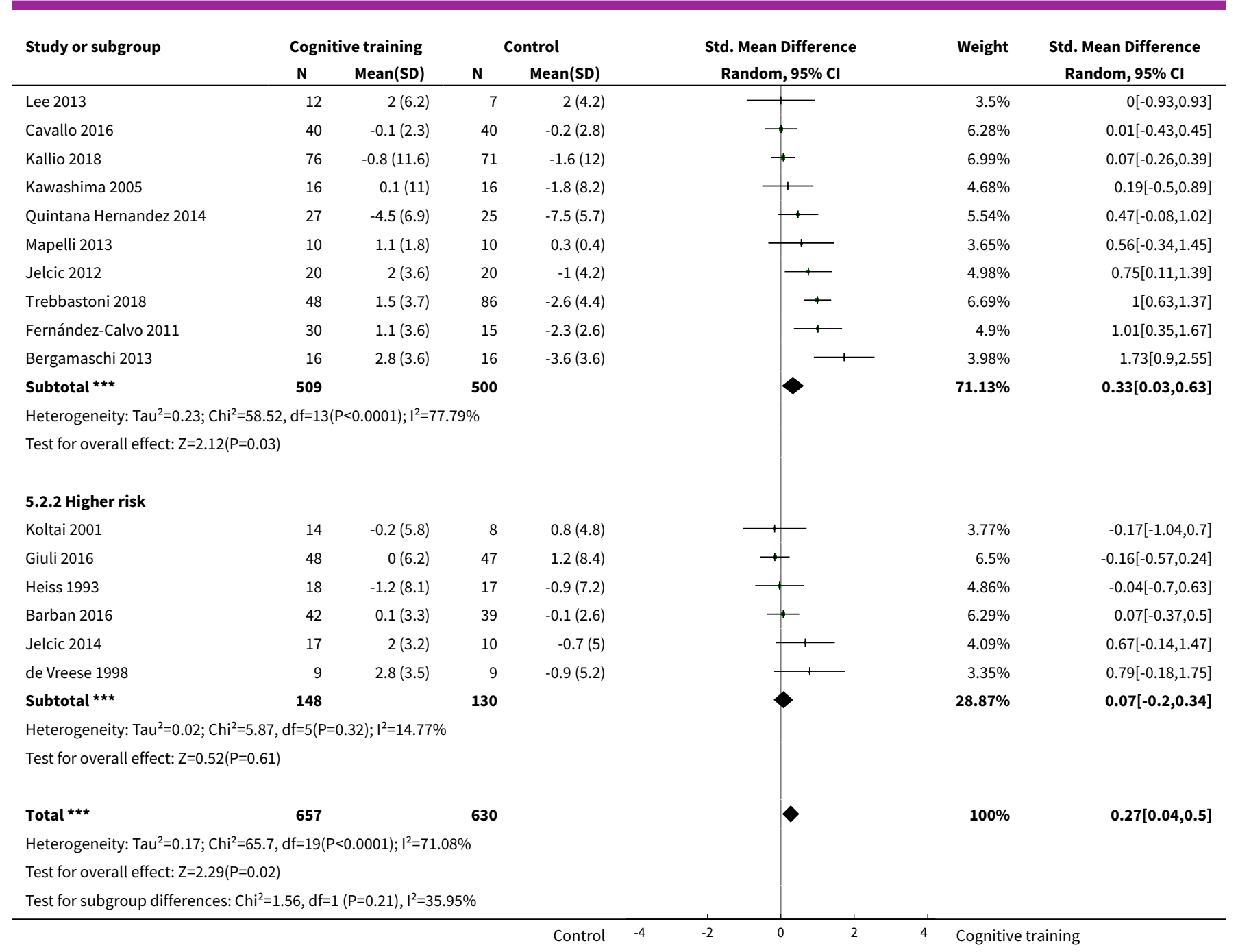

Analysis 5.3. Comparison 5 Cognitive training vs control immediately post intervention - risk of bias, Outcome 3 Change in a global measure of cognition (composite).

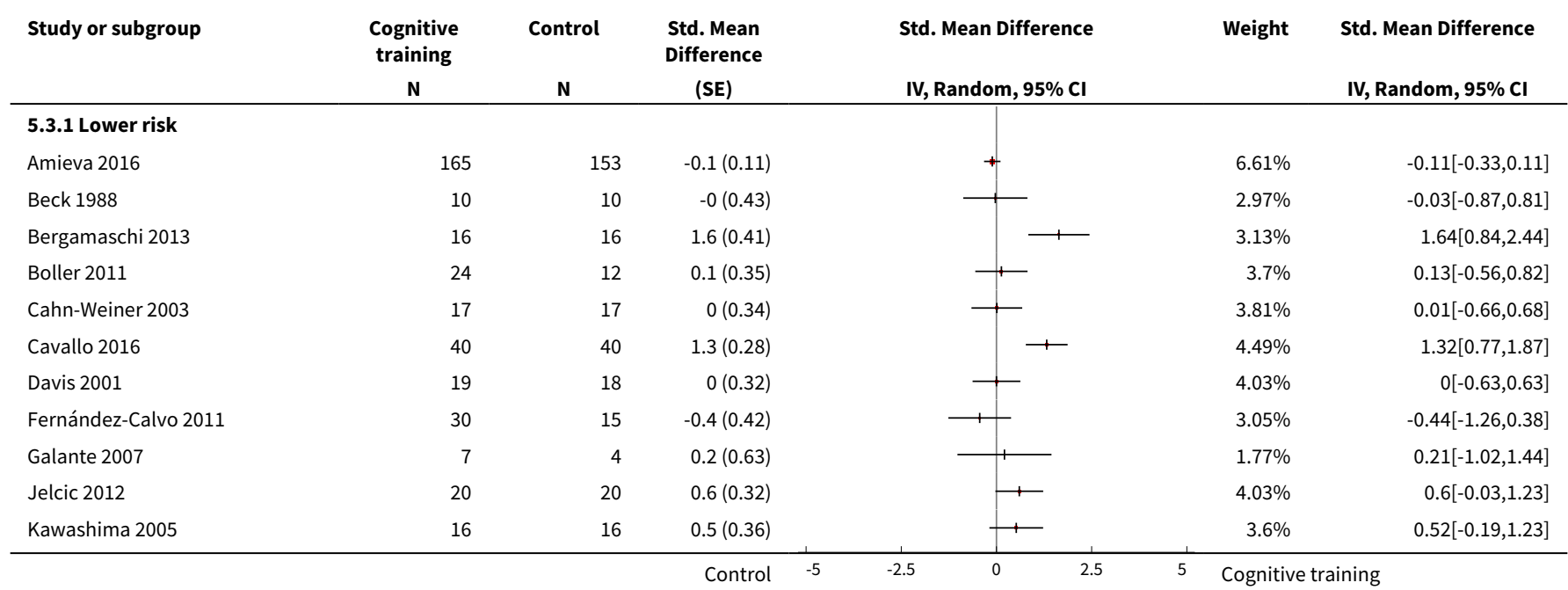




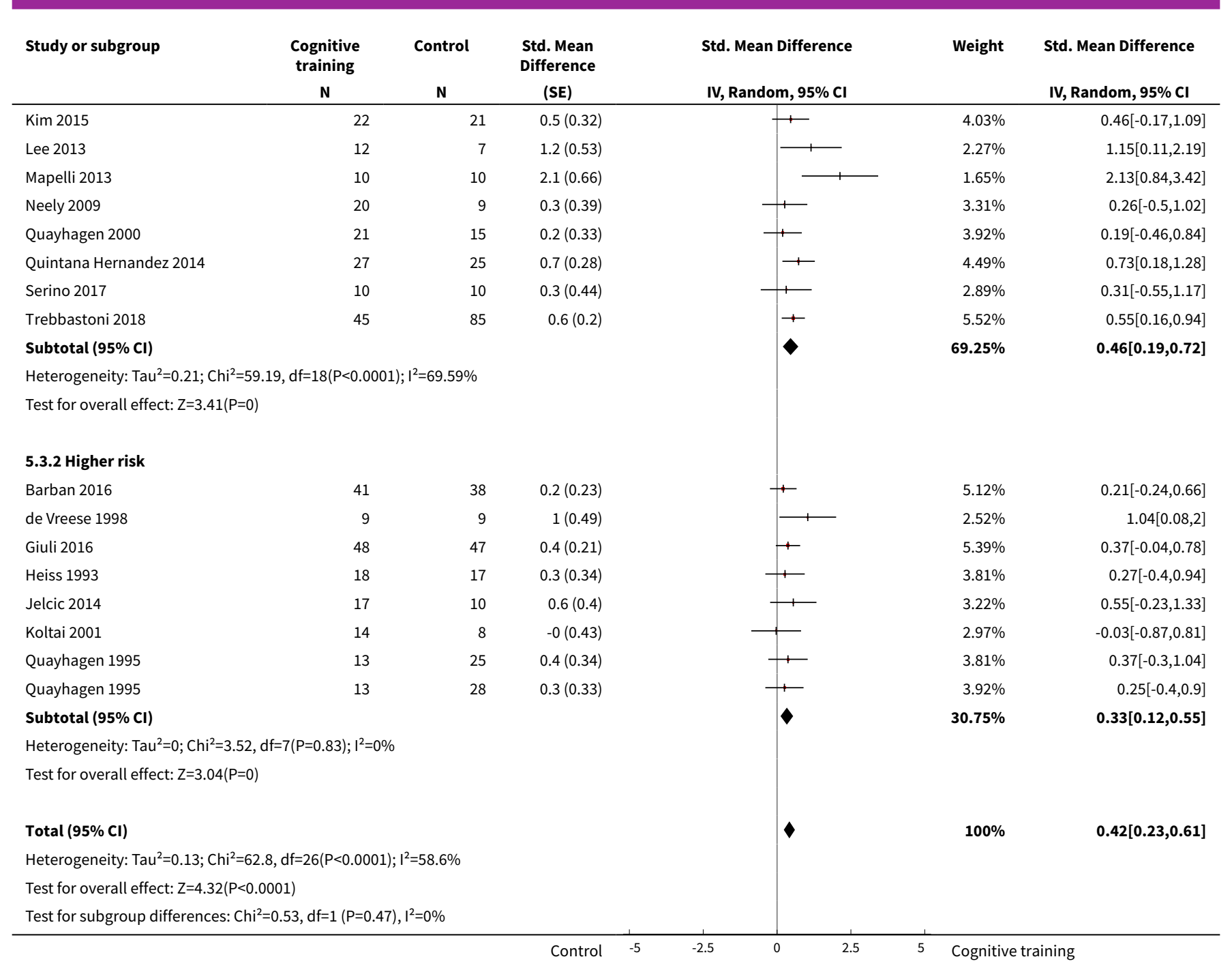

\section{Analysis 5.4. Comparison 5 Cognitive training vs control immediately post intervention - risk of bias, Outcome 4 Change in a global measure of cognition (composite)_zero correlation.}

\begin{tabular}{|c|c|c|c|c|c|c|}
\hline \multirow{2}{*}{$\begin{array}{l}\text { Study or subgroup } \\
\text { 5.4.1 Lower risk }\end{array}$} & \multirow[t]{2}{*}{$\begin{array}{c}\text { Cognitive } \\
\text { training } \\
\mathrm{N} \\
\end{array}$} & \multirow[t]{2}{*}{$\begin{array}{l}\text { Control } \\
\mathrm{N} \\
\end{array}$} & \multirow[t]{2}{*}{$\begin{array}{c}\text { Std. Mean } \\
\text { Difference } \\
(\mathrm{SE}) \\
\end{array}$} & $\begin{array}{l}\text { Std. Mean Difference } \\
\text { IV, Random, 95\% CI }\end{array}$ & \multirow[t]{2}{*}{ Weight } & \multirow[t]{2}{*}{$\begin{array}{l}\text { Std. Mean Difference } \\
\text { IV, Random, 95\% CI }\end{array}$} \\
\hline & & & & & & \\
\hline Amieva 2016 & 166 & 153 & $-0.1(0.11)$ & + & $15.93 \%$ & $-0.07[-0.29,0.15]$ \\
\hline Beck 1988 & 10 & 10 & $-0(0.43)$ & 1 & $1.92 \%$ & $-0.03[-0.87,0.81]$ \\
\hline Bergamaschi 2013 & 16 & 16 & $0.8(0.36)$ & 1 & $2.67 \%$ & $0.78[0.07,1.49]$ \\
\hline Cahn-Weiner 2003 & 17 & 17 & $0(0.34)$ & $\longrightarrow$ & $2.97 \%$ & $0.01[-0.66,0.68]$ \\
\hline Cavallo 2016 & 40 & 40 & $0.6(0.23)$ & $\rightarrow$ & $5.85 \%$ & $0.61[0.16,1.06]$ \\
\hline Davis 2001 & 19 & 18 & $0(0.32)$ & $\longrightarrow$ & $3.31 \%$ & $0[-0.63,0.63]$ \\
\hline Fernández-Calvo 2011 & 30 & 15 & $-0.3(0.34)$ & 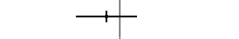 & $2.97 \%$ & $-0.29[-0.96,0.38]$ \\
\hline Galante 2007 & 7 & 4 & $0.1(0.59)$ & 1 & $1.05 \%$ & $0.12[-1.04,1.28]$ \\
\hline Jelcic 2012 & 20 & 20 & $0.3(0.31)$ & 1 & $3.51 \%$ & $0.28[-0.33,0.89]$ \\
\hline
\end{tabular}




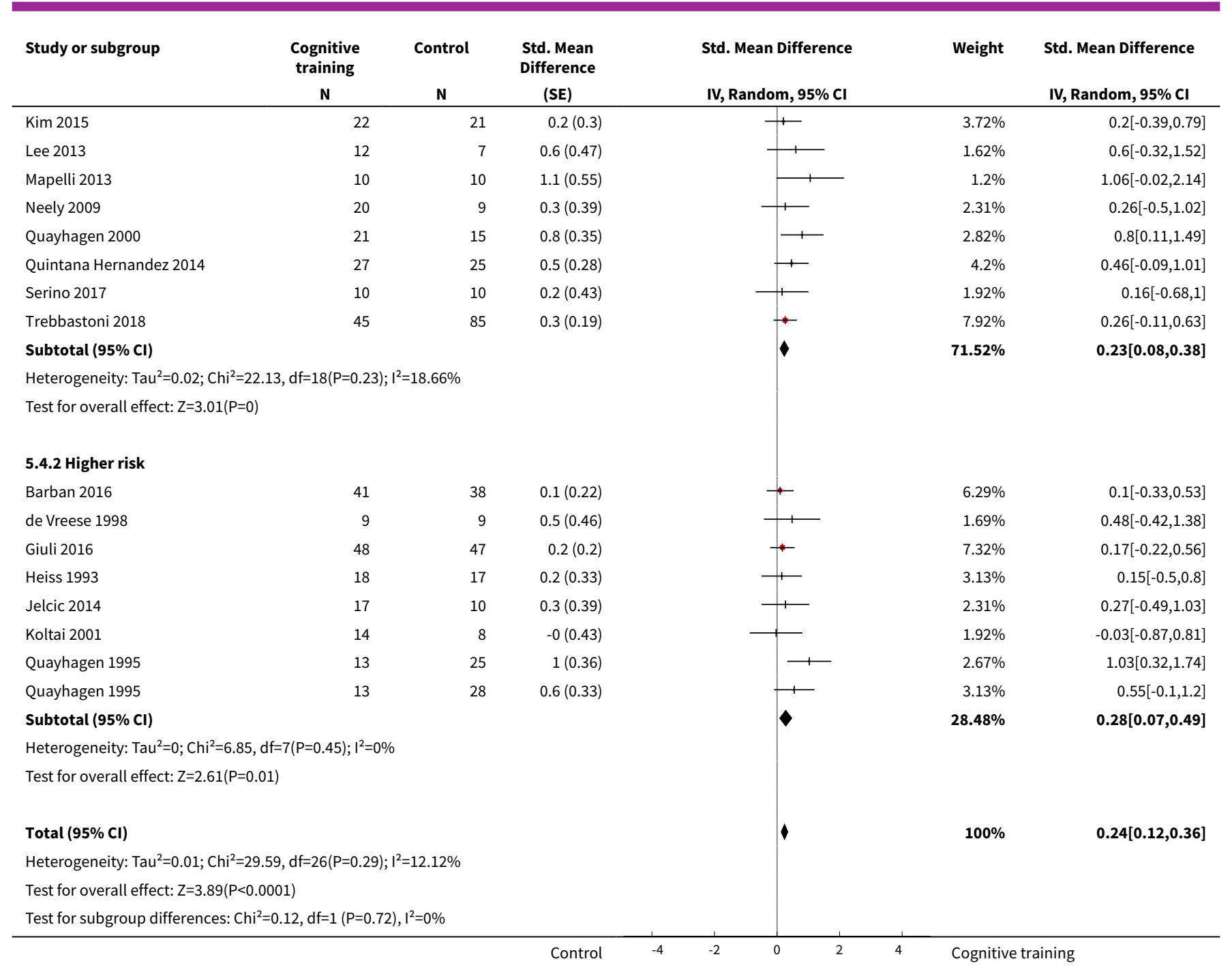

Analysis 5.5. Comparison 5 Cognitive training vs control immediately post intervention - risk of bias, Outcome 5 Change in immediate memory.

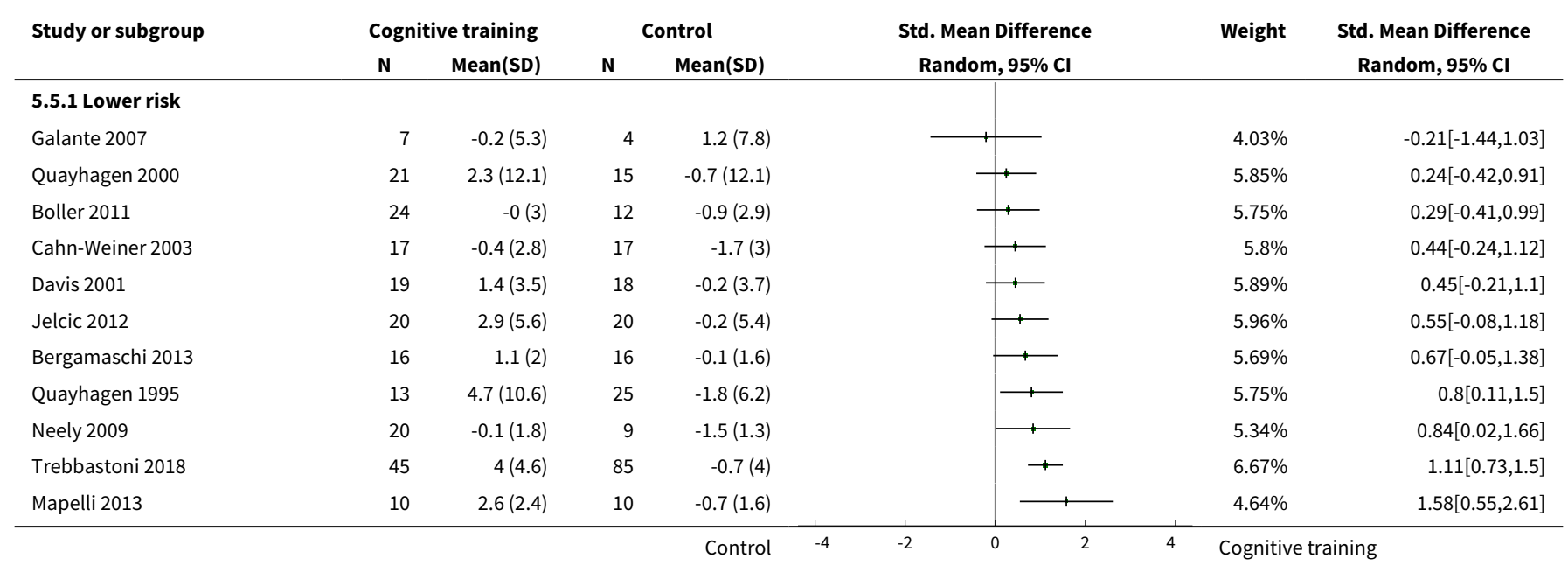




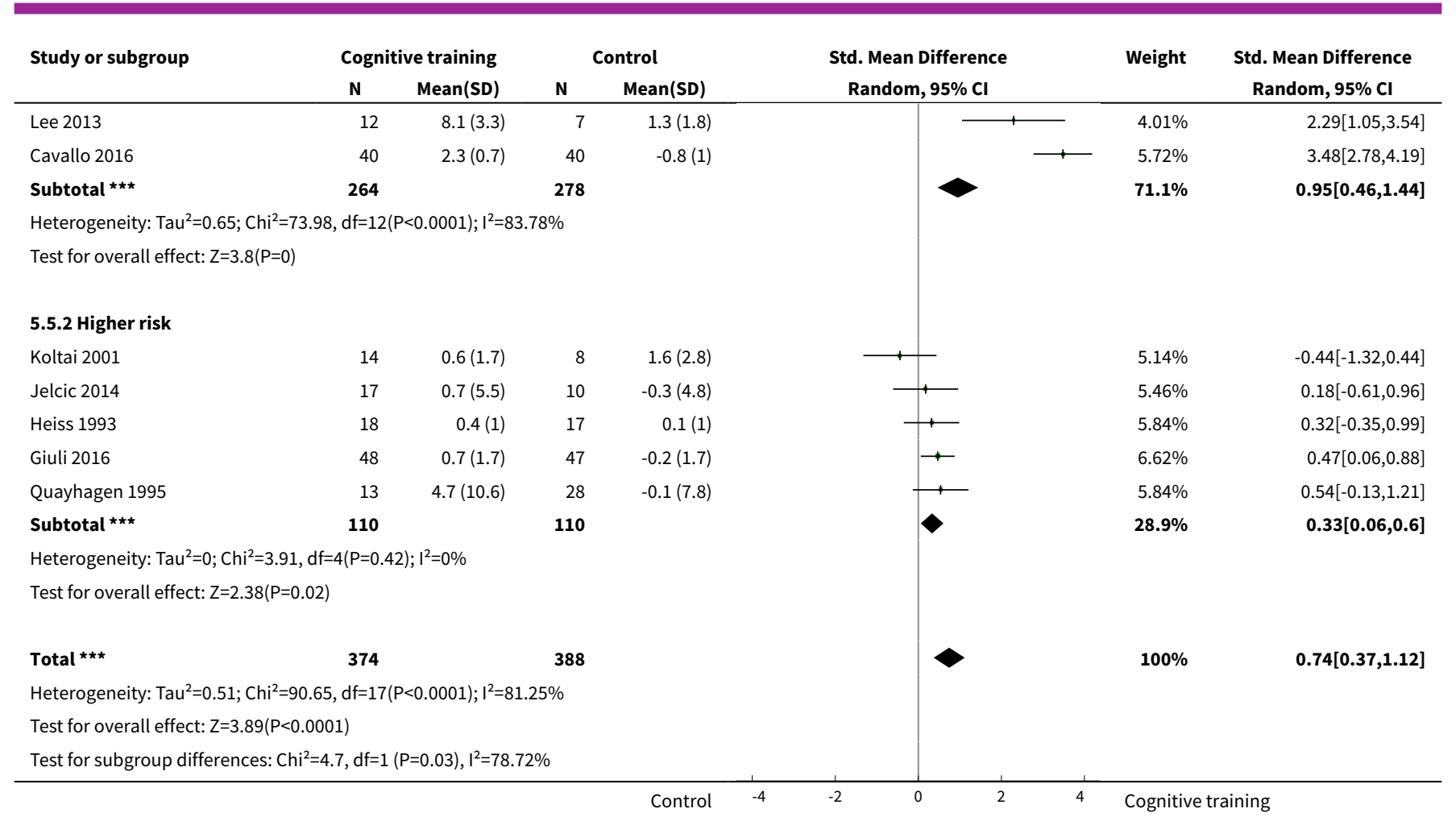

Analysis 5.6. Comparison 5 Cognitive training vs control immediately post intervention - risk of bias, Outcome 6 Change in delayed memory.

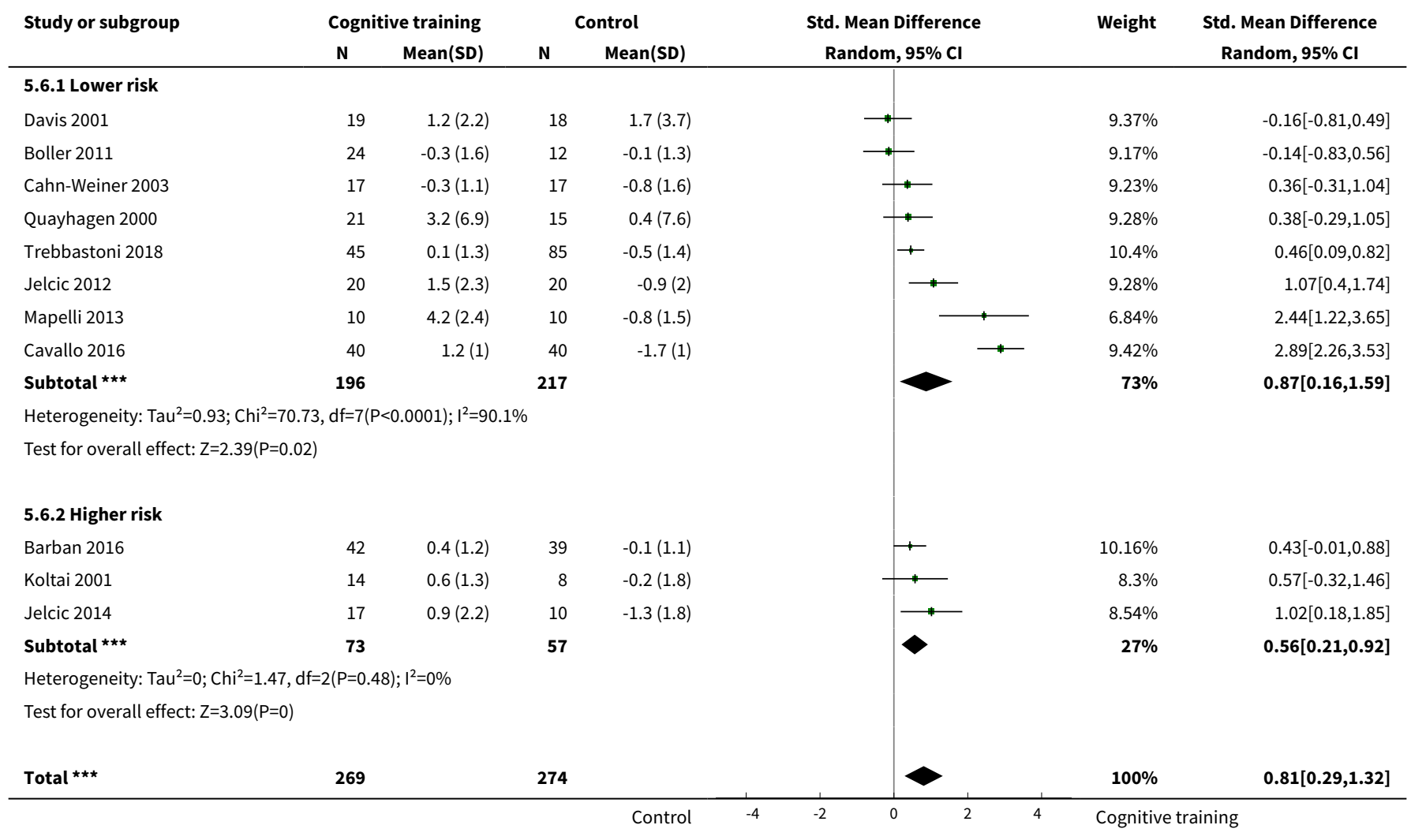




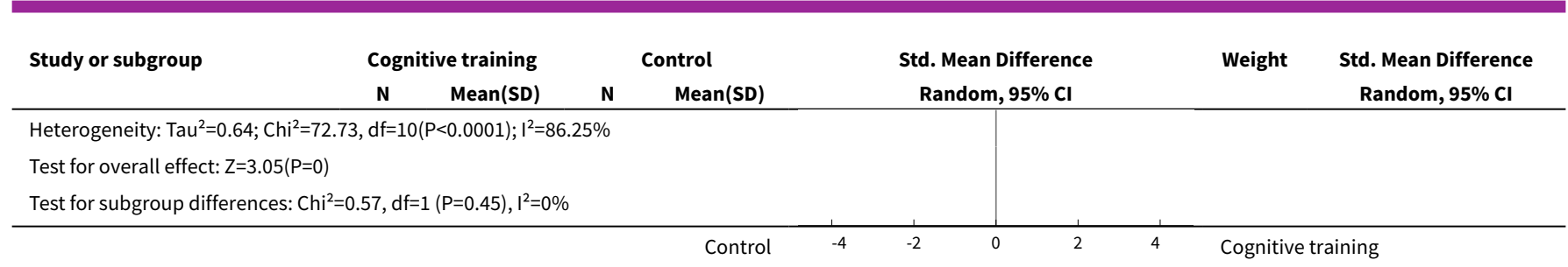

Analysis 5.7. Comparison 5 Cognitive training vs control immediately post intervention - risk of bias, Outcome 7 Change in attention and working memory.

\begin{tabular}{|c|c|c|c|c|c|c|c|}
\hline \multirow[t]{2}{*}{ Study or subgroup } & \multicolumn{2}{|c|}{ Cognitive training } & \multicolumn{2}{|c|}{ Control } & \multirow{2}{*}{$\begin{array}{c}\text { Std. Mean Difference } \\
\text { Random, } 95 \% \mathrm{CI}\end{array}$} & \multirow[t]{2}{*}{ Weight } & \multirow{2}{*}{$\begin{array}{c}\text { Std. Mean Difference } \\
\text { Random, } 95 \% \mathrm{CI}\end{array}$} \\
\hline & $\mathbf{N}$ & Mean(SD) & $\mathbf{N}$ & Mean(SD) & & & \\
\hline \multicolumn{8}{|l|}{ 5.7.1 Lower risk } \\
\hline Galante 2007 & 7 & $0.1(0.7)$ & 4 & $0.7(1.5)$ & $\longrightarrow$ & $6.15 \%$ & $-0.53[-1.79,0.73]$ \\
\hline Trebbastoni 2018 & 45 & $-0.3(0.6)$ & 85 & $-0.2(0.7)$ & $\leftarrow$ & $9.88 \%$ & $-0.1[-0.46,0.27]$ \\
\hline Boller 2011 & 24 & $0(1.1)$ & 12 & $0.1(0.9)$ & & $8.62 \%$ & $-0.07[-0.77,0.62]$ \\
\hline Davis 2001 & 19 & $-0.4(1.6)$ & 18 & $-0.5(1.6)$ & $\longrightarrow$ & $8.83 \%$ & $0.04[-0.61,0.68]$ \\
\hline Serino 2017 & 10 & $0(0.9)$ & 10 & $-0.3(0.6)$ & - & $7.75 \%$ & $0.41[-0.47,1.3]$ \\
\hline Beck 1988 & 10 & $0.7(1)$ & 10 & $-0.2(1.3)$ & & $7.63 \%$ & $0.75[-0.16,1.66]$ \\
\hline Jelcic 2012 & 20 & $0.2(0.6)$ & 20 & $-0.4(0.6)$ & + & $8.73 \%$ & $1.07[0.4,1.74]$ \\
\hline Mapelli 2013 & 10 & $1.7(1.2)$ & 10 & $-0.1(1.3)$ & $\longrightarrow$ & $7.27 \%$ & $1.36[0.37,2.36]$ \\
\hline Cavallo 2016 & 40 & $1.9(1)$ & 40 & $-0(0.9)$ & $\longrightarrow$ & $9.24 \%$ & $2.03[1.49,2.57]$ \\
\hline Subtotal $\star \star \star ~$ & 185 & & 209 & & & $74.1 \%$ & $0.57[-0.03,1.17]$ \\
\hline \multicolumn{8}{|c|}{ Heterogeneity: $\operatorname{Tau}^{2}=0.67 ; \mathrm{Chi}^{2}=53.99, \mathrm{df}=8(\mathrm{P}<0.0001) ; \mathrm{I}^{2}=85.18 \%$} \\
\hline \multicolumn{8}{|l|}{ 5.7.2 Higher risk } \\
\hline Heiss 1993 & 18 & $-0.6(1)$ & 17 & $-0(1)$ & $\longrightarrow$ & $8.68 \%$ & $-0.6[-1.28,0.08]$ \\
\hline Giuli 2016 & 48 & $0.3(0.9)$ & 47 & $-0.2(1)$ & $\rightarrow$ & $9.73 \%$ & $0.55[0.14,0.96]$ \\
\hline Jelcic 2014 & 17 & $0.3(1)$ & 10 & $-1.3(0.6)$ & $\longrightarrow$ & $7.49 \%$ & $1.85[0.9,2.8]$ \\
\hline 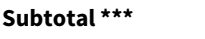 & 83 & & 74 & & & $25.9 \%$ & $0.56[-0.57,1.69]$ \\
\hline \multicolumn{8}{|c|}{ Heterogeneity: $\operatorname{Tau}^{2}=0.87 ; \mathrm{Chi}^{2}=17.87, \mathrm{df}=2(\mathrm{P}=0) ; \mathrm{I}^{2}=88.81 \%$} \\
\hline \multicolumn{8}{|c|}{ Test for overall effect: $Z=0.97(P=0.33)$} \\
\hline Total $\star \star \star ~$ & 268 & & 283 & & & $100 \%$ & $0.56[0.08,1.05]$ \\
\hline \multicolumn{8}{|c|}{ Heterogeneity: $\mathrm{Tau}^{2}=0.59 ; \mathrm{Chi}^{2}=71.95, \mathrm{df}=11(\mathrm{P}<0.0001) ; \mathrm{I}^{2}=84.71 \%$} \\
\hline \multicolumn{8}{|c|}{ Test for overall effect: $Z=2.27(P=0.02)$} \\
\hline \multicolumn{8}{|c|}{ Test for subgroup differences: $\mathrm{Chi}^{2}=0, \mathrm{df}=1(\mathrm{P}=0.98), \mathrm{I}^{2}=0 \%$} \\
\hline
\end{tabular}

Analysis 5.8. Comparison 5 Cognitive training vs control immediately post intervention - risk of bias, Outcome 8 Change in language (naming).

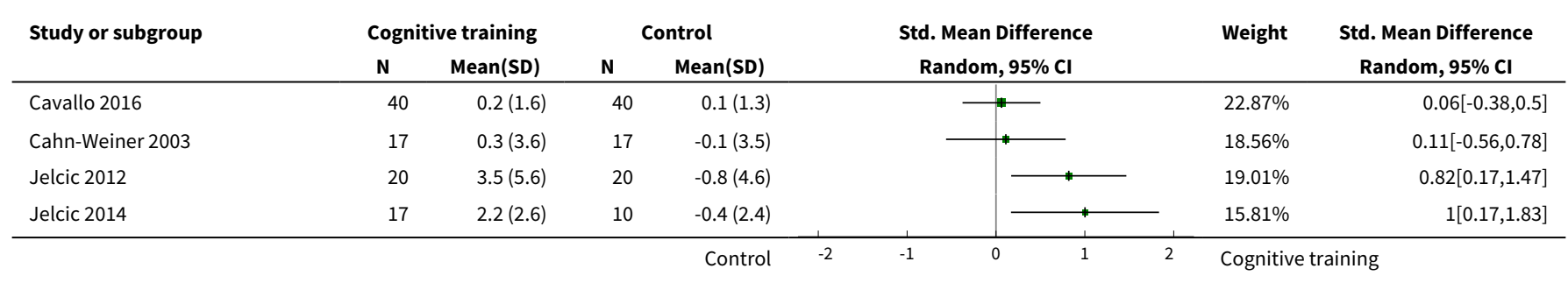




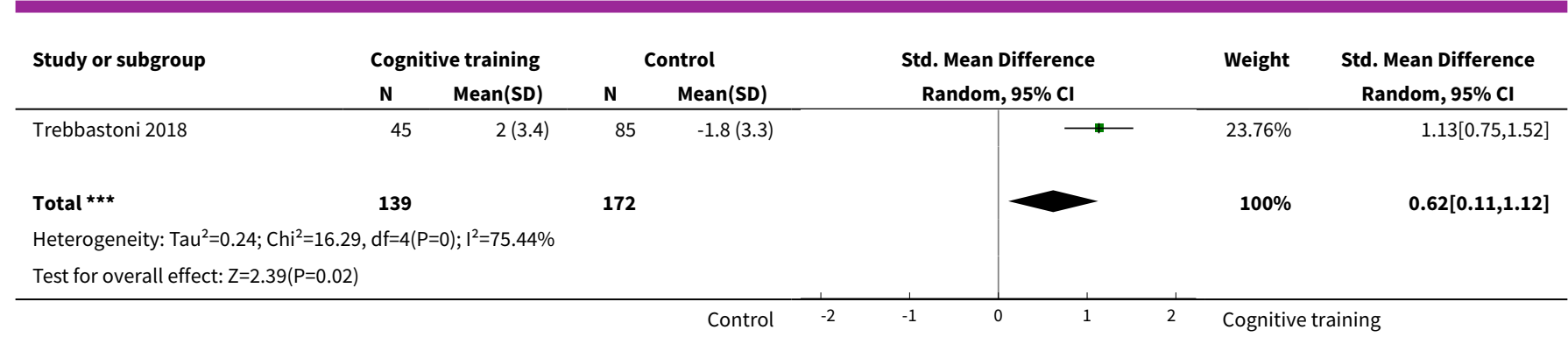

Analysis 5.9. Comparison 5 Cognitive training vs control immediately post intervention - risk of bias, Outcome 9 Change in verbal letter fluency.

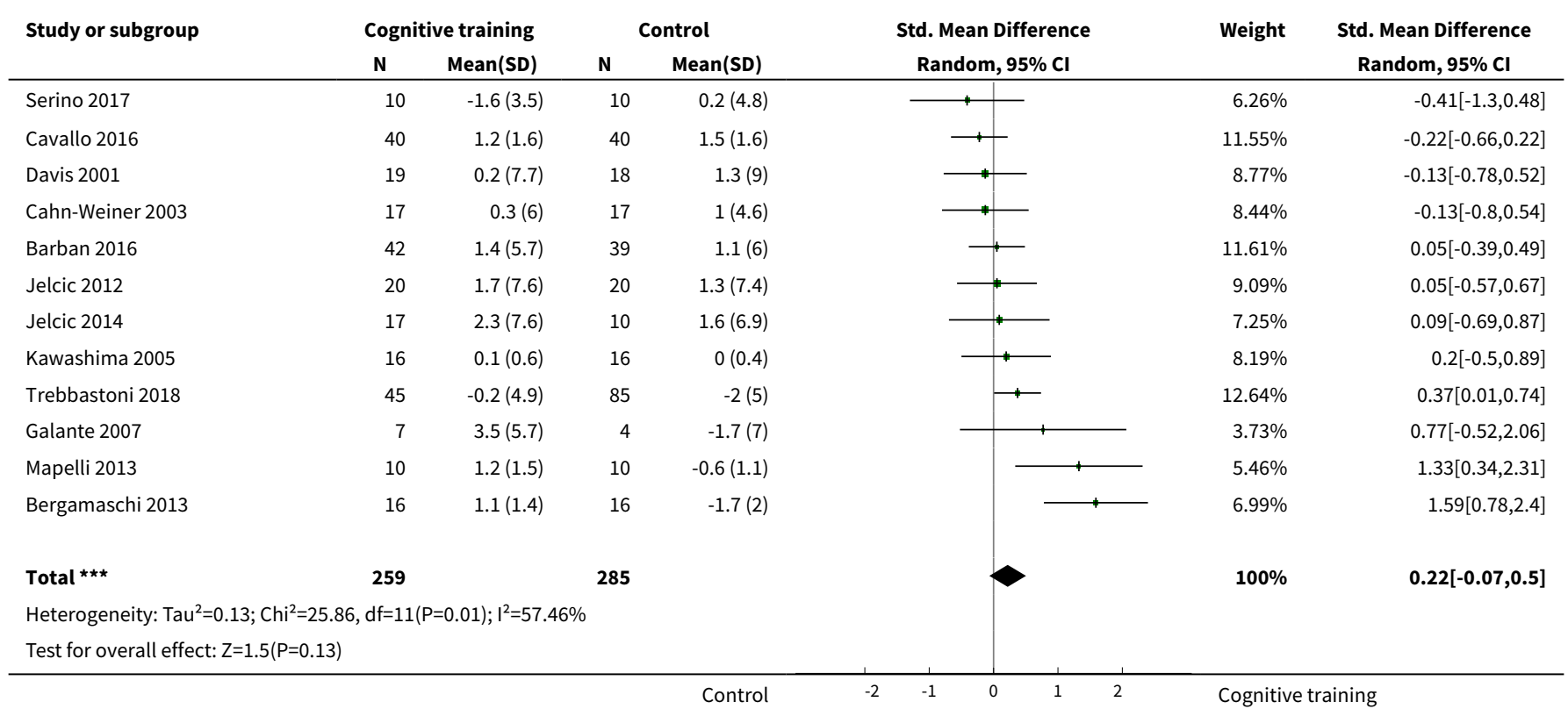

Analysis 5.10. Comparison 5 Cognitive training vs control immediately post intervention - risk of bias, Outcome 10 Change in speed of information processing.

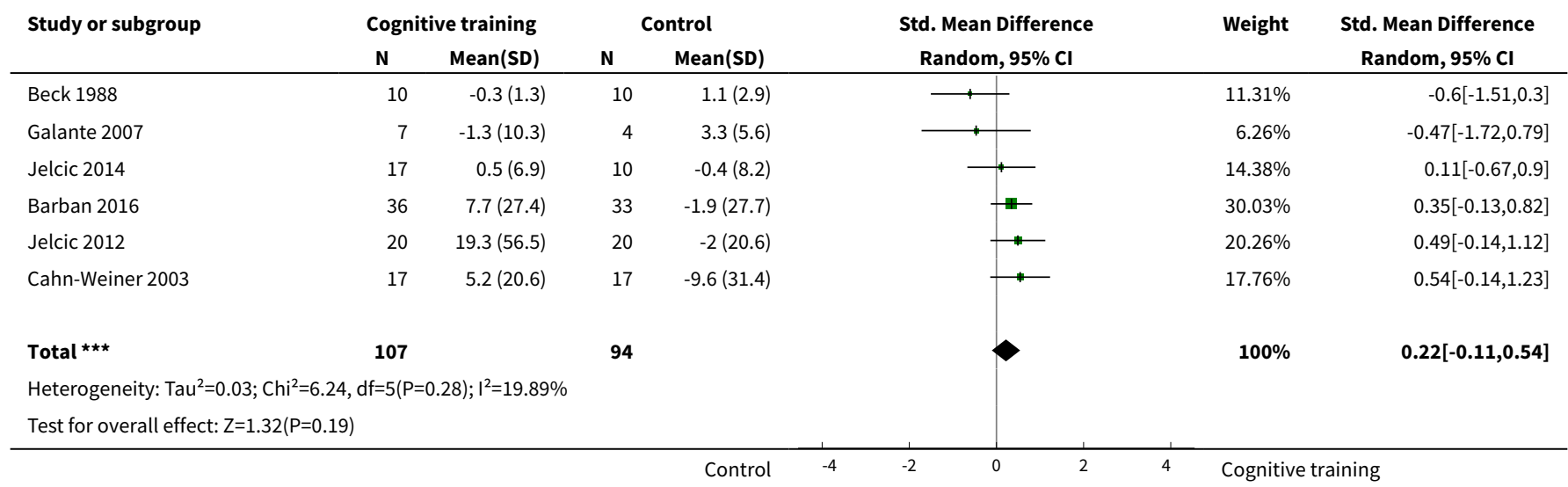


Analysis 5.11. Comparison 5 Cognitive training vs control immediately post intervention - risk of bias, Outcome 11 Change in executive function.

\begin{tabular}{|c|c|c|c|c|c|c|c|}
\hline \multirow[t]{2}{*}{ Study or subgroup } & \multicolumn{2}{|c|}{ Cognitive training } & \multicolumn{2}{|c|}{ Control } & \multirow{2}{*}{$\begin{array}{c}\text { Std. Mean Difference } \\
\text { Random, } 95 \% \mathrm{CI}\end{array}$} & \multirow[t]{2}{*}{ Weight } & \multirow{2}{*}{$\begin{array}{c}\text { Std. Mean Difference } \\
\text { Random, } 95 \% \mathrm{CI}\end{array}$} \\
\hline & $\mathbf{N}$ & Mean(SD) & $\mathbf{N}$ & $\operatorname{Mean}(S D)$ & & & \\
\hline Cahn-Weiner 2003 & 17 & $6(51.6)$ & 17 & $33.3(50)$ & 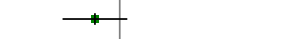 & $8.9 \%$ & $-0.52[-1.21,0.16]$ \\
\hline Galante 2007 & 7 & $-2.6(8.3)$ & 4 & $-2.3(3.3)$ & & $6.25 \%$ & $-0.04[-1.27,1.19]$ \\
\hline Kawashima 2005 & 16 & $15(18)$ & 16 & $-6(197)$ & - & $8.86 \%$ & $0.15[-0.55,0.84]$ \\
\hline Cavallo 2016 & 40 & $-0.1(0.8)$ & 40 & $-0.3(0.7)$ & + & $10.03 \%$ & $0.25[-0.19,0.69]$ \\
\hline Jelcic 2012 & 20 & $13.2(18.1)$ & 20 & $6.6(16.7)$ & + & $9.19 \%$ & $0.37[-0.25,1]$ \\
\hline Neely 2009 & 20 & $-0(0.2)$ & 9 & $-0.2(0.5)$ & $\rightarrow$ & $8.35 \%$ & $0.41[-0.39,1.2]$ \\
\hline Quayhagen 1995 & 13 & $4.8(7.3)$ & 28 & $0.4(6.6)$ & " & $8.96 \%$ & $0.63[-0.04,1.31]$ \\
\hline Trebbastoni 2018 & 45 & $1(2.1)$ & 85 & $-1.3(2.2)$ & + & $10.24 \%$ & $1.05[0.66,1.43]$ \\
\hline Quayhagen 1995 & 13 & $4.8(7.3)$ & 25 & $-3.6(6.4)$ & $\longrightarrow$ & $8.67 \%$ & $1.22[0.49,1.95]$ \\
\hline Quayhagen 2000 & 21 & $5.9(2.3)$ & 15 & $-2.6(3.8)$ & $\longrightarrow$ & $7.58 \%$ & $2.76[1.81,3.71]$ \\
\hline Mapelli 2013 & 10 & $2.3(0.5)$ & 10 & $-0.2(0.8)$ & & $5.1 \%$ & $3.58[2.06,5.1]$ \\
\hline Total $\star \star \star ~$ & 232 & & 279 & & & $100 \%$ & $0.75[0.28,1.22]$ \\
\hline \multicolumn{8}{|c|}{ Heterogeneity: $\mathrm{Tau}^{2}=0.52 ; \mathrm{Chi}^{2}=58.99, \mathrm{df}=11(\mathrm{P}<0.0001) ; \mathrm{I}^{2}=81.35 \%$} \\
\hline \multicolumn{5}{|c|}{ Test for overall effect: $Z=3.15(P=0)$} & & & \\
\hline
\end{tabular}

Analysis 5.12. Comparison 5 Cognitive training vs control immediately post intervention - risk of bias, Outcome 12 Change in verbal category fluency.

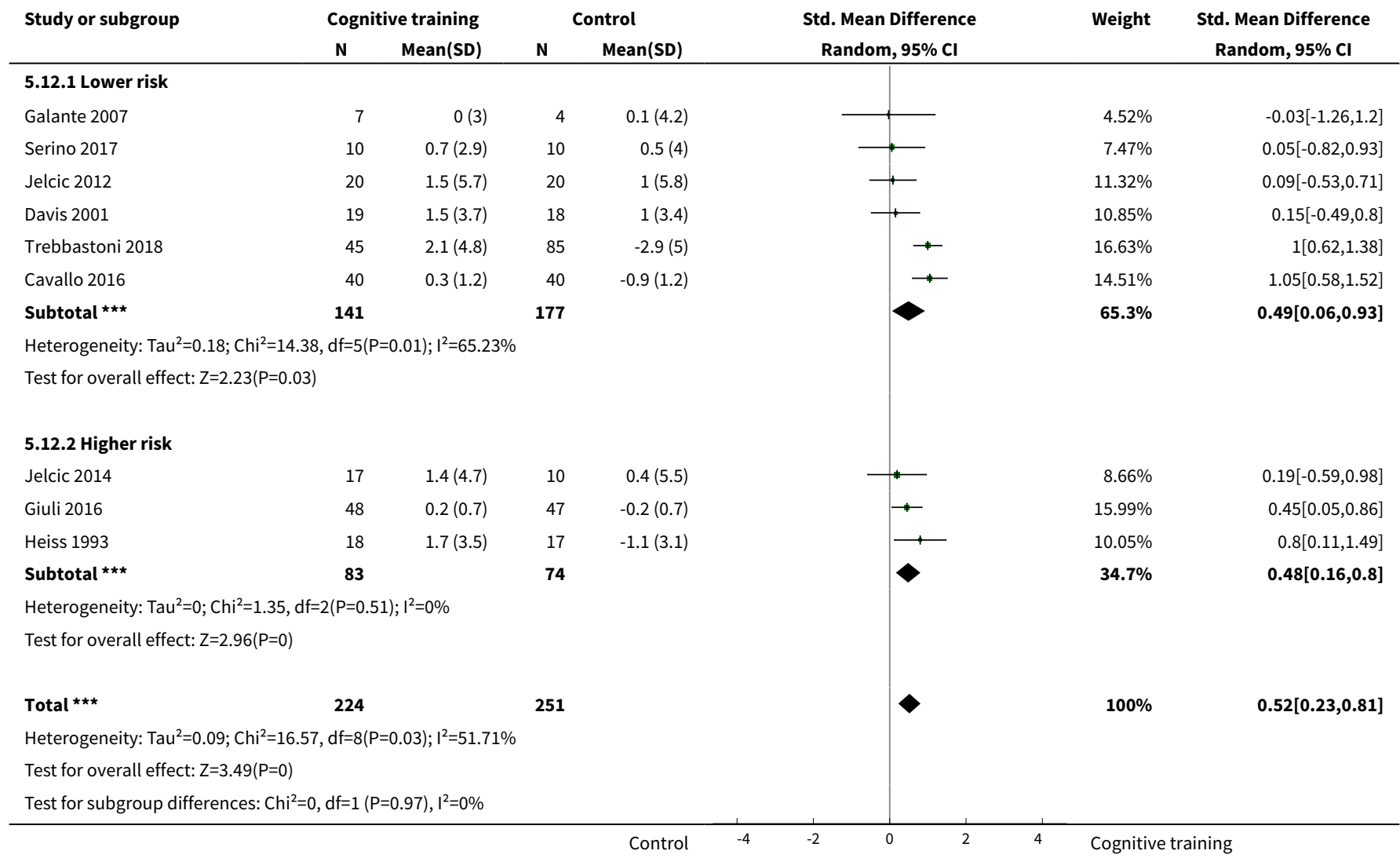


Analysis 5.13. Comparison 5 Cognitive training vs control immediately post intervention - risk of bias, Outcome 13 Change in meta cognition (self-reported).

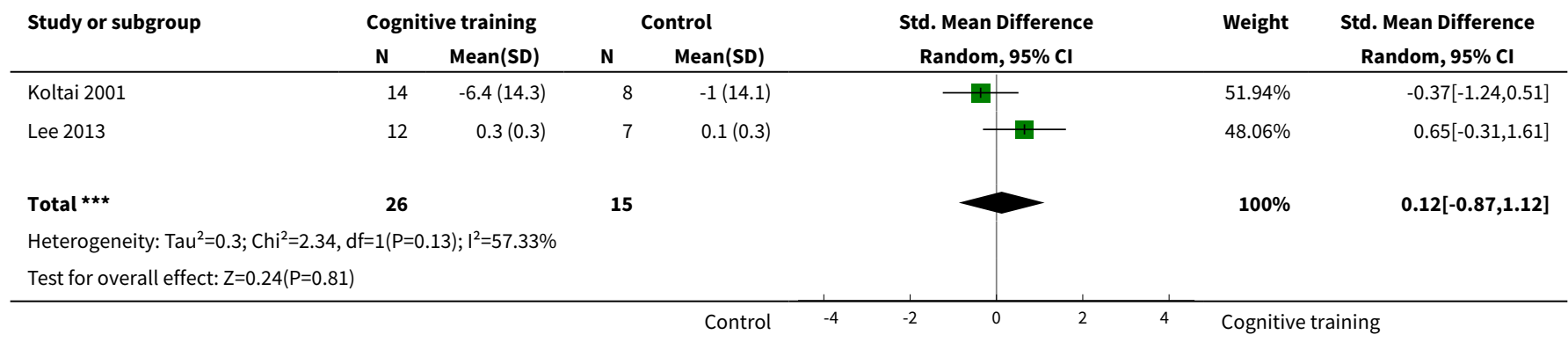

Analysis 5.14. Comparison 5 Cognitive training vs control immediately post intervention - risk of bias, Outcome 14 Change in meta cognition (informant-reported).

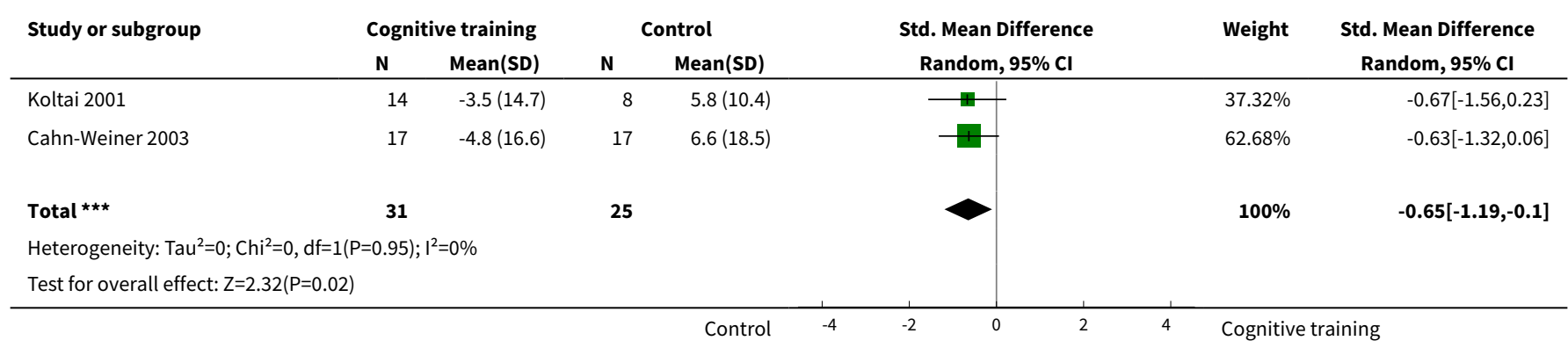

Analysis 5.15. Comparison 5 Cognitive training vs control immediately post intervention - risk of bias, Outcome 15 Change in participants' mood.

\begin{tabular}{|c|c|c|c|c|c|c|c|}
\hline \multirow[t]{2}{*}{ Study or subgroup } & \multicolumn{2}{|c|}{ Cognitive training } & \multicolumn{2}{|c|}{ Control } & \multirow{2}{*}{$\begin{array}{c}\text { Std. Mean Difference } \\
\text { Random, } 95 \% \mathrm{Cl}\end{array}$} & \multirow[t]{2}{*}{ Weight } & \multirow{2}{*}{$\begin{array}{c}\text { Std. Mean Difference } \\
\text { Random, } 95 \% \mathrm{Cl}\end{array}$} \\
\hline & $\mathbf{N}$ & $\operatorname{Mean}(\mathrm{SD})$ & $\mathbf{N}$ & Mean(SD) & & & \\
\hline Koltai 2001 & 14 & $-1.2(2.5)$ & 8 & $0.1(3)$ & $\longrightarrow$ & $12.23 \%$ & $-0.48[-1.36,0.4]$ \\
\hline Bergamaschi 2013 & 16 & $-1.1(3)$ & 16 & $0.4(5.1)$ & $\rightarrow$ & $12.91 \%$ & $-0.35[-1.05,0.35]$ \\
\hline Amieva 2016 & 164 & $-12.1(12.7)$ & 152 & $-10.8(12.2)$ & $\rightarrow$ & $14.11 \%$ & $-0.1[-0.32,0.12]$ \\
\hline Davis 2001 & 19 & $0.3(1.5)$ & 17 & $0.2(4.4)$ & $\longrightarrow$ & $13.07 \%$ & $0.05[-0.61,0.7]$ \\
\hline Lee 2013 & 12 & $-0.2(2.2)$ & 7 & $-1.9(2.8)$ & & $11.93 \%$ & $0.63[-0.33,1.59]$ \\
\hline Giuli 2016 & 48 & $-0.2(4.4)$ & 47 & $-7.3(3.8)$ & $\rightarrow$ & $13.6 \%$ & $1.73[1.25,2.2]$ \\
\hline Fernández-Calvo 2011 & 30 & $3.2(2)$ & 15 & $-4.3(1.9)$ & & $11.67 \%$ & $3.75[2.73,4.78]$ \\
\hline Total $\star \star \star$ & 310 & & 266 & & & $100 \%$ & $0.72[-0.1,1.54]$ \\
\hline \multicolumn{8}{|c|}{ Heterogeneity: $\mathrm{Tau}^{2}=1.23 ; \mathrm{Chi}^{2}=99.54, \mathrm{df}=7(\mathrm{P}<0.0001) ; \mathrm{I}^{2}=92.97 \%$} \\
\hline \multicolumn{8}{|c|}{ Test for overall effect: $Z=1.73(P=0.08)$} \\
\hline
\end{tabular}


Analysis 5.16. Comparison 5 Cognitive training vs control immediately post intervention - risk of bias, Outcome 16 Change in capacity for activities of daily living.

\begin{tabular}{|c|c|c|c|c|c|c|c|}
\hline \multirow[t]{2}{*}{ Study or subgroup } & \multicolumn{2}{|c|}{ Cognitive training } & \multicolumn{2}{|c|}{ Control } & \multirow{2}{*}{$\begin{array}{c}\text { Std. Mean Difference } \\
\text { Random, } 95 \% \mathrm{Cl}\end{array}$} & \multirow[t]{2}{*}{ Weight } & \multirow{2}{*}{$\begin{array}{c}\text { Std. Mean Difference } \\
\text { Random, } 95 \% \mathrm{Cl}\end{array}$} \\
\hline & $\mathbf{N}$ & $\operatorname{Mean}(S D)$ & $\mathbf{N}$ & Mean(SD) & & & \\
\hline \multicolumn{8}{|l|}{ 5.16.1 Lower risk } \\
\hline Galante 2007 & 7 & $-0.6(1.4)$ & 4 & $0.5(0.5)$ & & $2.63 \%$ & $-0.88[-2.19,0.43]$ \\
\hline Amieva 2016 & 167 & $-1.8(8.5)$ & 153 & $-1.2(8.3)$ & $\#$ & $23.78 \%$ & $-0.06[-0.28,0.16]$ \\
\hline Cahn-Weiner 2003 & 17 & $-0.2(3.2)$ & 17 & $0(3.1)$ & & $8.09 \%$ & $-0.06[-0.74,0.61]$ \\
\hline Kim 2015 & 21 & $0(0.9)$ & 21 & $0(1.1)$ & $\longrightarrow$ & $9.41 \%$ & $0[-0.6,0.6]$ \\
\hline Bergamaschi 2013 & 16 & $-0.4(1.5)$ & 16 & $-1.3(1.5)$ & 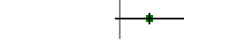 & $7.44 \%$ & $0.61[-0.1,1.32]$ \\
\hline Lee 2013 & 12 & $2.2(3.3)$ & 7 & $-2.1(4.5)$ & 1 & $4.18 \%$ & $1.1[0.09,2.11]$ \\
\hline Subtotal $* \star \star$ & 260 & & 256 & & & $66.45 \%$ & $0.09[-0.19,0.37]$ \\
\hline \multicolumn{8}{|c|}{ Heterogeneity: $\operatorname{Tau}^{2}=0.05 ; \mathrm{Chi}^{2}=9.51, \mathrm{df}=6(\mathrm{P}=0.15) ; \mathrm{I}^{2}=36.92 \%$} \\
\hline \multicolumn{8}{|c|}{ Test for overall effect: $Z=0.62(P=0.53)$} \\
\hline de Vreese 1998 & 9 & $-1.3(4.4)$ & 9 & $1(4)$ & 1 & $4.7 \%$ & $-0.53[-1.48,0.41]$ \\
\hline Barban 2016 & 38 & $-0.1(1)$ & 38 & $-0.3(1.1)$ & + & $13.63 \%$ & $0.19[-0.26,0.64]$ \\
\hline Giuli 2016 & 48 & $0.3(1.2)$ & 47 & $-0.2(1.3)$ & + & $15.22 \%$ & $0.4[-0,0.81]$ \\
\hline Subtotal $\star \star \star$ & 95 & & 94 & & & $33.55 \%$ & $0.18[-0.21,0.58]$ \\
\hline \multicolumn{8}{|c|}{ Heterogeneity: $\mathrm{Tau}^{2}=0.05 ; \mathrm{Chi}^{2}=3.23, \mathrm{df}=2(\mathrm{P}=0.2) ; \mathrm{I}^{2}=38.07 \%$} \\
\hline \multicolumn{8}{|c|}{ Test for overall effect: $\mathrm{Z}=0.92(\mathrm{P}=0.36)$} \\
\hline Total $\star \star \star$ & 355 & & 350 & & 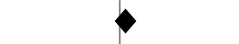 & $100 \%$ & $0.12[-0.1,0.34]$ \\
\hline \multicolumn{8}{|c|}{ Heterogeneity: $\operatorname{Tau}^{2}=0.04 ; \mathrm{Chi}^{2}=14.29, \mathrm{df}=9(\mathrm{P}=0.11) ; \mathrm{I}^{2}=37.04 \%$} \\
\hline \multicolumn{8}{|c|}{ Test for overall effect: $Z=1.05(P=0.29)$} \\
\hline Test for subgroup di & $.14, \mathrm{df}=$ & $P=0.71), I^{2}=09$ & & & & & \\
\hline
\end{tabular}

Analysis 5.17. Comparison 5 Cognitive training vs control immediately post intervention - risk of bias, Outcome 17 Change in general health and quality of life.

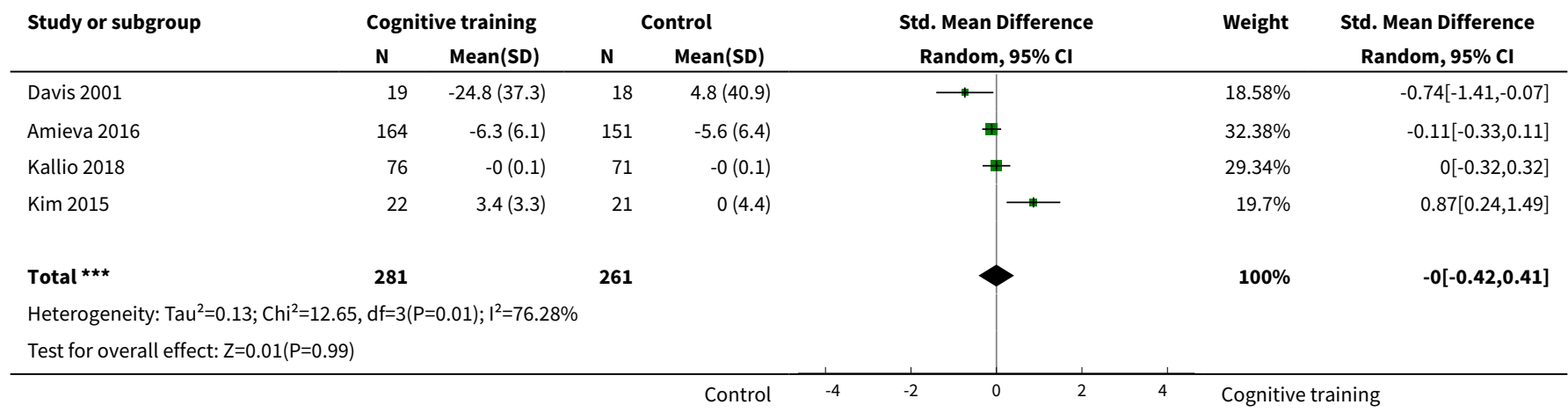


Analysis 5.18. Comparison 5 Cognitive training vs control immediately post intervention - risk of bias, Outcome 18 Change in behavioural and psychological symptoms of dementia (BPSD).

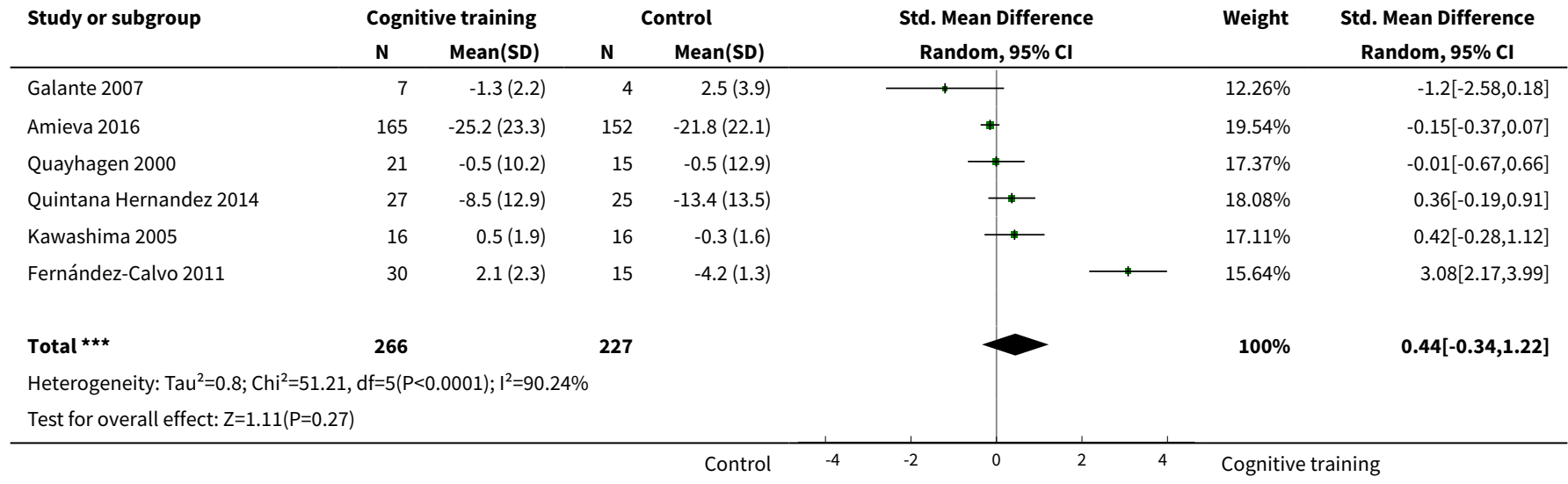

Analysis 5.19. Comparison 5 Cognitive training vs control immediately post intervention - risk of bias, Outcome 19 Change in disease progression.

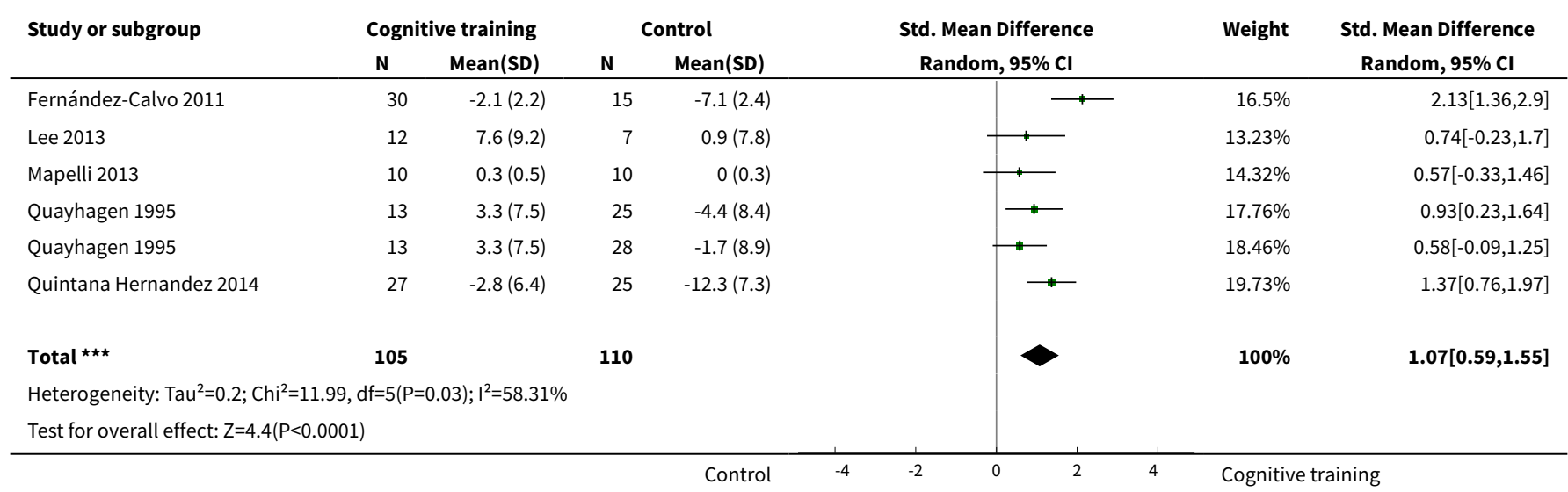

Analysis 5.20. Comparison 5 Cognitive training vs control immediately post intervention - risk of bias, Outcome 20 Change in burden of care (CAREGIVER).

\begin{tabular}{|c|c|c|c|c|c|c|c|}
\hline \multirow[t]{2}{*}{ Study or subgroup } & \multicolumn{2}{|c|}{ Cognitive training } & \multicolumn{2}{|c|}{ Control } & \multirow{2}{*}{$\begin{array}{c}\text { Std. Mean Difference } \\
\text { Random, } 95 \% \mathrm{Cl}\end{array}$} & \multirow[t]{2}{*}{ Weight } & \multirow{2}{*}{$\begin{array}{c}\text { Std. Mean Difference } \\
\text { Random, } 95 \% \mathrm{Cl}\end{array}$} \\
\hline & $\mathbf{N}$ & Mean(SD) & $\mathbf{N}$ & Mean(SD) & & & \\
\hline Amieva 2016 & 165 & $-21.3(19.1)$ & 152 & $-17.9(19)$ & & $76.98 \%$ & $-0.18[-0.4,0.04]$ \\
\hline Quayhagen 2000 & 21 & $-0.8(4.1)$ & 67 & $-1.3(3.7)$ & & $23.02 \%$ & $0.13[-0.36,0.62]$ \\
\hline Total ${ }^{\star \star \star}$ & 186 & & 219 & & & $100 \%$ & $-0.11[-0.36,0.15]$ \\
\hline \multicolumn{8}{|c|}{ Heterogeneity: $\operatorname{Tau}^{2}=0.01 ; \mathrm{Chi}^{2}=1.23, \mathrm{df}=1(\mathrm{P}=0.27) ; \mathrm{I}^{2}=18.62 \%$} \\
\hline
\end{tabular}


Analysis 5.21. Comparison 5 Cognitive training vs control immediately post intervention - risk of bias, Outcome 21 Change in quality of life (CAREGIVER).

\begin{tabular}{|c|c|c|c|c|c|c|c|c|}
\hline \multirow[t]{2}{*}{ Study or subgroup } & \multicolumn{2}{|c|}{ Cognitive training } & \multicolumn{2}{|c|}{ Control } & \multirow{2}{*}{\multicolumn{2}{|c|}{$\begin{array}{c}\text { Std. Mean Difference } \\
\text { Random, } 95 \% \mathrm{Cl}\end{array}$}} & \multirow[t]{2}{*}{ Weight } & \multirow{2}{*}{$\begin{array}{c}\text { Std. Mean Difference } \\
\text { Random, } 95 \% \mathrm{Cl}\end{array}$} \\
\hline & $\mathbf{N}$ & Mean(SD) & $\mathbf{N}$ & Mean(SD) & & & & \\
\hline Quayhagen 2000 & 21 & $-0.6(9.8)$ & 15 & $-2.8(16.5)$ & & & $100 \%$ & $0.16[-0.5,0.83]$ \\
\hline 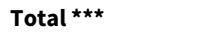 & 21 & & 15 & & & & $100 \%$ & $0.16[-0.5,0.83]$ \\
\hline \multicolumn{9}{|c|}{ Heterogeneity: Not applicable } \\
\hline \multicolumn{9}{|c|}{ Test for overall effect: $Z=0.48(P=0.63)$} \\
\hline
\end{tabular}

Analysis 5.22. Comparison 5 Cognitive training vs control immediately post intervention - risk of bias, Outcome 22 Change in mood and well-being (CAREGIVER).

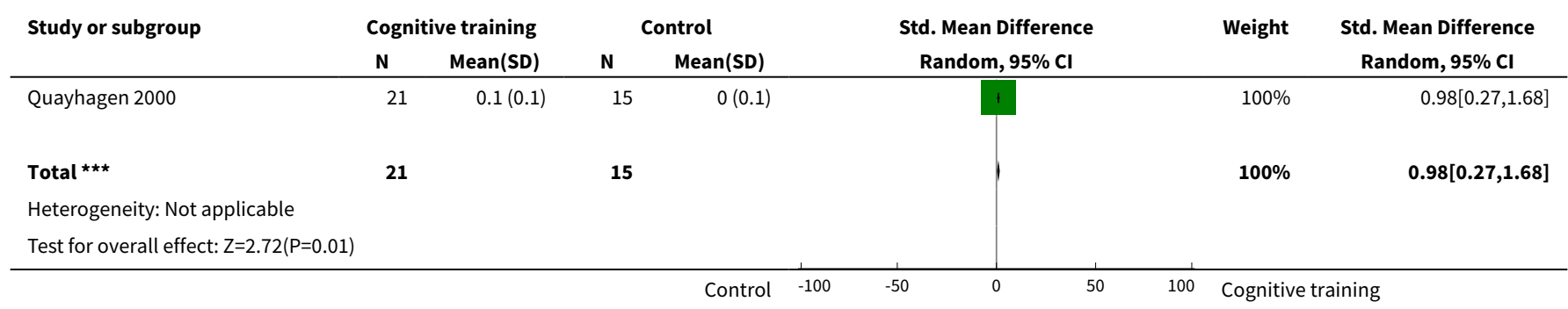

Analysis 5.23. Comparison 5 Cognitive training vs control immediately post intervention - risk of bias, Outcome 23 Participant burden (retention rates).

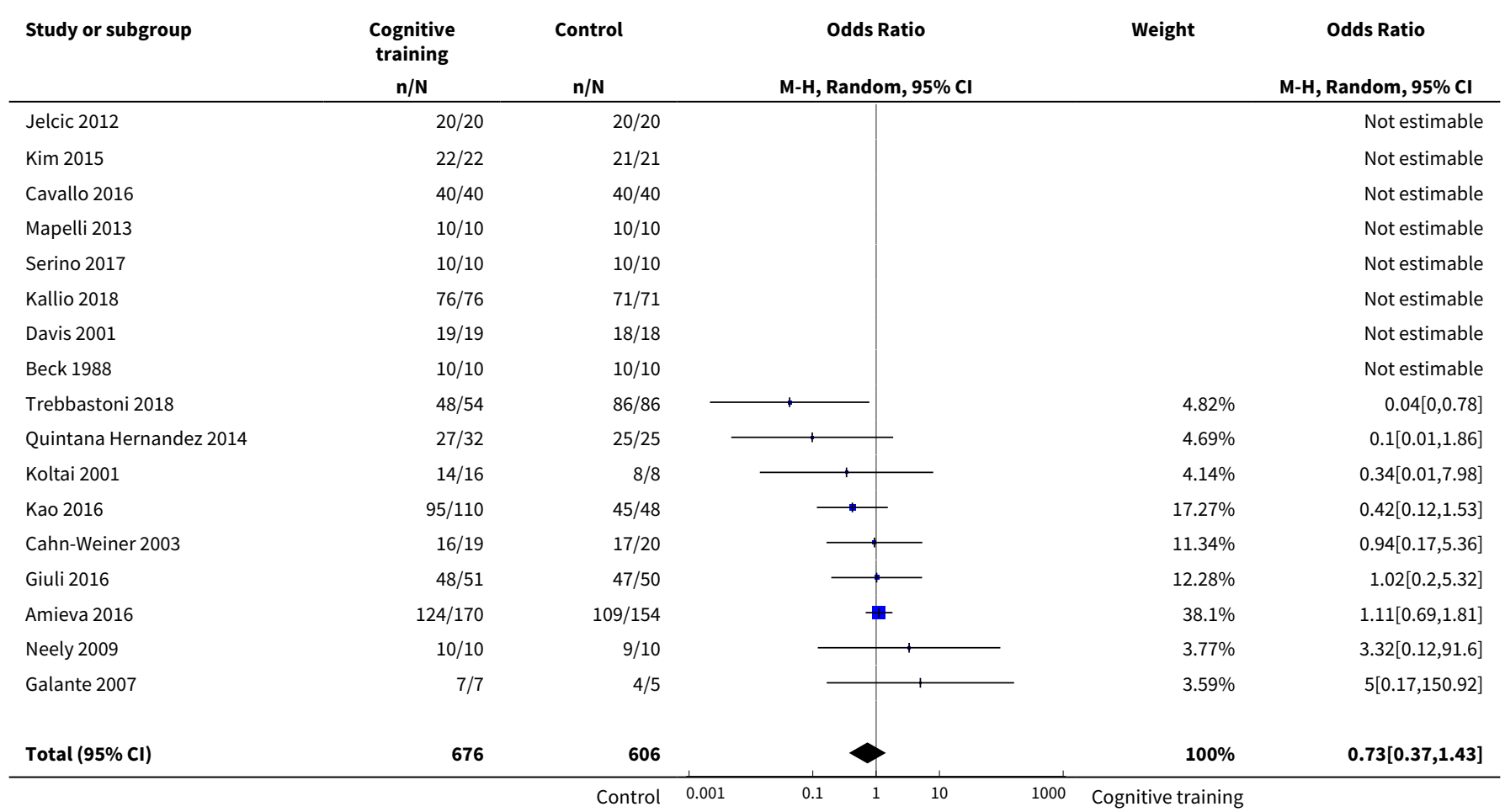




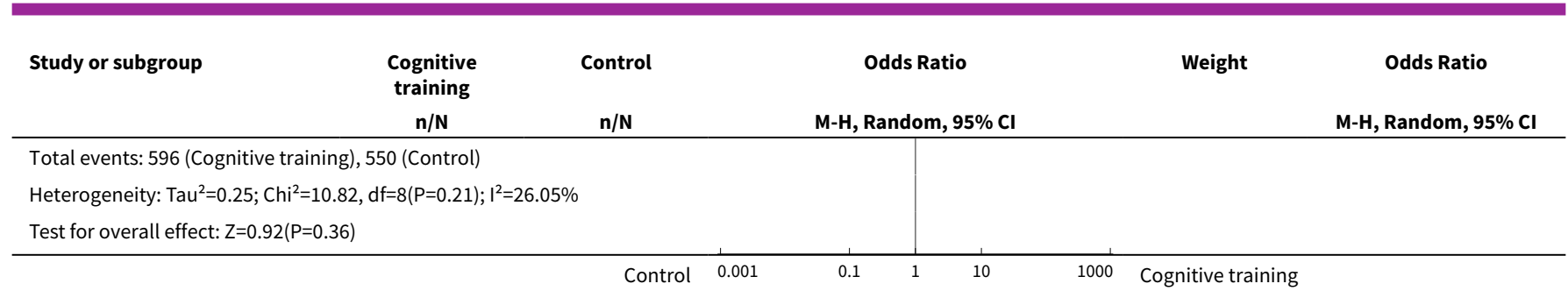

Analysis 5.24. Comparison 5 Cognitive training vs control immediately post intervention - risk of bias, Outcome 24 Change in general health and quality of life.

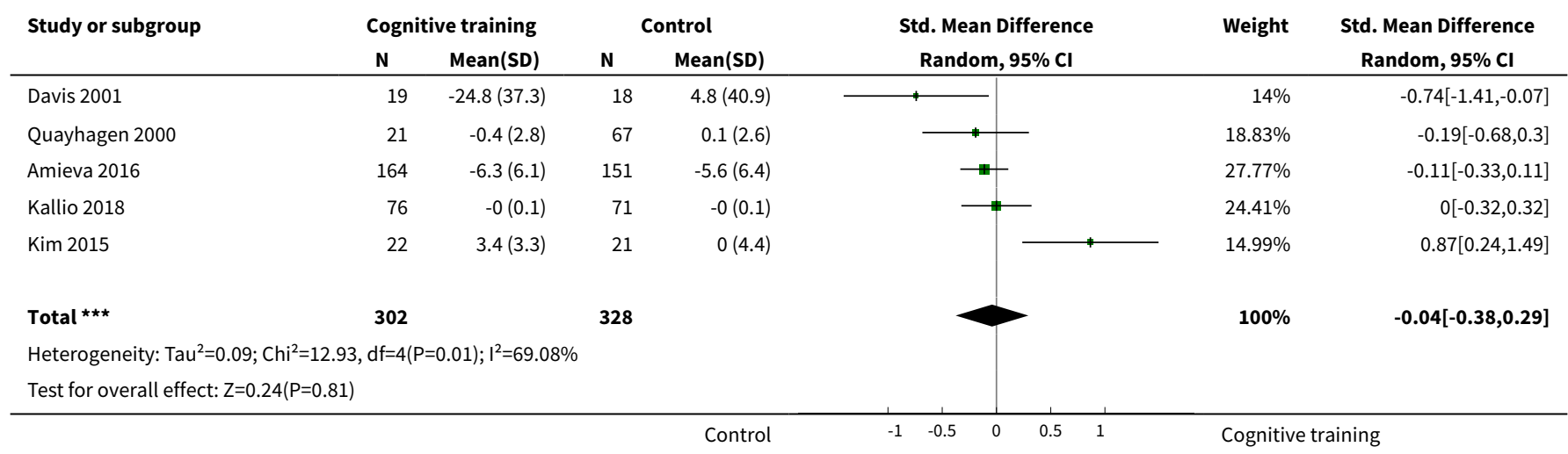

\section{Comparison 6. Cognitive training vs control immediately post intervention - intervention dose}

\begin{tabular}{|c|c|c|c|c|}
\hline Outcome or subgroup title & $\begin{array}{l}\text { No. of } \\
\text { studies }\end{array}$ & $\begin{array}{l}\text { No. of } \\
\text { partici- } \\
\text { pants }\end{array}$ & Statistical method & Effect size \\
\hline 1 Change in a global measure of cognition & 20 & 1288 & $\begin{array}{l}\text { Std. Mean Difference (IV, Random, } \\
95 \% \mathrm{CI})\end{array}$ & $0.65[0.26,1.05]$ \\
\hline 1.1 Up to 3 times & 15 & 1112 & $\begin{array}{l}\text { Std. Mean Difference (IV, Random, } \\
95 \% \mathrm{CI} \text { ) }\end{array}$ & $0.51[0.06,0.95]$ \\
\hline 1.2 More than 3 times & 5 & 176 & $\begin{array}{l}\text { Std. Mean Difference (IV, Random, } \\
95 \% \mathrm{CI})\end{array}$ & $1.14[0.27,2.01]$ \\
\hline $\begin{array}{l}2 \text { Change in a global measure of cogni- } \\
\text { tion_zero correlation }\end{array}$ & 20 & 1287 & $\begin{array}{l}\text { Std. Mean Difference (IV, Random, } \\
95 \% \mathrm{CI} \text { ) }\end{array}$ & $0.27[0.04,0.50]$ \\
\hline 2.1 Up to 3 times & 15 & 1111 & $\begin{array}{l}\text { Std. Mean Difference (IV, Random, } \\
95 \% \mathrm{CI} \text { ) }\end{array}$ & $0.23[-0.01,0.47]$ \\
\hline 2.2 More than 3 times & 5 & 176 & $\begin{array}{l}\text { Std. Mean Difference (IV, Random, } \\
95 \% \mathrm{CI} \text { ) }\end{array}$ & $0.42[-0.30,1.15]$ \\
\hline $\begin{array}{l}3 \text { Change in a global measure of cognition } \\
\text { (composite) }\end{array}$ & 26 & 1389 & $\begin{array}{l}\text { Std. Mean Difference (Random, } \\
95 \% \mathrm{CI} \text { ) }\end{array}$ & $0.42[0.23,0.61]$ \\
\hline
\end{tabular}




\begin{tabular}{|c|c|c|c|c|}
\hline Outcome or subgroup title & $\begin{array}{l}\text { No. of } \\
\text { studies }\end{array}$ & $\begin{array}{l}\text { No. of } \\
\text { partici- } \\
\text { pants }\end{array}$ & Statistical method & Effect size \\
\hline 3.1 Up to 3 times & 20 & 1138 & $\begin{array}{l}\text { Std. Mean Difference (Random, } \\
95 \% \mathrm{Cl} \text { ) }\end{array}$ & $0.33[0.13,0.53]$ \\
\hline 3.2 More than 3 times & 6 & 251 & $\begin{array}{l}\text { Std. Mean Difference (Random, } \\
95 \% \mathrm{Cl} \text { ) }\end{array}$ & $0.71[0.27,1.14]$ \\
\hline $\begin{array}{l}4 \text { Change in a global measure of cognition } \\
\text { (composite)_zero correlation }\end{array}$ & 26 & 1338 & $\begin{array}{l}\text { Std. Mean Difference (Random, } \\
95 \% \mathrm{Cl} \text { ) }\end{array}$ & $0.24[0.12,0.36]$ \\
\hline 4.1 Up to 3 times & 20 & 1139 & $\begin{array}{l}\text { Std. Mean Difference (Random, } \\
95 \% \mathrm{Cl} \text { ) }\end{array}$ & $0.14[0.02,0.26]$ \\
\hline 4.2 More than 3 times & 6 & 199 & $\begin{array}{l}\text { Std. Mean Difference (Random, } \\
95 \% \mathrm{Cl} \text { ) }\end{array}$ & $0.54[0.28,0.80]$ \\
\hline 5 Change in immediate memory & 17 & 762 & $\begin{array}{l}\text { Std. Mean Difference (IV, Random, } \\
95 \% \mathrm{CI} \text { ) }\end{array}$ & $0.74[0.37,1.12]$ \\
\hline 5.1 Up to 3 times & 14 & 636 & $\begin{array}{l}\text { Std. Mean Difference (IV, Random, } \\
95 \% \mathrm{CI})\end{array}$ & $0.73[0.27,1.19]$ \\
\hline 5.2 More than 3 times & 4 & 126 & $\begin{array}{l}\text { Std. Mean Difference (IV, Random, } \\
95 \% \mathrm{CI})\end{array}$ & $0.74[0.29,1.19]$ \\
\hline 6 Change in delayed memory & 11 & 543 & $\begin{array}{l}\text { Std. Mean Difference (IV, Random, } \\
95 \% \mathrm{CI})\end{array}$ & $0.81[0.29,1.32]$ \\
\hline 7 Change in attention and working memory & 12 & 551 & $\begin{array}{l}\text { Std. Mean Difference (IV, Random, } \\
95 \% \mathrm{CI} \text { ) }\end{array}$ & $0.56[0.08,1.05]$ \\
\hline 8 Change in language (naming) & 5 & 311 & $\begin{array}{l}\text { Std. Mean Difference (IV, Random, } \\
95 \% \mathrm{Cl} \text { ) }\end{array}$ & $0.62[0.11,1.12]$ \\
\hline 9 Change in verbal letter fluency & 12 & 544 & $\begin{array}{l}\text { Std. Mean Difference (IV, Random, } \\
95 \% \mathrm{Cl})\end{array}$ & $0.22[-0.07,0.50]$ \\
\hline 9.1 Up to 3 times & 9 & 460 & $\begin{array}{l}\text { Std. Mean Difference (IV, Random, } \\
95 \% \mathrm{CI} \text { ) }\end{array}$ & $0.05[-0.13,0.24]$ \\
\hline 9.2 More than 3 times & 3 & 84 & $\begin{array}{l}\text { Std. Mean Difference (IV, Random, } \\
95 \% \mathrm{Cl})\end{array}$ & $1.00[0.09,1.92]$ \\
\hline $\begin{array}{l}10 \text { Change in speed of information process- } \\
\text { ing }\end{array}$ & 6 & 201 & $\begin{array}{l}\text { Std. Mean Difference (IV, Random, } \\
95 \% \mathrm{CI})\end{array}$ & $0.22[-0.11,0.54]$ \\
\hline 11 Change in executive function & 11 & 511 & $\begin{array}{l}\text { Std. Mean Difference (IV, Random, } \\
95 \% \mathrm{CI} \text { ) }\end{array}$ & $0.75[0.28,1.22]$ \\
\hline 11.1 Up to 3 times & 8 & 380 & $\begin{array}{l}\text { Std. Mean Difference (IV, Random, } \\
95 \% \mathrm{Cl} \text { ) }\end{array}$ & $0.57[0.01,1.13]$ \\
\hline 11.2 More than 3 times & 3 & 131 & $\begin{array}{l}\text { Std. Mean Difference (IV, Random, } \\
95 \% \mathrm{Cl} \text { ) }\end{array}$ & $1.20[0.20,2.20]$ \\
\hline
\end{tabular}




\begin{tabular}{|c|c|c|c|c|}
\hline Outcome or subgroup title & $\begin{array}{l}\text { No. of } \\
\text { studies }\end{array}$ & $\begin{array}{l}\text { No. of } \\
\text { partici- } \\
\text { pants }\end{array}$ & Statistical method & Effect size \\
\hline 12 Change in verbal category fluency & 9 & 475 & $\begin{array}{l}\text { Std. Mean Difference (IV, Random, } \\
95 \% \mathrm{CI} \text { ) }\end{array}$ & $0.52[0.23,0.81]$ \\
\hline 13 Change in meta cognition (self-reported) & 2 & 41 & $\begin{array}{l}\text { Std. Mean Difference (IV, Random, } \\
95 \% \mathrm{CI})\end{array}$ & $0.12[-0.87,1.12]$ \\
\hline $\begin{array}{l}14 \text { Change in meta cognition (informant-re- } \\
\text { ported) }\end{array}$ & 2 & 56 & $\begin{array}{l}\text { Std. Mean Difference (IV, Random, } \\
95 \% \mathrm{CI})\end{array}$ & $-0.65[-1.19,-0.10]$ \\
\hline 15 Change in participants' mood & 8 & 576 & $\begin{array}{l}\text { Std. Mean Difference (IV, Random, } \\
95 \% \mathrm{Cl} \text { ) }\end{array}$ & $0.72[-0.10,1.54]$ \\
\hline $\begin{array}{l}16 \text { Change in capacity for activities of daily } \\
\text { living }\end{array}$ & 10 & 705 & $\begin{array}{l}\text { Std. Mean Difference (IV, Random, } \\
95 \% \mathrm{CI})\end{array}$ & $0.12[-0.10,0.34]$ \\
\hline 17 Change in disease progression & 5 & 215 & $\begin{array}{l}\text { Std. Mean Difference (IV, Random, } \\
95 \% \mathrm{CI})\end{array}$ & $1.07[0.59,1.55]$ \\
\hline $\begin{array}{l}18 \text { Change in behavioural and psychological } \\
\text { symptoms of dementia (BPSD) }\end{array}$ & 6 & 493 & $\begin{array}{l}\text { Std. Mean Difference (IV, Random, } \\
95 \% \mathrm{CI} \text { ) }\end{array}$ & $0.44[-0.34,1.22]$ \\
\hline 19 Change in attention and working memory & 12 & 551 & $\begin{array}{l}\text { Std. Mean Difference (IV, Random, } \\
95 \% \mathrm{Cl} \text { ) }\end{array}$ & $0.56[0.08,1.05]$ \\
\hline 20 Change in burden of care (CAREGIVER) & 2 & 405 & $\begin{array}{l}\text { Std. Mean Difference (IV, Random, } \\
95 \% \mathrm{Cl} \text { ) }\end{array}$ & $-0.11[-0.36,0.15]$ \\
\hline 21 Change in quality of life (CAREGIVER) & 1 & 36 & $\begin{array}{l}\text { Std. Mean Difference (IV, Random, } \\
95 \% \mathrm{CI} \text { ) }\end{array}$ & $0.16[-0.50,0.83]$ \\
\hline $\begin{array}{l}22 \text { Change in mood and well-being } \\
\text { (CAREGIVER) }\end{array}$ & 1 & 36 & $\begin{array}{l}\text { Std. Mean Difference (IV, Random, } \\
95 \% \mathrm{Cl} \text { ) }\end{array}$ & $0.98[0.27,1.68]$ \\
\hline 23 Participant burden (retention rates) & 17 & 1282 & $\begin{array}{l}\text { Odds Ratio (M-H, Random, 95\% } \\
\mathrm{Cl} \text { ) }\end{array}$ & $0.73[0.37,1.43]$ \\
\hline 23.1 Up to 3 times & 14 & 1047 & $\begin{array}{l}\text { Odds Ratio (M-H, Random, 95\% } \\
\text { Cl) }\end{array}$ & $0.98[0.53,1.81]$ \\
\hline 23.2 More than 3 times & 3 & 235 & $\begin{array}{l}\text { Odds Ratio (M-H, Random, 95\% } \\
\mathrm{Cl})\end{array}$ & $0.33[0.10,1.09]$ \\
\hline $\begin{array}{l}24 \text { Change in general health and quality of } \\
\text { life }\end{array}$ & 5 & 630 & $\begin{array}{l}\text { Std. Mean Difference (IV, Random, } \\
95 \% \mathrm{CI})\end{array}$ & $-0.04[-0.38,0.29]$ \\
\hline
\end{tabular}


Analysis 6.1. Comparison 6 Cognitive training vs control immediately post intervention - intervention dose, Outcome 1 Change in a global measure of cognition.

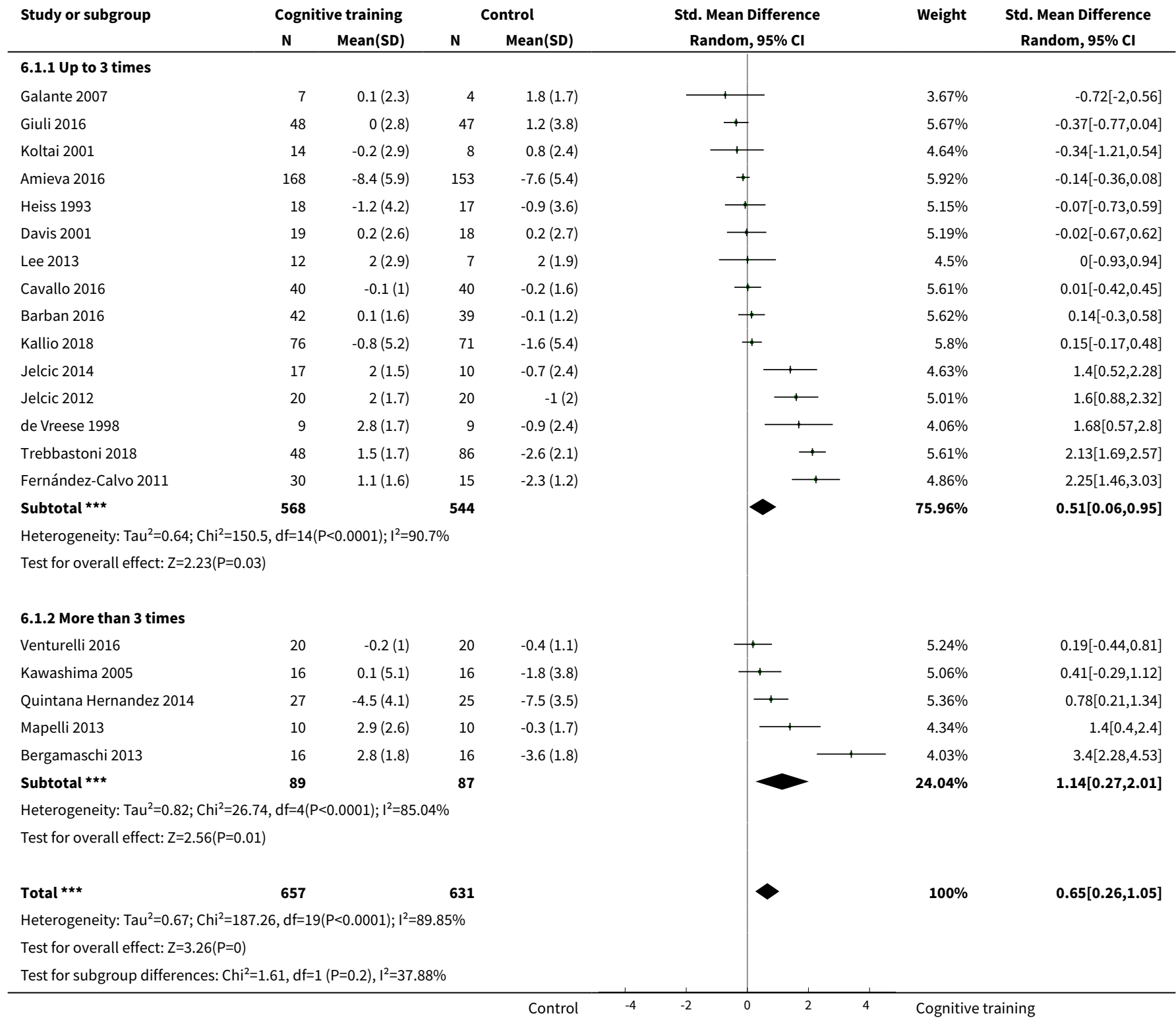

Analysis 6.2. Comparison 6 Cognitive training vs control immediately post intervention intervention dose, Outcome 2 Change in a global measure of cognition_zero correlation.

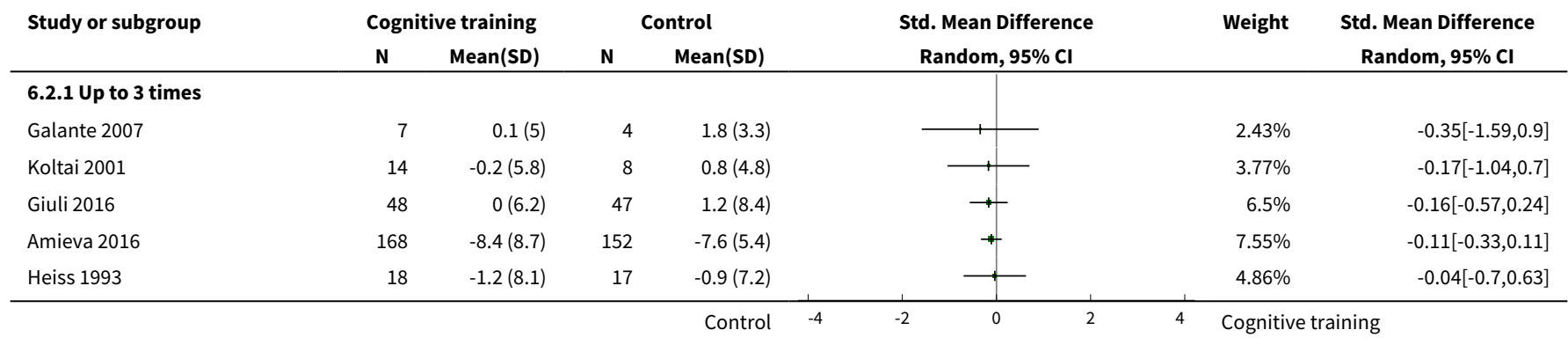




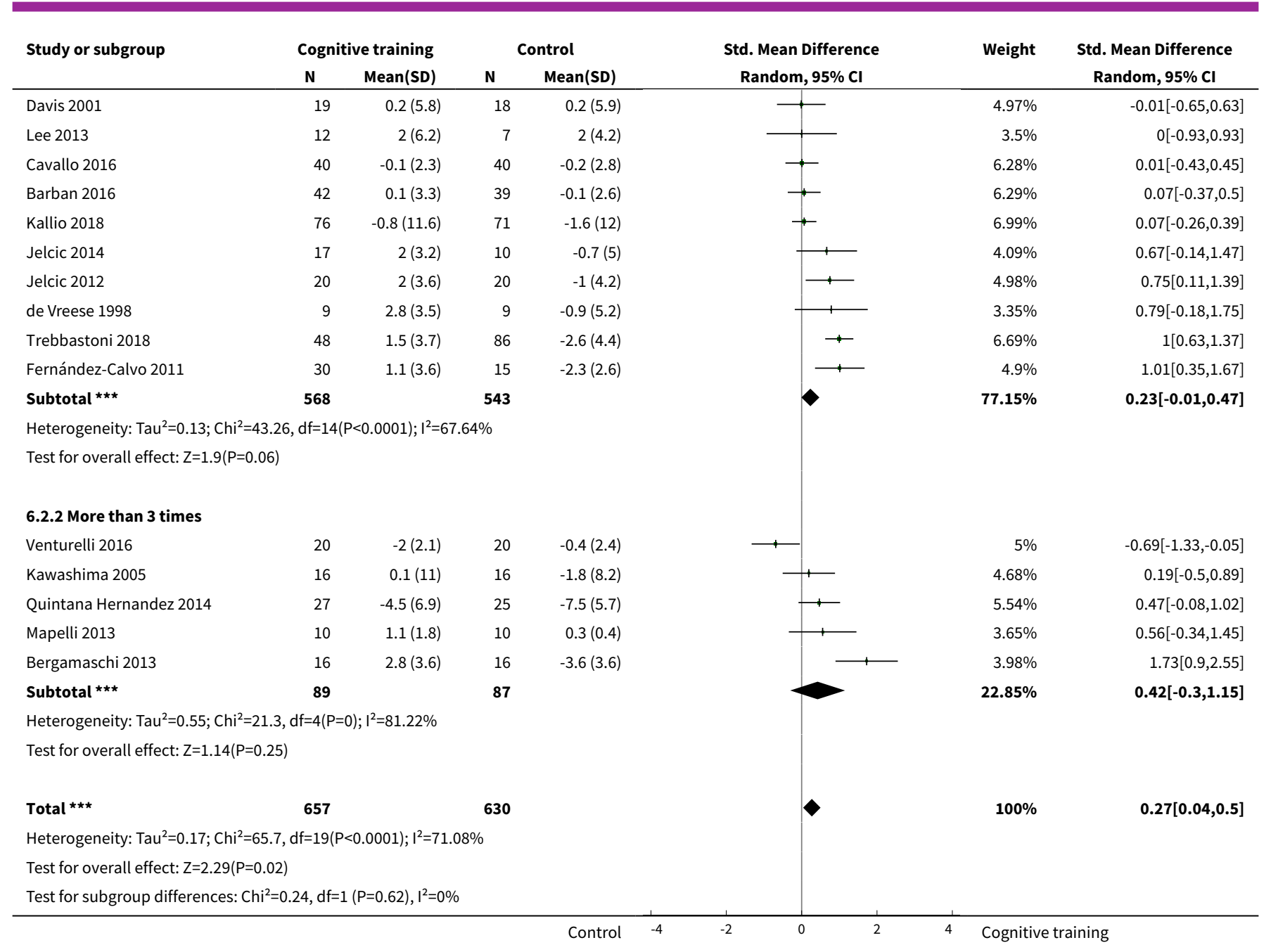

\section{Analysis 6.3. Comparison 6 Cognitive training vs control immediately post intervention} - intervention dose, Outcome 3 Change in a global measure of cognition (composite).

\begin{tabular}{|c|c|c|c|c|c|c|}
\hline \multirow{2}{*}{$\begin{array}{l}\text { Study or subgroup } \\
6.3 .1 \text { Up to } 3 \text { times }\end{array}$} & $\begin{array}{c}\text { Cognitive } \\
\text { training } \\
\mathrm{N}\end{array}$ & $\begin{array}{l}\text { Control } \\
\text { N }\end{array}$ & $\begin{array}{l}\text { Std. Mean } \\
\text { Difference } \\
\text { (SE) } \\
\end{array}$ & $\begin{array}{l}\text { Std. Mean Difference } \\
\text { IV, Random, 95\% CI }\end{array}$ & \multirow[t]{2}{*}{ Weight } & \multirow[t]{2}{*}{$\begin{array}{l}\text { Std. Mean Difference } \\
\text { IV, Random, 95\% CI }\end{array}$} \\
\hline & & & & & & \\
\hline Amieva 2016 & 165 & 153 & $-0.1(0.11)$ & + & $6.61 \%$ & $-0.11[-0.33,0.11]$ \\
\hline Barban 2016 & 41 & 38 & $0.2(0.23)$ & 十 & $5.12 \%$ & $0.21[-0.24,0.66]$ \\
\hline Beck 1988 & 10 & 10 & $-0(0.43)$ & 1 & $2.97 \%$ & $-0.03[-0.87,0.81]$ \\
\hline Cavallo 2016 & 40 & 40 & $1.3(0.28)$ & 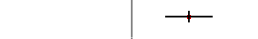 & $4.49 \%$ & $1.32[0.77,1.87]$ \\
\hline Davis 2001 & 19 & 18 & $0(0.32)$ & 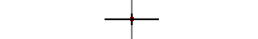 & $4.03 \%$ & $0[-0.63,0.63]$ \\
\hline de Vreese 1998 & 9 & 9 & $1(0.49)$ & 1 & $2.52 \%$ & $1.04[0.08,2]$ \\
\hline Fernández-Calvo 2011 & 30 & 15 & $-0.4(0.42)$ & - & $3.05 \%$ & $-0.44[-1.26,0.38]$ \\
\hline Galante 2007 & 7 & 4 & $0.2(0.63)$ & + & $1.77 \%$ & $0.21[-1.02,1.44]$ \\
\hline Giuli 2016 & 48 & 47 & $0.4(0.21)$ & + & $5.39 \%$ & $0.37[-0.04,0.78]$ \\
\hline Jelcic 2012 & 20 & 20 & $0.6(0.32)$ & 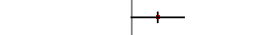 & $4.03 \%$ & $0.6[-0.03,1.23]$ \\
\hline
\end{tabular}




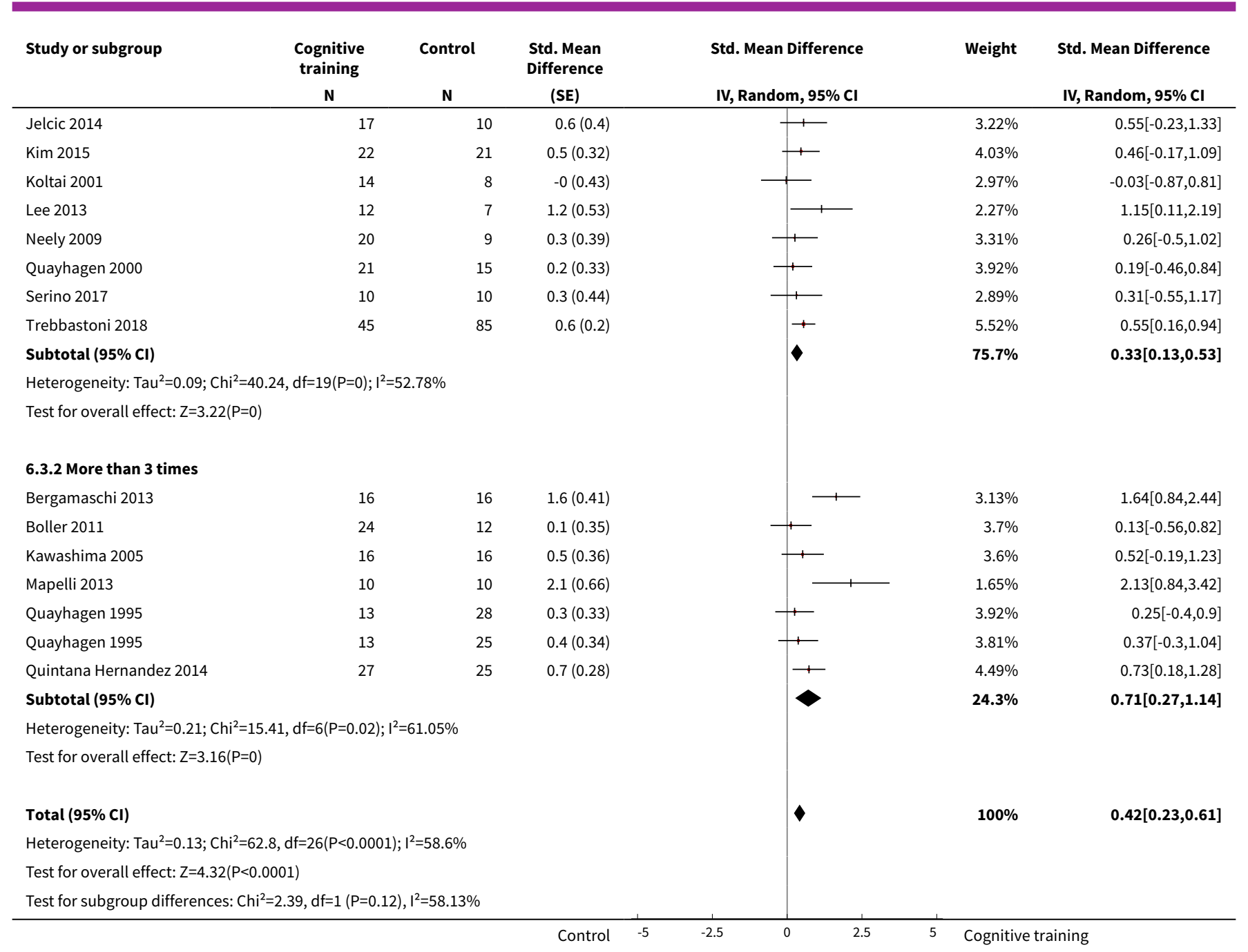

Analysis 6.4. Comparison 6 Cognitive training vs control immediately post intervention intervention dose, Outcome 4 Change in a global measure of cognition (composite)_zero correlation.

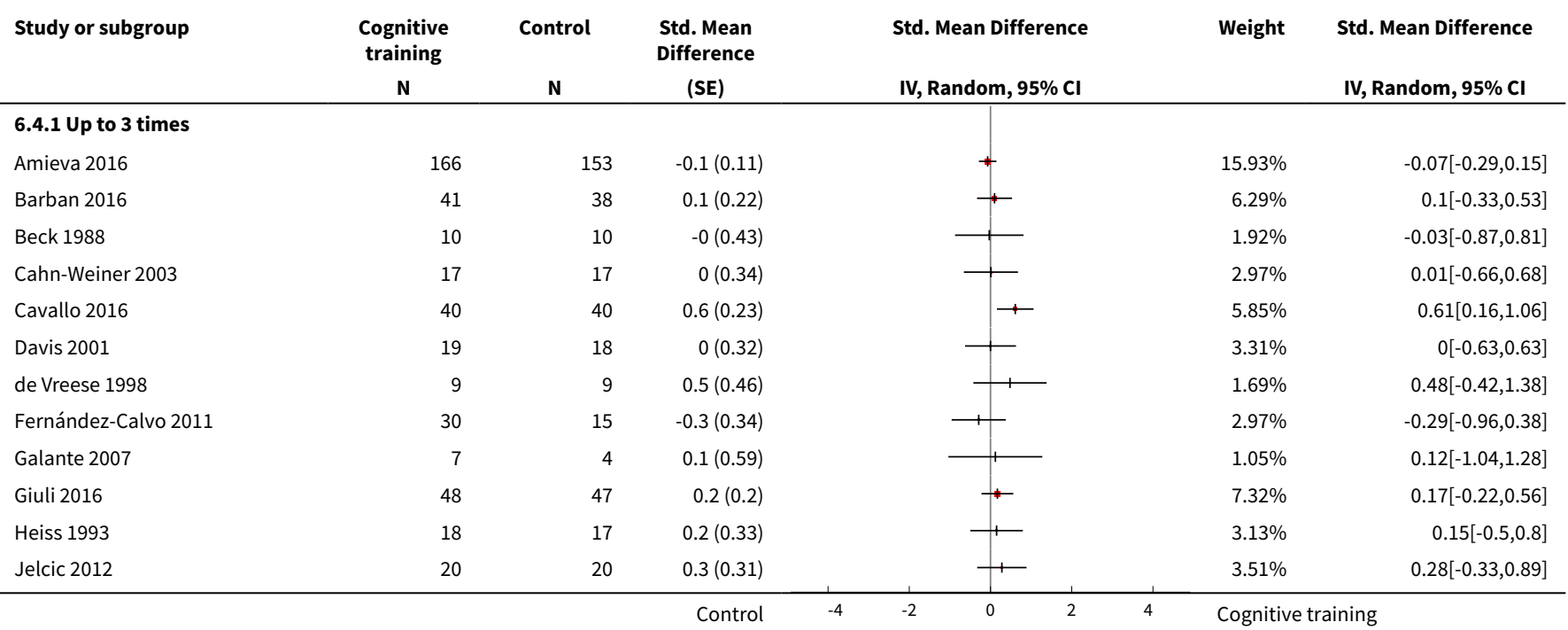




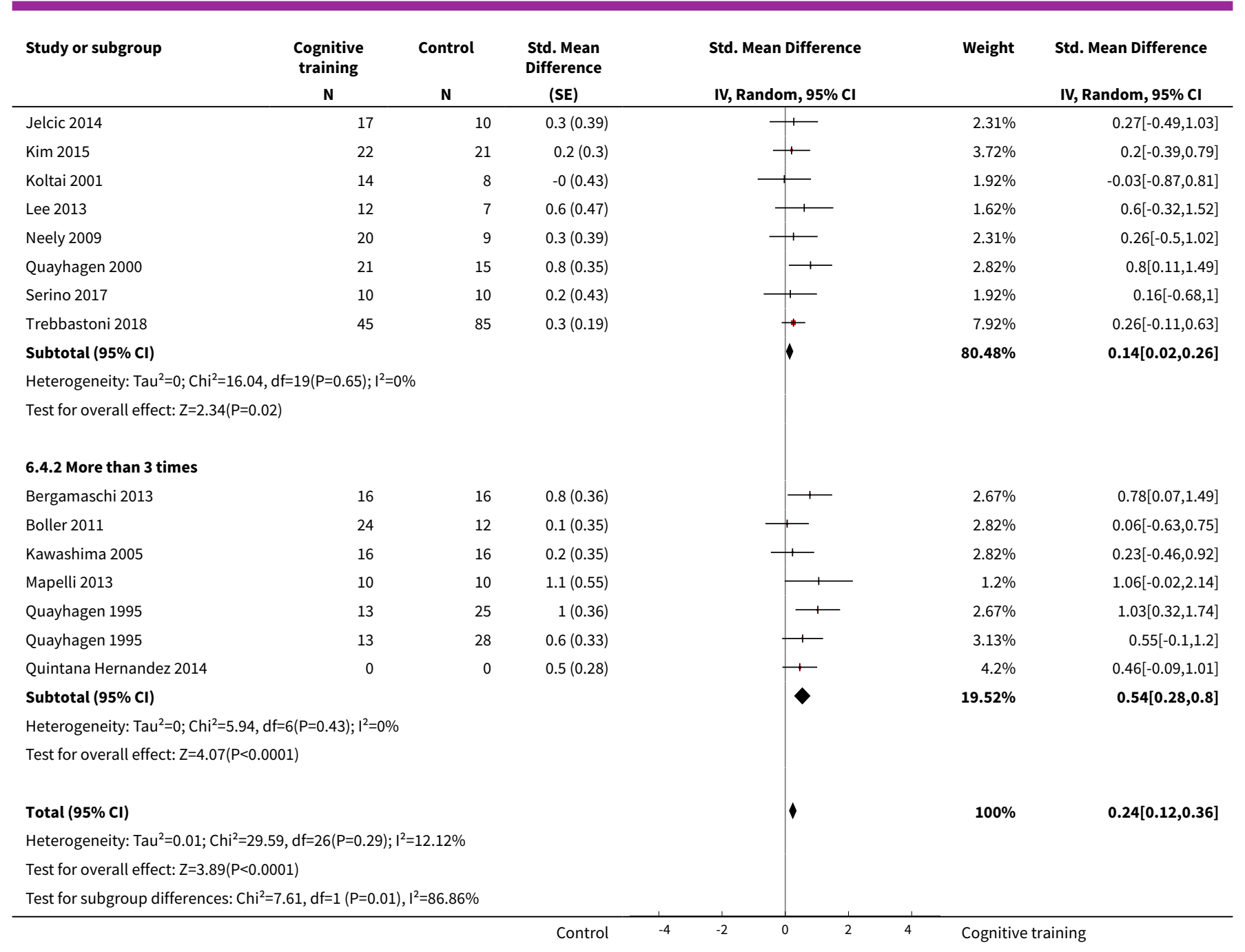

Analysis 6.5. Comparison 6 Cognitive training vs control immediately post intervention - intervention dose, Outcome 5 Change in immediate memory.

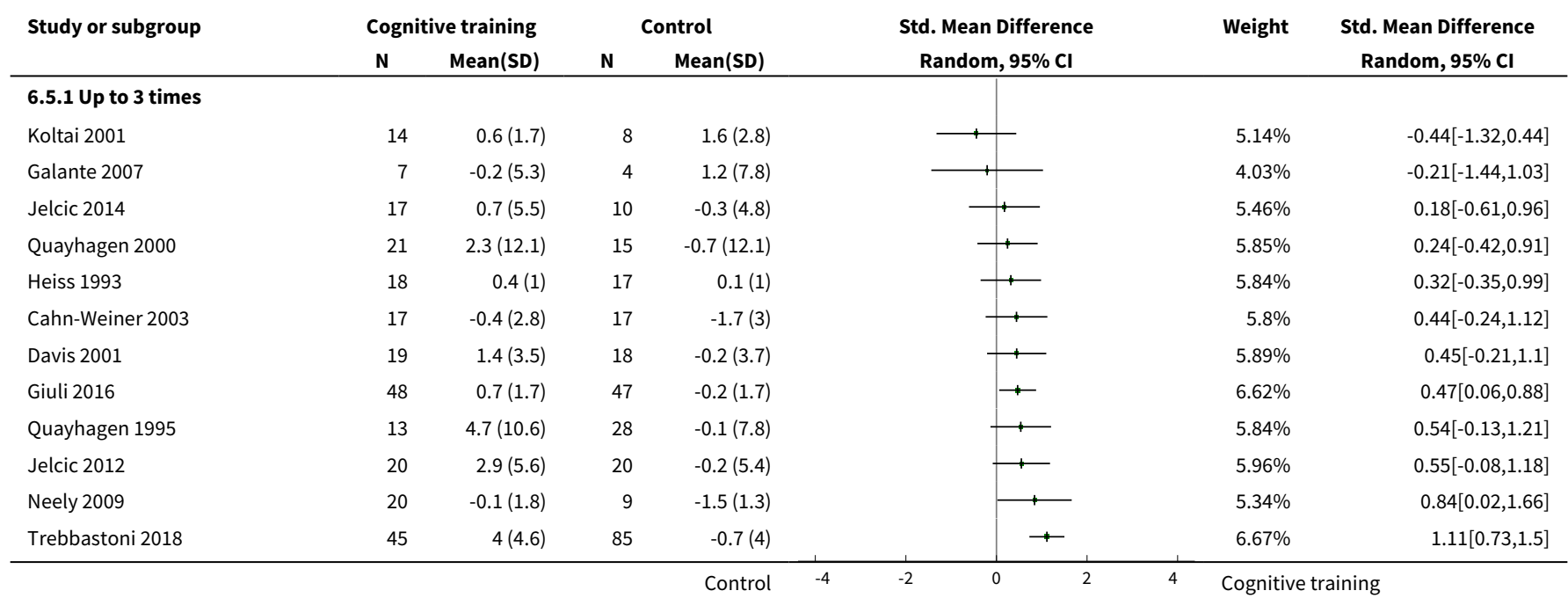




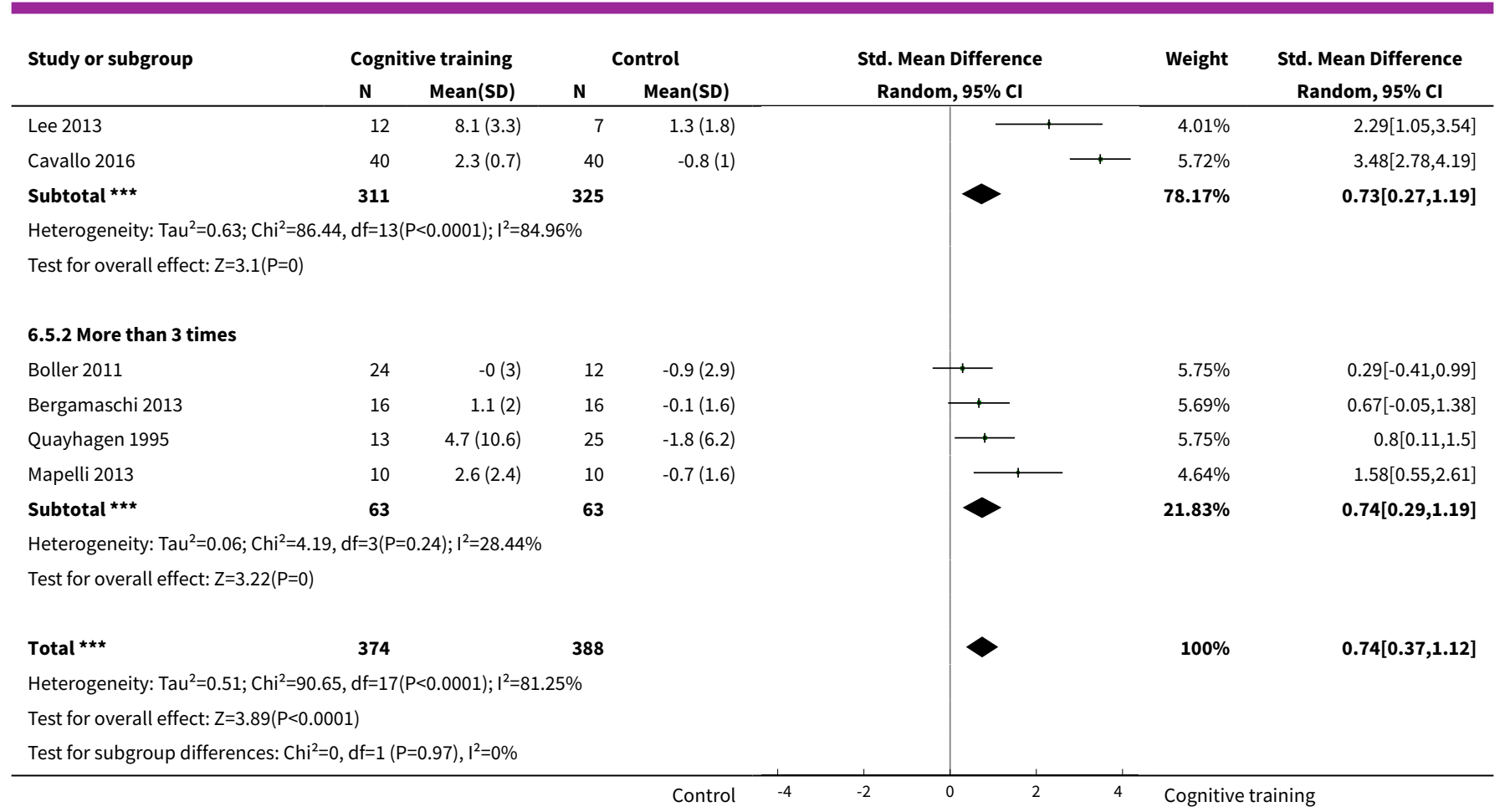

Analysis 6.6. Comparison 6 Cognitive training vs control immediately post intervention - intervention dose, Outcome 6 Change in delayed memory.

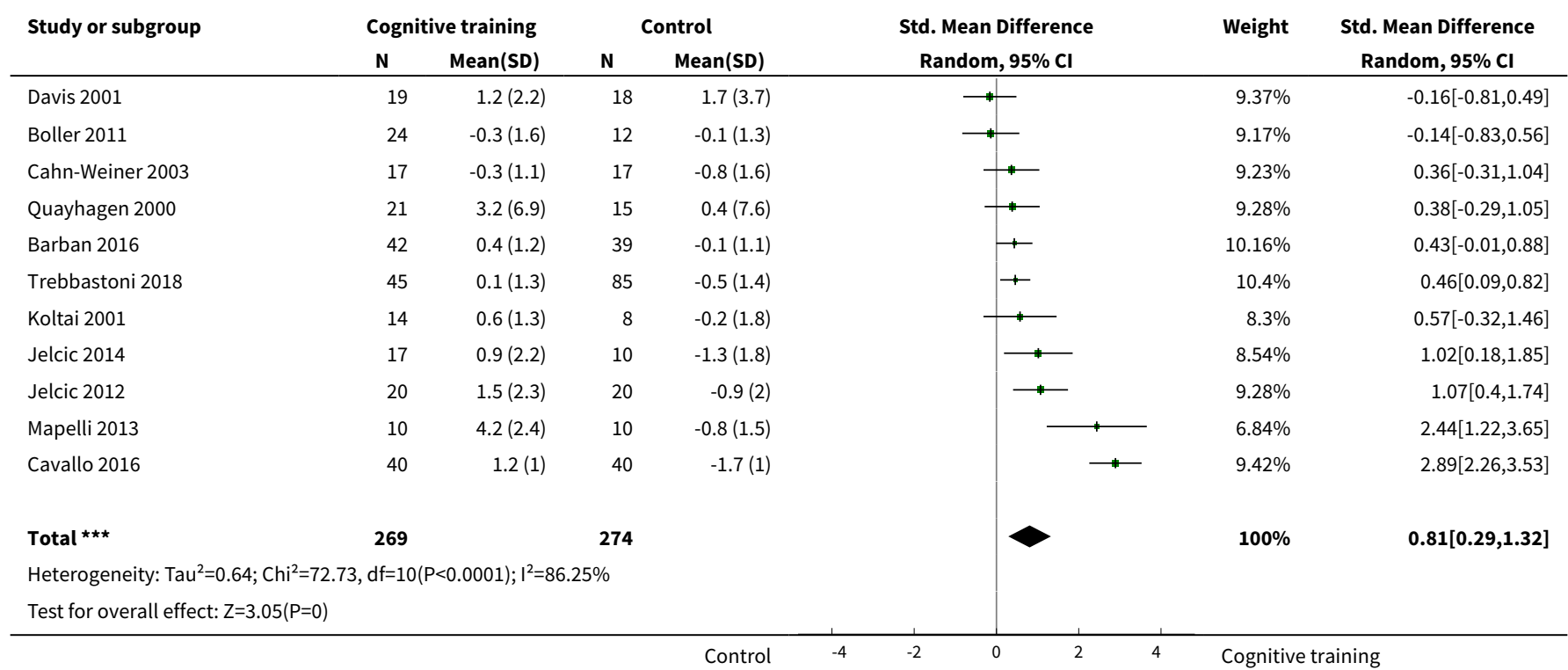


Analysis 6.7. Comparison 6 Cognitive training vs control immediately post intervention - intervention dose, Outcome 7 Change in attention and working memory.

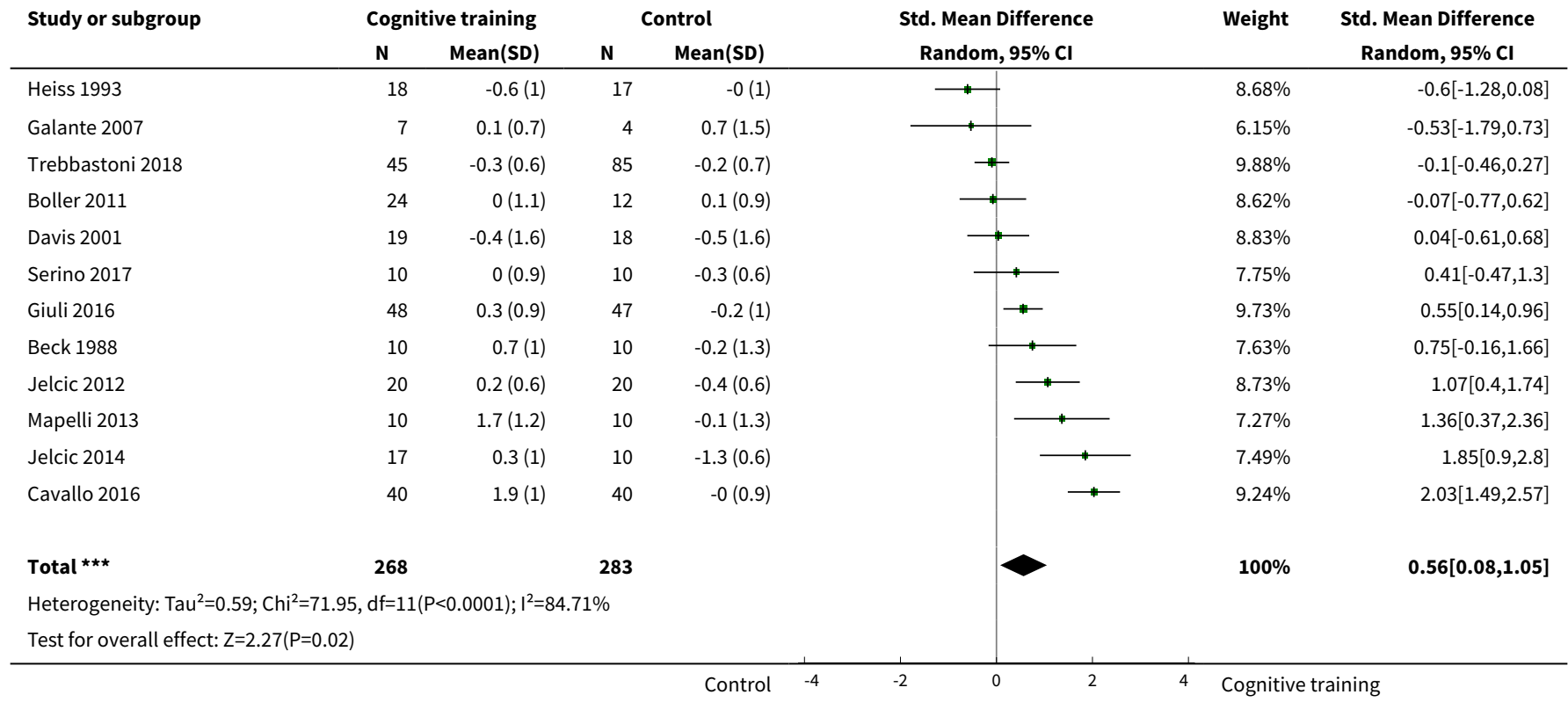

Analysis 6.8. Comparison 6 Cognitive training vs control immediately post intervention - intervention dose, Outcome 8 Change in language (naming).

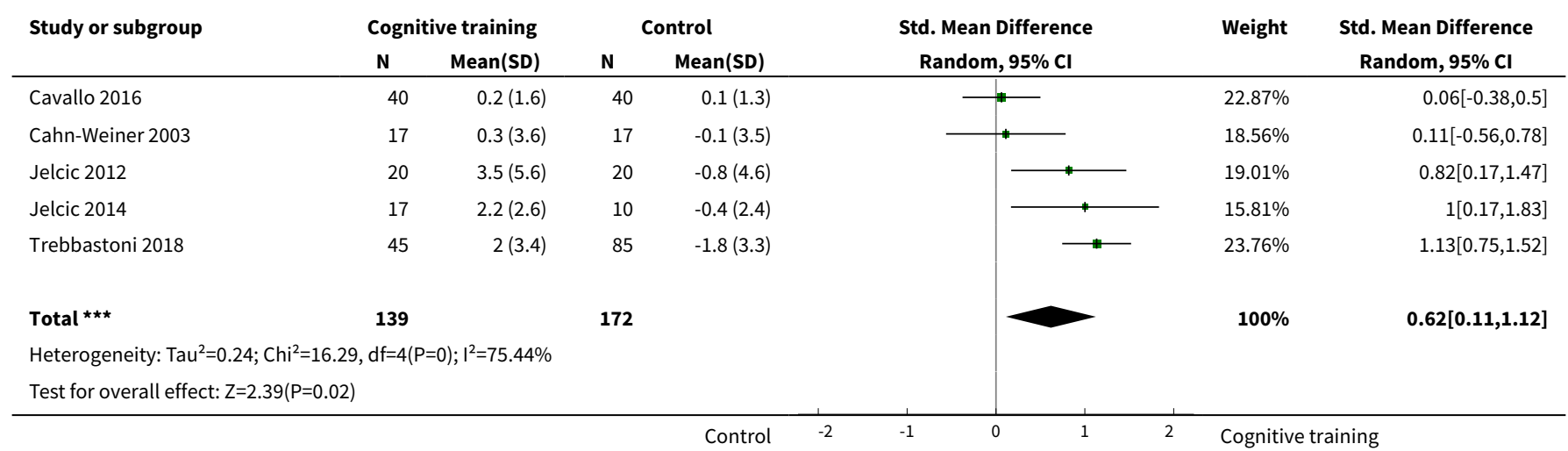

Analysis 6.9. Comparison 6 Cognitive training vs control immediately post intervention - intervention dose, Outcome 9 Change in verbal letter fluency.

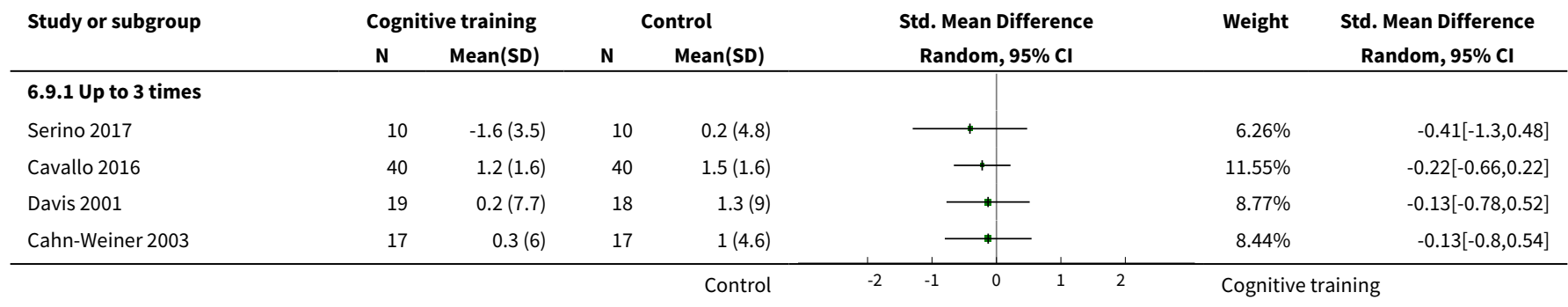




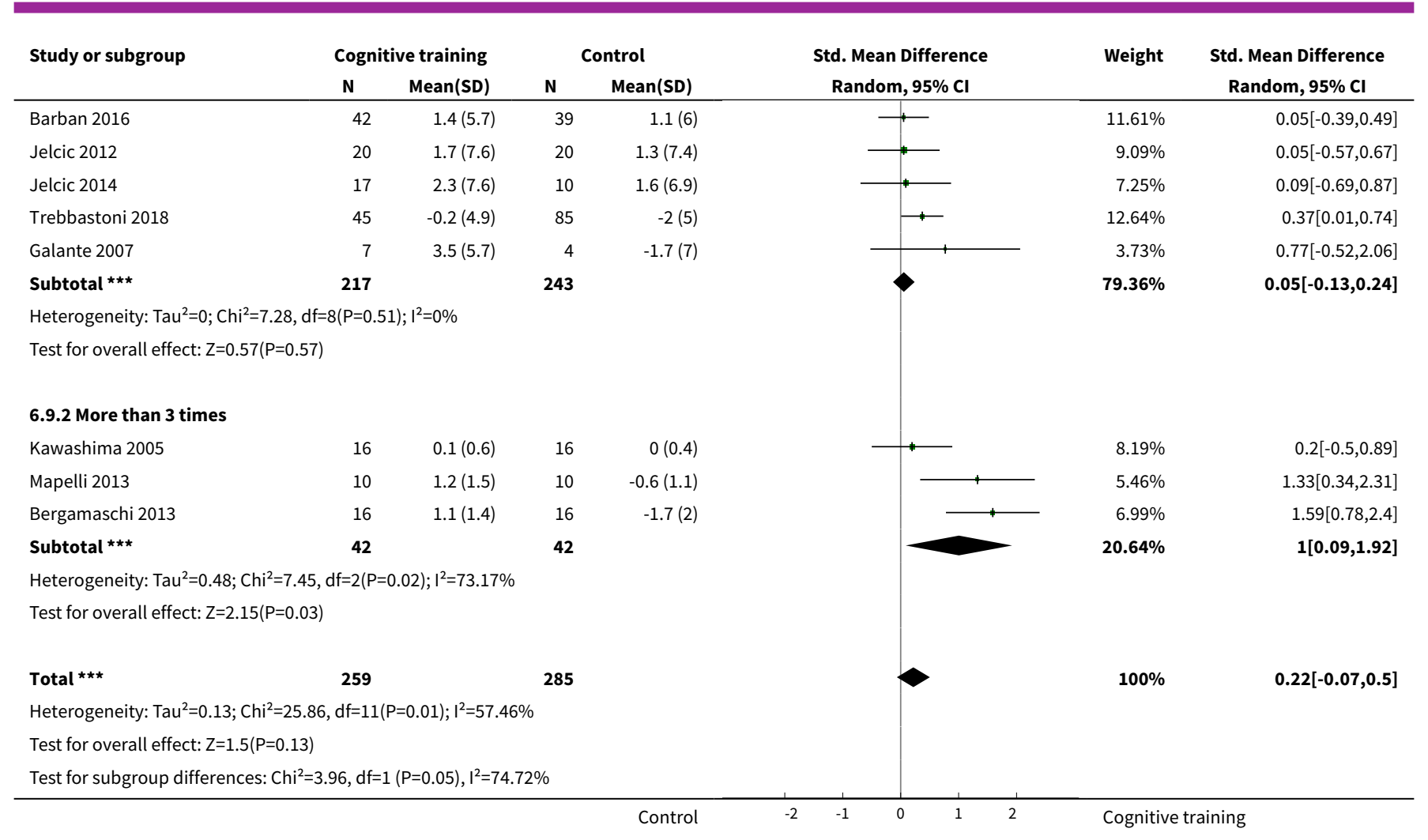

Analysis 6.10. Comparison 6 Cognitive training vs control immediately post intervention - intervention dose, Outcome 10 Change in speed of information processing.

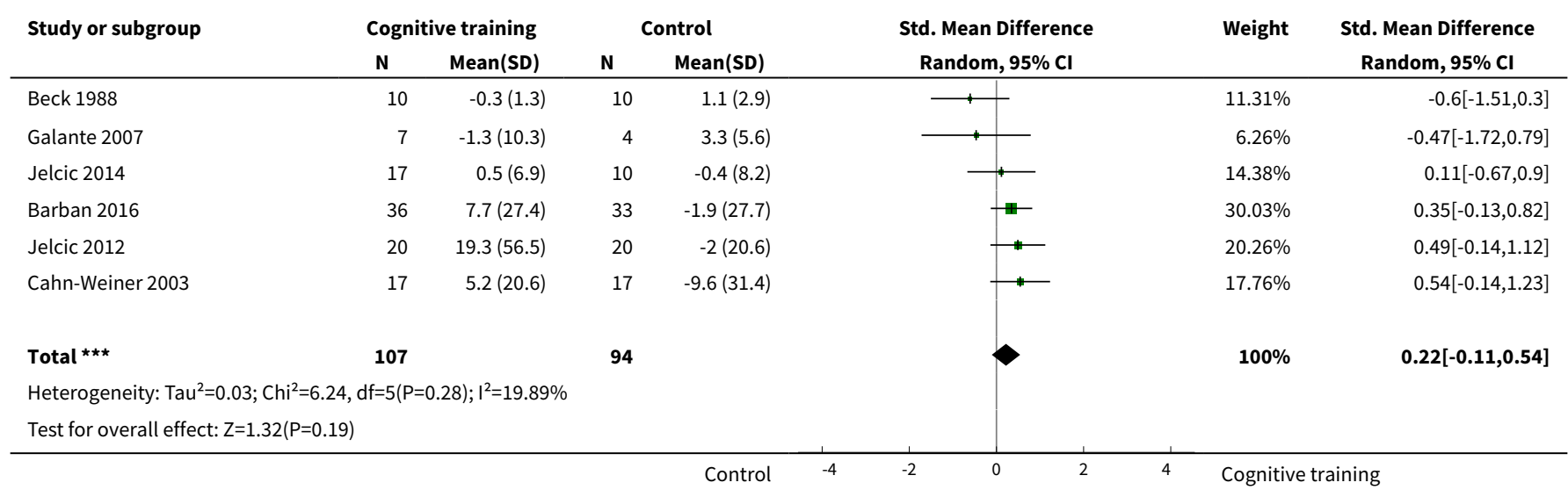

Analysis 6.11. Comparison 6 Cognitive training vs control immediately post intervention - intervention dose, Outcome 11 Change in executive function.

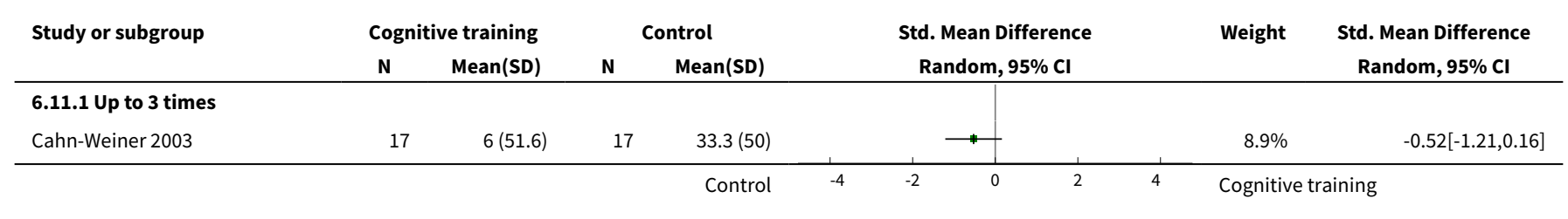




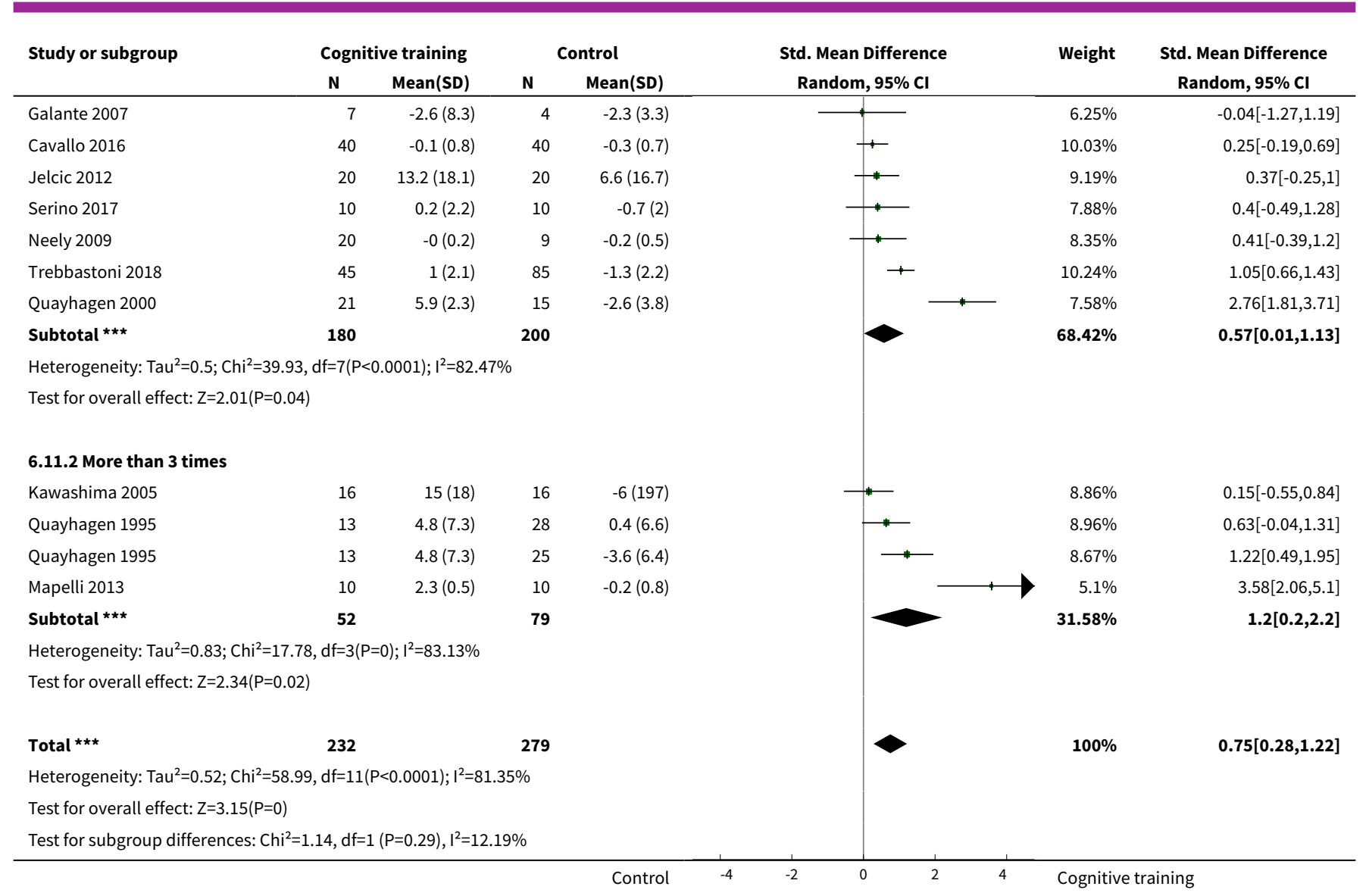

Analysis 6.12. Comparison 6 Cognitive training vs control immediately post intervention - intervention dose, Outcome 12 Change in verbal category fluency.

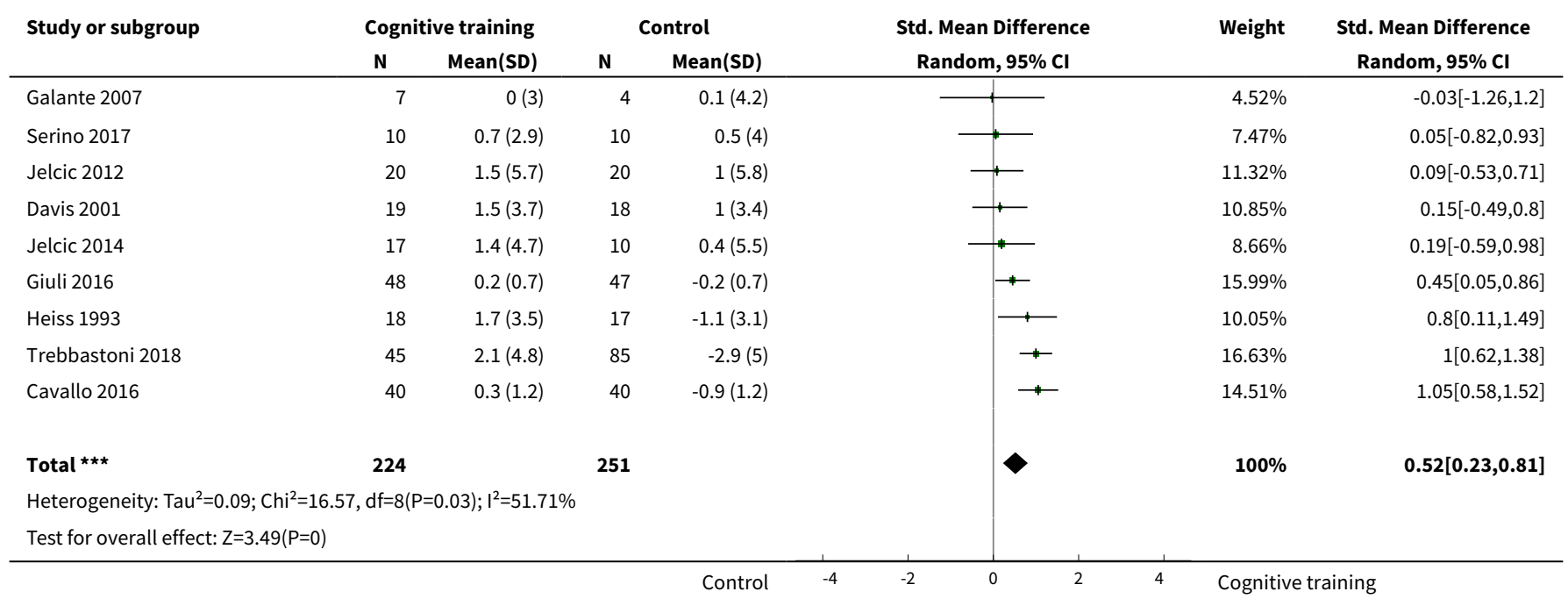


Analysis 6.13. Comparison 6 Cognitive training vs control immediately post intervention - intervention dose, Outcome 13 Change in meta cognition (self-reported).

\begin{tabular}{|c|c|c|c|c|c|c|c|}
\hline \multirow[t]{2}{*}{ Study or subgroup } & \multicolumn{2}{|c|}{ Cognitive training } & \multicolumn{2}{|c|}{ Control } & \multirow{2}{*}{$\begin{array}{c}\text { Std. Mean Difference } \\
\text { Random, } 95 \% \mathrm{Cl} \\
\end{array}$} & \multirow[t]{2}{*}{ Weight } & \multirow{2}{*}{$\begin{array}{c}\text { Std. Mean Difference } \\
\text { Random, } 95 \% \mathrm{Cl}\end{array}$} \\
\hline & $\mathbf{N}$ & Mean(SD) & $\mathbf{N}$ & $\operatorname{Mean}(S D)$ & & & \\
\hline Koltai 2001 & 14 & $-6.4(14.3)$ & 8 & $-1(14.1)$ & +1 & $51.94 \%$ & $-0.37[-1.24,0.51]$ \\
\hline Lee 2013 & 12 & $0.3(0.3)$ & 7 & $0.1(0.3)$ & + & $48.06 \%$ & $0.65[-0.31,1.61]$ \\
\hline Total $* \star \star$ & 26 & & 15 & & & $100 \%$ & $0.12[-0.87,1.12]$ \\
\hline \multicolumn{8}{|c|}{ Heterogeneity: $\operatorname{Tau}^{2}=0.3 ; \mathrm{Chi}^{2}=2.34, \mathrm{df}=1(\mathrm{P}=0.13) ; \mathrm{I}^{2}=57.33 \%$} \\
\hline
\end{tabular}

Analysis 6.14. Comparison 6 Cognitive training vs control immediately post intervention - intervention dose, Outcome 14 Change in meta cognition (informant-reported).

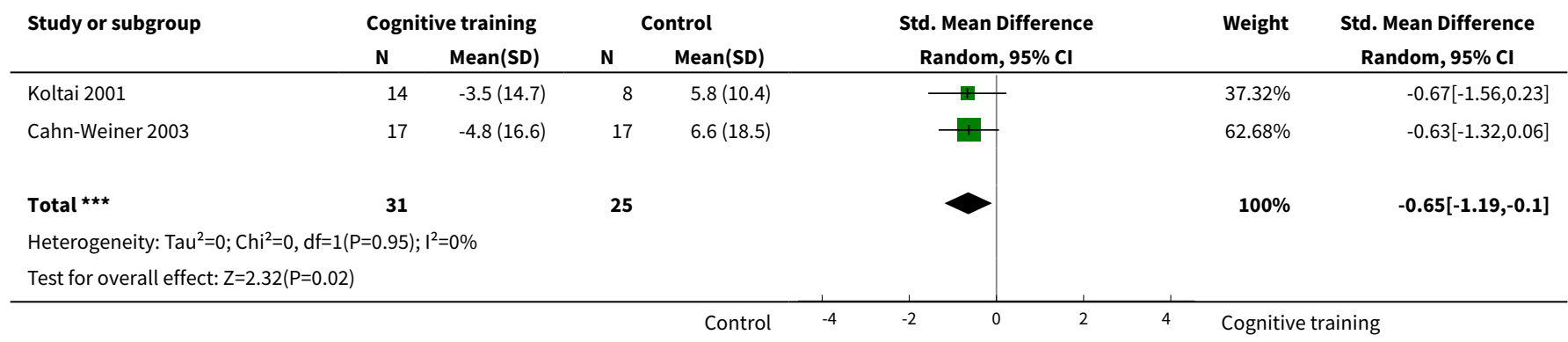

Analysis 6.15. Comparison 6 Cognitive training vs control immediately post intervention - intervention dose, Outcome 15 Change in participants' mood.

\begin{tabular}{|c|c|c|c|c|c|c|c|}
\hline \multirow[t]{2}{*}{ Study or subgroup } & \multicolumn{2}{|c|}{ Cognitive training } & \multicolumn{2}{|c|}{ Control } & \multirow{2}{*}{$\begin{array}{c}\text { Std. Mean Difference } \\
\text { Random, } 95 \% \mathrm{Cl}\end{array}$} & \multirow[t]{2}{*}{ Weight } & \multirow{2}{*}{$\begin{array}{c}\text { Std. Mean Difference } \\
\text { Random, } 95 \% \mathrm{Cl}\end{array}$} \\
\hline & $\mathbf{N}$ & Mean(SD) & $\mathbf{N}$ & Mean(SD) & & & \\
\hline Koltai 2001 & 14 & $-1.2(2.5)$ & 8 & $0.1(3)$ & $\longrightarrow$ & $12.23 \%$ & $-0.48[-1.36,0.4]$ \\
\hline Bergamaschi 2013 & 16 & $-1.1(3)$ & 16 & $0.4(5.1)$ & $\longrightarrow$ & $12.91 \%$ & $-0.35[-1.05,0.35]$ \\
\hline Amieva 2016 & 164 & $-12.1(12.7)$ & 152 & $-10.8(12.2)$ & + & $14.11 \%$ & $-0.1[-0.32,0.12]$ \\
\hline Davis 2001 & 19 & $0.3(1.5)$ & 17 & $0.2(4.4)$ & $\longrightarrow$ & $13.07 \%$ & $0.05[-0.61,0.7]$ \\
\hline Lee 2013 & 12 & $-0.2(2.2)$ & 7 & $-1.9(2.8)$ & - & $11.93 \%$ & $0.63[-0.33,1.59]$ \\
\hline Galante 2007 & 7 & $0.5(0.9)$ & 4 & $-0.3(0.8)$ & & $10.48 \%$ & $0.84[-0.47,2.14]$ \\
\hline Giuli 2016 & 48 & $-0.2(4.4)$ & 47 & $-7.3(3.8)$ & $\rightarrow$ & $13.6 \%$ & $1.73[1.25,2.2]$ \\
\hline Fernández-Calvo 2011 & 30 & $3.2(2)$ & 15 & $-4.3(1.9)$ & & $11.67 \%$ & $3.75[2.73,4.78]$ \\
\hline 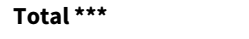 & 310 & & 266 & & & $100 \%$ & $0.72[-0.1,1.54]$ \\
\hline \multicolumn{8}{|c|}{ Heterogeneity: $\operatorname{Tau}^{2}=1.23 ; \mathrm{Chi}^{2}=99.54, \mathrm{df}=7(\mathrm{P}<0.0001) ; \mathrm{I}^{2}=92.97 \%$} \\
\hline \multicolumn{8}{|c|}{ Test for overall effect: $Z=1.73(P=0.08)$} \\
\hline
\end{tabular}


Analysis 6.16. Comparison 6 Cognitive training vs control immediately post intervention - intervention dose, Outcome 16 Change in capacity for activities of daily living.

\begin{tabular}{|c|c|c|c|c|c|c|c|}
\hline \multirow{2}{*}{ Study or subgroup } & \multicolumn{2}{|c|}{ Cognitive training } & \multicolumn{2}{|c|}{ Control } & \multirow{2}{*}{$\begin{array}{c}\text { Std. Mean Difference } \\
\text { Random, } 95 \% \mathrm{Cl}\end{array}$} & \multirow[t]{2}{*}{ Weight } & \multirow{2}{*}{$\begin{array}{c}\text { Std. Mean Difference } \\
\text { Random, } 95 \% \mathrm{Cl}\end{array}$} \\
\hline & $\mathbf{N}$ & Mean(SD) & $\mathbf{N}$ & Mean(SD) & & & \\
\hline Galante 2007 & 7 & $-0.6(1.4)$ & 4 & $0.5(0.5)$ & \begin{tabular}{l|l}
1 &
\end{tabular} & $2.63 \%$ & $-0.88[-2.19,0.43]$ \\
\hline de Vreese 1998 & 9 & $-1.3(4.4)$ & 9 & $1(4)$ & \begin{tabular}{l|l}
1 \\
1
\end{tabular} & $4.7 \%$ & $-0.53[-1.48,0.41]$ \\
\hline Amieva 2016 & 167 & $-1.8(8.5)$ & 153 & $-1.2(8.3)$ & \# & $23.78 \%$ & $-0.06[-0.28,0.16]$ \\
\hline Cahn-Weiner 2003 & 17 & $-0.2(3.2)$ & 17 & $0(3.1)$ & $\longrightarrow$ & $8.09 \%$ & $-0.06[-0.74,0.61]$ \\
\hline Kim 2015 & 21 & $0(0.9)$ & 21 & $0(1.1)$ & + & $9.41 \%$ & $0[-0.6,0.6]$ \\
\hline Barban 2016 & 38 & $-0.1(1)$ & 38 & $-0.3(1.1)$ & 4 & $13.63 \%$ & $0.19[-0.26,0.64]$ \\
\hline Giuli 2016 & 48 & $0.3(1.2)$ & 47 & $-0.2(1.3)$ & + & $15.22 \%$ & $0.4[-0,0.81]$ \\
\hline Bergamaschi 2013 & 16 & $-0.4(1.5)$ & 16 & $-1.3(1.5)$ & 廿 & $7.44 \%$ & $0.61[-0.1,1.32]$ \\
\hline Lee 2013 & 12 & $2.2(3.3)$ & 7 & $-2.1(4.5)$ & 1 & $4.18 \%$ & $1.1[0.09,2.11]$ \\
\hline Total $\star \star \star$ & 355 & & 350 & & $\gamma$ & $100 \%$ & $0.12[-0.1,0.34]$ \\
\hline Test for overall effec & & & & & & & \\
\hline
\end{tabular}

Analysis 6.17. Comparison 6 Cognitive training vs control immediately post intervention - intervention dose, Outcome 17 Change in disease progression.

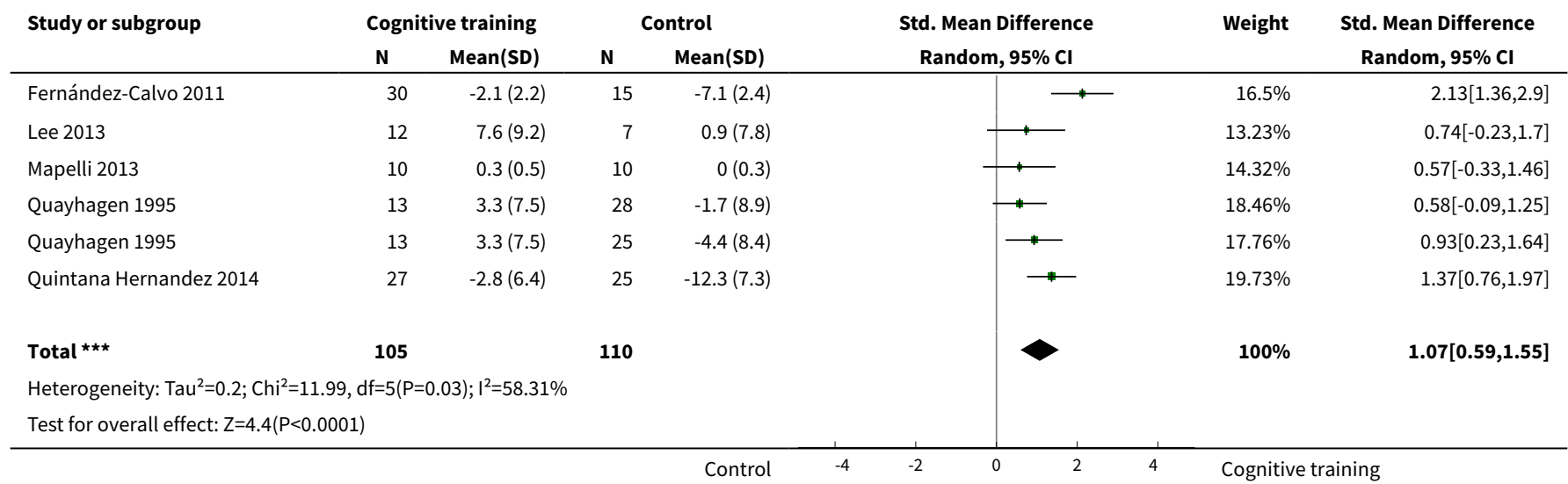

Analysis 6.18. Comparison 6 Cognitive training vs control immediately post intervention - intervention dose, Outcome 18 Change in behavioural and psychological symptoms of dementia (BPSD).

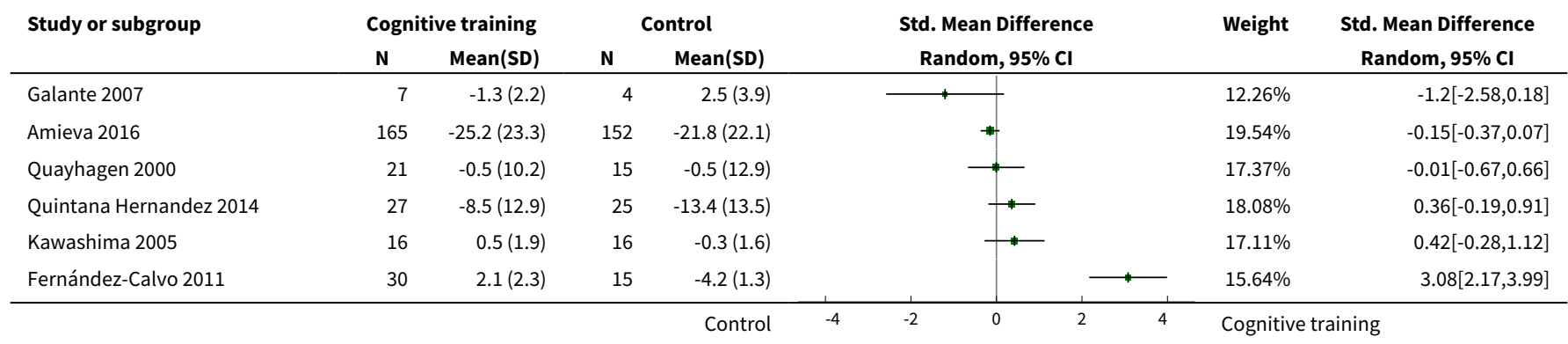




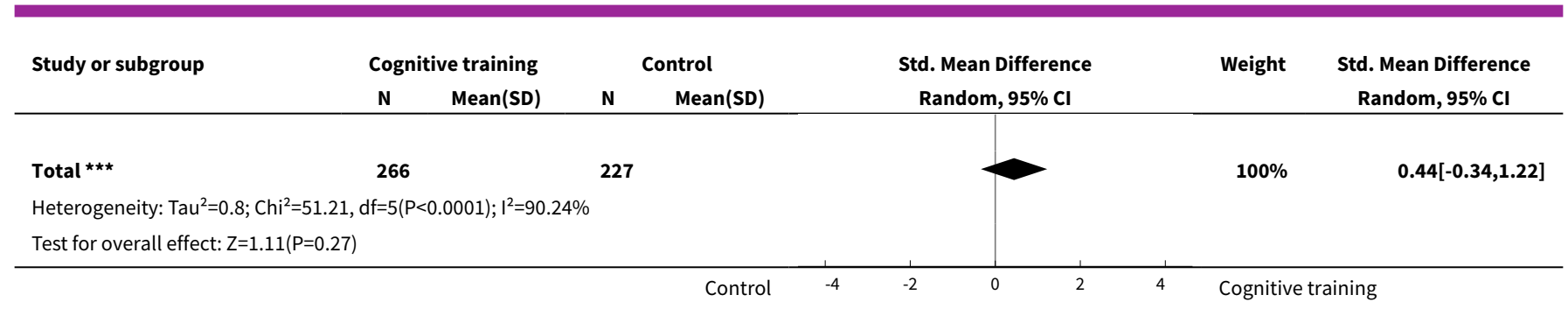

Analysis 6.19. Comparison 6 Cognitive training vs control immediately post intervention - intervention dose, Outcome 19 Change in attention and working memory.

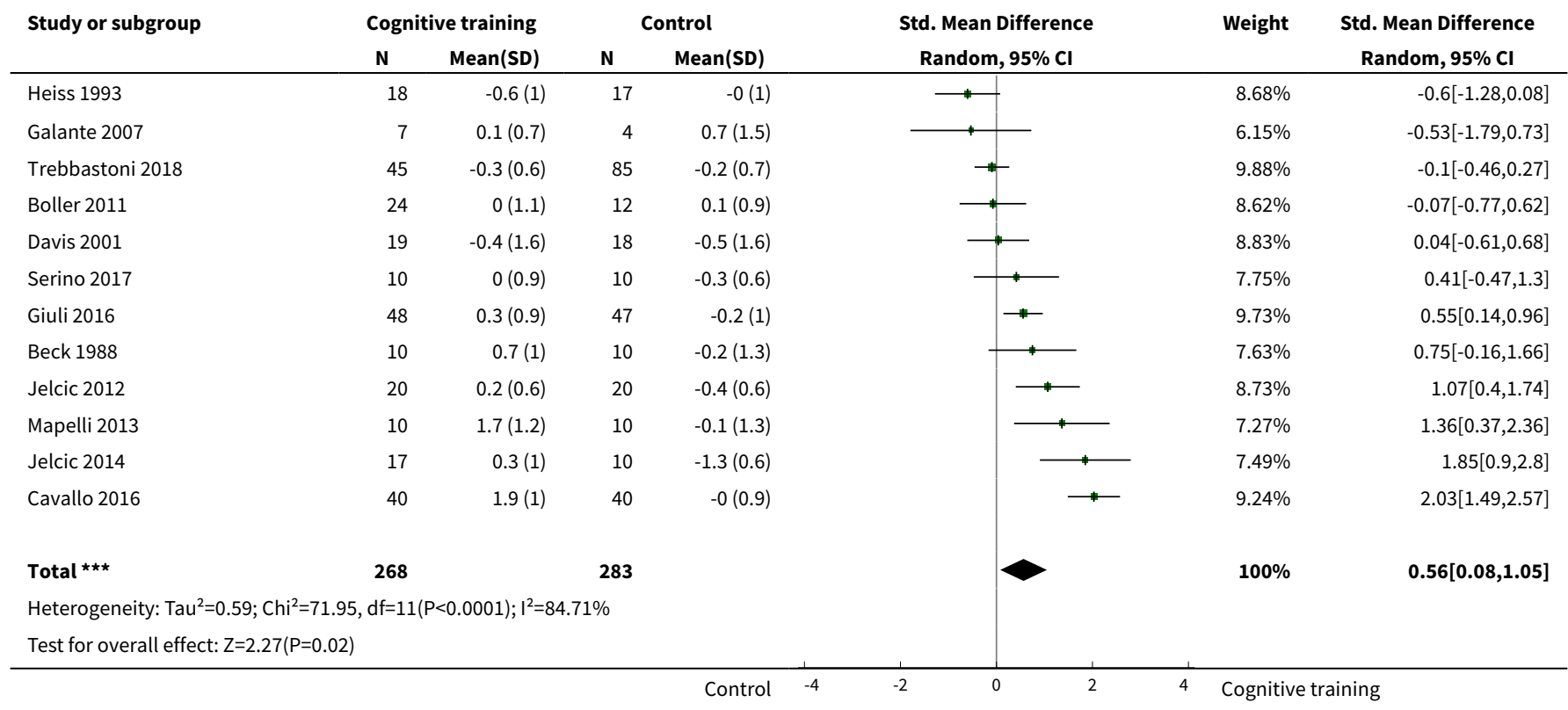

Analysis 6.20. Comparison 6 Cognitive training vs control immediately post intervention - intervention dose, Outcome 20 Change in burden of care (CAREGIVER).

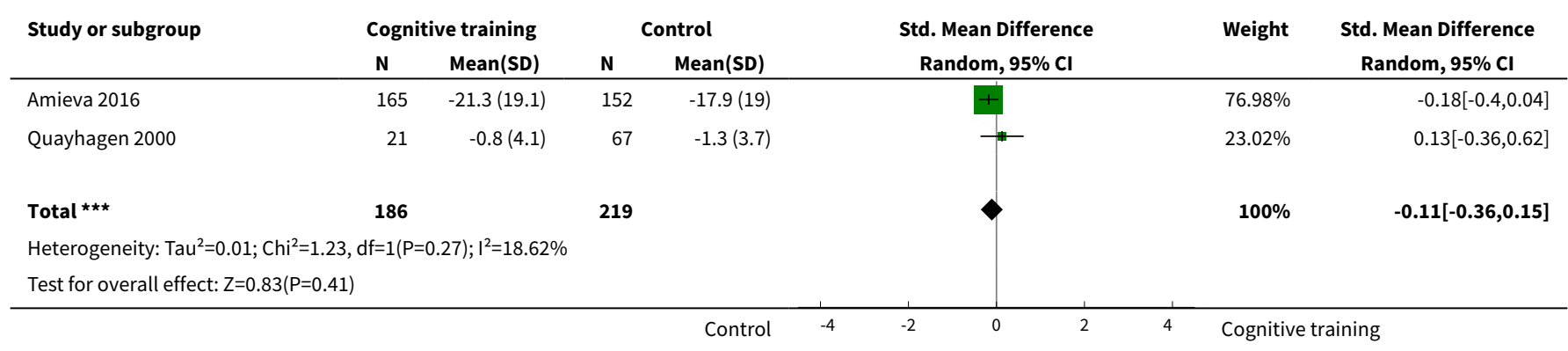


Analysis 6.21. Comparison 6 Cognitive training vs control immediately post intervention - intervention dose, Outcome 21 Change in quality of life (CAREGIVER).

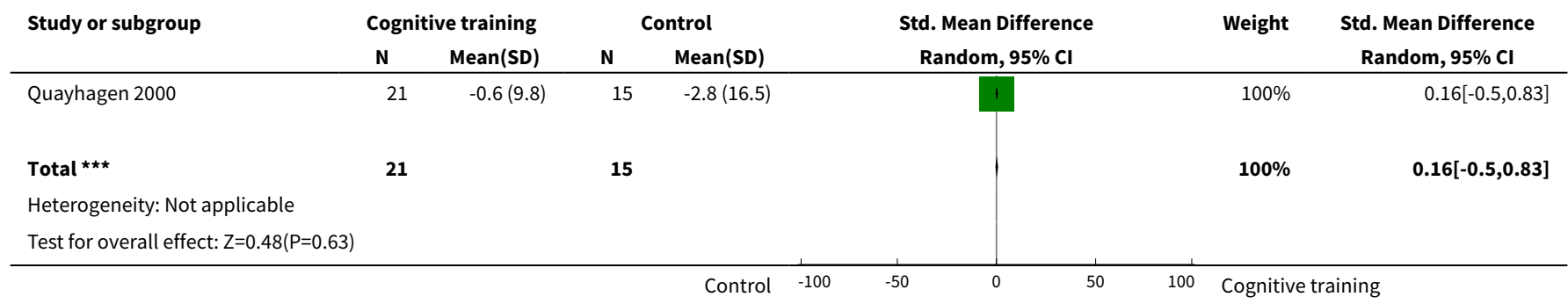

Analysis 6.22. Comparison 6 Cognitive training vs control immediately post intervention - intervention dose, Outcome 22 Change in mood and well-being (CAREGIVER).

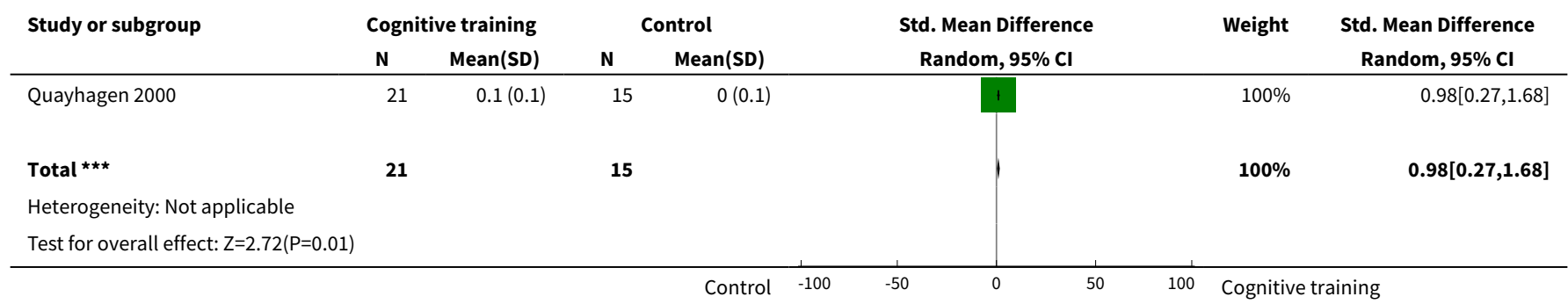

Analysis 6.23. Comparison 6 Cognitive training vs control immediately post intervention - intervention dose, Outcome 23 Participant burden (retention rates).

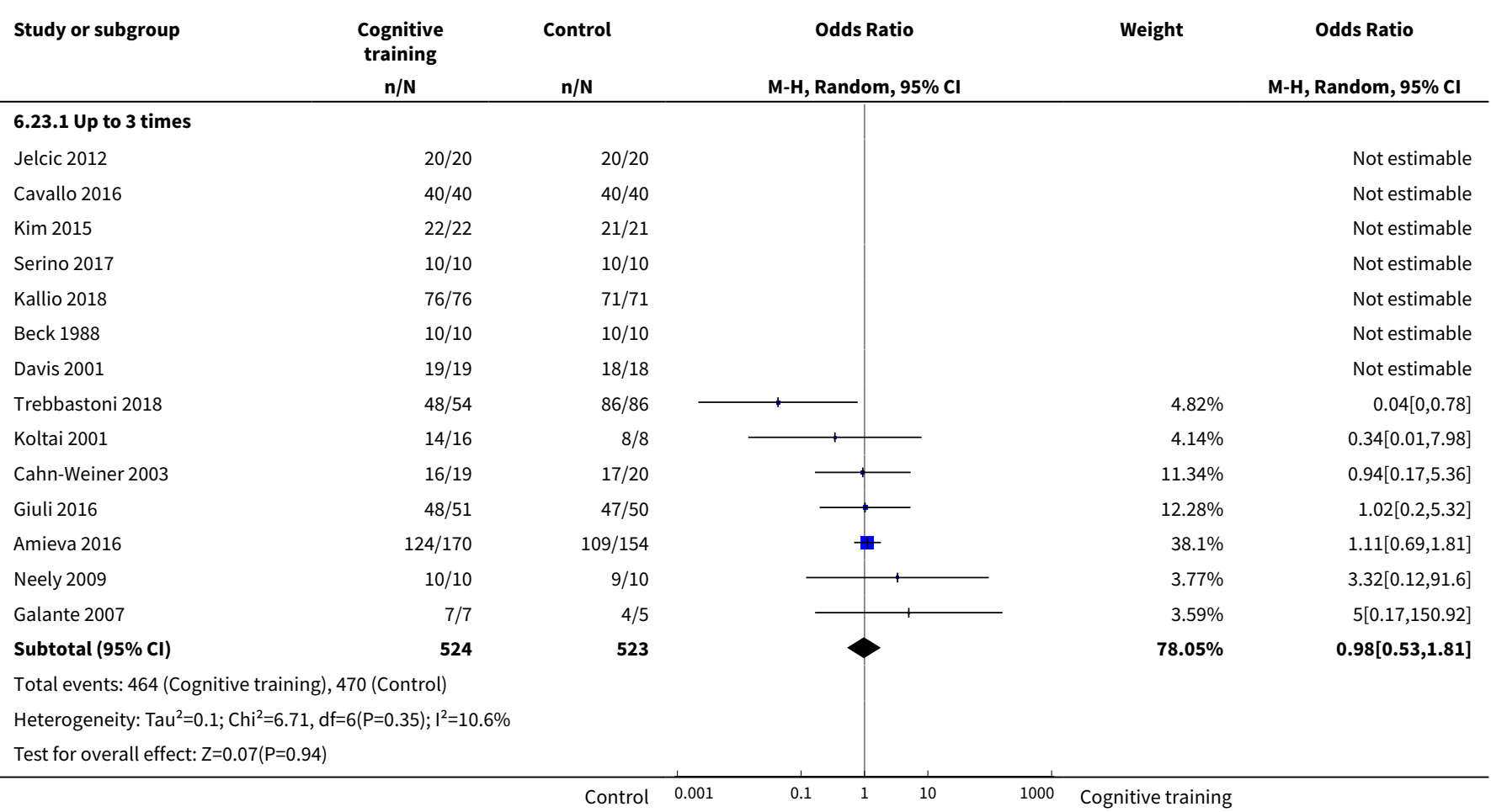




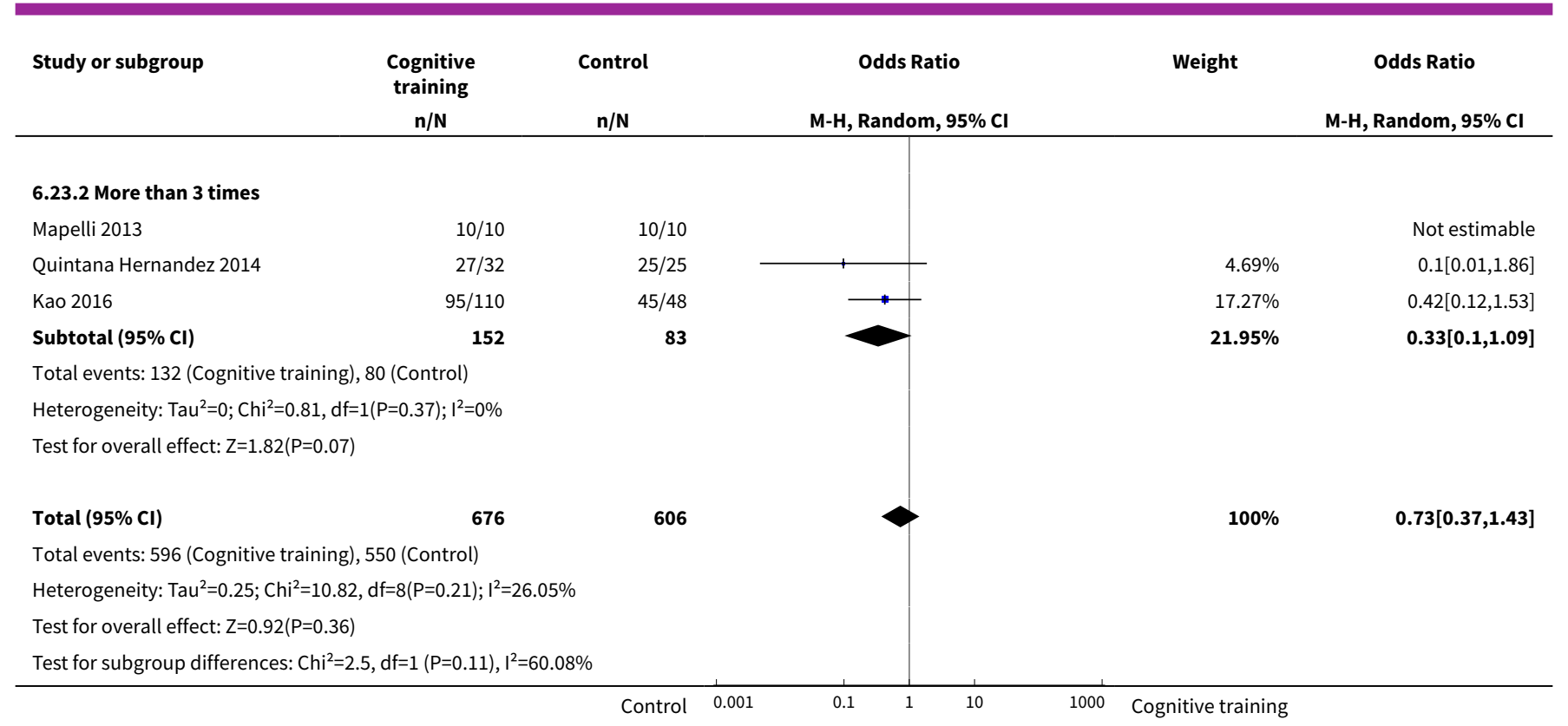

Analysis 6.24. Comparison 6 Cognitive training vs control immediately post intervention - intervention dose, Outcome 24 Change in general health and quality of life.

\begin{tabular}{|c|c|c|c|c|c|c|c|}
\hline \multirow{3}{*}{$\begin{array}{l}\text { Study or subgroup } \\
\text { Davis } 2001\end{array}$} & \multicolumn{2}{|c|}{ Cognitive training } & \multicolumn{2}{|c|}{ Control } & \multirow{2}{*}{$\begin{array}{c}\text { Std. Mean Difference } \\
\text { Random, } 95 \% \mathrm{Cl}\end{array}$} & \multirow[t]{2}{*}{ Weight } & \multirow{2}{*}{$\begin{array}{c}\text { Std. Mean Difference } \\
\text { Random, } 95 \% \mathrm{Cl}\end{array}$} \\
\hline & $\mathbf{N}$ & $\operatorname{Mean}(\mathrm{SD})$ & $\mathbf{N}$ & Mean(SD) & & & \\
\hline & 19 & $-24.8(37.3)$ & 18 & $4.8(40.9)$ & ఋ & $14 \%$ & $-0.74[-1.41,-0.07]$ \\
\hline Quayhagen 2000 & 21 & $-0.4(2.8)$ & 67 & $0.1(2.6)$ & $\longrightarrow$ & $18.83 \%$ & $-0.19[-0.68,0.3]$ \\
\hline Amieva 2016 & 164 & $-6.3(6.1)$ & 151 & $-5.6(6.4)$ & $\rightarrow$ & $27.77 \%$ & $-0.11[-0.33,0.11]$ \\
\hline Kallio 2018 & 76 & $-0(0.1)$ & 71 & $-0(0.1)$ & & $24.41 \%$ & $0[-0.32,0.32]$ \\
\hline Kim 2015 & 22 & $3.4(3.3)$ & 21 & $0(4.4)$ & & $14.99 \%$ & $0.87[0.24,1.49]$ \\
\hline 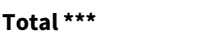 & 302 & & 328 & & & $100 \%$ & $-0.04[-0.38,0.29]$ \\
\hline \multicolumn{8}{|c|}{ Heterogeneity: $\mathrm{Tau}^{2}=0.09 ; \mathrm{Chi}^{2}=12.93, \mathrm{df}=4(\mathrm{P}=0.01) ; \mathrm{I}^{2}=69.08 \%$} \\
\hline \multicolumn{8}{|c|}{ Test for overall effect: $Z=0.24(P=0.81)$} \\
\hline
\end{tabular}

\section{Comparison 7. Cognitive training vs control immediately post intervention - intervention duration}

\begin{tabular}{lllll}
\hline Outcome or subgroup title & $\begin{array}{l}\text { No. of } \\
\text { studies }\end{array}$ & $\begin{array}{l}\text { No. of } \\
\text { partici- } \\
\text { pants }\end{array}$ & Statistical method & Effect size \\
\hline 1 Change in a global measure of cognition & 20 & 1288 & $\begin{array}{l}\text { Std. Mean Difference (IV, Random, } \\
95 \% \text { Cl) }\end{array}$ & $0.65[0.26,1.05]$ \\
\hline 1.1 Up to 3 months & 14 & 682 & $\begin{array}{l}\text { Std. Mean Difference (IV, Random, } \\
95 \% \text { Cl) }\end{array}$ & $0.49[0.09,0.89]$ \\
\hline 1.2 More than 3 months & 6 & 606 & $\begin{array}{l}\text { Std. Mean Difference (IV, Random, } \\
95 \% \text { Cl) }\end{array}$ & $1.03[0.04,2.02]$ \\
\hline
\end{tabular}




\begin{tabular}{|c|c|c|c|c|}
\hline Outcome or subgroup title & $\begin{array}{l}\text { No. of } \\
\text { studies }\end{array}$ & $\begin{array}{l}\text { No. of } \\
\text { partici- } \\
\text { pants }\end{array}$ & Statistical method & Effect size \\
\hline $\begin{array}{l}2 \text { Change in a global measure of cogni- } \\
\text { tion_zero correlation }\end{array}$ & 20 & 1287 & $\begin{array}{l}\text { Std. Mean Difference (IV, Random, } \\
95 \% \mathrm{CI})\end{array}$ & $0.27[0.04,0.50]$ \\
\hline 2.1 Up to 3 months & 14 & 682 & $\begin{array}{l}\text { Std. Mean Difference (IV, Random, } \\
95 \% \mathrm{CI})\end{array}$ & $0.15[-0.08,0.38]$ \\
\hline 2.2 More than 3 months & 6 & 605 & $\begin{array}{l}\text { Std. Mean Difference (IV, Random, } \\
95 \% \mathrm{CI})\end{array}$ & $0.51[-0.04,1.05]$ \\
\hline $\begin{array}{l}3 \text { Change in a global measure of cognition } \\
\text { (composite) }\end{array}$ & 26 & 1389 & $\begin{array}{l}\text { Std. Mean Difference (Random, } \\
95 \% \mathrm{Cl} \text { ) }\end{array}$ & $0.42[0.23,0.61]$ \\
\hline 3.1 Up to 3 months & 20 & 790 & $\begin{array}{l}\text { Std. Mean Difference (Random, } \\
95 \% \mathrm{Cl} \text { ) }\end{array}$ & $0.38[0.18,0.58]$ \\
\hline 3.2 More than 3 months & 6 & 599 & $\begin{array}{l}\text { Std. Mean Difference (Random, } \\
95 \% \mathrm{Cl} \text { ) }\end{array}$ & $0.54[0.07,1.01]$ \\
\hline $\begin{array}{l}4 \text { Change in a global measure of cognition } \\
\text { (composite)_zero correlation }\end{array}$ & 26 & 1390 & $\begin{array}{l}\text { Std. Mean Difference (Random, } \\
95 \% \mathrm{Cl} \text { ) }\end{array}$ & $0.24[0.12,0.36]$ \\
\hline 4.1 Up to 3 months & 20 & 790 & $\begin{array}{l}\text { Std. Mean Difference (Random, } \\
95 \% \mathrm{Cl} \text { ) }\end{array}$ & $0.28[0.14,0.42]$ \\
\hline 4.2 More than 3 months & 6 & 600 & $\begin{array}{l}\text { Std. Mean Difference (Random, } \\
95 \% \mathrm{Cl} \text { ) }\end{array}$ & $0.21[-0.03,0.46]$ \\
\hline 5 Change in immediate memory & 17 & 762 & $\begin{array}{l}\text { Std. Mean Difference (IV, Random, } \\
95 \% \mathrm{Cl})\end{array}$ & $0.74[0.37,1.12]$ \\
\hline 5.1 Up to 3 months & 14 & 565 & $\begin{array}{l}\text { Std. Mean Difference (IV, Random, } \\
95 \% \mathrm{Cl})\end{array}$ & $0.75[0.29,1.21]$ \\
\hline 5.2 More than 3 months & 3 & 197 & $\begin{array}{l}\text { Std. Mean Difference (IV, Random, } \\
95 \% \mathrm{Cl})\end{array}$ & $0.76[0.26,1.26]$ \\
\hline 6 Change in delayed memory & 11 & 543 & $\begin{array}{l}\text { Std. Mean Difference (IV, Random, } \\
95 \% \mathrm{Cl})\end{array}$ & $0.81[0.29,1.32]$ \\
\hline 7 Change in attention and working memory & 12 & 551 & $\begin{array}{l}\text { Std. Mean Difference (IV, Random, } \\
95 \% \mathrm{Cl})\end{array}$ & $0.56[0.08,1.05]$ \\
\hline 8 Change in language (naming) & 5 & 311 & $\begin{array}{l}\text { Std. Mean Difference (IV, Random, } \\
95 \% \mathrm{CI})\end{array}$ & $0.62[0.11,1.12]$ \\
\hline 9 Change in verbal letter fluency & 12 & 544 & $\begin{array}{l}\text { Std. Mean Difference (IV, Random, } \\
95 \% \mathrm{CI})\end{array}$ & $0.22[-0.07,0.50]$ \\
\hline 9.1 Up to 3 months & 9 & 350 & $\begin{array}{l}\text { Std. Mean Difference (IV, Random, } \\
95 \% \mathrm{CI})\end{array}$ & $0.03[-0.23,0.28]$ \\
\hline 9.2 More than 3 months & 3 & 194 & $\begin{array}{l}\text { Std. Mean Difference (IV, Random, } \\
95 \% \mathrm{CI} \text { ) }\end{array}$ & $0.66[-0.05,1.38]$ \\
\hline
\end{tabular}




\begin{tabular}{|c|c|c|c|c|}
\hline Outcome or subgroup title & $\begin{array}{l}\text { No. of } \\
\text { studies }\end{array}$ & $\begin{array}{l}\text { No. of } \\
\text { partici- } \\
\text { pants }\end{array}$ & Statistical method & Effect size \\
\hline $\begin{array}{l}10 \text { Change in speed of information process- } \\
\text { ing }\end{array}$ & 6 & 201 & $\begin{array}{l}\text { Std. Mean Difference (IV, Random, } \\
95 \% \mathrm{CI})\end{array}$ & $0.22[-0.11,0.54]$ \\
\hline 11 Change in executive function & 11 & 511 & $\begin{array}{l}\text { Std. Mean Difference (IV, Random, } \\
95 \% \mathrm{CI})\end{array}$ & $0.75[0.28,1.22]$ \\
\hline 12 Change in verbal category fluency & 9 & 475 & $\begin{array}{l}\text { Std. Mean Difference (IV, Random, } \\
95 \% \mathrm{CI})\end{array}$ & $0.52[0.23,0.81]$ \\
\hline 13 Change in meta cognition (self-reported) & 2 & 41 & $\begin{array}{l}\text { Std. Mean Difference (IV, Random, } \\
95 \% \mathrm{CI})\end{array}$ & $0.12[-0.87,1.12]$ \\
\hline $\begin{array}{l}14 \text { Change in meta cognition (informant-re- } \\
\text { ported) }\end{array}$ & 2 & 56 & $\begin{array}{l}\text { Std. Mean Difference (IV, Random, } \\
95 \% \mathrm{CI})\end{array}$ & $-0.65[-1.19,-0.10]$ \\
\hline 15 Change in participants' mood & 8 & 576 & $\begin{array}{l}\text { Std. Mean Difference (IV, Random, } \\
95 \% \mathrm{CI})\end{array}$ & $0.72[-0.10,1.54]$ \\
\hline $\begin{array}{l}16 \text { Change in capacity for activities of daily } \\
\text { living }\end{array}$ & 10 & 705 & $\begin{array}{l}\text { Std. Mean Difference (IV, Random, } \\
95 \% \mathrm{CI})\end{array}$ & $0.12[-0.10,0.34]$ \\
\hline 17 Change in disease progression & 5 & 215 & $\begin{array}{l}\text { Std. Mean Difference (IV, Random, } \\
95 \% \mathrm{CI})\end{array}$ & $1.07[0.59,1.55]$ \\
\hline $\begin{array}{l}18 \text { Change in behavioural and psychological } \\
\text { symptoms of dementia (BPSD) }\end{array}$ & 6 & 493 & $\begin{array}{l}\text { Std. Mean Difference (IV, Random, } \\
95 \% \mathrm{CI})\end{array}$ & $0.44[-0.34,1.22]$ \\
\hline 18.1 Up to 3 months & 3 & 92 & $\begin{array}{l}\text { Std. Mean Difference (IV, Random, } \\
95 \% \mathrm{CI})\end{array}$ & $0.66[-1.70,3.02]$ \\
\hline 18.2 More than 3 months & 3 & 401 & $\begin{array}{l}\text { Std. Mean Difference (IV, Random, } \\
95 \% \mathrm{CI})\end{array}$ & $0.12[-0.29,0.53]$ \\
\hline 19 Participant burden (retention rates) & 17 & 1282 & $\begin{array}{l}\text { Odds Ratio (M-H, Random, 95\% } \\
\mathrm{Cl})\end{array}$ & $0.73[0.37,1.43]$ \\
\hline 19.1 Up to 3 months & 14 & 761 & $\begin{array}{l}\text { Odds Ratio (M-H, Random, 95\% } \\
\mathrm{Cl})\end{array}$ & $0.78[0.35,1.73]$ \\
\hline 19.2 More than 3 months & 3 & 521 & $\begin{array}{l}\text { Odds Ratio (M-H, Random, 95\% } \\
\mathrm{Cl})\end{array}$ & $0.24[0.02,2.57]$ \\
\hline 20 Change in burden of care (CAREGIVER) & 2 & 405 & $\begin{array}{l}\text { Std. Mean Difference (IV, Random, } \\
95 \% \mathrm{CI})\end{array}$ & $-0.11[-0.36,0.15]$ \\
\hline 21 Change in quality of life (CAREGIVER) & 1 & 36 & $\begin{array}{l}\text { Std. Mean Difference (IV, Random, } \\
95 \% \mathrm{CI})\end{array}$ & $0.16[-0.50,0.83]$ \\
\hline $\begin{array}{l}22 \text { Change in mood and well-being } \\
\text { (CAREGIVER) }\end{array}$ & 1 & 36 & $\begin{array}{l}\text { Std. Mean Difference (IV, Random, } \\
95 \% \mathrm{CI})\end{array}$ & $0.98[0.27,1.68]$ \\
\hline $\begin{array}{l}23 \text { Change in general health and quality of } \\
\text { life }\end{array}$ & 5 & 630 & $\begin{array}{l}\text { Std. Mean Difference (IV, Random, } \\
95 \% \mathrm{CI})\end{array}$ & $-0.04[-0.38,0.29]$ \\
\hline
\end{tabular}


Analysis 7.1. Comparison 7 Cognitive training vs control immediately post intervention - intervention duration, Outcome 1 Change in a global measure of cognition.

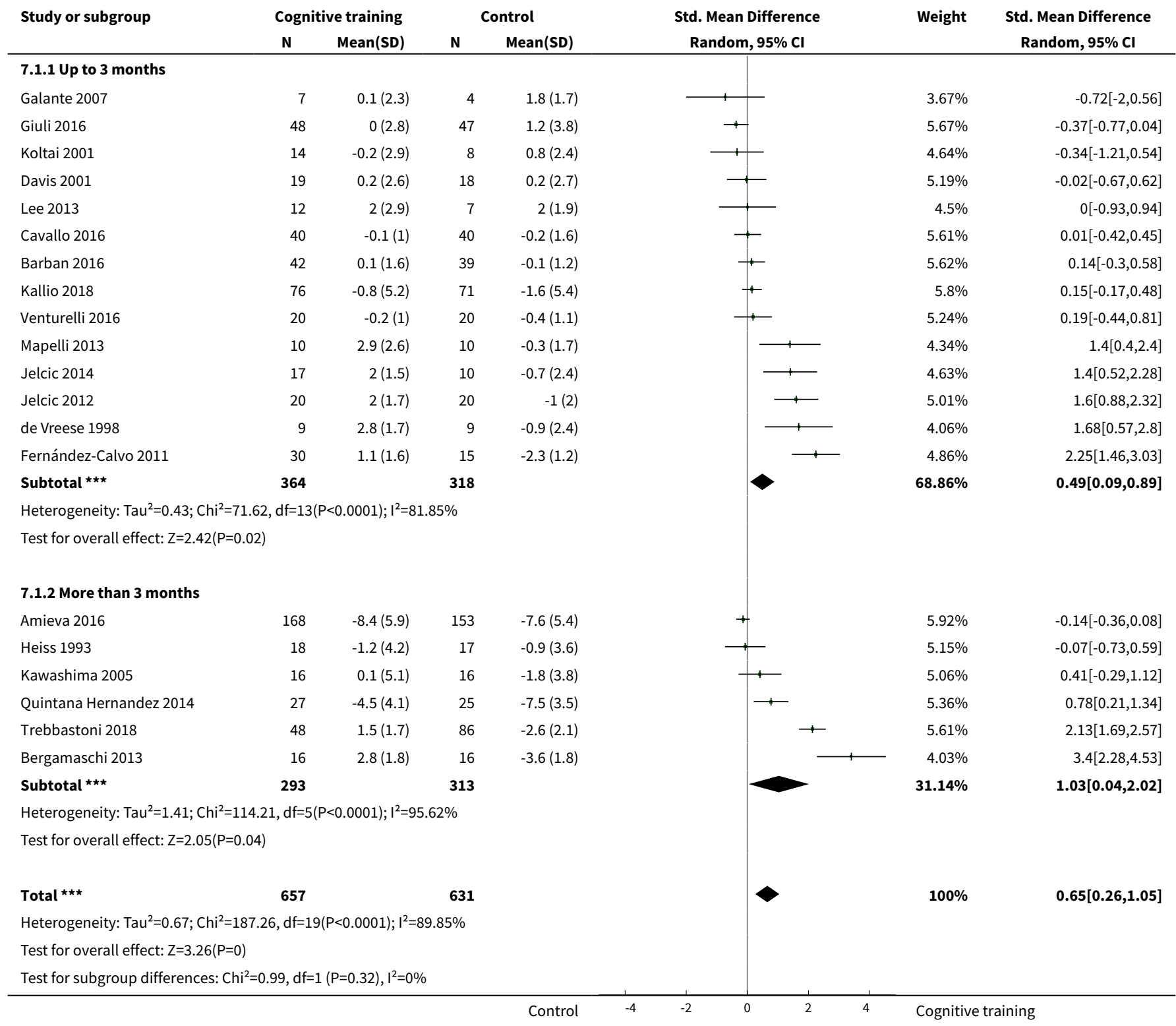

Analysis 7.2. Comparison 7 Cognitive training vs control immediately post intervention intervention duration, Outcome 2 Change in a global measure of cognition_zero correlation.

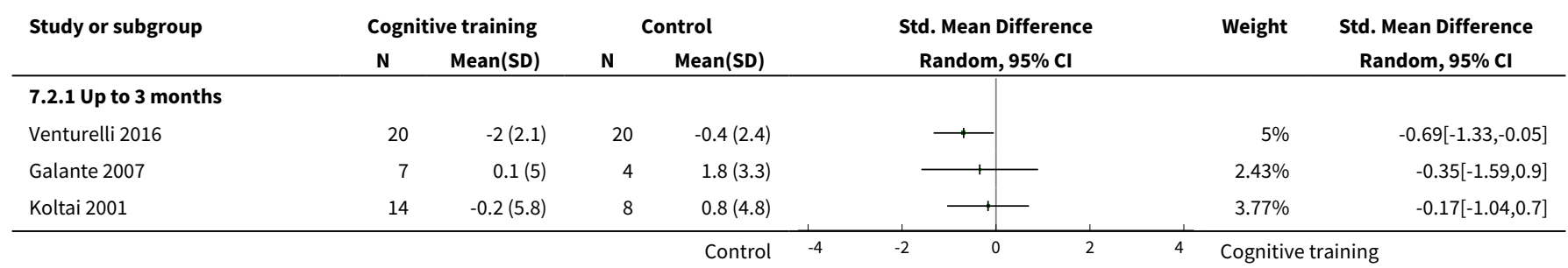




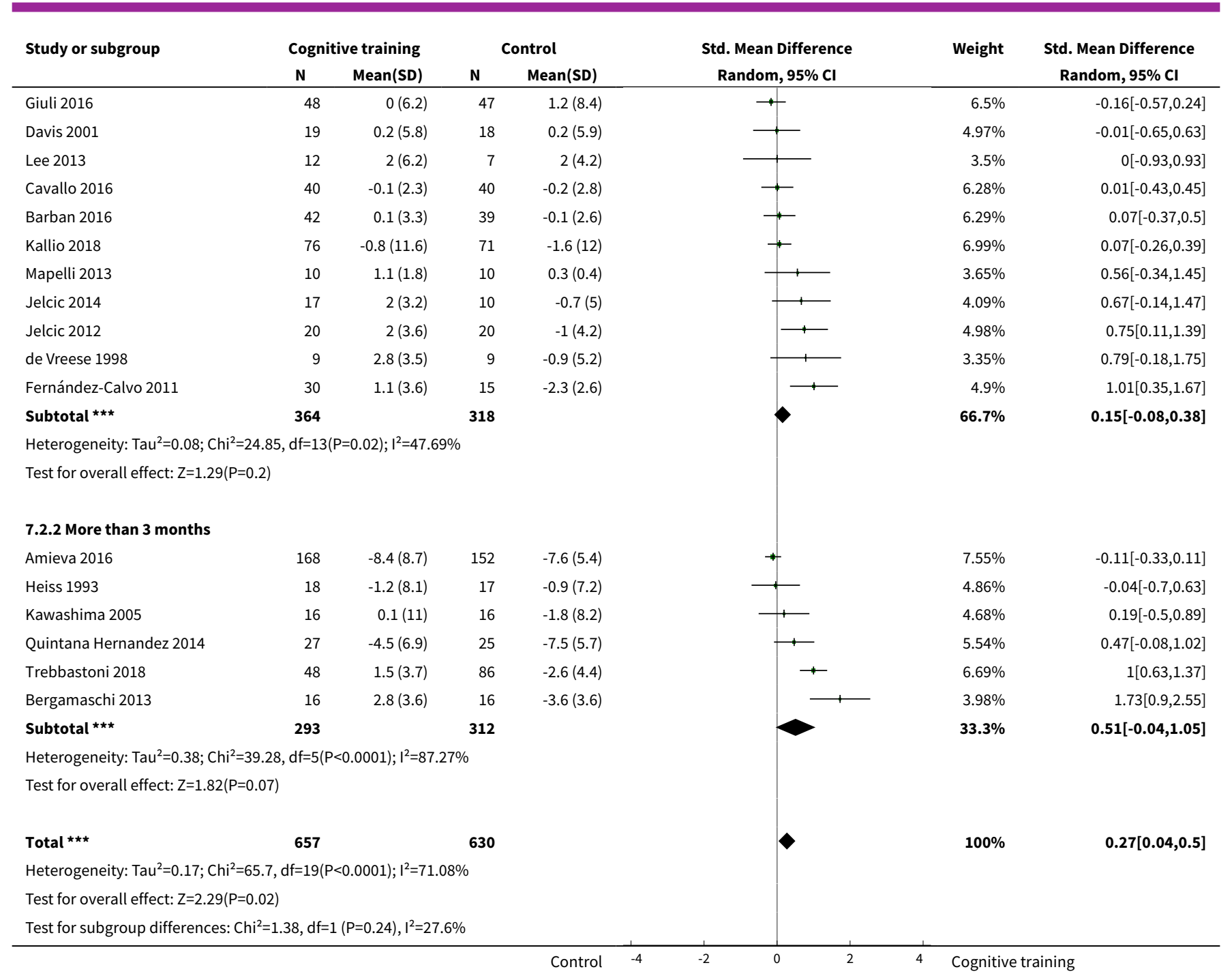

Analysis 7.3. Comparison 7 Cognitive training vs control immediately post intervention - intervention duration, Outcome 3 Change in a global measure of cognition (composite).

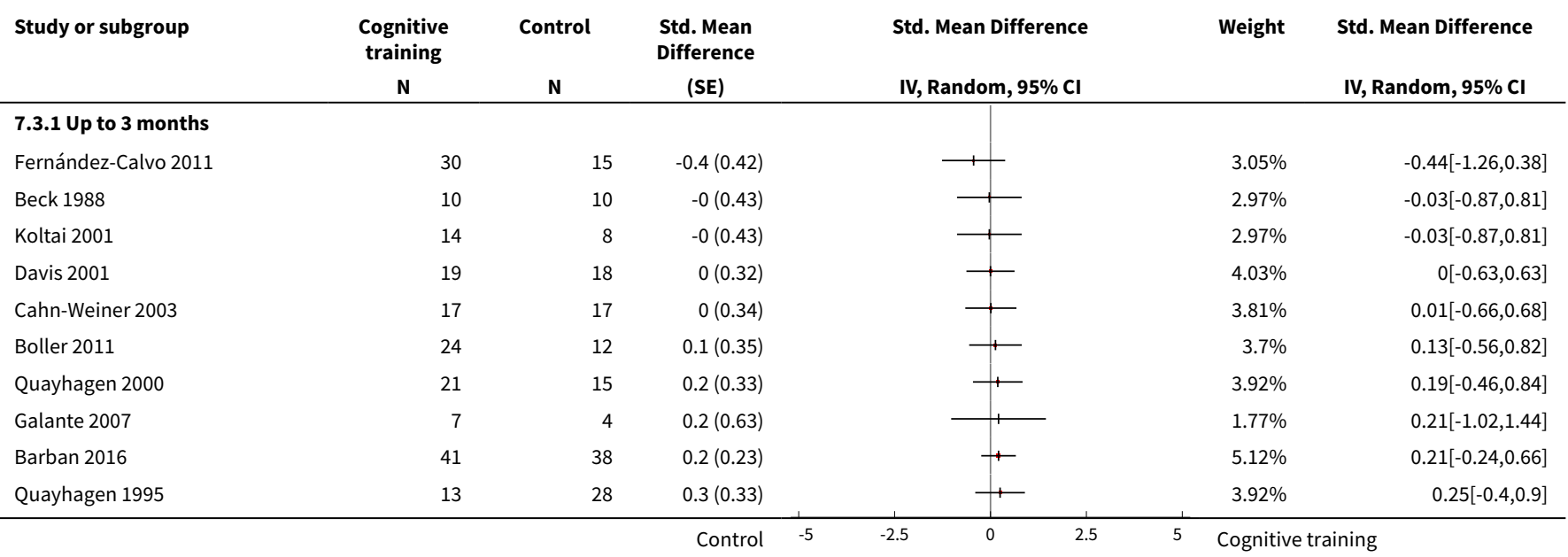




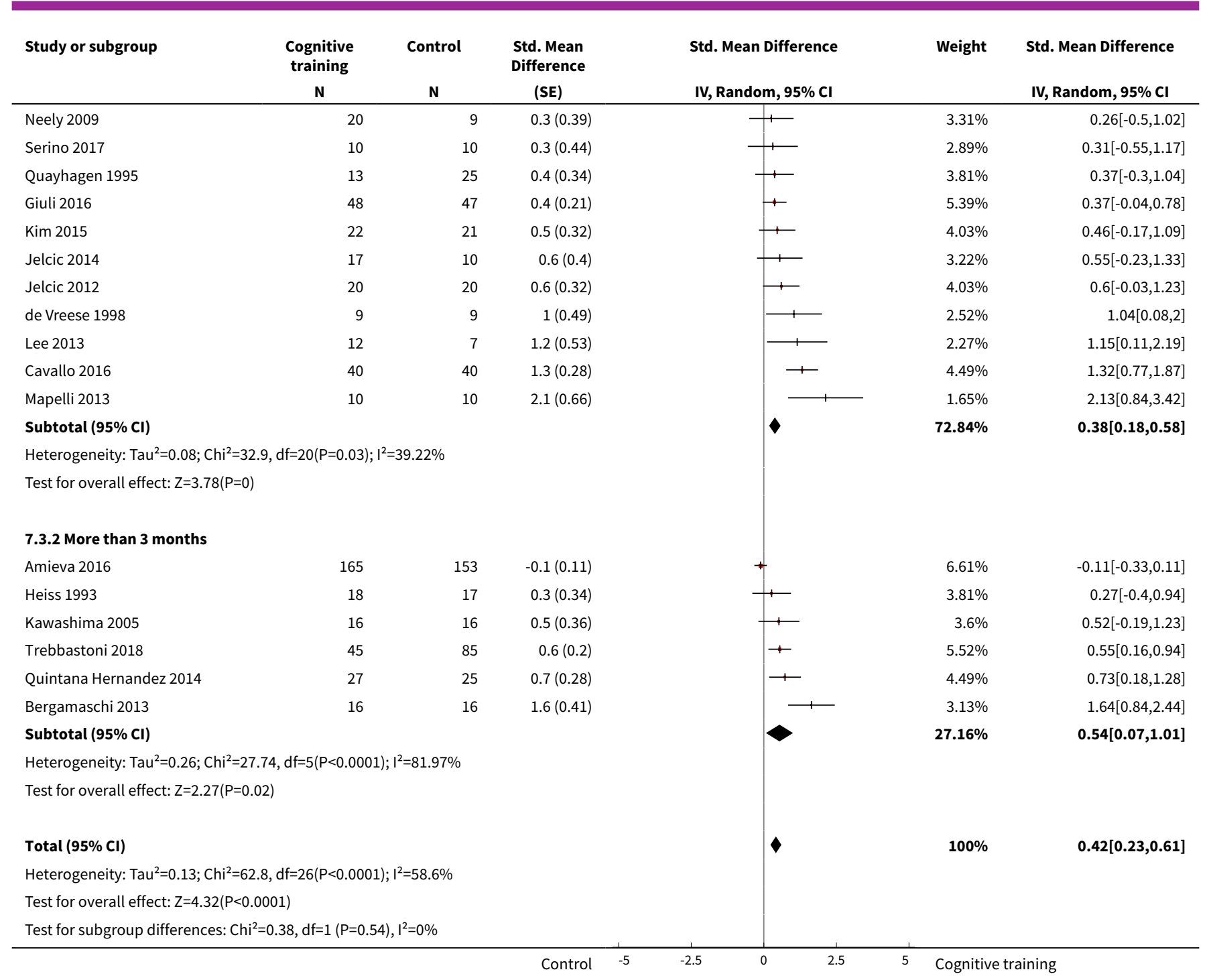

\section{Analysis 7.4. Comparison 7 Cognitive training vs control immediately post intervention - intervention duration, Outcome 4 Change in a global measure of cognition (composite)_zero correlation.}

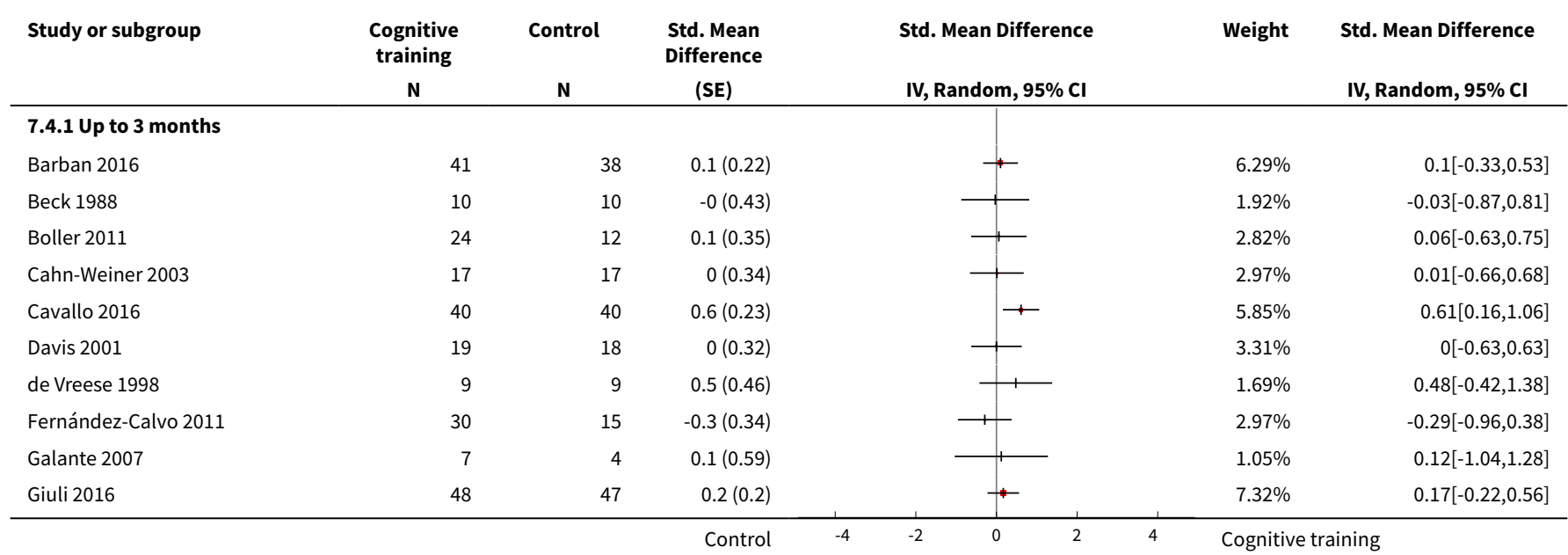




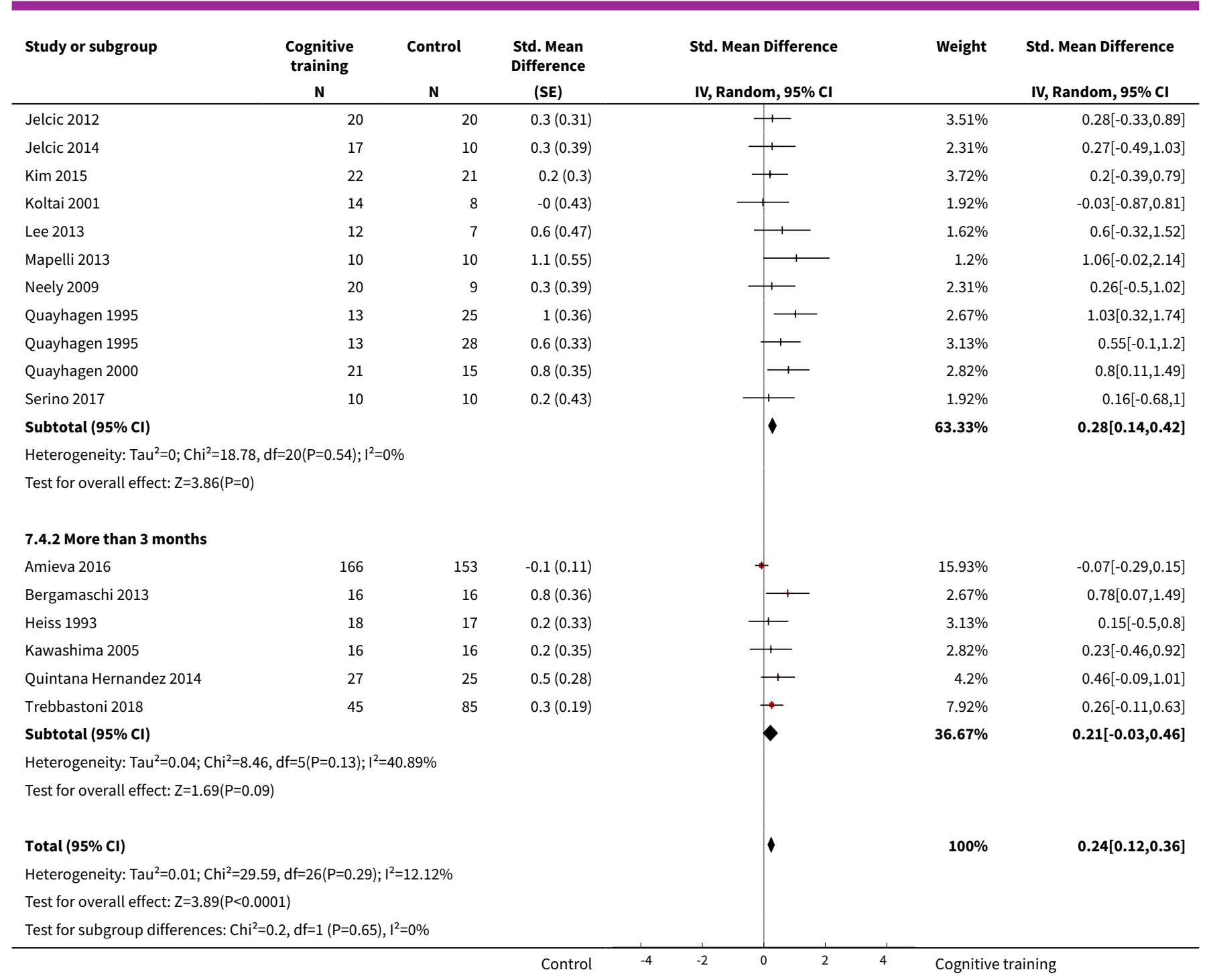

Analysis 7.5. Comparison 7 Cognitive training vs control immediately post intervention - intervention duration, Outcome 5 Change in immediate memory.

\begin{tabular}{|c|c|c|c|c|c|c|c|}
\hline \multirow{3}{*}{$\begin{array}{l}\text { Study or subgroup } \\
\text { 7.5.1 Up to } 3 \text { months }\end{array}$} & \multicolumn{2}{|c|}{ Cognitive training } & \multicolumn{2}{|c|}{ Control } & \multirow{2}{*}{$\begin{array}{c}\text { Std. Mean Difference } \\
\text { Random, } 95 \% \mathrm{Cl}\end{array}$} & \multirow[t]{2}{*}{ Weight } & \multirow{2}{*}{$\begin{array}{c}\text { Std. Mean Difference } \\
\text { Random, } 95 \% \mathrm{Cl}\end{array}$} \\
\hline & $\mathbf{N}$ & Mean(SD) & $\mathbf{N}$ & Mean(SD) & & & \\
\hline & & & & & & & \\
\hline Koltai 2001 & 14 & $0.6(1.7)$ & 8 & $1.6(2.8)$ & \begin{tabular}{l|l}
1 \\
\end{tabular} & $5.14 \%$ & $-0.44[-1.32,0.44]$ \\
\hline Galante 2007 & 7 & $-0.2(5.3)$ & 4 & $1.2(7.8)$ & & $4.03 \%$ & $-0.21[-1.44,1.03]$ \\
\hline Jelcic 2014 & 17 & $0.7(5.5)$ & 10 & $-0.3(4.8)$ & 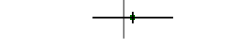 & $5.46 \%$ & $0.18[-0.61,0.96]$ \\
\hline Quayhagen 2000 & 21 & $2.3(12.1)$ & 15 & $-0.7(12.1)$ & + & $5.85 \%$ & $0.24[-0.42,0.91]$ \\
\hline Boller 2011 & 24 & $-0(3)$ & 12 & $-0.9(2.9)$ & 1 & $5.75 \%$ & $0.29[-0.41,0.99]$ \\
\hline Cahn-Weiner 2003 & 17 & $-0.4(2.8)$ & 17 & $-1.7(3)$ & $\longrightarrow$ & $5.8 \%$ & $0.44[-0.24,1.12]$ \\
\hline Davis 2001 & 19 & $1.4(3.5)$ & 18 & $-0.2(3.7)$ & ? & $5.89 \%$ & $0.45[-0.21,1.1]$ \\
\hline Giuli 2016 & 48 & $0.7(1.7)$ & 47 & $-0.2(1.7)$ & $\rightarrow$ & $6.62 \%$ & $0.47[0.06,0.88]$ \\
\hline Quayhagen 1995 & 13 & $4.7(10.6)$ & 28 & $-0.1(7.8)$ & + & $5.84 \%$ & $0.54[-0.13,1.21]$ \\
\hline Jelcic 2012 & 20 & $2.9(5.6)$ & 20 & $-0.2(5.4)$ & 1 & $5.96 \%$ & $0.55[-0.08,1.18]$ \\
\hline
\end{tabular}




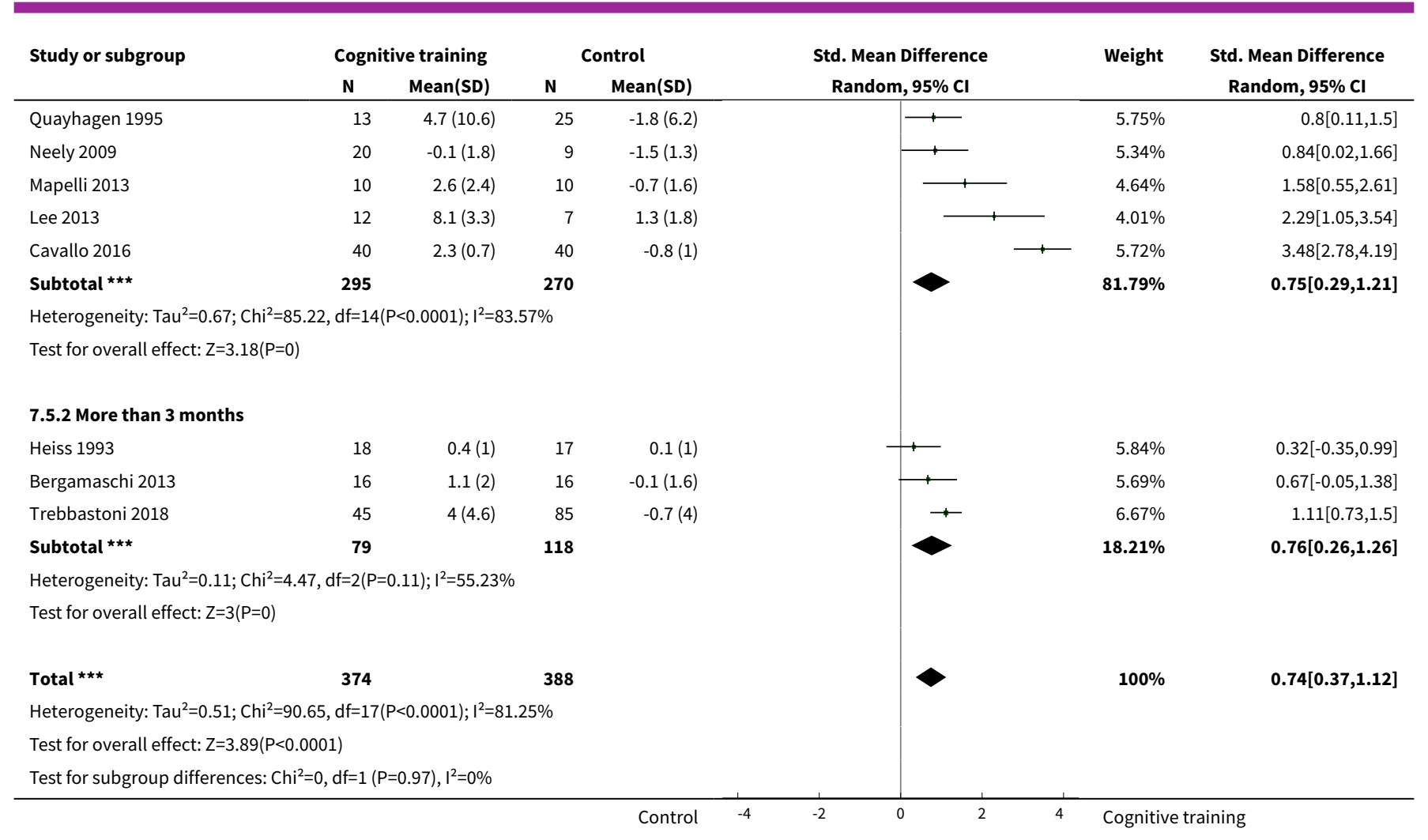

Analysis 7.6. Comparison 7 Cognitive training vs control immediately post intervention - intervention duration, Outcome 6 Change in delayed memory.

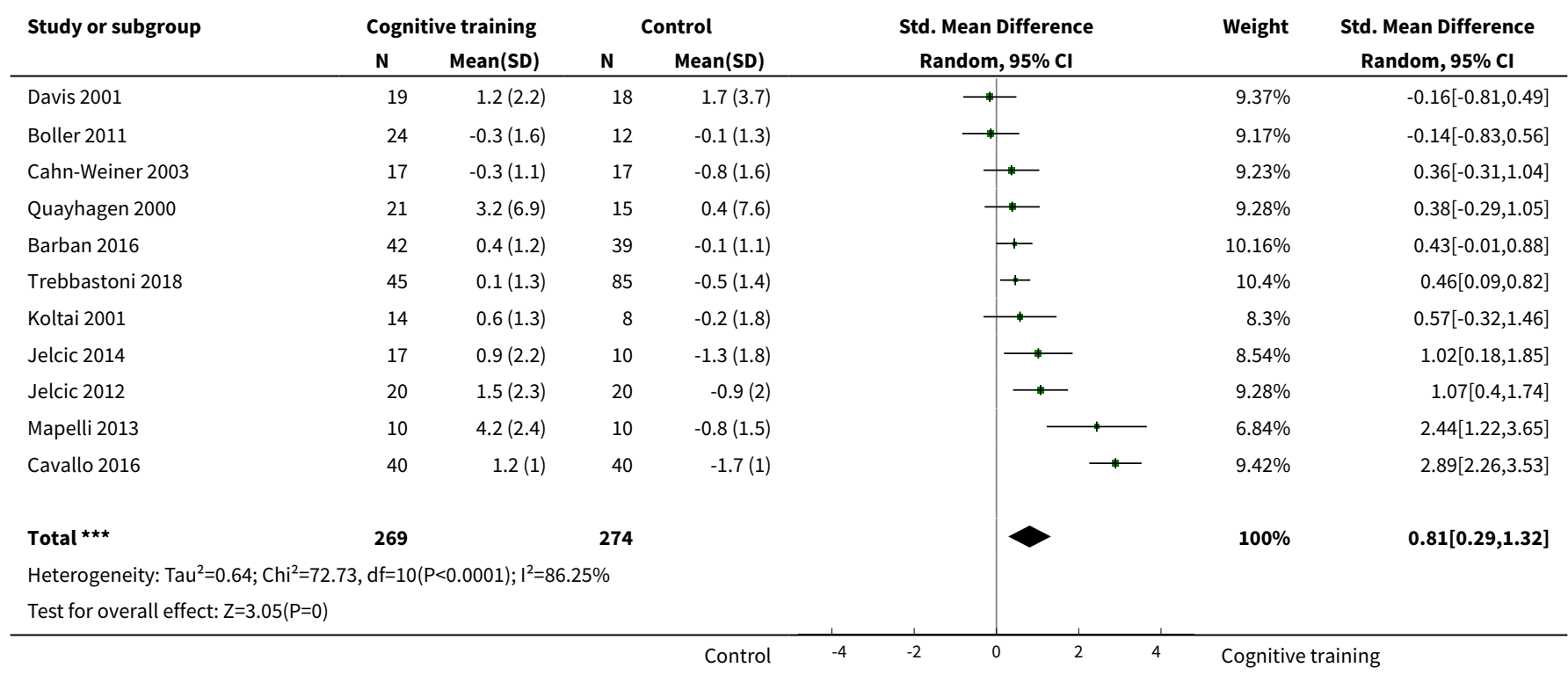


Analysis 7.7. Comparison 7 Cognitive training vs control immediately post intervention - intervention duration, Outcome 7 Change in attention and working memory.

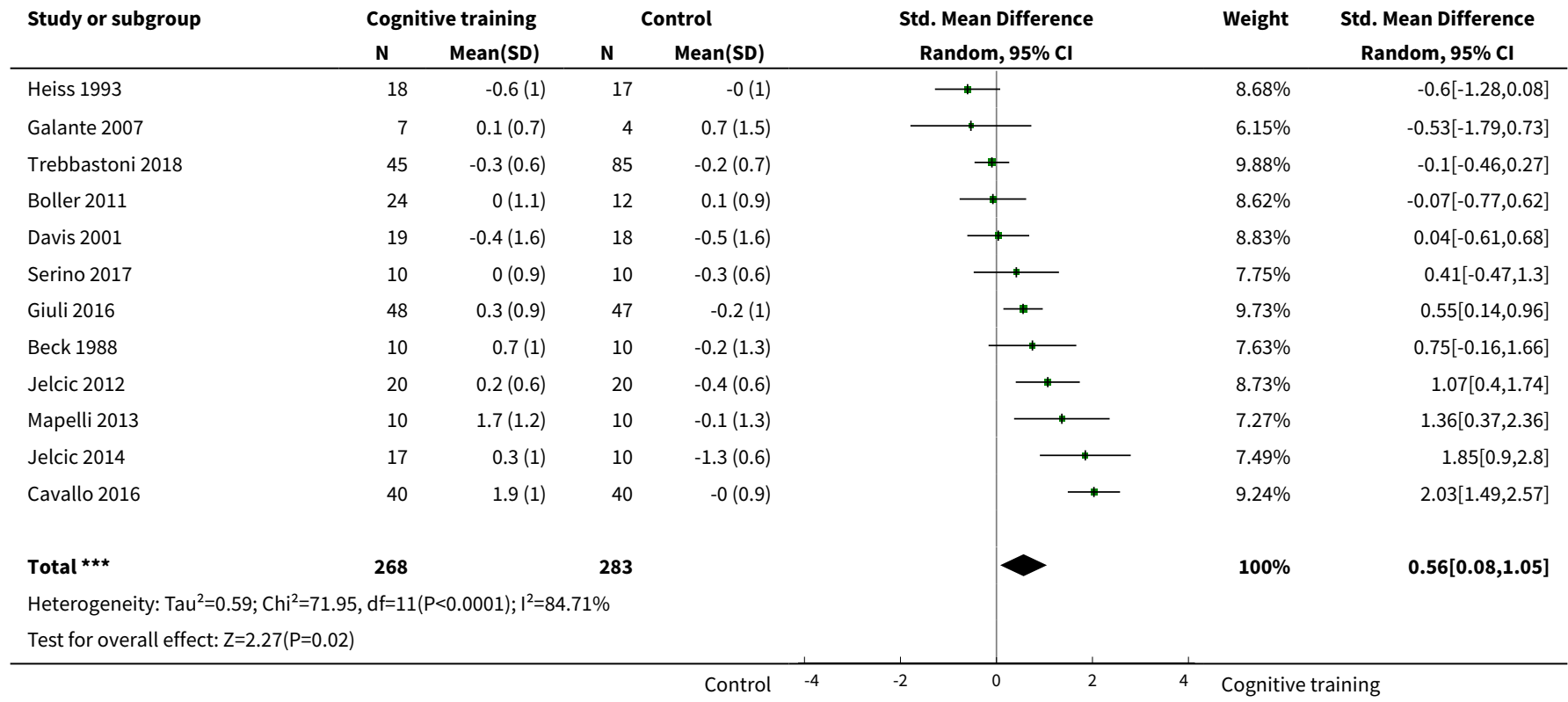

Analysis 7.8. Comparison 7 Cognitive training vs control immediately post intervention - intervention duration, Outcome 8 Change in language (naming).

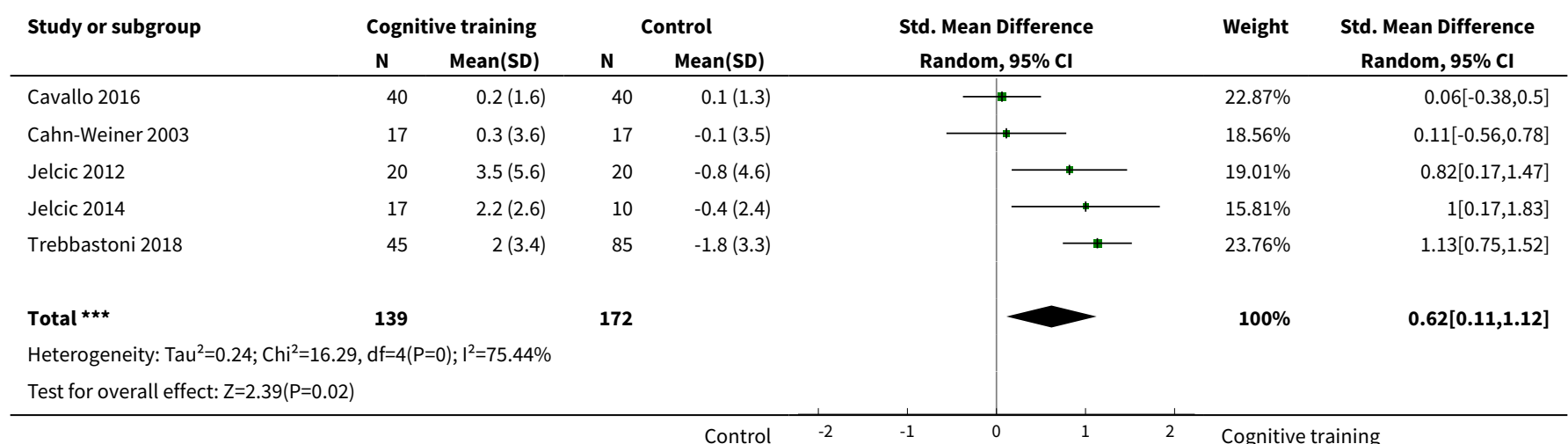

Analysis 7.9. Comparison 7 Cognitive training vs control immediately post intervention - intervention duration, Outcome 9 Change in verbal letter fluency.

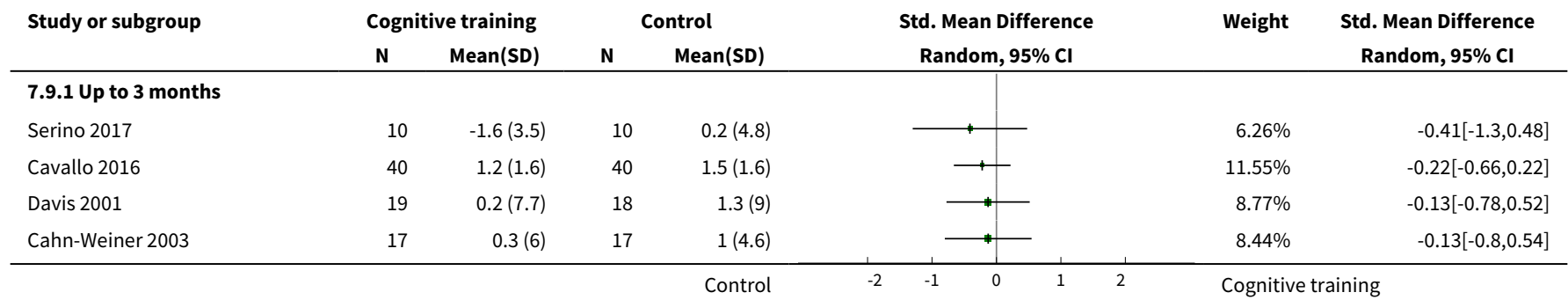




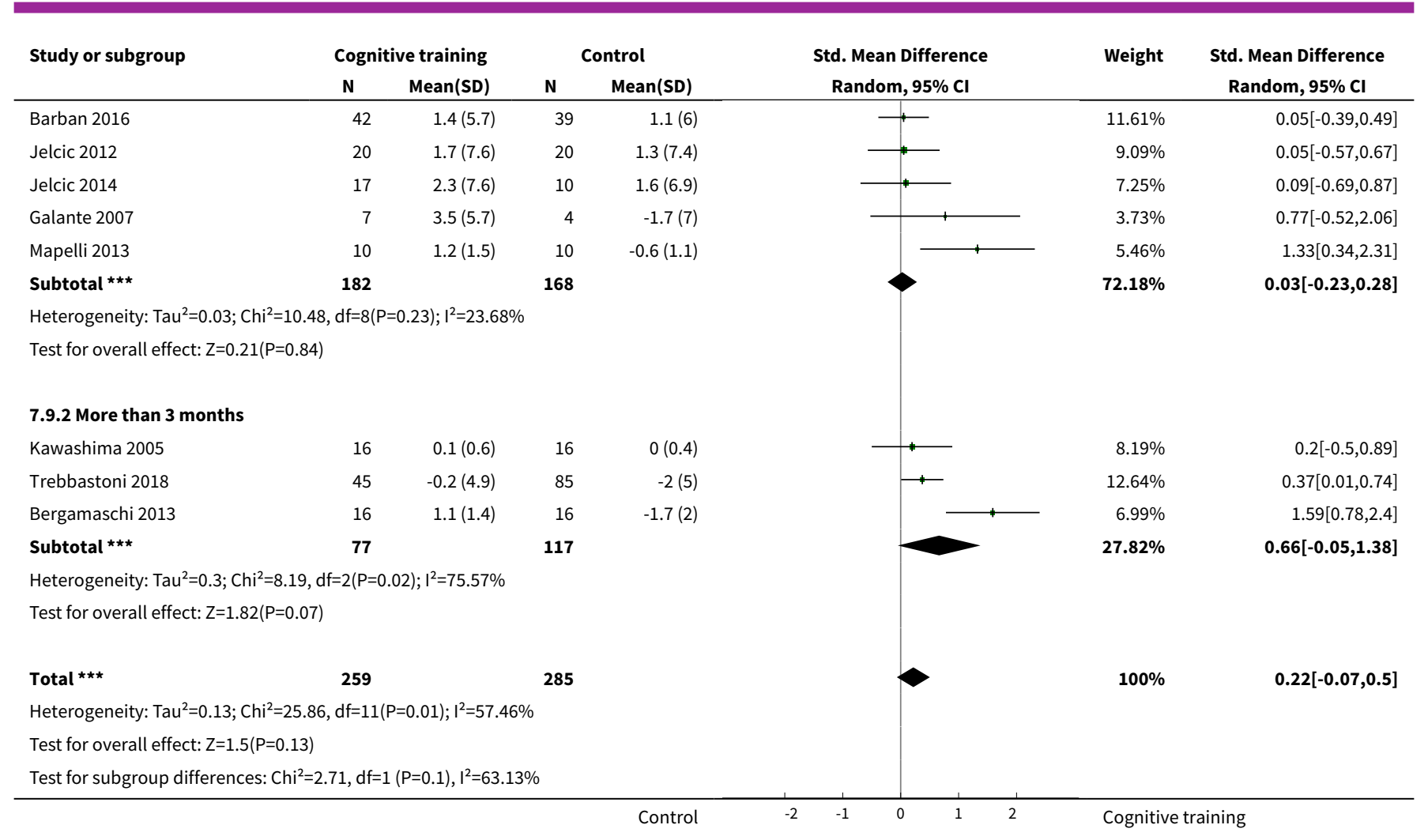

Analysis 7.10. Comparison 7 Cognitive training vs control immediately post intervention - intervention duration, Outcome 10 Change in speed of information processing.

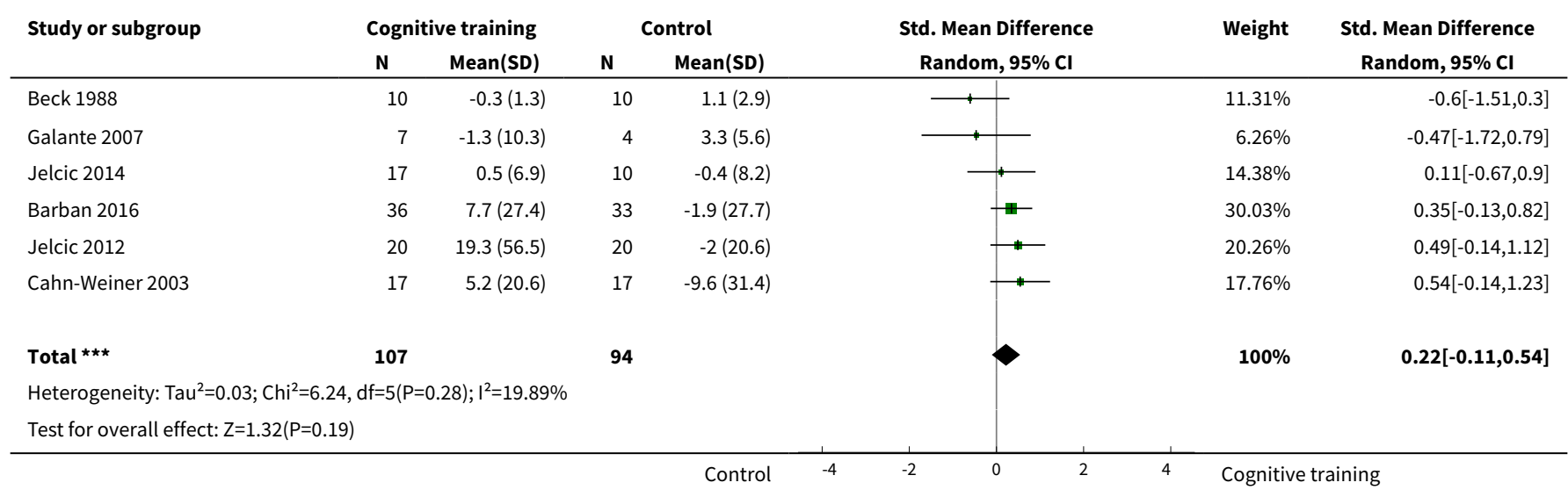

Analysis 7.11. Comparison 7 Cognitive training vs control immediately post intervention - intervention duration, Outcome 11 Change in executive function.

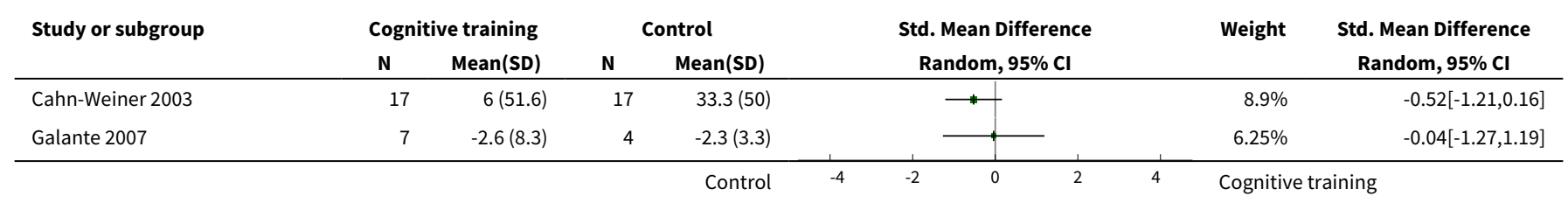




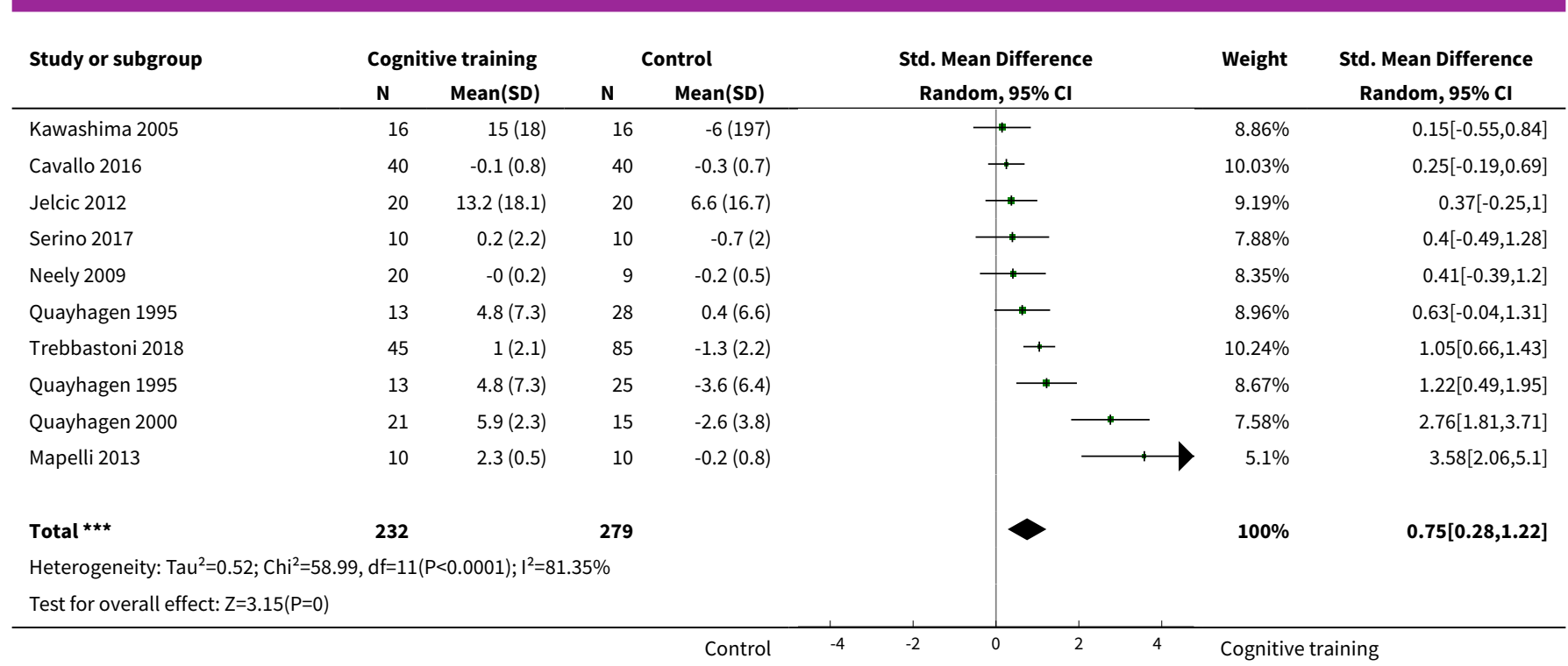

Analysis 7.12. Comparison 7 Cognitive training vs control immediately post intervention - intervention duration, Outcome 12 Change in verbal category fluency.

\begin{tabular}{|c|c|c|c|c|c|c|c|}
\hline \multirow{3}{*}{$\begin{array}{l}\text { Study or subgroup } \\
\text { Galante } 2007\end{array}$} & \multicolumn{2}{|c|}{ Cognitive training } & \multicolumn{2}{|c|}{ Control } & \multirow{2}{*}{$\begin{array}{c}\text { Std. Mean Difference } \\
\text { Random, } 95 \% \mathrm{CI}\end{array}$} & \multirow[t]{2}{*}{ Weight } & \multirow{2}{*}{$\begin{array}{c}\text { Std. Mean Difference } \\
\text { Random, } 95 \% \mathrm{Cl}\end{array}$} \\
\hline & $\mathbf{N}$ & Mean(SD) & $\mathbf{N}$ & Mean(SD) & & & \\
\hline & 7 & $0(3)$ & 4 & $0.1(4.2)$ & & $4.52 \%$ & $-0.03[-1.26,1.2]$ \\
\hline Serino 2017 & 10 & $0.7(2.9)$ & 10 & $0.5(4)$ & $\longrightarrow$ & $7.47 \%$ & $0.05[-0.82,0.93]$ \\
\hline Jelcic 2012 & 20 & $1.5(5.7)$ & 20 & $1(5.8)$ & 4 & $11.32 \%$ & $0.09[-0.53,0.71]$ \\
\hline Davis 2001 & 19 & $1.5(3.7)$ & 18 & $1(3.4)$ & 1 & $10.85 \%$ & $0.15[-0.49,0.8]$ \\
\hline Jelcic 2014 & 17 & $1.4(4.7)$ & 10 & $0.4(5.5)$ & - & $8.66 \%$ & $0.19[-0.59,0.98]$ \\
\hline Heiss 1993 & 18 & $1.7(3.5)$ & 17 & $-1.1(3.1)$ & $\longrightarrow$ & $10.05 \%$ & $0.8[0.11,1.49]$ \\
\hline Trebbastoni 2018 & 45 & $2.1(4.8)$ & 85 & $-2.9(5)$ & $\rightarrow$ & $16.63 \%$ & $1[0.62,1.38]$ \\
\hline Cavallo 2016 & 40 & $0.3(1.2)$ & 40 & $-0.9(1.2)$ & $\rightarrow$ & $14.51 \%$ & $1.05[0.58,1.52]$ \\
\hline 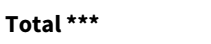 & 224 & & 251 & & $>$ & $100 \%$ & $0.52[0.23,0.81]$ \\
\hline \multicolumn{8}{|c|}{ Heterogeneity: $\mathrm{Tau}^{2}=0.09 ; \mathrm{Chi}^{2}=16.57, \mathrm{df}=8(\mathrm{P}=0.03) ; \mathrm{I}^{2}=51.71 \%$} \\
\hline
\end{tabular}

Analysis 7.13. Comparison 7 Cognitive training vs control immediately post intervention - intervention duration, Outcome 13 Change in meta cognition (self-reported).

\begin{tabular}{|c|c|c|c|c|c|c|c|}
\hline \multirow[t]{2}{*}{ Study or subgroup } & \multicolumn{2}{|c|}{ Cognitive training } & \multicolumn{2}{|c|}{ Control } & \multirow{2}{*}{$\begin{array}{c}\text { Std. Mean Difference } \\
\text { Random, } 95 \% \mathrm{Cl}\end{array}$} & \multirow[t]{2}{*}{ Weight } & \multirow{2}{*}{$\begin{array}{c}\text { Std. Mean Difference } \\
\text { Random, } 95 \% \mathrm{Cl}\end{array}$} \\
\hline & $\mathbf{N}$ & $\operatorname{Mean}(\mathrm{SD})$ & $\mathbf{N}$ & $\operatorname{Mean}(\mathrm{SD})$ & & & \\
\hline Koltai 2001 & 14 & $-6.4(14.3)$ & 8 & $-1(14.1)$ & \begin{tabular}{l|l}
1 \\
+1
\end{tabular} & $51.94 \%$ & $-0.37[-1.24,0.51]$ \\
\hline Lee 2013 & 12 & $0.3(0.3)$ & 7 & $0.1(0.3)$ & +1 & $48.06 \%$ & $0.65[-0.31,1.61]$ \\
\hline 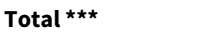 & 26 & & 15 & & & $100 \%$ & $0.12[-0.87,1.12]$ \\
\hline \multicolumn{8}{|c|}{ Heterogeneity: $\operatorname{Tau}^{2}=0.3 ; \mathrm{Chi}^{2}=2.34, \mathrm{df}=1(\mathrm{P}=0.13) ; \mathrm{I}^{2}=57.33 \%$} \\
\hline & & & & Control & -2 & Cognitiv & ining \\
\hline
\end{tabular}


Analysis 7.14. Comparison 7 Cognitive training vs control immediately post intervention - intervention duration, Outcome 14 Change in meta cognition (informant-reported).

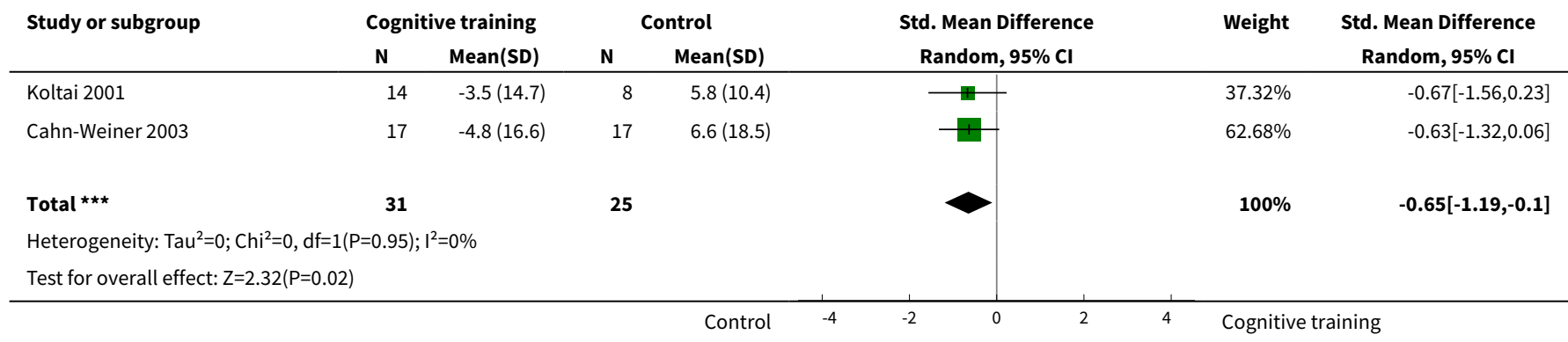

Analysis 7.15. Comparison 7 Cognitive training vs control immediately post intervention - intervention duration, Outcome 15 Change in participants' mood.

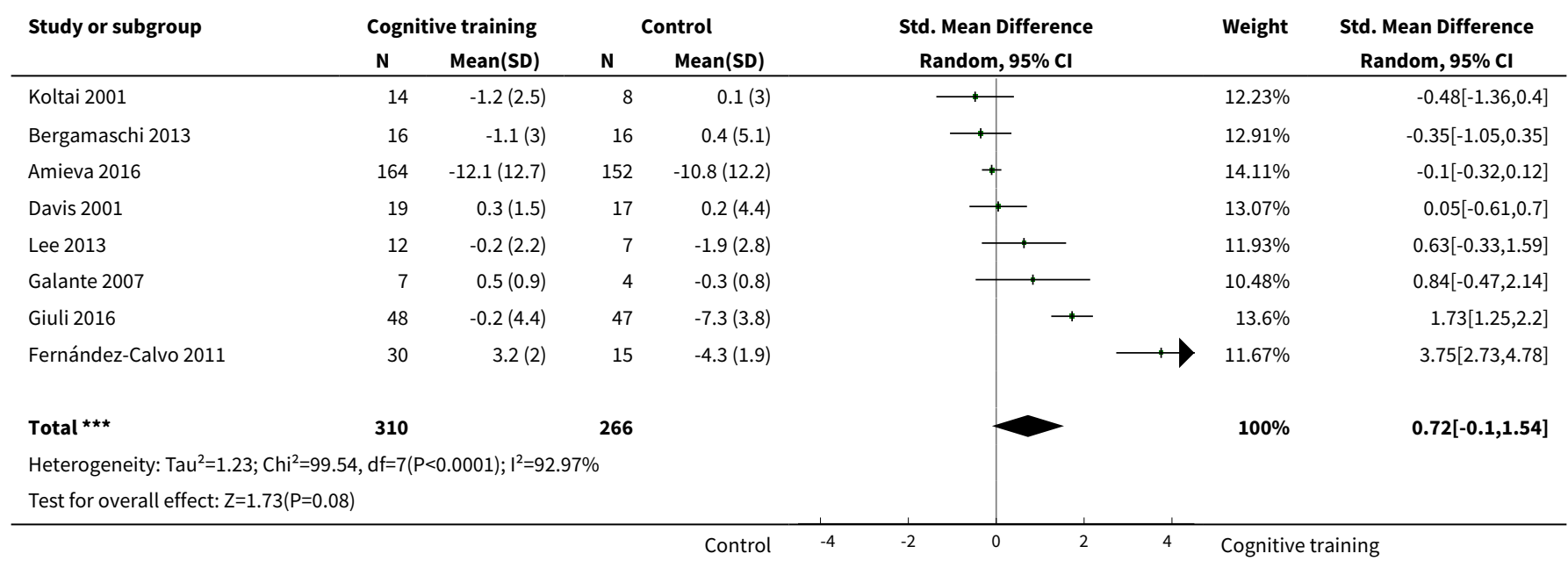

Analysis 7.16. Comparison 7 Cognitive training vs control immediately post intervention - intervention duration, Outcome 16 Change in capacity for activities of daily living.

\begin{tabular}{|c|c|c|c|c|c|c|c|}
\hline \multirow[t]{2}{*}{ Study or subgroup } & \multicolumn{2}{|c|}{ Cognitive training } & \multicolumn{2}{|c|}{ Control } & \multirow{2}{*}{$\begin{array}{c}\text { Std. Mean Difference } \\
\text { Random, } 95 \% \mathrm{CI}\end{array}$} & \multirow[t]{2}{*}{ Weight } & \multirow{2}{*}{$\begin{array}{c}\text { Std. Mean Difference } \\
\text { Random, } 95 \% \mathrm{Cl}\end{array}$} \\
\hline & $\mathbf{N}$ & Mean(SD) & $\mathbf{N}$ & Mean(SD) & & & \\
\hline Galante 2007 & 7 & $-0.6(1.4)$ & 4 & $0.5(0.5)$ & \begin{tabular}{l|l}
1 & \\
\end{tabular} & $2.63 \%$ & $-0.88[-2.19,0.43]$ \\
\hline de Vreese 1998 & 9 & $-1.3(4.4)$ & 9 & $1(4)$ & & $4.7 \%$ & $-0.53[-1.48,0.41]$ \\
\hline Amieva 2016 & 167 & $-1.8(8.5)$ & 153 & $-1.2(8.3)$ & + & $23.78 \%$ & $-0.06[-0.28,0.16]$ \\
\hline Cahn-Weiner 2003 & 17 & $-0.2(3.2)$ & 17 & $0(3.1)$ & $\longrightarrow$ & $8.09 \%$ & $-0.06[-0.74,0.61]$ \\
\hline Kim 2015 & 21 & $0(0.9)$ & 21 & $0(1.1)$ & $\rightarrow$ & $9.41 \%$ & $0[-0.6,0.6]$ \\
\hline Barban 2016 & 38 & $-0.1(1)$ & 38 & $-0.3(1.1)$ & + & $13.63 \%$ & $0.19[-0.26,0.64]$ \\
\hline Giuli 2016 & 48 & $0.3(1.2)$ & 47 & $-0.2(1.3)$ & + & $15.22 \%$ & $0.4[-0,0.81]$ \\
\hline Bergamaschi 2013 & 16 & $-0.4(1.5)$ & 16 & $-1.3(1.5)$ & $\longrightarrow$ & $7.44 \%$ & $0.61[-0.1,1.32]$ \\
\hline Lee 2013 & 12 & $2.2(3.3)$ & 7 & $-2.1(4.5)$ & - & $4.18 \%$ & $1.1[0.09,2.11]$ \\
\hline
\end{tabular}




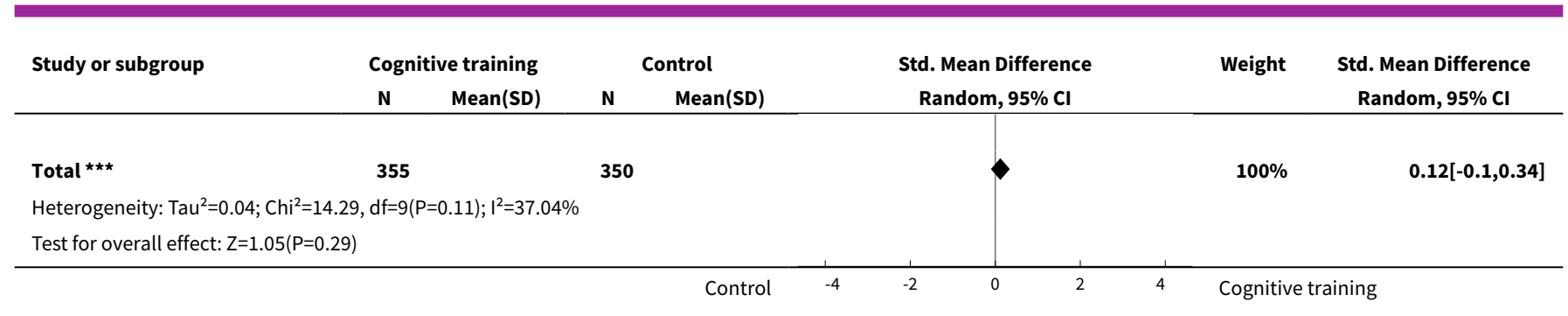

Analysis 7.17. Comparison 7 Cognitive training vs control immediately post intervention - intervention duration, Outcome 17 Change in disease progression.

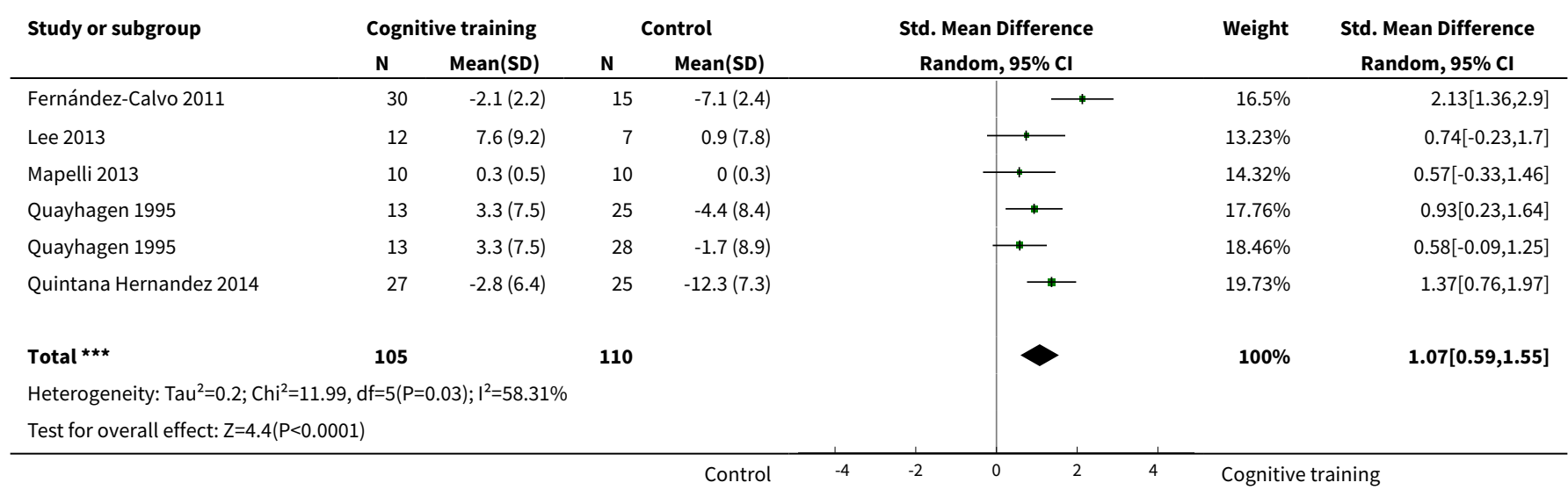
Analysis 7.18. Comparison 7 Cognitive training vs control immediately post intervention - intervention
duration, Outcome 18 Change in behavioural and psychological symptoms of dementia (BPSD).

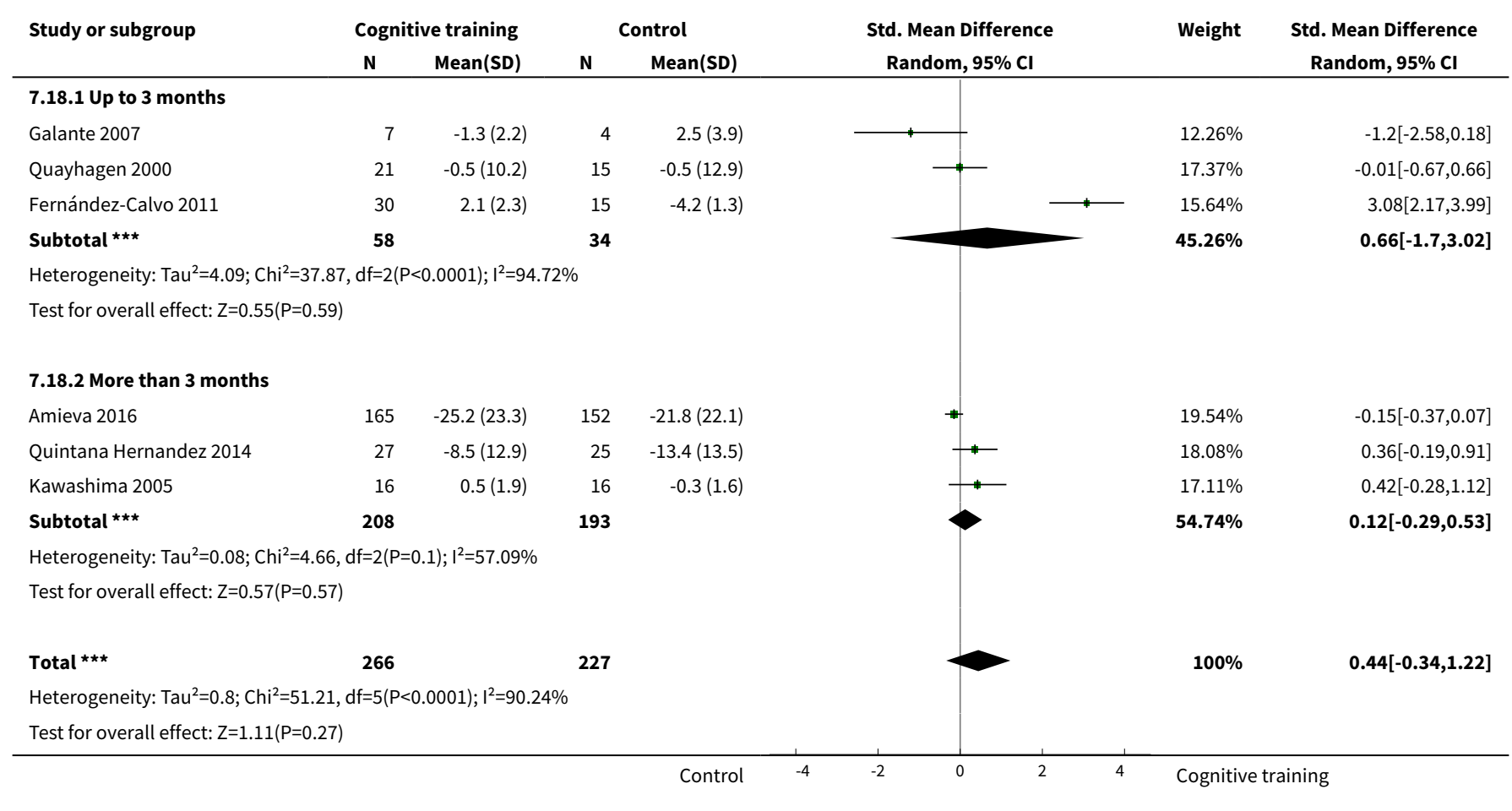




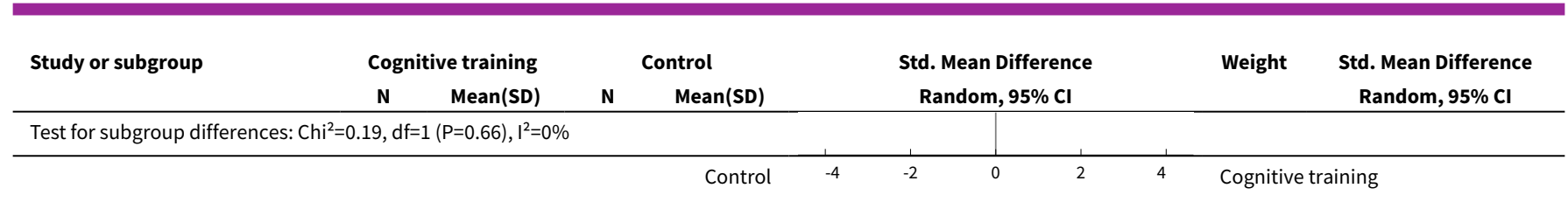

Analysis 7.19. Comparison 7 Cognitive training vs control immediately post intervention - intervention duration, Outcome 19 Participant burden (retention rates).

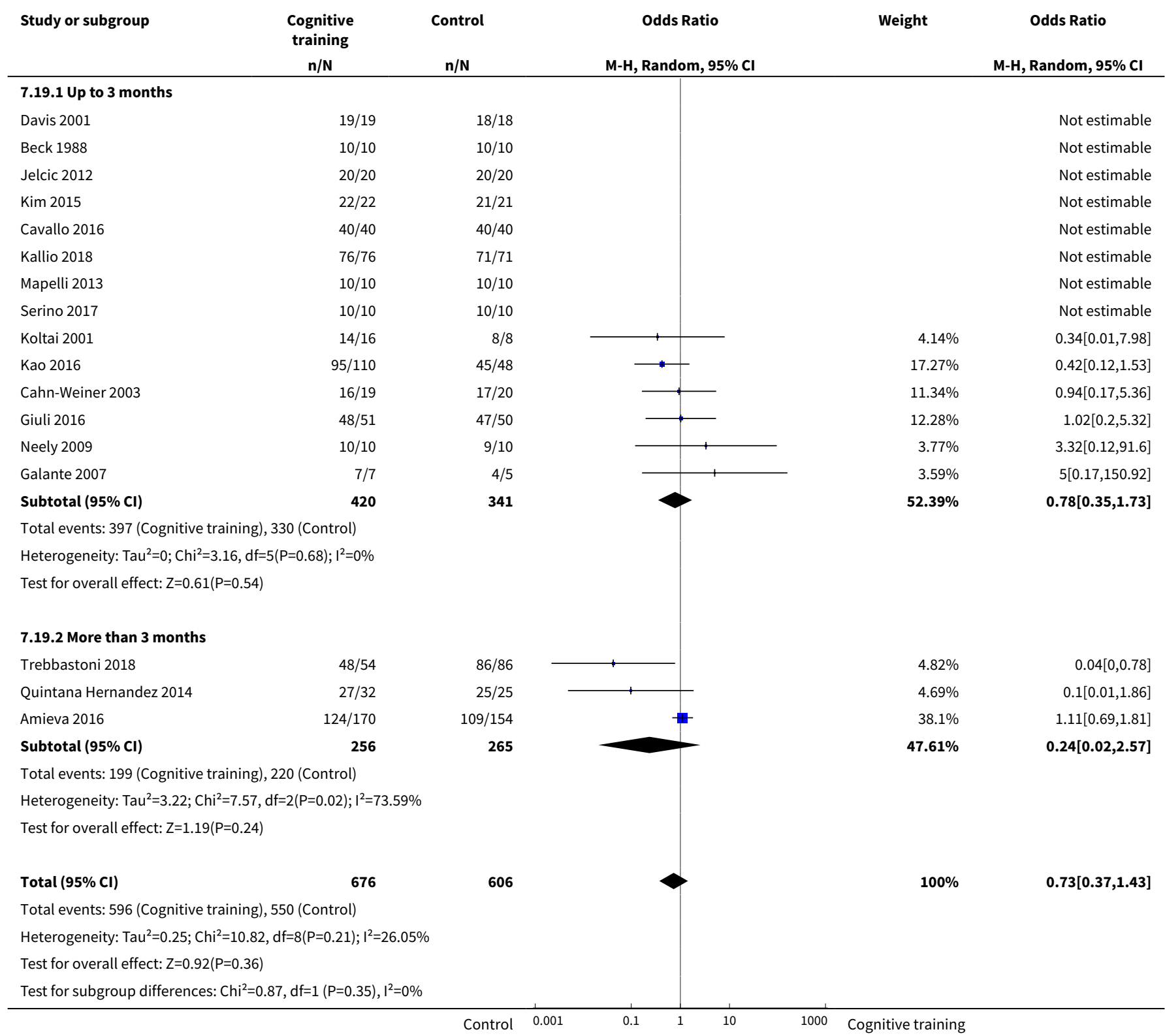


Analysis 7.20. Comparison 7 Cognitive training vs control immediately post intervention - intervention duration, Outcome 20 Change in burden of care (CAREGIVER).

\begin{tabular}{|c|c|c|c|c|c|c|c|}
\hline \multirow[t]{2}{*}{ Study or subgroup } & \multicolumn{2}{|c|}{ Cognitive training } & \multicolumn{2}{|c|}{ Control } & \multirow{2}{*}{$\begin{array}{c}\text { Std. Mean Difference } \\
\text { Random, } 95 \% \mathrm{CI}\end{array}$} & \multirow[t]{2}{*}{ Weight } & \multirow{2}{*}{$\begin{array}{c}\text { Std. Mean Difference } \\
\text { Random, } 95 \% \mathrm{Cl}\end{array}$} \\
\hline & $\mathbf{N}$ & Mean(SD) & $\mathbf{N}$ & $\operatorname{Mean}(S D)$ & & & \\
\hline Amieva 2016 & 165 & $-21.3(19.1)$ & 152 & $-17.9(19)$ & & $76.98 \%$ & $-0.18[-0.4,0.04]$ \\
\hline Quayhagen 2000 & 21 & $-0.8(4.1)$ & 67 & $-1.3(3.7)$ & 7 & $23.02 \%$ & $0.13[-0.36,0.62]$ \\
\hline Total $\star \star \star ~$ & 186 & & 219 & & & $100 \%$ & $-0.11[-0.36,0.15]$ \\
\hline \multicolumn{8}{|c|}{ Heterogeneity: $\mathrm{Tau}^{2}=0.01 ; \mathrm{Chi}^{2}=1.23, \mathrm{df}=1(\mathrm{P}=0.27) ; \mathrm{I}^{2}=18.62 \%$} \\
\hline
\end{tabular}

Analysis 7.21. Comparison 7 Cognitive training vs control immediately post intervention - intervention duration, Outcome 21 Change in quality of life (CAREGIVER).

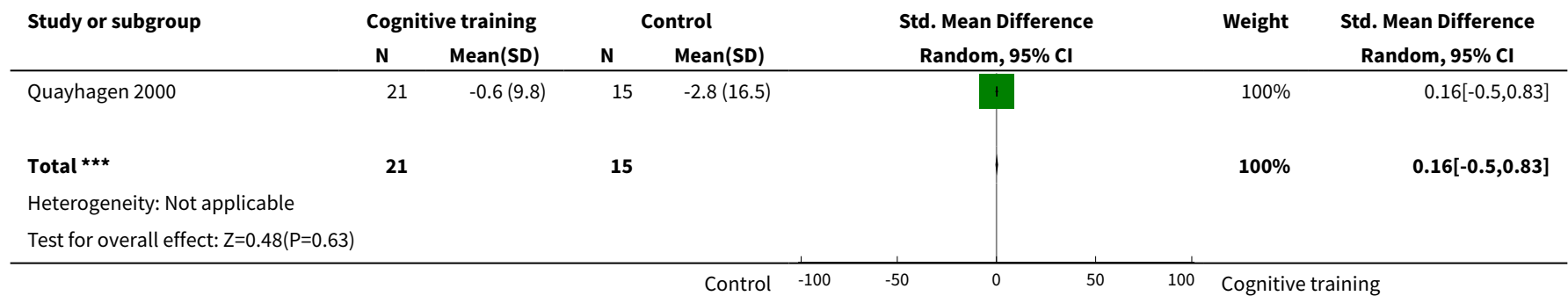

Analysis 7.22. Comparison 7 Cognitive training vs control immediately post intervention - intervention duration, Outcome 22 Change in mood and well-being (CAREGIVER).

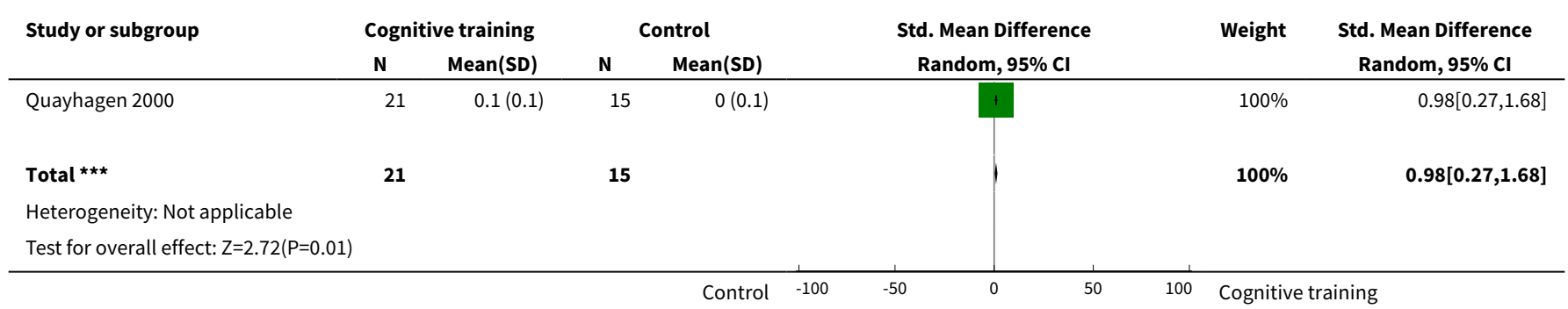

Analysis 7.23. Comparison 7 Cognitive training vs control immediately post intervention - intervention duration, Outcome 23 Change in general health and quality of life.

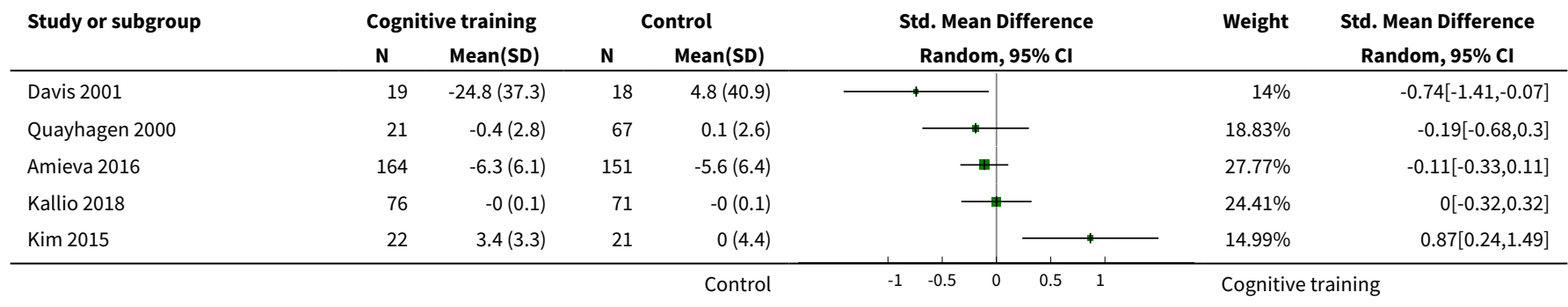




\begin{tabular}{|c|c|c|c|c|c|c|c|}
\hline \multirow[t]{2}{*}{ Study or subgroup } & \multicolumn{2}{|c|}{ Cognitive training } & \multicolumn{2}{|c|}{ Control } & \multirow{2}{*}{$\begin{array}{c}\text { Std. Mean Difference } \\
\text { Random, } 95 \% \mathrm{Cl}\end{array}$} & \multirow[t]{2}{*}{ Weight } & \multirow{2}{*}{$\begin{array}{c}\text { Std. Mean Difference } \\
\text { Random, } 95 \% \mathrm{Cl}\end{array}$} \\
\hline & $\mathbf{N}$ & Mean(SD) & $\mathbf{N}$ & Mean(SD) & & & \\
\hline 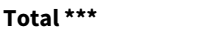 & 302 & & 328 & & & $100 \%$ & $-0.04[-0.38,0.29]$ \\
\hline \multicolumn{8}{|c|}{ Heterogeneity: $\mathrm{Tau}^{2}=0.09 ; \mathrm{Chi}^{2}=12.93, \mathrm{df}=4(\mathrm{P}=0.01) ; \mathrm{I}^{2}=69.08 \%$} \\
\hline \multicolumn{8}{|c|}{ Test for overall effect: $Z=0.24(P=0.81)$} \\
\hline & & & & Control & -0.5 & Cognitiv & ining \\
\hline
\end{tabular}

\section{Comparison 8. Cognitive training vs control immediately post intervention - type of CT (traditional vs augmented)}

\begin{tabular}{|c|c|c|c|c|}
\hline Outcome or subgroup title & $\begin{array}{l}\text { No. of } \\
\text { studies }\end{array}$ & $\begin{array}{l}\text { No. of } \\
\text { partici- } \\
\text { pants }\end{array}$ & Statistical method & Effect size \\
\hline $\begin{array}{l}1 \text { Change in a global measure of cogni- } \\
\text { tion }\end{array}$ & 20 & 1288 & $\begin{array}{l}\text { Std. Mean Difference (IV, Random, } \\
95 \% \mathrm{CI})\end{array}$ & $0.65[0.26,1.05]$ \\
\hline 1.1 Traditional & 13 & 975 & $\begin{array}{l}\text { Std. Mean Difference (IV, Random, } \\
95 \% \mathrm{CI})\end{array}$ & $0.84[0.30,1.39]$ \\
\hline 1.2 Augmented & 7 & 313 & $\begin{array}{l}\text { Std. Mean Difference (IV, Random, } \\
95 \% \mathrm{CI})\end{array}$ & $0.25[-0.21,0.70]$ \\
\hline $\begin{array}{l}2 \text { Change in a global measure of cogni- } \\
\text { tion_zero correlation }\end{array}$ & 20 & 1287 & $\begin{array}{l}\text { Std. Mean Difference (IV, Random, } \\
95 \% \mathrm{CI})\end{array}$ & $0.27[0.04,0.50]$ \\
\hline 2.1 Traditional & 13 & 974 & $\begin{array}{l}\text { Std. Mean Difference (IV, Random, } \\
95 \% \mathrm{CI})\end{array}$ & $0.41[0.11,0.71]$ \\
\hline 2.2 Augmented & 7 & 313 & $\begin{array}{l}\text { Std. Mean Difference (IV, Random, } \\
95 \% \mathrm{CI})\end{array}$ & $-0.03[-0.33,0.26]$ \\
\hline $\begin{array}{l}3 \text { Change in a global measure of cogni- } \\
\text { tion (composite) }\end{array}$ & 26 & 1389 & $\begin{array}{l}\text { Std. Mean Difference (Random, 95\% } \\
\mathrm{Cl} \text { ) }\end{array}$ & $0.42[0.23,0.61]$ \\
\hline 3.1 Traditional & 17 & 1026 & $\begin{array}{l}\text { Std. Mean Difference (Random, 95\% } \\
\mathrm{Cl} \text { ) }\end{array}$ & $0.43[0.18,0.68]$ \\
\hline 3.2 Augmented & 9 & 363 & $\begin{array}{l}\text { Std. Mean Difference (Random, 95\% } \\
\mathrm{Cl} \text { ) }\end{array}$ & $0.37[0.10,0.65]$ \\
\hline $\begin{array}{l}4 \text { Change in a global measure of cogni- } \\
\text { tion (composite)_zero correlation }\end{array}$ & 26 & 1390 & $\begin{array}{l}\text { Std. Mean Difference (Random, 95\% } \\
\mathrm{Cl} \text { ) }\end{array}$ & $0.24[0.12,0.36]$ \\
\hline 4.1 Traditional & 17 & 1027 & $\begin{array}{l}\text { Std. Mean Difference (Random, 95\% } \\
\mathrm{Cl} \text { ) }\end{array}$ & $0.30[0.12,0.47]$ \\
\hline 4.2 Augmented & 9 & 363 & $\begin{array}{l}\text { Std. Mean Difference (Random, 95\% } \\
\mathrm{Cl} \text { ) }\end{array}$ & $0.18[-0.02,0.39]$ \\
\hline 5 Change in immediate memory & 17 & 762 & $\begin{array}{l}\text { Std. Mean Difference (IV, Random, } \\
95 \% \mathrm{CI})\end{array}$ & $0.74[0.37,1.12]$ \\
\hline 5.1 Traditional & 12 & 559 & $\begin{array}{l}\text { Std. Mean Difference (IV, Random, } \\
95 \% \mathrm{CI})\end{array}$ & $0.82[0.33,1.30]$ \\
\hline
\end{tabular}




\begin{tabular}{|c|c|c|c|c|}
\hline Outcome or subgroup title & $\begin{array}{l}\text { No. of } \\
\text { studies }\end{array}$ & $\begin{array}{l}\text { No. of } \\
\text { partici- } \\
\text { pants }\end{array}$ & Statistical method & Effect size \\
\hline 5.2 Augmented & 5 & 203 & $\begin{array}{l}\text { Std. Mean Difference (IV, Random, } \\
95 \% \mathrm{CI})\end{array}$ & $0.53[0.04,1.02]$ \\
\hline 6 Change in delayed memory & 11 & 543 & $\begin{array}{l}\text { Std. Mean Difference (IV, Random, } \\
95 \% \mathrm{CI})\end{array}$ & $0.81[0.29,1.32]$ \\
\hline 6.1 Traditional & 7 & 383 & $\begin{array}{l}\text { Std. Mean Difference (IV, Random, } \\
95 \% \mathrm{CI})\end{array}$ & $0.86[0.15,1.58]$ \\
\hline 6.2 Augmented & 4 & 160 & $\begin{array}{l}\text { Std. Mean Difference (IV, Random, } \\
95 \% \mathrm{CI})\end{array}$ & $0.68[-0.11,1.46]$ \\
\hline $\begin{array}{l}7 \text { Change in attention and working } \\
\text { memory }\end{array}$ & 12 & 551 & $\begin{array}{l}\text { Std. Mean Difference (IV, Random, } \\
95 \% \mathrm{CI})\end{array}$ & $0.56[0.08,1.05]$ \\
\hline 7.1 Traditional & 8 & 379 & $\begin{array}{l}\text { Std. Mean Difference (IV, Random, } \\
95 \% \mathrm{CI})\end{array}$ & $0.56[-0.17,1.30]$ \\
\hline 7.2 Augmented & 4 & 172 & $\begin{array}{l}\text { Std. Mean Difference (IV, Random, } \\
95 \% \mathrm{CI})\end{array}$ & $0.51[0.07,0.95]$ \\
\hline 8 Change in language (naming) & 5 & 311 & $\begin{array}{l}\text { Std. Mean Difference (IV, Random, } \\
95 \% \mathrm{CI})\end{array}$ & $0.62[0.11,1.12]$ \\
\hline 9 Change in verbal letter fluency & 12 & 544 & $\begin{array}{l}\text { Std. Mean Difference (IV, Random, } \\
95 \% \mathrm{CI})\end{array}$ & $0.22[-0.07,0.50]$ \\
\hline 9.1 Traditional & 8 & 386 & $\begin{array}{l}\text { Std. Mean Difference (IV, Random, } \\
95 \% \mathrm{CI})\end{array}$ & $0.26[-0.09,0.62]$ \\
\hline 9.2 Augmented & 4 & 158 & $\begin{array}{l}\text { Std. Mean Difference (IV, Random, } \\
95 \% \mathrm{CI})\end{array}$ & $0.14[-0.42,0.70]$ \\
\hline $\begin{array}{l}10 \text { Change in speed of information pro- } \\
\text { cessing }\end{array}$ & 6 & 201 & $\begin{array}{l}\text { Std. Mean Difference (IV, Random, } \\
95 \% \mathrm{CI})\end{array}$ & $0.22[-0.11,0.54]$ \\
\hline 11 Change in executive function & 11 & 511 & $\begin{array}{l}\text { Std. Mean Difference (IV, Random, } \\
95 \% \mathrm{CI})\end{array}$ & $0.75[0.28,1.22]$ \\
\hline 11.1 Traditional & 8 & 442 & $\begin{array}{l}\text { Std. Mean Difference (IV, Random, } \\
95 \% \mathrm{CI})\end{array}$ & $0.64[0.14,1.14]$ \\
\hline 11.2 Augmented & 3 & 69 & $\begin{array}{l}\text { Std. Mean Difference (IV, Random, } \\
95 \% \mathrm{CI} \text { ) }\end{array}$ & $1.32[-0.26,2.91]$ \\
\hline 12 Change in verbal category fluency & 9 & 475 & $\begin{array}{l}\text { Std. Mean Difference (IV, Random, } \\
95 \% \mathrm{CI})\end{array}$ & $0.52[0.23,0.81]$ \\
\hline 12.1 Traditional & 6 & 323 & $\begin{array}{l}\text { Std. Mean Difference (IV, Random, } \\
95 \% \mathrm{CI})\end{array}$ & $0.64[0.26,1.01]$ \\
\hline 12.2 Augmented & 3 & 152 & $\begin{array}{l}\text { Std. Mean Difference (IV, Random, } \\
95 \% \mathrm{CI} \text { ) }\end{array}$ & $0.33[0.00,0.65]$ \\
\hline
\end{tabular}




\begin{tabular}{|c|c|c|c|c|}
\hline Outcome or subgroup title & $\begin{array}{l}\text { No. of } \\
\text { studies }\end{array}$ & $\begin{array}{l}\text { No. of } \\
\text { partici- } \\
\text { pants }\end{array}$ & Statistical method & Effect size \\
\hline $\begin{array}{l}13 \text { Change in meta cognition (self-re- } \\
\text { ported) }\end{array}$ & 2 & 41 & $\begin{array}{l}\text { Std. Mean Difference (IV, Random, } \\
95 \% \mathrm{CI})\end{array}$ & $0.12[-0.87,1.12]$ \\
\hline $\begin{array}{l}14 \text { Change in meta cognition (infor- } \\
\text { mant-reported) }\end{array}$ & 2 & 56 & $\begin{array}{l}\text { Std. Mean Difference (IV, Random, } \\
95 \% \mathrm{CI})\end{array}$ & $-0.65[-1.19,-0.10]$ \\
\hline 15 Change in participants' mood & 8 & 576 & $\begin{array}{l}\text { Std. Mean Difference (IV, Random, } \\
95 \% \mathrm{CI})\end{array}$ & $0.72[-0.10,1.54]$ \\
\hline 15.1 Traditional & 5 & 423 & $\begin{array}{l}\text { Std. Mean Difference (IV, Random, } \\
95 \% \mathrm{CI})\end{array}$ & $0.90[-0.30,2.10]$ \\
\hline 15.2 Augmented & 3 & 153 & $\begin{array}{l}\text { Std. Mean Difference (IV, Random, } \\
95 \% \mathrm{CI})\end{array}$ & $0.46[-0.94,1.86]$ \\
\hline $\begin{array}{l}16 \text { Change in capacity for activities of } \\
\text { daily living }\end{array}$ & 10 & 705 & $\begin{array}{l}\text { Std. Mean Difference (IV, Random, } \\
95 \% \mathrm{CI})\end{array}$ & $0.12[-0.10,0.34]$ \\
\hline 17 Change in disease progression & 5 & 215 & $\begin{array}{l}\text { Std. Mean Difference (IV, Random, } \\
95 \% \mathrm{CI})\end{array}$ & $1.07[0.59,1.55]$ \\
\hline $\begin{array}{l}18 \text { Change in behavioural and psycho- } \\
\text { logical symptoms of dementia (BPSD) }\end{array}$ & 6 & 493 & $\begin{array}{l}\text { Std. Mean Difference (IV, Random, } \\
95 \% \mathrm{CI})\end{array}$ & $0.44[-0.34,1.22]$ \\
\hline 19 Participant burden (retention rates) & 17 & 1282 & Odds Ratio (M-H, Random, 95\% Cl) & $0.73[0.37,1.43]$ \\
\hline 19.1 Traditional & 10 & 1017 & Odds Ratio (M-H, Random, 95\% Cl) & $0.59[0.23,1.53]$ \\
\hline 19.2 Augmented & 7 & 265 & Odds Ratio (M-H, Random, 95\% Cl) & $1.02[0.27,3.87]$ \\
\hline $\begin{array}{l}20 \text { Change in burden of care } \\
\text { (CAREGIVER) }\end{array}$ & 2 & 405 & $\begin{array}{l}\text { Std. Mean Difference (IV, Random, } \\
95 \% \mathrm{CI})\end{array}$ & $-0.11[-0.36,0.15]$ \\
\hline $\begin{array}{l}21 \text { Change in quality of life } \\
\text { (CAREGIVER) }\end{array}$ & 1 & 36 & $\begin{array}{l}\text { Std. Mean Difference (IV, Random, } \\
95 \% \mathrm{CI})\end{array}$ & $0.16[-0.50,0.83]$ \\
\hline $\begin{array}{l}22 \text { Change in mood and well-being } \\
\text { (CAREGIVER) }\end{array}$ & 1 & 36 & $\begin{array}{l}\text { Std. Mean Difference (IV, Random, } \\
95 \% \mathrm{CI})\end{array}$ & $0.98[0.27,1.68]$ \\
\hline $\begin{array}{l}23 \text { Change in general health and quali- } \\
\text { ty of life }\end{array}$ & 5 & 630 & $\begin{array}{l}\text { Std. Mean Difference (IV, Random, } \\
95 \% \mathrm{CI})\end{array}$ & $-0.04[-0.38,0.29]$ \\
\hline
\end{tabular}

\section{Analysis 8.1. Comparison 8 Cognitive training vs control immediately post intervention - type of CT (traditional vs augmented), Outcome 1 Change in a global measure of cognition.}

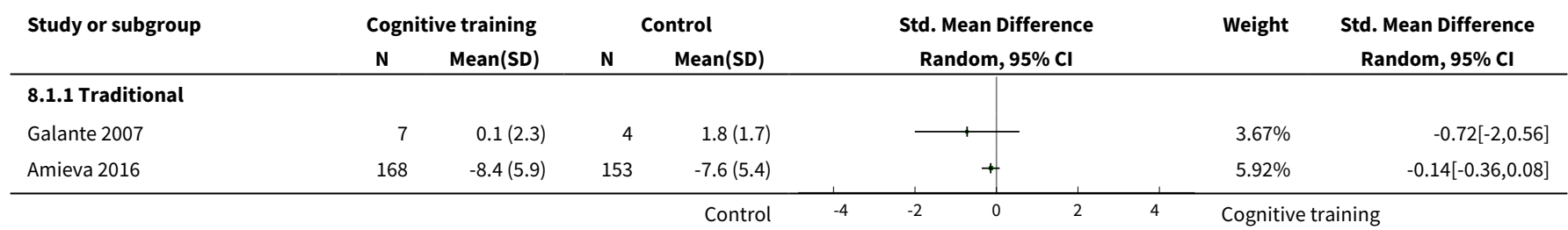




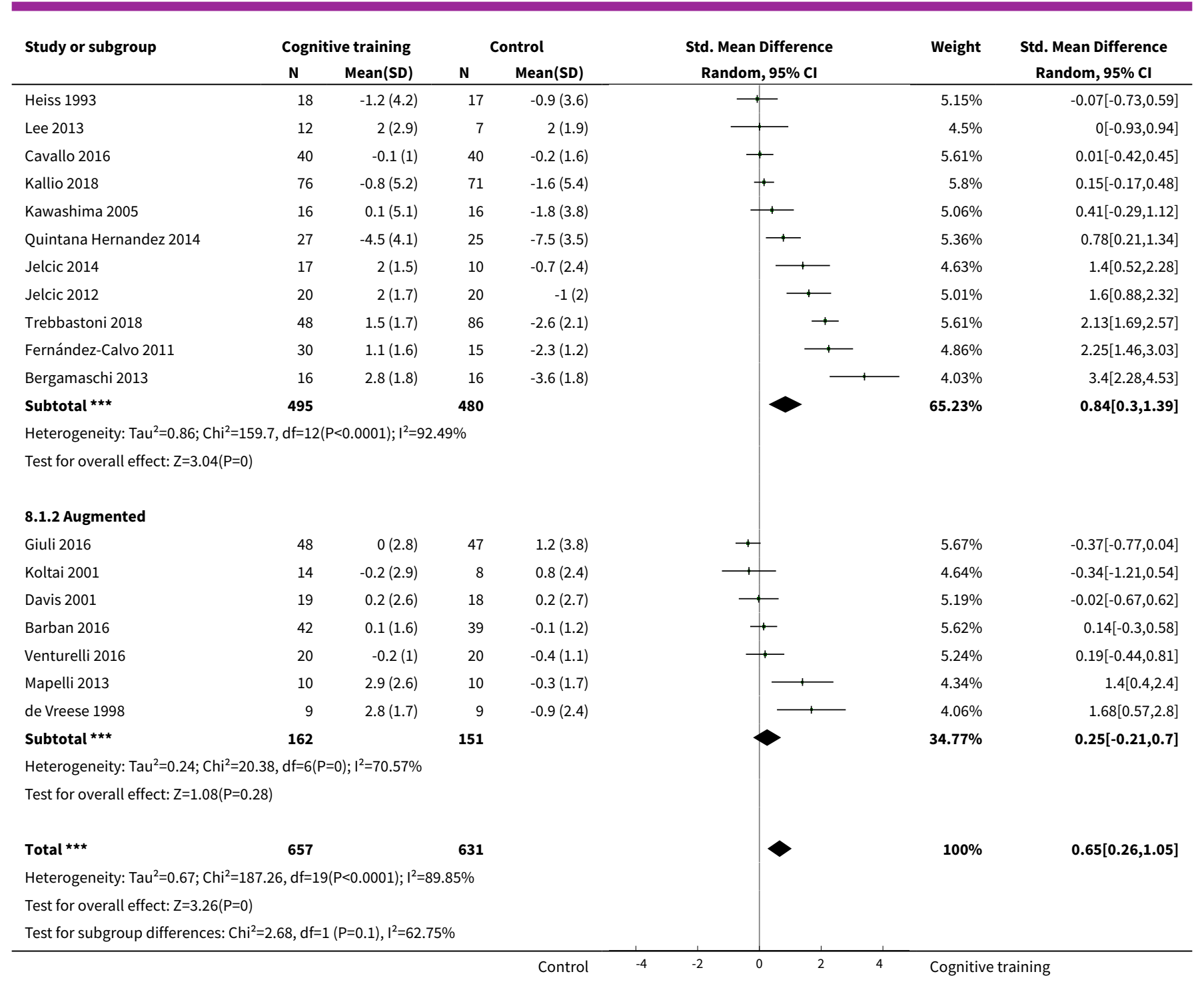

\section{Analysis 8.2. Comparison 8 Cognitive training vs control immediately post intervention - type of CT (traditional vs augmented), Outcome 2 Change in a global measure of cognition_zero correlation.}

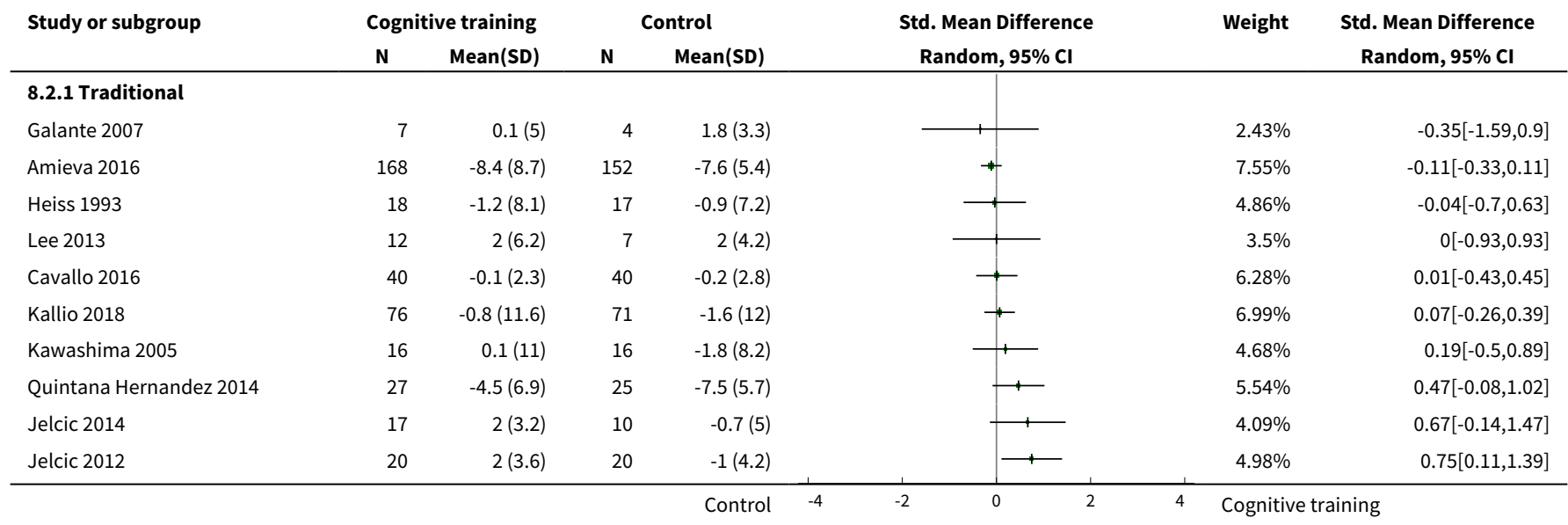




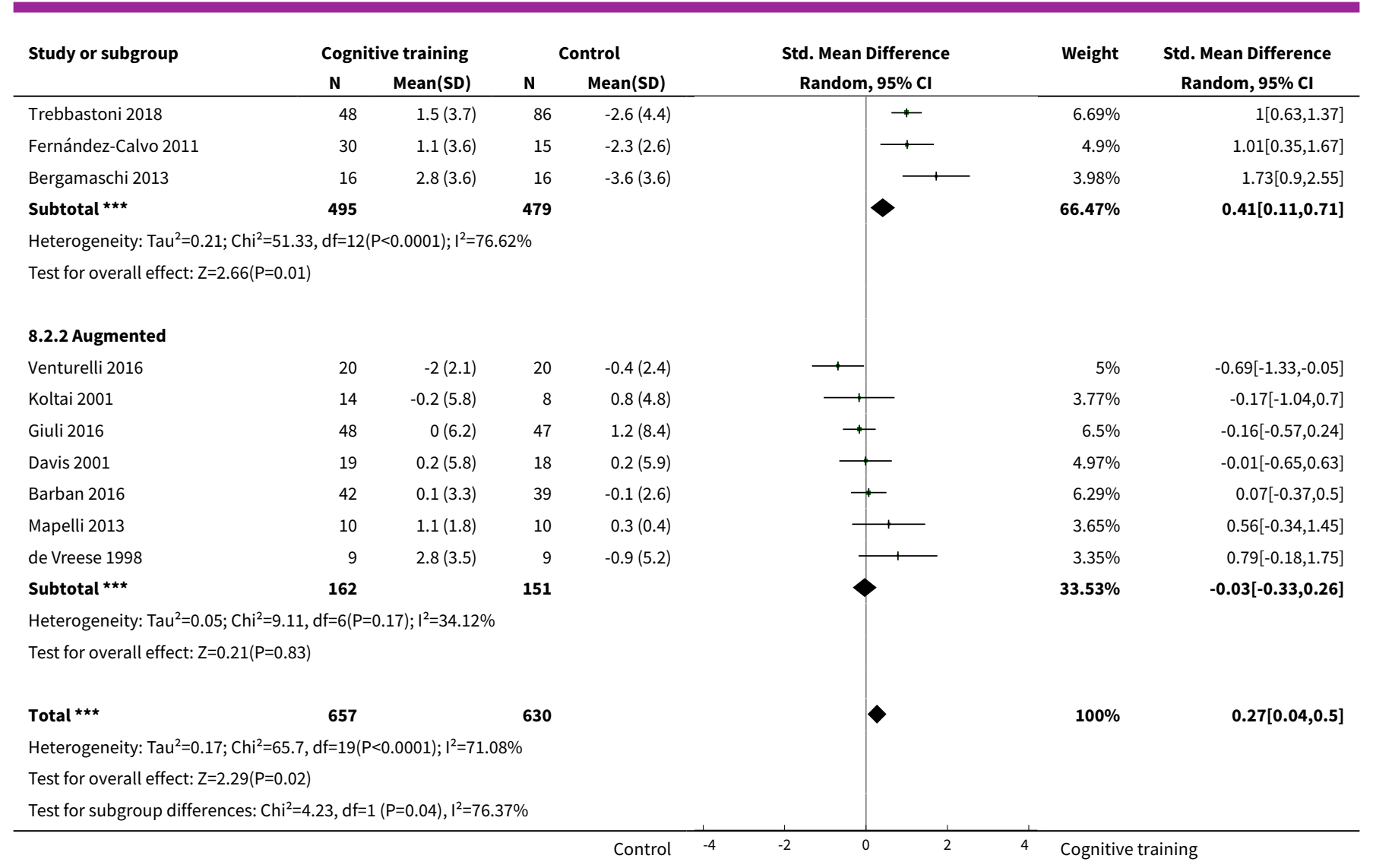

Analysis 8.3. Comparison 8 Cognitive training vs control immediately post intervention - type of CT (traditional vs augmented), Outcome 3 Change in a global measure of cognition (composite).

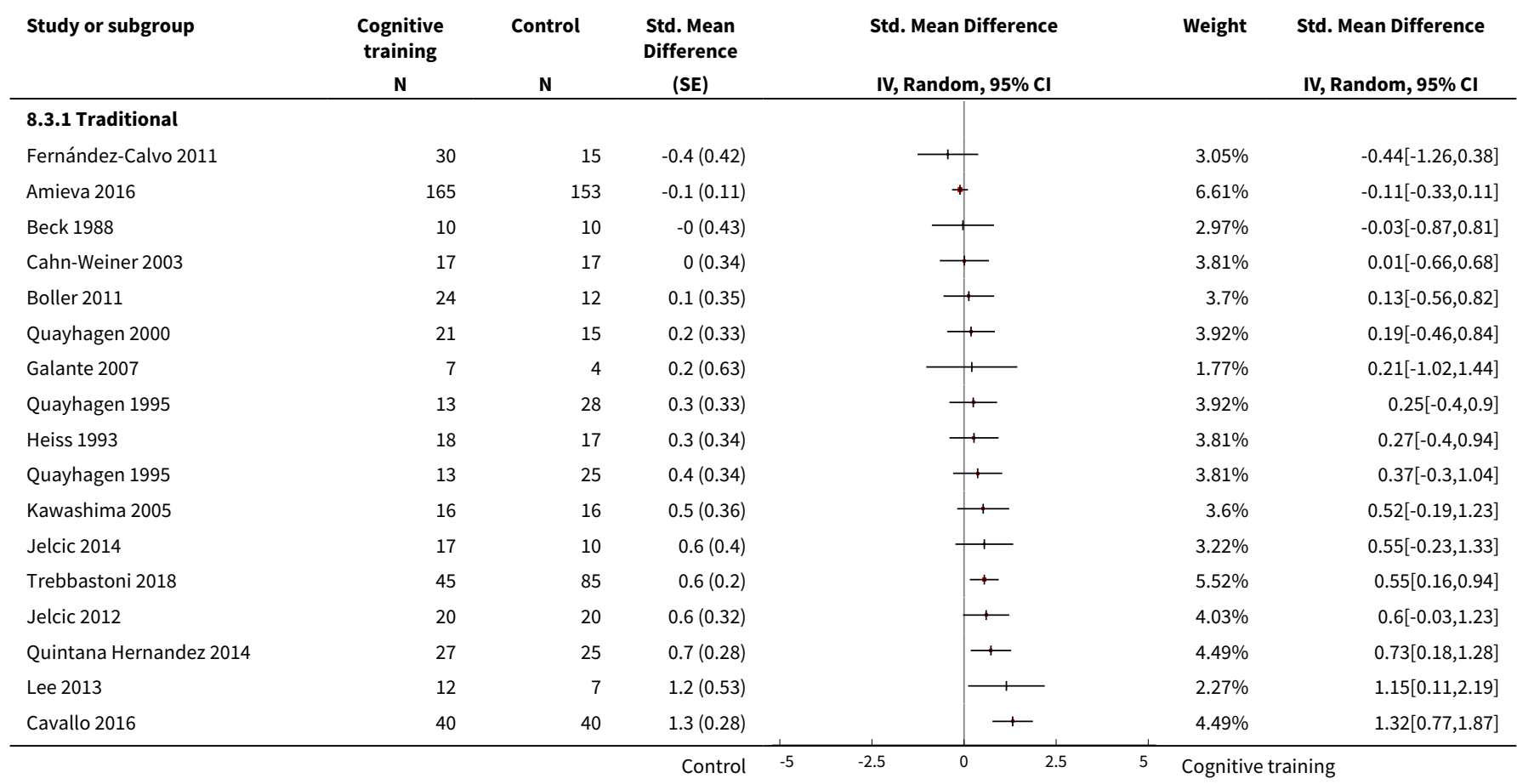




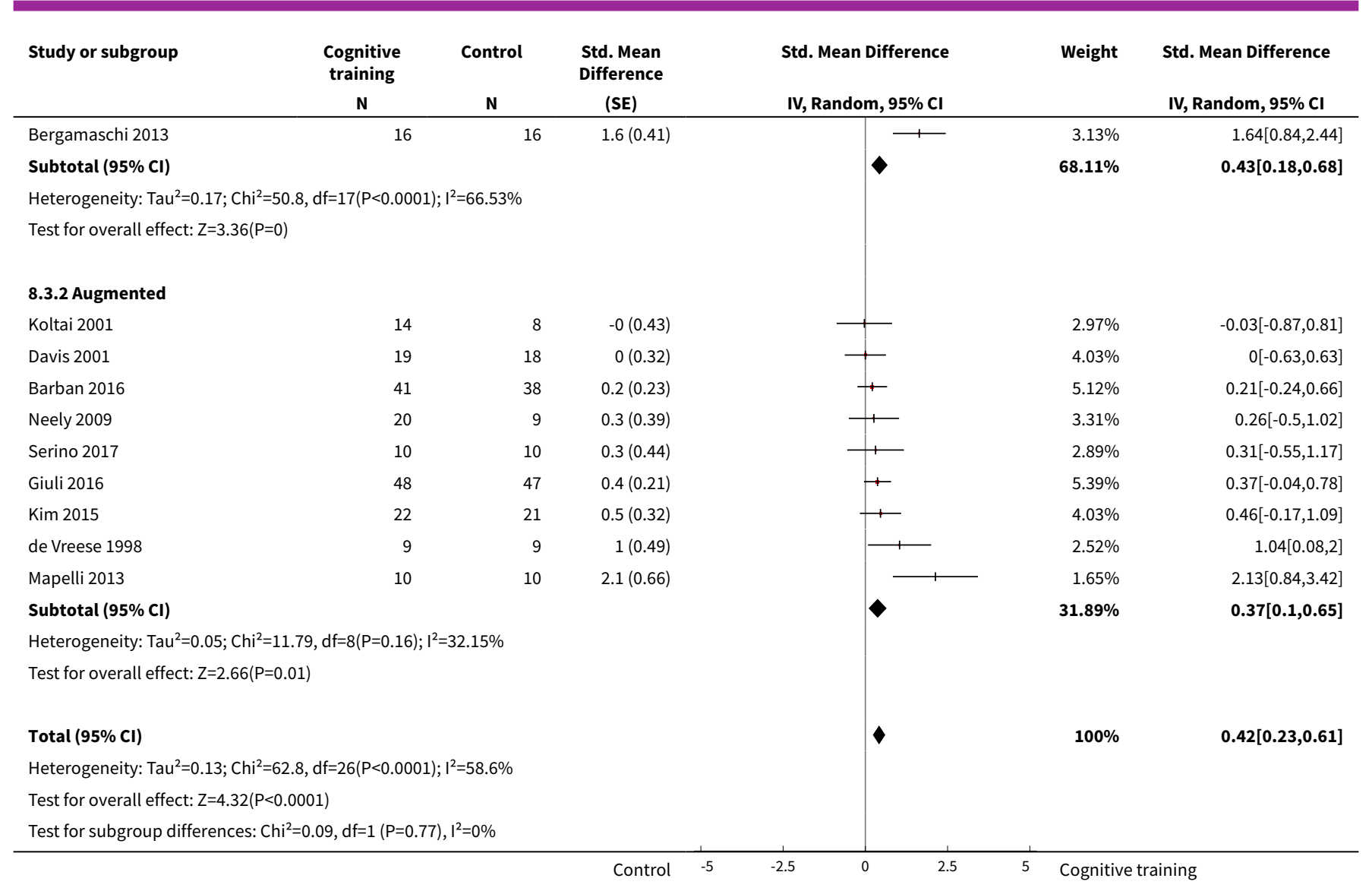

Analysis 8.4. Comparison 8 Cognitive training vs control immediately post intervention - type of CT (traditional vs augmented), Outcome 4 Change in a global measure of cognition (composite)_zero correlation.

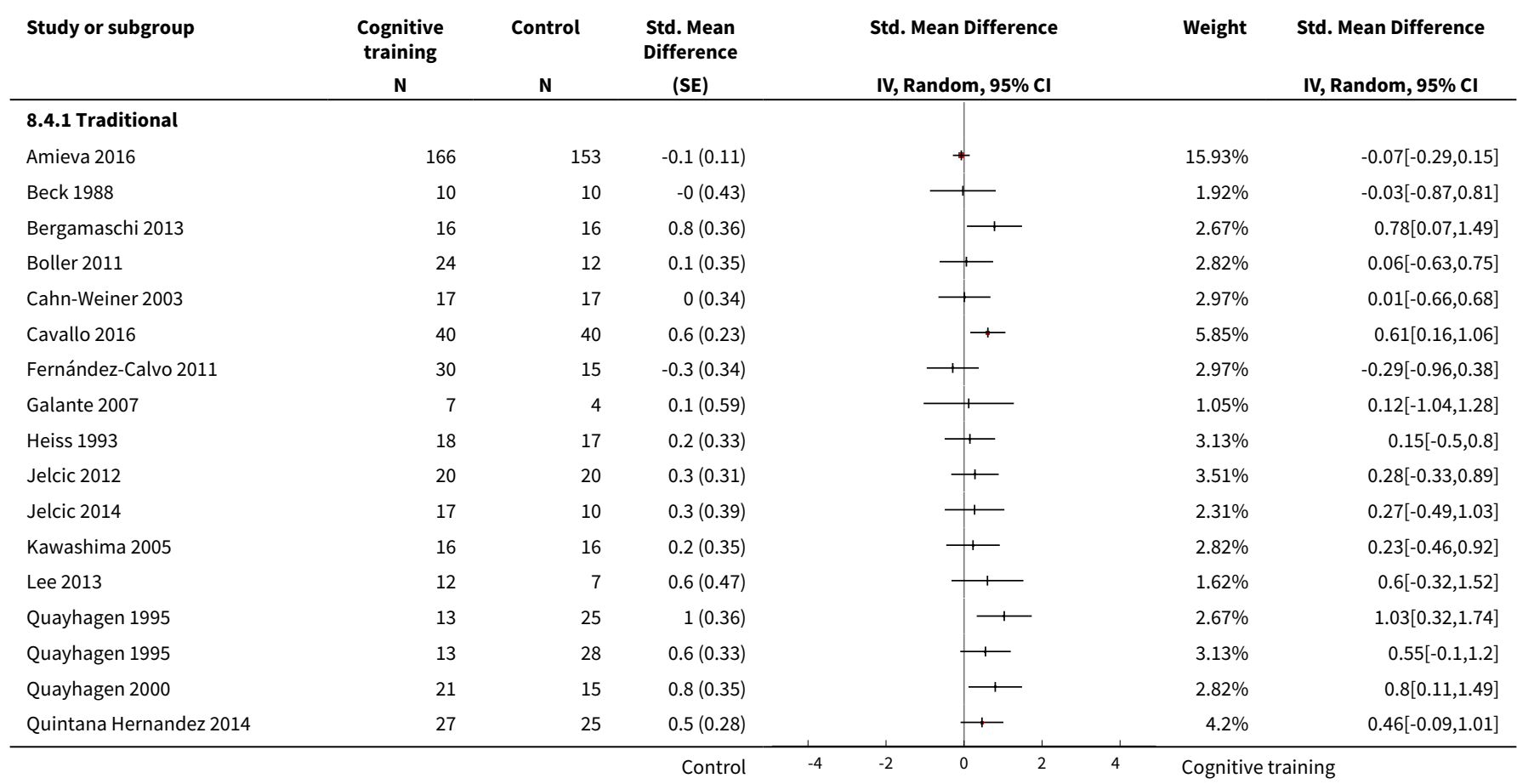




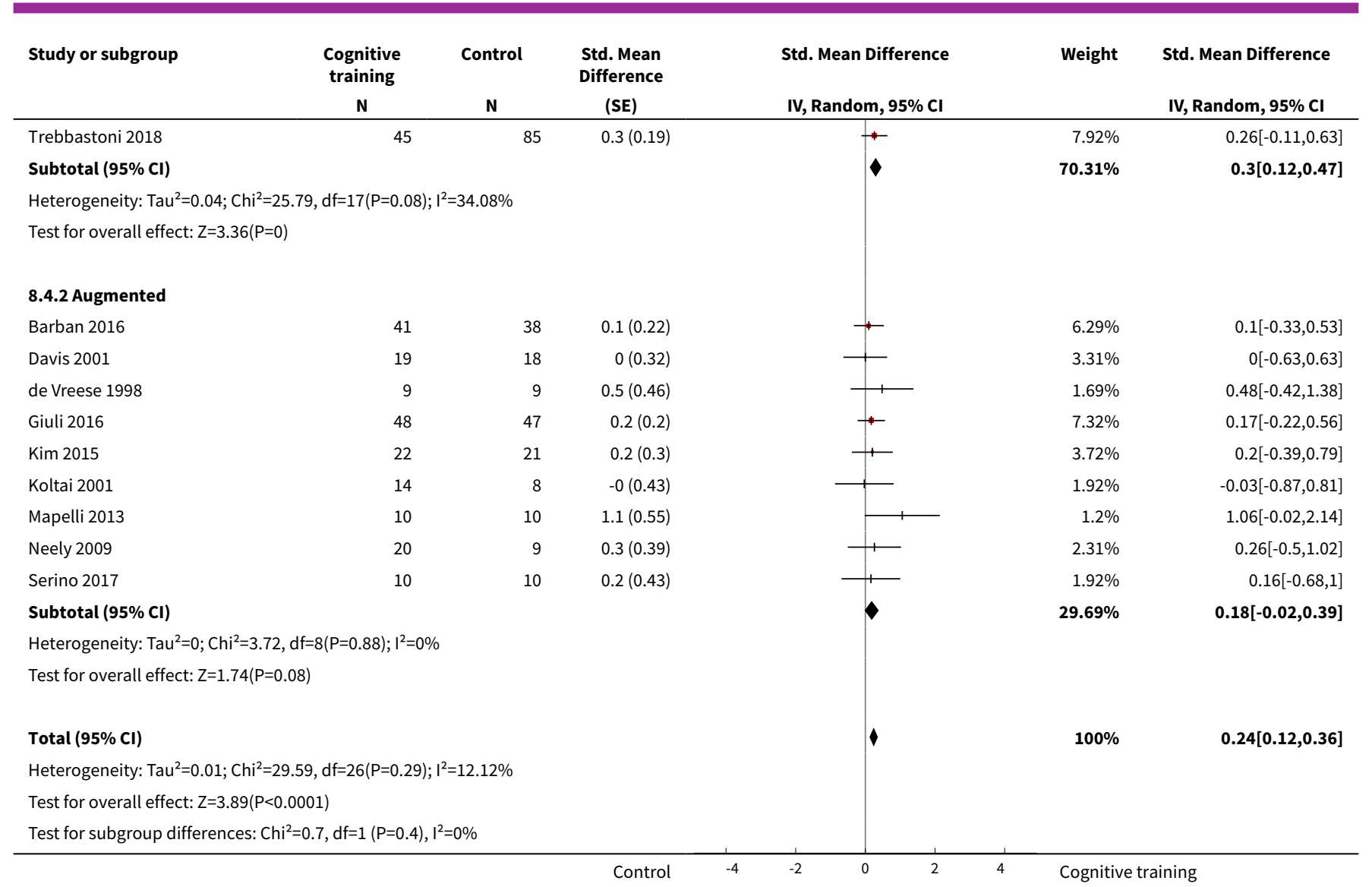

\section{Analysis 8.5. Comparison 8 Cognitive training vs control immediately post intervention - type of CT (traditional vs augmented), Outcome 5 Change in immediate memory.}

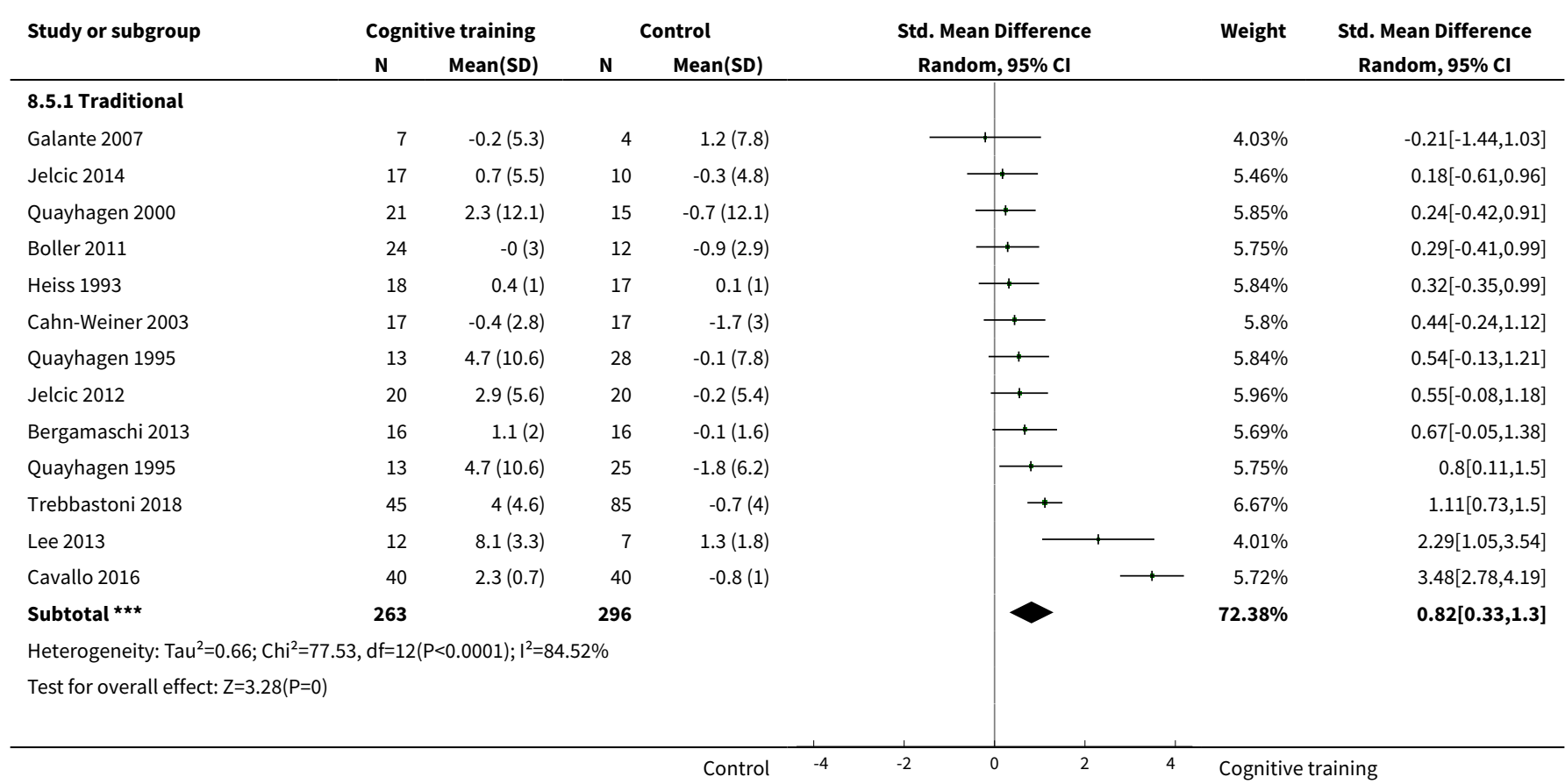




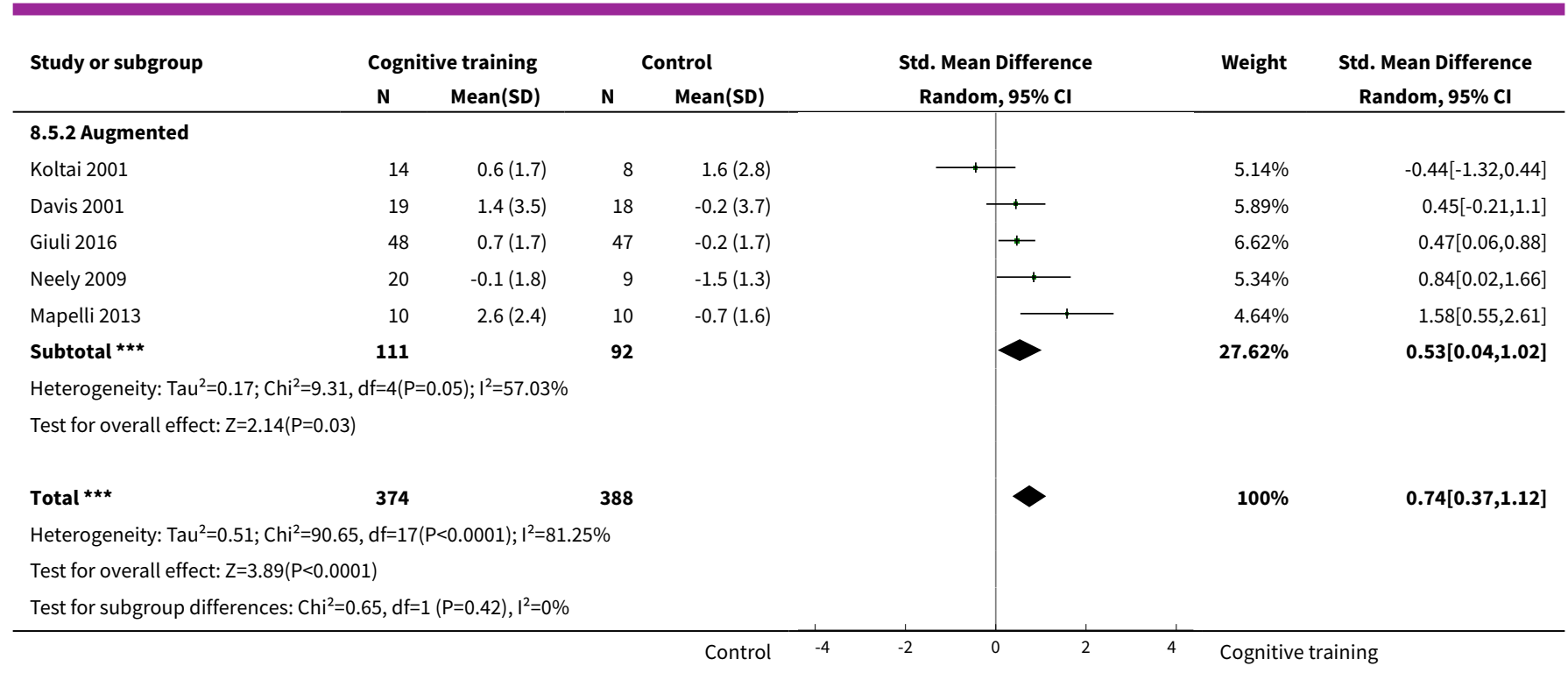

\section{Analysis 8.6. Comparison 8 Cognitive training vs control immediately post intervention - type of CT (traditional vs augmented), Outcome 6 Change in delayed memory.}

\begin{tabular}{|c|c|c|c|c|c|c|c|}
\hline \multirow[t]{2}{*}{ Study or subgroup } & \multicolumn{2}{|c|}{ Cognitive training } & \multicolumn{2}{|c|}{ Control } & \multirow{2}{*}{$\begin{array}{c}\text { Std. Mean Difference } \\
\text { Random, } 95 \% \mathrm{Cl}\end{array}$} & \multirow[t]{2}{*}{ Weight } & \multirow{2}{*}{$\begin{array}{c}\text { Std. Mean Difference } \\
\text { Random, } 95 \% \mathrm{Cl}\end{array}$} \\
\hline & $\mathbf{N}$ & $\operatorname{Mean}(S D)$ & $\mathbf{N}$ & $\operatorname{Mean}(\mathrm{SD})$ & & & \\
\hline \multicolumn{8}{|l|}{ 8.6.1 Traditional } \\
\hline Boller 2011 & 24 & $-0.3(1.6)$ & 12 & $-0.1(1.3)$ & $\div$ & $9.17 \%$ & $-0.14[-0.83,0.56]$ \\
\hline Cahn-Weiner 2003 & 17 & $-0.3(1.1)$ & 17 & $-0.8(1.6)$ & + & $9.23 \%$ & $0.36[-0.31,1.04]$ \\
\hline Quayhagen 2000 & 21 & $3.2(6.9)$ & 15 & $0.4(7.6)$ & $\rightarrow$ & $9.28 \%$ & $0.38[-0.29,1.05]$ \\
\hline Trebbastoni 2018 & 45 & $0.1(1.3)$ & 85 & $-0.5(1.4)$ & + & $10.4 \%$ & $0.46[0.09,0.82]$ \\
\hline Jelcic 2012 & 20 & $1.5(2.3)$ & 20 & $-0.9(2)$ & $\div$ & $9.28 \%$ & $1.07[0.4,1.74]$ \\
\hline Cavallo 2016 & 40 & $1.2(1)$ & 40 & $-1.7(1)$ & 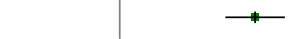 & $9.42 \%$ & $2.89[2.26,3.53]$ \\
\hline 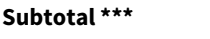 & 184 & & 199 & & & $65.33 \%$ & $0.86[0.15,1.58]$ \\
\hline \multicolumn{8}{|c|}{ Heterogeneity: $\operatorname{Tau}^{2}=0.82 ; \mathrm{Chi}^{2}=56.07, \mathrm{df}=6(\mathrm{P}<0.0001) ; \mathrm{I}^{2}=89.3 \%$} \\
\hline \multicolumn{8}{|c|}{ Test for overall effect: $Z=2.37(P=0.02)$} \\
\hline Davis 2001 & 19 & $1.2(2.2)$ & 18 & $1.7(3.7)$ & & $9.37 \%$ & $-0.16[-0.81,0.49]$ \\
\hline Barban 2016 & 42 & $0.4(1.2)$ & 39 & $-0.1(1.1)$ & + & $10.16 \%$ & $0.43[-0.01,0.88]$ \\
\hline Koltai 2001 & 14 & $0.6(1.3)$ & 8 & $-0.2(1.8)$ & & $8.3 \%$ & $0.57[-0.32,1.46]$ \\
\hline Mapelli 2013 & 10 & $4.2(2.4)$ & 10 & $-0.8(1.5)$ & $\longrightarrow$ & $6.84 \%$ & $2.44[1.22,3.65]$ \\
\hline Subtotal $\star \star \star$ & 85 & & 75 & & & $34.67 \%$ & $0.68[-0.11,1.46]$ \\
\hline \multicolumn{8}{|c|}{ Heterogeneity: $\mathrm{Tau}^{2}=0.48 ; \mathrm{Chi}^{2}=13.78, \mathrm{df}=3(\mathrm{P}=0) ; \mathrm{I}^{2}=78.23 \%$} \\
\hline \multicolumn{8}{|c|}{ Test for overall effect: $Z=1.69(P=0.09)$} \\
\hline 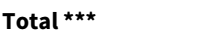 & 269 & & 274 & & & $100 \%$ & $0.81[0.29,1.32]$ \\
\hline \multicolumn{8}{|c|}{ Heterogeneity: $\mathrm{Tau}^{2}=0.64 ; \mathrm{Chi}^{2}=72.73, \mathrm{df}=10(\mathrm{P}<0.0001) ; \mathrm{I}^{2}=86.25 \%$} \\
\hline \multicolumn{8}{|c|}{ Test for overall effect: $Z=3.05(P=0)$} \\
\hline Test for subgroup dif & $12, \mathrm{df}=$ & $P=0.73), I^{2}=0$ & & & & & \\
\hline
\end{tabular}


Analysis 8.7. Comparison 8 Cognitive training vs control immediately post intervention type of CT (traditional vs augmented), Outcome 7 Change in attention and working memory.

\begin{tabular}{|c|c|c|c|c|c|c|c|}
\hline \multirow{3}{*}{$\begin{array}{l}\text { Study or subgroup } \\
\text { 8.7.1 Traditional }\end{array}$} & \multicolumn{2}{|c|}{ Cognitive training } & \multicolumn{2}{|c|}{ Control } & \multirow{2}{*}{$\begin{array}{c}\text { Std. Mean Difference } \\
\text { Random, } 95 \% \mathrm{Cl}\end{array}$} & \multirow[t]{2}{*}{ Weight } & \multirow{2}{*}{$\begin{array}{c}\text { Std. Mean Difference } \\
\text { Random, } 95 \% \mathrm{Cl}\end{array}$} \\
\hline & \multirow[t]{2}{*}{$\mathbf{N}$} & \multirow[t]{2}{*}{ Mean(SD) } & \multirow[t]{2}{*}{$\mathbf{N}$} & \multirow[t]{2}{*}{ Mean(SD) } & & & \\
\hline & & & & & & & \\
\hline Heiss 1993 & 18 & $-0.6(1)$ & 17 & $-0(1)$ & $\longrightarrow$ & $8.68 \%$ & $-0.6[-1.28,0.08]$ \\
\hline Galante 2007 & 7 & $0.1(0.7)$ & 4 & $0.7(1.5)$ & $\longrightarrow$ & $6.15 \%$ & $-0.53[-1.79,0.73]$ \\
\hline Trebbastoni 2018 & 45 & $-0.3(0.6)$ & 85 & $-0.2(0.7)$ & & $9.88 \%$ & $-0.1[-0.46,0.27]$ \\
\hline Boller 2011 & 24 & $0(1.1)$ & 12 & $0.1(0.9)$ & & $8.62 \%$ & $-0.07[-0.77,0.62]$ \\
\hline Jelcic 2012 & 20 & $0.2(0.6)$ & 20 & $-0.4(0.6)$ & 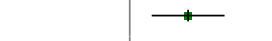 & $8.73 \%$ & $1.07[0.4,1.74]$ \\
\hline Jelcic 2014 & 17 & $0.3(1)$ & 10 & $-1.3(0.6)$ & $\longrightarrow$ & $7.49 \%$ & $1.85[0.9,2.8]$ \\
\hline Cavallo 2016 & 40 & $1.9(1)$ & 40 & $-0(0.9)$ & $\longrightarrow$ & $9.24 \%$ & $2.03[1.49,2.57]$ \\
\hline Subtotal $\star \star \star$ & 181 & & 198 & & & $66.42 \%$ & $0.56[-0.17,1.3]$ \\
\hline \multicolumn{8}{|c|}{ Heterogeneity: Tau $^{2}=0.96 ; \mathrm{Chi}^{2}=66.97, \mathrm{df}=7(\mathrm{P}<0.0001) ; \mathrm{I}^{2}=89.55 \%$} \\
\hline \multicolumn{8}{|c|}{ Test for overall effect: $Z=1.5(P=0.13)$} \\
\hline \multicolumn{8}{|l|}{ 8.7.2 Augmented } \\
\hline Davis 2001 & 19 & $-0.4(1.6)$ & 18 & $-0.5(1.6)$ & + & $8.83 \%$ & $0.04[-0.61,0.68]$ \\
\hline Serino 2017 & 10 & $0(0.9)$ & 10 & $-0.3(0.6)$ & + & $7.75 \%$ & $0.41[-0.47,1.3]$ \\
\hline Giuli 2016 & 48 & $0.3(0.9)$ & 47 & $-0.2(1)$ & $\rightarrow$ & $9.73 \%$ & $0.55[0.14,0.96]$ \\
\hline Mapelli 2013 & 10 & $1.7(1.2)$ & 10 & $-0.1(1.3)$ & $\longrightarrow$ & $7.27 \%$ & $1.36[0.37,2.36]$ \\
\hline Subtotal $\star \star \star$ & 87 & & 85 & & & $33.58 \%$ & $0.51[0.07,0.95]$ \\
\hline \multicolumn{8}{|c|}{ Heterogeneity: $\mathrm{Tau}^{2}=0.08 ; \mathrm{Chi}^{2}=4.96, \mathrm{df}=3(\mathrm{P}=0.17) ; \mathrm{I}^{2}=39.56 \%$} \\
\hline \multicolumn{8}{|c|}{ Test for overall effect: $Z=2.29(P=0.02)$} \\
\hline 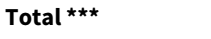 & 268 & & 283 & & & $100 \%$ & $0.56[0.08,1.05]$ \\
\hline \multicolumn{8}{|c|}{ Heterogeneity: Tau $^{2}=0.59 ; \mathrm{Chi}^{2}=71.95, \mathrm{df}=11(\mathrm{P}<0.0001) ; \mathrm{I}^{2}=84.71 \%$} \\
\hline \multicolumn{8}{|c|}{ Test for overall effect: $Z=2.27(P=0.02)$} \\
\hline \multicolumn{8}{|c|}{ Test for subgroup differences: $\mathrm{Chi}^{2}=0.01, \mathrm{df}=1(\mathrm{P}=0.91), \mathrm{I}^{2}=0 \%$} \\
\hline
\end{tabular}

Analysis 8.8. Comparison 8 Cognitive training vs control immediately post intervention - type of CT (traditional vs augmented), Outcome 8 Change in language (naming).

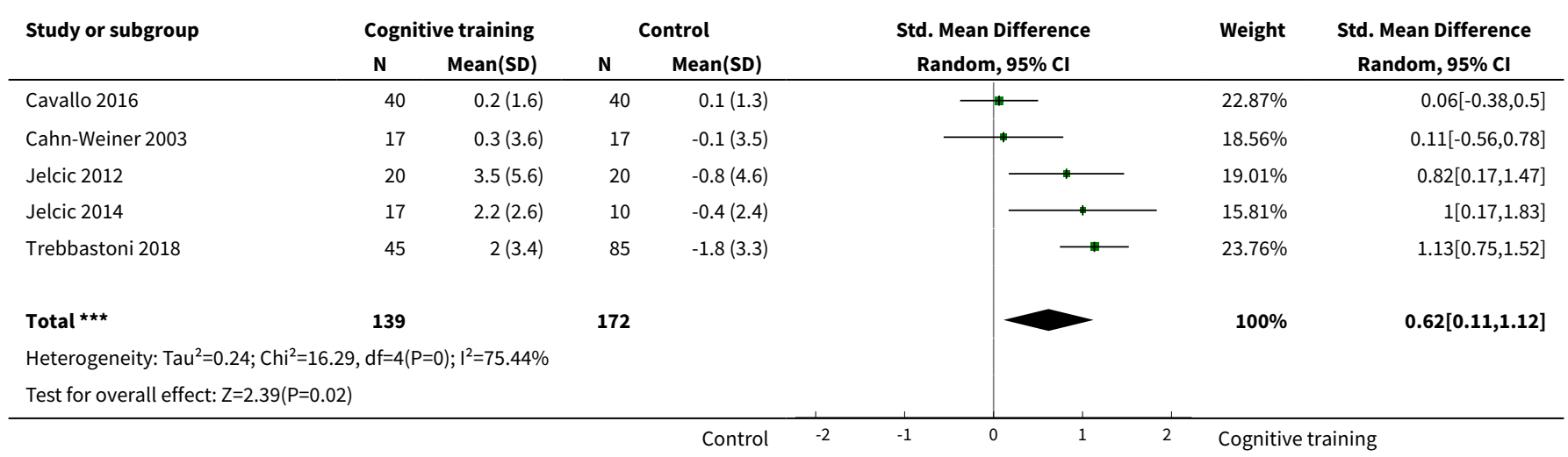


Analysis 8.9. Comparison 8 Cognitive training vs control immediately post intervention - type of CT (traditional vs augmented), Outcome 9 Change in verbal letter fluency.

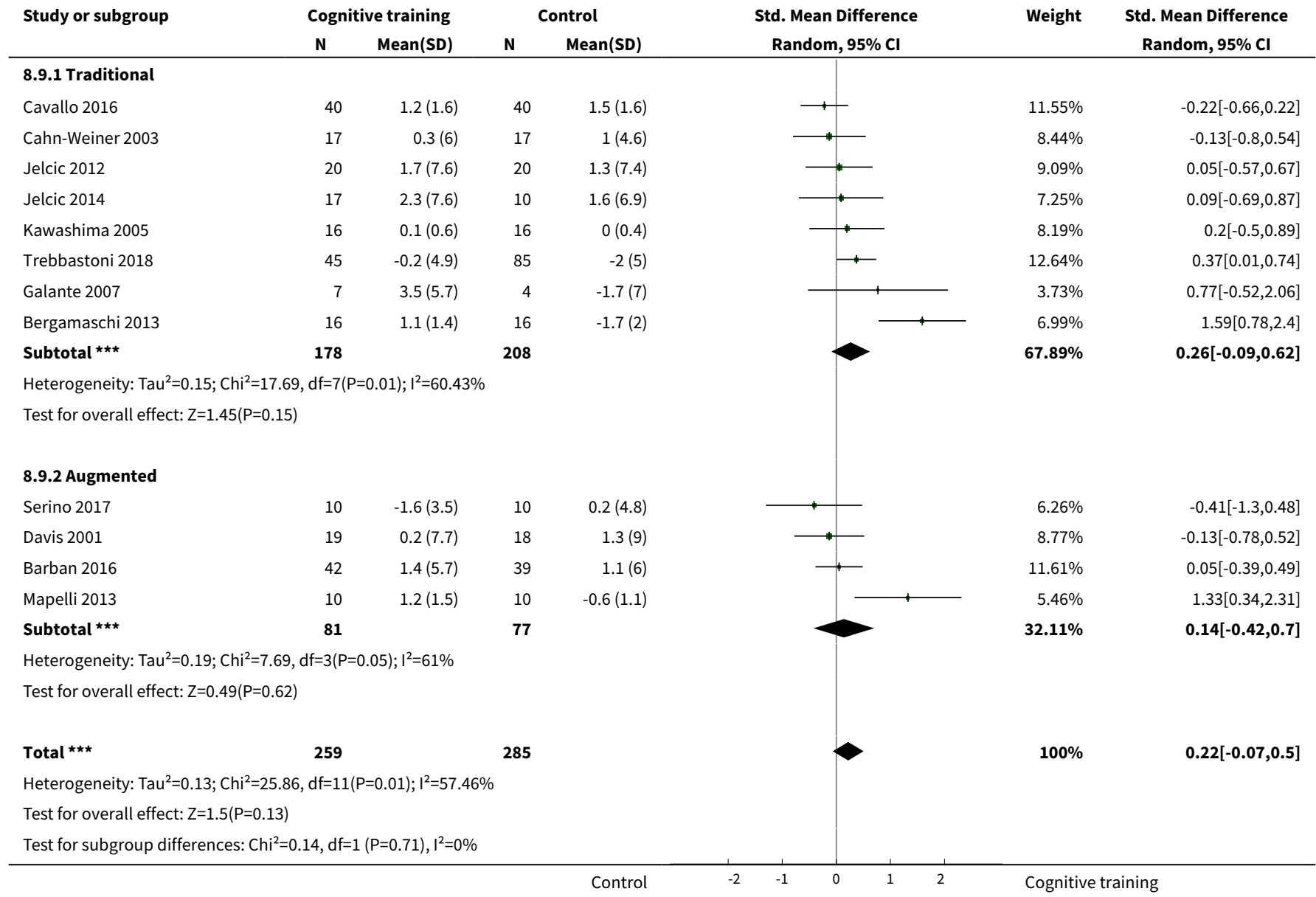

Analysis 8.10. Comparison 8 Cognitive training vs control immediately post intervention type of CT (traditional vs augmented), Outcome 10 Change in speed of information processing.

\begin{tabular}{|c|c|c|c|c|c|c|c|}
\hline \multirow[t]{2}{*}{ Study or subgroup } & \multicolumn{2}{|c|}{ Cognitive training } & \multicolumn{2}{|c|}{ Control } & \multirow{2}{*}{$\begin{array}{c}\text { Std. Mean Difference } \\
\text { Random, } 95 \% \mathrm{CI}\end{array}$} & \multirow[t]{2}{*}{ Weight } & \multirow{2}{*}{$\begin{array}{c}\text { Std. Mean Difference } \\
\text { Random, } 95 \% \mathrm{CI}\end{array}$} \\
\hline & $\mathbf{N}$ & Mean(SD) & $\mathbf{N}$ & Mean(SD) & & & \\
\hline Beck 1988 & 10 & $-0.3(1.3)$ & 10 & $1.1(2.9)$ & $\longrightarrow$ & $11.31 \%$ & $-0.6[-1.51,0.3]$ \\
\hline Galante 2007 & 7 & $-1.3(10.3)$ & 4 & $3.3(5.6)$ & & $6.26 \%$ & $-0.47[-1.72,0.79]$ \\
\hline Jelcic 2014 & 17 & $0.5(6.9)$ & 10 & $-0.4(8.2)$ & & $14.38 \%$ & $0.11[-0.67,0.9]$ \\
\hline Barban 2016 & 36 & $7.7(27.4)$ & 33 & $-1.9(27.7)$ & - & $30.03 \%$ & $0.35[-0.13,0.82]$ \\
\hline Jelcic 2012 & 20 & $19.3(56.5)$ & 20 & $-2(20.6)$ & 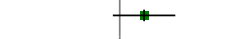 & $20.26 \%$ & $0.49[-0.14,1.12]$ \\
\hline Cahn-Weiner 2003 & 17 & $5.2(20.6)$ & 17 & $-9.6(31.4)$ & 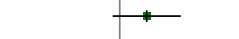 & $17.76 \%$ & $0.54[-0.14,1.23]$ \\
\hline Total $\star \star \star$ & 107 & & 94 & & & $100 \%$ & $0.22[-0.11,0.54]$ \\
\hline \multicolumn{8}{|c|}{ Heterogeneity: $\mathrm{Tau}^{2}=0.03 ; \mathrm{Chi}^{2}=6.24, \mathrm{df}=5(\mathrm{P}=0.28) ; \mathrm{I}^{2}=19.89 \%$} \\
\hline \multicolumn{3}{|c|}{ Test for overall effect: $Z=1.32(P=0.19)$} & & & & & \\
\hline
\end{tabular}


Analysis 8.11. Comparison 8 Cognitive training vs control immediately post intervention - type of CT (traditional vs augmented), Outcome 11 Change in executive function.

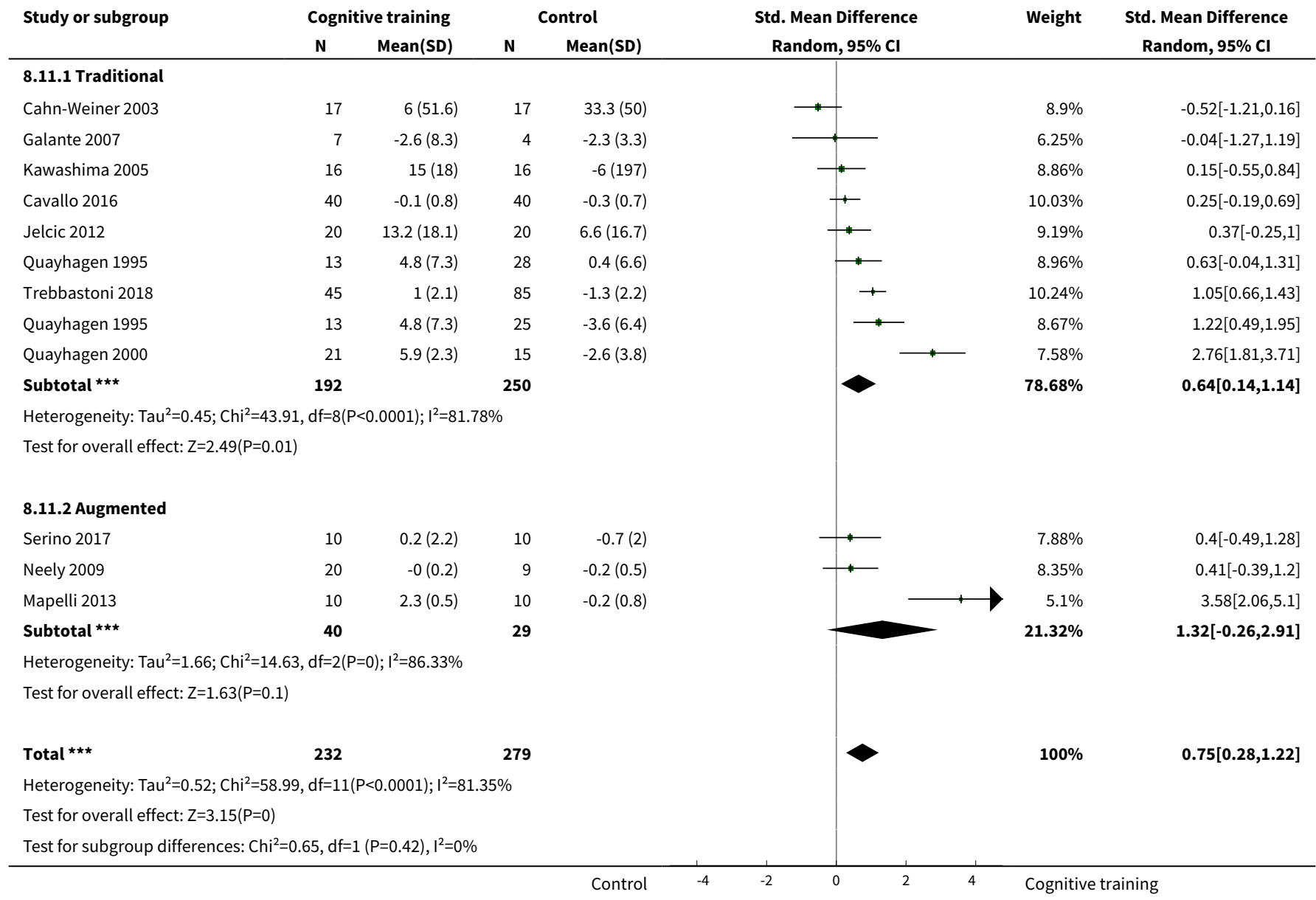

Analysis 8.12. Comparison 8 Cognitive training vs control immediately post intervention - type of CT (traditional vs augmented), Outcome 12 Change in verbal category fluency.

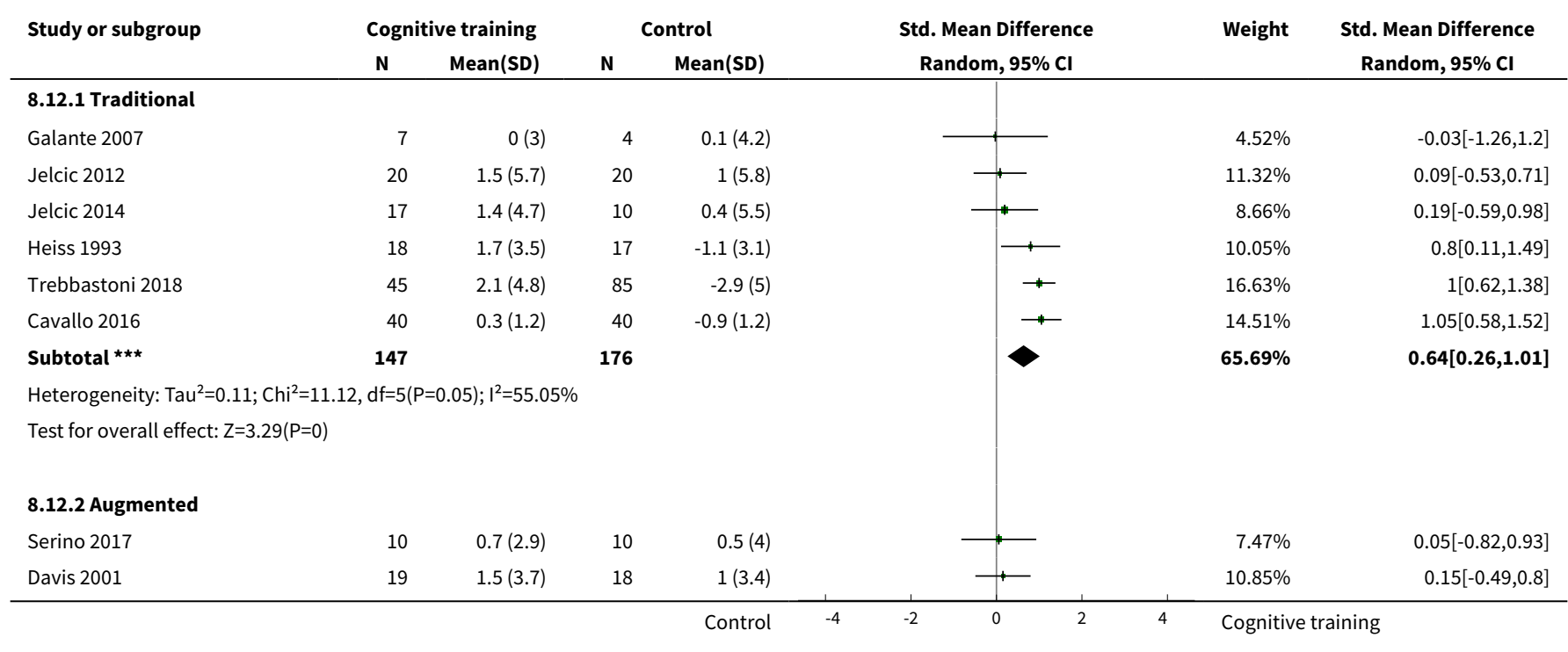




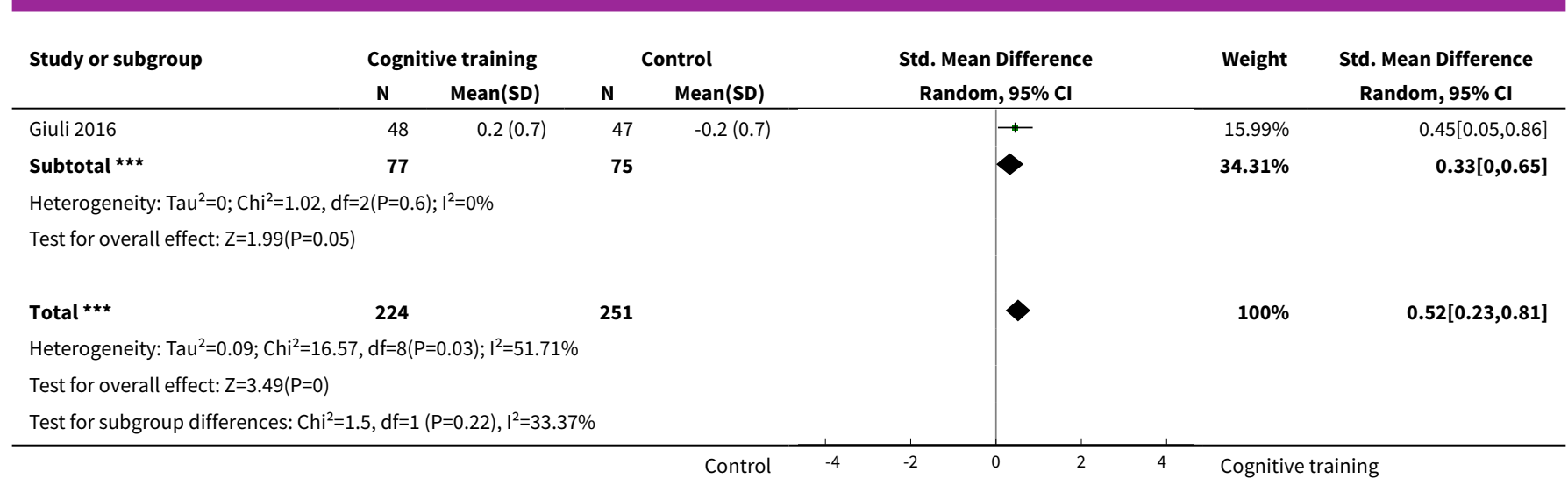

Analysis 8.13. Comparison 8 Cognitive training vs control immediately post intervention type of CT (traditional vs augmented), Outcome 13 Change in meta cognition (self-reported).

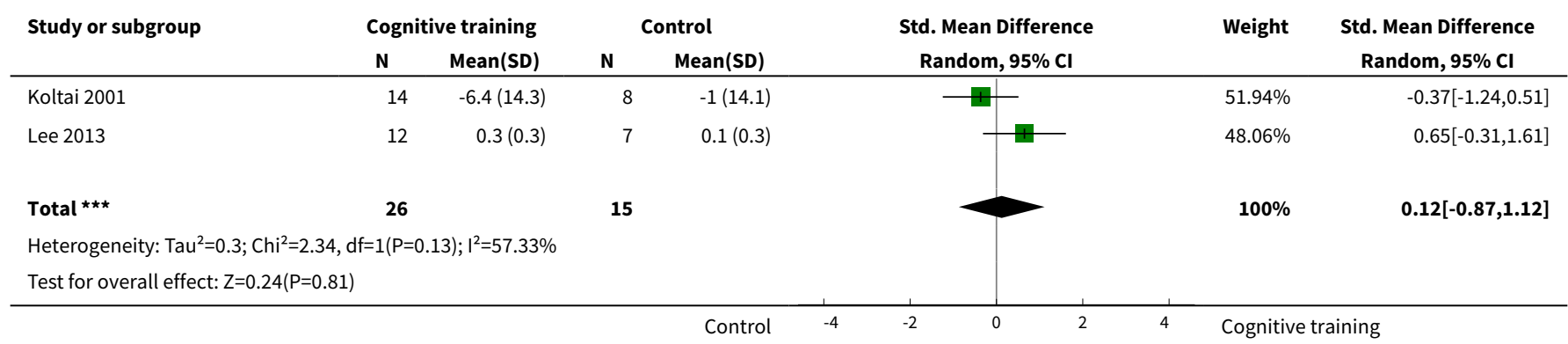

Analysis 8.14. Comparison 8 Cognitive training vs control immediately post intervention - type of CT (traditional vs augmented), Outcome 14 Change in meta cognition (informant-reported).

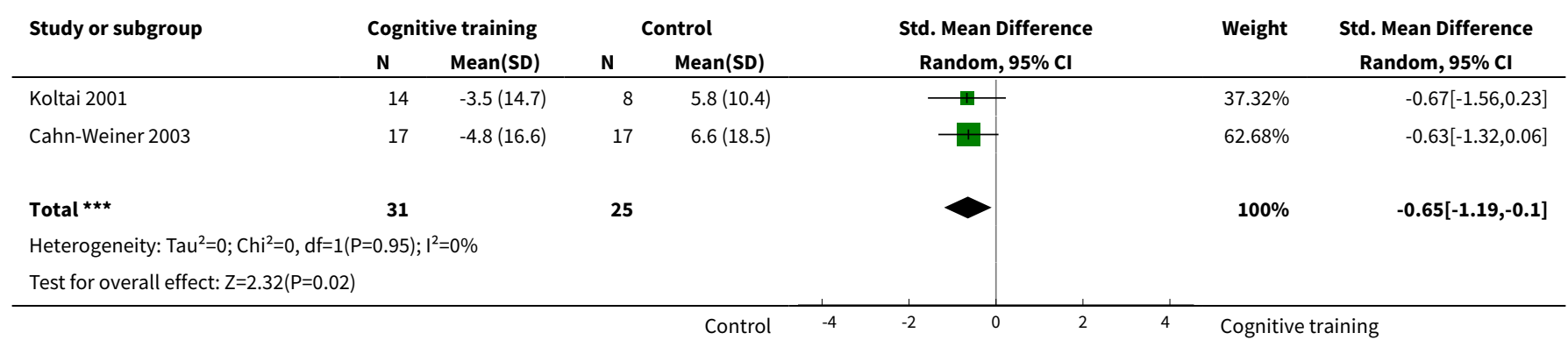

Analysis 8.15. Comparison 8 Cognitive training vs control immediately post intervention - type of CT (traditional vs augmented), Outcome 15 Change in participants' mood.

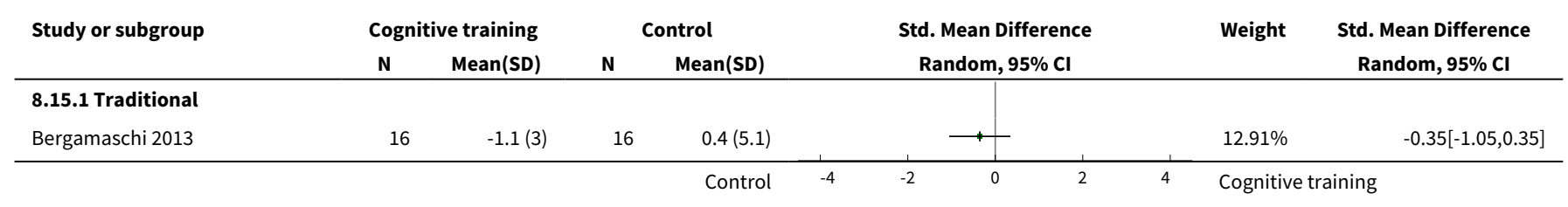




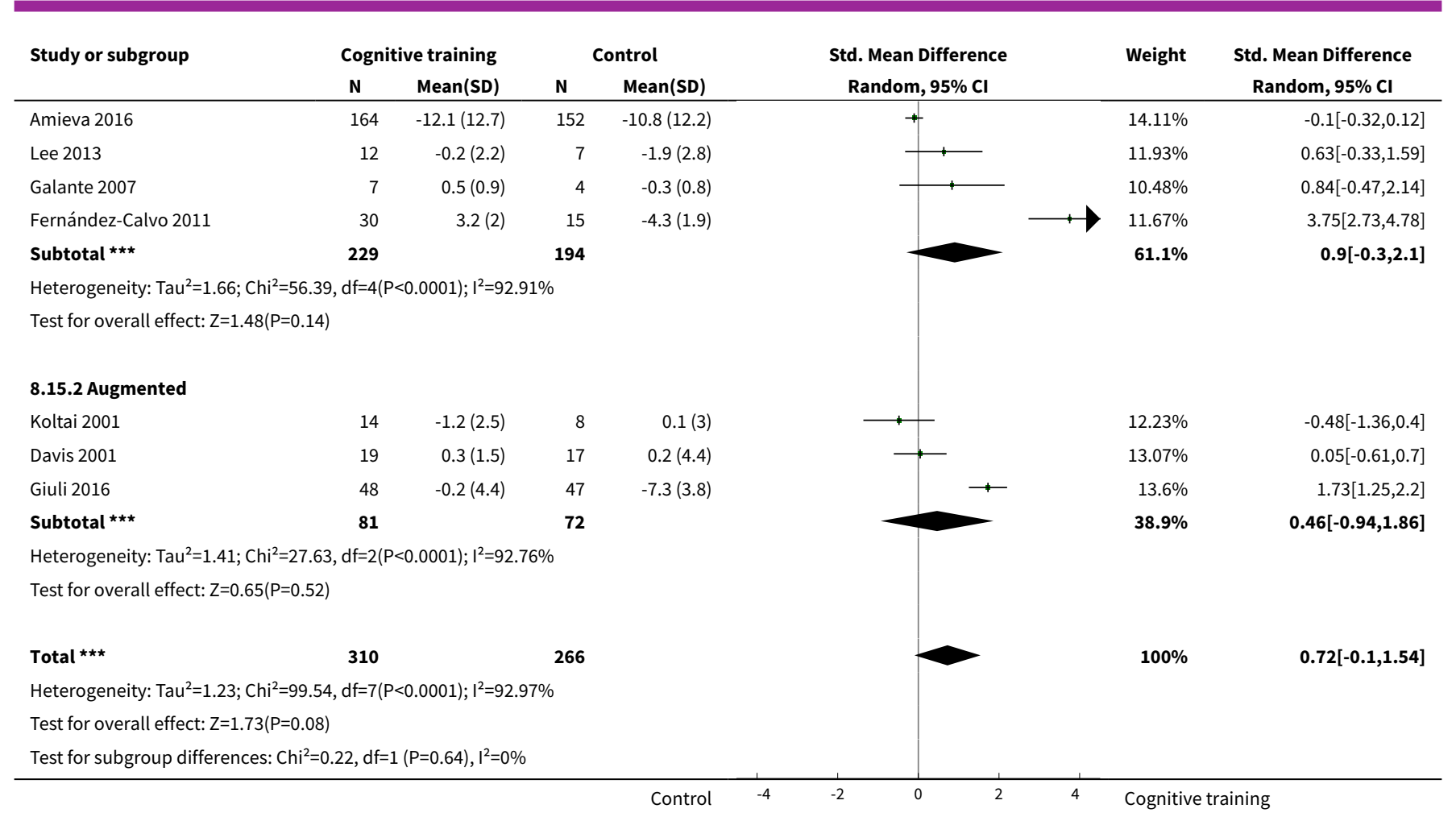

\section{Analysis 8.16. Comparison 8 Cognitive training vs control immediately post intervention - type of CT (traditional vs augmented), Outcome 16 Change in capacity for activities of daily living.}

\begin{tabular}{|c|c|c|c|c|c|c|c|}
\hline \multirow[t]{2}{*}{ Study or subgroup } & \multicolumn{2}{|c|}{ Cognitive training } & \multicolumn{2}{|c|}{ Control } & \multirow{2}{*}{$\begin{array}{c}\text { Std. Mean Difference } \\
\text { Random, } 95 \% \mathrm{Cl}\end{array}$} & \multirow[t]{2}{*}{ Weight } & \multirow{2}{*}{$\begin{array}{c}\text { Std. Mean Difference } \\
\text { Random, } 95 \% \mathrm{Cl}\end{array}$} \\
\hline & $\mathbf{N}$ & Mean(SD) & $\mathbf{N}$ & $\operatorname{Mean}(\mathrm{SD})$ & & & \\
\hline Galante 2007 & 7 & $-0.6(1.4)$ & 4 & $0.5(0.5)$ & - & $2.63 \%$ & $-0.88[-2.19,0.43]$ \\
\hline de Vreese 1998 & 9 & $-1.3(4.4)$ & 9 & $1(4)$ & \begin{tabular}{l|l}
1 \\
+
\end{tabular} & $4.7 \%$ & $-0.53[-1.48,0.41]$ \\
\hline Amieva 2016 & 167 & $-1.8(8.5)$ & 153 & $-1.2(8.3)$ & & $23.78 \%$ & $-0.06[-0.28,0.16]$ \\
\hline Cahn-Weiner 2003 & 17 & $-0.2(3.2)$ & 17 & $0(3.1)$ & $\longrightarrow$ & $8.09 \%$ & $-0.06[-0.74,0.61]$ \\
\hline Kim 2015 & 21 & $0(0.9)$ & 21 & $0(1.1)$ & 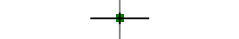 & $9.41 \%$ & $0[-0.6,0.6]$ \\
\hline Barban 2016 & 38 & $-0.1(1)$ & 38 & $-0.3(1.1)$ & + & $13.63 \%$ & $0.19[-0.26,0.64]$ \\
\hline Giuli 2016 & 48 & $0.3(1.2)$ & 47 & $-0.2(1.3)$ & + & $15.22 \%$ & $0.4[-0,0.81]$ \\
\hline Bergamaschi 2013 & 16 & $-0.4(1.5)$ & 16 & $-1.3(1.5)$ & 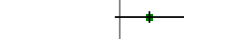 & $7.44 \%$ & $0.61[-0.1,1.32]$ \\
\hline Lee 2013 & 12 & $2.2(3.3)$ & 7 & $-2.1(4.5)$ & $\longrightarrow$ & $4.18 \%$ & $1.1[0.09,2.11]$ \\
\hline Total *** & 355 & & 350 & & 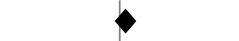 & $100 \%$ & $0.12[-0.1,0.34]$ \\
\hline \multicolumn{8}{|c|}{ Test for overall effect: $Z=1.05(P=0.29)$} \\
\hline
\end{tabular}


Analysis 8.17. Comparison 8 Cognitive training vs control immediately post intervention - type of CT (traditional vs augmented), Outcome 17 Change in disease progression.

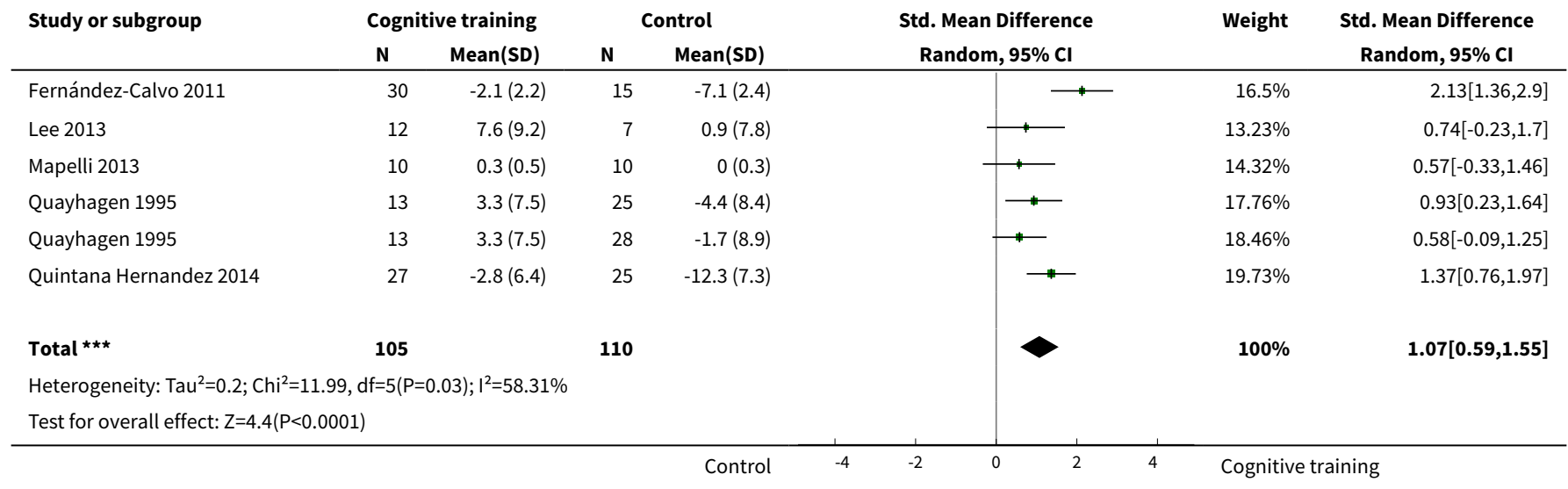

Analysis 8.18. Comparison 8 Cognitive training vs control immediately post intervention - type of CT (traditional vs augmented), Outcome 18 Change in behavioural and psychological symptoms of dementia (BPSD).

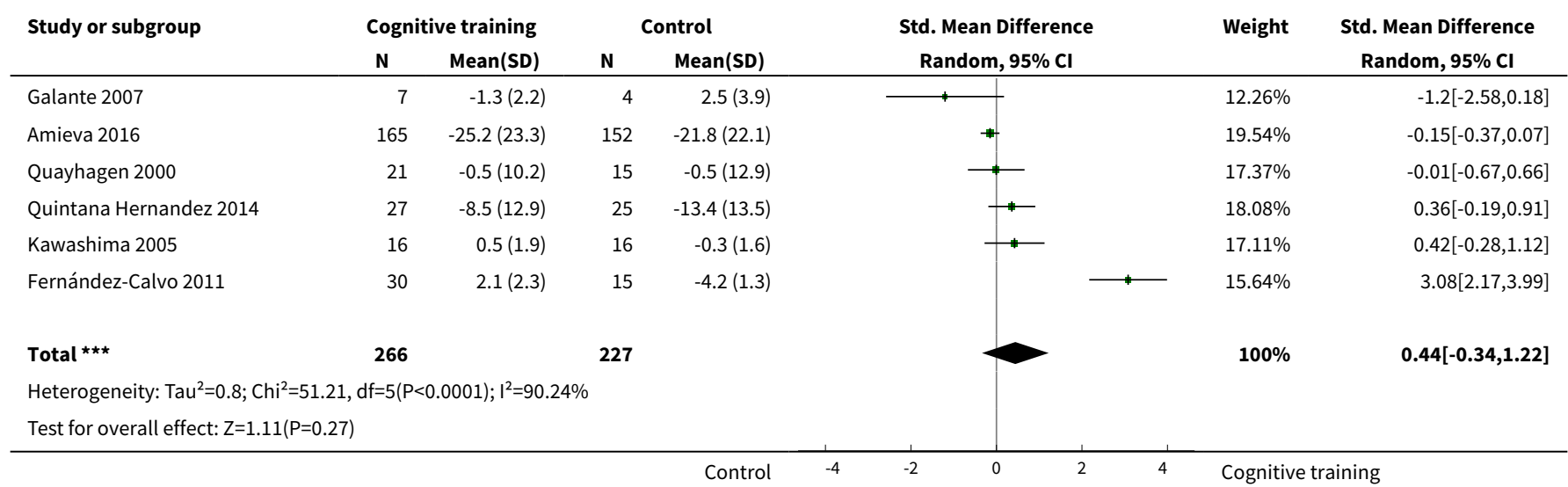

Analysis 8.19. Comparison 8 Cognitive training vs control immediately post intervention - type of CT (traditional vs augmented), Outcome 19 Participant burden (retention rates).

\begin{tabular}{ccccc} 
Study or subgroup & $\begin{array}{c}\text { Cognitive } \\
\text { training } \\
\mathrm{n} / \mathrm{N}\end{array}$ & $\mathrm{n} / \mathrm{N}$ & Odds Ratio & Odds Ratio \\
\hline
\end{tabular}

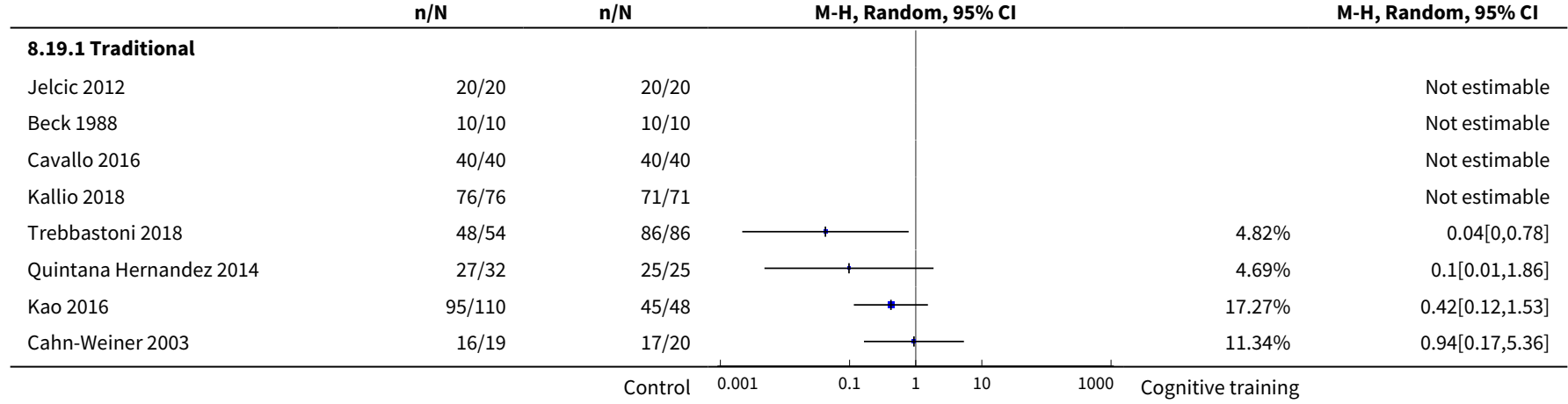




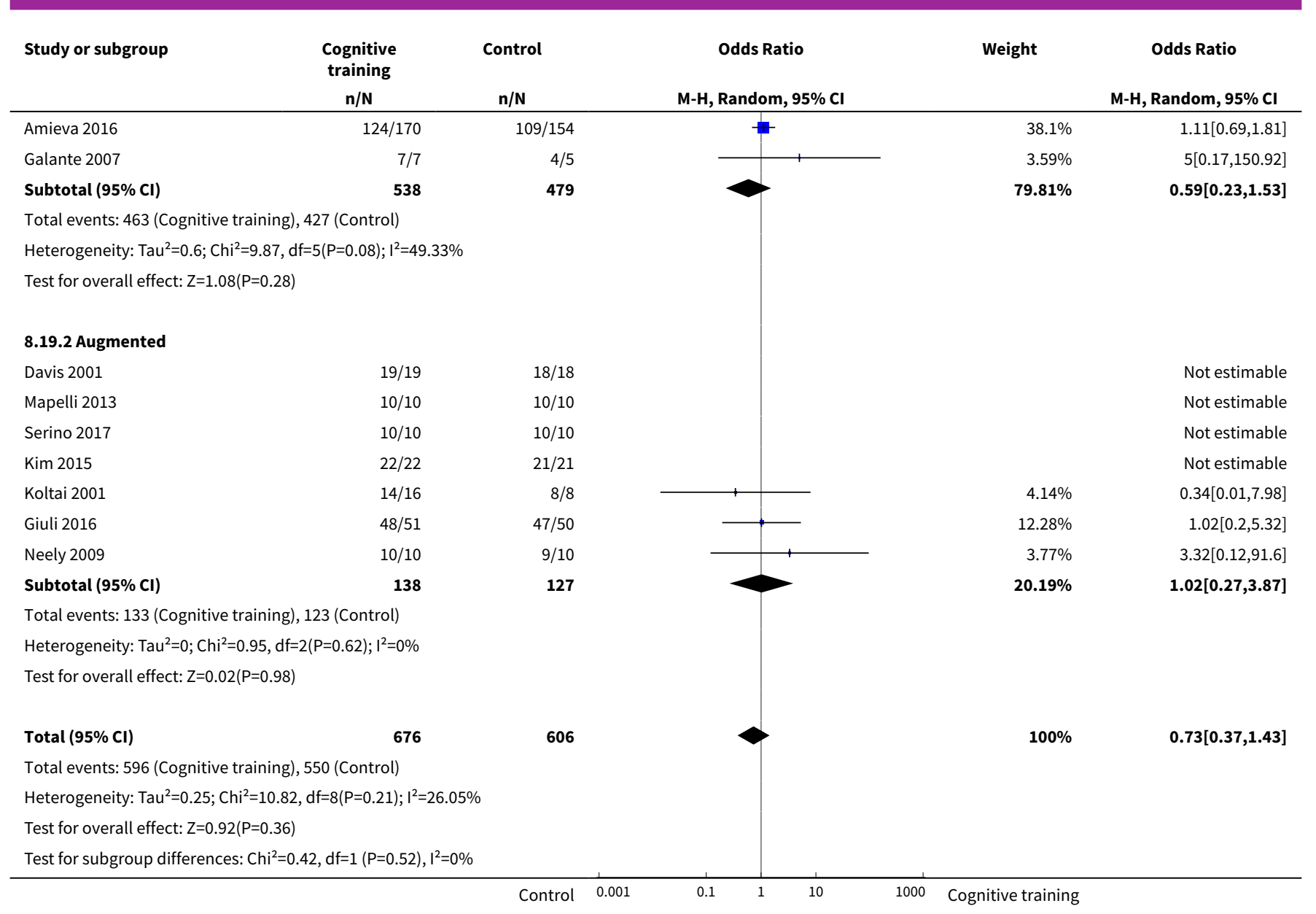

Analysis 8.20. Comparison 8 Cognitive training vs control immediately post intervention type of CT (traditional vs augmented), Outcome 20 Change in burden of care (CAREGIVER).

\begin{tabular}{|c|c|c|c|c|c|c|c|}
\hline \multirow[t]{2}{*}{ Study or subgroup } & \multicolumn{2}{|c|}{ Cognitive training } & \multicolumn{2}{|c|}{ Control } & \multirow{2}{*}{$\begin{array}{c}\text { Std. Mean Difference } \\
\text { Random, } 95 \% \mathrm{Cl}\end{array}$} & \multirow[t]{2}{*}{ Weight } & \multirow{2}{*}{$\begin{array}{c}\text { Std. Mean Difference } \\
\text { Random, } 95 \% \mathrm{Cl}\end{array}$} \\
\hline & $\mathbf{N}$ & Mean(SD) & $\mathbf{N}$ & Mean(SD) & & & \\
\hline Amieva 2016 & 165 & $-21.3(19.1)$ & 152 & $-17.9(19)$ & + & $76.98 \%$ & $-0.18[-0.4,0.04]$ \\
\hline Quayhagen 2000 & 21 & $-0.8(4.1)$ & 67 & $-1.3(3.7)$ & $\rightarrow$ & $23.02 \%$ & $0.13[-0.36,0.62]$ \\
\hline 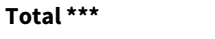 & 186 & & 219 & & & $100 \%$ & $-0.11[-0.36,0.15]$ \\
\hline \multicolumn{8}{|c|}{ Heterogeneity: $\mathrm{Tau}^{2}=0.01 ; \mathrm{Chi}^{2}=1.23, \mathrm{df}=1(\mathrm{P}=0.27) ; \mathrm{I}^{2}=18.62 \%$} \\
\hline \multicolumn{8}{|c|}{ Test for overall effect: $Z=0.83(P=0.41)$} \\
\hline
\end{tabular}

Analysis 8.21. Comparison 8 Cognitive training vs control immediately post intervention - type of CT (traditional vs augmented), Outcome 21 Change in quality of life (CAREGIVER).

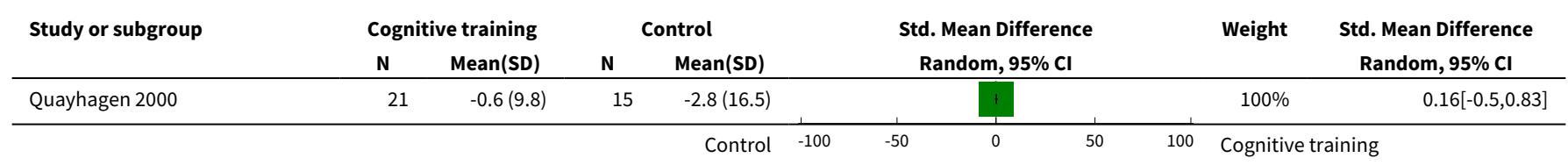




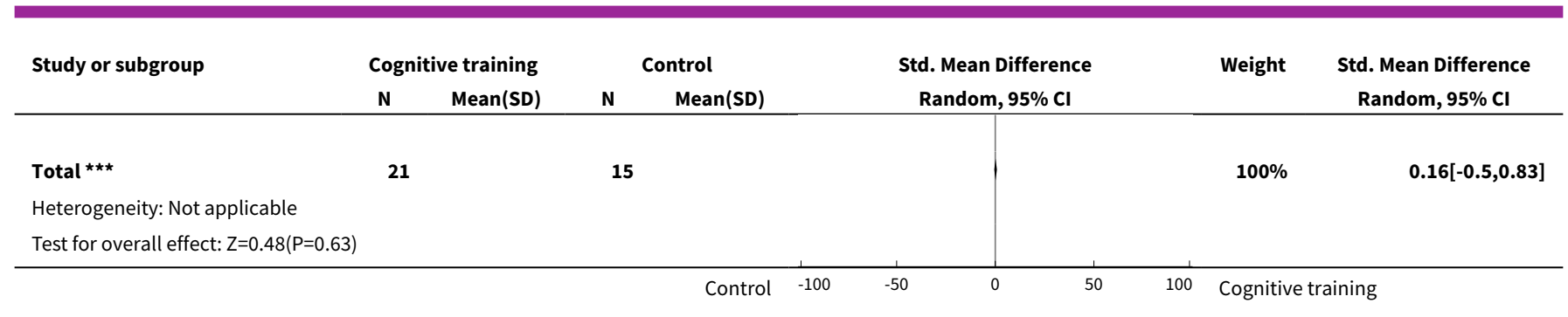

Analysis 8.22. Comparison 8 Cognitive training vs control immediately post intervention - type of CT (traditional vs augmented), Outcome 22 Change in mood and well-being (CAREGIVER).

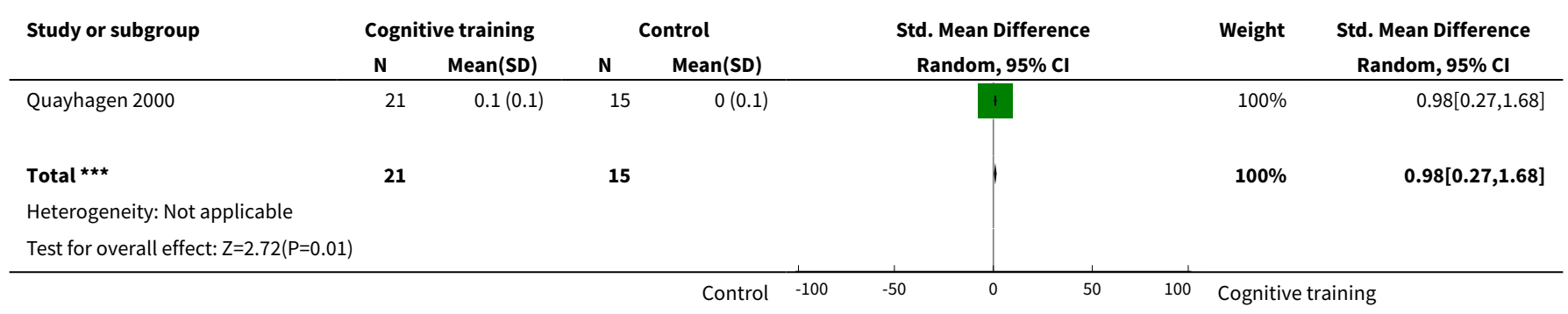

Analysis 8.23. Comparison 8 Cognitive training vs control immediately post intervention type of CT (traditional vs augmented), Outcome 23 Change in general health and quality of life.

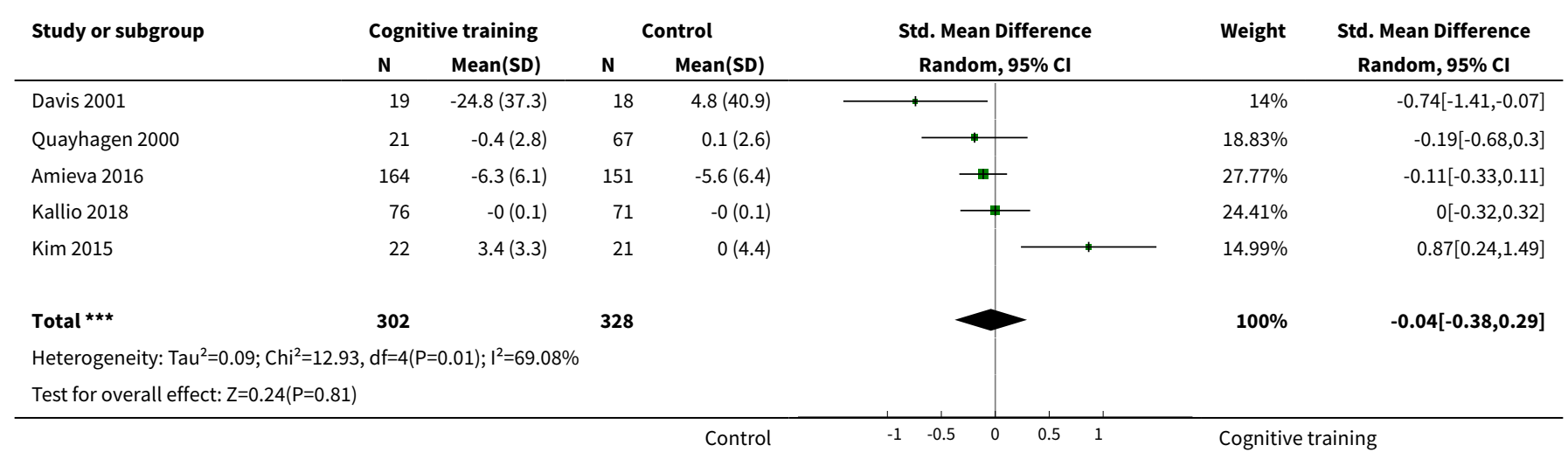

Comparison 9. Cognitive training vs control immediately post intervention - type of $\mathrm{CT}$ (multi-domain vs single domain)

\begin{tabular}{lllll}
\hline Outcome or subgroup title & $\begin{array}{l}\text { No. of } \\
\text { studies }\end{array}$ & $\begin{array}{l}\text { No. of } \\
\text { partici- } \\
\text { pants }\end{array}$ & Statistical method & Effect size \\
\hline $\begin{array}{l}1 \text { Change in a global measure of cogni- } \\
\text { tion }\end{array}$ & 20 & 1288 & $\begin{array}{l}\text { Std. Mean Difference (IV, Random, } \\
95 \% \text { CI) }\end{array}$ & $0.65[0.26,1.05]$ \\
\hline \begin{tabular}{l}
1.1 Multidomain \\
\hline \hline
\end{tabular} & 16 & 1165 & $\begin{array}{l}\text { Std. Mean Difference (IV, Random, } \\
95 \% \text { CI) }\end{array}$ & $0.63[0.19,1.07]$ \\
\hline
\end{tabular}




\begin{tabular}{|c|c|c|c|c|}
\hline Outcome or subgroup title & $\begin{array}{l}\text { No. of } \\
\text { studies }\end{array}$ & $\begin{array}{l}\text { No. of } \\
\text { partici- } \\
\text { pants }\end{array}$ & Statistical method & Effect size \\
\hline 1.2 Single domain & 4 & 123 & $\begin{array}{l}\text { Std. Mean Difference (IV, Random, } \\
95 \% \mathrm{CI} \text { ) }\end{array}$ & $0.75[-0.14,1.63]$ \\
\hline $\begin{array}{l}2 \text { Change in a global measure of cogni- } \\
\text { tion_zero correlation }\end{array}$ & 20 & 1287 & $\begin{array}{l}\text { Std. Mean Difference (IV, Random, } \\
95 \% \mathrm{CI} \text { ) }\end{array}$ & $0.27[0.04,0.50]$ \\
\hline 2.1 Multidomain & 16 & 1164 & $\begin{array}{l}\text { Std. Mean Difference (IV, Random, } \\
95 \% \mathrm{CI} \text { ) }\end{array}$ & $0.25[-0.01,0.52]$ \\
\hline 2.2 Single domain & 4 & 123 & $\begin{array}{l}\text { Std. Mean Difference (IV, Random, } \\
95 \% \mathrm{CI} \text { ) }\end{array}$ & $0.37[-0.04,0.79]$ \\
\hline $\begin{array}{l}3 \text { Change in a global measure of cogni- } \\
\text { tion (composite) }\end{array}$ & 26 & 1389 & $\begin{array}{l}\text { Std. Mean Difference (Random, 95\% } \\
\mathrm{Cl} \text { ) }\end{array}$ & $0.42[0.23,0.61]$ \\
\hline 3.1 Multidomain & 16 & 990 & $\begin{array}{l}\text { Std. Mean Difference (Random, 95\% } \\
\mathrm{Cl} \text { ) }\end{array}$ & $0.44[0.18,0.71]$ \\
\hline 3.2 Single domain & 10 & 399 & $\begin{array}{l}\text { Std. Mean Difference (Random, 95\% } \\
\mathrm{Cl} \text { ) }\end{array}$ & $0.35[0.10,0.59]$ \\
\hline $\begin{array}{l}4 \text { Change in a global measure of cogni- } \\
\text { tion (composite)_zero correlation }\end{array}$ & 26 & 1390 & $\begin{array}{l}\text { Std. Mean Difference (Random, 95\% } \\
\mathrm{CI} \text { ) }\end{array}$ & $0.24[0.12,0.36]$ \\
\hline 4.1 Multidomain & 16 & 991 & $\begin{array}{l}\text { Std. Mean Difference (Random, 95\% } \\
\mathrm{Cl} \text { ) }\end{array}$ & $0.27[0.10,0.44]$ \\
\hline 4.2 Single domain & 10 & 399 & $\begin{array}{l}\text { Std. Mean Difference (Random, 95\% } \\
\mathrm{Cl} \text { ) }\end{array}$ & $0.23[0.04,0.43]$ \\
\hline 5 Change in immediate memory & 17 & 762 & $\begin{array}{l}\text { Std. Mean Difference (IV, Random, } \\
95 \% \mathrm{CI} \text { ) }\end{array}$ & $0.74[0.37,1.12]$ \\
\hline 5.1 Multidomain & 10 & 540 & $\begin{array}{l}\text { Std. Mean Difference (IV, Random, } \\
95 \% \mathrm{Cl})\end{array}$ & $0.80[0.24,1.35]$ \\
\hline 5.2 Single domain & 7 & 222 & $\begin{array}{l}\text { Std. Mean Difference (IV, Random, } \\
95 \% \mathrm{Cl})\end{array}$ & $0.58[0.22,0.94]$ \\
\hline 6 Change in delayed memory & 11 & 543 & $\begin{array}{l}\text { Std. Mean Difference (IV, Random, } \\
95 \% \mathrm{Cl})\end{array}$ & $0.81[0.29,1.32]$ \\
\hline 6.1 Multidomain & 6 & 369 & $\begin{array}{l}\text { Std. Mean Difference (IV, Random, } \\
95 \% \mathrm{CI})\end{array}$ & $1.15[0.32,1.97]$ \\
\hline 6.2 Single domain & 5 & 174 & $\begin{array}{l}\text { Std. Mean Difference (IV, Random, } \\
95 \% \mathrm{CI})\end{array}$ & $0.41[-0.11,0.94]$ \\
\hline $\begin{array}{l}7 \text { Change in attention and working } \\
\text { memory }\end{array}$ & 12 & 551 & $\begin{array}{l}\text { Std. Mean Difference (IV, Random, } \\
95 \% \mathrm{CI} \text { ) }\end{array}$ & $0.56[0.08,1.05]$ \\
\hline 7.1 Multidomain & 8 & 411 & $\begin{array}{l}\text { Std. Mean Difference (IV, Random, } \\
95 \% \mathrm{CI})\end{array}$ & $0.50[-0.14,1.15]$ \\
\hline
\end{tabular}




\begin{tabular}{|c|c|c|c|c|}
\hline Outcome or subgroup title & $\begin{array}{l}\text { No. of } \\
\text { studies }\end{array}$ & $\begin{array}{l}\text { No. of } \\
\text { partici- } \\
\text { pants }\end{array}$ & Statistical method & Effect size \\
\hline 7.2 Single domain & 4 & 140 & $\begin{array}{l}\text { Std. Mean Difference (IV, Random, } \\
95 \% \mathrm{CI})\end{array}$ & $0.68[-0.14,1.49]$ \\
\hline 8 Change in language (naming) & 5 & 311 & $\begin{array}{l}\text { Std. Mean Difference (IV, Random, } \\
95 \% \mathrm{CI} \text { ) }\end{array}$ & $0.62[0.11,1.12]$ \\
\hline 9 Change in verbal letter fluency & 12 & 544 & $\begin{array}{l}\text { Std. Mean Difference (IV, Random, } \\
95 \% \mathrm{CI} \text { ) }\end{array}$ & $0.22[-0.07,0.50]$ \\
\hline 9.1 Multidomain & 8 & 406 & $\begin{array}{l}\text { Std. Mean Difference (IV, Random, } \\
95 \% \mathrm{CI})\end{array}$ & $0.37[-0.04,0.78]$ \\
\hline 9.2 Single domain & 4 & 138 & $\begin{array}{l}\text { Std. Mean Difference (IV, Random, } \\
95 \% \mathrm{CI})\end{array}$ & $-0.04[-0.37,0.30]$ \\
\hline $\begin{array}{l}10 \text { Change in speed of information pro- } \\
\text { cessing }\end{array}$ & 6 & 201 & $\begin{array}{l}\text { Std. Mean Difference (IV, Random, } \\
95 \% \mathrm{CI})\end{array}$ & $0.22[-0.11,0.54]$ \\
\hline 11 Change in executive function & 11 & 511 & $\begin{array}{l}\text { Std. Mean Difference (IV, Random, } \\
95 \% \mathrm{CI})\end{array}$ & $0.75[0.28,1.22]$ \\
\hline 11.1 Multidomain & 8 & 408 & $\begin{array}{l}\text { Std. Mean Difference (IV, Random, } \\
95 \% \mathrm{CI} \text { ) }\end{array}$ & $0.99[0.44,1.55]$ \\
\hline 11.2 Single domain & 3 & 103 & $\begin{array}{l}\text { Std. Mean Difference (IV, Random, } \\
95 \% \mathrm{CI})\end{array}$ & $0.08[-0.53,0.68]$ \\
\hline 12 Change in verbal category fluency & 9 & 475 & $\begin{array}{l}\text { Std. Mean Difference (IV, Random, } \\
95 \% \mathrm{CI})\end{array}$ & $0.52[0.23,0.81]$ \\
\hline 12.1 Multidomain & 6 & 371 & $\begin{array}{l}\text { Std. Mean Difference (IV, Random, } \\
95 \% \mathrm{CI} \text { ) }\end{array}$ & $0.70[0.38,1.02]$ \\
\hline 12.2 Single domain & 3 & 104 & $\begin{array}{l}\text { Std. Mean Difference (IV, Random, } \\
95 \% \mathrm{Cl})\end{array}$ & $0.14[-0.25,0.52]$ \\
\hline $\begin{array}{l}13 \text { Change in meta cognition (self-re- } \\
\text { ported) }\end{array}$ & 2 & 41 & $\begin{array}{l}\text { Std. Mean Difference (IV, Random, } \\
95 \% \mathrm{Cl})\end{array}$ & $0.12[-0.87,1.12]$ \\
\hline $\begin{array}{l}14 \text { Change in meta cognition (infor- } \\
\text { mant-reported) }\end{array}$ & 2 & 56 & $\begin{array}{l}\text { Std. Mean Difference (IV, Random, } \\
95 \% \mathrm{Cl})\end{array}$ & $-0.65[-1.19,-0.10]$ \\
\hline 15 Change in participants' mood & 8 & 576 & $\begin{array}{l}\text { Std. Mean Difference (IV, Random, } \\
95 \% \mathrm{CI})\end{array}$ & $0.72[-0.10,1.54]$ \\
\hline $\begin{array}{l}16 \text { Change in capacity for activities of } \\
\text { daily living }\end{array}$ & 10 & 705 & $\begin{array}{l}\text { Std. Mean Difference (IV, Random, } \\
95 \% \mathrm{CI})\end{array}$ & $0.12[-0.10,0.34]$ \\
\hline 17 Change in disease progression & 5 & 215 & $\begin{array}{l}\text { Std. Mean Difference (IV, Random, } \\
95 \% \mathrm{CI} \text { ) }\end{array}$ & $1.07[0.59,1.55]$ \\
\hline $\begin{array}{l}18 \text { Change in behavioural and psycho- } \\
\text { logical symptoms of dementia (BPSD) }\end{array}$ & 6 & 493 & $\begin{array}{l}\text { Std. Mean Difference (IV, Random, } \\
95 \% \mathrm{CI} \text { ) }\end{array}$ & $0.44[-0.34,1.22]$ \\
\hline
\end{tabular}




\begin{tabular}{|c|c|c|c|c|}
\hline Outcome or subgroup title & $\begin{array}{l}\text { No. of } \\
\text { studies }\end{array}$ & $\begin{array}{l}\text { No. of } \\
\text { partici- } \\
\text { pants }\end{array}$ & Statistical method & Effect size \\
\hline 19 Participant burden (retention rates) & 17 & 1282 & Odds Ratio (M-H, Random, 95\% Cl) & $0.73[0.37,1.43]$ \\
\hline 19.1 Multidomain & 12 & 988 & Odds Ratio (M-H, Random, 95\% Cl) & $0.61[0.21,1.81]$ \\
\hline 19.2 Single domain & 5 & 294 & Odds Ratio (M-H, Random, 95\% Cl) & $0.66[0.24,1.77]$ \\
\hline $\begin{array}{l}20 \text { Change in burden of care } \\
\text { (CAREGIVER) }\end{array}$ & 2 & 405 & $\begin{array}{l}\text { Std. Mean Difference (IV, Random, } \\
95 \% \mathrm{CI})\end{array}$ & $-0.11[-0.36,0.15]$ \\
\hline 21 Change in quality of life (CAREGIVER) & 1 & 36 & $\begin{array}{l}\text { Std. Mean Difference (IV, Random, } \\
95 \% \mathrm{CI} \text { ) }\end{array}$ & $0.16[-0.50,0.83]$ \\
\hline $\begin{array}{l}22 \text { Change in mood and well-being } \\
\text { (CAREGIVER) }\end{array}$ & 1 & 36 & $\begin{array}{l}\text { Std. Mean Difference (IV, Random, } \\
95 \% \mathrm{CI})\end{array}$ & $0.98[0.27,1.68]$ \\
\hline $\begin{array}{l}23 \text { Change in general health and quality } \\
\text { of life }\end{array}$ & 5 & 630 & $\begin{array}{l}\text { Std. Mean Difference (IV, Random, } \\
95 \% \mathrm{CI})\end{array}$ & $-0.04[-0.38,0.29]$ \\
\hline
\end{tabular}

\section{Analysis 9.1. Comparison 9 Cognitive training vs control immediately post intervention - type of CT (multi-domain vs single domain), Outcome 1 Change in a global measure of cognition.}

\begin{tabular}{|c|c|c|c|c|c|c|c|}
\hline \multirow{3}{*}{$\begin{array}{l}\text { Study or subgroup } \\
\text { 9.1.1 Multidomain }\end{array}$} & \multicolumn{2}{|c|}{ Cognitive training } & \multicolumn{2}{|c|}{ Control } & \multirow{2}{*}{$\begin{array}{c}\text { Std. Mean Difference } \\
\text { Random, } 95 \% \mathrm{CI}\end{array}$} & \multirow[t]{2}{*}{ Weight } & \multirow{2}{*}{$\begin{array}{l}\text { Std. Mean Difference } \\
\text { Random, } 95 \% \mathrm{Cl}\end{array}$} \\
\hline & \multirow[t]{2}{*}{$\mathbf{N}$} & \multirow[t]{2}{*}{ Mean(SD) } & \multirow[t]{2}{*}{$\mathbf{N}$} & \multirow[t]{2}{*}{$\operatorname{Mean}(S D)$} & & & \\
\hline & & & & & & & \\
\hline Galante 2007 & 7 & $0.1(2.3)$ & 4 & $1.8(1.7)$ & \begin{tabular}{l|l}
1 & \\
1
\end{tabular} & $3.67 \%$ & $-0.72[-2,0.56]$ \\
\hline Giuli 2016 & 48 & $0(2.8)$ & 47 & $1.2(3.8)$ & $\rightarrow$ & $5.67 \%$ & $-0.37[-0.77,0.04]$ \\
\hline Koltai 2001 & 14 & $-0.2(2.9)$ & 8 & $0.8(2.4)$ & $\longrightarrow$ & $4.64 \%$ & $-0.34[-1.21,0.54]$ \\
\hline Amieva 2016 & 168 & $-8.4(5.9)$ & 153 & $-7.6(5.4)$ & + & $5.92 \%$ & $-0.14[-0.36,0.08]$ \\
\hline Cavallo 2016 & 40 & $-0.1(1)$ & 40 & $-0.2(1.6)$ & + & $5.61 \%$ & $0.01[-0.42,0.45]$ \\
\hline Barban 2016 & 42 & $0.1(1.6)$ & 39 & $-0.1(1.2)$ & + & $5.62 \%$ & $0.14[-0.3,0.58]$ \\
\hline Kallio 2018 & 76 & $-0.8(5.2)$ & 71 & $-1.6(5.4)$ & + & $5.8 \%$ & $0.15[-0.17,0.48]$ \\
\hline Venturelli 2016 & 20 & $-0.2(1)$ & 20 & $-0.4(1.1)$ & 1 & $5.24 \%$ & $0.19[-0.44,0.81]$ \\
\hline Kawashima 2005 & 16 & $0.1(5.1)$ & 16 & $-1.8(3.8)$ & + & $5.06 \%$ & $0.41[-0.29,1.12]$ \\
\hline Quintana Hernandez 2014 & 27 & $-4.5(4.1)$ & 25 & $-7.5(3.5)$ & $\longrightarrow$ & $5.36 \%$ & $0.78[0.21,1.34]$ \\
\hline de Vreese 1998 & 9 & $2.8(1.7)$ & 9 & $-0.9(2.4)$ & + & $4.06 \%$ & $1.68[0.57,2.8]$ \\
\hline Trebbastoni 2018 & 48 & $1.5(1.7)$ & 86 & $-2.6(2.1)$ & $\rightarrow$ & $5.61 \%$ & $2.13[1.69,2.57]$ \\
\hline Fernández-Calvo 2011 & 30 & $1.1(1.6)$ & 15 & $-2.3(1.2)$ & $\longrightarrow$ & $4.86 \%$ & $2.25[1.46,3.03]$ \\
\hline Bergamaschi 2013 & 16 & $2.8(1.8)$ & 16 & $-3.6(1.8)$ & & $4.03 \%$ & $3.4[2.28,4.53]$ \\
\hline 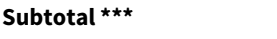 & 589 & & 576 & & & $80.66 \%$ & $0.63[0.19,1.07]$ \\
\hline \multicolumn{8}{|c|}{ Heterogeneity: Tau $^{2}=0.68 ; \mathrm{Chi}^{2}=167.63, \mathrm{df}=15(\mathrm{P}<0.0001) ; \mathrm{I}^{2}=91.05 \%$} \\
\hline \multicolumn{8}{|c|}{ Test for overall effect: $Z=2.81(P=0)$} \\
\hline \multicolumn{8}{|l|}{ 9.1.2 Single domain } \\
\hline Davis 2001 & 19 & $0.2(2.6)$ & 18 & $0.2(2.7)$ & $\longrightarrow$ & $5.19 \%$ & $-0.02[-0.67,0.62]$ \\
\hline Lee 2013 & 12 & $2(2.9)$ & 7 & $2(1.9)$ & & $4.5 \%$ & $0[-0.93,0.94]$ \\
\hline Jelcic 2014 & 17 & $2(1.5)$ & 10 & $-0.7(2.4)$ & $\longrightarrow$ & $4.63 \%$ & $1.4[0.52,2.28]$ \\
\hline
\end{tabular}




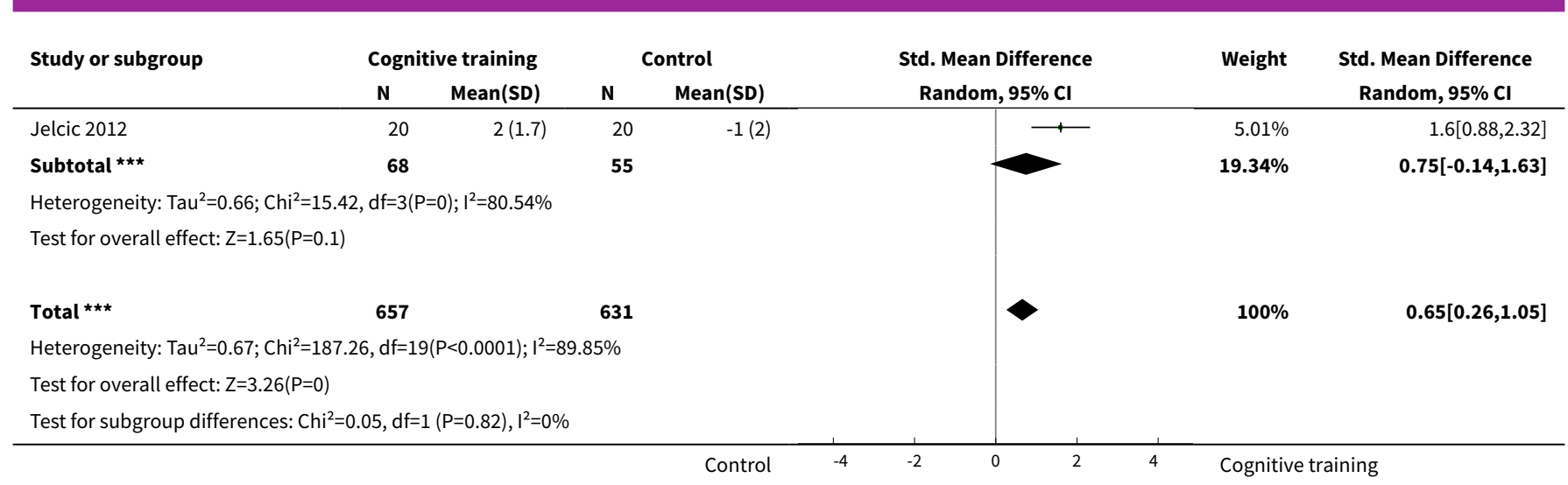

Analysis 9.2. Comparison 9 Cognitive training vs control immediately post intervention - type of CT (multi-domain vs single domain), Outcome 2 Change in a global measure of cognition_zero correlation.

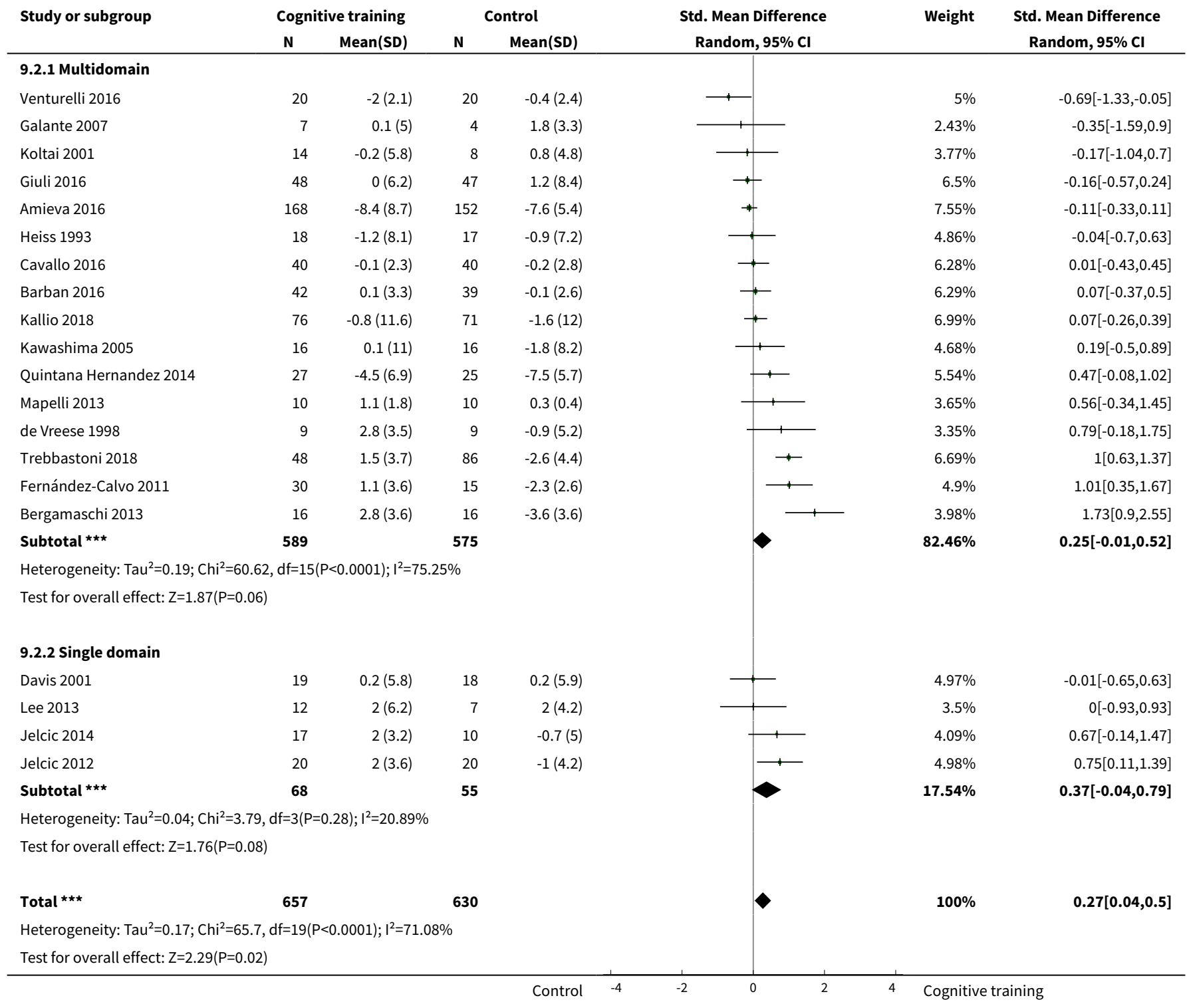




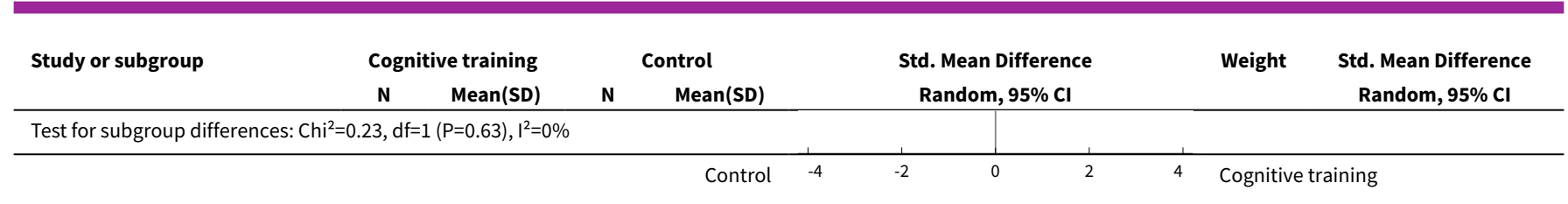

Analysis 9.3. Comparison 9 Cognitive training vs control immediately post intervention - type of CT
(multi-domain vs single domain), Outcome 3 Change in a global measure of cognition (composite).

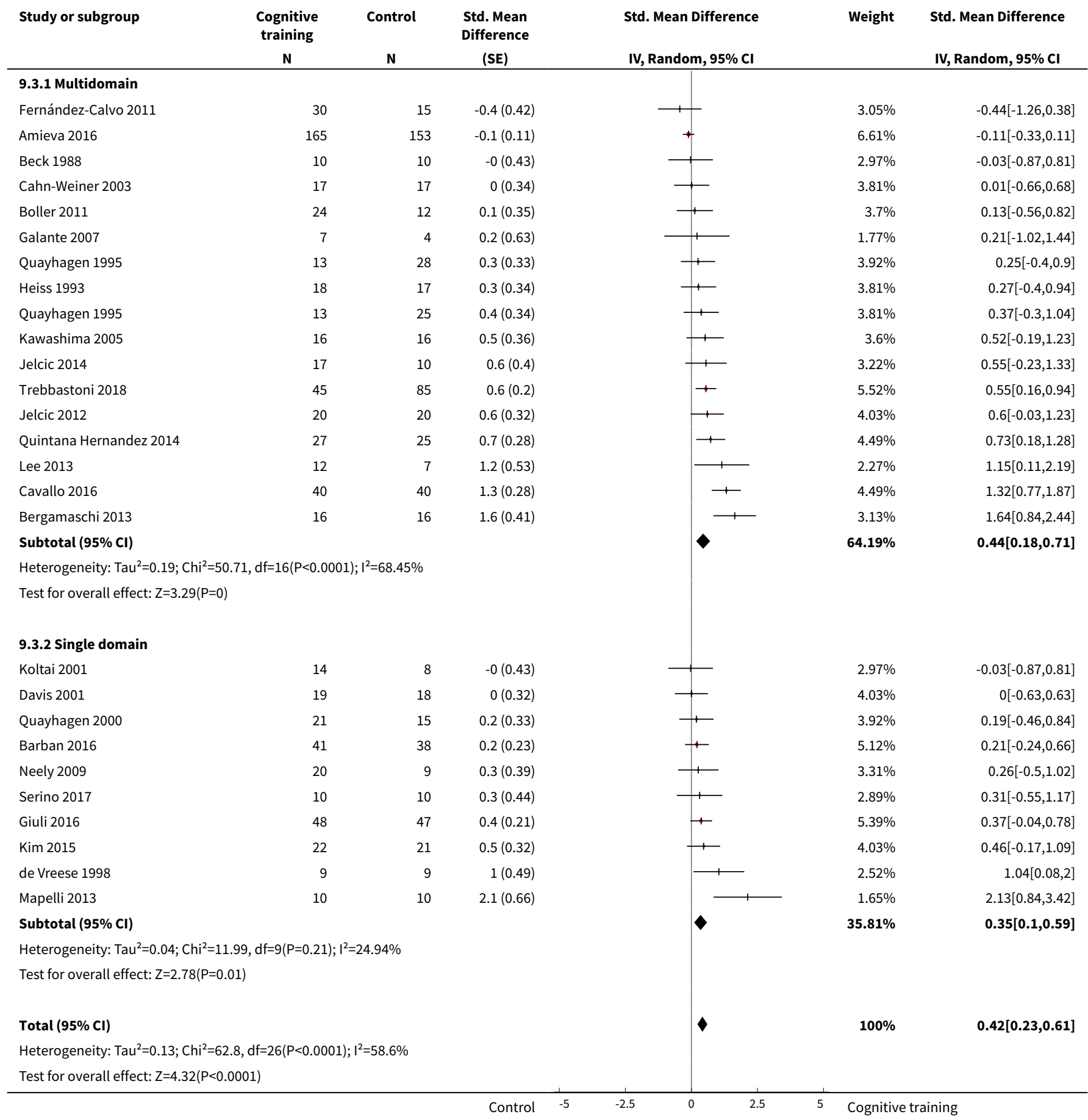




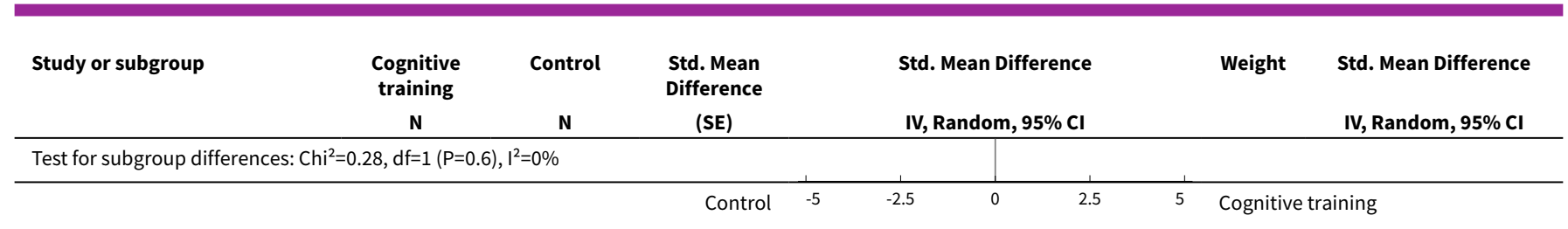

\section{Analysis 9.4. Comparison 9 Cognitive training vs control immediately post intervention - type of CT (multi- domain vs single domain), Outcome $\mathbf{4}$ Change in a global measure of cognition (composite)_zero correlation.}

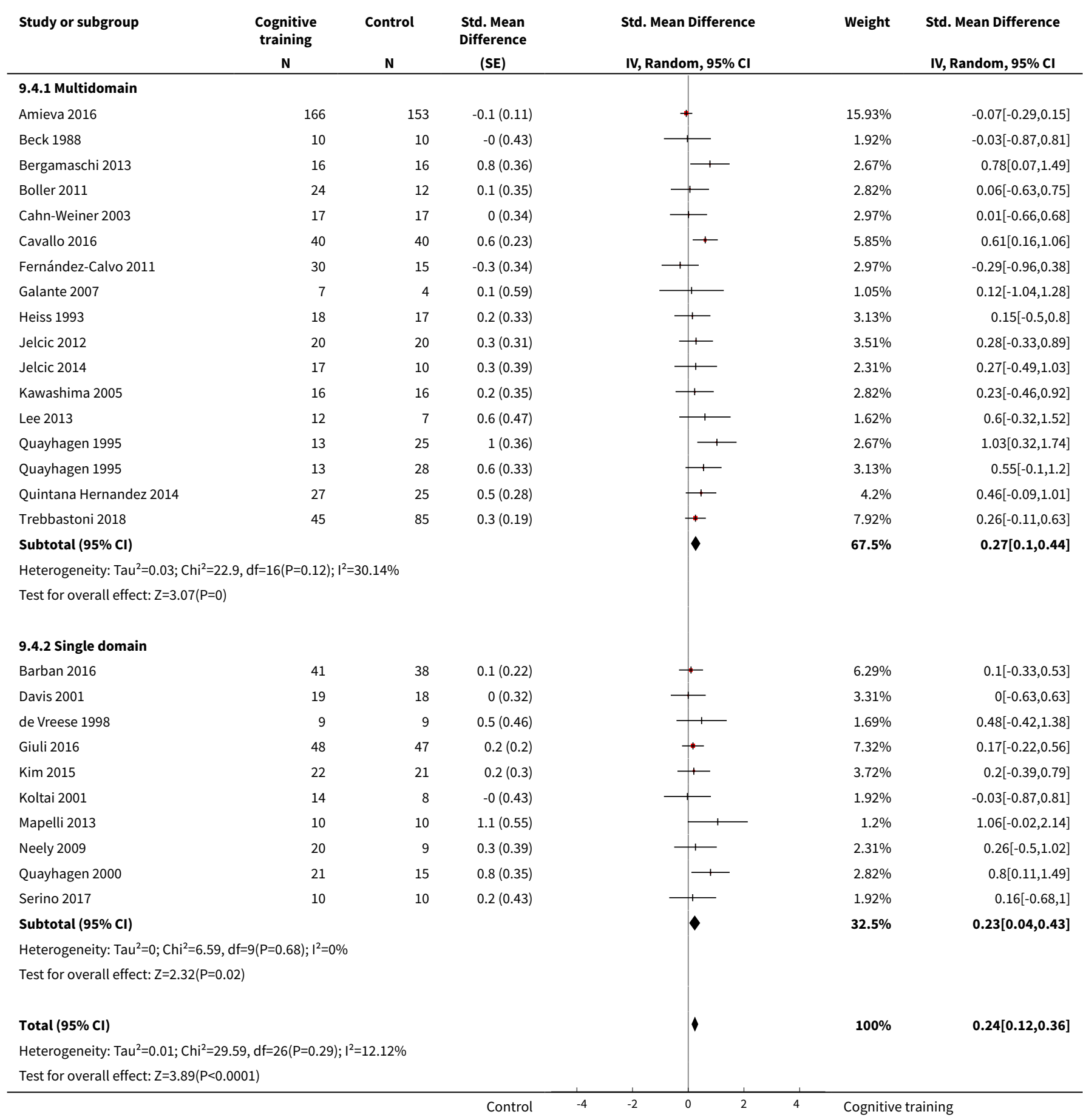




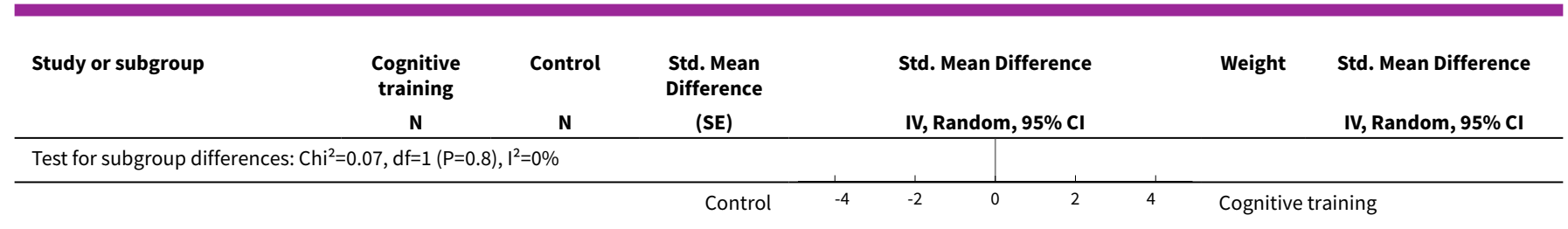

Analysis 9.5. Comparison 9 Cognitive training vs control immediately post intervention - type of CT (multi-domain vs single domain), Outcome 5 Change in immediate memory.

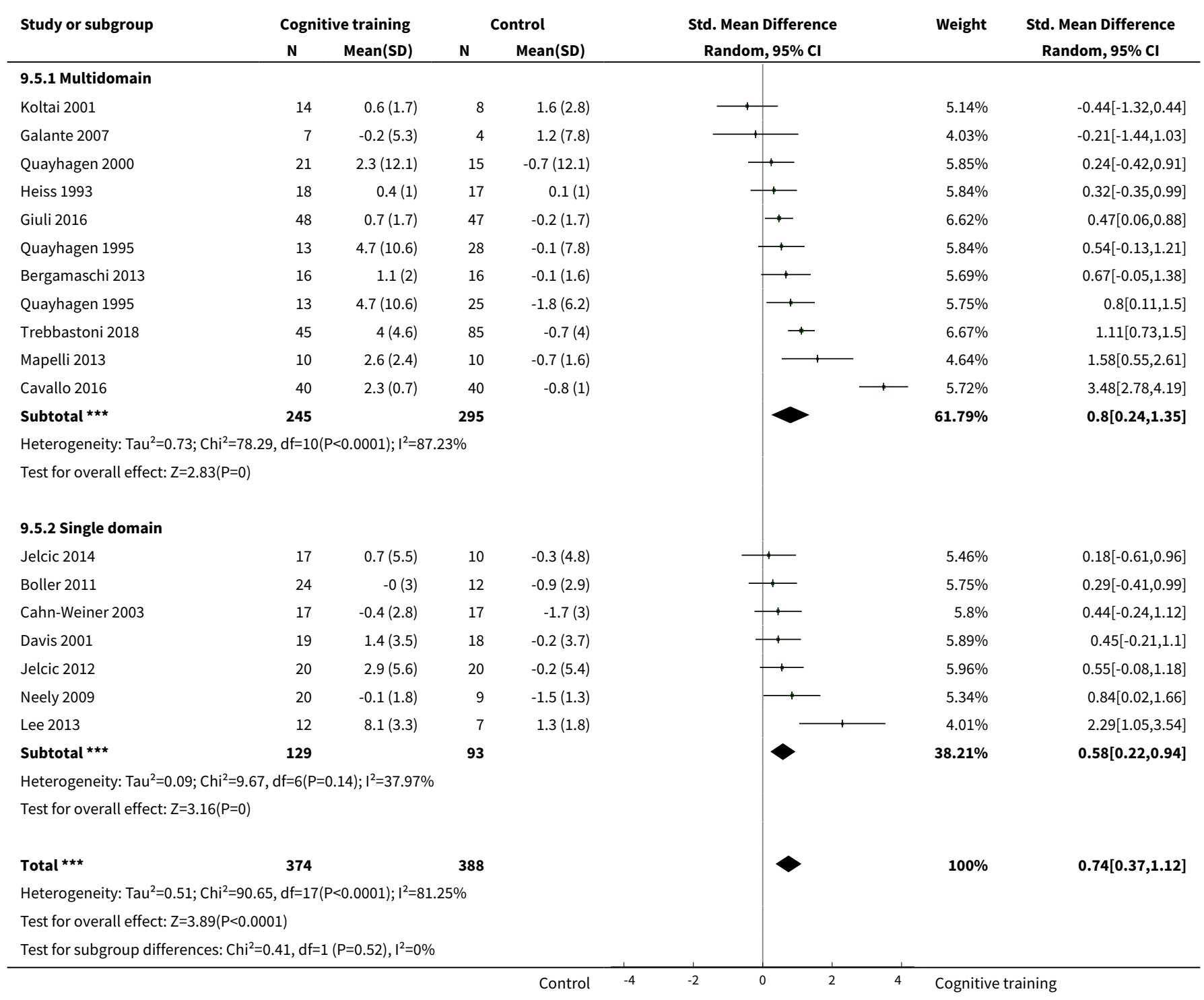


Analysis 9.6. Comparison 9 Cognitive training vs control immediately post intervention - type of CT (multi-domain vs single domain), Outcome 6 Change in delayed memory.

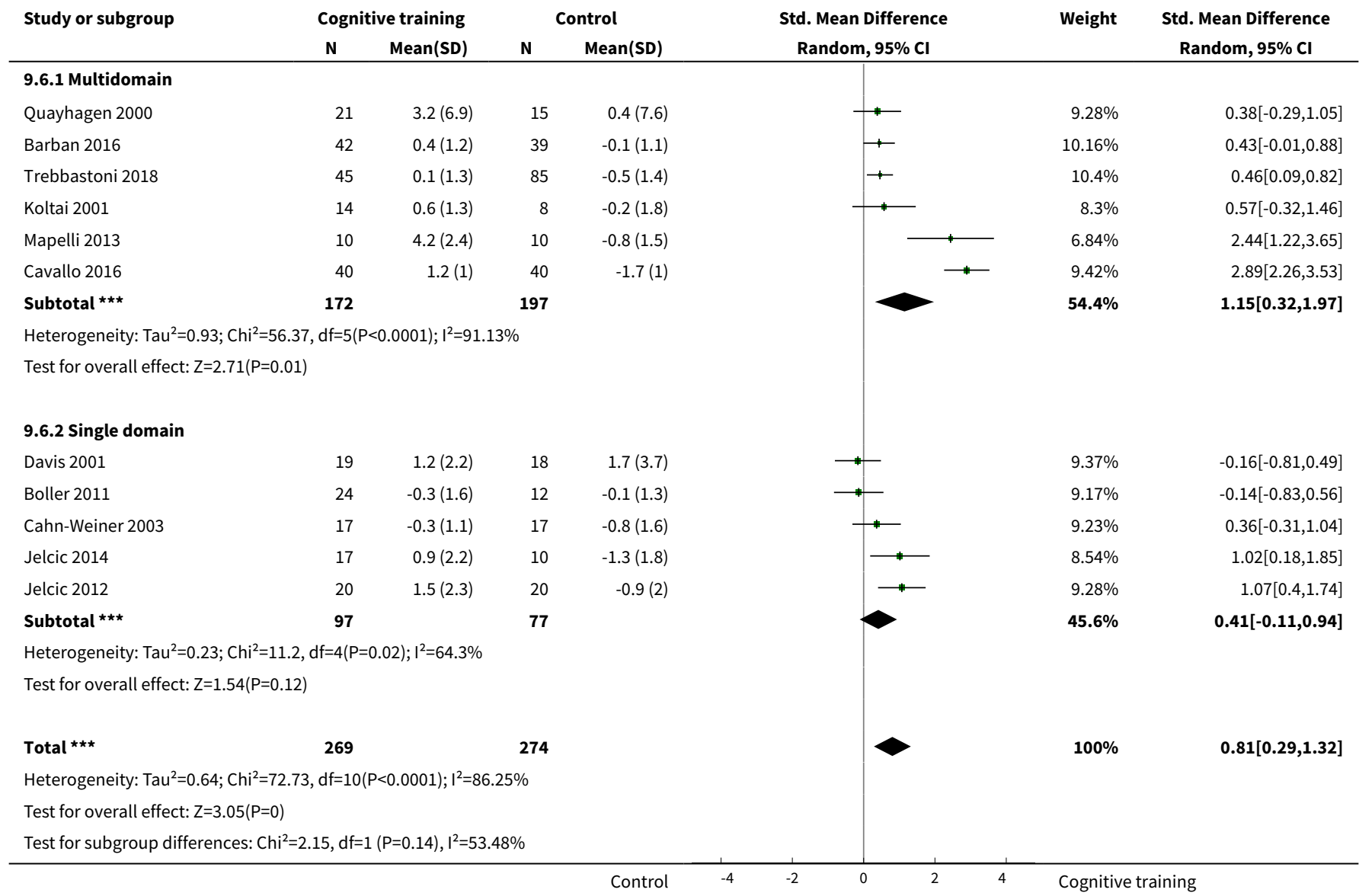

Analysis 9.7. Comparison 9 Cognitive training vs control immediately post intervention - type of CT (multi-domain vs single domain), Outcome 7 Change in attention and working memory.

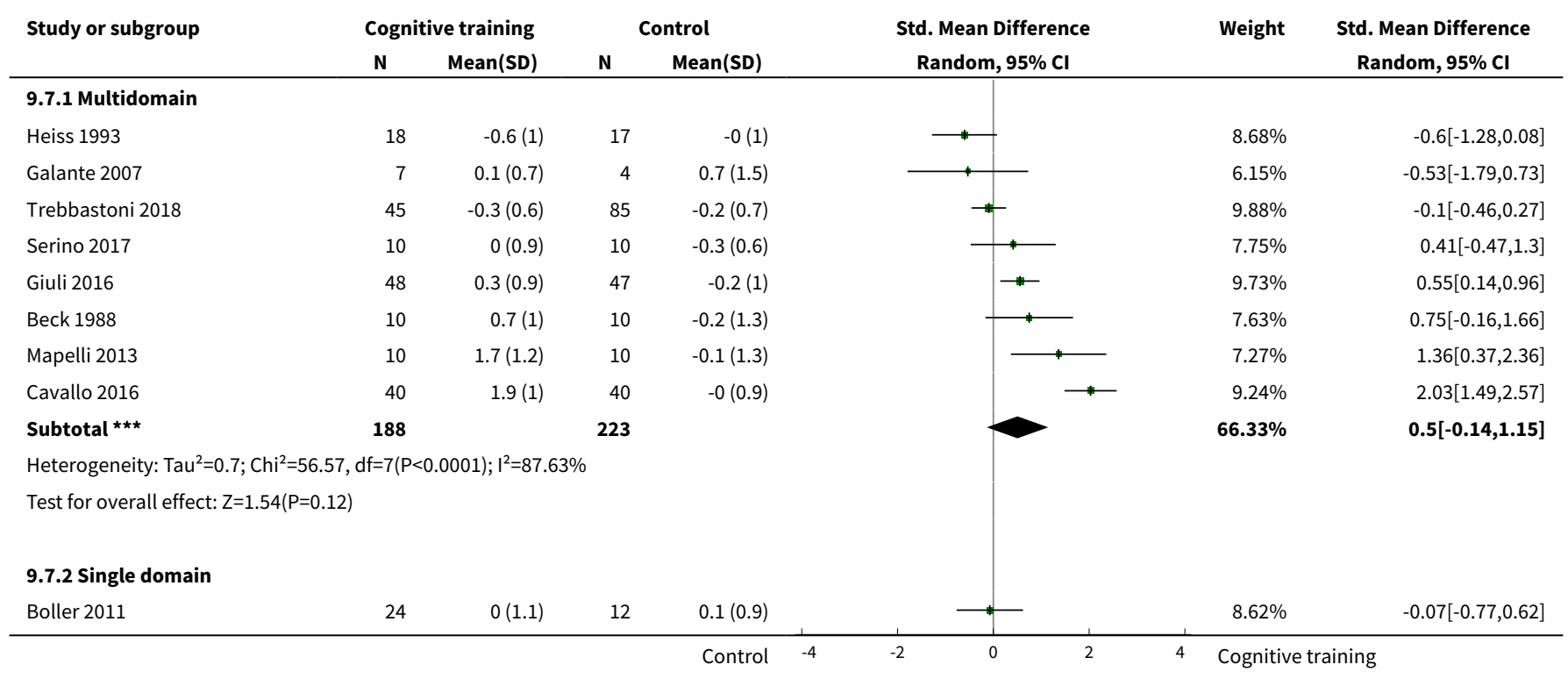




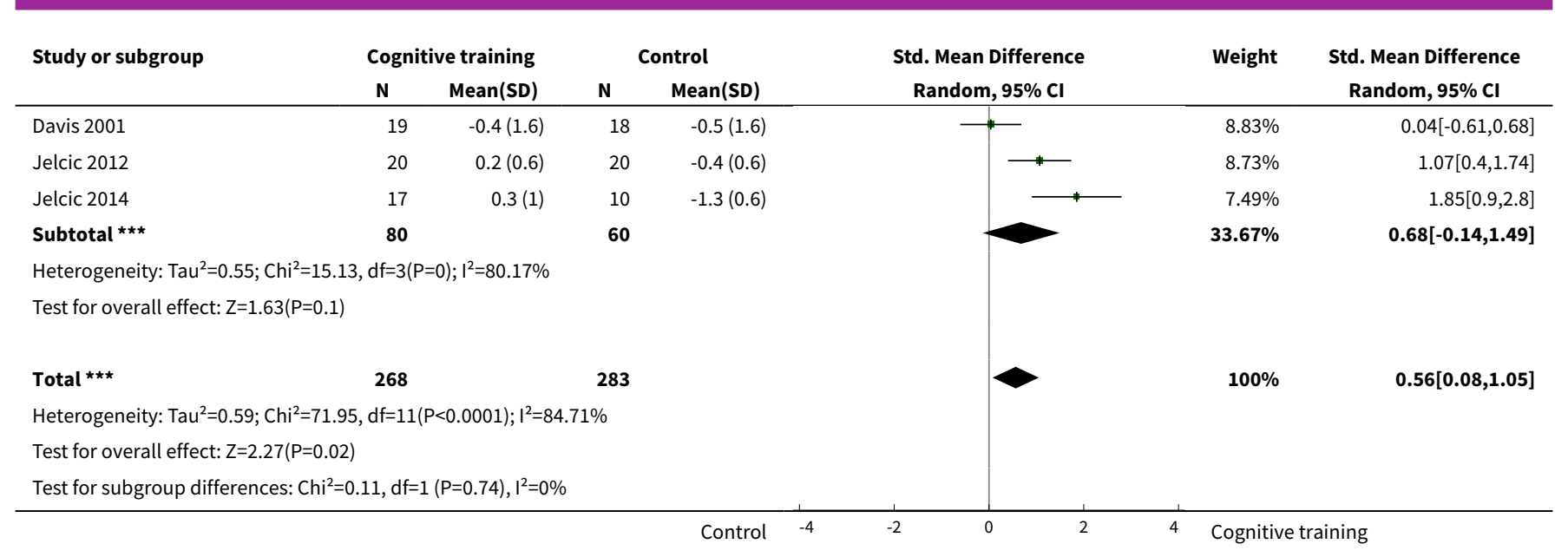

Analysis 9.8. Comparison 9 Cognitive training vs control immediately post intervention - type of CT (multi-domain vs single domain), Outcome 8 Change in language (naming).

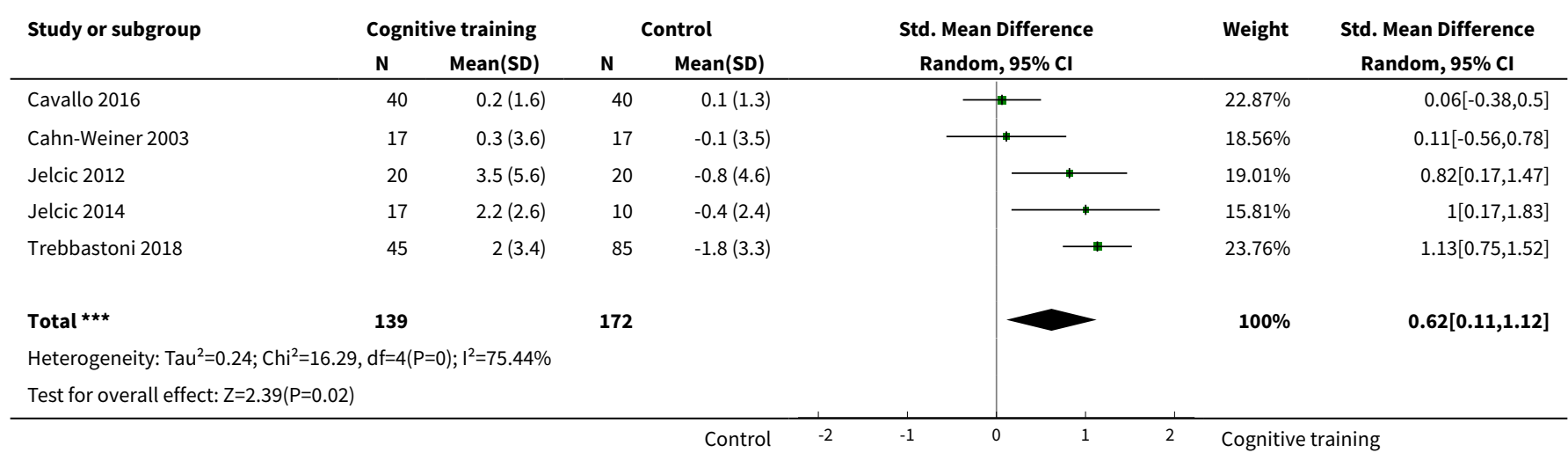

Analysis 9.9. Comparison 9 Cognitive training vs control immediately post intervention - type of CT (multi-domain vs single domain), Outcome 9 Change in verbal letter fluency.

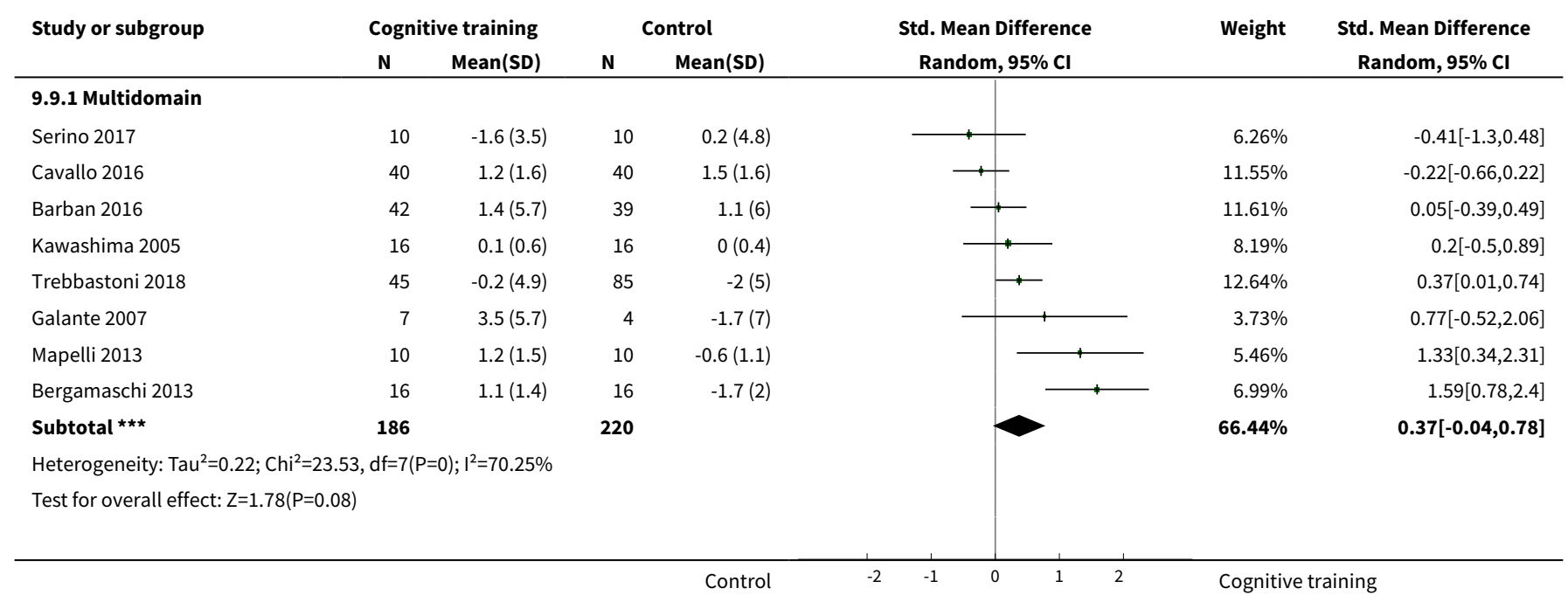




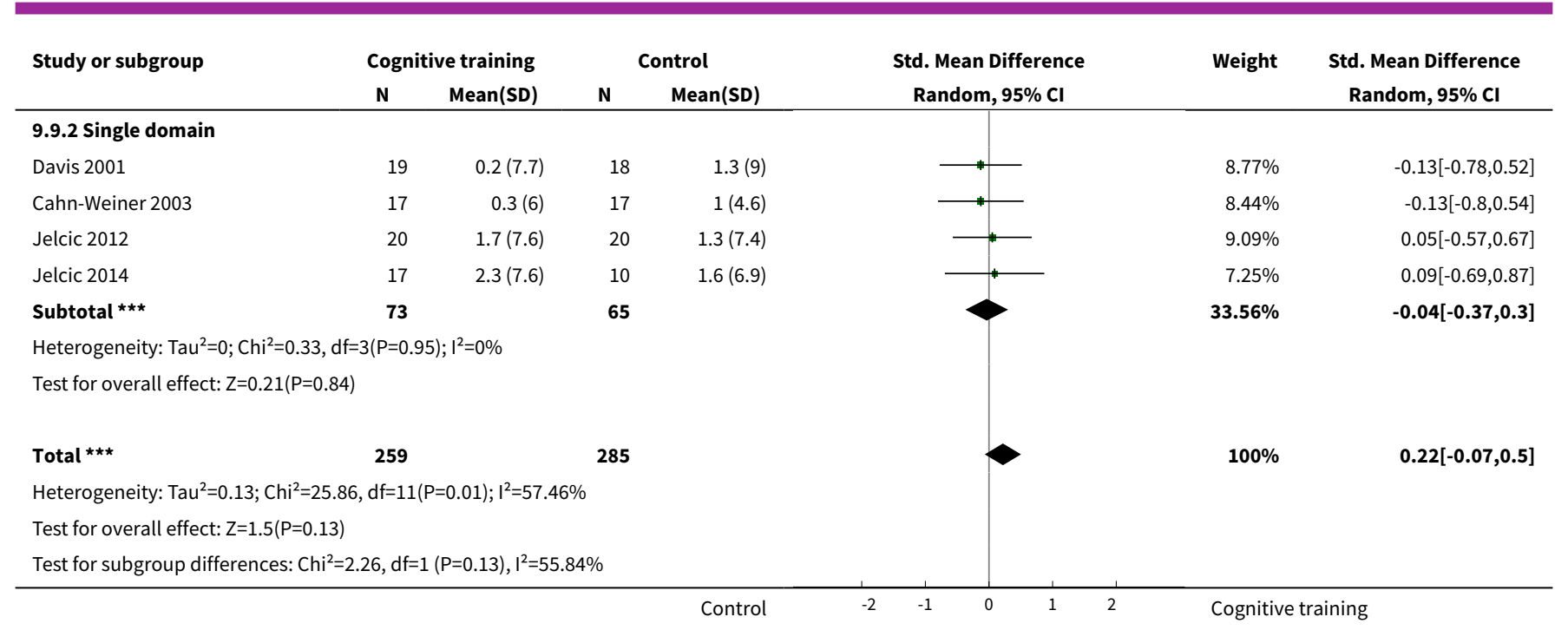

Analysis 9.10. Comparison 9 Cognitive training vs control immediately post intervention - type of CT (multi-domain vs single domain), Outcome 10 Change in speed of information processing.

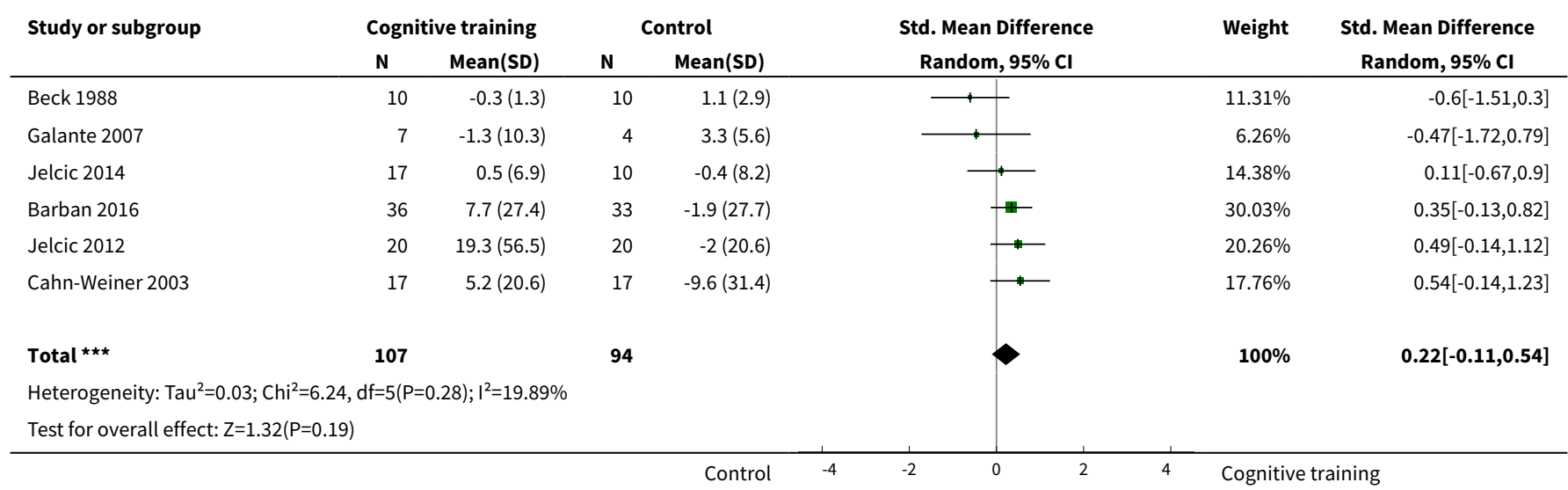

Analysis 9.11. Comparison 9 Cognitive training vs control immediately post intervention - type of CT (multi-domain vs single domain), Outcome 11 Change in executive function.

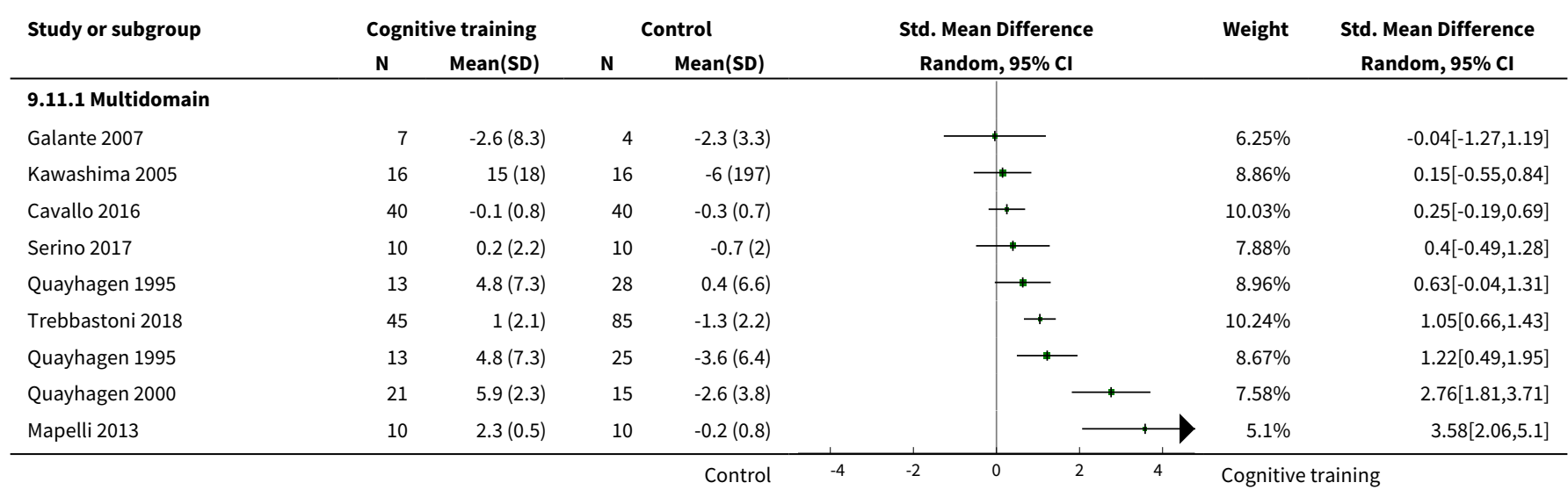




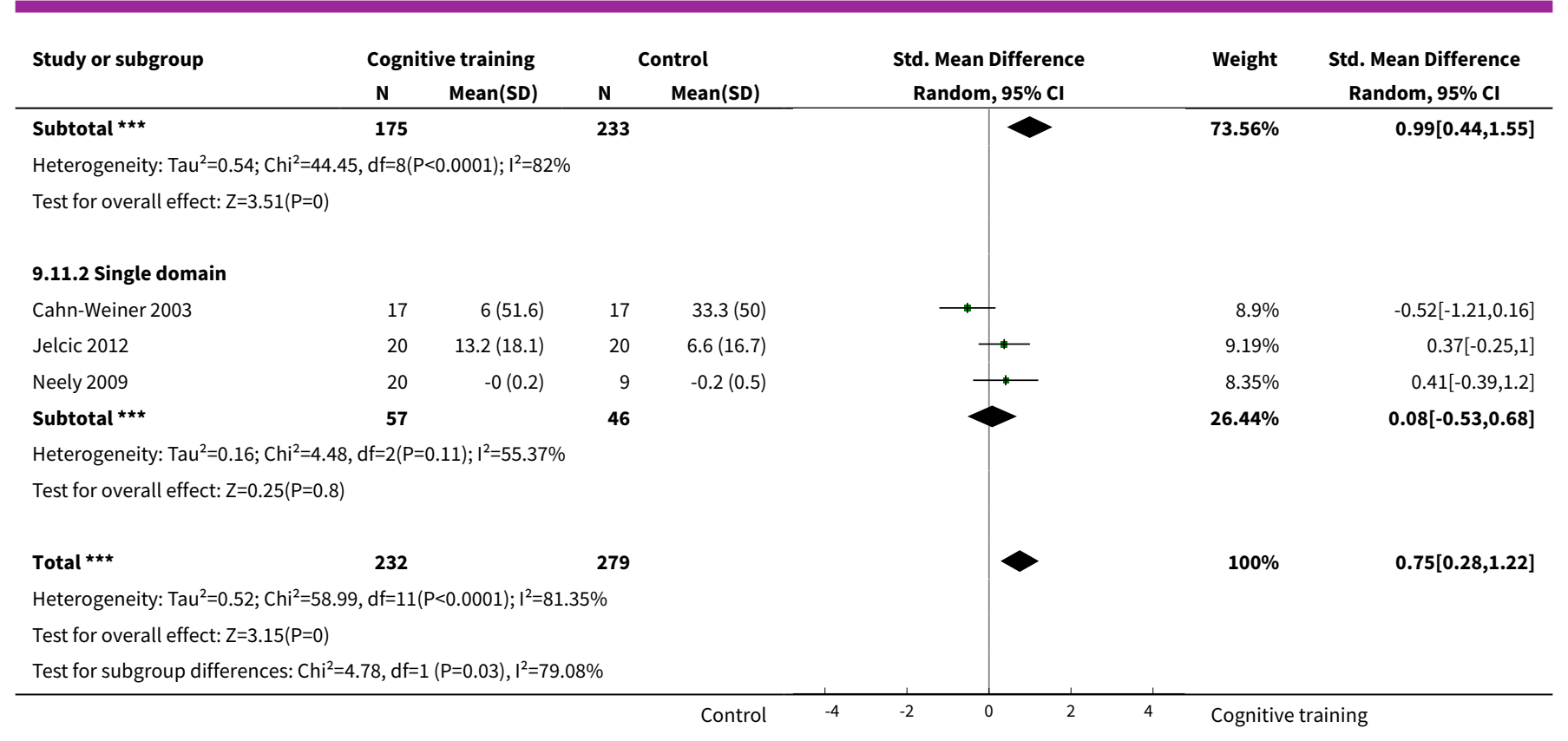

Analysis 9.12. Comparison 9 Cognitive training vs control immediately post intervention type of $\mathrm{CT}$ (multi-domain vs single domain), Outcome 12 Change in verbal category fluency.

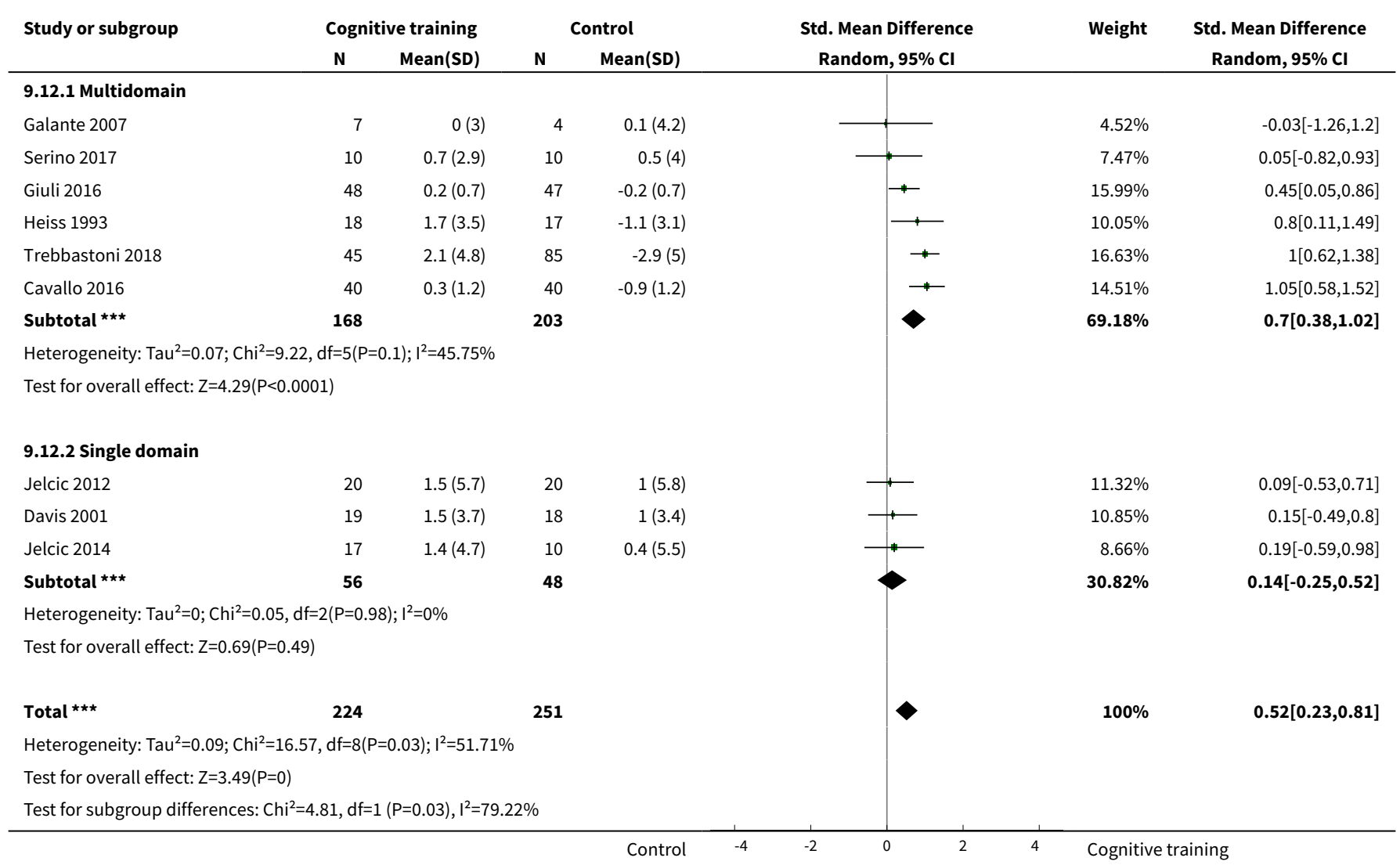


Analysis 9.13. Comparison 9 Cognitive training vs control immediately post intervention - type of CT (multi-domain vs single domain), Outcome 13 Change in meta cognition (self-reported).

\begin{tabular}{|c|c|c|c|c|c|c|c|}
\hline \multirow[t]{2}{*}{ Study or subgroup } & \multicolumn{2}{|c|}{ Cognitive training } & \multicolumn{2}{|c|}{ Control } & \multirow{2}{*}{$\begin{array}{c}\text { Std. Mean Difference } \\
\text { Random, } 95 \% \mathrm{Cl}\end{array}$} & \multirow[t]{2}{*}{ Weight } & \multirow{2}{*}{$\begin{array}{c}\text { Std. Mean Difference } \\
\text { Random, } 95 \% \mathrm{Cl}\end{array}$} \\
\hline & $\mathbf{N}$ & $\operatorname{Mean}(S D)$ & $\mathbf{N}$ & Mean(SD) & & & \\
\hline Koltai 2001 & 14 & $-6.4(14.3)$ & 8 & $-1(14.1)$ & 1 & $51.94 \%$ & $-0.37[-1.24,0.51]$ \\
\hline Lee 2013 & 12 & $0.3(0.3)$ & 7 & $0.1(0.3)$ & -1 - & $48.06 \%$ & $0.65[-0.31,1.61]$ \\
\hline 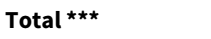 & 26 & & 15 & & & $100 \%$ & $0.12[-0.87,1.12]$ \\
\hline \multicolumn{8}{|c|}{ Heterogeneity: $\mathrm{Tau}^{2}=0.3 ; \mathrm{Chi}^{2}=2.34, \mathrm{df}=1(\mathrm{P}=0.13) ; \mathrm{I}^{2}=57.33 \%$} \\
\hline
\end{tabular}

Analysis 9.14. Comparison 9 Cognitive training vs control immediately post intervention - type of CT (multi-domain vs single domain), Outcome 14 Change in meta cognition (informant-reported).

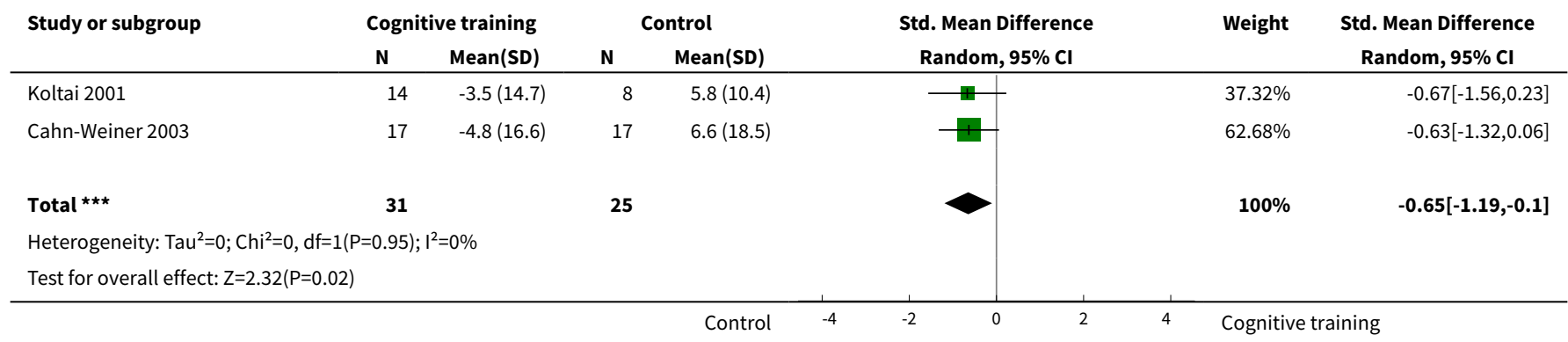

Analysis 9.15. Comparison 9 Cognitive training vs control immediately post intervention - type of CT (multi-domain vs single domain), Outcome 15 Change in participants' mood.

\begin{tabular}{|c|c|c|c|c|c|c|c|}
\hline \multirow[t]{2}{*}{ Study or subgroup } & \multicolumn{2}{|c|}{ Cognitive training } & \multicolumn{2}{|c|}{ Control } & \multirow{2}{*}{$\begin{array}{c}\text { Std. Mean Difference } \\
\text { Random, } 95 \% \mathrm{Cl}\end{array}$} & \multirow[t]{2}{*}{ Weight } & \multirow{2}{*}{$\begin{array}{c}\text { Std. Mean Difference } \\
\text { Random, } 95 \% \mathrm{Cl}\end{array}$} \\
\hline & $\mathbf{N}$ & Mean(SD) & $\mathbf{N}$ & Mean(SD) & & & \\
\hline Koltai 2001 & 14 & $-1.2(2.5)$ & 8 & $0.1(3)$ & $\longrightarrow$ & $12.23 \%$ & $-0.48[-1.36,0.4]$ \\
\hline Bergamaschi 2013 & 16 & $-1.1(3)$ & 16 & $0.4(5.1)$ & $\because$ & $12.91 \%$ & $-0.35[-1.05,0.35]$ \\
\hline Amieva 2016 & 164 & $-12.1(12.7)$ & 152 & $-10.8(12.2)$ & + & $14.11 \%$ & $-0.1[-0.32,0.12]$ \\
\hline Davis 2001 & 19 & $0.3(1.5)$ & 17 & $0.2(4.4)$ & 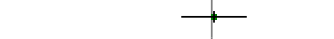 & $13.07 \%$ & $0.05[-0.61,0.7]$ \\
\hline Lee 2013 & 12 & $-0.2(2.2)$ & 7 & $-1.9(2.8)$ & & $11.93 \%$ & $0.63[-0.33,1.59]$ \\
\hline Giuli 2016 & 48 & $-0.2(4.4)$ & 47 & $-7.3(3.8)$ & $\rightarrow$ & $13.6 \%$ & $1.73[1.25,2.2]$ \\
\hline Fernández-Calvo 2011 & 30 & $3.2(2)$ & 15 & $-4.3(1.9)$ & & $11.67 \%$ & $3.75[2.73,4.78]$ \\
\hline 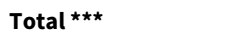 & 310 & & 266 & & & $100 \%$ & $0.72[-0.1,1.54]$ \\
\hline \multicolumn{8}{|c|}{ Heterogeneity: $\mathrm{Tau}^{2}=1.23 ; \mathrm{Chi}^{2}=99.54, \mathrm{df}=7(\mathrm{P}<0.0001) ; \mathrm{I}^{2}=92.97 \%$} \\
\hline \multicolumn{8}{|c|}{ Test for overall effect: $Z=1.73(P=0.08)$} \\
\hline
\end{tabular}


Analysis 9.16. Comparison 9 Cognitive training vs control immediately post intervention - type of CT (multi-domain vs single domain), Outcome 16 Change in capacity for activities of daily living.

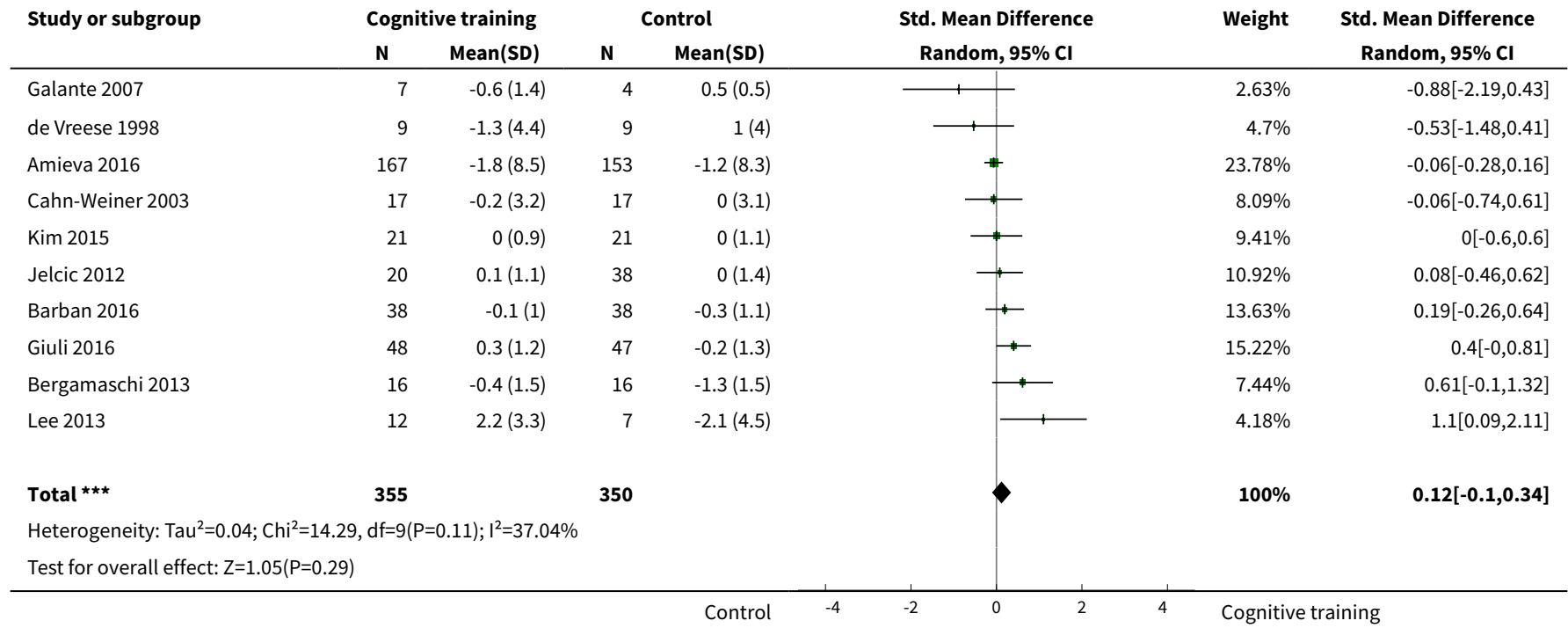

Analysis 9.17. Comparison 9 Cognitive training vs control immediately post intervention - type of CT (multi-domain vs single domain), Outcome 17 Change in disease progression.

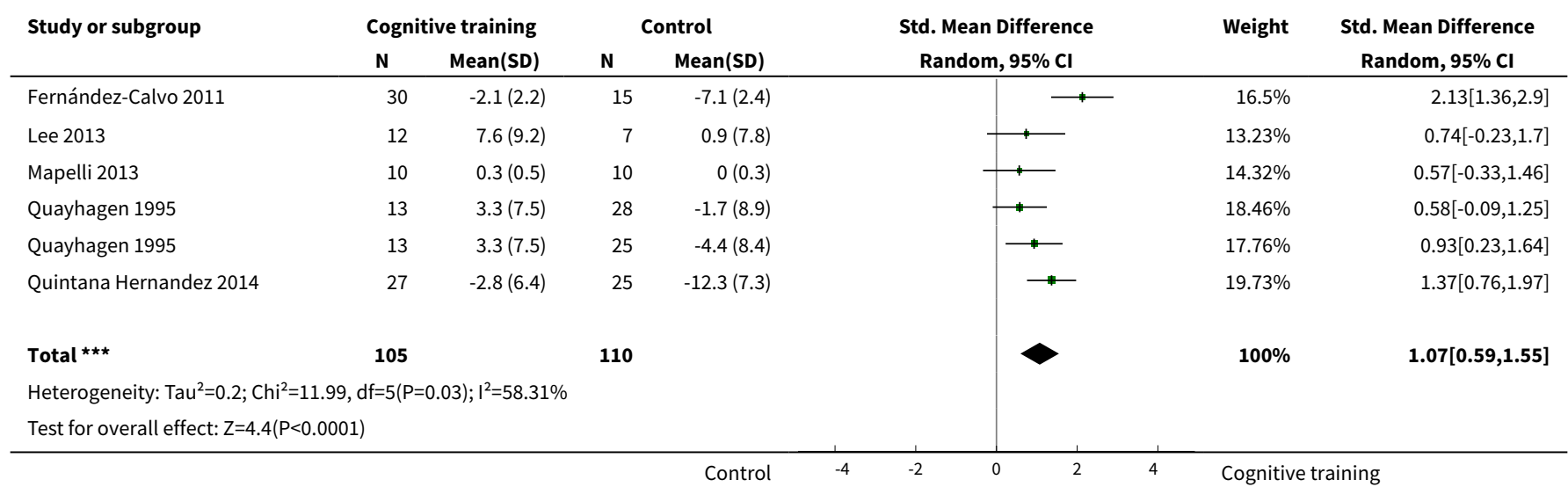

Analysis 9.18. Comparison 9 Cognitive training vs control immediately post intervention - type of CT (multidomain vs single domain), Outcome 18 Change in behavioural and psychological symptoms of dementia (BPSD).

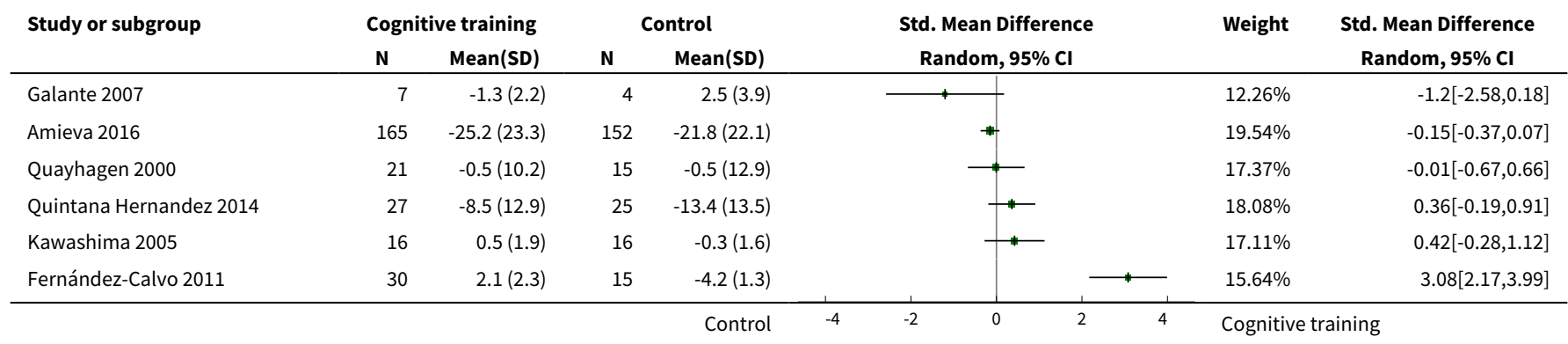




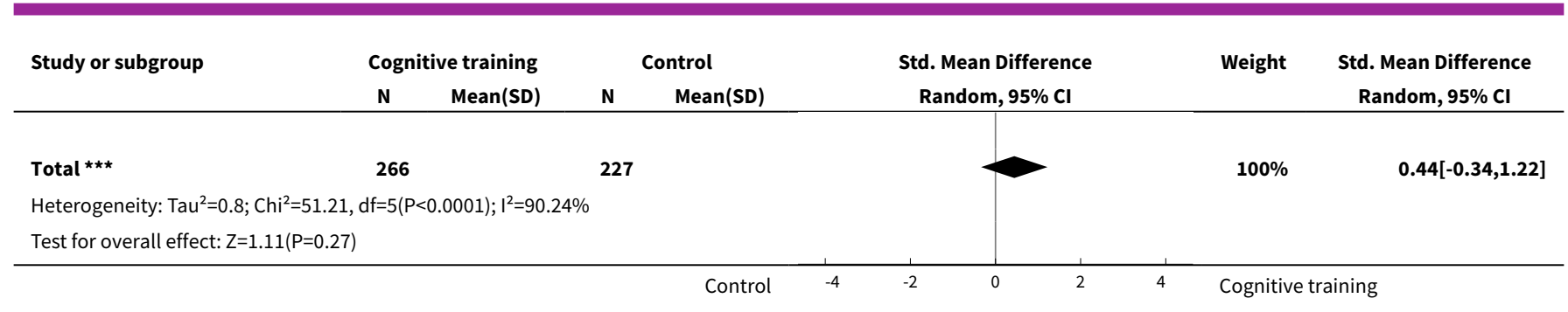

\section{Analysis 9.19. Comparison 9 Cognitive training vs control immediately post intervention - type of CT (multi-domain vs single domain), Outcome 19 Participant burden (retention rates).}

$\begin{array}{ccccc}\text { Study or subgroup } & \begin{array}{c}\text { Cognitive } \\ \text { training } \\ \mathrm{n} / \mathrm{N}\end{array} & \mathrm{n} / \mathrm{N} & \text { Odds Ratio } & \text { Odds Ratio } \\ & \mathrm{M} & \mathrm{M}-\mathrm{H}, \mathrm{Random}, 95 \% \mathrm{Cl} & \mathrm{M}-\mathrm{H}, \mathrm{Random}, 95 \% \mathrm{Cl}\end{array}$

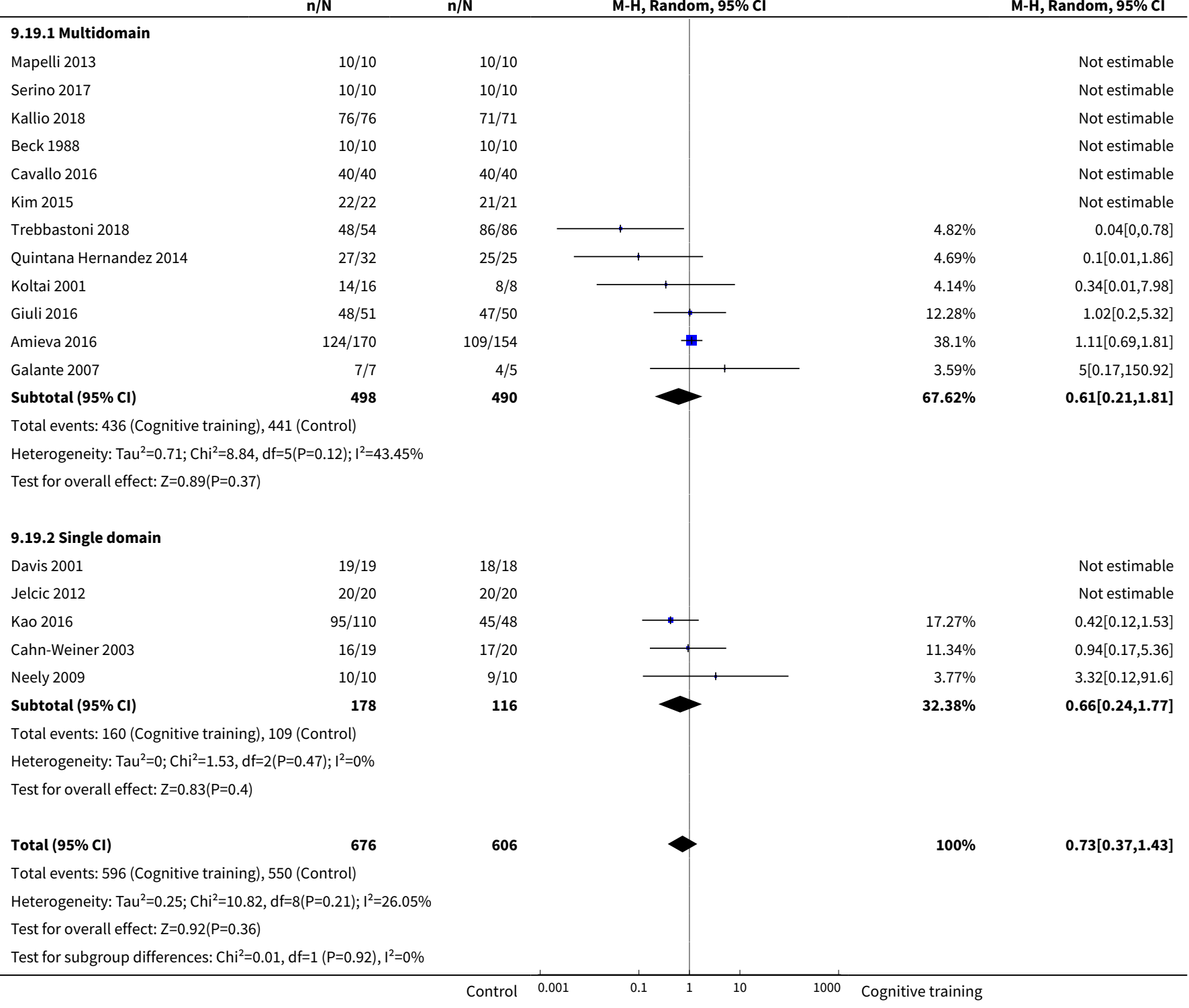


Analysis 9.20. Comparison 9 Cognitive training vs control immediately post intervention - type of CT (multi-domain vs single domain), Outcome 20 Change in burden of care (CAREGIVER).

\begin{tabular}{|c|c|c|c|c|c|c|c|}
\hline \multirow[t]{2}{*}{ Study or subgroup } & \multicolumn{2}{|c|}{ Cognitive training } & \multicolumn{2}{|c|}{ Control } & \multirow{2}{*}{$\begin{array}{c}\text { Std. Mean Difference } \\
\text { Random, } 95 \% \mathrm{CI}\end{array}$} & \multirow[t]{2}{*}{ Weight } & \multirow{2}{*}{$\begin{array}{c}\text { Std. Mean Difference } \\
\text { Random, } 95 \% \mathrm{Cl}\end{array}$} \\
\hline & $\mathbf{N}$ & Mean(SD) & $\mathbf{N}$ & Mean(SD) & & & \\
\hline Amieva 2016 & 165 & $-21.3(19.1)$ & 152 & $-17.9(19)$ & -1 & $76.98 \%$ & $-0.18[-0.4,0.04]$ \\
\hline Quayhagen 2000 & 21 & $-0.8(4.1)$ & 67 & $-1.3(3.7)$ & 7 & $23.02 \%$ & $0.13[-0.36,0.62]$ \\
\hline Total $\star \star \star$ & 186 & & 219 & & & $100 \%$ & $-0.11[-0.36,0.15]$ \\
\hline \multicolumn{8}{|c|}{ Heterogeneity: $\mathrm{Tau}^{2}=0.01 ; \mathrm{Chi}^{2}=1.23, \mathrm{df}=1(\mathrm{P}=0.27) ; \mathrm{I}^{2}=18.62 \%$} \\
\hline
\end{tabular}

Analysis 9.21. Comparison 9 Cognitive training vs control immediately post intervention type of CT (multi-domain vs single domain), Outcome 21 Change in quality of life (CAREGIVER).

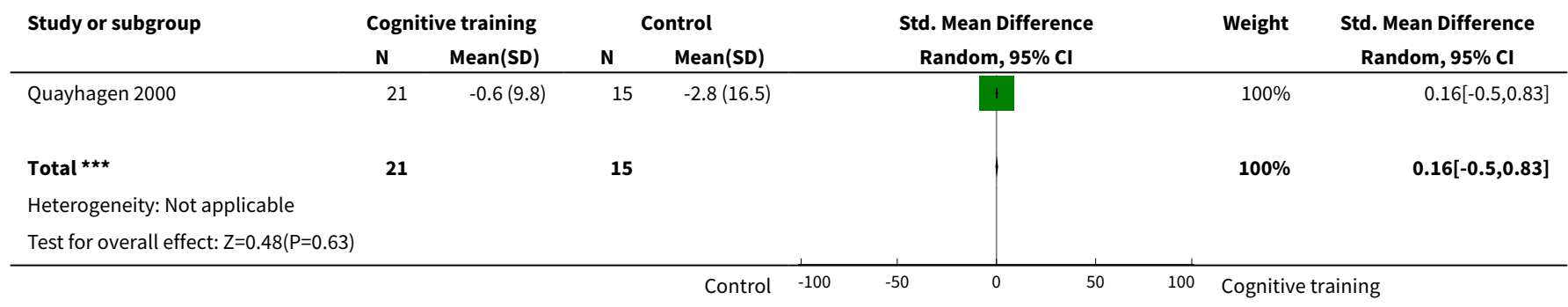

Analysis 9.22. Comparison 9 Cognitive training vs control immediately post intervention - type of CT (multi-domain vs single domain), Outcome 22 Change in mood and well-being (CAREGIVER).

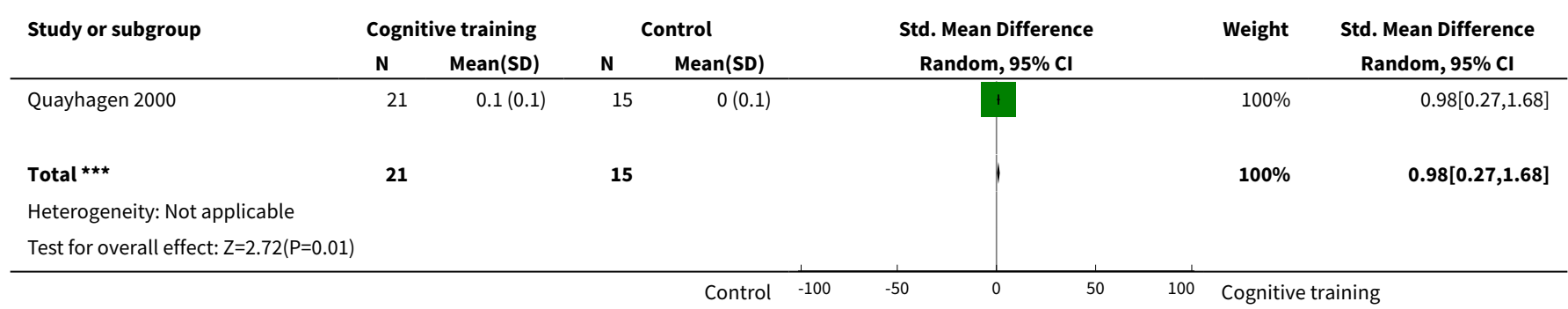

Analysis 9.23. Comparison 9 Cognitive training vs control immediately post intervention - type of CT (multi-domain vs single domain), Outcome 23 Change in general health and quality of life.

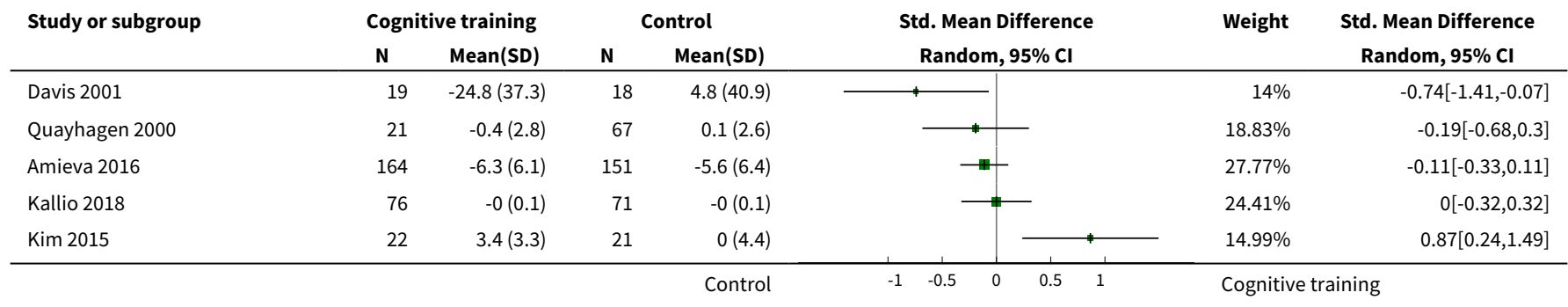




\begin{tabular}{|c|c|c|c|c|c|c|c|}
\hline \multirow[t]{2}{*}{ Study or subgroup } & \multicolumn{2}{|c|}{ Cognitive training } & \multicolumn{2}{|c|}{ Control } & \multirow{2}{*}{$\begin{array}{c}\text { Std. Mean Difference } \\
\text { Random, } 95 \% \mathrm{Cl}\end{array}$} & \multirow[t]{2}{*}{ Weight } & \multirow{2}{*}{$\begin{array}{c}\text { Std. Mean Difference } \\
\text { Random, } 95 \% \mathrm{Cl}\end{array}$} \\
\hline & $\mathbf{N}$ & Mean(SD) & $\mathbf{N}$ & Mean(SD) & & & \\
\hline 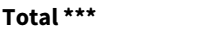 & 302 & & 328 & & & $100 \%$ & $-0.04[-0.38,0.29]$ \\
\hline \multicolumn{8}{|c|}{ Heterogeneity: $\mathrm{Tau}^{2}=0.09 ; \mathrm{Chi}^{2}=12.93, \mathrm{df}=4(\mathrm{P}=0.01) ; \mathrm{I}^{2}=69.08 \%$} \\
\hline \multicolumn{8}{|c|}{ Test for overall effect: $Z=0.24(P=0.81)$} \\
\hline & & & & Control & $-1 \quad-0.5$ & Cognitiv & ining \\
\hline
\end{tabular}

\section{Comparison 10. Cognitive training vs control immediately post intervention - type of control (passive vs active)}

\begin{tabular}{|c|c|c|c|c|}
\hline Outcome or subgroup title & $\begin{array}{l}\text { No. of } \\
\text { studies }\end{array}$ & $\begin{array}{l}\text { No. of } \\
\text { partici- } \\
\text { pants }\end{array}$ & Statistical method & Effect size \\
\hline $\begin{array}{l}1 \text { Change in a global measure of cog- } \\
\text { nition }\end{array}$ & 20 & 1288 & $\begin{array}{l}\text { Std. Mean Difference (IV, Random, } \\
95 \% \mathrm{CI} \text { ) }\end{array}$ & $0.65[0.26,1.05]$ \\
\hline 1.1 Passive & 11 & 912 & $\begin{array}{l}\text { Std. Mean Difference (IV, Random, } \\
95 \% \mathrm{CI} \text { ) }\end{array}$ & $0.75[0.22,1.28]$ \\
\hline 1.2 Active & 9 & 376 & $\begin{array}{l}\text { Std. Mean Difference (IV, Random, } \\
95 \% \mathrm{CI} \text { ) }\end{array}$ & $0.54[-0.11,1.19]$ \\
\hline $\begin{array}{l}2 \text { Change in a global measure of cog- } \\
\text { nition_zero correlation }\end{array}$ & 20 & 1287 & $\begin{array}{l}\text { Std. Mean Difference (IV, Random, } \\
95 \% \mathrm{CI} \text { ) }\end{array}$ & $0.27[0.04,0.50]$ \\
\hline 2.1 Passive & 11 & 911 & $\begin{array}{l}\text { Std. Mean Difference (IV, Random, } \\
95 \% \mathrm{CI} \text { ) }\end{array}$ & $0.27[-0.04,0.58]$ \\
\hline 2.2 Active & 9 & 376 & $\begin{array}{l}\text { Std. Mean Difference (IV, Random, } \\
95 \% \mathrm{CI} \text { ) }\end{array}$ & $0.27[-0.10,0.65]$ \\
\hline $\begin{array}{l}3 \text { Change in a global measure of cog- } \\
\text { nition (composite) }\end{array}$ & 26 & 1389 & $\begin{array}{l}\text { Std. Mean Difference (Random, 95\% } \\
\mathrm{Cl} \text { ) }\end{array}$ & $0.42[0.23,0.61]$ \\
\hline 3.1 Passive & 14 & 875 & $\begin{array}{l}\text { Std. Mean Difference (Random, 95\% } \\
\mathrm{Cl} \text { ) }\end{array}$ & $0.31[0.06,0.55]$ \\
\hline 3.2 Active & 13 & 514 & $\begin{array}{l}\text { Std. Mean Difference (Random, 95\% } \\
\mathrm{Cl} \text { ) }\end{array}$ & $0.53[0.26,0.81]$ \\
\hline $\begin{array}{l}4 \text { Change in a global measure of cog- } \\
\text { nition (composite)_zero correlation }\end{array}$ & 26 & 1390 & $\begin{array}{l}\text { Std. Mean Difference (Random, 95\% } \\
\mathrm{Cl} \text { ) }\end{array}$ & $0.24[0.12,0.36]$ \\
\hline 4.1 Passive & 14 & 876 & $\begin{array}{l}\text { Std. Mean Difference (Random, 95\% } \\
\mathrm{Cl} \text { ) }\end{array}$ & $0.24[0.04,0.43]$ \\
\hline 4.2 Active & 13 & 514 & $\begin{array}{l}\text { Std. Mean Difference (Random, 95\% } \\
\mathrm{Cl} \text { ) }\end{array}$ & $0.30[0.13,0.48]$ \\
\hline 5 Change in immediate memory & 17 & 762 & $\begin{array}{l}\text { Std. Mean Difference (IV, Random, } \\
95 \% \mathrm{CI})\end{array}$ & $0.74[0.37,1.12]$ \\
\hline 5.1 Passive & 7 & 311 & $\begin{array}{l}\text { Std. Mean Difference (IV, Random, } \\
95 \% \mathrm{CI})\end{array}$ & $0.64[0.20,1.08]$ \\
\hline
\end{tabular}




\begin{tabular}{|c|c|c|c|c|}
\hline Outcome or subgroup title & $\begin{array}{l}\text { No. of } \\
\text { studies }\end{array}$ & $\begin{array}{l}\text { No. of } \\
\text { partici- } \\
\text { pants }\end{array}$ & Statistical method & Effect size \\
\hline 5.2 Active & 11 & 451 & $\begin{array}{l}\text { Std. Mean Difference (IV, Random, } \\
95 \% \mathrm{CI})\end{array}$ & $0.82[0.25,1.39]$ \\
\hline 6 Change in delayed memory & 11 & 543 & $\begin{array}{l}\text { Std. Mean Difference (IV, Random, } \\
95 \% \mathrm{Cl} \text { ) }\end{array}$ & $0.81[0.29,1.32]$ \\
\hline 6.1 Passive & 6 & 325 & $\begin{array}{l}\text { Std. Mean Difference (IV, Random, } \\
95 \% \mathrm{CI} \text { ) }\end{array}$ & $0.52[0.11,0.94]$ \\
\hline 6.2 Active & 5 & 218 & $\begin{array}{l}\text { Std. Mean Difference (IV, Random, } \\
95 \% \mathrm{CI} \text { ) }\end{array}$ & $1.04[-0.04,2.12]$ \\
\hline $\begin{array}{l}7 \text { Change in attention and working } \\
\text { memory }\end{array}$ & 12 & 551 & $\begin{array}{l}\text { Std. Mean Difference (IV, Random, } \\
95 \% \mathrm{CI})\end{array}$ & $0.56[0.08,1.05]$ \\
\hline 7.1 Passive & 4 & 206 & $\begin{array}{l}\text { Std. Mean Difference (IV, Random, } \\
95 \% \mathrm{CI})\end{array}$ & $0.37[-0.25,0.99]$ \\
\hline 7.2 Active & 8 & 345 & $\begin{array}{l}\text { Std. Mean Difference (IV, Random, } \\
95 \% \mathrm{CI})\end{array}$ & $0.63[-0.03,1.29]$ \\
\hline 8 Change in language (naming) & 5 & 311 & $\begin{array}{l}\text { Std. Mean Difference (IV, Random, } \\
95 \% \mathrm{CI} \text { ) }\end{array}$ & $0.62[0.11,1.12]$ \\
\hline 9 Change in verbal letter fluency & 12 & 544 & $\begin{array}{l}\text { Std. Mean Difference (IV, Random, } \\
95 \% \mathrm{CI} \text { ) }\end{array}$ & $0.22[-0.07,0.50]$ \\
\hline 9.1 Passive & 4 & 263 & $\begin{array}{l}\text { Std. Mean Difference (IV, Random, } \\
95 \% \mathrm{CI})\end{array}$ & $0.35[-0.03,0.72]$ \\
\hline 9.2 Active & 8 & 281 & $\begin{array}{l}\text { Std. Mean Difference (IV, Random, } \\
95 \% \mathrm{Cl} \text { ) }\end{array}$ & $0.14[-0.27,0.55]$ \\
\hline $\begin{array}{l}10 \text { Change in speed of information } \\
\text { processing }\end{array}$ & 6 & 201 & $\begin{array}{l}\text { Std. Mean Difference (IV, Random, } \\
95 \% \mathrm{CI})\end{array}$ & $0.22[-0.11,0.54]$ \\
\hline 11 Change in executive function & 11 & 511 & $\begin{array}{l}\text { Std. Mean Difference (IV, Random, } \\
95 \% \mathrm{CI})\end{array}$ & $0.75[0.28,1.22]$ \\
\hline 11.1 Passive & 6 & 285 & $\begin{array}{l}\text { Std. Mean Difference (IV, Random, } \\
95 \% \mathrm{Cl} \text { ) }\end{array}$ & $1.37[0.60,2.14]$ \\
\hline 11.2 Active & 6 & 226 & $\begin{array}{l}\text { Std. Mean Difference (IV, Random, } \\
95 \% \mathrm{CI} \text { ) }\end{array}$ & $0.21[-0.11,0.53]$ \\
\hline 12 Change in verbal category fluency & 9 & 475 & $\begin{array}{l}\text { Std. Mean Difference (IV, Random, } \\
95 \% \mathrm{CI} \text { ) }\end{array}$ & $0.52[0.23,0.81]$ \\
\hline $\begin{array}{l}13 \text { Change in meta cognition (self-re- } \\
\text { ported) }\end{array}$ & 2 & 41 & $\begin{array}{l}\text { Std. Mean Difference (IV, Random, } \\
95 \% \mathrm{Cl} \text { ) }\end{array}$ & $0.12[-0.87,1.12]$ \\
\hline $\begin{array}{l}14 \text { Change in meta cognition (infor- } \\
\text { mant-reported) }\end{array}$ & 2 & 56 & $\begin{array}{l}\text { Std. Mean Difference (IV, Random, } \\
95 \% \mathrm{CI} \text { ) }\end{array}$ & $-0.65[-1.19,-0.10]$ \\
\hline
\end{tabular}




\begin{tabular}{|c|c|c|c|c|}
\hline Outcome or subgroup title & $\begin{array}{l}\text { No. of } \\
\text { studies }\end{array}$ & $\begin{array}{l}\text { No. of } \\
\text { partici- } \\
\text { pants }\end{array}$ & Statistical method & Effect size \\
\hline 15 Change in participants' mood & 8 & 576 & $\begin{array}{l}\text { Std. Mean Difference (IV, Random, } \\
95 \% \mathrm{Cl} \text { ) }\end{array}$ & $0.72[-0.10,1.54]$ \\
\hline 15.1 Passive & 3 & 383 & $\begin{array}{l}\text { Std. Mean Difference (IV, Random, } \\
95 \% \mathrm{CI} \text { ) }\end{array}$ & $1.02[-1.07,3.10]$ \\
\hline 15.2 Active & 5 & 193 & $\begin{array}{l}\text { Std. Mean Difference (IV, Random, } \\
95 \% \mathrm{CI} \text { ) }\end{array}$ & $0.58[-0.34,1.49]$ \\
\hline $\begin{array}{l}16 \text { Change in capacity for activities of } \\
\text { daily living }\end{array}$ & 10 & 705 & $\begin{array}{l}\text { Std. Mean Difference (IV, Random, } \\
95 \% \mathrm{CI} \text { ) }\end{array}$ & $0.12[-0.10,0.34]$ \\
\hline 17 Change in disease progression & 5 & 215 & $\begin{array}{l}\text { Std. Mean Difference (IV, Random, } \\
95 \% \mathrm{Cl} \text { ) }\end{array}$ & $1.07[0.59,1.55]$ \\
\hline $\begin{array}{l}18 \text { Change in behavioural and psy- } \\
\text { chological symptoms of dementia } \\
\text { (BPSD) }\end{array}$ & 6 & 493 & $\begin{array}{l}\text { Std. Mean Difference (IV, Random, } \\
95 \% \mathrm{CI} \text { ) }\end{array}$ & $0.44[-0.34,1.22]$ \\
\hline $\begin{array}{l}19 \text { Participant burden (retention } \\
\text { rates) }\end{array}$ & 17 & 1282 & Odds Ratio (M-H, Random, 95\% Cl) & $0.73[0.37,1.43]$ \\
\hline 19.1 Passive & 9 & 910 & Odds Ratio (M-H, Random, 95\% Cl) & $0.49[0.17,1.40]$ \\
\hline 19.2 Active & 8 & 372 & Odds Ratio (M-H, Random, 95\% Cl) & $1.17[0.38,3.64]$ \\
\hline $\begin{array}{l}20 \text { Change in burden of care } \\
\text { (CAREGIVER) }\end{array}$ & 2 & 405 & $\begin{array}{l}\text { Std. Mean Difference (IV, Random, } \\
95 \% \mathrm{CI})\end{array}$ & $-0.11[-0.36,0.15]$ \\
\hline $\begin{array}{l}21 \text { Change in quality of life } \\
\text { (CAREGIVER) }\end{array}$ & 1 & 36 & $\begin{array}{l}\text { Std. Mean Difference (IV, Random, } \\
95 \% \mathrm{CI} \text { ) }\end{array}$ & $0.16[-0.50,0.83]$ \\
\hline $\begin{array}{l}22 \text { Change in mood and well-being } \\
\text { (CAREGIVER) }\end{array}$ & 1 & 36 & $\begin{array}{l}\text { Std. Mean Difference (IV, Random, } \\
95 \% \mathrm{Cl} \text { ) }\end{array}$ & $0.98[0.27,1.68]$ \\
\hline $\begin{array}{l}23 \text { Change in general health and qual- } \\
\text { ity of life }\end{array}$ & 5 & 630 & $\begin{array}{l}\text { Std. Mean Difference (IV, Random, } \\
95 \% \mathrm{CI} \text { ) }\end{array}$ & $-0.04[-0.38,0.29]$ \\
\hline
\end{tabular}

\section{Analysis 10.1. Comparison 10 Cognitive training vs control immediately post intervention - type of control (passive vs active), Outcome 1 Change in a global measure of cognition.}

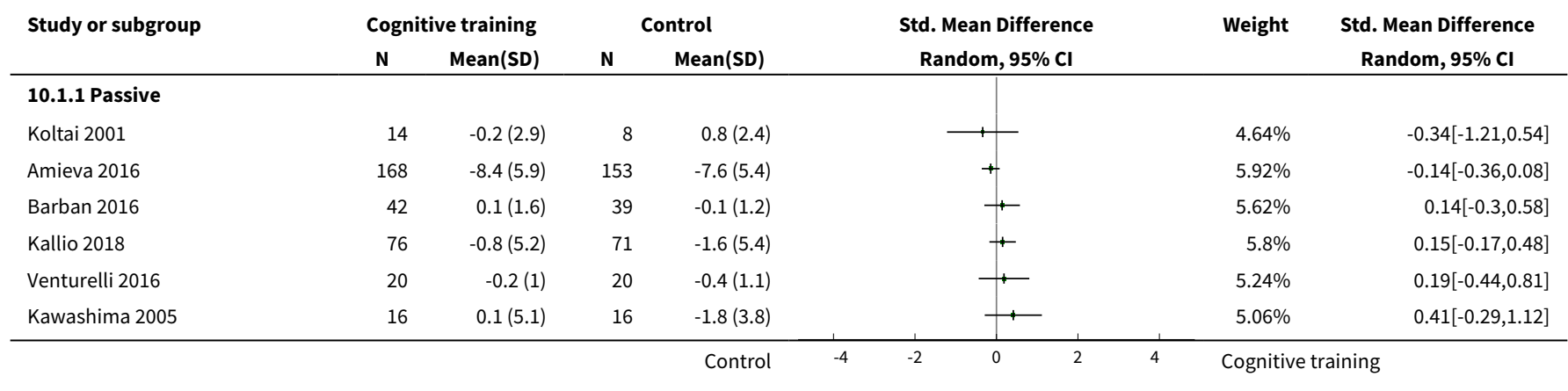




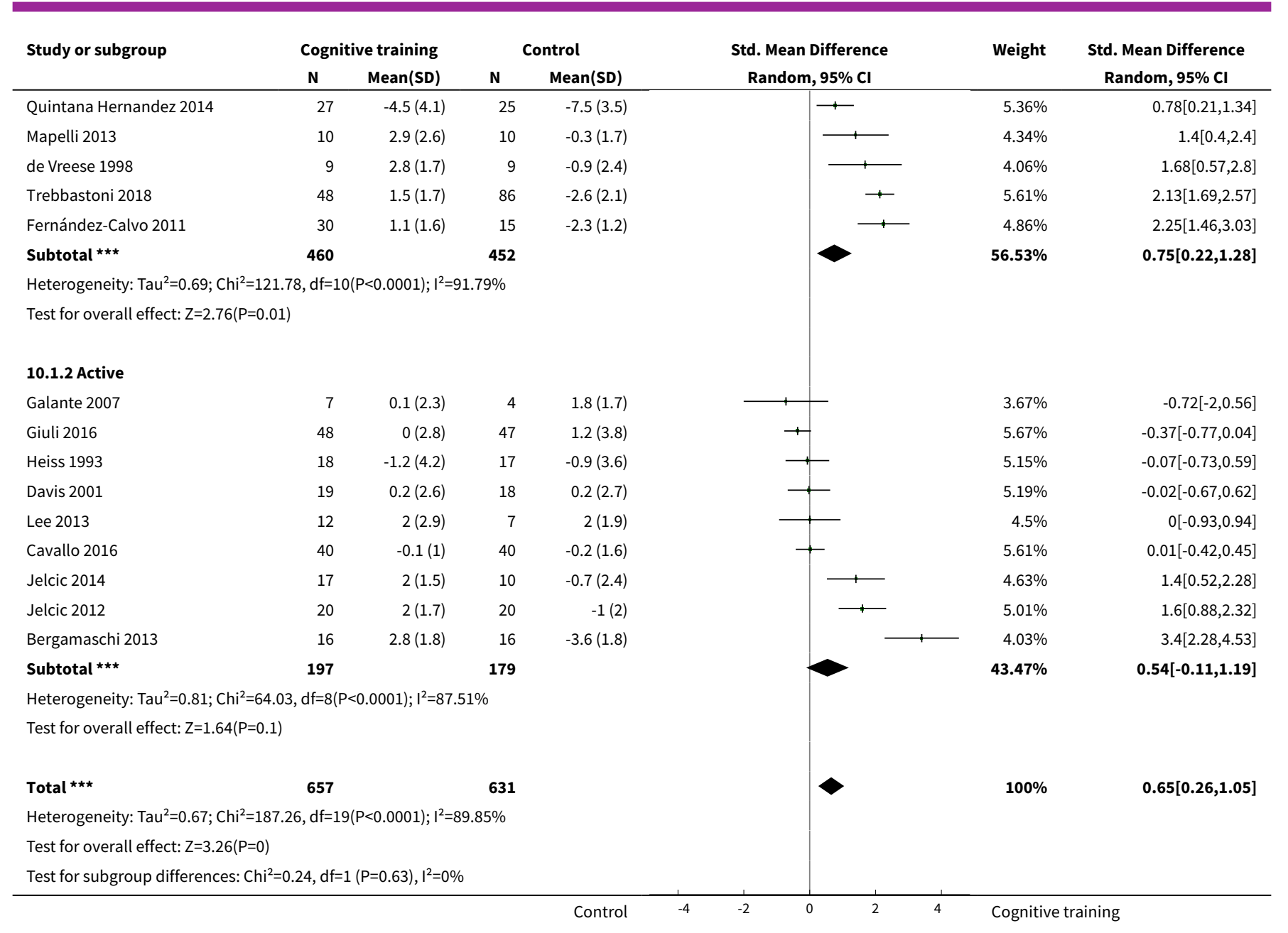

\section{Analysis 10.2. Comparison 10 Cognitive training vs control immediately post intervention - type} of control (passive vs active), Outcome 2 Change in a global measure of cognition_zero correlation.

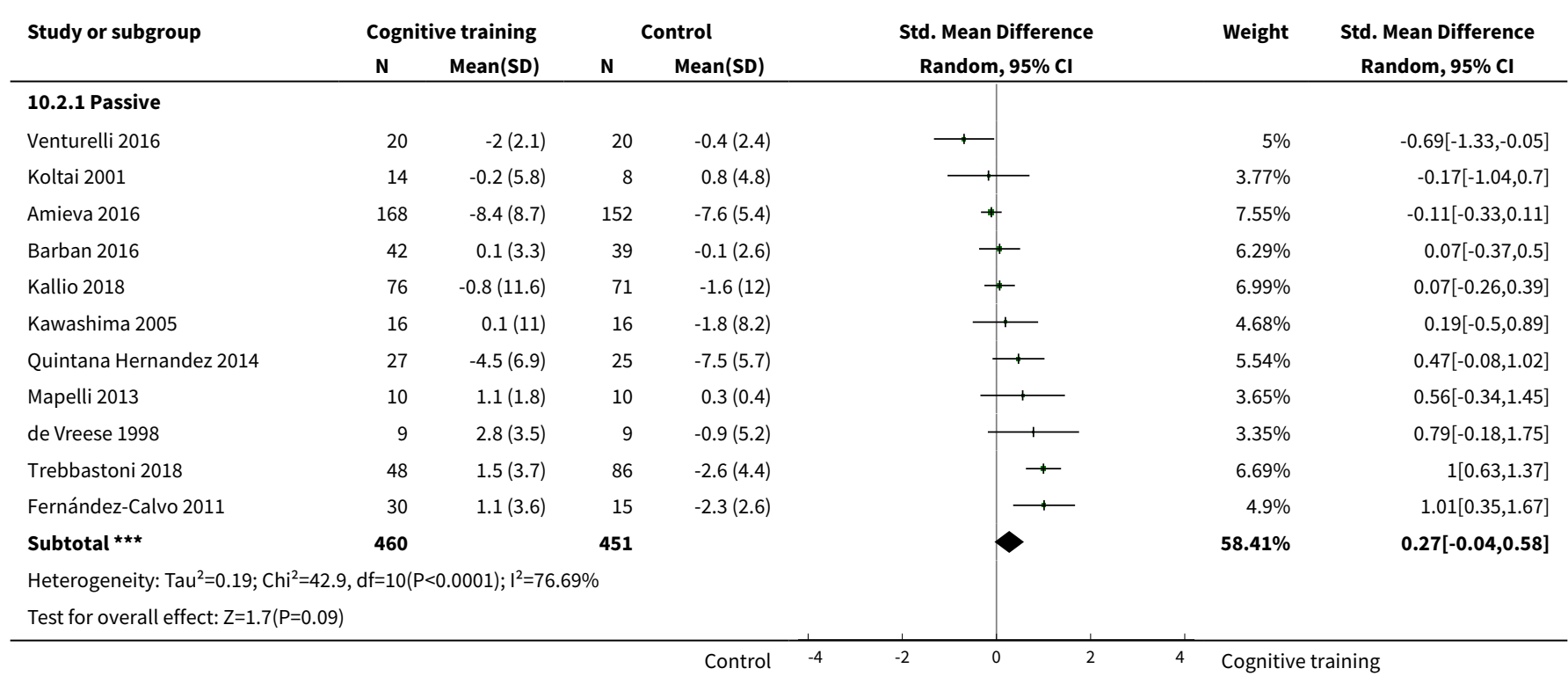




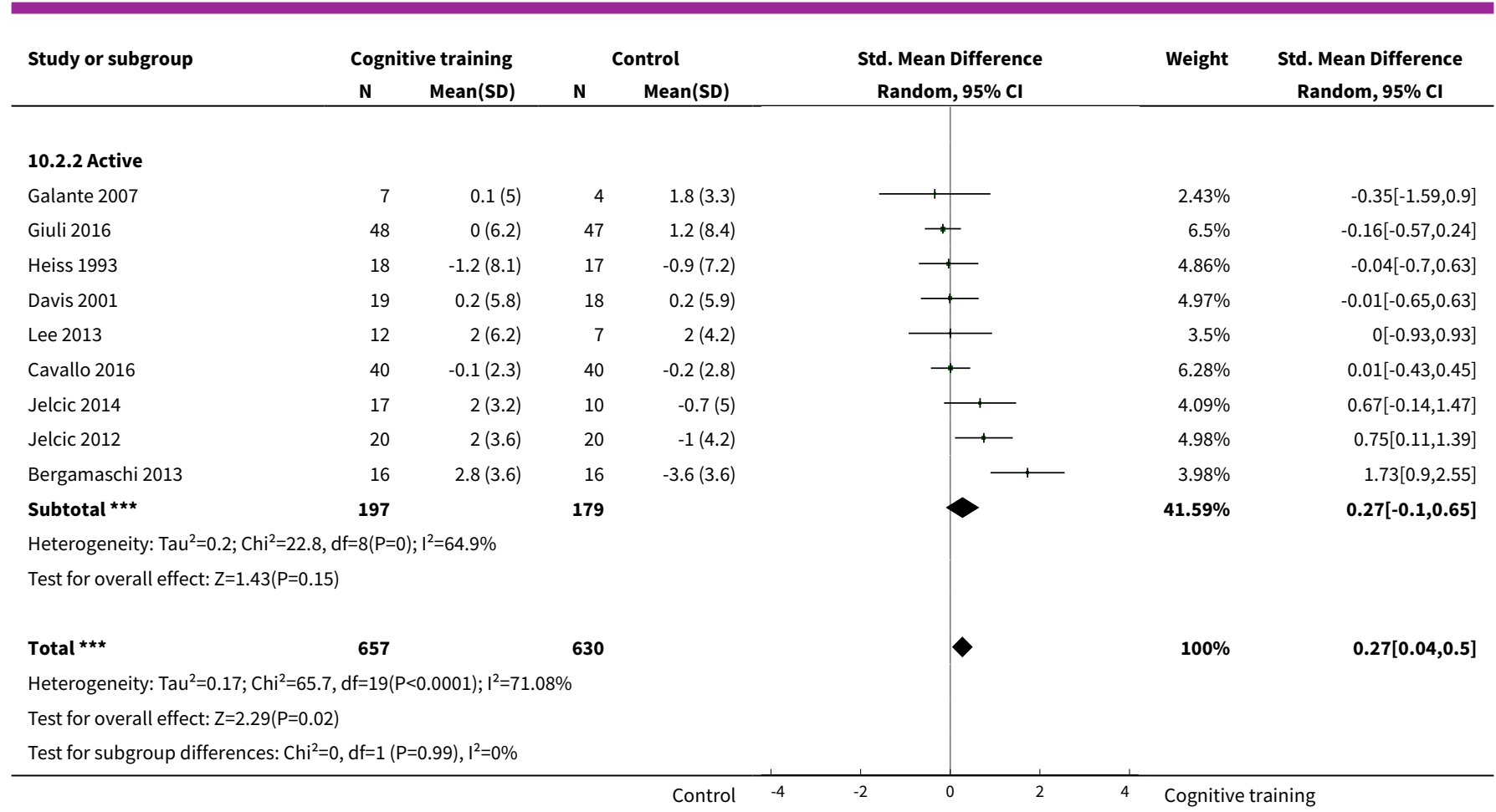

\section{Analysis 10.3. Comparison 10 Cognitive training vs control immediately post intervention - type of control (passive vs active), Outcome 3 Change in a global measure of cognition (composite).}

\begin{tabular}{|c|c|c|c|c|c|c|}
\hline \multirow{2}{*}{$\begin{array}{l}\text { Study or subgroup } \\
\text { 10.3.1 Passive }\end{array}$} & $\begin{array}{c}\text { Cognitive } \\
\text { training } \\
\mathbf{N} \\
\end{array}$ & $\begin{array}{l}\text { Control } \\
\text { N }\end{array}$ & $\begin{array}{c}\text { Std. Mean } \\
\text { Difference } \\
(\mathrm{SE}) \\
\end{array}$ & $\begin{array}{l}\text { Std. Mean Difference } \\
\text { IV, Random, } 95 \% \text { CI }\end{array}$ & \multirow[t]{2}{*}{ Weight } & \multirow[t]{2}{*}{$\begin{array}{l}\text { Std. Mean Difference } \\
\text { IV, Random, } 95 \% \text { CI }\end{array}$} \\
\hline & & & & & & \\
\hline Fernández-Calvo 2011 & 30 & 15 & $-0.4(0.42)$ & 1 & $3.05 \%$ & $-0.44[-1.26,0.38]$ \\
\hline Amieva 2016 & 165 & 153 & $-0.1(0.11)$ & + & $6.61 \%$ & $-0.11[-0.33,0.11]$ \\
\hline Beck 1988 & 10 & 10 & $-0(0.43)$ & $\longrightarrow$ & $2.97 \%$ & $-0.03[-0.87,0.81]$ \\
\hline Boller 2011 & 24 & 12 & $0.1(0.35)$ & + & $3.7 \%$ & $0.13[-0.56,0.82]$ \\
\hline Quayhagen 2000 & 21 & 15 & $0.2(0.33)$ & 1 & $3.92 \%$ & $0.19[-0.46,0.84]$ \\
\hline Barban 2016 & 41 & 38 & $0.2(0.23)$ & 1 & $5.12 \%$ & $0.21[-0.24,0.66]$ \\
\hline Neely 2009 & 20 & 9 & $0.3(0.39)$ & 1 & $3.31 \%$ & $0.26[-0.5,1.02]$ \\
\hline Quayhagen 1995 & 13 & 25 & $0.4(0.34)$ & 1 & $3.81 \%$ & $0.37[-0.3,1.04]$ \\
\hline Kawashima 2005 & 16 & 16 & $0.5(0.36)$ & 1 & $3.6 \%$ & $0.52[-0.19,1.23]$ \\
\hline Quintana Hernandez 2014 & 27 & 25 & $0.7(0.28)$ & 十 & $4.49 \%$ & $0.73[0.18,1.28]$ \\
\hline de Vreese 1998 & 9 & 9 & $1(0.49)$ & 1 & $2.52 \%$ & $1.04[0.08,2]$ \\
\hline Mapelli 2013 & 10 & 10 & $2.1(0.66)$ & $\longrightarrow$ & $1.65 \%$ & $2.13[0.84,3.42]$ \\
\hline Subtotal $(95 \% \mathrm{Cl})$ & & & & $\diamond$ & $53.23 \%$ & $0.31[0.06,0.55]$ \\
\hline \multicolumn{7}{|c|}{ Heterogeneity: $\mathrm{Tau}^{2}=0.11 ; \mathrm{Chi}^{2}=29.98, \mathrm{df}=13(\mathrm{P}=0) ; \mathrm{I}^{2}=56.64 \%$} \\
\hline \multicolumn{7}{|c|}{ Test for overall effect: $Z=2.46(P=0.01)$} \\
\hline \multicolumn{7}{|l|}{ 10.3.2 Active } \\
\hline Davis 2001 & 19 & 18 & $0(0.32)$ & 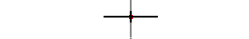 & $4.03 \%$ & $0[-0.63,0.63]$ \\
\hline Cahn-Weiner 2003 & 17 & 17 & $0(0.34)$ & $\longrightarrow$ & $3.81 \%$ & $0.01[-0.66,0.68]$ \\
\hline
\end{tabular}




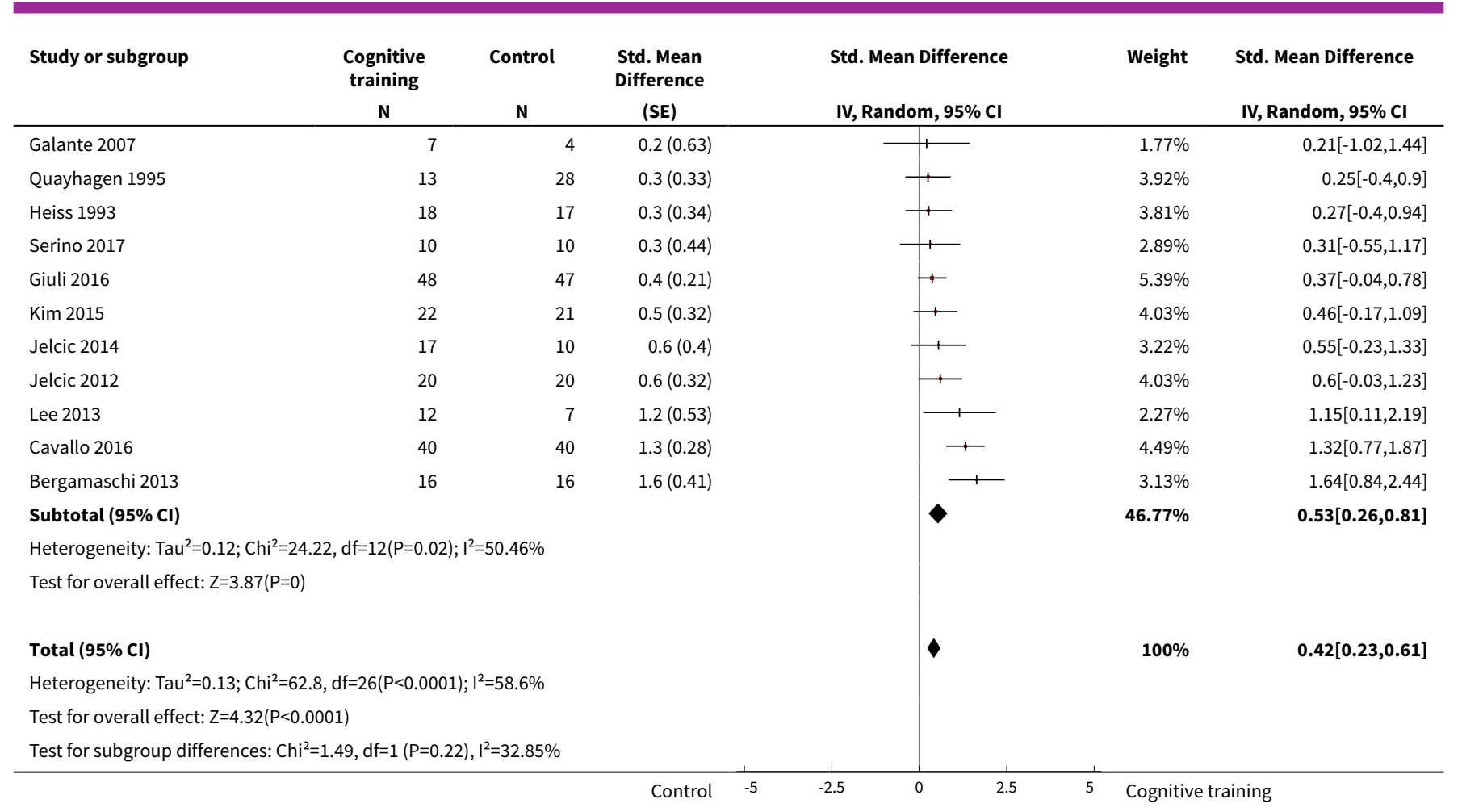

\section{Analysis 10.4. Comparison 10 Cognitive training vs control immediately post intervention - type of control (passive vs active), Outcome 4 Change in a global measure of cognition (composite)_zero correlation.}

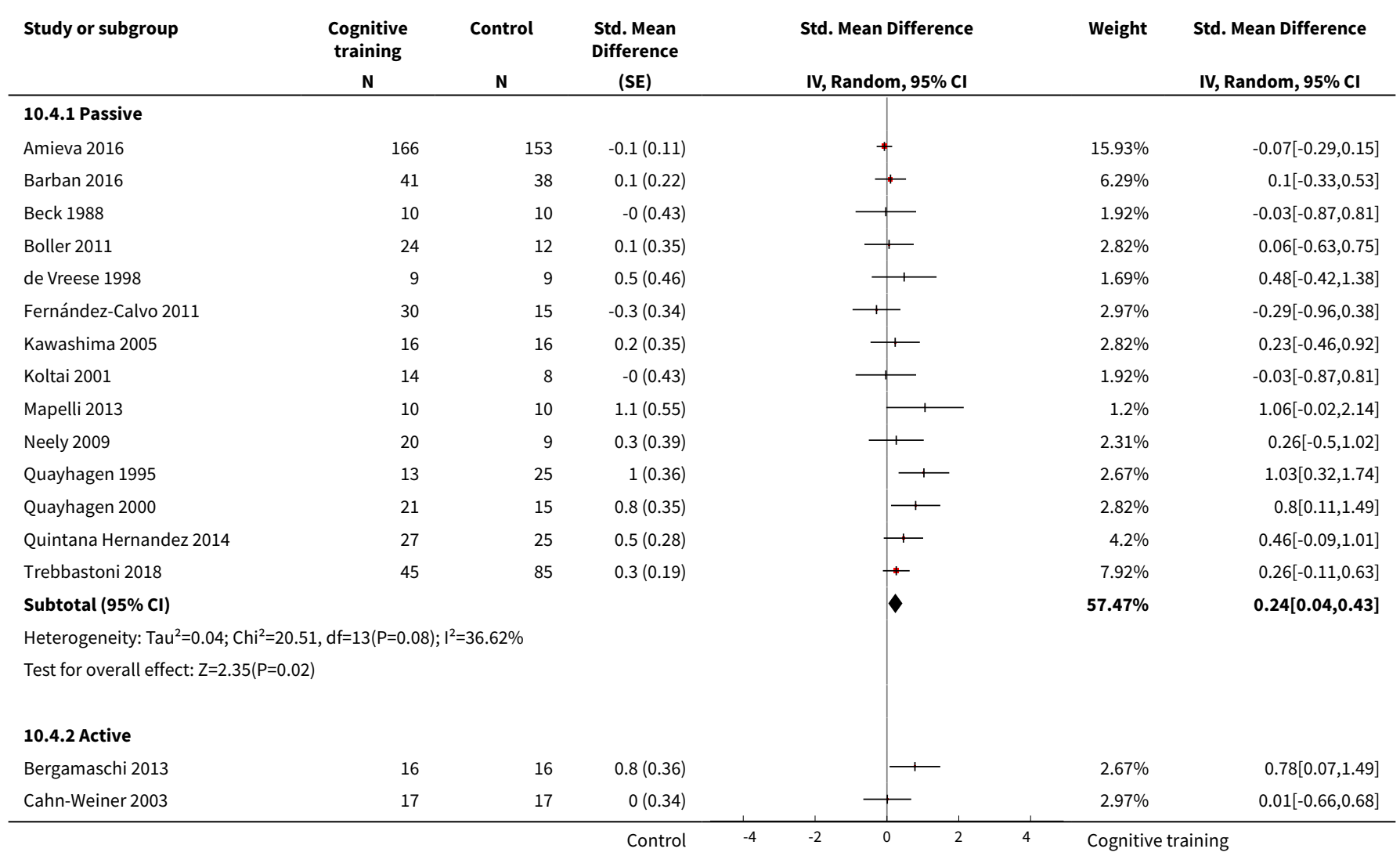




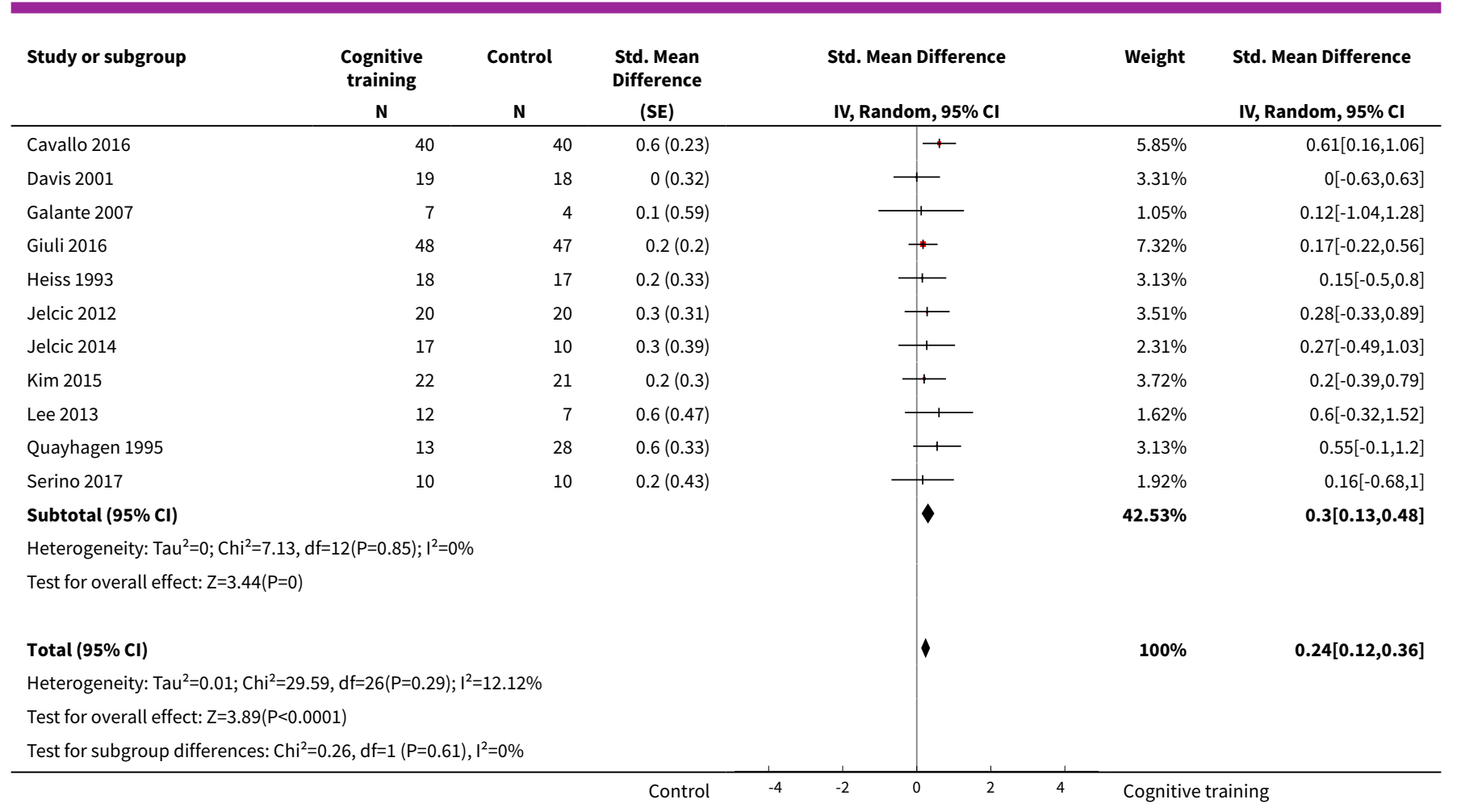

\section{Analysis 10.5. Comparison 10 Cognitive training vs control immediately post intervention - type of control (passive vs active), Outcome 5 Change in immediate memory.}

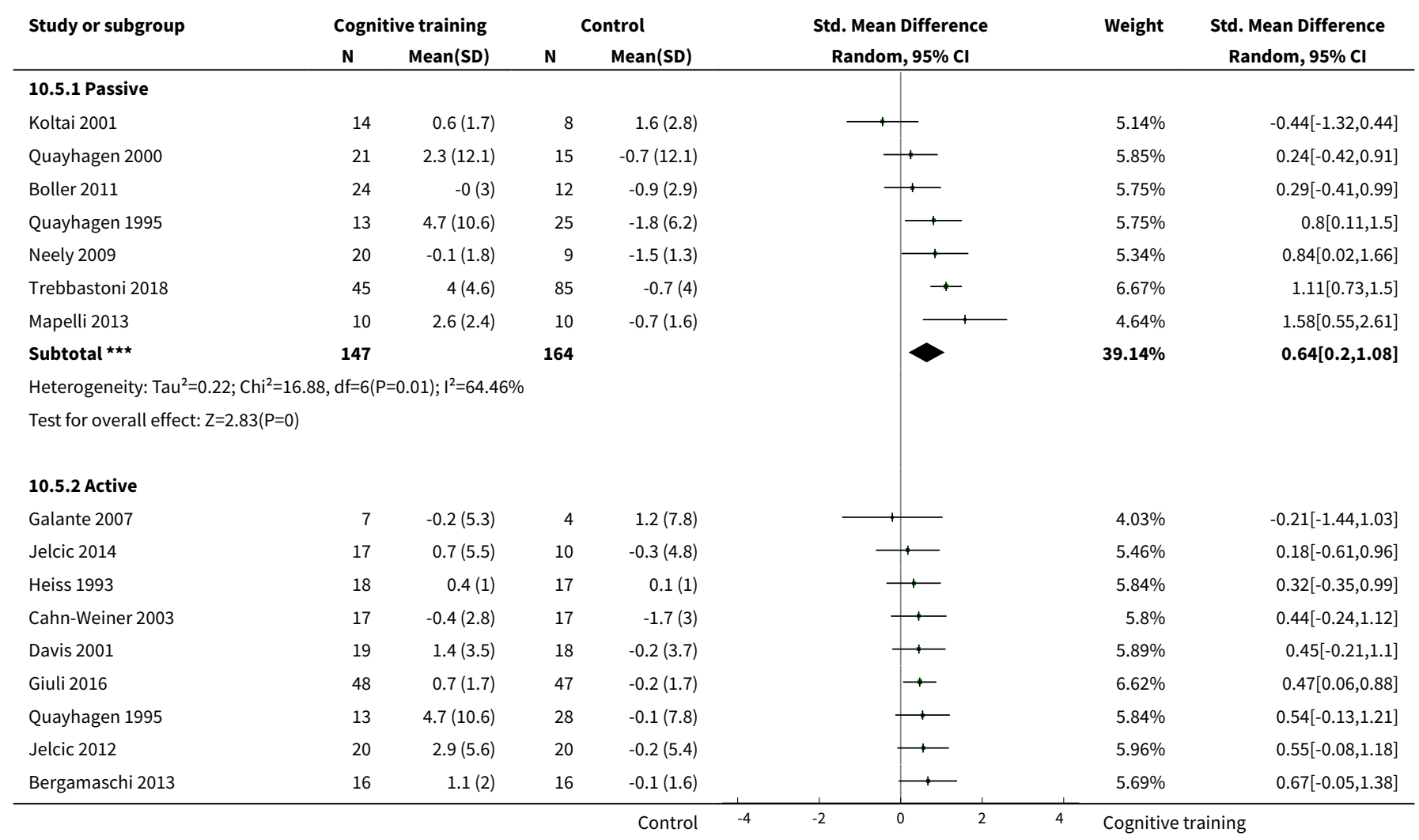




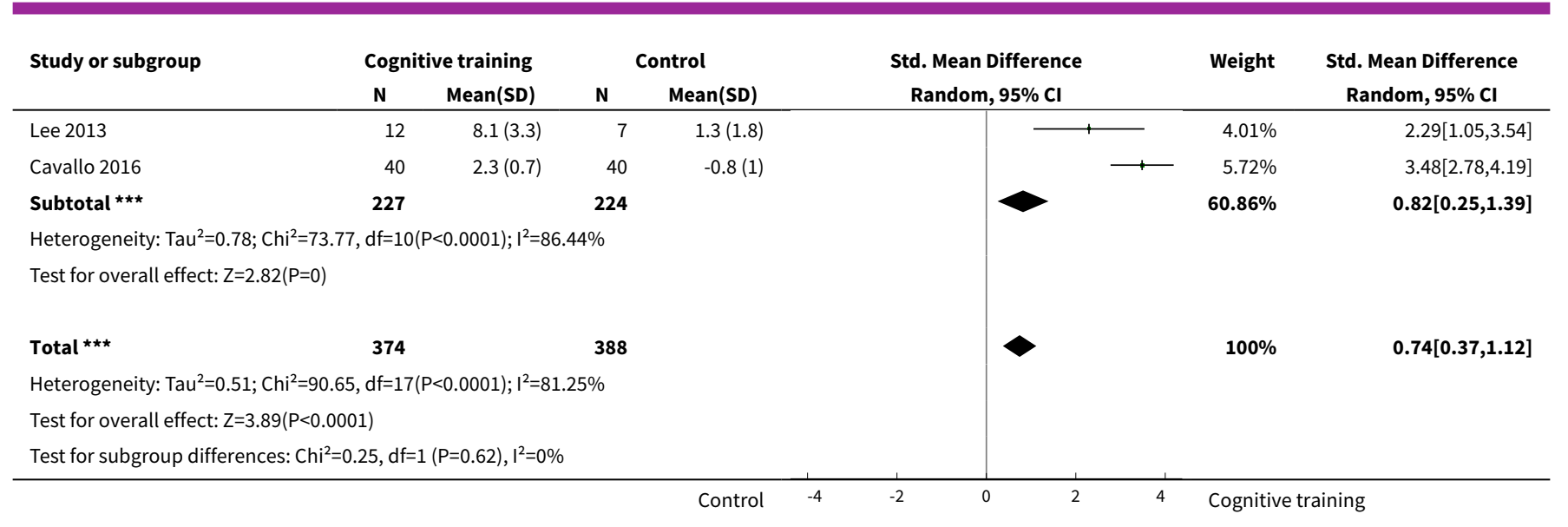

Analysis 10.6. Comparison 10 Cognitive training vs control immediately post intervention - type of control (passive vs active), Outcome 6 Change in delayed memory.

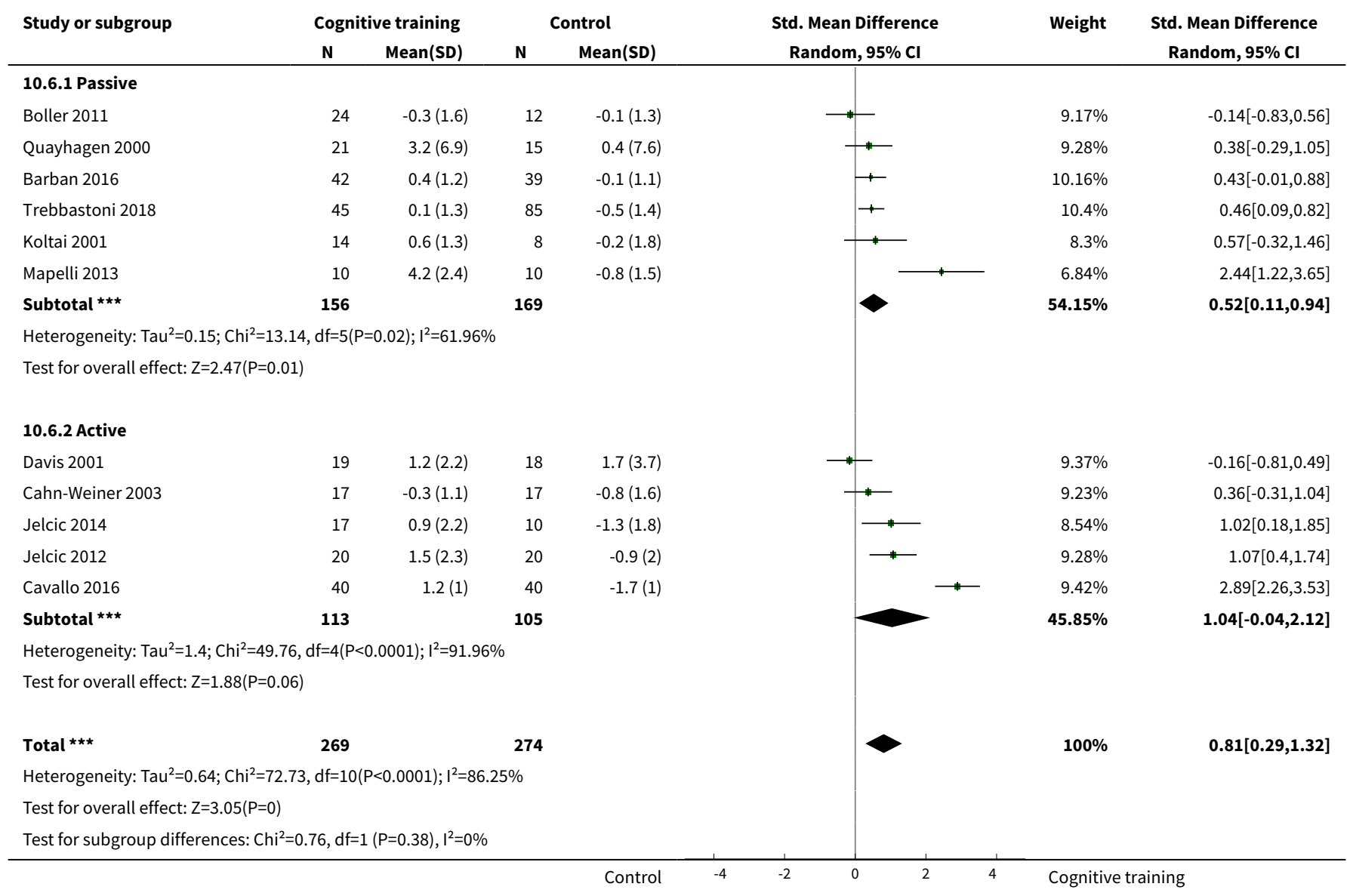


Analysis 10.7. Comparison 10 Cognitive training vs control immediately post intervention - type of control (passive vs active), Outcome 7 Change in attention and working memory.

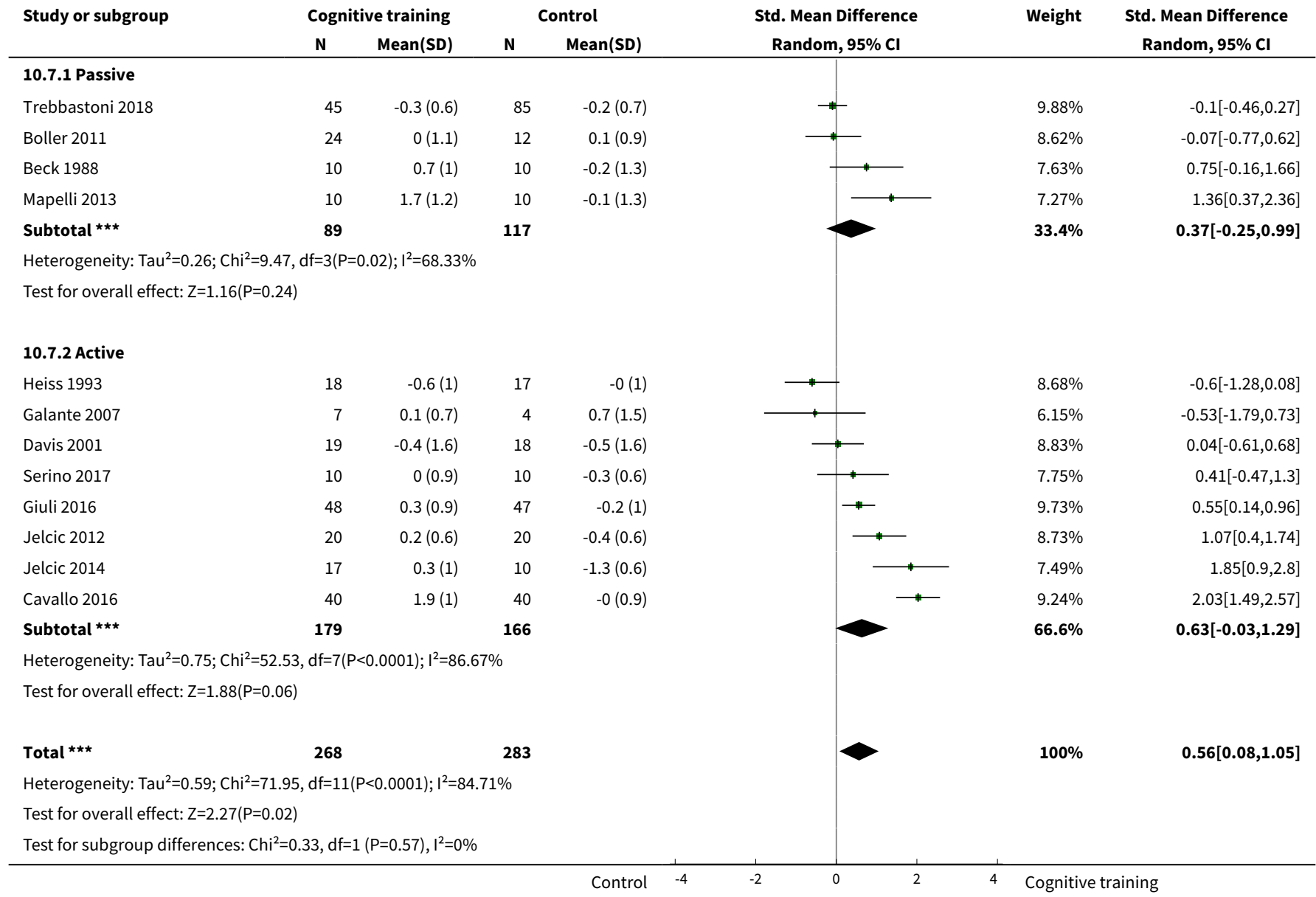

Analysis 10.8. Comparison 10 Cognitive training vs control immediately post intervention - type of control (passive vs active), Outcome 8 Change in language (naming).

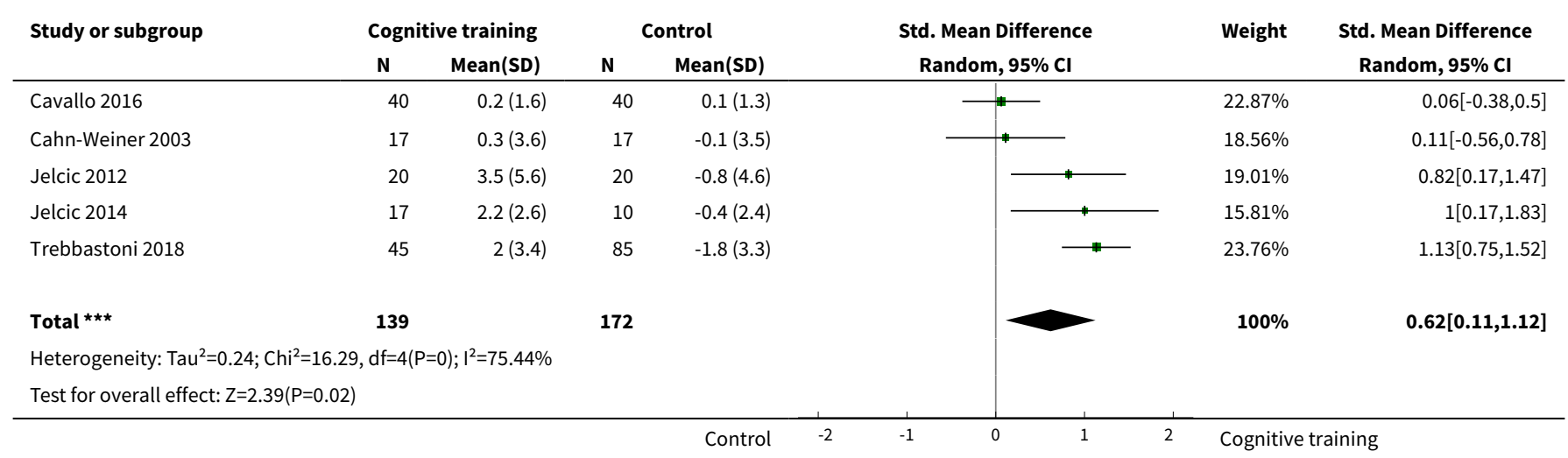


Analysis 10.9. Comparison 10 Cognitive training vs control immediately post intervention - type of control (passive vs active), Outcome 9 Change in verbal letter fluency.

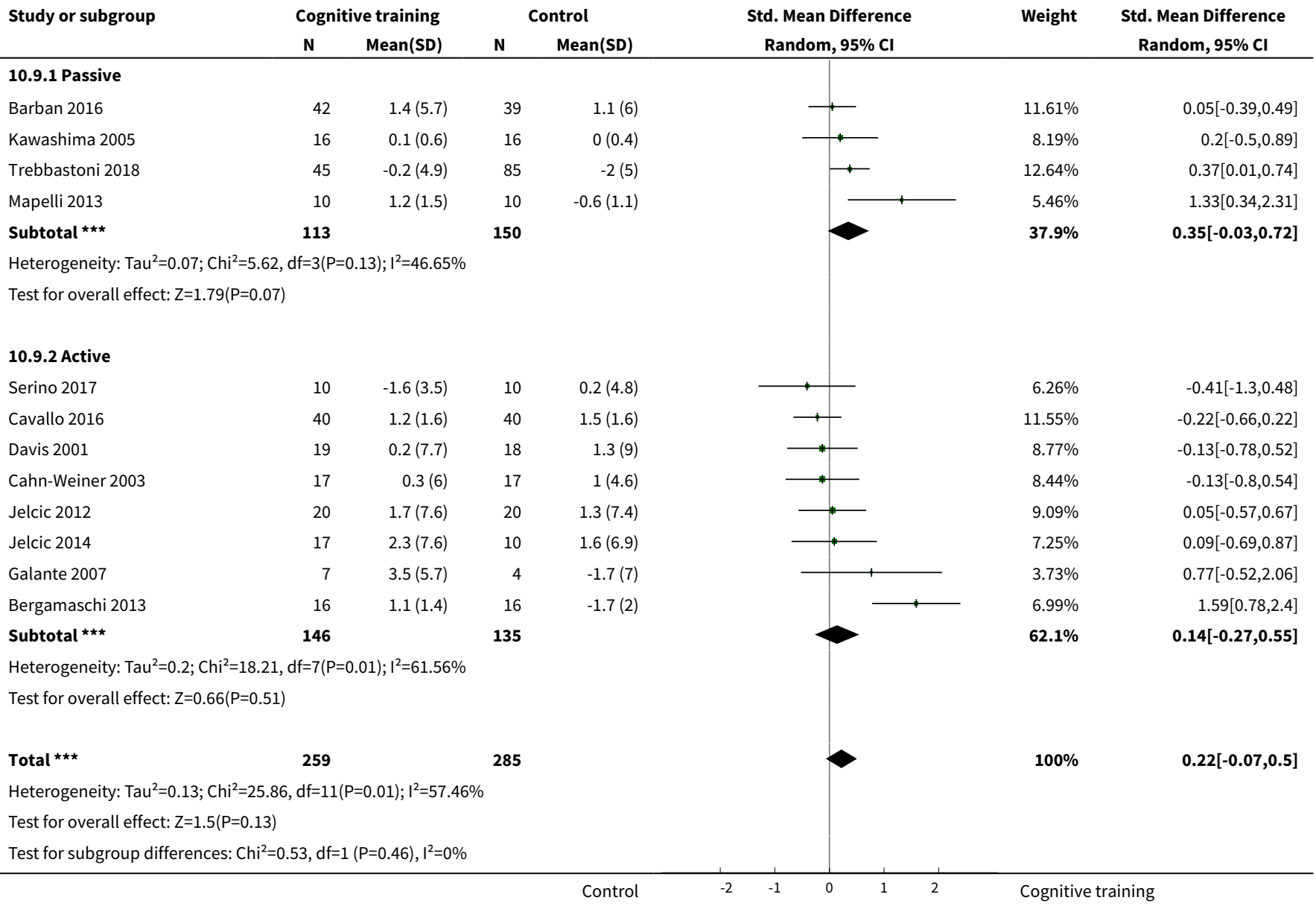

Analysis 10.10. Comparison 10 Cognitive training vs control immediately post intervention - type of control (passive vs active), Outcome 10 Change in speed of information processing.

\begin{tabular}{|c|c|c|c|c|c|c|c|}
\hline \multirow[t]{2}{*}{ Study or subgroup } & \multicolumn{2}{|c|}{ Cognitive training } & \multicolumn{2}{|c|}{ Control } & \multirow{2}{*}{$\begin{array}{c}\text { Std. Mean Difference } \\
\text { Random, } 95 \% \mathrm{CI}\end{array}$} & \multirow[t]{2}{*}{ Weight } & \multirow{2}{*}{$\begin{array}{c}\text { Std. Mean Difference } \\
\text { Random, } 95 \% \mathrm{CI}\end{array}$} \\
\hline & $\mathbf{N}$ & Mean(SD) & $\mathbf{N}$ & Mean(SD) & & & \\
\hline Beck 1988 & 10 & $-0.3(1.3)$ & 10 & $1.1(2.9)$ & $\longrightarrow$ & $11.31 \%$ & $-0.6[-1.51,0.3]$ \\
\hline Galante 2007 & 7 & $-1.3(10.3)$ & 4 & $3.3(5.6)$ & & $6.26 \%$ & $-0.47[-1.72,0.79]$ \\
\hline Jelcic 2014 & 17 & $0.5(6.9)$ & 10 & $-0.4(8.2)$ & & $14.38 \%$ & $0.11[-0.67,0.9]$ \\
\hline Barban 2016 & 36 & $7.7(27.4)$ & 33 & $-1.9(27.7)$ & - & $30.03 \%$ & $0.35[-0.13,0.82]$ \\
\hline Jelcic 2012 & 20 & $19.3(56.5)$ & 20 & $-2(20.6)$ & 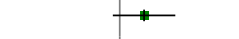 & $20.26 \%$ & $0.49[-0.14,1.12]$ \\
\hline Cahn-Weiner 2003 & 17 & $5.2(20.6)$ & 17 & $-9.6(31.4)$ & 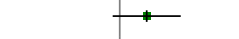 & $17.76 \%$ & $0.54[-0.14,1.23]$ \\
\hline Total $\star \star \star$ & 107 & & 94 & & & $100 \%$ & $0.22[-0.11,0.54]$ \\
\hline \multicolumn{8}{|c|}{ Heterogeneity: $\mathrm{Tau}^{2}=0.03 ; \mathrm{Chi}^{2}=6.24, \mathrm{df}=5(\mathrm{P}=0.28) ; \mathrm{I}^{2}=19.89 \%$} \\
\hline \multicolumn{3}{|c|}{ Test for overall effect: $Z=1.32(P=0.19)$} & & & & & \\
\hline
\end{tabular}


Analysis 10.11. Comparison 10 Cognitive training vs control immediately post intervention - type of control (passive vs active), Outcome 11 Change in executive function.

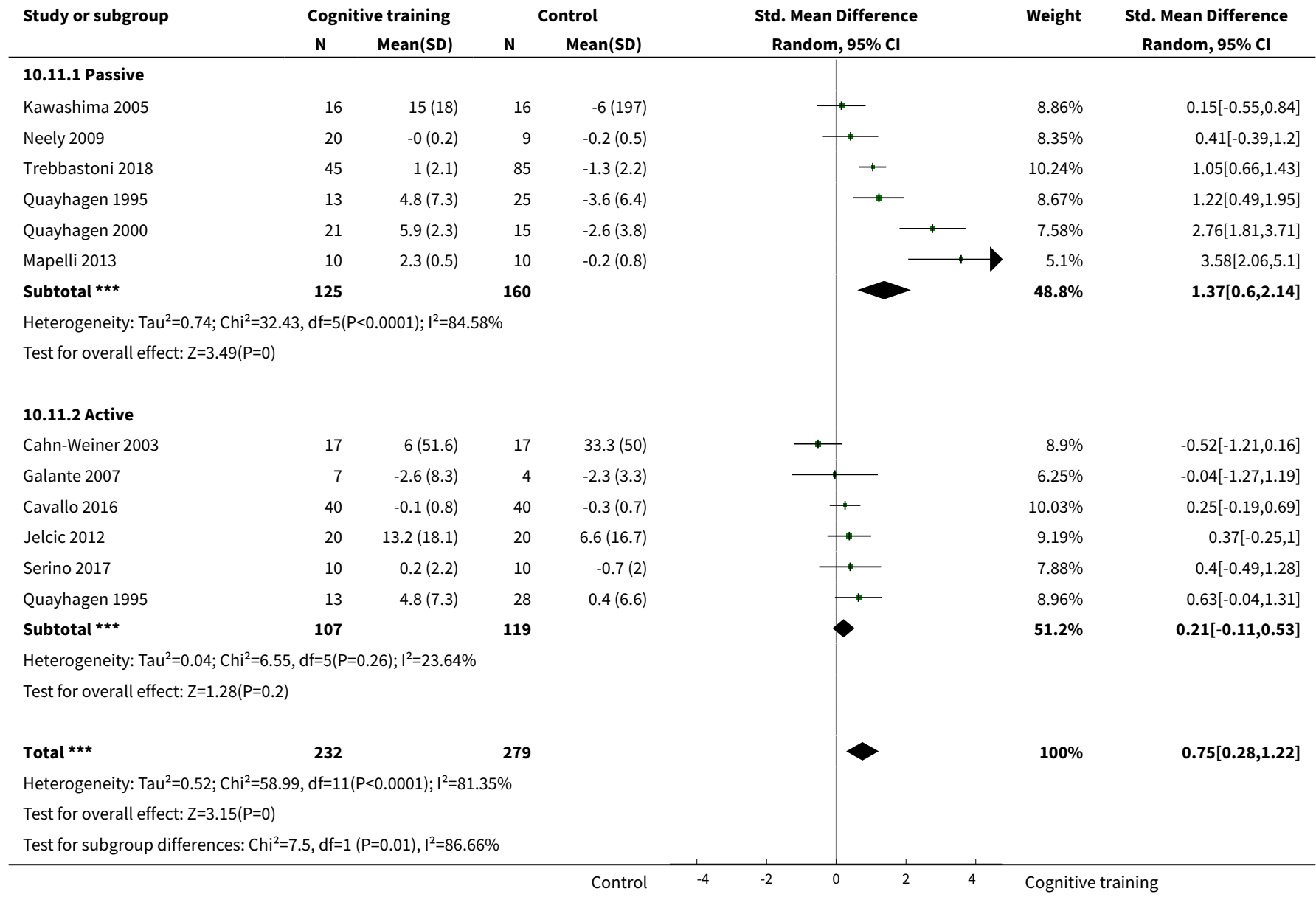

\section{Analysis 10.12. Comparison 10 Cognitive training vs control immediately post intervention - type of control (passive vs active), Outcome 12 Change in verbal category fluency.}

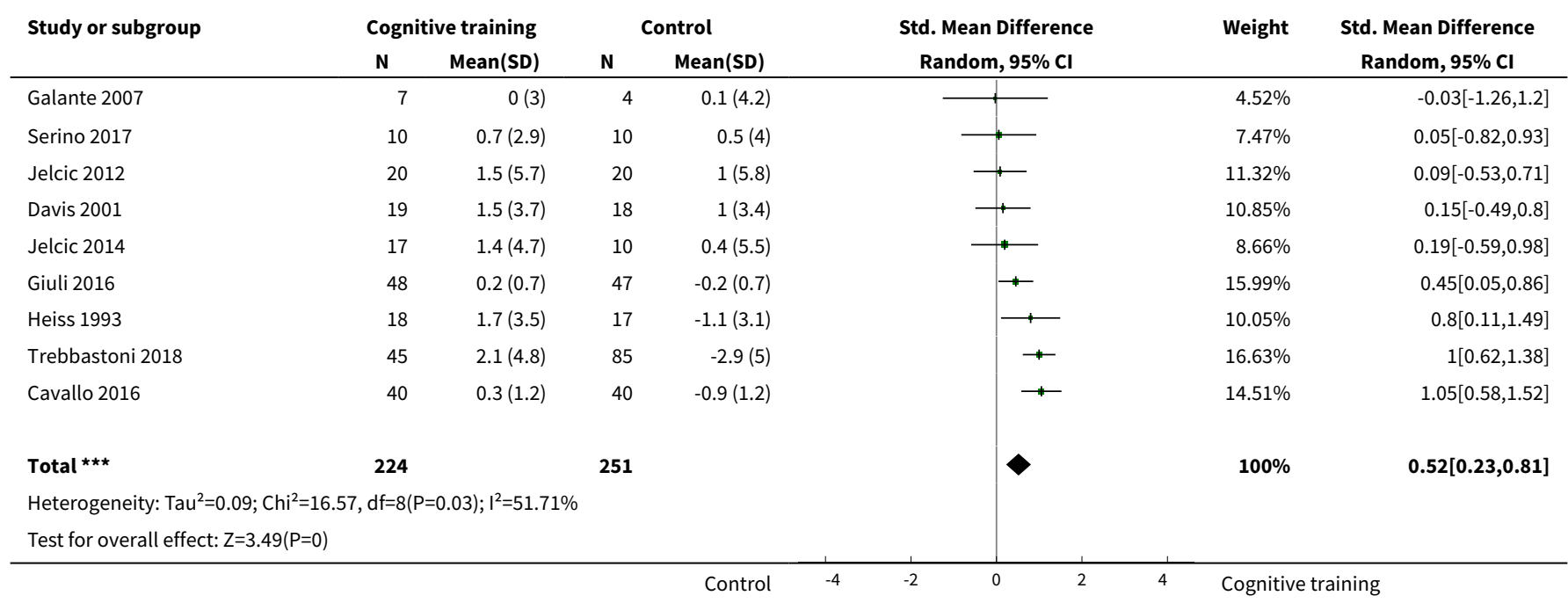


Analysis 10.13. Comparison 10 Cognitive training vs control immediately post intervention - type of control (passive vs active), Outcome 13 Change in meta cognition (self-reported).

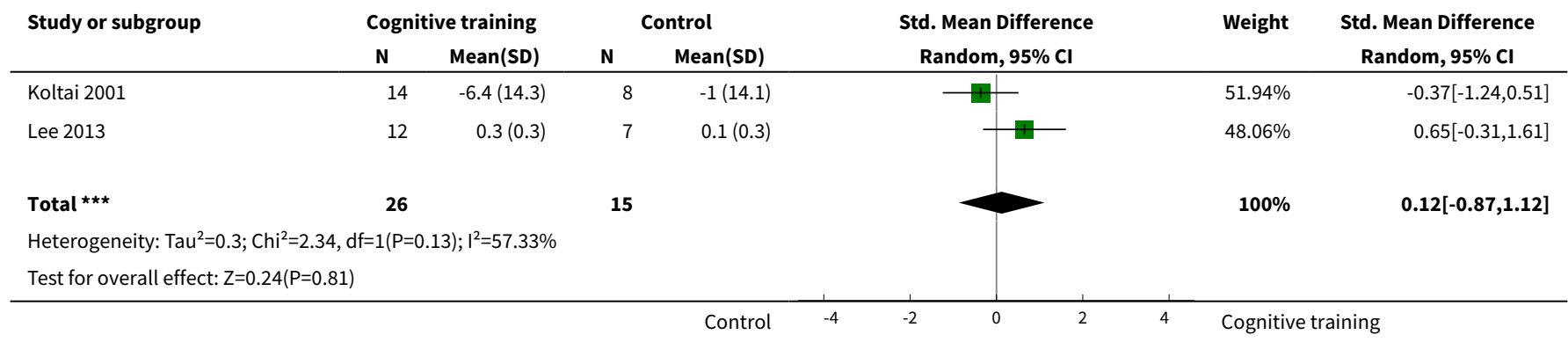

Analysis 10.14. Comparison 10 Cognitive training vs control immediately post intervention type of control (passive vs active), Outcome 14 Change in meta cognition (informant-reported).

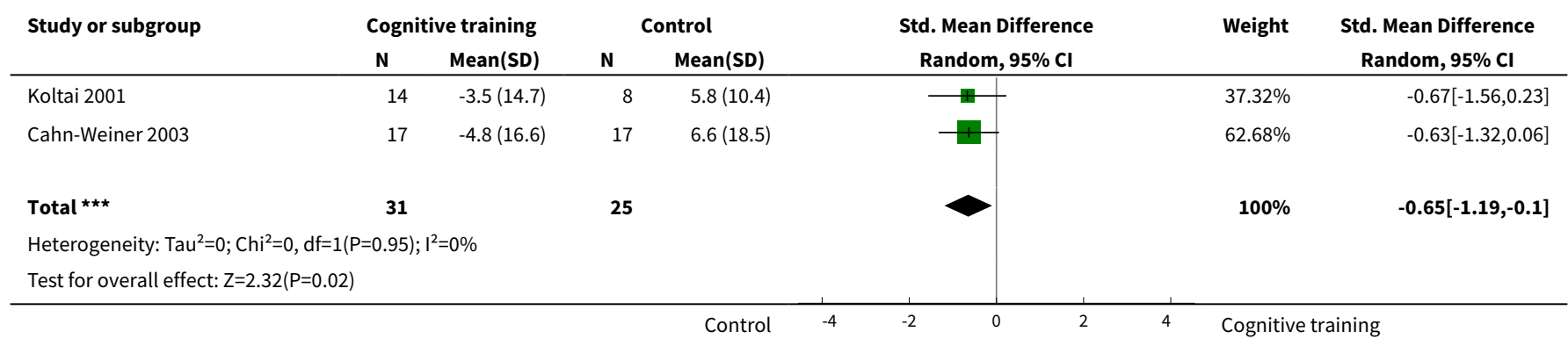

Analysis 10.15. Comparison 10 Cognitive training vs control immediately post intervention - type of control (passive vs active), Outcome 15 Change in participants' mood.

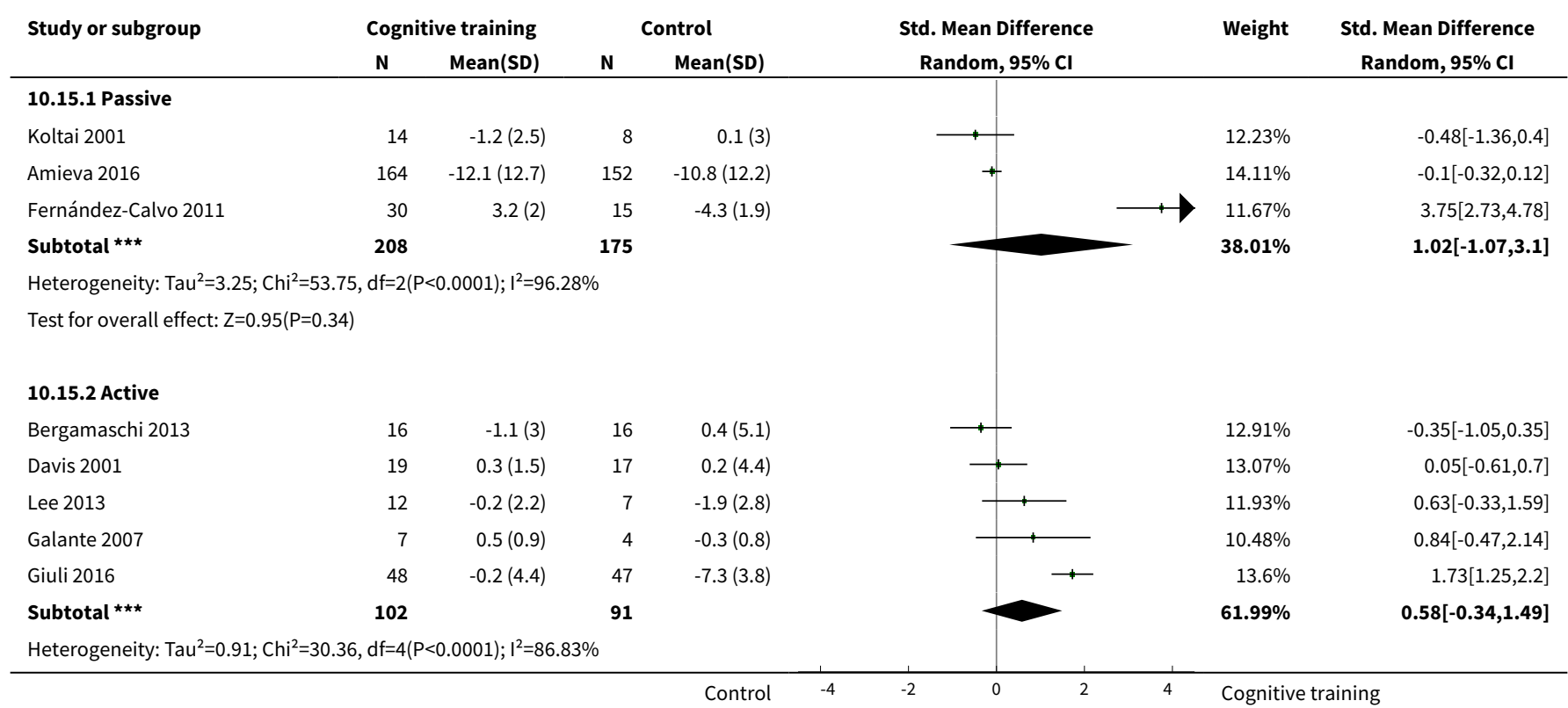




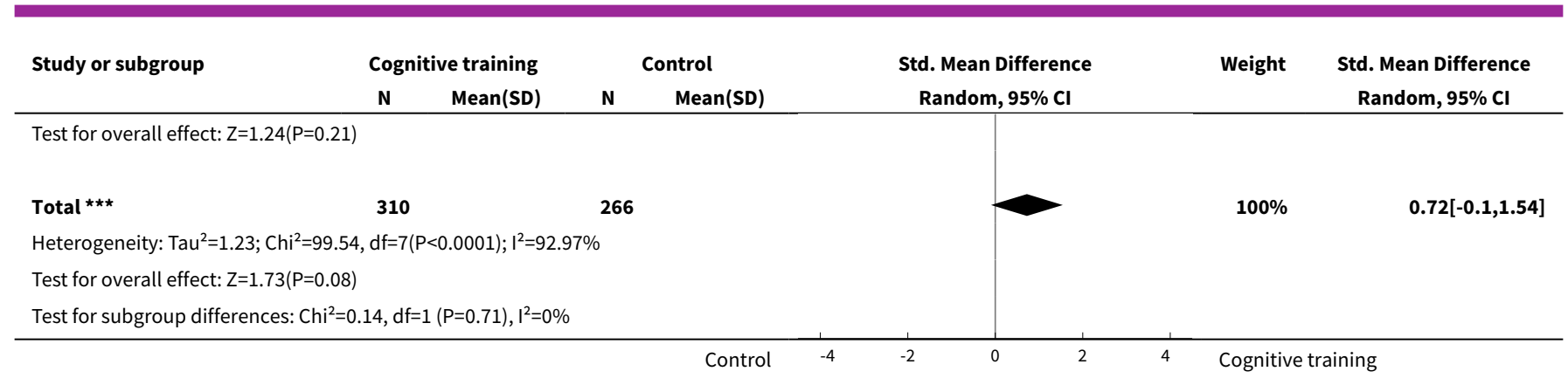

Analysis 10.16. Comparison 10 Cognitive training vs control immediately post intervention type of control (passive vs active), Outcome 16 Change in capacity for activities of daily living.

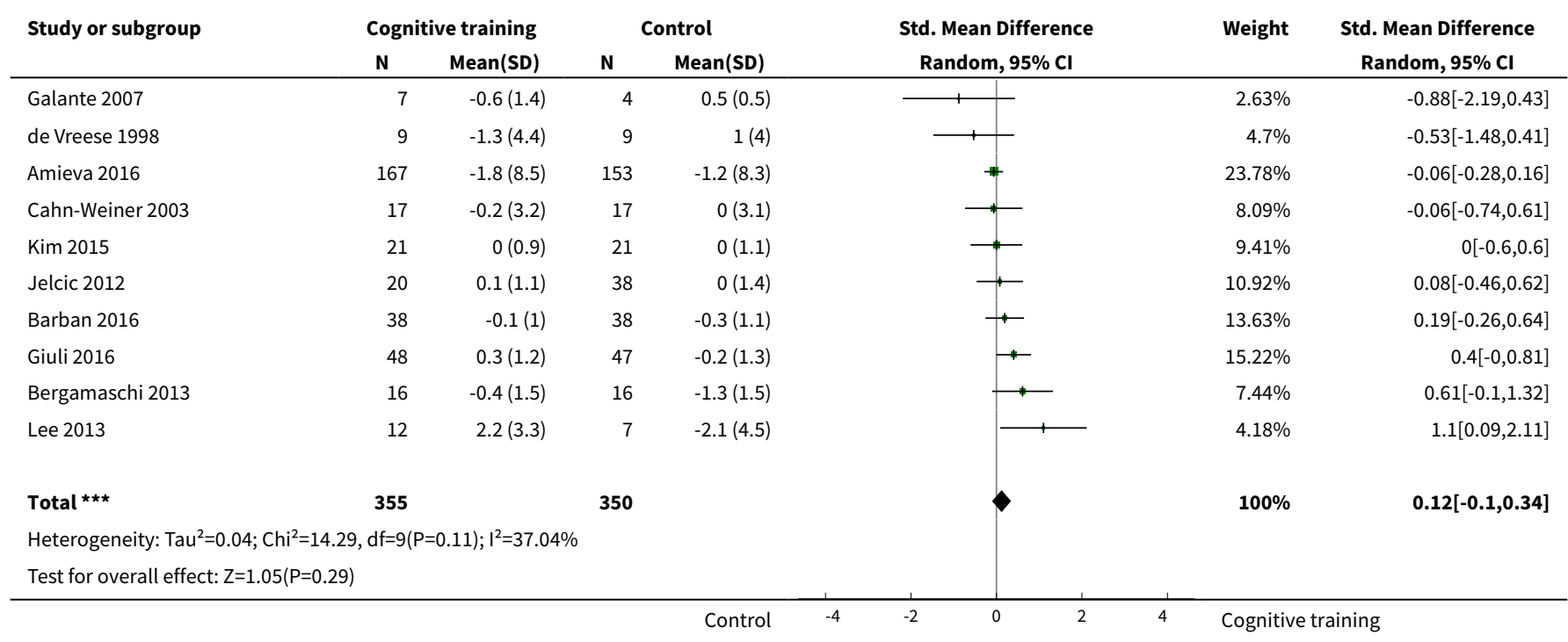

Analysis 10.17. Comparison 10 Cognitive training vs control immediately post intervention - type of control (passive vs active), Outcome 17 Change in disease progression.

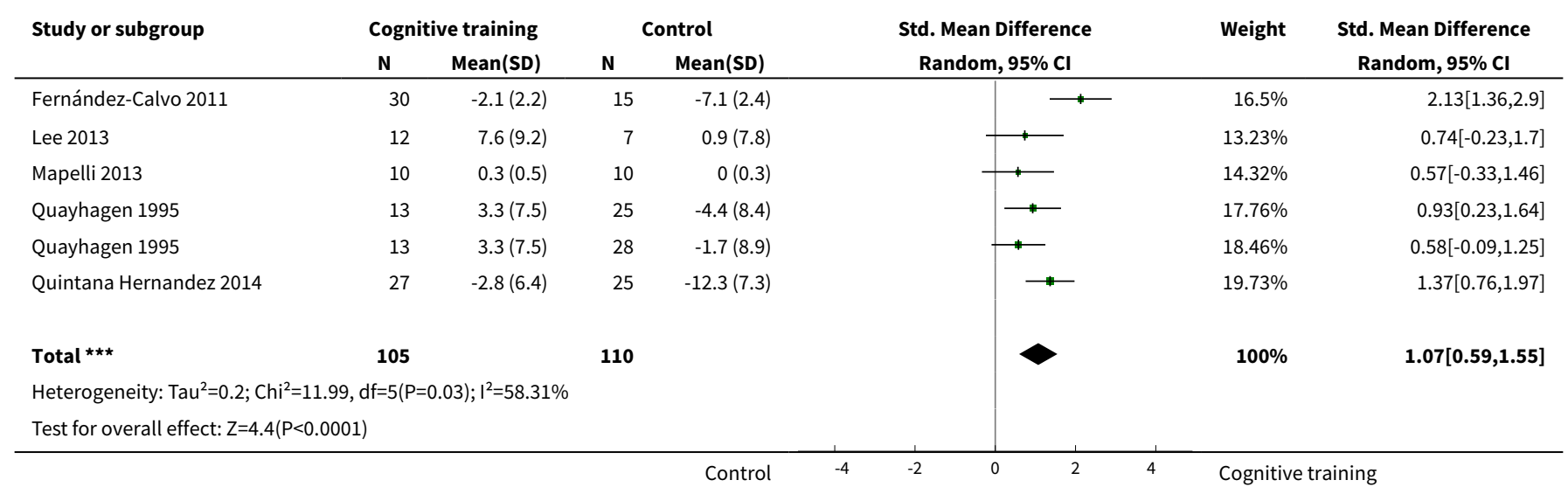


Analysis 10.18. Comparison 10 Cognitive training vs control immediately post intervention - type of control (passive vs active), Outcome 18 Change in behavioural and psychological symptoms of dementia (BPSD).

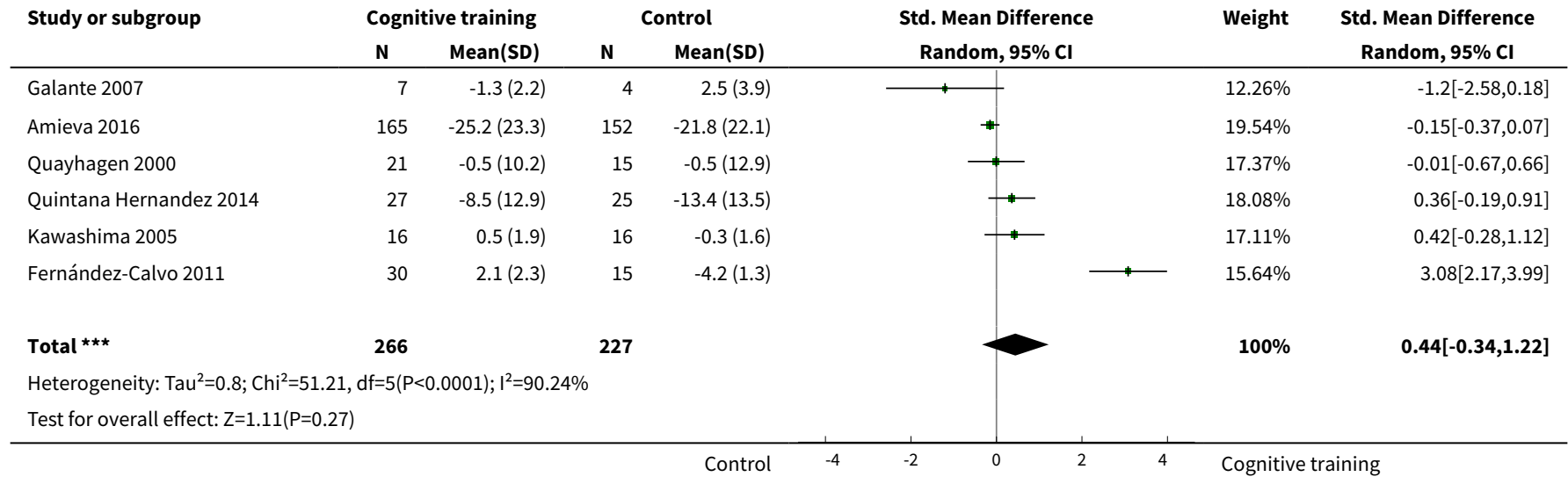

\section{Analysis 10.19. Comparison 10 Cognitive training vs control immediately post intervention - type of control (passive vs active), Outcome 19 Participant burden (retention rates).}

\begin{tabular}{|c|c|c|c|c|c|}
\hline Study or subgroup & $\begin{array}{c}\text { Cognitive } \\
\text { training } \\
n / N\end{array}$ & $\begin{array}{l}\text { Control } \\
\mathrm{n} / \mathrm{N} \\
\end{array}$ & $\begin{array}{c}\text { Odds Ratio } \\
\text { M-H, Random, } 95 \% \mathrm{CI}\end{array}$ & Weight & $\begin{array}{c}\text { Odds Ratio } \\
\text { M-H, Random, } 95 \% \mathrm{Cl}\end{array}$ \\
\hline \multicolumn{6}{|l|}{ 10.19.1 Passive } \\
\hline Kallio 2018 & $76 / 76$ & $71 / 71$ & & & Not estimable \\
\hline Mapelli 2013 & $10 / 10$ & $10 / 10$ & & & Not estimable \\
\hline Beck 1988 & $10 / 10$ & $10 / 10$ & & & Not estimable \\
\hline Trebbastoni 2018 & $48 / 54$ & $86 / 86$ & & $4.82 \%$ & $0.04[0,0.78]$ \\
\hline Quintana Hernandez 2014 & $27 / 32$ & $25 / 25$ & - & $4.69 \%$ & $0.1[0.01,1.86]$ \\
\hline Koltai 2001 & $14 / 16$ & $8 / 8$ & & $4.14 \%$ & $0.34[0.01,7.98]$ \\
\hline Kao 2016 & $95 / 110$ & $45 / 48$ & - & $17.27 \%$ & $0.42[0.12,1.53]$ \\
\hline Amieva 2016 & $124 / 170$ & $109 / 154$ & $=$ & $38.1 \%$ & $1.11[0.69,1.81]$ \\
\hline Neely 2009 & $10 / 10$ & $9 / 10$ & 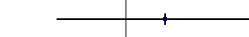 & $3.77 \%$ & $3.32[0.12,91.6]$ \\
\hline Subtotal $(95 \% \mathrm{Cl})$ & 488 & 422 & & $72.79 \%$ & $0.49[0.17,1.4]$ \\
\hline \multicolumn{6}{|c|}{ Total events: 414 (Cognitive training), 373 (Control) } \\
\hline \multicolumn{6}{|c|}{ Heterogeneity: $\mathrm{Tau}^{2}=0.71 ; \mathrm{Chi}^{2}=9.86, \mathrm{df}=5(\mathrm{P}=0.08) ; \mathrm{I}^{2}=49.27 \%$} \\
\hline \multicolumn{6}{|c|}{ Test for overall effect: $Z=1.33(P=0.18)$} \\
\hline \multicolumn{6}{|l|}{ 10.19.2 Active } \\
\hline Jelcic 2012 & $20 / 20$ & $20 / 20$ & & & Not estimable \\
\hline Davis 2001 & $19 / 19$ & $18 / 18$ & & & Not estimable \\
\hline Cavallo 2016 & $40 / 40$ & $40 / 40$ & & & Not estimable \\
\hline Kim 2015 & $22 / 22$ & $21 / 21$ & & & Not estimable \\
\hline Serino 2017 & $10 / 10$ & $10 / 10$ & & & Not estimable \\
\hline Cahn-Weiner 2003 & $16 / 19$ & $17 / 20$ & & $11.34 \%$ & $0.94[0.17,5.36]$ \\
\hline Giuli 2016 & $48 / 51$ & $47 / 50$ & & $12.28 \%$ & $1.02[0.2,5.32]$ \\
\hline Galante 2007 & $7 / 7$ & $4 / 5$ & & $3.59 \%$ & $5[0.17,150.92]$ \\
\hline Subtotal $(95 \% \mathrm{Cl})$ & 188 & 184 & 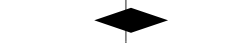 & $27.21 \%$ & $1.17[0.38,3.64]$ \\
\hline \multicolumn{6}{|c|}{ Total events: 182 (Cognitive training), 177 (Control) } \\
\hline \multicolumn{6}{|c|}{ Heterogeneity: $\mathrm{Tau}^{2}=0 ; \mathrm{Chi}^{2}=0.79, \mathrm{df}=2(\mathrm{P}=0.67) ; \mathrm{I}^{2}=0 \%$} \\
\hline Test for overall effect: $Z=0.2$ & & & & & \\
\hline
\end{tabular}




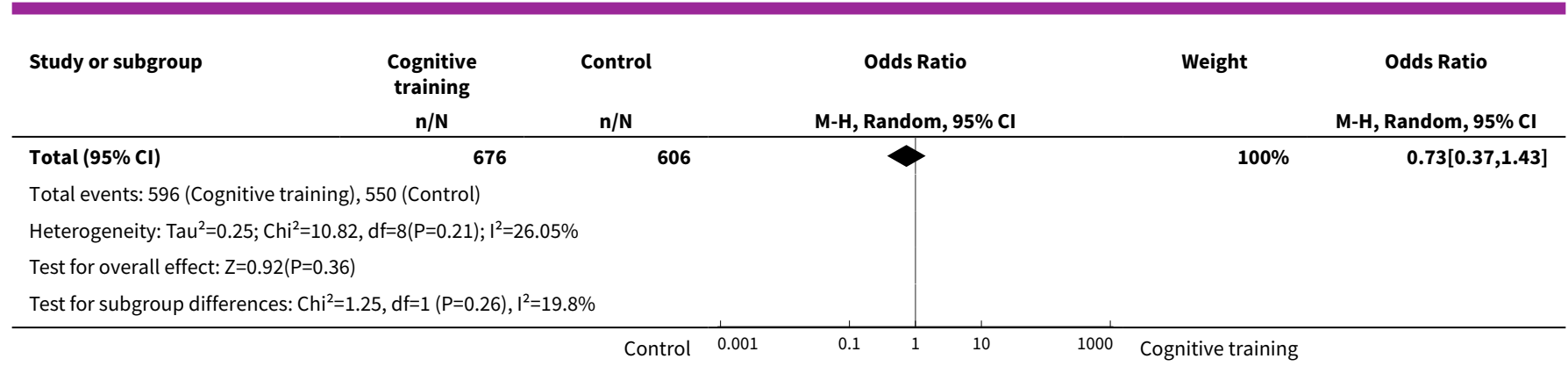

\section{Analysis 10.20. Comparison 10 Cognitive training vs control immediately post intervention - type of control (passive vs active), Outcome 20 Change in burden of care (CAREGIVER).}

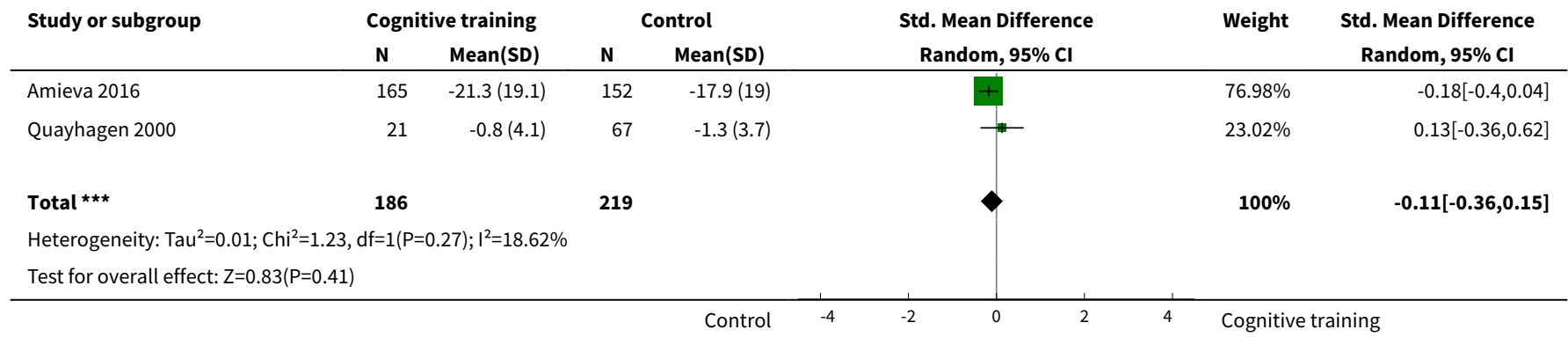

\section{Analysis 10.21. Comparison 10 Cognitive training vs control immediately post intervention - type of control (passive vs active), Outcome 21 Change in quality of life (CAREGIVER).}

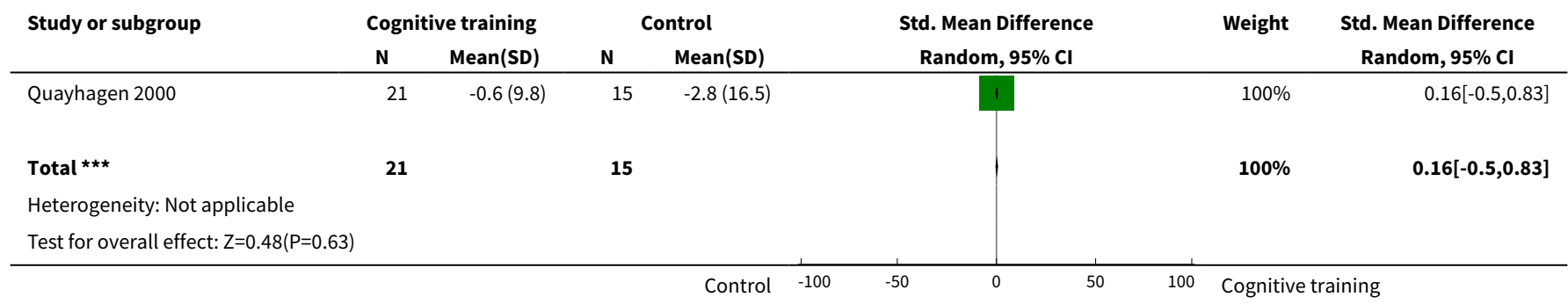

Analysis 10.22. Comparison 10 Cognitive training vs control immediately post intervention type of control (passive vs active), Outcome 22 Change in mood and well-being (CAREGIVER).

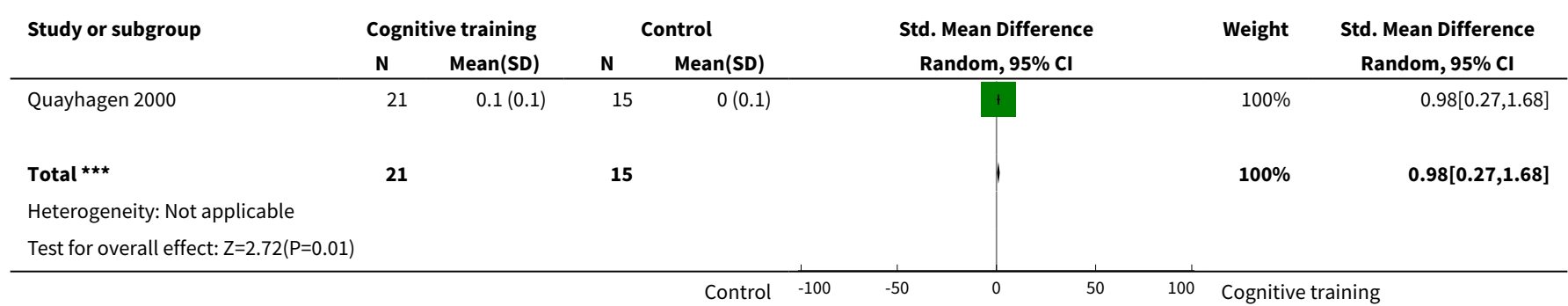


Analysis 10.23. Comparison 10 Cognitive training vs control immediately post intervention - type of control (passive vs active), Outcome 23 Change in general health and quality of life.

\begin{tabular}{|c|c|c|c|c|c|c|c|}
\hline \multirow{3}{*}{$\begin{array}{l}\text { Study or subgroup } \\
\text { Davis } 2001\end{array}$} & \multicolumn{2}{|c|}{ Cognitive training } & \multicolumn{2}{|c|}{ Control } & \multirow{2}{*}{$\begin{array}{c}\text { Std. Mean Difference } \\
\text { Random, } 95 \% \mathrm{Cl}\end{array}$} & \multirow[t]{2}{*}{ Weight } & \multirow{2}{*}{$\begin{array}{c}\text { Std. Mean Difference } \\
\text { Random, } 95 \% \mathrm{CI}\end{array}$} \\
\hline & $\mathbf{N}$ & $\operatorname{Mean}(S D)$ & $\mathbf{N}$ & Mean(SD) & & & \\
\hline & 19 & $-24.8(37.3)$ & 18 & $4.8(40.9)$ & * & $14 \%$ & $-0.74[-1.41,-0.07]$ \\
\hline Quayhagen 2000 & 21 & $-0.4(2.8)$ & 67 & $0.1(2.6)$ & & $18.83 \%$ & $-0.19[-0.68,0.3]$ \\
\hline Amieva 2016 & 164 & $-6.3(6.1)$ & 151 & $-5.6(6.4)$ & - & $27.77 \%$ & $-0.11[-0.33,0.11]$ \\
\hline Kallio 2018 & 76 & $-0(0.1)$ & 71 & $-0(0.1)$ & & $24.41 \%$ & $0[-0.32,0.32]$ \\
\hline Kim 2015 & 22 & $3.4(3.3)$ & 21 & $0(4.4)$ & & $14.99 \%$ & $0.87[0.24,1.49]$ \\
\hline 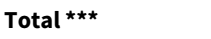 & 302 & & 328 & & & $100 \%$ & $-0.04[-0.38,0.29]$ \\
\hline \multicolumn{8}{|c|}{ Heterogeneity: $\mathrm{Tau}^{2}=0.09 ; \mathrm{Chi}^{2}=12.93, \mathrm{df}=4(\mathrm{P}=0.01) ; \mathrm{I}^{2}=69.08 \%$} \\
\hline \multicolumn{8}{|c|}{ Test for overall effect: $Z=0.24(P=0.81)$} \\
\hline & & & & Control & -0.5 & Cognitiv & ning \\
\hline
\end{tabular}

Comparison 11. Cognitive training vs alternative treatment immediately post intervention - intervention dose

\begin{tabular}{|c|c|c|c|c|}
\hline Outcome or subgroup title & $\begin{array}{l}\text { No. of } \\
\text { studies }\end{array}$ & $\begin{array}{l}\text { No. of } \\
\text { partici- } \\
\text { pants }\end{array}$ & Statistical method & Effect size \\
\hline 1 Change in a global measure of cognition & 7 & 724 & $\begin{array}{l}\text { Std. Mean Difference (IV, Ran- } \\
\text { dom, } 95 \% \mathrm{CI} \text { ) }\end{array}$ & $0.16[-0.28,0.60]$ \\
\hline 1.1 Up to 3 times & 3 & 551 & $\begin{array}{l}\text { Std. Mean Difference (IV, Ran- } \\
\text { dom, } 95 \% \mathrm{CI} \text { ) }\end{array}$ & $0.32[-0.48,1.12]$ \\
\hline 1.2 More than 3 times & 4 & 173 & $\begin{array}{l}\text { Std. Mean Difference (IV, Ran- } \\
\text { dom, } 95 \% \mathrm{Cl} \text { ) }\end{array}$ & $0.13[-0.63,0.89]$ \\
\hline $\begin{array}{l}2 \text { Change in a global measure of cognition_zero } \\
\text { correlation }\end{array}$ & 7 & 724 & $\begin{array}{l}\text { Std. Mean Difference (IV, Ran- } \\
\text { dom, } 95 \% \mathrm{Cl} \text { ) }\end{array}$ & $-0.02[-0.24,0.20]$ \\
\hline 2.1 Up to 3 times & 3 & 551 & $\begin{array}{l}\text { Std. Mean Difference (IV, Ran- } \\
\text { dom, } 95 \% \mathrm{Cl} \text { ) }\end{array}$ & $0.02[-0.35,0.40]$ \\
\hline 2.2 More than 3 times & 4 & 173 & $\begin{array}{l}\text { Std. Mean Difference (IV, Ran- } \\
\text { dom, } 95 \% \mathrm{Cl} \text { ) }\end{array}$ & $0.01[-0.41,0.43]$ \\
\hline $\begin{array}{l}3 \text { Change in a global measure of cognition } \\
\text { (composite) }\end{array}$ & 7 & 769 & $\begin{array}{l}\text { Std. Mean Difference (Random, } \\
95 \% \mathrm{Cl} \text { ) }\end{array}$ & $0.21[-0.23,0.64]$ \\
\hline 3.1 Up to 3 times & 4 & 636 & $\begin{array}{l}\text { Std. Mean Difference (Random, } \\
95 \% \mathrm{Cl} \text { ) }\end{array}$ & $0.23[-0.27,0.74]$ \\
\hline 3.2 More than 3 times & 3 & 133 & $\begin{array}{l}\text { Std. Mean Difference (Random, } \\
95 \% \mathrm{Cl} \text { ) }\end{array}$ & $0.32[-0.90,1.55]$ \\
\hline $\begin{array}{l}4 \text { Change in a global measure of cognition } \\
\text { (composite)_zero correlation }\end{array}$ & 7 & 769 & $\begin{array}{l}\text { Std. Mean Difference (Random, } \\
95 \% \mathrm{Cl} \text { ) }\end{array}$ & $-0.03[-0.23,0.17]$ \\
\hline
\end{tabular}




\begin{tabular}{|c|c|c|c|c|}
\hline Outcome or subgroup title & $\begin{array}{l}\text { No. of } \\
\text { studies }\end{array}$ & $\begin{array}{l}\text { No. of } \\
\text { partici- } \\
\text { pants }\end{array}$ & Statistical method & Effect size \\
\hline 4.1 Up to 3 times & 4 & 636 & $\begin{array}{l}\text { Std. Mean Difference (Random, } \\
95 \% \mathrm{Cl} \text { ) }\end{array}$ & $-0.04[-0.22,0.14]$ \\
\hline 4.2 More than 3 times & 3 & 133 & $\begin{array}{l}\text { Std. Mean Difference (Random, } \\
95 \% \mathrm{Cl} \text { ) }\end{array}$ & $0.05[-0.59,0.70]$ \\
\hline 5 Change in immediate memory & 3 & 147 & $\begin{array}{l}\text { Std. Mean Difference (IV, Ran- } \\
\text { dom, } 95 \% \mathrm{CI} \text { ) }\end{array}$ & $0.51[-0.19,1.21]$ \\
\hline 6 Change in delayed memory & 3 & 147 & $\begin{array}{l}\text { Std. Mean Difference (IV, Ran- } \\
\text { dom, } 95 \% \mathrm{CI} \text { ) }\end{array}$ & $0.71[-0.33,1.75]$ \\
\hline 7 Change in attention and working memory & 2 & 69 & $\begin{array}{l}\text { Std. Mean Difference (IV, Ran- } \\
\text { dom, 95\% CI) }\end{array}$ & $0.91[-0.46,2.27]$ \\
\hline 8 Change in language (naming) & 1 & 16 & $\begin{array}{l}\text { Std. Mean Difference (IV, Ran- } \\
\text { dom, } 95 \% \mathrm{CI} \text { ) }\end{array}$ & $-0.63[-1.65,0.38]$ \\
\hline 9 Change in verbal letter fluency & 3 & 75 & $\begin{array}{l}\text { Std. Mean Difference (IV, Ran- } \\
\text { dom, } 95 \% \mathrm{CI} \text { ) }\end{array}$ & $0.34[-0.38,1.05]$ \\
\hline 10 Change in verbal category fluency & 2 & 55 & $\begin{array}{l}\text { Std. Mean Difference (IV, Ran- } \\
\text { dom, 95\% CI) }\end{array}$ & $-0.28[-1.46,0.89]$ \\
\hline 11 Change in executive function & 4 & 163 & $\begin{array}{l}\text { Std. Mean Difference (IV, Ran- } \\
\text { dom, } 95 \% \mathrm{CI} \text { ) }\end{array}$ & $1.44[-0.26,3.14]$ \\
\hline 12 Change in meta cognition (self-reported) & 0 & 0 & $\begin{array}{l}\text { Std. Mean Difference (IV, Ran- } \\
\text { dom, 95\% CI) }\end{array}$ & $0.0[0.0,0.0]$ \\
\hline $\begin{array}{l}13 \text { Change in meta cognition (informant-re- } \\
\text { ported) }\end{array}$ & 0 & 0 & $\begin{array}{l}\text { Std. Mean Difference (IV, Ran- } \\
\text { dom, } 95 \% \mathrm{CI} \text { ) }\end{array}$ & $0.0[0.0,0.0]$ \\
\hline 14 Change in participants' mood & 3 & 543 & $\begin{array}{l}\text { Std. Mean Difference (IV, Ran- } \\
\text { dom, } 95 \% \mathrm{CI} \text { ) }\end{array}$ & $-0.11[-0.29,0.07]$ \\
\hline $\begin{array}{l}15 \text { Change in capacity for activities of daily liv- } \\
\text { ing }\end{array}$ & 3 & 525 & $\begin{array}{l}\text { Std. Mean Difference (IV, Ran- } \\
\text { dom, 95\% CI) }\end{array}$ & $-0.25[-0.43,-0.07]$ \\
\hline $\begin{array}{l}16 \text { Change in behavioural and psychological } \\
\text { symptoms of dementia (BPSD) }\end{array}$ & 3 & 672 & $\begin{array}{l}\text { Std. Mean Difference (IV, Ran- } \\
\text { dom, 95\% CI) }\end{array}$ & $-0.11[-0.27,0.06]$ \\
\hline 17 Change in disease progression & 3 & 131 & $\begin{array}{l}\text { Std. Mean Difference (IV, Ran- } \\
\text { dom, } 95 \% \mathrm{CI} \text { ) }\end{array}$ & $0.15[-0.33,0.63]$ \\
\hline 18 Participant burden (retention rates) & 4 & 639 & $\begin{array}{l}\text { Odds Ratio (M-H, Random, } \\
95 \% \mathrm{Cl} \text { ) }\end{array}$ & $0.78[0.24,2.57]$ \\
\hline $\begin{array}{l}19 \text { Change in mood and well-being } \\
\text { (CAREGIVER) }\end{array}$ & 1 & 88 & $\begin{array}{l}\text { Std. Mean Difference (IV, Ran- } \\
\text { dom, 95\% CI) }\end{array}$ & $1.50[0.96,2.04]$ \\
\hline 20 Change in burden of care (CAREGIVER) & 3 & 591 & $\begin{array}{l}\text { Std. Mean Difference (IV, Ran- } \\
\text { dom, } 95 \% \mathrm{CI} \text { ) }\end{array}$ & $-0.15[-0.47,0.17]$ \\
\hline
\end{tabular}




\begin{tabular}{llllll}
\hline Outcome or subgroup title & $\begin{array}{l}\text { No. of } \\
\text { studies }\end{array}$ & $\begin{array}{l}\text { No. of } \\
\text { partici- } \\
\text { pants }\end{array}$ & Statistical method & Effect size \\
\hline 21 Change in quality of life (CAREGIVER) & 1 & 88 & $\begin{array}{l}\text { Std. Mean Difference (IV, Ran- } \\
\text { dom, 95\% CI) }\end{array}$ & $-0.25[-0.74,0.24]$ \\
\hline 22 Change in speed of information processing & 2 & 55 & $\begin{array}{l}\text { Std. Mean Difference (IV, Ran- } \\
\text { dom, 95\% CI) }\end{array}$ & $-0.21[-0.77,0.34]$ \\
\hline 23 Change in general health and quality of life & 4 & 631 & $\begin{array}{l}\text { Std. Mean Difference (IV, Ran- } \\
\text { dom, 95\% CI) }\end{array}$ & -0.49 [1.00, 0.02] \\
\hline
\end{tabular}

Analysis 11.1. Comparison 11 Cognitive training vs alternative treatment immediately post intervention - intervention dose, Outcome 1 Change in a global measure of cognition.

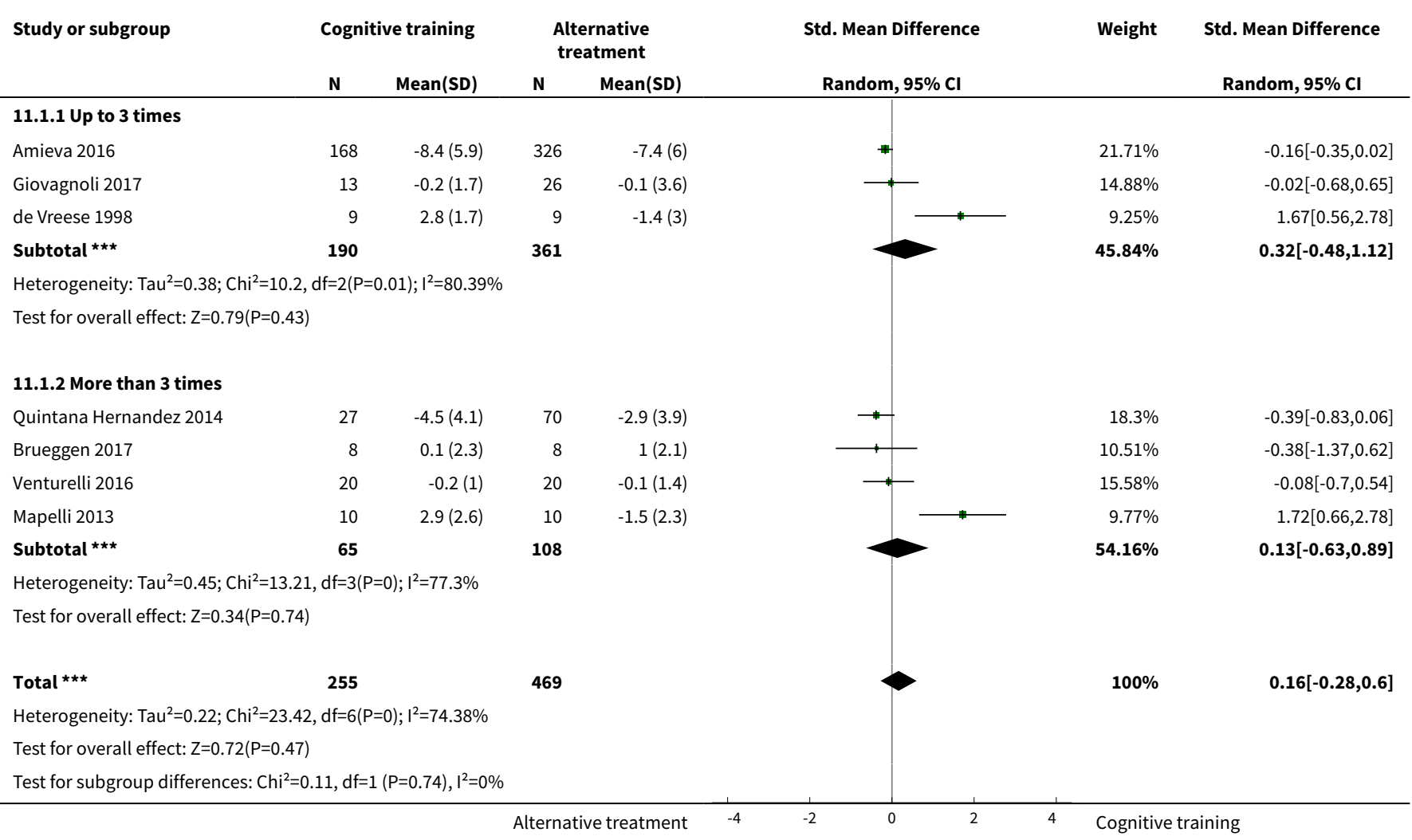

Analysis 11.2. Comparison 11 Cognitive training vs alternative treatment immediately post intervention - intervention dose, Outcome 2 Change in a global measure of cognition_zero correlation.

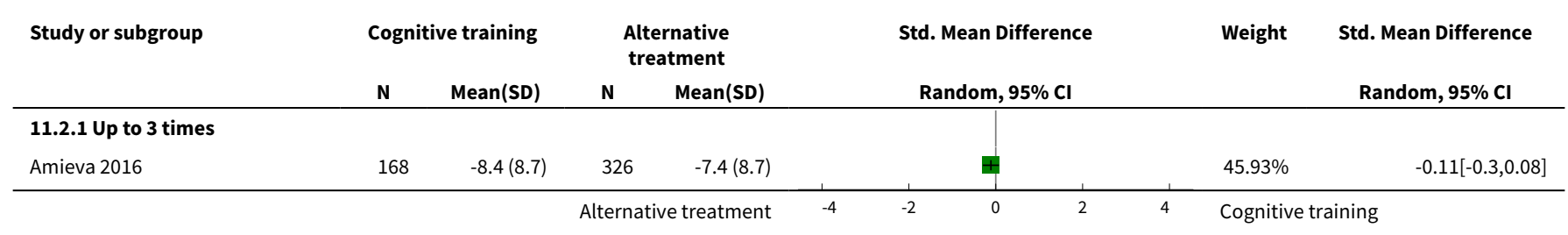




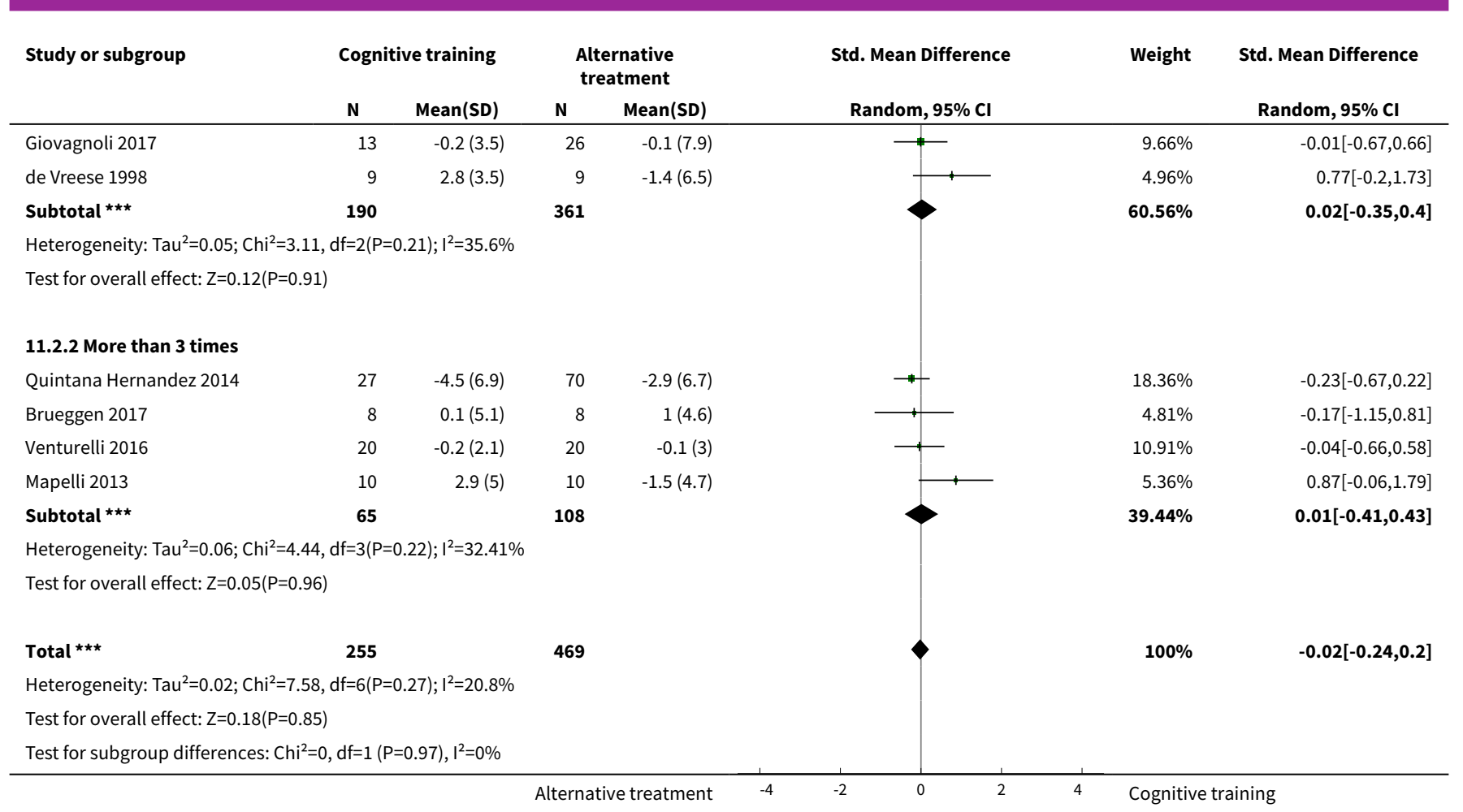

\section{Analysis 11.3. Comparison 11 Cognitive training vs alternative treatment immediately post intervention - intervention dose, Outcome 3 Change in a global measure of cognition (composite).}

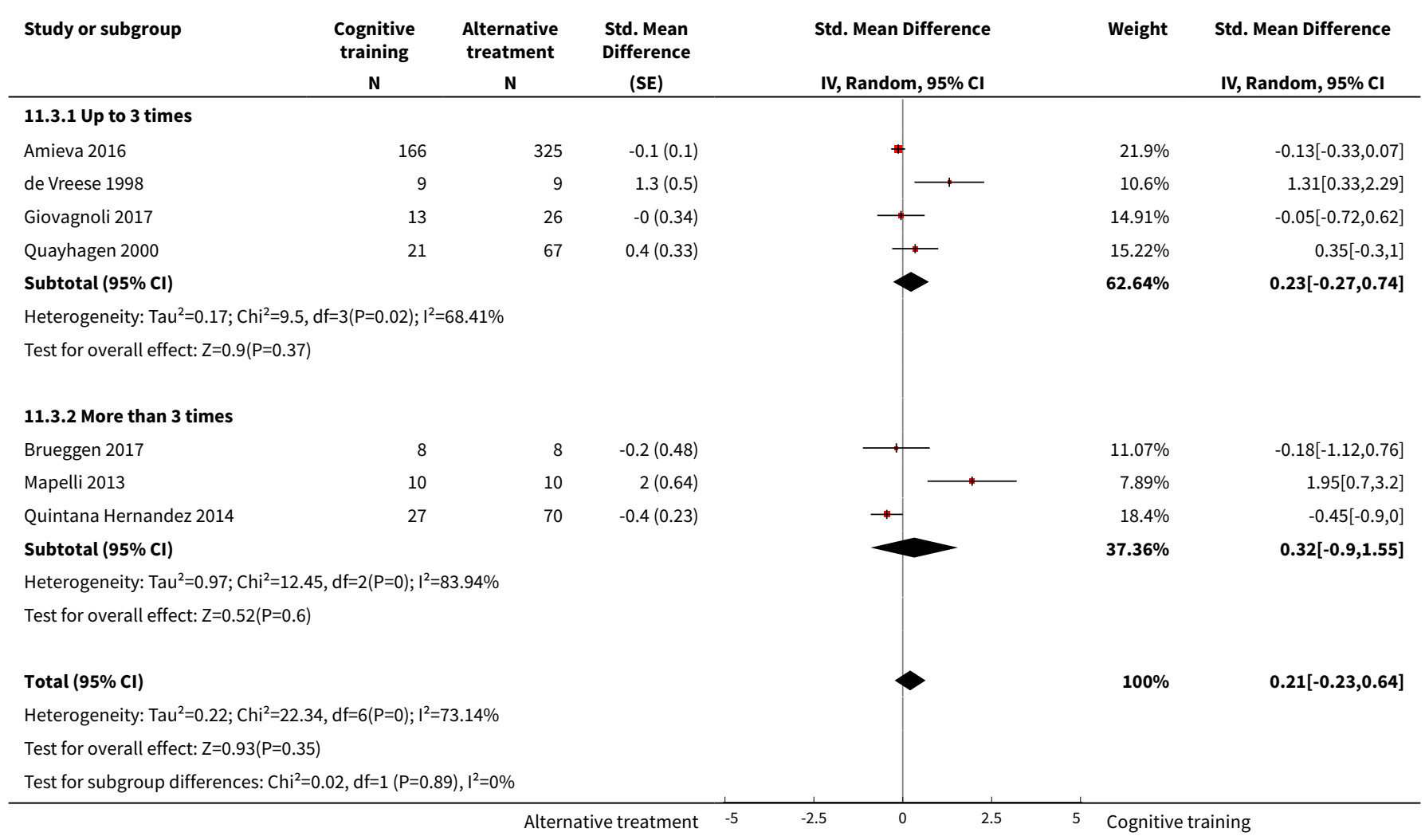




\section{Analysis 11.4. Comparison 11 Cognitive training vs alternative treatment immediately post intervention} - intervention dose, Outcome 4 Change in a global measure of cognition (composite)_zero correlation.

\begin{tabular}{|c|c|c|c|c|c|c|}
\hline Study or subgroup & $\begin{array}{c}\text { Cognitive } \\
\text { training } \\
\mathbf{N} \\
\end{array}$ & $\begin{array}{c}\text { Alternative } \\
\text { treatment } \\
\mathbf{N} \\
\end{array}$ & $\begin{array}{c}\text { Std. Mean } \\
\text { Difference } \\
(\mathrm{SE}) \\
\end{array}$ & IV, Random, 95\% CI & Weight & IV, Random, $95 \% \mathrm{CI}$ \\
\hline \multicolumn{7}{|l|}{ 11.4.1 Up to 3 times } \\
\hline Amieva 2016 & 166 & 325 & $-0.1(0.1)$ & + & $55.07 \%$ & $-0.09[-0.29,0.11]$ \\
\hline de Vreese 1998 & 9 & 9 & $0.6(0.46)$ & & $4.66 \%$ & $0.6[-0.3,1.5]$ \\
\hline Giovagnoli 2017 & 13 & 26 & $-0(0.37)$ & & $7.06 \%$ & $-0.04[-0.77,0.69]$ \\
\hline Subtotal $(95 \% \mathrm{CI})$ & & & & & $75.53 \%$ & $-0.04[-0.22,0.14]$ \\
\hline \multicolumn{7}{|c|}{ Heterogeneity: $\mathrm{Tau}^{2}=0 ; \mathrm{Chi}^{2}=2.55, \mathrm{df}=3(\mathrm{P}=0.47) ; \mathrm{I}^{2}=0 \%$} \\
\hline \multicolumn{7}{|c|}{ Test for overall effect: $Z=0.45(P=0.65)$} \\
\hline \multicolumn{7}{|l|}{ 11.4.2 More than 3 times } \\
\hline Brueggen 2017 & 8 & 8 & $-0.1(0.47)$ & & $4.48 \%$ & $-0.1[-1.02,0.82]$ \\
\hline Quintana Hernandez 2014 & 27 & 70 & $-0.3(0.23)$ & $\rightarrow+$ & $16.69 \%$ & $-0.27[-0.72,0.18]$ \\
\hline Subtotal $(95 \% \mathrm{Cl})$ & & & & & $24.47 \%$ & $0.05[-0.59,0.7]$ \\
\hline \multicolumn{7}{|c|}{ Heterogeneity: $\mathrm{Tau}^{2}=0.17 ; \mathrm{Chi}^{2}=4.05, \mathrm{df}=2(\mathrm{P}=0.13) ; \mathrm{I}^{2}=50.64 \%$} \\
\hline \multicolumn{7}{|c|}{ Test for overall effect: $Z=0.16(P=0.87)$} \\
\hline Total $(95 \% \mathrm{Cl})$ & & & & $>$ & $100 \%$ & $-0.03[-0.23,0.17]$ \\
\hline \multicolumn{7}{|c|}{ Heterogeneity: $\mathrm{Tau}^{2}=0.01 ; \mathrm{Chi}^{2}=6.66, \mathrm{df}=6(\mathrm{P}=0.35) ; \mathrm{I}^{2}=9.96 \%$} \\
\hline \multicolumn{7}{|c|}{ Test for overall effect: $Z=0.29(P=0.77)$} \\
\hline Test for subgroup differenc & $08, \mathrm{df}=1(\mathrm{P}=0$. & ), $1^{2}=0 \%$ & & & & \\
\hline
\end{tabular}

\section{Analysis 11.5. Comparison 11 Cognitive training vs alternative treatment immediately post intervention - intervention dose, Outcome 5 Change in immediate memory.}

\begin{tabular}{|c|c|c|c|c|c|c|c|c|}
\hline \multirow[t]{2}{*}{ Study or subgroup } & \multicolumn{2}{|c|}{ Cognitive training } & \multicolumn{2}{|c|}{$\begin{array}{l}\text { Alternative } \\
\text { treatment }\end{array}$} & \multirow{2}{*}{\multicolumn{2}{|c|}{$\begin{array}{l}\text { Std. Mean Difference } \\
\text { Random, } 95 \% \mathrm{CI}\end{array}$}} & \multirow[t]{2}{*}{ Weight } & \multirow{2}{*}{$\begin{array}{l}\text { Std. Mean Difference } \\
\text { Random, } 95 \% \mathrm{Cl}\end{array}$} \\
\hline & $\mathbf{N}$ & Mean(SD) & $\mathbf{N}$ & $\operatorname{Mean}(S D)$ & & & & \\
\hline Giovagnoli 2017 & 13 & $1(4.4)$ & 26 & $0.3(6.2)$ & & 1 & $34.91 \%$ & $0.12[-0.55,0.78]$ \\
\hline Quayhagen 2000 & 21 & $2.3(12.1)$ & 67 & $0(9.3)$ & & $\square$ & $40.81 \%$ & $0.22[-0.27,0.71]$ \\
\hline Mapelli 2013 & 10 & $2.6(2.4)$ & 10 & $-0.5(1.3)$ & & i & $24.28 \%$ & $1.56[0.53,2.59]$ \\
\hline Total $\star \star \star ~$ & 44 & & 103 & & & 1 & $100 \%$ & $0.51[-0.19,1.21]$ \\
\hline \multicolumn{9}{|c|}{ Heterogeneity: $\mathrm{Tau}^{2}=0.25 ; \mathrm{Chi}^{2}=6.02, \mathrm{df}=2(\mathrm{P}=0.05) ; \mathrm{I}^{2}=66.76 \%$} \\
\hline \multicolumn{9}{|c|}{ Test for overall effect: $Z=1.43(P=0.15)$} \\
\hline & & & Alterna & treatment & -100 & -50 & Cognitive & ining \\
\hline
\end{tabular}


Analysis 11.6. Comparison 11 Cognitive training vs alternative treatment immediately post intervention - intervention dose, Outcome 6 Change in delayed memory.

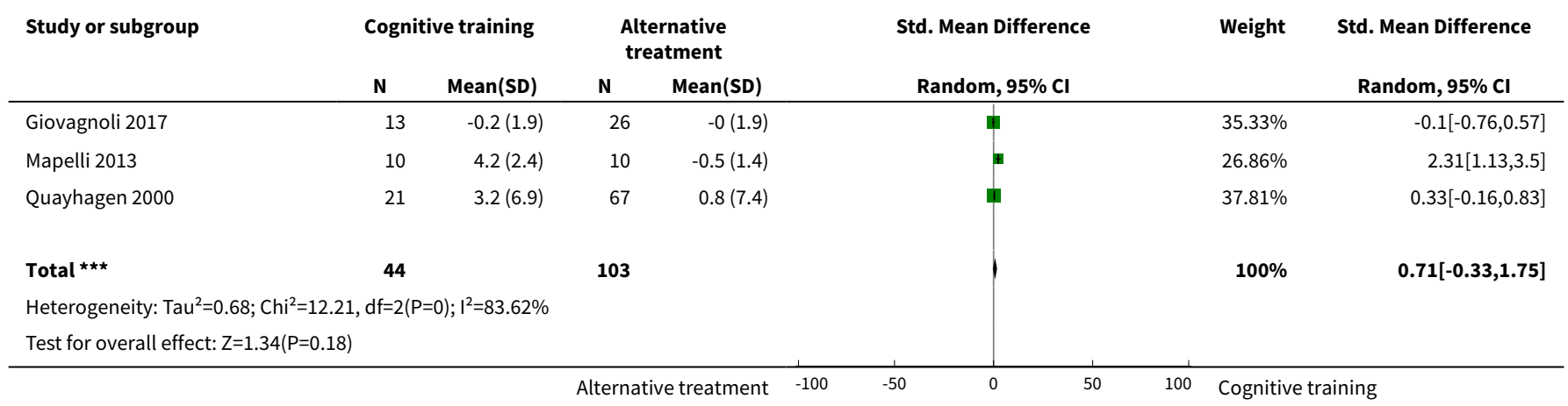

Analysis 11.7. Comparison 11 Cognitive training vs alternative treatment immediately post intervention - intervention dose, Outcome 7 Change in attention and working memory.

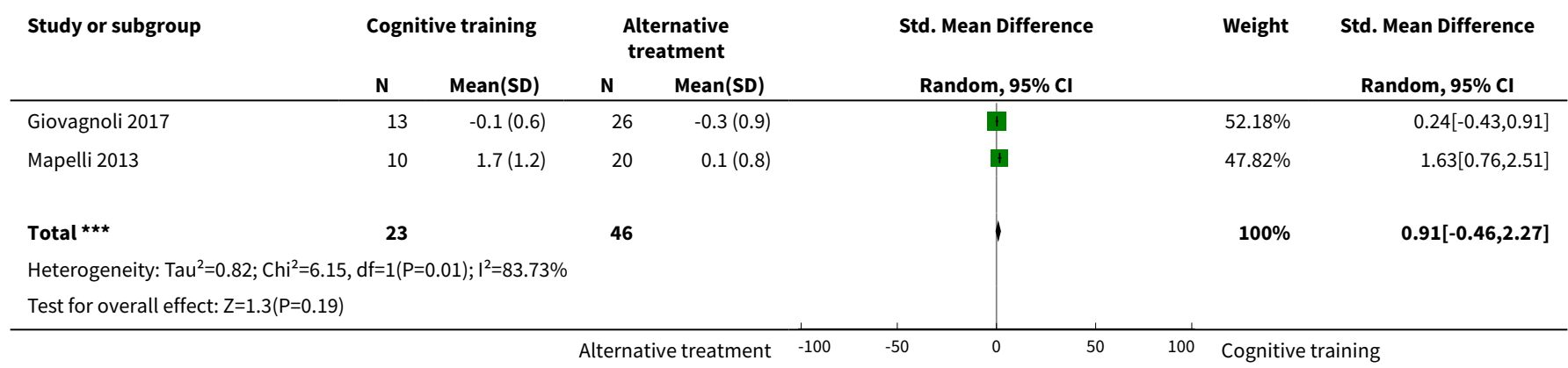

Analysis 11.8. Comparison 11 Cognitive training vs alternative treatment immediately post intervention - intervention dose, Outcome 8 Change in language (naming).

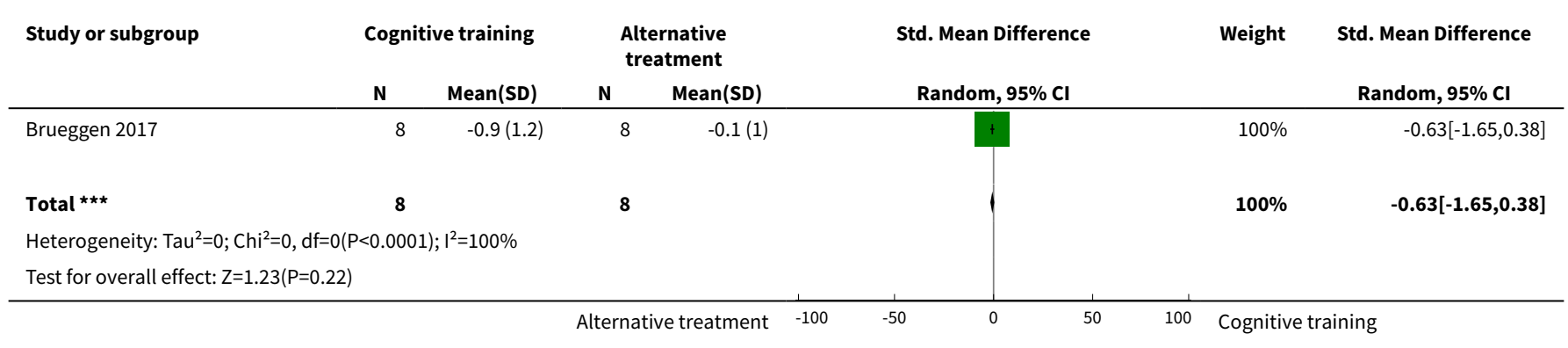


Analysis 11.9. Comparison 11 Cognitive training vs alternative treatment immediately post intervention - intervention dose, Outcome 9 Change in verbal letter fluency.

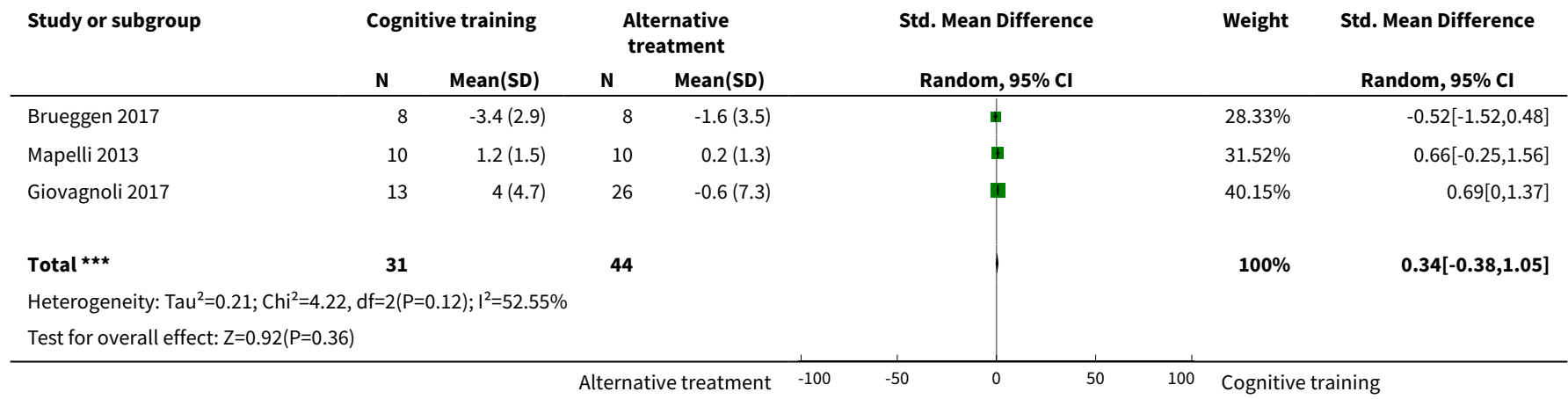

\section{Analysis 11.10. Comparison 11 Cognitive training vs alternative treatment immediately post intervention - intervention dose, Outcome 10 Change in verbal category fluency.}

\begin{tabular}{|c|c|c|c|c|c|c|c|}
\hline \multirow[t]{2}{*}{ Study or subgroup } & \multicolumn{2}{|c|}{ Cognitive training } & \multicolumn{2}{|c|}{$\begin{array}{l}\text { Alternative } \\
\text { treatment }\end{array}$} & \multirow{2}{*}{$\begin{array}{l}\text { Std. Mean Difference } \\
\text { Random, 95\% Cl } \\
\end{array}$} & \multirow[t]{2}{*}{ Weight } & \multirow{2}{*}{$\begin{array}{l}\text { Std. Mean Difference } \\
\text { Random, 95\% Cl } \\
\end{array}$} \\
\hline & $\mathbf{N}$ & Mean(SD) & $\mathbf{N}$ & Mean(SD) & & & \\
\hline Brueggen 2017 & 8 & $-2.4(4.3)$ & 8 & $1.5(3.4)$ & + & $44.12 \%$ & $-0.96[-2.01,0.1]$ \\
\hline Giovagnoli 2017 & 13 & $-0.5(5.8)$ & 26 & $-1.9(5.6)$ & 1 & $55.88 \%$ & $0.25[-0.42,0.92]$ \\
\hline 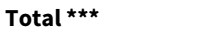 & 21 & & 34 & & 1 & $100 \%$ & $-0.28[-1.46,0.89]$ \\
\hline \multicolumn{8}{|c|}{ Heterogeneity: $\mathrm{Tau}^{2}=0.53 ; \mathrm{Chi}^{2}=3.61, \mathrm{df}=1(\mathrm{P}=0.06) ; \mathrm{I}^{2}=72.31 \%$} \\
\hline \multicolumn{3}{|c|}{ Test for overall effect: $Z=0.47(P=0.64)$} & & & & & \\
\hline
\end{tabular}

Analysis 11.11. Comparison 11 Cognitive training vs alternative treatment immediately post intervention - intervention dose, Outcome 11 Change in executive function.

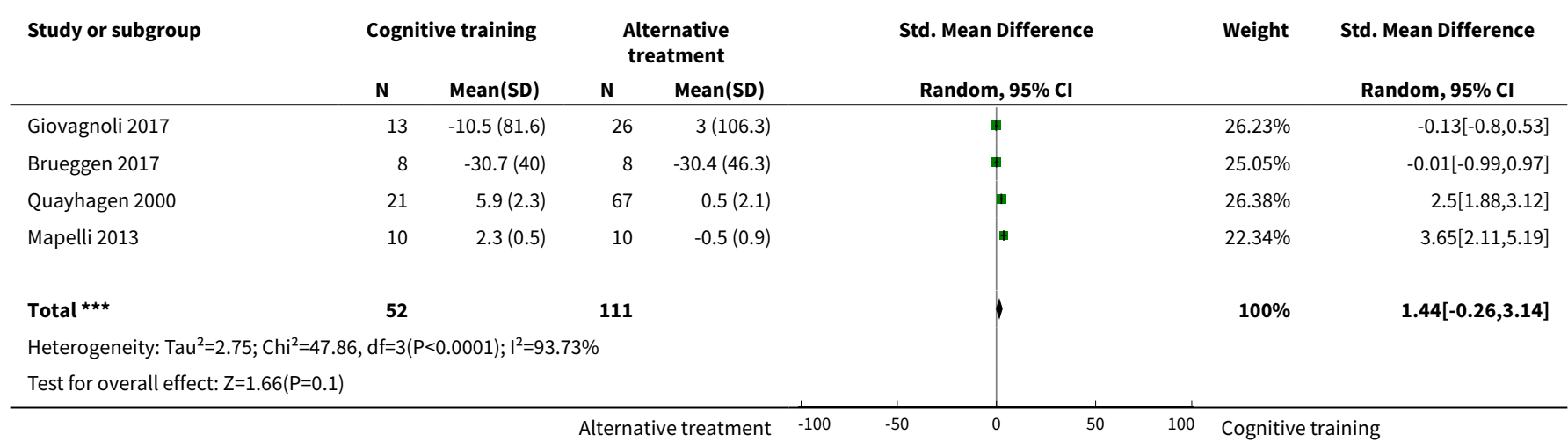


Analysis 11.14. Comparison 11 Cognitive training vs alternative treatment immediately post intervention - intervention dose, Outcome 14 Change in participants' mood.

\begin{tabular}{|c|c|c|c|c|c|c|c|}
\hline \multirow[t]{2}{*}{ Study or subgroup } & \multicolumn{2}{|c|}{ Cognitive training } & \multicolumn{2}{|c|}{$\begin{array}{c}\text { Alternative } \\
\text { treatment }\end{array}$} & \multirow{2}{*}{$\begin{array}{c}\text { Std. Mean Difference } \\
\text { Random, } 95 \% \mathrm{CI} \\
\end{array}$} & \multirow[t]{2}{*}{ Weight } & \multirow{2}{*}{$\begin{array}{l}\text { Std. Mean Difference } \\
\text { Random, } 95 \% \mathrm{Cl} \\
\end{array}$} \\
\hline & $\mathbf{N}$ & Mean(SD) & $\mathbf{N}$ & Mean(SD) & & & \\
\hline Giovagnoli 2017 & 13 & $2.2(2.9)$ & 26 & $3.9(5.7)$ & \& & $7.05 \%$ & $-0.33[-1,0.34]$ \\
\hline Brueggen 2017 & 8 & $-0.9(3.9)$ & 8 & $-0.1(3.4)$ & & $3.28 \%$ & $-0.19[-1.18,0.79]$ \\
\hline Amieva 2016 & 164 & $-12.1(12.7)$ & 324 & $-10.9(12.8)$ & & $89.67 \%$ & $-0.09[-0.28,0.1]$ \\
\hline 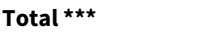 & 185 & & 358 & & & $100 \%$ & $-0.11[-0.29,0.07]$ \\
\hline \multicolumn{8}{|c|}{ Heterogeneity: $\operatorname{Tau}^{2}=0 ; \mathrm{Chi}^{2}=0.48, \mathrm{df}=2(\mathrm{P}=0.79) ; \mathrm{I}^{2}=0 \%$} \\
\hline \multicolumn{3}{|c|}{ Test for overall effect: $\mathrm{Z}=1.23(\mathrm{P}=0.22)$} & & & & & \\
\hline
\end{tabular}

Analysis 11.15. Comparison 11 Cognitive training vs alternative treatment immediately post intervention - intervention dose, Outcome 15 Change in capacity for activities of daily living.

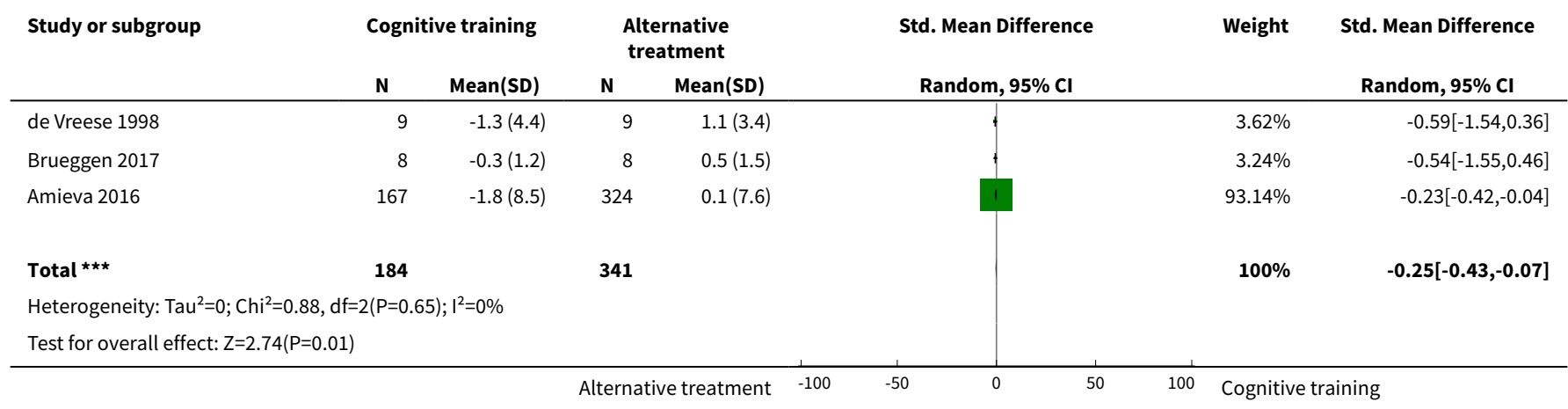

Analysis 11.16. Comparison 11 Cognitive training vs alternative treatment immediately post intervention - intervention dose, Outcome 16 Change in behavioural and psychological symptoms of dementia (BPSD).

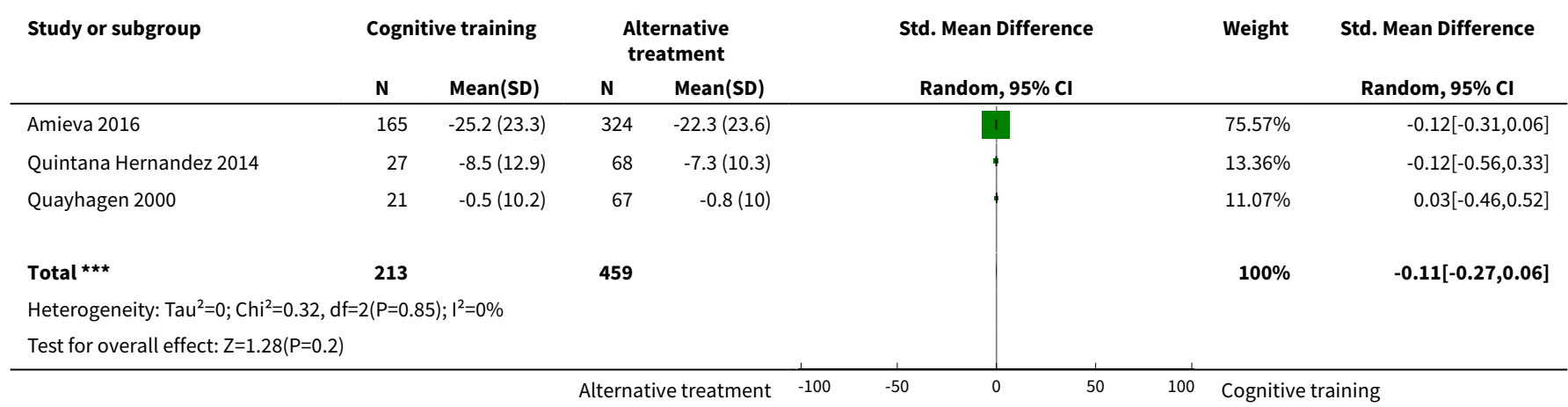


Analysis 11.17. Comparison 11 Cognitive training vs alternative treatment immediately post intervention - intervention dose, Outcome 17 Change in disease progression.

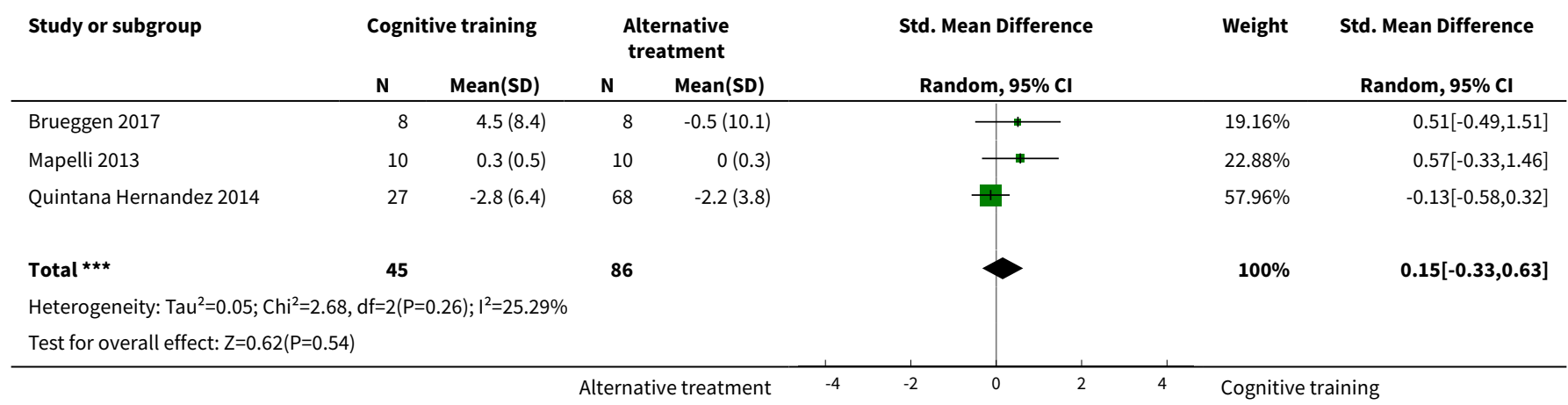

Analysis 11.18. Comparison 11 Cognitive training vs alternative treatment immediately post intervention - intervention dose, Outcome 18 Participant burden (retention rates).

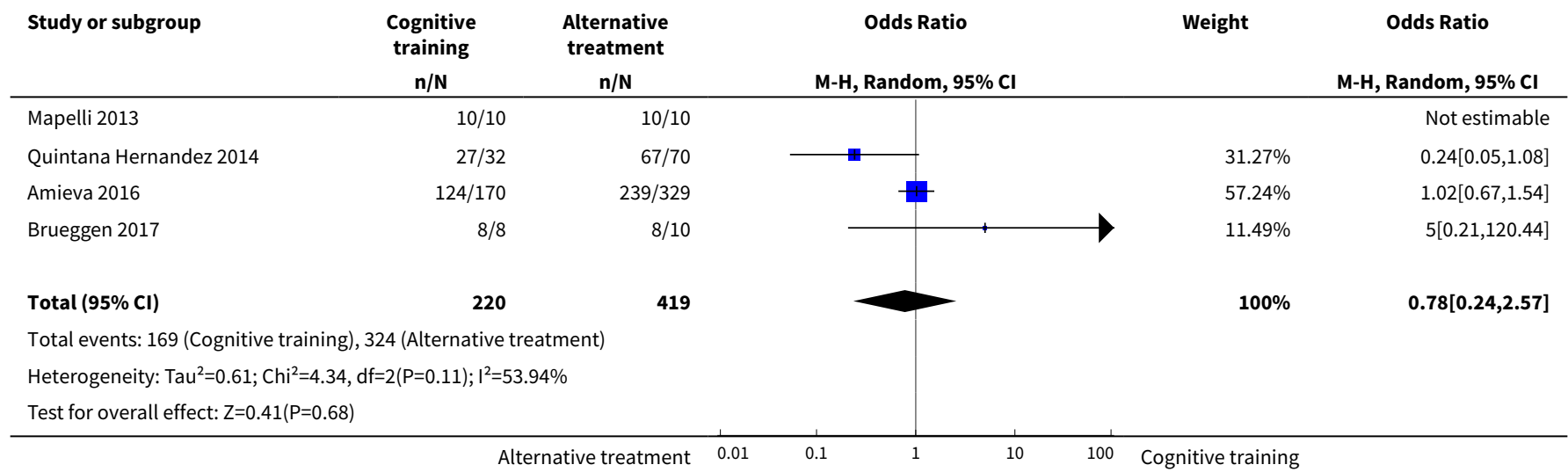

Analysis 11.19. Comparison 11 Cognitive training vs alternative treatment immediately post intervention - intervention dose, Outcome 19 Change in mood and well-being (CAREGIVER).

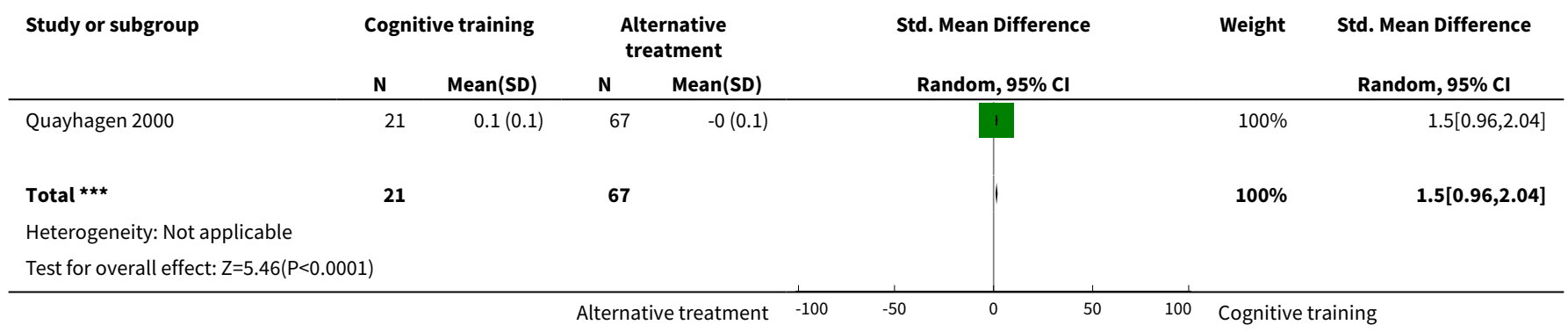


Analysis 11.20. Comparison 11 Cognitive training vs alternative treatment immediately post intervention - intervention dose, Outcome 20 Change in burden of care (CAREGIVER).

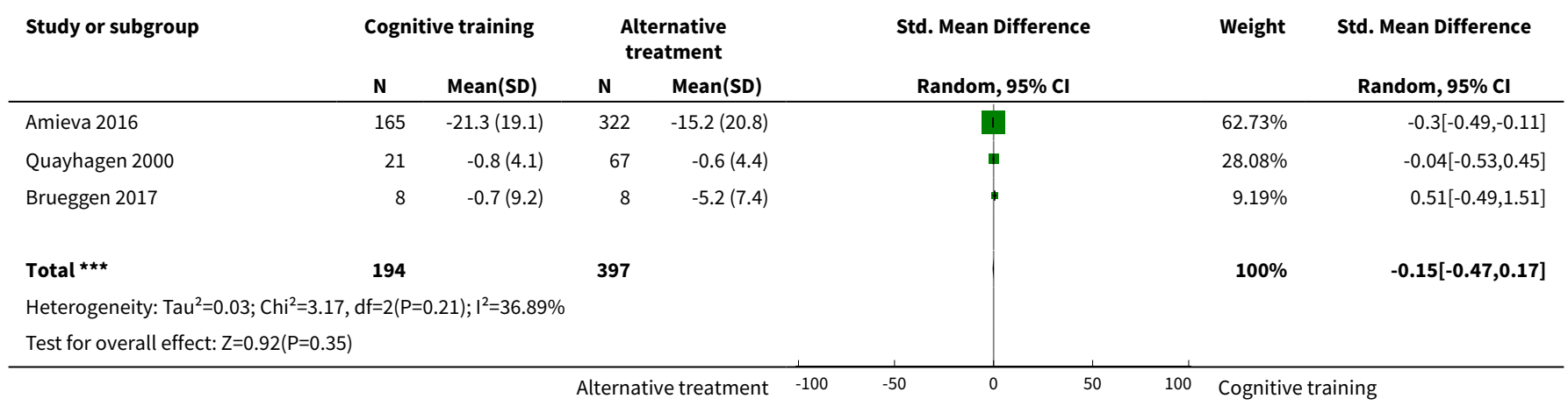

Analysis 11.21. Comparison 11 Cognitive training vs alternative treatment immediately post intervention - intervention dose, Outcome 21 Change in quality of life (CAREGIVER).

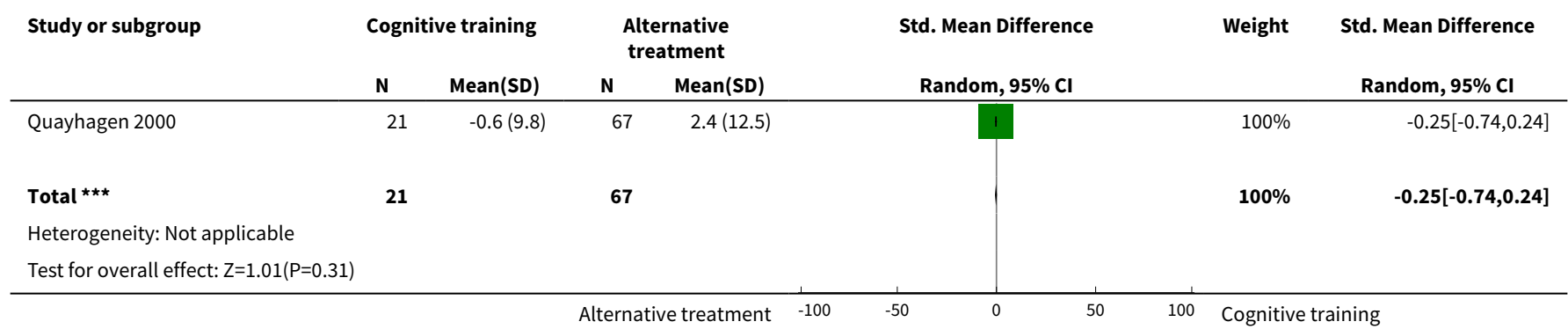

Analysis 11.22. Comparison 11 Cognitive training vs alternative treatment immediately post intervention - intervention dose, Outcome 22 Change in speed of information processing.

\begin{tabular}{|c|c|c|c|c|c|c|c|c|}
\hline \multirow[t]{2}{*}{ Study or subgroup } & \multicolumn{2}{|c|}{ Cognitive training } & \multicolumn{2}{|c|}{$\begin{array}{c}\text { Alternative } \\
\text { treatment }\end{array}$} & \multirow{2}{*}{\multicolumn{2}{|c|}{$\begin{array}{c}\text { Std. Mean Difference } \\
\text { Random, 95\% Cl } \\
\end{array}$}} & \multirow[t]{2}{*}{ Weight } & \multirow{2}{*}{$\begin{array}{l}\text { Std. Mean Difference } \\
\text { Random, } 95 \% \mathrm{Cl} \\
\end{array}$} \\
\hline & $\mathbf{N}$ & Mean(SD) & $\mathbf{N}$ & Mean(SD) & & & & \\
\hline Brueggen 2017 & 8 & $-15.6(33.6)$ & 8 & $0(33.5)$ & & 甲 & $30.93 \%$ & $-0.44[-1.44,0.56]$ \\
\hline Giovagnoli 2017 & 13 & $-12.5(21.2)$ & 26 & $-7.6(51.3)$ & & & $69.07 \%$ & $-0.11[-0.78,0.56]$ \\
\hline 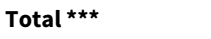 & 21 & & 34 & & & & $100 \%$ & $-0.21[-0.77,0.34]$ \\
\hline \multicolumn{9}{|c|}{ Heterogeneity: $\operatorname{Tau}^{2}=0 ; \mathrm{Chi}^{2}=0.29, \mathrm{df}=1(\mathrm{P}=0.59) ; \mathrm{I}^{2}=0 \%$} \\
\hline \multicolumn{9}{|c|}{ Test for overall effect: $Z=0.75(P=0.45)$} \\
\hline & & & Alterna & treatment & -100 & -50 & Cognitiv & ining \\
\hline
\end{tabular}


Analysis 11.23. Comparison 11 Cognitive training vs alternative treatment immediately post intervention - intervention dose, Outcome 23 Change in general health and quality of life.

\begin{tabular}{|c|c|c|c|c|c|c|c|}
\hline \multirow[t]{2}{*}{ Study or subgroup } & \multicolumn{2}{|c|}{ Cognitive training } & \multicolumn{2}{|c|}{$\begin{array}{l}\text { Alternative } \\
\text { treatment }\end{array}$} & \multirow{2}{*}{$\begin{array}{l}\text { Std. Mean Difference } \\
\text { Random, } 95 \% \mathrm{CI}\end{array}$} & \multirow[t]{2}{*}{ Weight } & \multirow{2}{*}{$\begin{array}{l}\text { Std. Mean Difference } \\
\text { Random, } 95 \% \mathrm{Cl}\end{array}$} \\
\hline & $\mathbf{N}$ & Mean(SD) & $\mathbf{N}$ & $\operatorname{Mean}(S D)$ & & & \\
\hline Brueggen 2017 & 8 & $-4.4(4.6)$ & 8 & $3.1(4.8)$ & 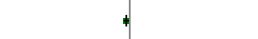 & $12.91 \%$ & $-1.52[-2.67,-0.36]$ \\
\hline Giovagnoli 2017 & 13 & $-0.1(12.7)$ & 26 & $8.8(6)$ & 曲 & $22.11 \%$ & $-0.99[-1.69,-0.28]$ \\
\hline Quayhagen 2000 & 21 & $-0.4(2.8)$ & 67 & $0.1(3.2)$ & 4 & $28.35 \%$ & $-0.14[-0.63,0.35]$ \\
\hline Amieva 2016 & 164 & $-6.3(6.1)$ & 324 & $-5.7(6.3)$ & $\Phi$ & $36.64 \%$ & $-0.1[-0.29,0.09]$ \\
\hline 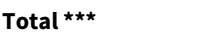 & 206 & & 425 & & & $100 \%$ & $-0.49[-1,0.02]$ \\
\hline \multicolumn{8}{|c|}{ Heterogeneity: $\mathrm{Tau}^{2}=0.17 ; \mathrm{Chi}^{2}=10.91, \mathrm{df}=3(\mathrm{P}=0.01) ; \mathrm{I}^{2}=72.51 \%$} \\
\hline \multicolumn{8}{|c|}{ Test for overall effect: $Z=1.9(P=0.06)$} \\
\hline
\end{tabular}

Comparison 12. Cognitive training vs alternative treatment immediately post intervention - type of CT (traditional vs augmented)

\begin{tabular}{|c|c|c|c|c|}
\hline Outcome or subgroup title & $\begin{array}{l}\text { No. of } \\
\text { studies }\end{array}$ & $\begin{array}{l}\text { No. of } \\
\text { partici- } \\
\text { pants }\end{array}$ & Statistical method & Effect size \\
\hline 1 Change in a global measure of cognition & 7 & 724 & $\begin{array}{l}\text { Std. Mean Difference (IV, Ran- } \\
\text { dom, } 95 \% \mathrm{CI} \text { ) }\end{array}$ & $0.16[-0.28,0.60]$ \\
\hline 1.1 Traditional & 4 & 646 & $\begin{array}{l}\text { Std. Mean Difference (IV, Ran- } \\
\text { dom, } 95 \% \mathrm{CI} \text { ) }\end{array}$ & $-0.19[-0.35,-0.02]$ \\
\hline 1.2 Augmented & 3 & 78 & $\begin{array}{l}\text { Std. Mean Difference (IV, Ran- } \\
\text { dom, } 95 \% \mathrm{CI} \text { ) }\end{array}$ & $1.04[-0.29,2.36]$ \\
\hline $\begin{array}{l}2 \text { Change in a global measure of cognition_ze- } \\
\text { ro correlation }\end{array}$ & 7 & 724 & $\begin{array}{l}\text { Std. Mean Difference (IV, Ran- } \\
\text { dom, } 95 \% \mathrm{CI} \text { ) }\end{array}$ & $-0.02[-0.24,0.20]$ \\
\hline 2.1 Traditional & 4 & 646 & $\begin{array}{l}\text { Std. Mean Difference (IV, Ran- } \\
\text { dom, } 95 \% \mathrm{CI} \text { ) }\end{array}$ & $-0.12[-0.29,0.04]$ \\
\hline 2.2 Augmented & 3 & 78 & $\begin{array}{l}\text { Std. Mean Difference (IV, Ran- } \\
\text { dom, } 95 \% \mathrm{CI} \text { ) }\end{array}$ & $0.44[-0.18,1.06]$ \\
\hline $\begin{array}{l}3 \text { Change in a global measure of cognition } \\
\text { (composite) }\end{array}$ & 7 & 769 & $\begin{array}{l}\text { Std. Mean Difference (Random, } \\
95 \% \mathrm{Cl})\end{array}$ & $0.21[-0.23,0.64]$ \\
\hline 3.1 Traditional & 4 & 643 & $\begin{array}{l}\text { Std. Mean Difference (Random, } \\
95 \% \mathrm{Cl} \text { ) }\end{array}$ & $-0.17[-0.34,-0.00]$ \\
\hline 3.2 Augmented & 3 & 126 & $\begin{array}{l}\text { Std. Mean Difference (Random, } \\
95 \% \mathrm{Cl} \text { ) }\end{array}$ & $1.09[0.14,2.04]$ \\
\hline $\begin{array}{l}4 \text { Change in a global measure of cognition } \\
\text { (composite)_zero correlation }\end{array}$ & 7 & 769 & $\begin{array}{l}\text { Std. Mean Difference (Random, } \\
95 \% \mathrm{Cl})\end{array}$ & $-0.03[-0.23,0.17]$ \\
\hline 4.1 Traditional & 4 & 643 & $\begin{array}{l}\text { Std. Mean Difference (Random, } \\
95 \% \mathrm{Cl} \text { ) }\end{array}$ & $-0.11[-0.28,0.06]$ \\
\hline
\end{tabular}




\begin{tabular}{|c|c|c|c|c|}
\hline Outcome or subgroup title & $\begin{array}{l}\text { No. of } \\
\text { studies }\end{array}$ & $\begin{array}{l}\text { No. of } \\
\text { partici- } \\
\text { pants }\end{array}$ & Statistical method & Effect size \\
\hline 4.2 Augmented & 3 & 126 & $\begin{array}{l}\text { Std. Mean Difference (Random, } \\
95 \% \mathrm{Cl} \text { ) }\end{array}$ & $0.43[-0.04,0.90]$ \\
\hline 5 Change in immediate memory & 3 & 147 & $\begin{array}{l}\text { Std. Mean Difference (IV, Ran- } \\
\text { dom, } 95 \% \mathrm{CI} \text { ) }\end{array}$ & $0.51[-0.19,1.21]$ \\
\hline 6 Change in delayed memory & 3 & 147 & $\begin{array}{l}\text { Std. Mean Difference (IV, Ran- } \\
\text { dom, } 95 \% \mathrm{CI} \text { ) }\end{array}$ & $0.71[-0.33,1.75]$ \\
\hline 7 Change in attention and working memory & 2 & 69 & $\begin{array}{l}\text { Std. Mean Difference (IV, Ran- } \\
\text { dom, } 95 \% \mathrm{CI} \text { ) }\end{array}$ & $0.91[-0.46,2.27]$ \\
\hline 8 Change in language (naming) & 1 & 16 & $\begin{array}{l}\text { Std. Mean Difference (IV, Ran- } \\
\text { dom, } 95 \% \mathrm{CI} \text { ) }\end{array}$ & $-0.63[-1.65,0.38]$ \\
\hline 9 Change in verbal letter fluency & 3 & 75 & $\begin{array}{l}\text { Std. Mean Difference (IV, Ran- } \\
\text { dom, } 95 \% \mathrm{CI} \text { ) }\end{array}$ & $0.34[-0.38,1.05]$ \\
\hline 10 Change in verbal category fluency & 2 & 55 & $\begin{array}{l}\text { Std. Mean Difference (IV, Ran- } \\
\text { dom, } 95 \% \mathrm{CI} \text { ) }\end{array}$ & $-0.28[-1.46,0.89]$ \\
\hline 11 Change in executive function & 4 & 163 & $\begin{array}{l}\text { Std. Mean Difference (IV, Ran- } \\
\text { dom, } 95 \% \mathrm{CI} \text { ) }\end{array}$ & $1.44[-0.26,3.14]$ \\
\hline 12 Change in meta cognition (self-reported) & 0 & 0 & $\begin{array}{l}\text { Std. Mean Difference (IV, Ran- } \\
\text { dom, } 95 \% \mathrm{CI} \text { ) }\end{array}$ & $0.0[0.0,0.0]$ \\
\hline $\begin{array}{l}13 \text { Change in meta cognition (informant-re- } \\
\text { ported) }\end{array}$ & 0 & 0 & $\begin{array}{l}\text { Std. Mean Difference (IV, Ran- } \\
\text { dom, } 95 \% \mathrm{CI} \text { ) }\end{array}$ & $0.0[0.0,0.0]$ \\
\hline 14 Change in participants' mood & 3 & 543 & $\begin{array}{l}\text { Std. Mean Difference (IV, Ran- } \\
\text { dom, } 95 \% \mathrm{CI} \text { ) }\end{array}$ & $-0.11[-0.29,0.07]$ \\
\hline $\begin{array}{l}15 \text { Change in capacity for activities of daily liv- } \\
\text { ing }\end{array}$ & 3 & 525 & $\begin{array}{l}\text { Std. Mean Difference (IV, Ran- } \\
\text { dom, } 95 \% \mathrm{CI} \text { ) }\end{array}$ & $-0.25[-0.43,-0.07]$ \\
\hline $\begin{array}{l}16 \text { Change in behavioural and psychological } \\
\text { symptoms of dementia (BPSD) }\end{array}$ & 3 & 672 & $\begin{array}{l}\text { Std. Mean Difference (IV, Ran- } \\
\text { dom, } 95 \% \mathrm{Cl} \text { ) }\end{array}$ & $-0.11[-0.27,0.06]$ \\
\hline 17 Change in disease progression & 3 & 131 & $\begin{array}{l}\text { Std. Mean Difference (IV, Ran- } \\
\text { dom, } 95 \% \mathrm{CI} \text { ) }\end{array}$ & $0.15[-0.33,0.63]$ \\
\hline 18 Participant burden (retention rates) & 4 & 639 & $\begin{array}{l}\text { Odds Ratio (M-H, Random, } \\
95 \% \mathrm{Cl})\end{array}$ & $0.78[0.24,2.57]$ \\
\hline $\begin{array}{l}19 \text { Change in mood and well-being } \\
\text { (CAREGIVER) }\end{array}$ & 1 & 88 & $\begin{array}{l}\text { Std. Mean Difference (IV, Ran- } \\
\text { dom, } 95 \% \mathrm{Cl} \text { ) }\end{array}$ & $1.50[0.96,2.04]$ \\
\hline 20 Change in burden of care (CAREGIVER) & 3 & 591 & $\begin{array}{l}\text { Std. Mean Difference (IV, Ran- } \\
\text { dom, } 95 \% \mathrm{CI} \text { ) }\end{array}$ & $-0.15[-0.47,0.17]$ \\
\hline 21 Change in quality of life (CAREGIVER) & 1 & 88 & $\begin{array}{l}\text { Std. Mean Difference (IV, Ran- } \\
\text { dom, } 95 \% \mathrm{CI} \text { ) }\end{array}$ & $-0.25[-0.74,0.24]$ \\
\hline
\end{tabular}




\begin{tabular}{llllll}
\hline Outcome or subgroup title & $\begin{array}{l}\text { No. of } \\
\text { studies }\end{array}$ & $\begin{array}{l}\text { No. of } \\
\text { partici- } \\
\text { pants }\end{array}$ & Statistical method & Effect size \\
\hline 22 Change in speed of information processing & 2 & 55 & $\begin{array}{l}\text { Std. Mean Difference (IV, Ran- } \\
\text { dom, 95\% CI) }\end{array}$ & $-0.21[-0.77,0.34]$ \\
\hline 23 Change in general health and quality of life & 4 & 631 & $\begin{array}{l}\text { Std. Mean Difference (IV, Ran- } \\
\text { dom, 95\% CI) }\end{array}$ & $-0.49[1.00,0.02]$ \\
\hline
\end{tabular}

Analysis 12.1. Comparison 12 Cognitive training vs alternative treatment immediately post intervention - type of CT (traditional vs augmented), Outcome 1 Change in a global measure of cognition.

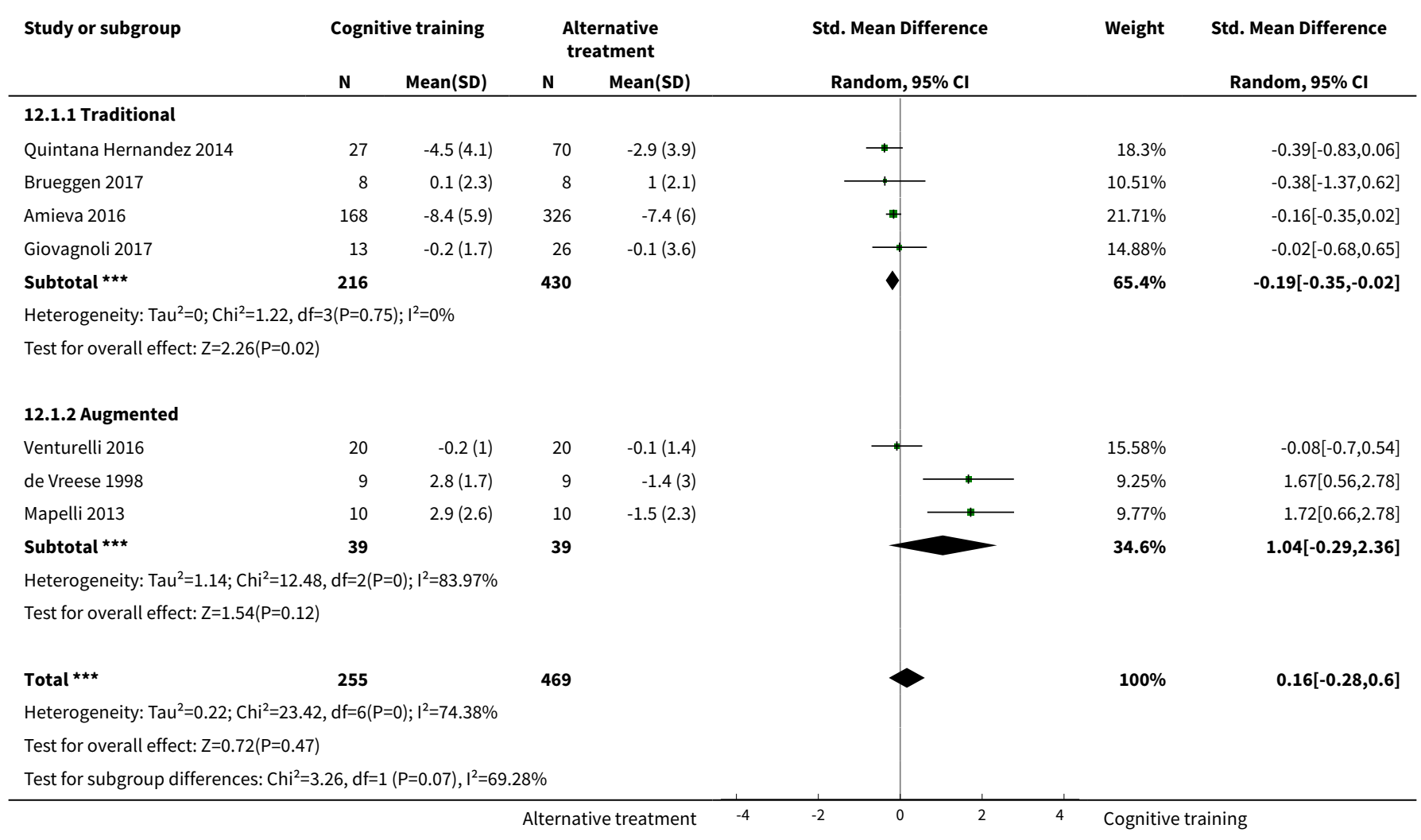

Analysis 12.2. Comparison 12 Cognitive training vs alternative treatment immediately post intervention type of CT (traditional vs augmented), Outcome 2 Change in a global measure of cognition_zero correlation.

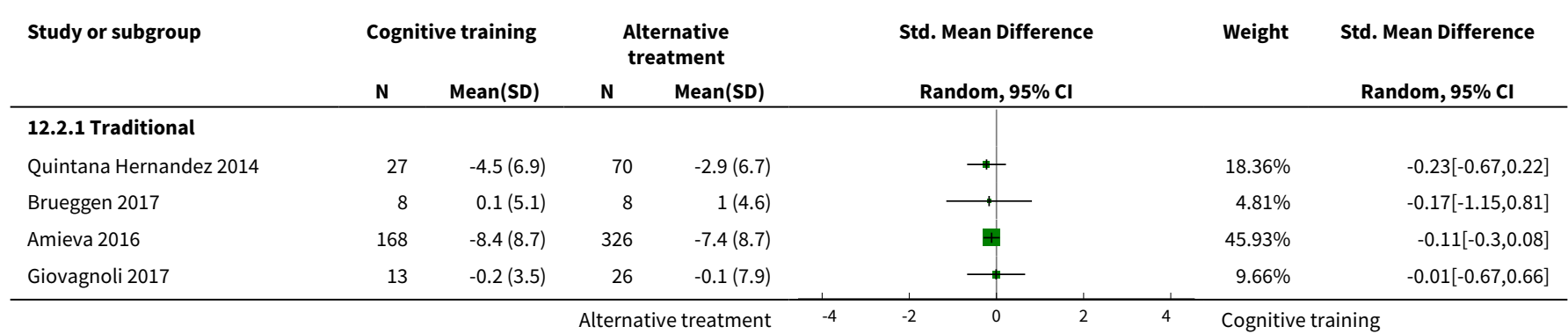




\begin{tabular}{|c|c|c|c|c|c|c|c|}
\hline \multirow[t]{2}{*}{ Study or subgroup } & \multicolumn{2}{|c|}{ Cognitive training } & \multicolumn{2}{|c|}{$\begin{array}{l}\text { Alternative } \\
\text { treatment }\end{array}$} & \multirow{2}{*}{$\begin{array}{l}\text { Std. Mean Difference } \\
\text { Random, } 95 \% \mathrm{Cl} \\
\end{array}$} & \multirow[t]{2}{*}{ Weight } & \multirow{2}{*}{$\begin{array}{l}\text { Std. Mean Difference } \\
\text { Random, } 95 \% \mathrm{Cl} \\
\end{array}$} \\
\hline & $\mathbf{N}$ & $\operatorname{Mean}(S D)$ & $\mathbf{N}$ & $\operatorname{Mean}(S D)$ & & & \\
\hline Subtotal $\star \star \star ~$ & 216 & & 430 & & 4 & $78.77 \%$ & $-0.12[-0.29,0.04]$ \\
\hline \multicolumn{8}{|c|}{ Heterogeneity: $\mathrm{Tau}^{2}=0 ; \mathrm{Chi}^{2}=0.35, \mathrm{df}=3(\mathrm{P}=0.95) ; \mathrm{I}^{2}=0 \%$} \\
\hline \multicolumn{8}{|c|}{ Test for overall effect: $Z=1.46(P=0.14)$} \\
\hline Venturelli 2016 & 20 & $-0.2(2.1)$ & 20 & $-0.1(3)$ & $\longrightarrow$ & $10.91 \%$ & $-0.04[-0.66,0.58]$ \\
\hline de Vreese 1998 & 9 & $2.8(3.5)$ & 9 & $-1.4(6.5)$ & 1 & $4.96 \%$ & $0.77[-0.2,1.73]$ \\
\hline Mapelli 2013 & 10 & $2.9(5)$ & 10 & $-1.5(4.7)$ & & $5.36 \%$ & $0.87[-0.06,1.79]$ \\
\hline Subtotal $\star \star \star ~$ & 39 & & 39 & & & $21.23 \%$ & $0.44[-0.18,1.06]$ \\
\hline \multicolumn{8}{|c|}{ Heterogeneity: $\operatorname{Tau}^{2}=0.13 ; \mathrm{Chi}^{2}=3.42, \mathrm{df}=2(\mathrm{P}=0.18) ; \mathrm{I}^{2}=41.58 \%$} \\
\hline \multicolumn{8}{|c|}{ Test for overall effect: $Z=1.39(P=0.16)$} \\
\hline Total $\star \star \star ~$ & 255 & & 469 & & 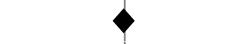 & $100 \%$ & $-0.02[-0.24,0.2]$ \\
\hline \multicolumn{8}{|c|}{ Heterogeneity: $\mathrm{Tau}^{2}=0.02 ; \mathrm{Chi}^{2}=7.58, \mathrm{df}=6(\mathrm{P}=0.27) ; \mathrm{I}^{2}=20.8 \%$} \\
\hline \multicolumn{8}{|c|}{ Test for overall effect: $Z=0.18(P=0.85)$} \\
\hline \multicolumn{8}{|c|}{ Test for subgroup differences: $\mathrm{Chi}^{2}=2.95, \mathrm{df}=1(\mathrm{P}=0.09), \mathrm{I}^{2}=66.05 \%$} \\
\hline & & & Altern & treatment & -2 & Cognitiv & ning \\
\hline
\end{tabular}

\section{Analysis 12.3. Comparison 12 Cognitive training vs alternative treatment immediately post intervention - type of CT (traditional vs augmented), Outcome 3 Change in a global measure of cognition (composite).}

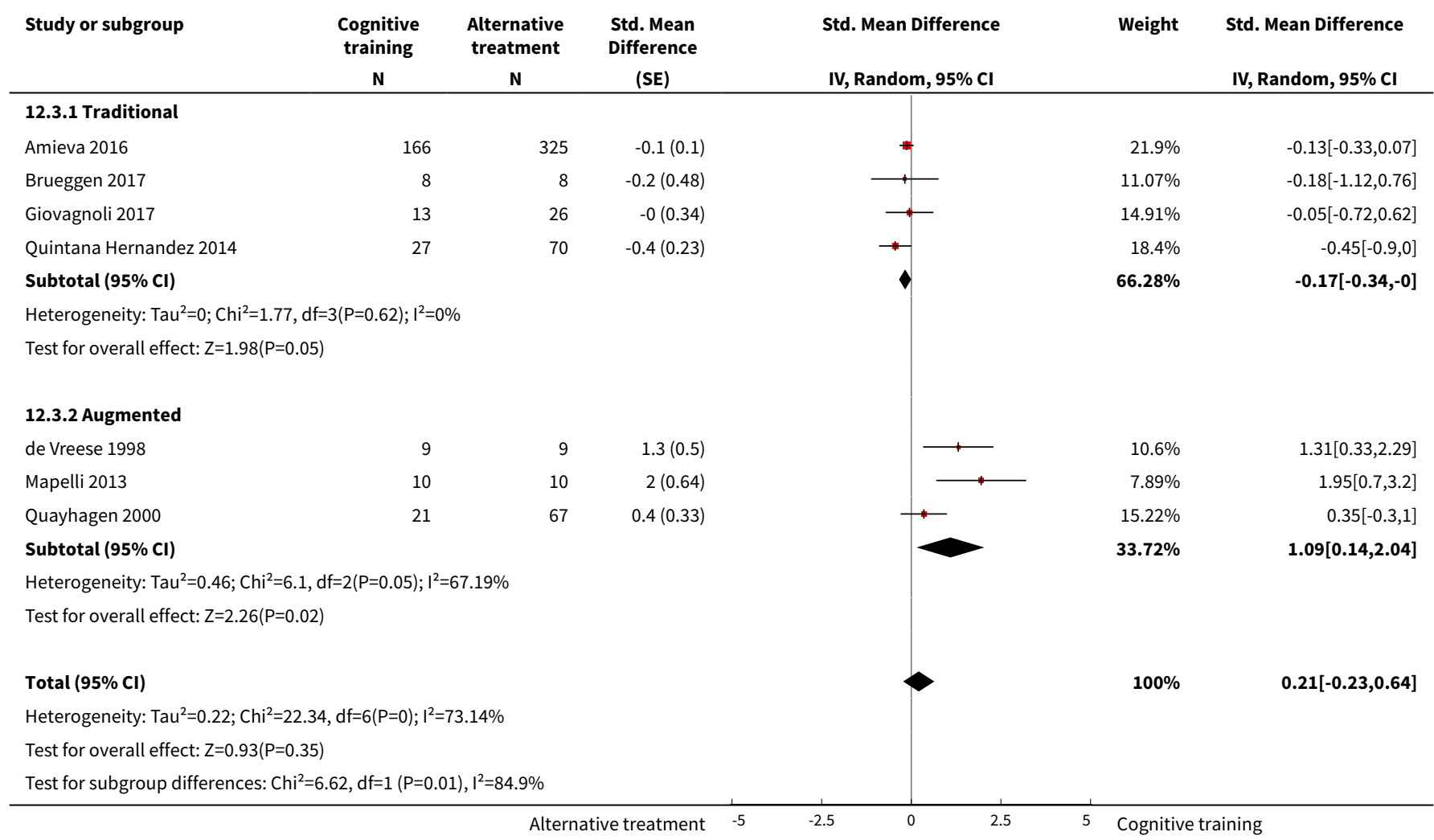


Analysis 12.4. Comparison 12 Cognitive training vs alternative treatment immediately post intervention - type of CT (traditional vs augmented), Outcome 4 Change in a global measure of cognition (composite)_zero correlation.

\begin{tabular}{|c|c|c|c|c|c|c|}
\hline Study or subgroup & $\begin{array}{c}\text { Cognitive } \\
\text { training } \\
\mathrm{N}\end{array}$ & $\begin{array}{c}\text { Alternative } \\
\text { treatment } \\
\mathbf{N}\end{array}$ & $\begin{array}{l}\text { Std. Mean } \\
\text { Difference } \\
\text { (SE) }\end{array}$ & IV, Random, 95\% CI & Weight & $\begin{array}{l}\text { Std. Mean Difference } \\
\text { IV, Random, } 95 \% \mathrm{CI}\end{array}$ \\
\hline \multicolumn{7}{|l|}{ 12.4.1 Traditional } \\
\hline Amieva 2016 & 166 & 325 & $-0.1(0.1)$ & + & $55.07 \%$ & $-0.09[-0.29,0.11]$ \\
\hline Brueggen 2017 & 8 & 8 & $-0.1(0.47)$ & $\longrightarrow$ & $4.48 \%$ & $-0.1[-1.02,0.82]$ \\
\hline Giovagnoli 2017 & 13 & 26 & $-0(0.37)$ & $\longrightarrow$ & $7.06 \%$ & $-0.04[-0.77,0.69]$ \\
\hline Subtotal $(95 \% \mathrm{Cl})$ & & & & $\checkmark$ & $83.29 \%$ & $-0.11[-0.28,0.06]$ \\
\hline \multicolumn{7}{|c|}{ Heterogeneity: $\operatorname{Tau}^{2}=0 ; \mathrm{Chi}^{2}=0.56, \mathrm{df}=3(\mathrm{P}=0.91) ; \mathrm{I}^{2}=0 \%$} \\
\hline \multicolumn{7}{|c|}{ Test for overall effect: $Z=1.3(P=0.19)$} \\
\hline \multicolumn{7}{|l|}{ 12.4.2 Augmented } \\
\hline de Vreese 1998 & 9 & 9 & $0.6(0.46)$ & & $4.66 \%$ & $0.6[-0.3,1.5]$ \\
\hline Quayhagen 2000 & 21 & 67 & $0.2(0.33)$ & - & $8.74 \%$ & $0.16[-0.49,0.81]$ \\
\hline Subtotal $(95 \% \mathrm{CI})$ & & & & & $16.71 \%$ & $0.43[-0.04,0.9]$ \\
\hline \multicolumn{7}{|c|}{ Heterogeneity: $\operatorname{Tau}^{2}=0 ; \mathrm{Chi}^{2}=1.63, \mathrm{df}=2(\mathrm{P}=0.44) ; \mathrm{I}^{2}=0 \%$} \\
\hline \multicolumn{7}{|c|}{ Test for overall effect: $Z=1.78(P=0.08)$} \\
\hline Total $(95 \% \mathrm{Cl})$ & & & & $\checkmark$ & $100 \%$ & $-0.03[-0.23,0.17]$ \\
\hline \multicolumn{7}{|c|}{ Heterogeneity: $\mathrm{Tau}^{2}=0.01 ; \mathrm{Chi}^{2}=6.66, \mathrm{df}=6(\mathrm{P}=0.35) ; \mathrm{I}^{2}=9.96 \%$} \\
\hline \multicolumn{7}{|c|}{ Test for overall effect: $Z=0.29(P=0.77)$} \\
\hline Test for subgroup differenc & $47, d f=1(P=0$. & $I^{2}=77.64 \%$ & & & & \\
\hline
\end{tabular}

Analysis 12.5. Comparison 12 Cognitive training vs alternative treatment immediately post intervention - type of CT (traditional vs augmented), Outcome 5 Change in immediate memory.

\begin{tabular}{|c|c|c|c|c|c|c|c|}
\hline \multirow[t]{2}{*}{ Study or subgroup } & \multicolumn{2}{|c|}{ Cognitive training } & \multicolumn{2}{|c|}{$\begin{array}{l}\text { Alternative } \\
\text { treatment }\end{array}$} & \multirow{2}{*}{$\begin{array}{l}\text { Std. Mean Difference } \\
\text { Random, } 95 \% \mathrm{Cl}\end{array}$} & \multirow[t]{2}{*}{ Weight } & \multirow{2}{*}{$\begin{array}{l}\text { Std. Mean Difference } \\
\text { Random, } 95 \% \mathrm{Cl}\end{array}$} \\
\hline & $\mathbf{N}$ & Mean(SD) & $\mathbf{N}$ & $\operatorname{Mean}(S D)$ & & & \\
\hline Giovagnoli 2017 & 13 & $1(4.4)$ & 26 & $0.3(6.2)$ & $\phi$ & $34.91 \%$ & $0.12[-0.55,0.78]$ \\
\hline Quayhagen 2000 & 21 & $2.3(12.1)$ & 67 & $0(9.3)$ & $\Pi$ & $40.81 \%$ & $0.22[-0.27,0.71]$ \\
\hline Mapelli 2013 & 10 & $2.6(2.4)$ & 10 & $-0.5(1.3)$ & i & $24.28 \%$ & $1.56[0.53,2.59]$ \\
\hline \multicolumn{8}{|c|}{ Heterogeneity: $\mathrm{Tau}^{2}=0.25 ; \mathrm{Chi}^{2}=6.02, \mathrm{df}=2(\mathrm{P}=0.05) ; \mathrm{I}^{2}=66.76 \%$} \\
\hline \multicolumn{3}{|c|}{ Test for overall effect: $Z=1.43(P=0.15)$} & & & & & \\
\hline
\end{tabular}


Analysis 12.6. Comparison 12 Cognitive training vs alternative treatment immediately post intervention - type of CT (traditional vs augmented), Outcome 6 Change in delayed memory.

\begin{tabular}{|c|c|c|c|c|c|c|c|}
\hline \multirow[t]{2}{*}{ Study or subgroup } & \multicolumn{2}{|c|}{ Cognitive training } & \multicolumn{2}{|c|}{$\begin{array}{c}\text { Alternative } \\
\text { treatment }\end{array}$} & \multirow{2}{*}{$\begin{array}{c}\text { Std. Mean Difference } \\
\text { Random, } 95 \% \mathrm{CI} \\
\end{array}$} & \multirow[t]{2}{*}{ Weight } & \multirow{2}{*}{$\begin{array}{c}\text { Std. Mean Difference } \\
\text { Random, 95\% Cl } \\
\end{array}$} \\
\hline & $\mathbf{N}$ & Mean(SD) & $\mathbf{N}$ & Mean(SD) & & & \\
\hline Giovagnoli 2017 & 13 & $-0.2(1.9)$ & 26 & $-0(1.9)$ & 由 & $35.33 \%$ & $-0.1[-0.76,0.57]$ \\
\hline Mapelli 2013 & 10 & $4.2(2.4)$ & 10 & $-0.5(1.4)$ & * & $26.86 \%$ & $2.31[1.13,3.5]$ \\
\hline Quayhagen 2000 & 21 & $3.2(6.9)$ & 67 & $0.8(7.4)$ & $\phi$ & $37.81 \%$ & $0.33[-0.16,0.83]$ \\
\hline 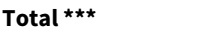 & 44 & & 103 & & 1 & $100 \%$ & $0.71[-0.33,1.75]$ \\
\hline \multicolumn{8}{|c|}{ Heterogeneity: $\mathrm{Tau}^{2}=0.68 ; \mathrm{Chi}^{2}=12.21, \mathrm{df}=2(\mathrm{P}=0) ; \mathrm{I}^{2}=83.62 \%$} \\
\hline \multicolumn{3}{|c|}{ Test for overall effect: $Z=1.34(P=0.18)$} & & & & & \\
\hline
\end{tabular}

Analysis 12.7. Comparison 12 Cognitive training vs alternative treatment immediately post intervention - type of CT (traditional vs augmented), Outcome 7 Change in attention and working memory.

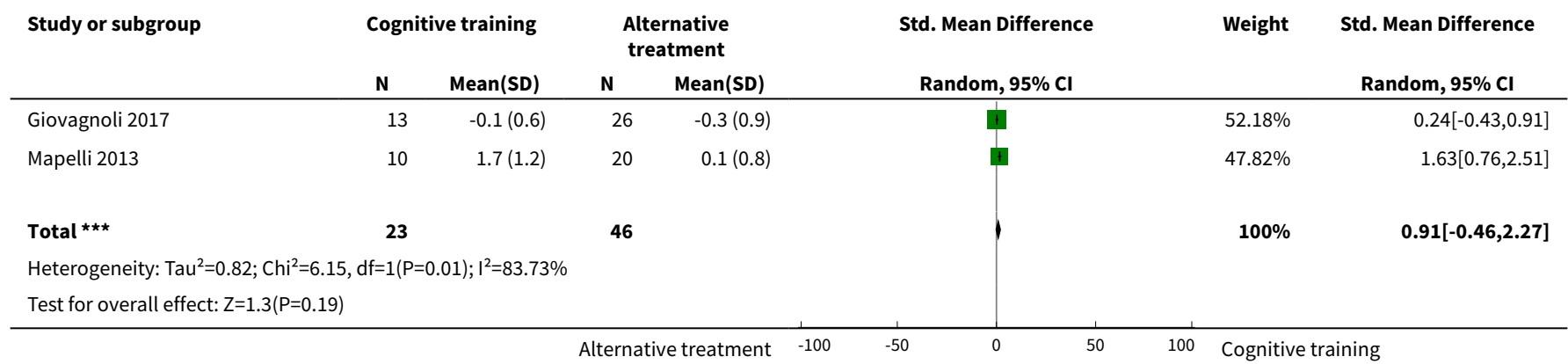

Analysis 12.8. Comparison 12 Cognitive training vs alternative treatment immediately post intervention - type of CT (traditional vs augmented), Outcome 8 Change in language (naming).

\begin{tabular}{|c|c|c|c|c|c|c|c|c|}
\hline \multirow[t]{2}{*}{ Study or subgroup } & \multicolumn{2}{|c|}{ Cognitive training } & \multicolumn{2}{|c|}{$\begin{array}{l}\text { Alternative } \\
\text { treatment }\end{array}$} & \multirow{2}{*}{\multicolumn{2}{|c|}{$\begin{array}{c}\text { Std. Mean Difference } \\
\text { Random, } 95 \% \mathrm{Cl} \\
\end{array}$}} & \multirow{2}{*}{ Weight } & \multirow{2}{*}{$\begin{array}{l}\text { Std. Mean Difference } \\
\text { Random, } 95 \% \mathrm{Cl} \\
\end{array}$} \\
\hline & $\mathbf{N}$ & $\operatorname{Mean}(S D)$ & $\mathbf{N}$ & Mean(SD) & & & & \\
\hline Brueggen 2017 & 8 & $-0.9(1.2)$ & 8 & $-0.1(1)$ & & $t$ & $100 \%$ & $-0.63[-1.65,0.38]$ \\
\hline 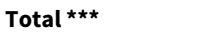 & 8 & & 8 & & & 1 & $100 \%$ & $-0.63[-1.65,0.38]$ \\
\hline \multicolumn{9}{|c|}{ Heterogeneity: $\operatorname{Tau}^{2}=0 ; \mathrm{Chi}^{2}=0, \mathrm{df}=0(\mathrm{P}<0.0001) ; \mathrm{I}^{2}=100 \%$} \\
\hline
\end{tabular}


Analysis 12.9. Comparison 12 Cognitive training vs alternative treatment immediately post intervention - type of CT (traditional vs augmented), Outcome 9 Change in verbal letter fluency.

\begin{tabular}{|c|c|c|c|c|c|c|c|}
\hline \multirow[t]{2}{*}{ Study or subgroup } & \multicolumn{2}{|c|}{ Cognitive training } & \multicolumn{2}{|c|}{$\begin{array}{c}\text { Alternative } \\
\text { treatment }\end{array}$} & \multirow{2}{*}{$\begin{array}{l}\text { Std. Mean Difference } \\
\text { Random, } 95 \% \mathrm{Cl}\end{array}$} & \multirow[t]{2}{*}{ Weight } & \multirow{2}{*}{$\begin{array}{l}\text { Std. Mean Difference } \\
\text { Random, } 95 \% \mathrm{Cl} \\
\end{array}$} \\
\hline & $\mathbf{N}$ & Mean(SD) & $\mathbf{N}$ & Mean(SD) & & & \\
\hline Brueggen 2017 & 8 & $-3.4(2.9)$ & 8 & $-1.6(3.5)$ & 电 & $28.33 \%$ & $-0.52[-1.52,0.48]$ \\
\hline Mapelli 2013 & 10 & $1.2(1.5)$ & 10 & $0.2(1.3)$ & 甲 & $31.52 \%$ & $0.66[-0.25,1.56]$ \\
\hline Giovagnoli 2017 & 13 & $4(4.7)$ & 26 & $-0.6(7.3)$ & 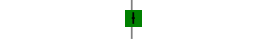 & $40.15 \%$ & $0.69[0,1.37]$ \\
\hline 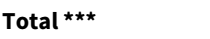 & 31 & & 44 & & & $100 \%$ & $0.34[-0.38,1.05]$ \\
\hline \multicolumn{8}{|c|}{ Heterogeneity: Tau $^{2}=0.21 ; \mathrm{Chi}^{2}=4.22, \mathrm{df}=2(\mathrm{P}=0.12) ; \mathrm{I}^{2}=52.55 \%$} \\
\hline \multicolumn{3}{|c|}{ Test for overall effect: $\mathrm{Z}=0.92(\mathrm{P}=0.36)$} & & & & & \\
\hline
\end{tabular}

Analysis 12.10. Comparison 12 Cognitive training vs alternative treatment immediately post intervention - type of $\mathrm{CT}$ (traditional vs augmented), Outcome 10 Change in verbal category fluency.

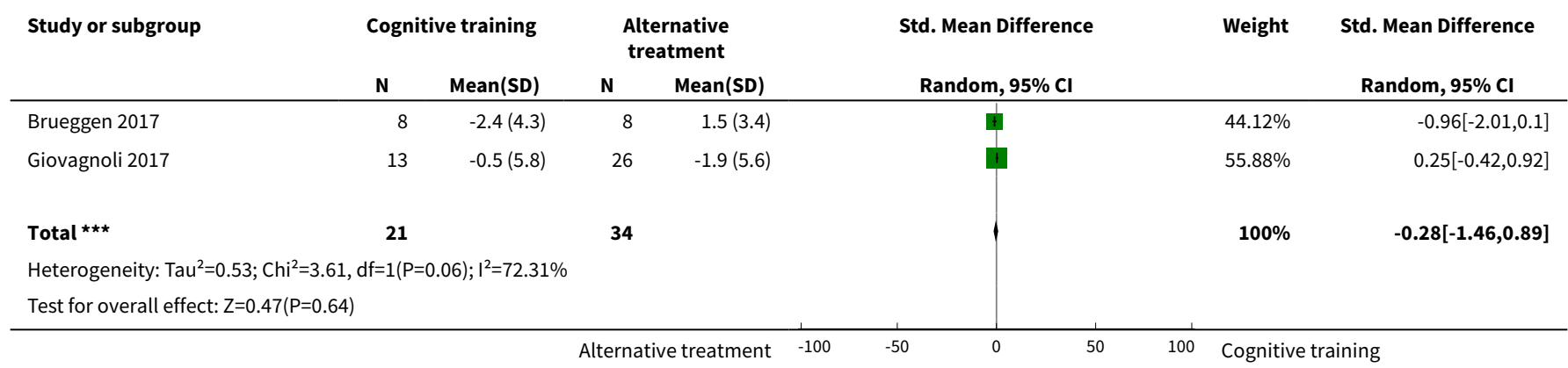

Analysis 12.11. Comparison 12 Cognitive training vs alternative treatment immediately post intervention - type of CT (traditional vs augmented), Outcome 11 Change in executive function.

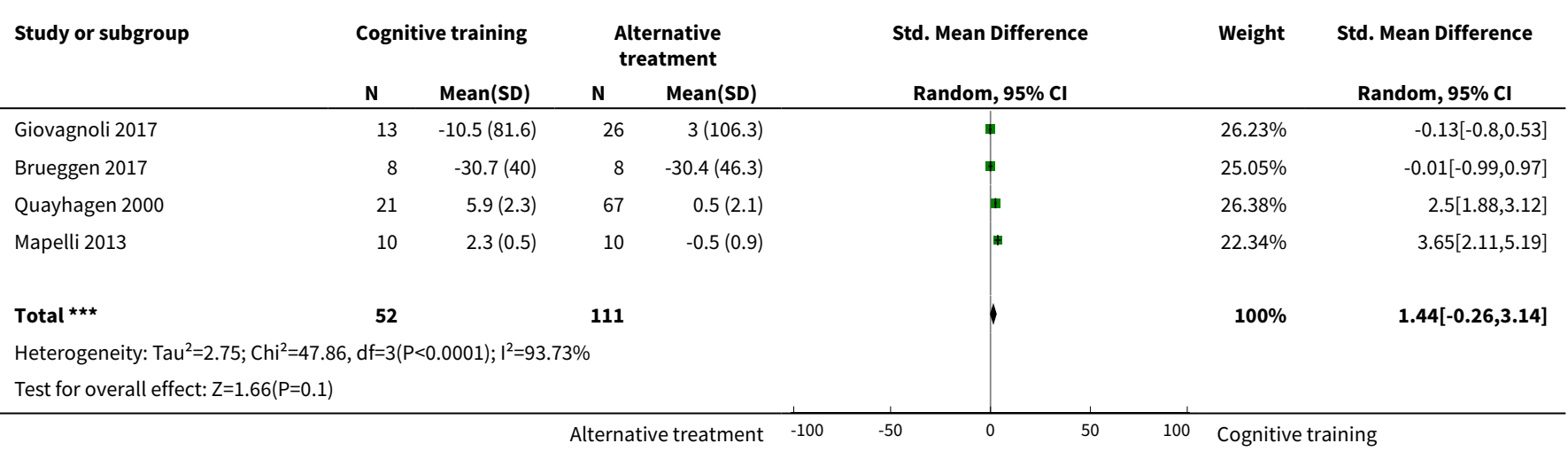


Analysis 12.14. Comparison 12 Cognitive training vs alternative treatment immediately post intervention - type of CT (traditional vs augmented), Outcome 14 Change in participants' mood.

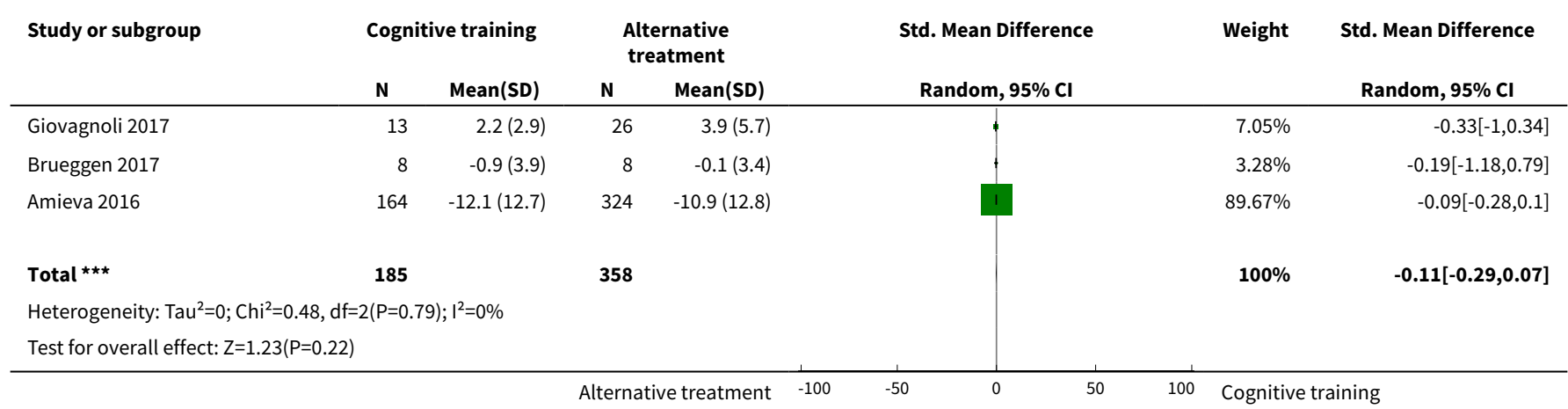

Analysis 12.15. Comparison 12 Cognitive training vs alternative treatment immediately post intervention - type of CT (traditional vs augmented), Outcome 15 Change in capacity for activities of daily living.

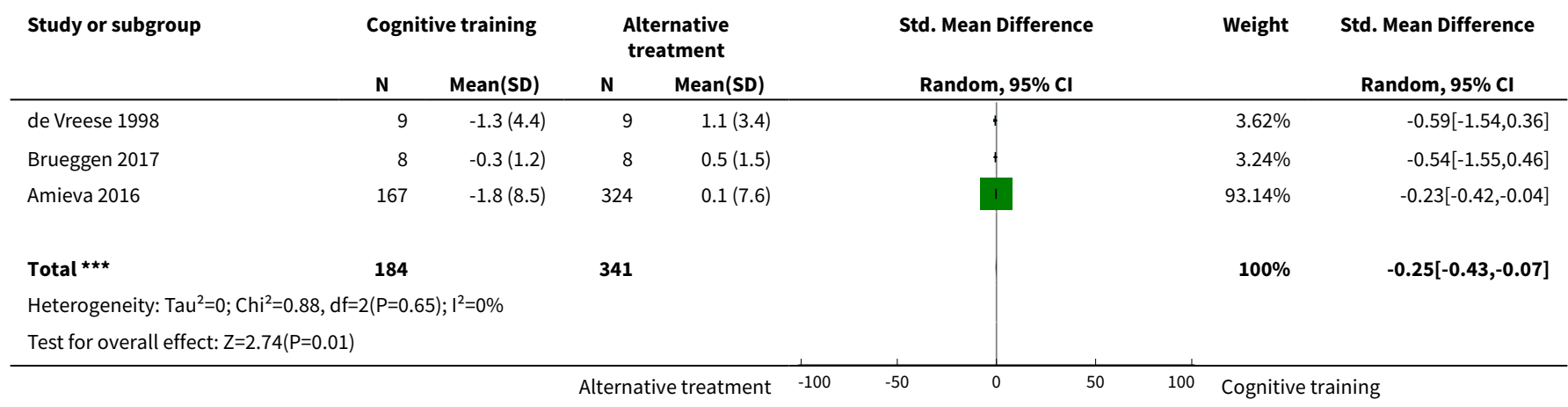

Analysis 12.16. Comparison 12 Cognitive training vs alternative treatment immediately post intervention - type of CT (traditional vs augmented), Outcome 16 Change in behavioural and psychological symptoms of dementia (BPSD).

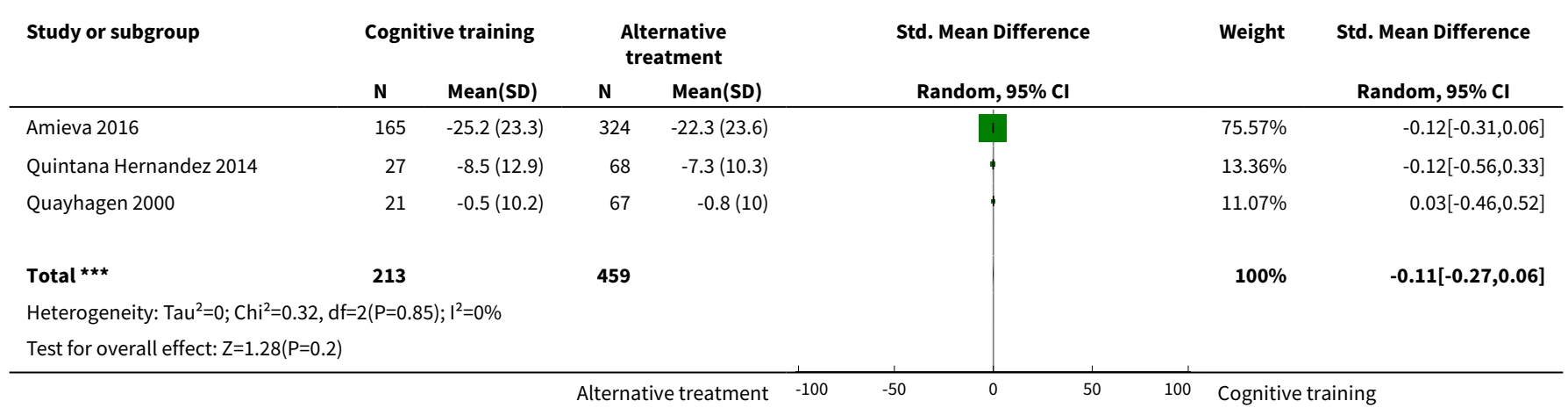


Analysis 12.17. Comparison 12 Cognitive training vs alternative treatment immediately post intervention - type of CT (traditional vs augmented), Outcome 17 Change in disease progression.

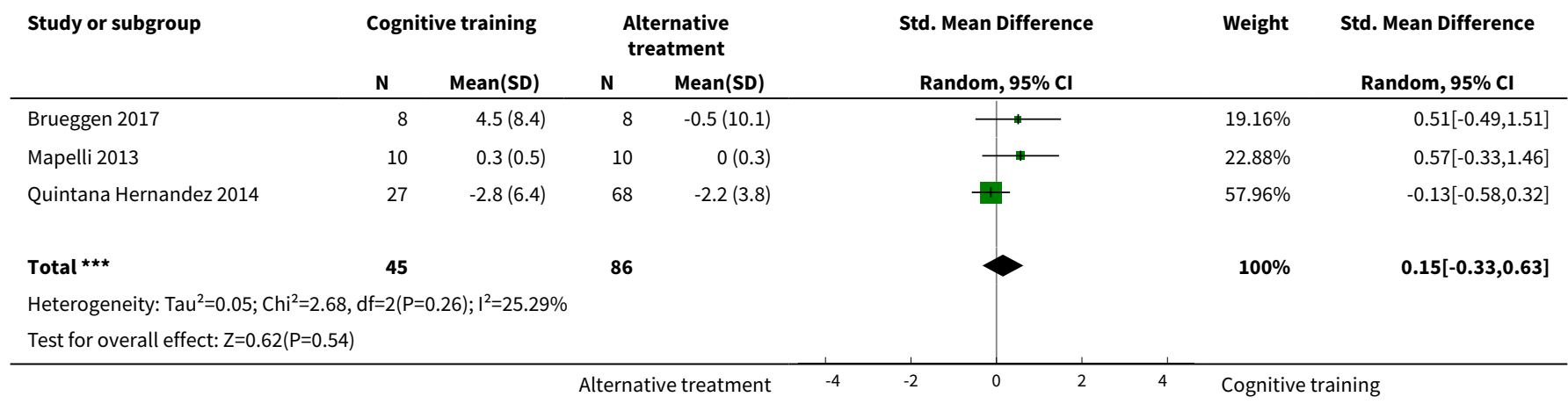

Analysis 12.18. Comparison 12 Cognitive training vs alternative treatment immediately post intervention - type of CT (traditional vs augmented), Outcome 18 Participant burden (retention rates).

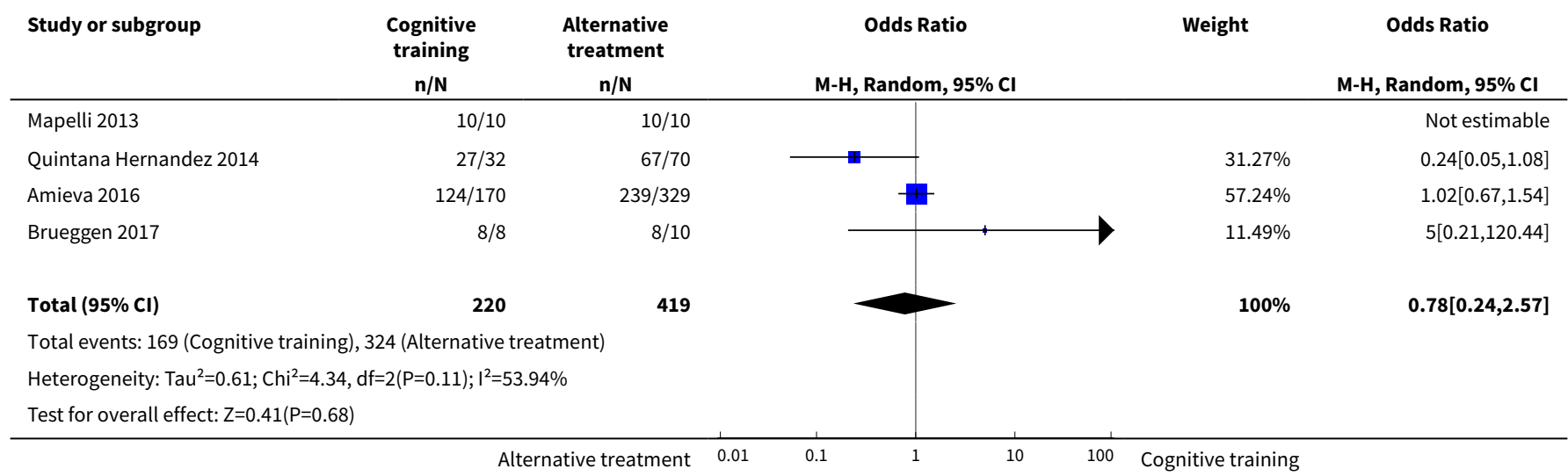
Analysis 12.19. Comparison 12 Cognitive training vs alternative treatment immediately post intervention
- type of CT (traditional vs augmented), Outcome 19 Change in mood and well-being (CAREGIVER).

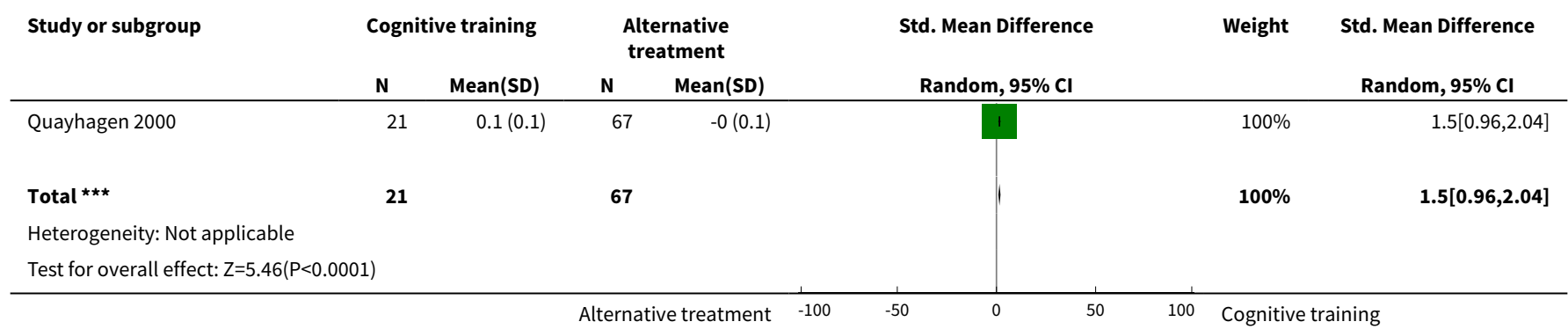


Analysis 12.20. Comparison 12 Cognitive training vs alternative treatment immediately post intervention - type of CT (traditional vs augmented), Outcome 20 Change in burden of care (CAREGIVER).

\begin{tabular}{|c|c|c|c|c|c|c|c|}
\hline \multirow[t]{2}{*}{ Study or subgroup } & \multicolumn{2}{|c|}{ Cognitive training } & \multicolumn{2}{|c|}{$\begin{array}{l}\text { Alternative } \\
\text { treatment }\end{array}$} & \multirow{2}{*}{$\begin{array}{l}\text { Std. Mean Difference } \\
\text { Random, } 95 \% \mathrm{Cl}\end{array}$} & \multirow[t]{2}{*}{ Weight } & \multirow{2}{*}{$\begin{array}{l}\text { Std. Mean Difference } \\
\text { Random, } 95 \% \mathrm{Cl} \\
\end{array}$} \\
\hline & $\mathbf{N}$ & $\operatorname{Mean}(\mathrm{SD})$ & $\mathbf{N}$ & Mean(SD) & & & \\
\hline Amieva 2016 & 165 & $-21.3(19.1)$ & 322 & $-15.2(20.8)$ & 1 & $62.73 \%$ & $-0.3[-0.49,-0.11]$ \\
\hline Quayhagen 2000 & 21 & $-0.8(4.1)$ & 67 & $-0.6(4.4)$ & 申 & $28.08 \%$ & $-0.04[-0.53,0.45]$ \\
\hline Brueggen 2017 & 8 & $-0.7(9.2)$ & 8 & $-5.2(7.4)$ & 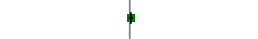 & $9.19 \%$ & $0.51[-0.49,1.51]$ \\
\hline 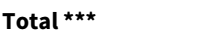 & 194 & & 397 & & & $100 \%$ & $-0.15[-0.47,0.17]$ \\
\hline \multicolumn{8}{|c|}{ Heterogeneity: $\mathrm{Tau}^{2}=0.03 ; \mathrm{Chi}^{2}=3.17, \mathrm{df}=2(\mathrm{P}=0.21) ; \mathrm{I}^{2}=36.89 \%$} \\
\hline \multicolumn{3}{|c|}{ Test for overall effect: $Z=0.92(P=0.35)$} & & & & & \\
\hline
\end{tabular}

Analysis 12.21. Comparison 12 Cognitive training vs alternative treatment immediately post intervention - type of CT (traditional vs augmented), Outcome 21 Change in quality of life (CAREGIVER).

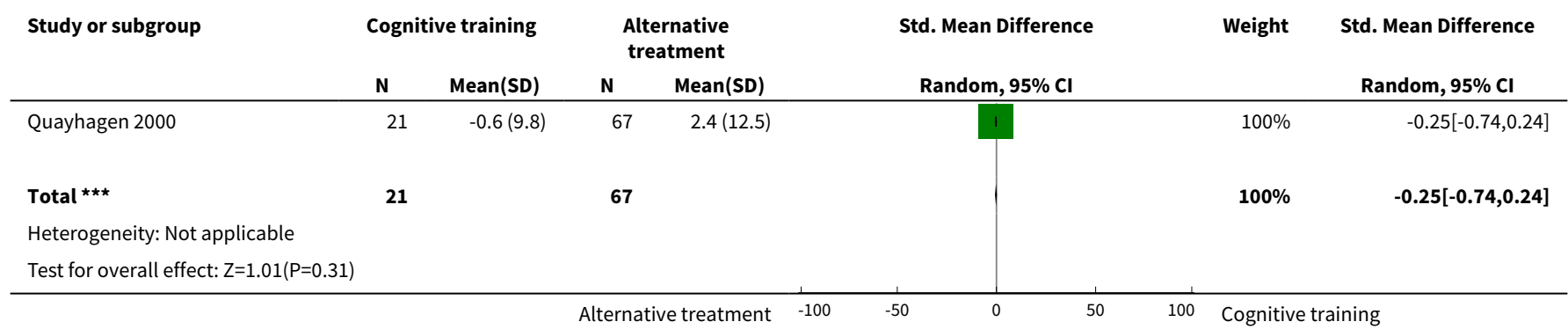

Analysis 12.22. Comparison 12 Cognitive training vs alternative treatment immediately post intervention - type of CT (traditional vs augmented), Outcome 22 Change in speed of information processing.

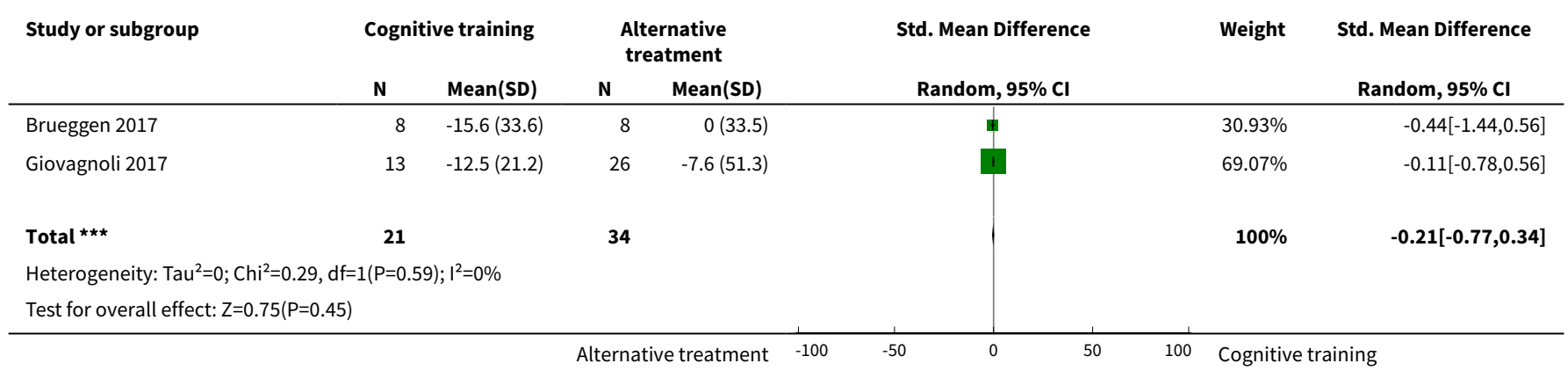


Analysis 12.23. Comparison 12 Cognitive training vs alternative treatment immediately post intervention - type of CT (traditional vs augmented), Outcome 23 Change in general health and quality of life.

\begin{tabular}{|c|c|c|c|c|c|c|c|}
\hline \multirow[t]{2}{*}{ Study or subgroup } & \multicolumn{2}{|c|}{ Cognitive training } & \multicolumn{2}{|c|}{$\begin{array}{c}\text { Alternative } \\
\text { treatment }\end{array}$} & \multirow{2}{*}{$\begin{array}{c}\text { Std. Mean Difference } \\
\text { Random, } 95 \% \mathrm{CI} \\
\end{array}$} & \multirow[t]{2}{*}{ Weight } & \multirow{2}{*}{$\begin{array}{l}\text { Std. Mean Difference } \\
\text { Random, } 95 \% \mathrm{CI} \\
\end{array}$} \\
\hline & $\mathbf{N}$ & $\operatorname{Mean}(\mathrm{SD})$ & $\mathbf{N}$ & Mean(SD) & & & \\
\hline Brueggen 2017 & 8 & $-4.4(4.6)$ & 8 & $3.1(4.8)$ & 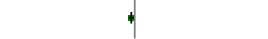 & $12.91 \%$ & $-1.52[-2.67,-0.36]$ \\
\hline Giovagnoli 2017 & 13 & $-0.1(12.7)$ & 26 & $8.8(6)$ & 米 & $22.11 \%$ & $-0.99[-1.69,-0.28]$ \\
\hline Quayhagen 2000 & 21 & $-0.4(2.8)$ & 67 & $0.1(3.2)$ & 4 & $28.35 \%$ & $-0.14[-0.63,0.35]$ \\
\hline Amieva 2016 & 164 & $-6.3(6.1)$ & 324 & $-5.7(6.3)$ & 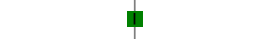 & $36.64 \%$ & $-0.1[-0.29,0.09]$ \\
\hline 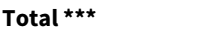 & 206 & & 425 & & & $100 \%$ & $-0.49[-1,0.02]$ \\
\hline \multicolumn{8}{|c|}{ Heterogeneity: $\operatorname{Tau}^{2}=0.17 ; \mathrm{Chi}^{2}=10.91, \mathrm{df}=3(\mathrm{P}=0.01) ; \mathrm{I}^{2}=72.51 \%$} \\
\hline \multicolumn{3}{|c|}{ Test for overall effect: $Z=1.9(P=0.06)$} & & & & & \\
\hline
\end{tabular}

Comparison 13. Cognitive training vs control in the medium term ( 3 to 12 months post intervention) - follow-up period

\begin{tabular}{|c|c|c|c|c|}
\hline Outcome or subgroup title & $\begin{array}{l}\text { No. of } \\
\text { studies }\end{array}$ & $\begin{array}{l}\text { No. of } \\
\text { partici- } \\
\text { pants }\end{array}$ & Statistical method & Effect size \\
\hline 1 Change in a global measure of cognition & 6 & 387 & $\begin{array}{l}\text { Std. Mean Difference (IV, Ran- } \\
\text { dom, } 95 \% \mathrm{CI} \text { ) }\end{array}$ & $1.33[0.31,2.34]$ \\
\hline $\begin{array}{l}2 \text { Change in a global measure of cognition (zero } \\
\text { correlation) }\end{array}$ & 6 & 387 & $\begin{array}{l}\text { Std. Mean Difference (IV, Ran- } \\
\text { dom, } 95 \% \mathrm{CI} \text { ) }\end{array}$ & $0.68[0.06,1.30]$ \\
\hline $\begin{array}{l}3 \text { Change in a global measure of cognition (com- } \\
\text { posite) }\end{array}$ & 7 & & $\begin{array}{l}\text { Std. Mean Difference (Ran- } \\
\text { dom, } 95 \% \mathrm{Cl} \text { ) }\end{array}$ & $0.65[0.11,1.20]$ \\
\hline 3.1 Up to 3 months & 3 & & $\begin{array}{l}\text { Std. Mean Difference (Ran- } \\
\text { dom, } 95 \% \mathrm{Cl} \text { ) }\end{array}$ & $-0.05[-0.54,0.43]$ \\
\hline 3.2 More than 3 months & 4 & & $\begin{array}{l}\text { Std. Mean Difference (Ran- } \\
\text { dom, } 95 \% \mathrm{Cl} \text { ) }\end{array}$ & $1.03[0.33,1.72]$ \\
\hline $\begin{array}{l}4 \text { Change in a global measure of cognition (com- } \\
\text { posite)_zero correlation }\end{array}$ & 7 & & $\begin{array}{l}\text { Std. Mean Difference (Ran- } \\
\text { dom, } 95 \% \mathrm{Cl} \text { ) }\end{array}$ & $0.40[0.09,0.71]$ \\
\hline 4.1 Up to 3 months & 3 & & $\begin{array}{l}\text { Std. Mean Difference (Ran- } \\
\text { dom, } 95 \% \mathrm{Cl} \text { ) }\end{array}$ & $-0.06[-0.54,0.42]$ \\
\hline 4.2 More than 4 months & 4 & & $\begin{array}{l}\text { Std. Mean Difference (Ran- } \\
\text { dom, } 95 \% \mathrm{Cl} \text { ) }\end{array}$ & $0.57[0.21,0.93]$ \\
\hline $\begin{array}{l}5 \text { Change in disease progression (zero correla- } \\
\text { tion) }\end{array}$ & 2 & 98 & $\begin{array}{l}\text { Std. Mean Difference (IV, Ran- } \\
\text { dom, } 95 \% \mathrm{Cl} \text { ) }\end{array}$ & $0.28[-0.14,0.71]$ \\
\hline 6 Change in disease progression & 2 & 98 & $\begin{array}{l}\text { Std. Mean Difference (IV, Ran- } \\
\text { dom, } 95 \% \mathrm{CI} \text { ) }\end{array}$ & $0.55[0.12,0.98]$ \\
\hline 7 Change in immediate memory & 7 & 383 & $\begin{array}{l}\text { Std. Mean Difference (IV, Ran- } \\
\text { dom, } 95 \% \mathrm{CI} \text { ) }\end{array}$ & $0.62[0.00,1.24]$ \\
\hline
\end{tabular}




\begin{tabular}{|c|c|c|c|c|}
\hline Outcome or subgroup title & $\begin{array}{l}\text { No. of } \\
\text { studies }\end{array}$ & $\begin{array}{l}\text { No. of } \\
\text { partici- } \\
\text { pants }\end{array}$ & Statistical method & Effect size \\
\hline 7.1 Up to 3 months & 3 & 64 & $\begin{array}{l}\text { Std. Mean Difference (IV, Ran- } \\
\text { dom, } 95 \% \mathrm{CI} \text { ) }\end{array}$ & $-0.34[-1.12,0.43]$ \\
\hline 7.2 More than 3 months & 4 & 319 & $\begin{array}{l}\text { Std. Mean Difference (IV, Ran- } \\
\text { dom, } 95 \% \mathrm{CI} \text { ) }\end{array}$ & $1.14[0.53,1.74]$ \\
\hline 8 Change in delayed memory & 4 & 270 & $\begin{array}{l}\text { Std. Mean Difference (IV, Ran- } \\
\text { dom, } 95 \% \mathrm{CI} \text { ) }\end{array}$ & $0.97[0.02,1.92]$ \\
\hline 9 Change in language (naming) & 4 & 274 & $\begin{array}{l}\text { Std. Mean Difference (IV, Ran- } \\
\text { dom, } 95 \% \mathrm{CI} \text { ) }\end{array}$ & $-0.87[-3.81,2.08]$ \\
\hline 10 Change in verbal letter fluency & 4 & 247 & $\begin{array}{l}\text { Std. Mean Difference (IV, Ran- } \\
\text { dom, } 95 \% \mathrm{CI} \text { ) }\end{array}$ & $0.47[-0.28,1.23]$ \\
\hline 11 Change in verbal category fluency & 3 & 213 & $\begin{array}{l}\text { Std. Mean Difference (IV, Ran- } \\
\text { dom, } 95 \% \mathrm{CI} \text { ) }\end{array}$ & $0.78[0.38,1.18]$ \\
\hline 12 Change in attention and working memory & 3 & 215 & $\begin{array}{l}\text { Std. Mean Difference (IV, Ran- } \\
\text { dom, } 95 \% \mathrm{CI} \text { ) }\end{array}$ & $0.50[-0.43,1.43]$ \\
\hline 13 Change in speed of information processing & 2 & 45 & $\begin{array}{l}\text { Std. Mean Difference (IV, Ran- } \\
\text { dom, } 95 \% \mathrm{CI} \text { ) }\end{array}$ & $0.30[-0.44,1.04]$ \\
\hline 14 Change in meta cognition (self-reported) & 1 & 19 & $\begin{array}{l}\text { Std. Mean Difference (IV, Ran- } \\
\text { dom, } 95 \% \mathrm{CI} \text { ) }\end{array}$ & $0.99[-0.01,1.99]$ \\
\hline $\begin{array}{l}15 \text { Change in meta cognition (informant-report- } \\
\text { ed) }\end{array}$ & 1 & 34 & $\begin{array}{l}\text { Std. Mean Difference (IV, Ran- } \\
\text { dom, } 95 \% \mathrm{Cl} \text { ) }\end{array}$ & $-0.06[-0.73,0.62]$ \\
\hline 16 Change in capacity for activities of daily living & 3 & 64 & $\begin{array}{l}\text { Std. Mean Difference (IV, Ran- } \\
\text { dom, } 95 \% \mathrm{CI} \text { ) }\end{array}$ & $0.22[-0.50,0.94]$ \\
\hline $\begin{array}{l}17 \text { Change in behavioural and psychological } \\
\text { symptoms of dementia (BPSD) }\end{array}$ & 1 & 11 & $\begin{array}{l}\text { Std. Mean Difference (IV, Ran- } \\
\text { dom, } 95 \% \mathrm{Cl} \text { ) }\end{array}$ & $-1.34[-2.75,0.07]$ \\
\hline 18 Change in general health and quality of life & 1 & 117 & $\begin{array}{l}\text { Std. Mean Difference (IV, Ran- } \\
\text { dom, } 95 \% \mathrm{Cl} \text { ) }\end{array}$ & $-0.02[-0.39,0.35]$ \\
\hline 19 Change in participants' mood & 2 & 30 & $\begin{array}{l}\text { Std. Mean Difference (IV, Ran- } \\
\text { dom, } 95 \% \mathrm{CI} \text { ) }\end{array}$ & $0.21[-0.54,0.96]$ \\
\hline 20 Change in mood and well-being (CAREGIVER) & 0 & 0 & $\begin{array}{l}\text { Std. Mean Difference (IV, Ran- } \\
\text { dom, } 95 \% \mathrm{CI} \text { ) }\end{array}$ & $0.0[0.0,0.0]$ \\
\hline 21 Change in burden of care (CAREGIVER) & 0 & 0 & $\begin{array}{l}\text { Std. Mean Difference (IV, Ran- } \\
\text { dom, } 95 \% \mathrm{CI} \text { ) }\end{array}$ & $0.0[0.0,0.0]$ \\
\hline 22 Change in quality of life (CAREGIVER) & 0 & 0 & $\begin{array}{l}\text { Std. Mean Difference (IV, Ran- } \\
\text { dom, } 95 \% \mathrm{CI} \text { ) }\end{array}$ & $0.0[0.0,0.0]$ \\
\hline 23 Change in executive function & 5 & 330 & $\begin{array}{l}\text { Std. Mean Difference (IV, Ran- } \\
\text { dom, } 95 \% \mathrm{CI} \text { ) }\end{array}$ & $0.56[0.02,1.10]$ \\
\hline
\end{tabular}


Analysis 13.1. Comparison 13 Cognitive training vs control in the medium term ( 3 to 12 months post intervention) - follow-up period, Outcome 1 Change in a global measure of cognition.

\begin{tabular}{|c|c|c|c|c|c|c|c|c|}
\hline \multirow[t]{2}{*}{ Study or subgroup } & \multicolumn{2}{|c|}{ Cognitive training } & \multicolumn{2}{|c|}{ Control } & \multirow{2}{*}{\multicolumn{2}{|c|}{$\begin{array}{c}\text { Std. Mean Difference } \\
\text { Random, } 95 \% \mathrm{Cl}\end{array}$}} & \multirow[t]{2}{*}{ Weight } & \multirow{2}{*}{$\begin{array}{c}\text { Std. Mean Difference } \\
\text { Random, } 95 \% \mathrm{Cl}\end{array}$} \\
\hline & $\mathbf{N}$ & Mean(SD) & $\mathbf{N}$ & Mean(SD) & & & & \\
\hline Lee 2013 & 12 & $0.5(2.9)$ & 7 & $1.7(2)$ & $\longrightarrow$ & - & $16.43 \%$ & $-0.45[-1.39,0.5]$ \\
\hline Kallio 2018 & 68 & $-1.3(5.4)$ & 49 & $-1.2(5.3)$ & & - & $18.69 \%$ & $-0.02[-0.39,0.35]$ \\
\hline Cavallo 2016 & 36 & $-2.2(1.1)$ & 36 & $-3.8(2.1)$ & & $\rightarrow$ & $18.35 \%$ & $0.92[0.44,1.41]$ \\
\hline Galante 2007 & 7 & $-1.5(2.3)$ & 4 & $-4.7(1.8)$ & & + & $13.95 \%$ & $1.37[-0.05,2.79]$ \\
\hline Trebbastoni 2018 & 45 & $-1.4(2.3)$ & 85 & $-5.5(2.6)$ & & $\rightarrow$ & $18.56 \%$ & $1.63[1.22,2.04]$ \\
\hline 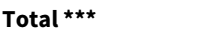 & 185 & & 202 & & & & $100 \%$ & $1.33[0.31,2.34]$ \\
\hline \multicolumn{9}{|c|}{ Heterogeneity: $\operatorname{Tau}^{2}=1.41 ; \mathrm{Chi}^{2}=80.97, \mathrm{df}=5(\mathrm{P}<0.0001) ; \mathrm{I}^{2}=93.82 \%$} \\
\hline \multicolumn{9}{|c|}{ Test for overall effect: $Z=2.55(P=0.01)$} \\
\hline
\end{tabular}

Analysis 13.2. Comparison 13 Cognitive training vs control in the medium term ( 3 to 12 months post intervention) - follow-up period, Outcome 2 Change in a global measure of cognition (zero correlation).

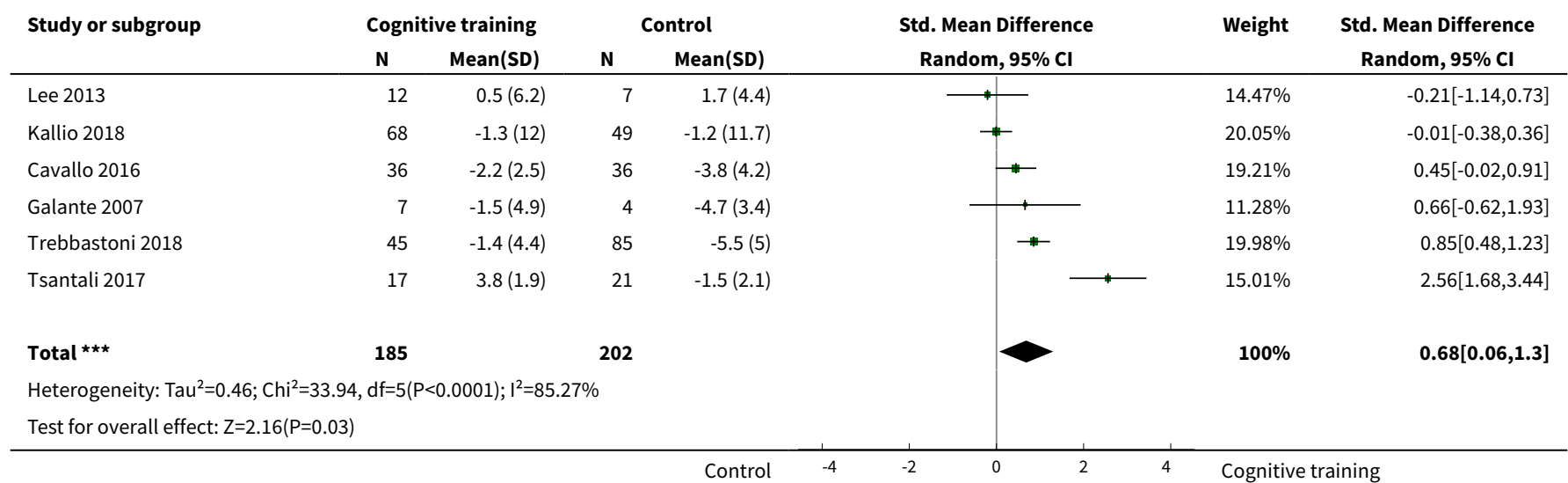

Analysis 13.3. Comparison 13 Cognitive training vs control in the medium term ( 3 to 12 months post intervention) - follow-up period, Outcome 3 Change in a global measure of cognition (composite).

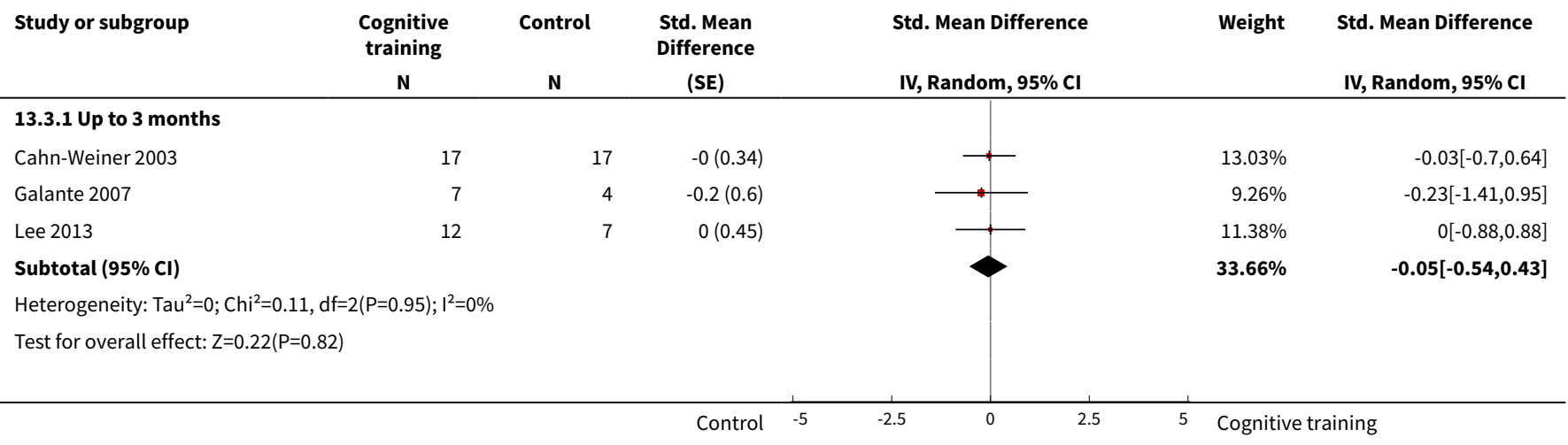




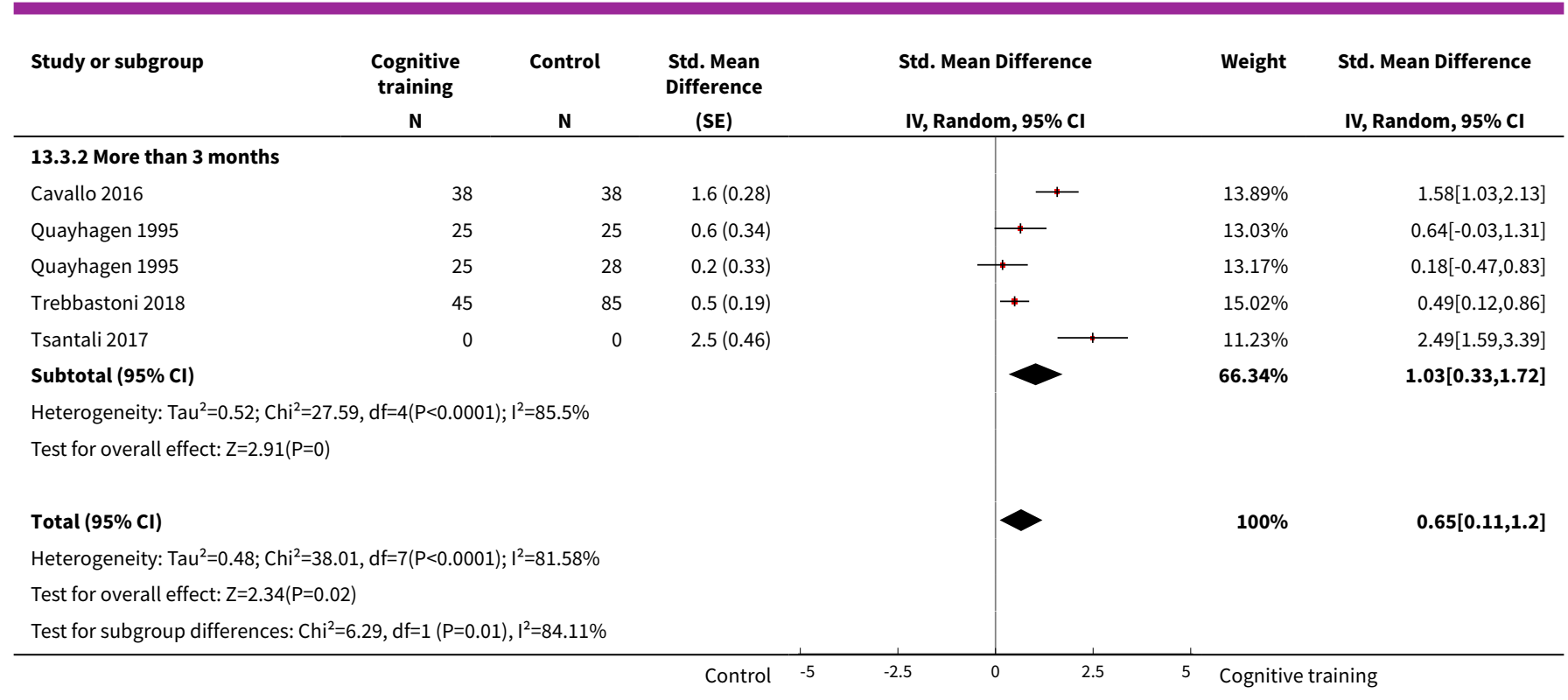

Analysis 13.4. Comparison 13 Cognitive training vs control in the medium term ( 3 to 12 months post intervention) - follow-up period, Outcome 4 Change in a global measure of cognition (composite)_zero correlation.

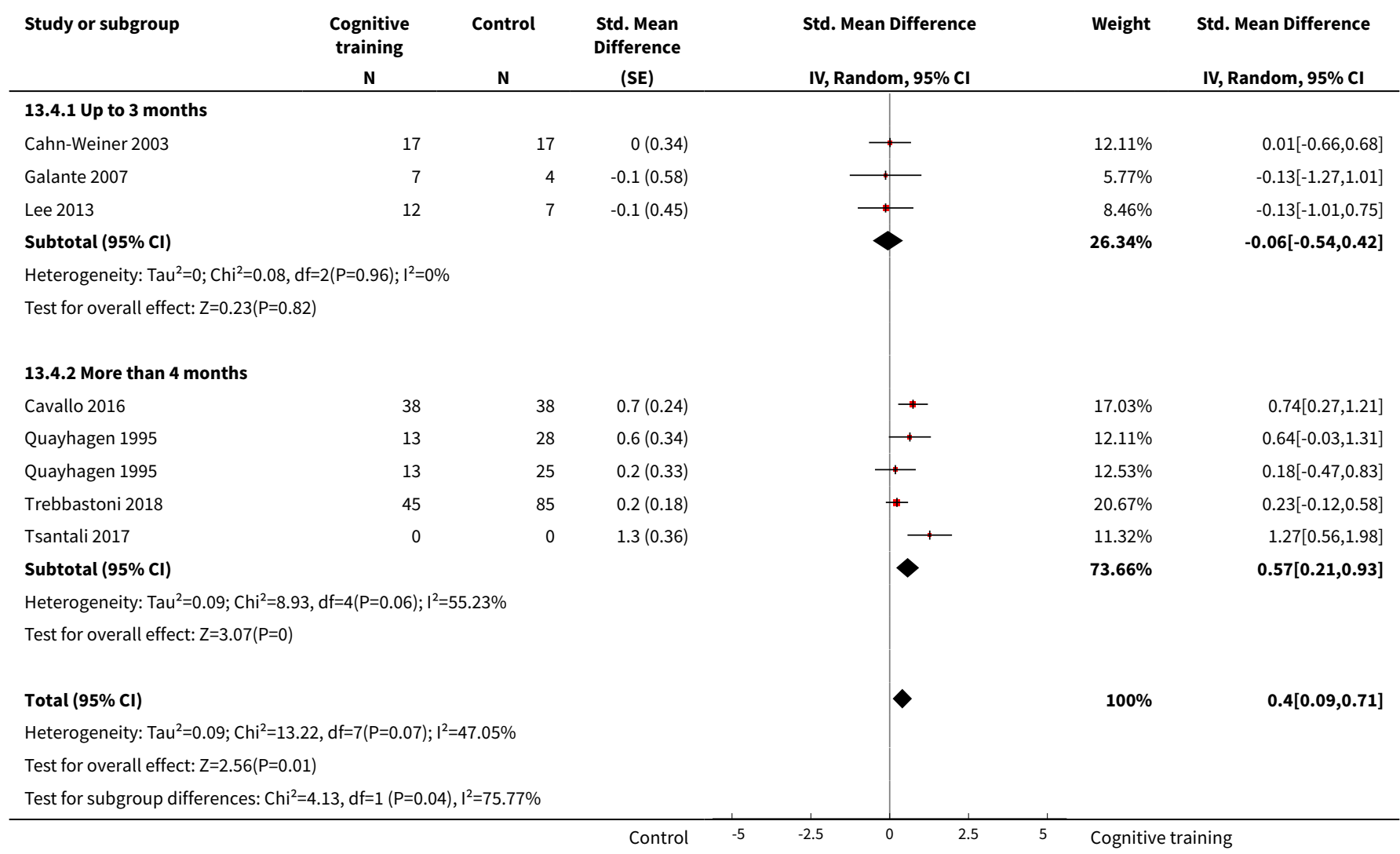


Analysis 13.5. Comparison 13 Cognitive training vs control in the medium term ( 3 to 12 months post intervention) - follow-up period, Outcome 5 Change in disease progression (zero correlation).

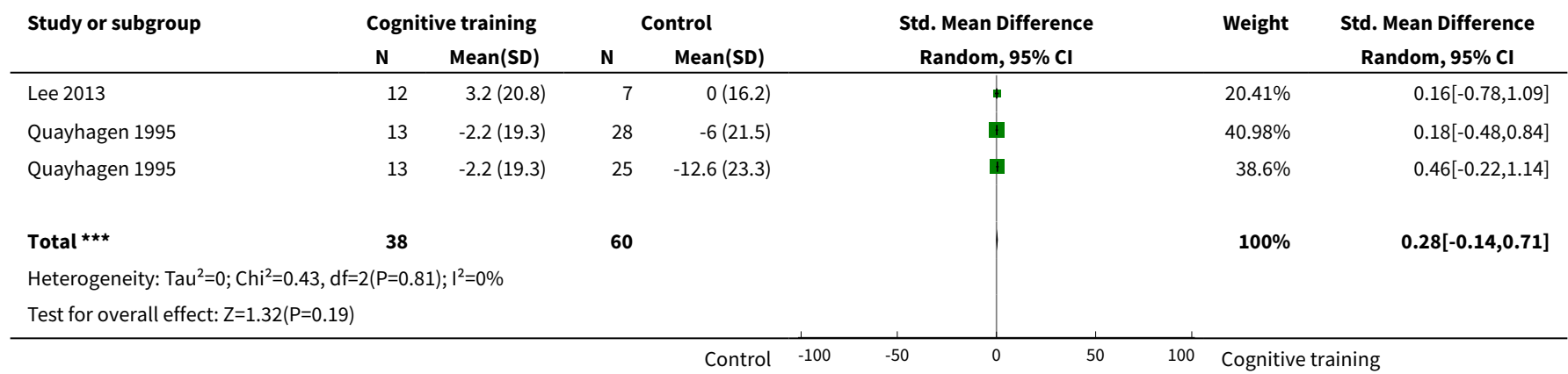

Analysis 13.6. Comparison 13 Cognitive training vs control in the medium term ( 3 to 12 months post intervention) - follow-up period, Outcome 6 Change in disease progression.

\begin{tabular}{|c|c|c|c|c|c|c|c|}
\hline \multirow[t]{2}{*}{ Study or subgroup } & \multicolumn{2}{|c|}{ Cognitive training } & \multicolumn{2}{|c|}{ Control } & \multirow{2}{*}{$\begin{array}{c}\text { Std. Mean Difference } \\
\text { Random, } 95 \% \mathrm{Cl} \\
\end{array}$} & \multirow[t]{2}{*}{ Weight } & \multirow{2}{*}{$\begin{array}{c}\text { Std. Mean Difference } \\
\text { Random, } 95 \% \mathrm{Cl}\end{array}$} \\
\hline & $\mathbf{N}$ & $\operatorname{Mean}(\mathrm{SD})$ & $\mathbf{N}$ & Mean(SD) & & & \\
\hline Lee 2013 & 12 & $3.2(9.3)$ & 7 & $0(7.3)$ & 申 & $20.78 \%$ & $0.35[-0.59,1.29]$ \\
\hline Quayhagen 1995 & 13 & $-2.2(9.1)$ & 28 & $-6(10.8)$ & + & $41.82 \%$ & $0.36[-0.3,1.02]$ \\
\hline Quayhagen 1995 & 13 & $-2.2(9.1)$ & 25 & $-12.6(12.9)$ & 1 & $37.4 \%$ & $0.87[0.16,1.57]$ \\
\hline Total $\star \star \star ~$ & 38 & & 60 & & & $100 \%$ & $0.55[0.12,0.98]$ \\
\hline \multicolumn{3}{|c|}{ Test for overall effect: $Z=2.5(P=0.01)$} & & & & & \\
\hline
\end{tabular}

Analysis 13.7. Comparison 13 Cognitive training vs control in the medium term ( 3 to 12 months post intervention) - follow-up period, Outcome 7 Change in immediate memory.

\begin{tabular}{|c|c|c|c|c|c|c|c|}
\hline \multirow[t]{2}{*}{ Study or subgroup } & \multicolumn{2}{|c|}{ Cognitive training } & \multicolumn{2}{|c|}{ Control } & \multirow{2}{*}{$\begin{array}{c}\text { Std. Mean Difference } \\
\text { Random, } 95 \% \mathrm{Cl}\end{array}$} & \multirow[t]{2}{*}{ Weight } & \multirow{2}{*}{$\begin{array}{c}\text { Std. Mean Difference } \\
\text { Random, } 95 \% \mathrm{Cl}\end{array}$} \\
\hline & $\mathbf{N}$ & $\operatorname{Mean}(S D)$ & $\mathbf{N}$ & $\operatorname{Mean}(S D)$ & & & \\
\hline \multicolumn{8}{|c|}{ 13.7.1 Up to 3 months } \\
\hline Galante 2007 & 7 & $-11(9)$ & 4 & $3.7(6.9)$ & * & $8.18 \%$ & $-1.6[-3.09,-0.12]$ \\
\hline Lee 2013 & 12 & $3.3(2.7)$ & 7 & $3.6(4.5)$ & 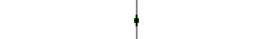 & $11.47 \%$ & $-0.09[-1.02,0.84]$ \\
\hline Cahn-Weiner 2003 & 17 & $-1.1(2.8)$ & 17 & $-1.1(2.9)$ & 1 & $13.12 \%$ & $0[-0.67,0.67]$ \\
\hline 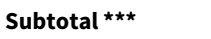 & 36 & & 28 & & r & $32.78 \%$ & $-0.34[-1.12,0.43]$ \\
\hline \multicolumn{8}{|c|}{ Test for overall effect: $Z=0.87(P=0.38)$} \\
\hline \multicolumn{8}{|c|}{ 13.7.2 More than 3 months } \\
\hline Quayhagen 1995 & 13 & $0.1(11)$ & 28 & $-2.1(8.1)$ & 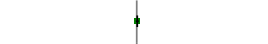 & $13.2 \%$ & $0.24[-0.42,0.9]$ \\
\hline Quayhagen 1995 & 13 & $0.1(11)$ & 25 & $-6.3(6.4)$ & , & $12.99 \%$ & $0.76[0.07,1.46]$ \\
\hline Trebbastoni 2018 & 45 & $2.8(4.6)$ & 85 & $-1.7(4.5)$ & , & $14.67 \%$ & $0.99[0.61,1.38]$ \\
\hline Cavallo 2016 & 36 & $-1(0.7)$ & 36 & $-3(1.1)$ & * & $13.63 \%$ & $2.14[1.56,2.73]$ \\
\hline 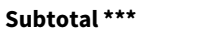 & 124 & & 195 & & 1 & $67.22 \%$ & $1.14[0.53,1.74]$ \\
\hline \multicolumn{8}{|c|}{ Heterogeneity: $\mathrm{Tau}^{2}=0.38 ; \mathrm{Chi}^{2}=21.16, \mathrm{df}=4(\mathrm{P}=0) ; \mathrm{I}^{2}=81.1 \%$} \\
\hline & & & & Control & -50 & Cognitiv & ing \\
\hline
\end{tabular}




\begin{tabular}{|c|c|c|c|c|c|c|c|c|}
\hline \multirow[t]{2}{*}{ Study or subgroup } & \multicolumn{2}{|c|}{ Cognitive training } & \multicolumn{2}{|c|}{ Control } & \multirow{2}{*}{\multicolumn{2}{|c|}{$\begin{array}{c}\text { Std. Mean Difference } \\
\text { Random, } 95 \% \mathrm{Cl}\end{array}$}} & \multirow[t]{2}{*}{ Weight } & \multirow{2}{*}{$\begin{array}{c}\text { Std. Mean Difference } \\
\text { Random, } 95 \% \mathrm{Cl}\end{array}$} \\
\hline & $\mathbf{N}$ & Mean(SD) & $\mathbf{N}$ & $\operatorname{Mean}(S D)$ & & & & \\
\hline \multicolumn{9}{|c|}{ Test for overall effect: $Z=3.67(P=0)$} \\
\hline Total $\star \star \star$ & 160 & & 223 & & & & $100 \%$ & $0.62[0,1.24]$ \\
\hline \multicolumn{9}{|c|}{ Heterogeneity: $\mathrm{Tau}^{2}=0.64 ; \mathrm{Chi}^{2}=46.37, \mathrm{df}=7(\mathrm{P}<0.0001) ; \mathrm{I}^{2}=84.91 \%$} \\
\hline \multicolumn{9}{|c|}{ Test for overall effect: $Z=1.97(P=0.05)$} \\
\hline \multicolumn{9}{|c|}{ Test for subgroup differences: $\mathrm{Chi}^{2}=8.72, \mathrm{df}=1(\mathrm{P}=0), \mathrm{I}^{2}=88.53 \%$} \\
\hline & & & & Control & -100 & -50 & Cognitive & aining \\
\hline
\end{tabular}

Analysis 13.8. Comparison 13 Cognitive training vs control in the medium term ( 3 to 12 months post intervention) - follow-up period, Outcome 8 Change in delayed memory.

\begin{tabular}{|c|c|c|c|c|c|c|c|c|}
\hline \multirow[t]{2}{*}{ Study or subgroup } & \multicolumn{2}{|c|}{ Cognitive training } & \multicolumn{2}{|c|}{ Control } & \multirow{2}{*}{\multicolumn{2}{|c|}{$\begin{array}{c}\text { Std. Mean Difference } \\
\text { Random, } 95 \% \mathrm{Cl}\end{array}$}} & \multirow[t]{2}{*}{ Weight } & \multirow{2}{*}{$\begin{array}{c}\text { Std. Mean Difference } \\
\text { Random, } 95 \% \mathrm{Cl}\end{array}$} \\
\hline & $\mathbf{N}$ & Mean(SD) & $\mathbf{N}$ & Mean(SD) & & & & \\
\hline Trebbastoni 2018 & 45 & $-0.4(1.3)$ & 85 & $-0.5(1.4)$ & & + & $26.78 \%$ & $0.06[-0.3,0.42]$ \\
\hline Cahn-Weiner 2003 & 17 & $-0.2(0.8)$ & 17 & $-0.8(1.7)$ & & $\phi$ & $24.36 \%$ & $0.45[-0.23,1.13]$ \\
\hline Tsantali 2017 & 17 & $1.8(2.5)$ & 17 & $-2.2(2.4)$ & & , & $23.42 \%$ & $1.59[0.81,2.38]$ \\
\hline Cavallo 2016 & 36 & $-2(1.1)$ & 36 & $-4(1)$ & & i & $25.44 \%$ & $1.84[1.28,2.39]$ \\
\hline \multicolumn{9}{|c|}{ Heterogeneity: Tau $^{2}=0.84 ; \mathrm{Chi}^{2}=33.73, \mathrm{df}=3(\mathrm{P}<0.0001) ; \mathrm{I}^{2}=91.1 \%$} \\
\hline \multicolumn{9}{|c|}{ Test for overall effect: $Z=1.99(P=0.05)$} \\
\hline & & & & Control & -100 & -50 & Cognitiv & ning \\
\hline
\end{tabular}

Analysis 13.9. Comparison 13 Cognitive training vs control in the medium term ( 3 to 12 months post intervention) - follow-up period, Outcome 9 Change in language (naming).

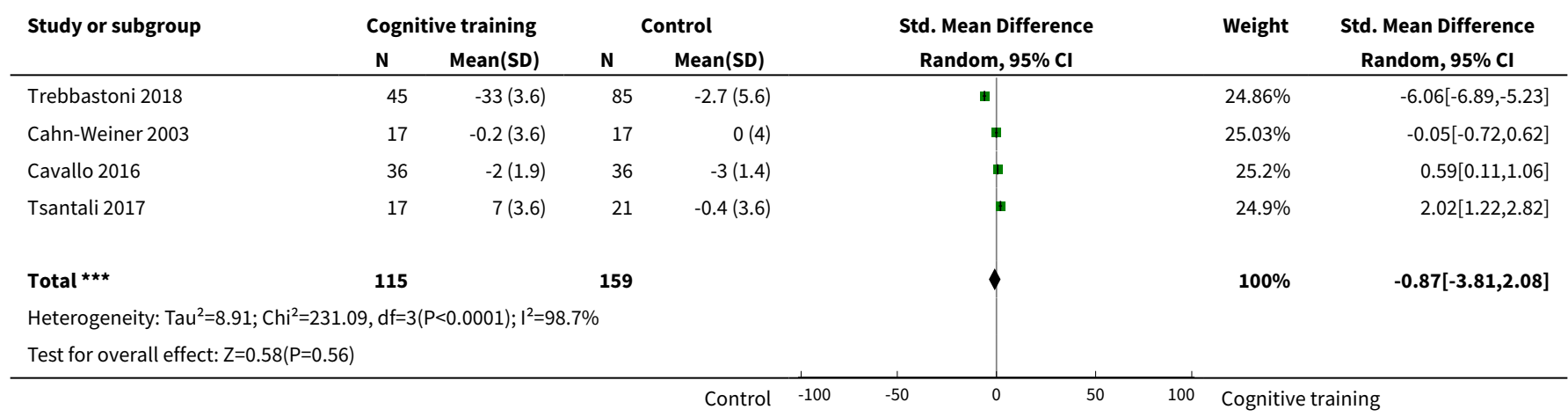

Analysis 13.10. Comparison 13 Cognitive training vs control in the medium term ( 3 to 12 months post intervention) - follow-up period, Outcome 10 Change in verbal letter fluency.

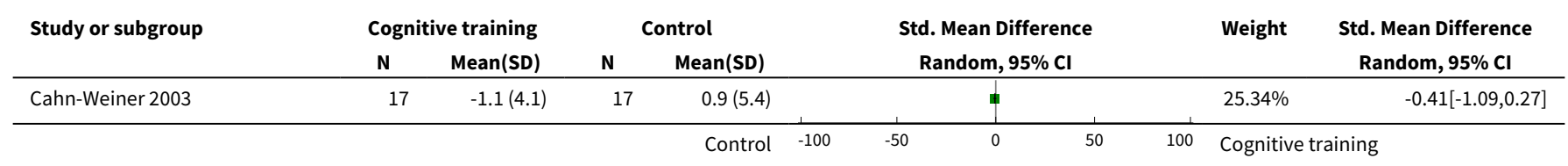




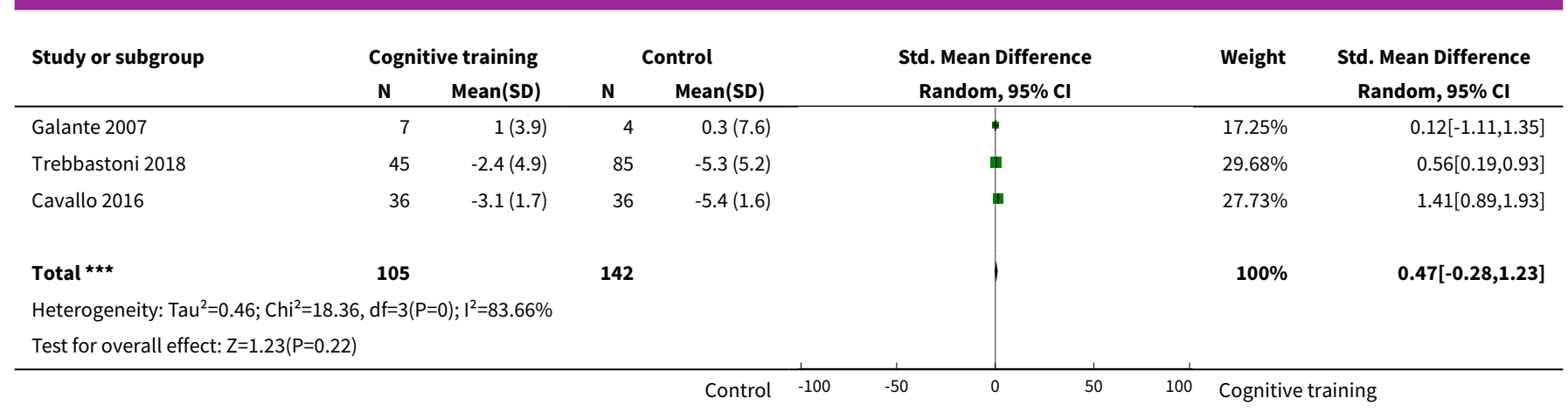

Analysis 13.11. Comparison 13 Cognitive training vs control in the medium term ( 3 to 12 months post intervention) - follow-up period, Outcome 11 Change in verbal category fluency.

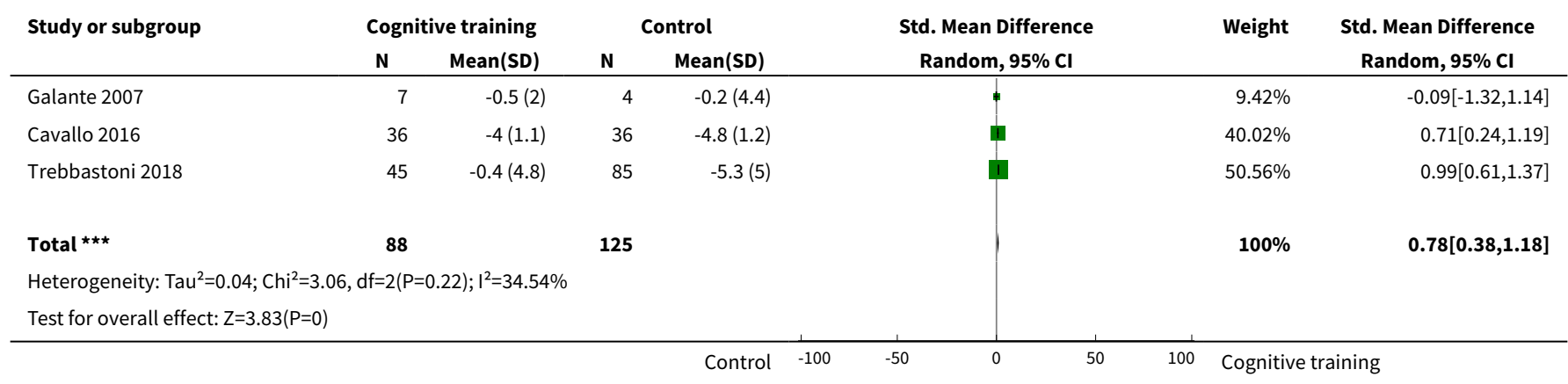

Analysis 13.12. Comparison 13 Cognitive training vs control in the medium term ( 3 to 12 months post intervention) - follow-up period, Outcome 12 Change in attention and working memory.

\begin{tabular}{|c|c|c|c|c|c|c|c|c|}
\hline \multirow[t]{2}{*}{ Study or subgroup } & \multicolumn{2}{|c|}{ Cognitive training } & \multicolumn{2}{|c|}{ Control } & & \multirow{2}{*}{$\begin{array}{c}\text { Std. Mean Difference } \\
\text { Random, } 95 \% \mathrm{Cl}\end{array}$} & \multirow[t]{2}{*}{ Weight } & \multirow{2}{*}{$\begin{array}{c}\text { Std. Mean Difference } \\
\text { Random, } 95 \% \mathrm{CI}\end{array}$} \\
\hline & $\mathbf{N}$ & $\operatorname{Mean}(S D)$ & $\mathbf{N}$ & Mean(SD) & & & & \\
\hline Galante 2007 & 7 & $-0.3(0.7)$ & 4 & $1(1.6)$ & & 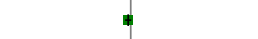 & $22.32 \%$ & $-1.12[-2.48,0.24]$ \\
\hline Trebbastoni 2018 & 45 & $-0.4(0.5)$ & 85 & $-0.7(0.7)$ & & 1 & $39.92 \%$ & $0.58[0.21,0.95]$ \\
\hline Cavallo 2016 & 36 & $-0.1(1)$ & 38 & $-1.5(1.1)$ & & $\Pi$ & $37.76 \%$ & $1.38[0.87,1.89]$ \\
\hline 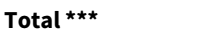 & 88 & & 127 & & & 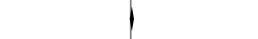 & $100 \%$ & $0.5[-0.43,1.43]$ \\
\hline \multicolumn{9}{|c|}{ Test for overall effect: $Z=1.05(P=0.29)$} \\
\hline & & & & Control & -100 & -50 & Cognitiv & ning \\
\hline
\end{tabular}

Analysis 13.13. Comparison 13 Cognitive training vs control in the medium term ( 3 to 12 months post intervention) - follow-up period, Outcome 13 Change in speed of information processing.

\begin{tabular}{|c|c|c|c|c|c|c|c|c|c|c|}
\hline \multirow[t]{2}{*}{ Study or subgroup } & \multicolumn{2}{|c|}{ Cognitive training } & \multicolumn{2}{|c|}{ Control } & \multirow{2}{*}{\multicolumn{3}{|c|}{$\begin{array}{c}\text { Std. Mean Difference } \\
\text { Random, } 95 \% \mathrm{Cl}\end{array}$}} & & \multirow[t]{2}{*}{ Weight } & \multirow{2}{*}{$\begin{array}{c}\text { Std. Mean Difference } \\
\text { Random, } 95 \% \mathrm{CI}\end{array}$} \\
\hline & $\mathbf{N}$ & $\operatorname{Mean}(S D)$ & $\mathbf{N}$ & Mean(SD) & & & & & & \\
\hline Galante 2007 & 7 & $0(8.9)$ & 4 & $2.5(6.7)$ & & & & & $29.86 \%$ & $-0.28[-1.51,0.96]$ \\
\hline Cahn-Weiner 2003 & 17 & $11.7(21.1)$ & 17 & $-1.4(25.3)$ & & & & & $70.14 \%$ & $0.55[-0.14,1.24]$ \\
\hline
\end{tabular}




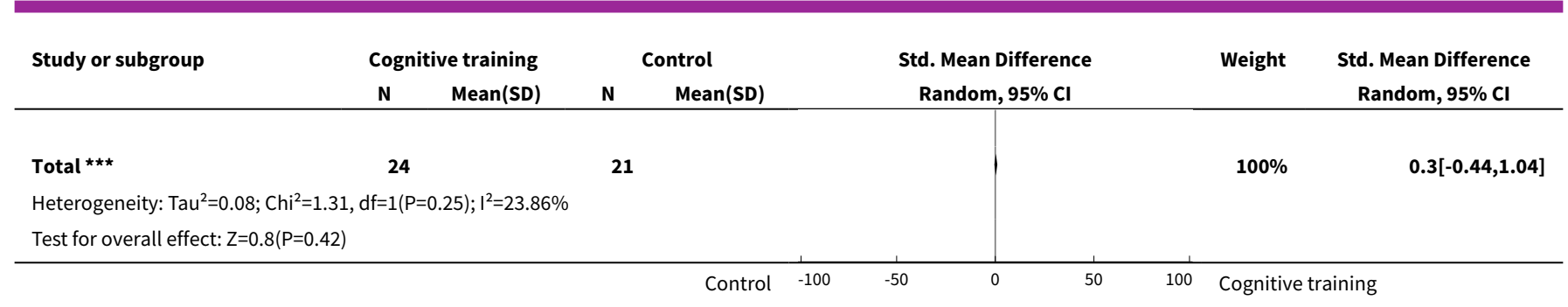

Analysis 13.14. Comparison 13 Cognitive training vs control in the medium term ( 3 to 12 months post intervention) - follow-up period, Outcome 14 Change in meta cognition (self-reported).

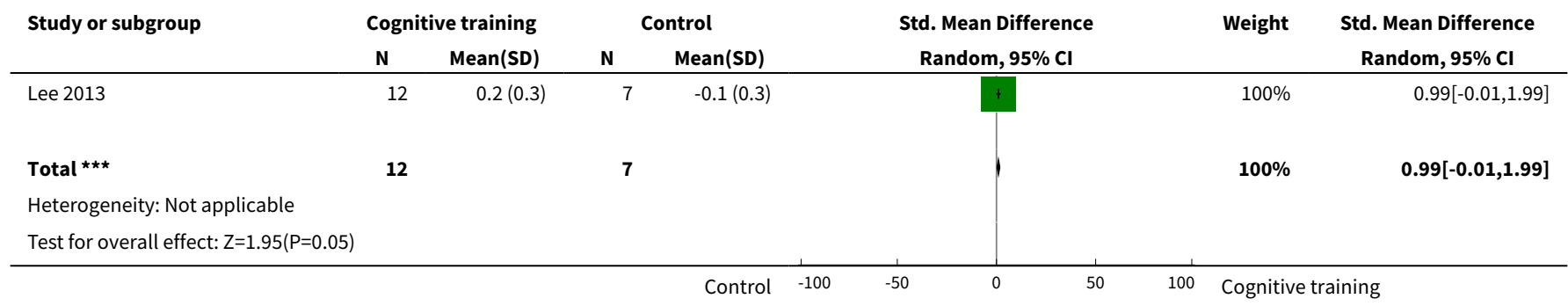

Analysis 13.15. Comparison 13 Cognitive training vs control in the medium term ( 3 to 12 months post intervention) - follow-up period, Outcome 15 Change in meta cognition (informant-reported).

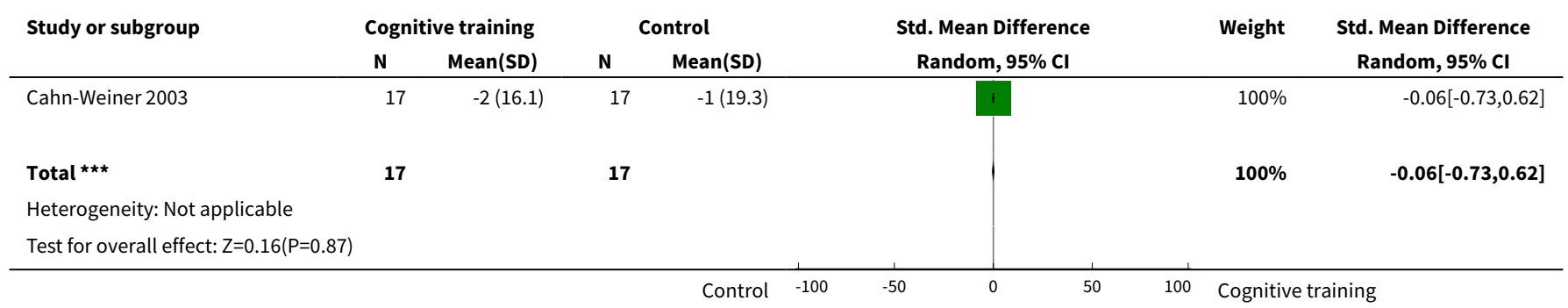

Analysis 13.16. Comparison 13 Cognitive training vs control in the medium term ( 3 to 12 months post intervention) - follow-up period, Outcome 16 Change in capacity for activities of daily living.

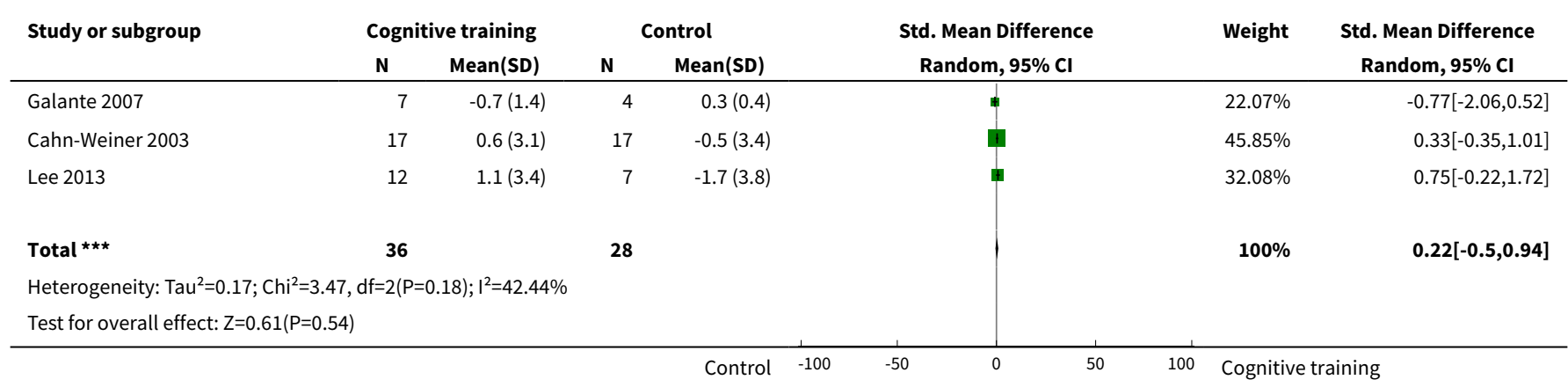


Analysis 13.17. Comparison 13 Cognitive training vs control in the medium term ( 3 to 12 months post intervention) - follow-up period, Outcome 17 Change in behavioural and psychological symptoms of dementia (BPSD).

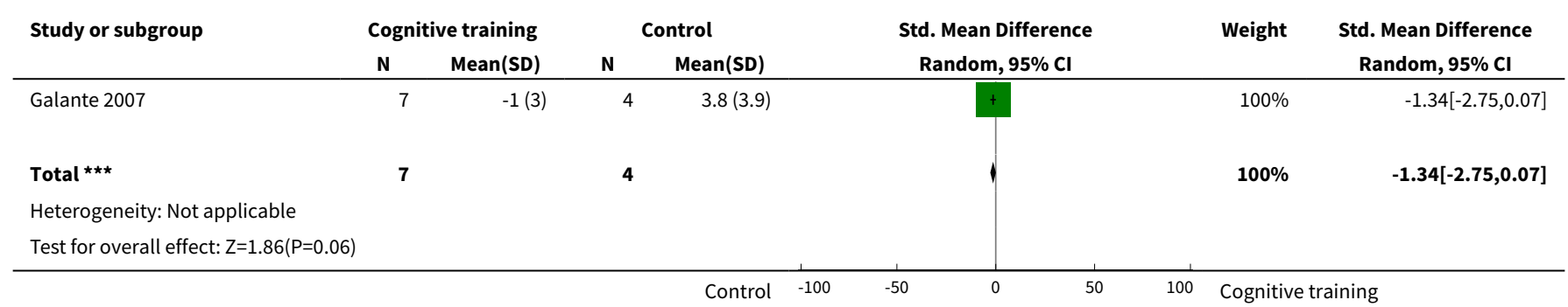

Analysis 13.18. Comparison 13 Cognitive training vs control in the medium term ( 3 to 12 months post intervention) - follow-up period, Outcome 18 Change in general health and quality of life.

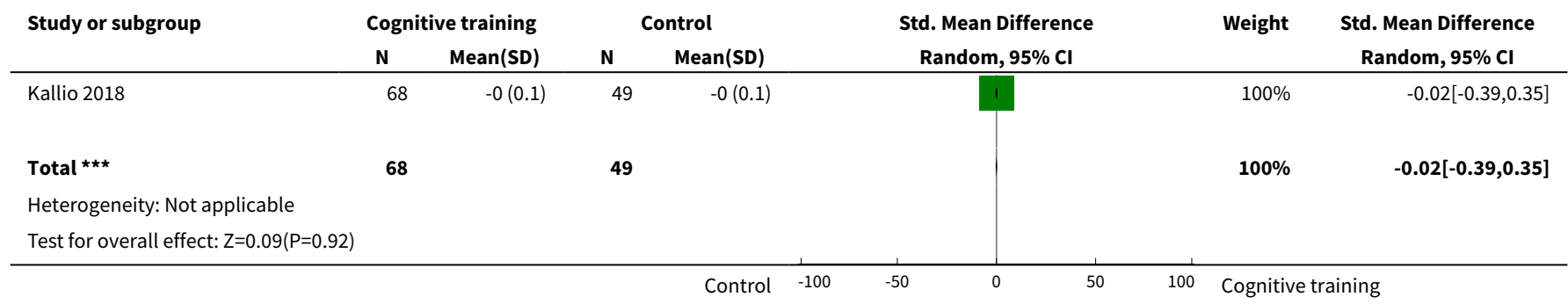

Analysis 13.19. Comparison 13 Cognitive training vs control in the medium term ( 3 to 12 months post intervention) - follow-up period, Outcome 19 Change in participants' mood.

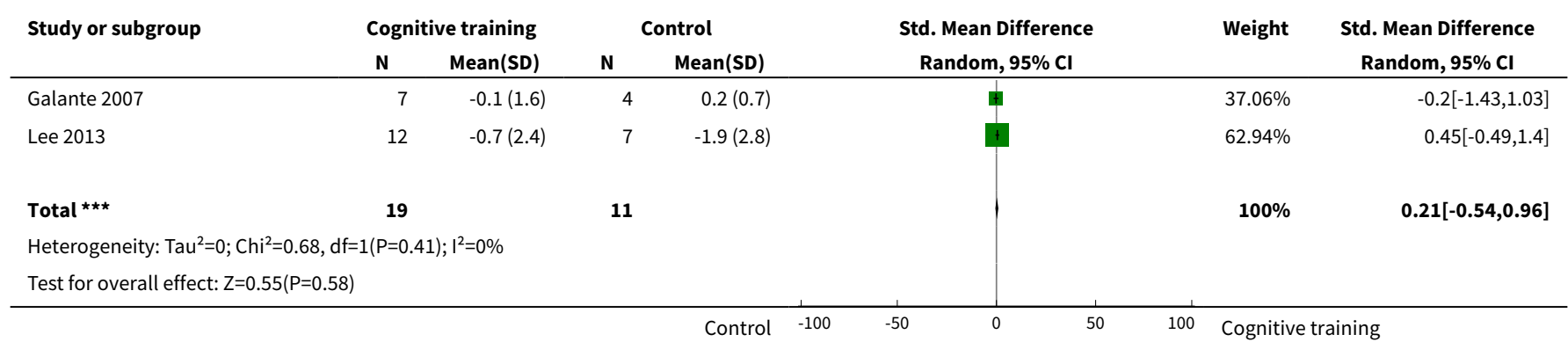

Analysis 13.23. Comparison 13 Cognitive training vs control in the medium term ( 3 to 12 months post intervention) - follow-up period, Outcome 23 Change in executive function.

\begin{tabular}{|c|c|c|c|c|c|c|c|c|}
\hline \multirow[t]{2}{*}{ Study or subgroup } & \multicolumn{2}{|c|}{ Cognitive training } & \multicolumn{2}{|c|}{ Control } & & \multirow{2}{*}{$\begin{array}{c}\text { Std. Mean Difference } \\
\text { Random, } 95 \% \mathrm{CI}\end{array}$} & \multirow[t]{2}{*}{ Weight } & \multirow{2}{*}{$\begin{array}{c}\text { Std. Mean Difference } \\
\text { Random, } 95 \% \mathrm{Cl}\end{array}$} \\
\hline & $\mathbf{N}$ & Mean(SD) & $\mathbf{N}$ & Mean(SD) & & & & \\
\hline Cahn-Weiner 2003 & 17 & $3(50.5)$ & 17 & $28.8(60.1)$ & & 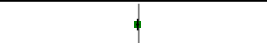 & $16.68 \%$ & $-0.45[-1.14,0.23]$ \\
\hline Galante 2007 & 7 & $0.4(4.9)$ & 4 & $-0.7(3.3)$ & & + & $10.38 \%$ & $0.23[-1.01,1.46]$ \\
\hline Quayhagen 1995 & 13 & $1.1(6.9)$ & 28 & $-1.4(7.8)$ & & $\phi$ & $16.94 \%$ & $0.33[-0.34,0.99]$ \\
\hline Quayhagen 1995 & 13 & $1.1(6.9)$ & 25 & $-3.2(7.5)$ & & , & $16.65 \%$ & $0.58[-0.11,1.26]$ \\
\hline
\end{tabular}




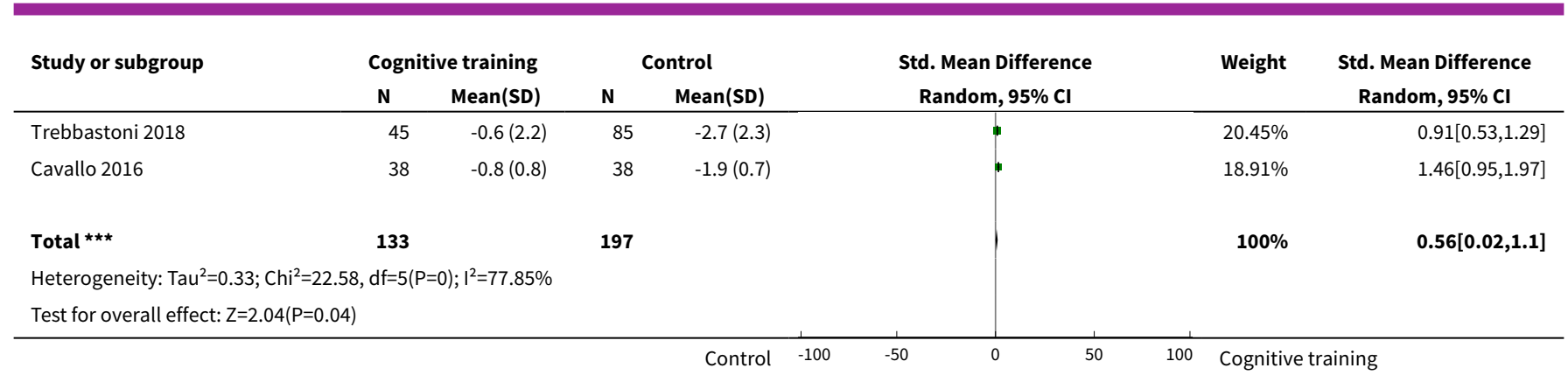

ADDITIONAL TABLES 


\begin{tabular}{|c|c|c|c|c|c|c|c|c|c|}
\hline Study & Condition & $\begin{array}{l}\text { Sample } \\
\text { size (at } \\
\text { base- } \\
\text { line) }\end{array}$ & $\begin{array}{l}\text { Age, mean } \\
\text { (SD), } \\
\text { range }\end{array}$ & $\begin{array}{l}\text { Gender } \\
\text { balance } \\
\text { (m:f) }\end{array}$ & Education & $\begin{array}{l}\text { Medications } \\
\text { (number or } \\
\text { proportion on } \\
\text { dementia-re- } \\
\text { lated medica- } \\
\text { tion) }\end{array}$ & $\begin{array}{l}\text { Baseline } \\
\text { MMSE } \\
\text { score }\end{array}$ & $\begin{array}{l}\text { Retention } \\
\text { rates }\end{array}$ & Adverse reactions \\
\hline $\begin{array}{l}\text { Beck } \\
1988\end{array}$ & Cognitive training & 10 & $\begin{array}{l}74 \text { (range } 68 \\
\text { to } 75 \text { ) }\end{array}$ & $5: 5$ & $\begin{array}{l}\text { Attended col- } \\
\text { lege }=2\end{array}$ & None & $\begin{array}{l}\text { Not report- } \\
\text { ed }\end{array}$ & $100 \%$ & $\begin{array}{l}\text { "many subjects } \\
\text { complained of tir- } \\
\text { ing" }\end{array}$ \\
\hline & Control & 10 & $\begin{array}{l}76 \text { (range } 70 \\
\text { to } 93 \text { ) }\end{array}$ & $3: 7$ & $\begin{array}{l}\text { Attended col- } \\
\text { lege }=1\end{array}$ & None & $\begin{array}{l}\text { Not report- } \\
\text { ed }\end{array}$ & $100 \%$ & Not specified \\
\hline \multirow[t]{4}{*}{$\begin{array}{l}\text { Heiss } \\
1993\end{array}$} & Cognitive training & $\begin{array}{l}\text { Not re- } \\
\text { ported } \\
\text { (18 } \\
\text { complet- } \\
\text { ed the } \\
\text { study) }\end{array}$ & $65.9(6.28)$ & $9: 9$ & Not reported & None & $20.55(4.42)$ & Not reported & Not specified \\
\hline & $\begin{array}{l}\text { Control (social sup- } \\
\text { port) }\end{array}$ & $\begin{array}{l}\text { Not re- } \\
\text { ported } \\
\text { (17 } \\
\text { complet- } \\
\text { ed the } \\
\text { study) }\end{array}$ & $66.63(10.17)$ & $10: 7$ & Not reported & None & $20.23(4.10)$ & Not reported & Not specified \\
\hline & Control (CT+pyritinol) & 17 & $67.18(8.51)$ & $\begin{array}{l}\text { Not re- } \\
\text { ported }\end{array}$ & 88.88 & Not reported & $21.64(4.55)$ & Not reported & Not specified \\
\hline & $\begin{array}{l}\text { Control (CT+phos- } \\
\text { phatidylserine) }\end{array}$ & 18 & $66.74(6.93)$ & $\begin{array}{l}\text { Not re- } \\
\text { ported }\end{array}$ & 125 & Not reported & $20.88(4.73)$ & Not reported & Not specified \\
\hline \multirow[t]{2}{*}{$\begin{array}{l}\text { Quayha- } \\
\text { gen } 1995\end{array}$} & Cognitive training & 25 & $\begin{array}{l}\text { Not report- } \\
\text { ed }\end{array}$ & $\begin{array}{l}\text { Not re- } \\
\text { ported }\end{array}$ & Not reported & Not reported & $\begin{array}{l}109.8(12.0) \\
\text { DRS }\end{array}$ & Not reported & Not specified \\
\hline & Passive control & 25 & $\begin{array}{l}\text { Not report- } \\
\text { ed }\end{array}$ & $\begin{array}{l}\text { Not re- } \\
\text { ported }\end{array}$ & Not reported & Not reported & $\begin{array}{l}109.2(11.7) \\
\text { DRS }\end{array}$ & Not reported & Not specified \\
\hline
\end{tabular}




\begin{tabular}{|c|c|c|c|c|c|c|c|c|c|}
\hline & Active control & 28 & $\begin{array}{l}\text { Not report- } \\
\text { ed }\end{array}$ & $\begin{array}{l}\text { not re- } \\
\text { ported }\end{array}$ & Not reported & Not reported & $\begin{array}{l}110(12.2) \\
\text { DRS }\end{array}$ & Not reported & Not specified \\
\hline \multirow[t]{4}{*}{$\begin{array}{l}\text { de } \\
\text { Vreese } \\
1998\end{array}$} & Cognitive training & 12 & NA & NA & NA & NA & NA & NA & NA \\
\hline & $\begin{array}{l}\text { Cognitive train- } \\
\text { ing }+A C h E-I\end{array}$ & 12 & $\begin{array}{l}\text { Not report- } \\
\text { ed }\end{array}$ & $\begin{array}{l}\text { Not re- } \\
\text { ported }\end{array}$ & Not reported & All & $17.33(3.39)$ & $100 \%$ & Not specified \\
\hline & Control (placebo drug) & 12 & $\begin{array}{l}\text { Not report- } \\
\text { ed }\end{array}$ & $\begin{array}{l}\text { Not re- } \\
\text { ported }\end{array}$ & Not reported & All & $17.44(4.67)$ & $100 \%$ & Not specified \\
\hline & Control (AChE-I) & 12 & $\begin{array}{l}\text { Not report- } \\
\text { ed }\end{array}$ & $\begin{array}{l}\text { Not re- } \\
\text { ported }\end{array}$ & Not reported & All & $17(3.20)$ & $100 \%$ & Not specified \\
\hline \multirow[t]{5}{*}{$\begin{array}{l}\text { Quayha- } \\
\text { gen } 2000\end{array}$} & Cognitive training & 21 & $\begin{array}{l}\text { Not report- } \\
\text { ed }\end{array}$ & $\begin{array}{l}\text { Not re- } \\
\text { ported }\end{array}$ & Not reported & Not reported & $\begin{array}{l}\text { Not as- } \\
\text { sessed }\end{array}$ & Not reported & Not specified \\
\hline & Passive control & 15 & $\begin{array}{l}\text { Not report- } \\
\text { ed }\end{array}$ & $\begin{array}{l}\text { Not re- } \\
\text { ported }\end{array}$ & Not reported & Not reported & $\begin{array}{l}\text { Not as- } \\
\text { sessed }\end{array}$ & Not reported & Not specified \\
\hline & $\begin{array}{l}\text { Dyadic counselling } \\
\text { control }\end{array}$ & 29 & $\begin{array}{l}\text { Not report- } \\
\text { ed }\end{array}$ & $\begin{array}{l}\text { Not re- } \\
\text { ported }\end{array}$ & Not reported & Not reported & $\begin{array}{l}\text { Not as- } \\
\text { sessed }\end{array}$ & Not reported & Not specified \\
\hline & $\begin{array}{l}\text { Seminar groups con- } \\
\text { trol }\end{array}$ & 22 & $\begin{array}{l}\text { Not report- } \\
\text { ed }\end{array}$ & $\begin{array}{l}\text { Not re- } \\
\text { ported }\end{array}$ & Not reported & Not reported & $\begin{array}{l}\text { Not as- } \\
\text { sessed }\end{array}$ & Not reported & Not specified \\
\hline & $\begin{array}{l}\text { Early-stage daycare } \\
\text { control }\end{array}$ & 16 & $\begin{array}{l}\text { Not report- } \\
\text { ed }\end{array}$ & $\begin{array}{l}\text { Not re- } \\
\text { ported }\end{array}$ & Not reported & Not reported & $\begin{array}{l}\text { Not as- } \\
\text { sessed }\end{array}$ & Not reported & Not specified \\
\hline \multirow[t]{2}{*}{$\begin{array}{l}\text { Davis } \\
2001\end{array}$} & Cognitive training & 19 & $68.67(3.86)$ & $10: 9$ & $15.06(3.86)$ & 5 & $21.84(4.03)$ & $100 \%$ & Not specified \\
\hline & Control & 18 & $72.56(7.62)$ & $6: 12$ & $12.97(2.56)$ & 4 & $22.78(4.45)$ & $100 \%$ & Not specified \\
\hline \multirow[t]{2}{*}{$\begin{array}{l}\text { Koltai } \\
2001\end{array}$} & $\begin{array}{l}\text { Cognitive training } \\
\text { (group and individual) } \\
\text { - shared data }\end{array}$ & 16 & $72.9(6.7)$ & $\begin{array}{l}\text { Not re- } \\
\text { ported }\end{array}$ & $15.0(4.0)$ & Not reported & $22.9(3.6)$ & $\begin{array}{l}87.5 \% \text { (missed: } \\
2 \text { participants } \\
\text { from group CT) }\end{array}$ & Not specified \\
\hline & Control & 8 & $73.9(7.2)$ & $\begin{array}{l}\text { Not re- } \\
\text { ported }\end{array}$ & $15.0(4.0)$ & Not reported & $26.6(2.5)$ & $100 \%$ & Not specified \\
\hline
\end{tabular}




\begin{tabular}{|c|c|c|c|c|c|c|c|c|c|}
\hline $\begin{array}{l}\text { Cahn- } \\
\text { Weiner } \\
2003\end{array}$ & Cognitive training & 19 & $77.8(6.9)$ & $9: 8$ & $12.7(2.1)$ & $\begin{array}{l}\text { All participants: } \\
\text { donepezil }\end{array}$ & $24.3(2.2)$ & $\begin{array}{l}89.4 \% \text { (missed: } \\
3 \text { participants) }\end{array}$ & Not specified \\
\hline & Control & 20 & $76.0(7.7)$ & $5: 12$ & $13.1(3.5)$ & $\begin{array}{l}\text { All participants: } \\
\text { donepezil }\end{array}$ & $25.1(1.7)$ & $\begin{array}{l}85 \% \text { (missed: } 3 \\
\text { participants) }\end{array}$ & Not specified \\
\hline $\begin{array}{l}\text { Galante } \\
2007\end{array}$ & Cognitive training & 7 & $\begin{array}{l}\text { Not report- } \\
\text { ed }\end{array}$ & $\begin{array}{l}\text { Not re- } \\
\text { ported }\end{array}$ & Not reported & All & $22.9(3.1)$ & $100 \%$ & Not specified \\
\hline & Control & 5 & $\begin{array}{l}\text { Not report- } \\
\text { ed }\end{array}$ & $\begin{array}{l}\text { Not re- } \\
\text { ported }\end{array}$ & Not reported & All & $23.1(1.8)$ & $\begin{array}{l}80 \% \text { (missed: } 1 \\
\text { participant) }\end{array}$ & Not specified \\
\hline $\begin{array}{l}\text { Neely } \\
2009\end{array}$ & Cognitive training & 10 & $74.8(6.7)$ & $6: 4$ & Not reported & Not reported & $22.9(4.15)$ & $100 \%$ & Not specified \\
\hline & Control & 10 & $77.0(6.6)$ & $6: 4$ & Not reported & Not reported & $18.6(5.7)$ & $\begin{array}{l}\text { 90\% (missed: } 1 \\
\text { participant) }\end{array}$ & Not specified \\
\hline \multicolumn{2}{|c|}{$\begin{array}{l}\text { Kawashima Cognitive training } \\
2005\end{array}$} & 16 & $\begin{array}{l}85.1(5.4) \\
\text { (range } 76 \text { to } \\
96 \text { ) }\end{array}$ & $\begin{array}{l}\text { Not re- } \\
\text { ported }\end{array}$ & Not reported & Not reported & $19.9(7.0)$ & $100 \%$ & Not specified \\
\hline & Control & 16 & $\begin{array}{l}86.3(4.9) \\
\text { (range } 78 \text { to } \\
96)\end{array}$ & $\begin{array}{l}\text { Not re- } \\
\text { ported }\end{array}$ & Not reported & Not reported & $19.6(5.4)$ & Not reported & Not specified \\
\hline \multirow[t]{3}{*}{$\begin{array}{l}\text { Boller } \\
2011\end{array}$} & $\begin{array}{l}\text { Cognitive training (rec- } \\
\text { ollection) }\end{array}$ & 12 & 81.58 & $4: 8$ & $10.92(2.94)$ & Not reported & $24(3.05)$ & Not reported & Not specified \\
\hline & $\begin{array}{l}\text { Cognitive training } \\
\text { (recognition) }\end{array}$ & 12 & 82.67 & $4: 8$ & $12.08(2.07)$ & Not reported & $24.83(2.12)$ & Not reported & Not specified \\
\hline & Control & 12 & $79.33(3.85)$ & $5: 7$ & $11.08(3.85)$ & Not reported & $25.83(1.40)$ & Not reported & Not specified \\
\hline \multirow[t]{2}{*}{$\begin{array}{l}\text { Fernán- } \\
\text { dez-Cal- } \\
\text { vo } 2011\end{array}$} & $\begin{array}{l}\text { Cognitive training } \\
\text { (BBA) }\end{array}$ & 15 & $75.80(4.27)$ & $9: 6$ & $7.46(1.84)$ & $\begin{array}{l}\text { All participants } \\
\text { on } \mathrm{laCHe}\end{array}$ & $19.33(2.48)$ & Not reported & Not specified \\
\hline & Cognitive training (IPP) & 15 & $75.60(4.55)$ & $8: 7$ & $8.40(2.77)$ & $\begin{array}{l}\text { All participants } \\
\text { on } \mathrm{laCHe}\end{array}$ & $20(2.92)$ & Not reported & Not specified \\
\hline
\end{tabular}




\begin{tabular}{|c|c|c|c|c|c|c|c|c|c|c|}
\hline & Control & 15 & $75.86(4.15)$ & $8: 7$ & $7.26(3.34)$ & $\begin{array}{l}\text { All participants } \\
\text { on } \mathrm{IaCHe}\end{array}$ & $20.44(1.90)$ & Not reported & Not specified & \\
\hline $\begin{array}{l}\text { Goudour } \\
2011\end{array}$ & Cognitive training & 5 & $68.8(10.0)$ & $2: 3$ & $9.0(1.2)$ & Not reported & $20.2(2.8)$ & Not reported & Not specified & ํㅜㄹ \\
\hline & Control & 5 & $70.0(5.9)$ & $2: 3$ & $9.6(1.9)$ & Not reported & $20.6(4.1)$ & Not reported & Not specified & \\
\hline $\begin{array}{l}\text { Jelcic } \\
2012\end{array}$ & Cognitive training & 20 & $82.9(3.6)$ & $2: 18$ & $6.7(2.9)$ & Not reported & $24.4(2.8)$ & $100 \%$ & Not specified & 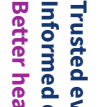 \\
\hline & Control & 20 & $81.8(5.5)$ & $5: 15$ & $8.25(3.6)$ & Not reported & $25(2.6)$ & $100 \%$ & Not specified & \\
\hline $\begin{array}{l}\text { Berga- } \\
\text { maschi } \\
2013\end{array}$ & Cognitive training & 16 & $78.19(5.50)$ & $\begin{array}{l}\text { Not re- } \\
\text { ported }\end{array}$ & $7.25(3.24)$ & $\begin{array}{l}\text { All participants: } \\
\text { donepezil } 5 \text { or } \\
10 \mathrm{mg} / \mathrm{d}\end{array}$ & $20.25(2.95)$ & Not reported & Not specified & \\
\hline & Control & 16 & $77.72(5.06)$ & $\begin{array}{l}\text { Not re- } \\
\text { ported }\end{array}$ & $5.61(2.30)$ & $\begin{array}{l}\text { All participants: } \\
\text { donepezil } 5 \text { or } \\
10 \mathrm{mg} / \mathrm{d}\end{array}$ & $21.94(2.01)$ & Not reported & Not specified & \\
\hline Lee 2013 & $\begin{array}{l}\text { Cognitive training } \\
\text { (CELP) }\end{array}$ & 6 & $\begin{array}{l}\text { Not report- } \\
\text { ed }\end{array}$ & $1: 6$ & $\begin{array}{l}\text { Nil: } 42.8 \% \\
\text { <2 years: } 14.3 \% \\
3 \text { to } 6 \text { years: } \\
28.6 \% \\
\text { Secondary: } \\
14.3 \% \\
\text { University: } 0 \%\end{array}$ & Not reported & $15.3(2.7)$ & Not reported & Not specified & \\
\hline & $\begin{array}{l}\text { Cognitive training } \\
\text { (TELP) }\end{array}$ & 6 & $\begin{array}{l}\text { Not report- } \\
\text { ed }\end{array}$ & $3: 3$ & $\begin{array}{l}\text { Nil: } 16.7 \% \\
\text { < } 2 \text { years: } 16.7 \% \\
3 \text { to } 6 \text { years: } \\
33.2 \% \\
\text { Secondary: } \\
16.7 \% \\
\text { University: } \\
16.7 \%\end{array}$ & Not reported & $17.6(4.7)$ & Not reported & Not specified & 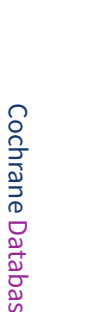 \\
\hline & Control & 7 & Reported & $2: 4$ & $\begin{array}{l}\text { Nil: } 33.3 \% \\
\text { <2 years: } 16.7 \% \\
3 \text { to } 6 \text { years: } \\
16.7 \% \\
\text { Secondary: } \\
33.3 \%\end{array}$ & Not reported & $17(3.5)$ & Not reported & Not specified & 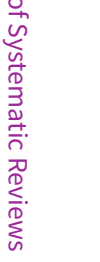 \\
\hline
\end{tabular}




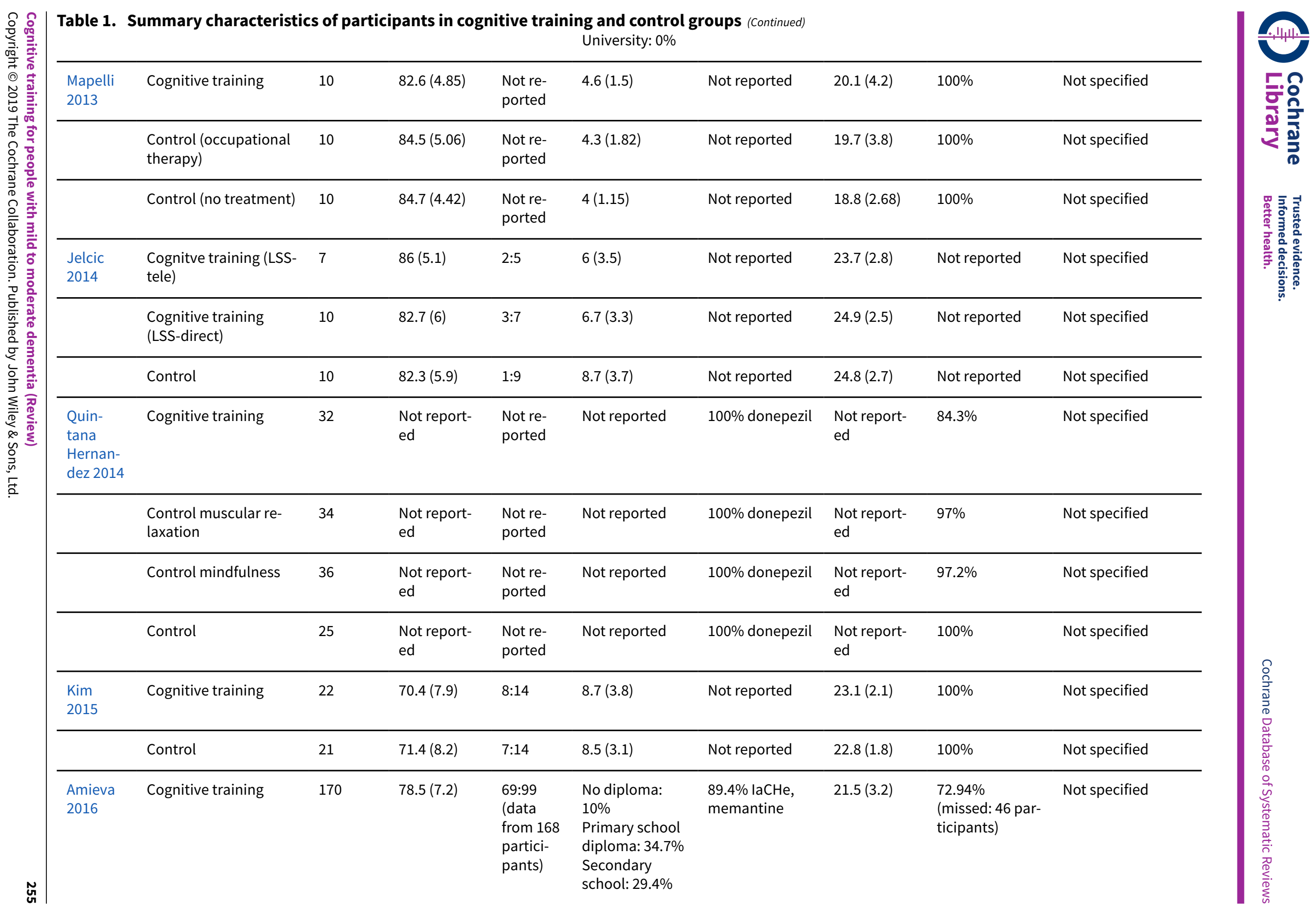




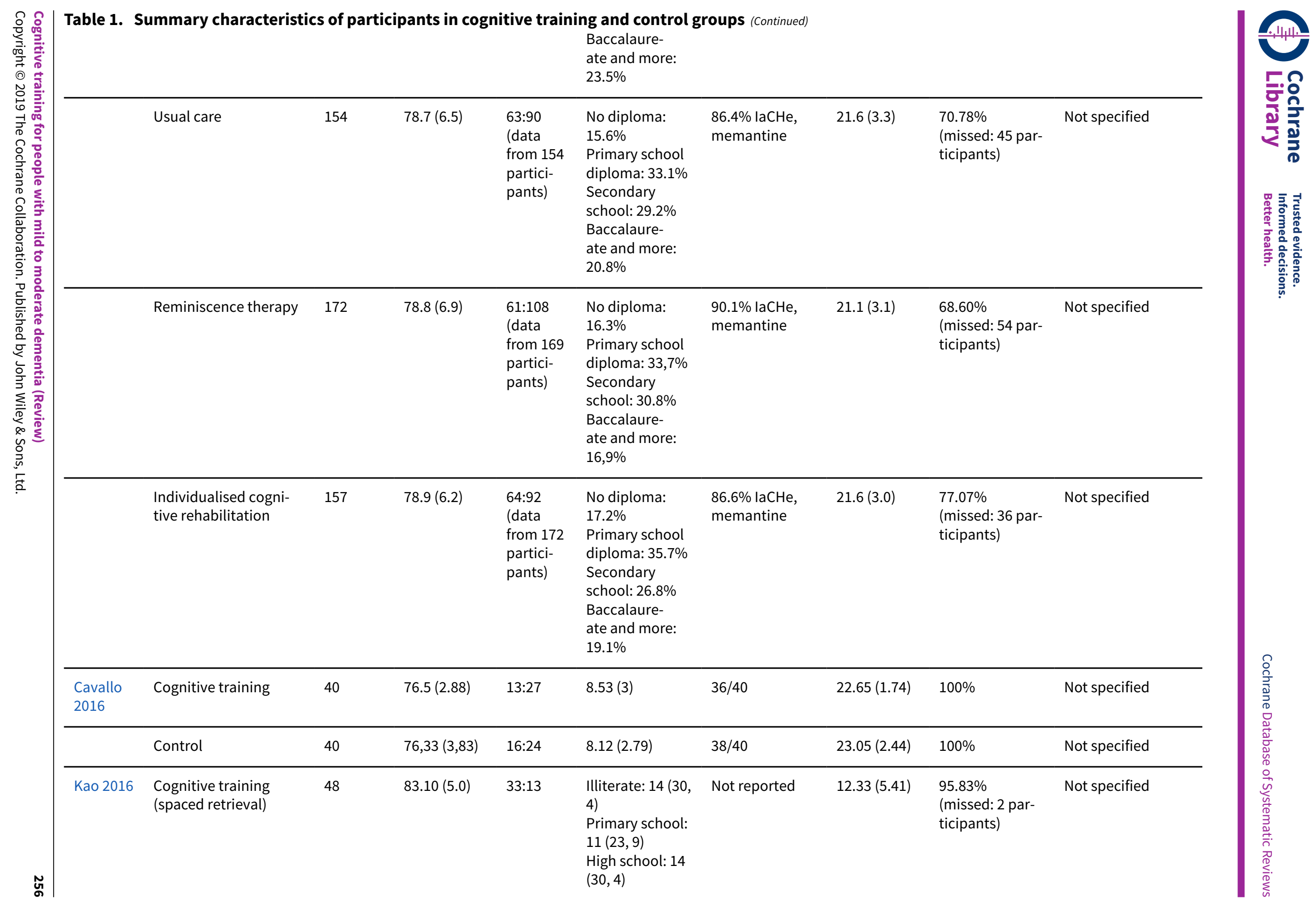




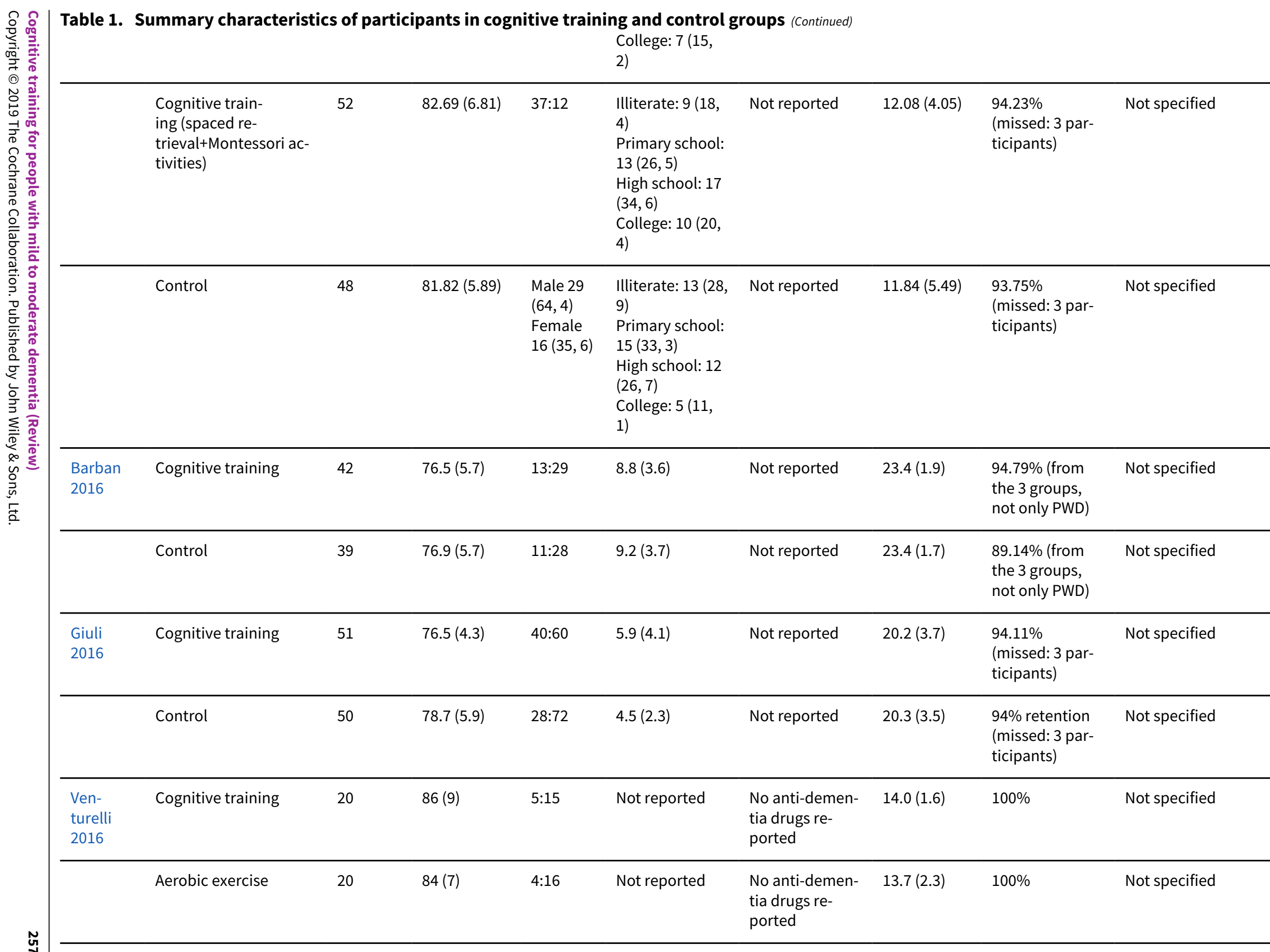


Table 1. Summary characteristics of participants in cognitive training and control groups (Continued)

Aerobic exercise+Cog- 20 nitive training

$6: 14$

Not reported

No anti-demen-

$13.8(1.5) \quad 100 \%$

Not specified

tia drugs re-

ported

\begin{tabular}{|c|c|c|c|c|c|c|c|c|c|}
\hline & Control & 20 & $84(10)$ & $17: 13$ & Not reported & $\begin{array}{l}\text { No anti-demen- } \\
\text { tia drugs re- } \\
\text { ported }\end{array}$ & $14.2(1.5)$ & $100 \%$ & Not specified \\
\hline \multirow[t]{3}{*}{$\begin{array}{l}\text { Tsantali } \\
2017\end{array}$} & Cognitive training & 17 & $73.4(5.7)$ & $\begin{array}{l}\text { Not re- } \\
\text { ported }\end{array}$ & $9.9(4.2)$ & $\begin{array}{l}\text { All were on } \\
\text { inhibitors of } \\
\text { cholinesterase, } \\
\text { used for at least } \\
2 \text { years }\end{array}$ & $23.2(1.6)$ & $100 \%$ & Not specified \\
\hline & $\begin{array}{l}\text { Alternative treatment } \\
\text { (cognitive stimulation) }\end{array}$ & 21 & $74.2(5.6)$ & $\begin{array}{l}\text { Not re- } \\
\text { ported }\end{array}$ & $9.5(4.1)$ & $\begin{array}{l}\text { All were on } \\
\text { inhibitors of } \\
\text { cholinesterase, } \\
\text { used for at least } \\
2 \text { years }\end{array}$ & $23.1(1.4)$ & $100 \%$ & Not specified \\
\hline & Control & 17 & $73.3(4.9)$ & $\begin{array}{l}\text { Not re- } \\
\text { ported }\end{array}$ & $9.8(4.0)$ & $\begin{array}{l}\text { All were on } \\
\text { inhibitors of } \\
\text { cholinesterase, } \\
\text { used for at least } \\
2 \text { years }\end{array}$ & $22.5(0.9)$ & $100 \%$ & Not specified \\
\hline $\begin{array}{l}\text { Brueggen } \\
2017\end{array}$ & Cognitive training & 8 & (53 to 80$)$ & $4: 4$ & (10 to 17 ) & $\begin{array}{l}5 \text { participants } \\
\text { on anti-demen- } \\
\text { tia medication }\end{array}$ & $24(3.55)$ & $100 \%$ & Not specified \\
\hline & Control & 10 & (59 to 83 ) & $5: 3$ & (11 to 17 ) & $\begin{array}{l}\text { All } 8 \text { partici- } \\
\text { pants were on } \\
\text { anti-dementia } \\
\text { medication }\end{array}$ & $21.75(3.24)$ & $\begin{array}{l}80 \% \text { (missed: } 2 \\
\text { participants) }\end{array}$ & Not specified \\
\hline \multirow[t]{3}{*}{$\begin{array}{l}\text { Serino } \\
2017\end{array}$} & VR group - AD & 10 & $\begin{array}{l}86.60 \\
(6.13)\end{array}$ & $1: 9$ & $9.80(3.97)$ & Not reported & $22.05(1.62)$ & $100 \%$ & Not specified \\
\hline & $\begin{array}{l}\text { VR group - normal age- } \\
\text { ing }\end{array}$ & 8 & $86.62(6.19)$ & $4: 4$ & $9.12(5.05)$ & Not reported & $27.73(2.02)$ & $100 \%$ & Not specified \\
\hline & Control & 10 & 88.70 (3.59) & $2: 8$ & $7.00(5.00)$ & Not reported & $20.79(1.80)$ & $100 \%$ & Not specified \\
\hline
\end{tabular}




\begin{tabular}{|c|c|c|c|c|c|c|c|c|c|c|c|}
\hline 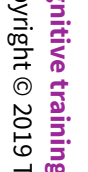 & $\begin{array}{l}\text { Gio- } \\
\text { vagnoli } \\
2017\end{array}$ & Cognitive training & 13 & $71.69(7.88)$ & $3: 10$ & $6.92(2.46)$ & Not reported & $23.62(1.94)$ & $\begin{array}{l}100 \% \text { ( } 4 \\
\text { dropped out } \\
\text { before treat- } \\
\text { ment) }\end{array}$ & No adverse effects & 드용 \\
\hline 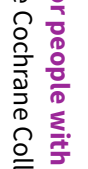 & & $\begin{array}{l}\text { Alternative treatment } \\
\text { (music therapy) }\end{array}$ & 13 & $73.92(7.74)$ & $7: 6$ & $10.46(5.3)$ & Not reported & $22.85(6.28)$ & $\begin{array}{l}100 \% \text { ( } 4 \\
\text { dropped out } \\
\text { before treat- } \\
\text { ment) }\end{array}$ & No adverse effects & D \\
\hline 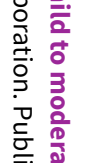 & & $\begin{array}{l}\text { Alternative treatment } \\
\text { (neuroeducation) }\end{array}$ & 13 & $75.31(5.56)$ & $8: 5$ & $7.31(4.01)$ & Not reported & $21.15(3.48)$ & $\begin{array}{l}100 \% \text { ( } 3 \\
\text { dropped out } \\
\text { before treat- } \\
\text { ment) }\end{array}$ & No adverse effects & 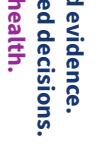 \\
\hline 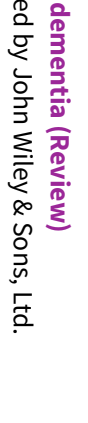 & $\begin{array}{l}\text { Treb- } \\
\text { bastoni } \\
2018\end{array}$ & Cognitive training & 54 & $74.26(6.97)$ & $28: 26$ & $8.64(4.21)$ & $\begin{array}{l}78 \% \text { AChEls } \\
27 \% \text { donezepil } \\
5 \mathrm{mg} \\
24 \% \text { donezepil } \\
10 \mathrm{mg} \\
20 \% \text { rivastig- } \\
\text { mine } 9.5 \mathrm{mg} \\
7 \% \text { rivastigmine } \\
4.6 \mathrm{mg} \\
2 \% \text { memantine } \\
20 \mathrm{mg}\end{array}$ & $22.20(2.37)$ & $\begin{array}{l}88.80 \% \\
\text { (missed: } 6 \text { par- } \\
\text { ticipants) }\end{array}$ & $\begin{array}{l}2 \text { falls (group is } \\
\text { not reported) and } \\
2 \text { deaths ( } 1 \text { from } \\
\text { each group). Study } \\
\text { authors stated that } \\
\text { these events were } \\
\text { not related to any } \\
\text { of the experimen- } \\
\text { tal procedures per- } \\
\text { formed }\end{array}$ & \\
\hline & & Control & 86 & $76.01(6.46)$ & $34: 52$ & $8.40(4.12)$ & $\begin{array}{l}88 \% \text { AChEls, } \\
40 \% \text { donepeziil } \\
5 \mathrm{mg} \\
20 \% \text { rivastig- } \\
\text { mine } 9.5 \mathrm{mg} \\
18 \% \text { donepezil } \\
10 \mathrm{mg} \\
12 \% \text { rivastig- } \\
\text { mine } 4.6 \mathrm{mg} \\
2 \% \text { memantine } \\
20 \mathrm{mg}\end{array}$ & $22.89(2.72)$ & $100 \%$ & $\begin{array}{l}2 \text { falls (group is } \\
\text { not reported) and } \\
2 \text { deaths ( } 1 \text { from } \\
\text { each group). Study } \\
\text { authors stated that } \\
\text { these events were } \\
\text { not related to any } \\
\text { of the experimen- } \\
\text { tal procedures per- } \\
\text { formed }\end{array}$ & 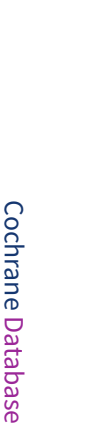 \\
\hline กั & $\begin{array}{l}\text { Kallio } \\
2018\end{array}$ & Cognitive training & 76 & $82.6(5.5)$ & $\begin{array}{l}34.2 \% \\
\text { male }\end{array}$ & $32<8$ years & $\begin{array}{l}60(78.9 \%) \text { tak- } \\
\text { ing AD med- } \\
\text { ications; } 33 \\
(43.4 \%) \text { taking } \\
\text { anticholinergics }\end{array}$ & $21.0(4.3)$ & 68 of 76 & Not specified & . \\
\hline
\end{tabular}




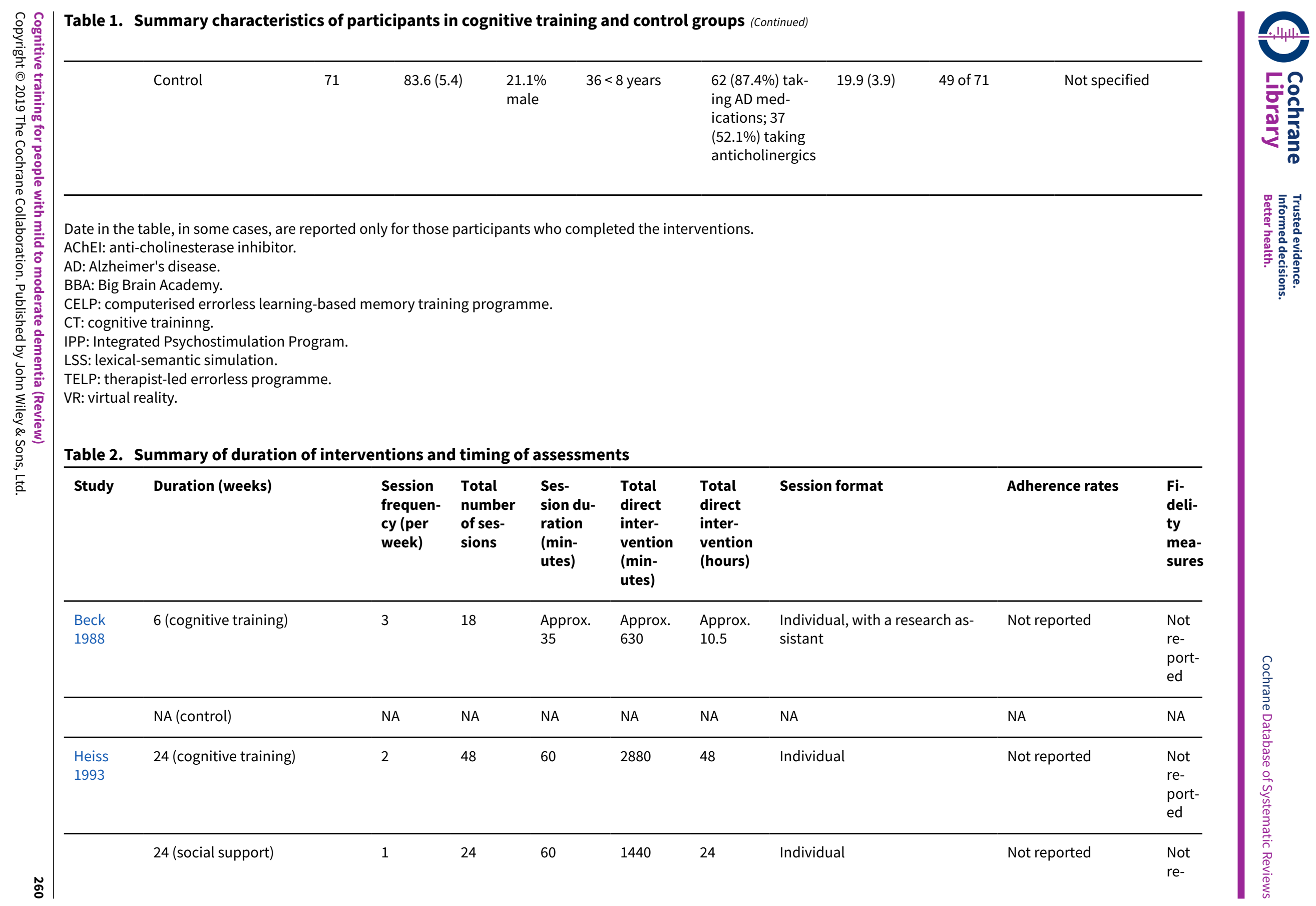




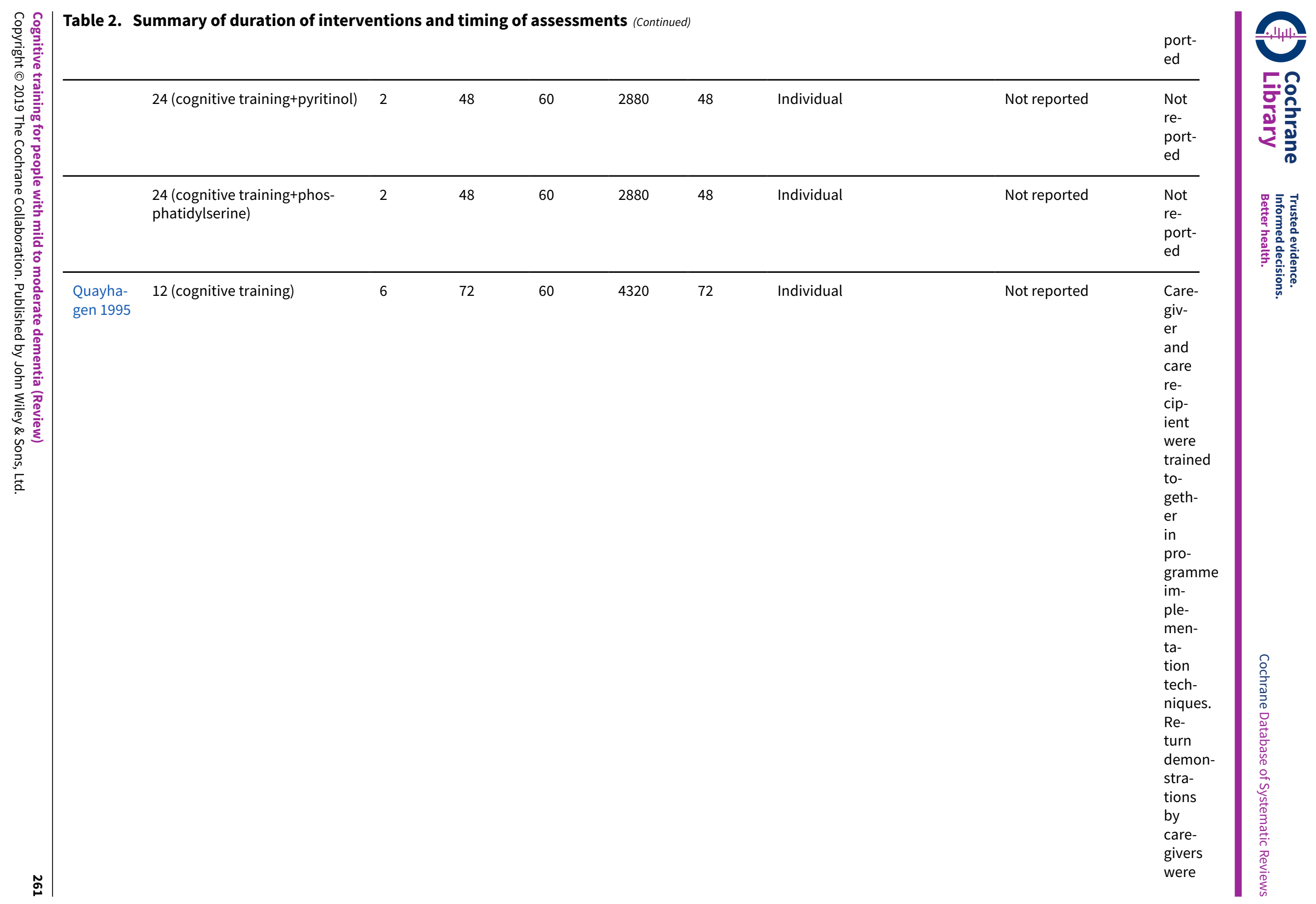




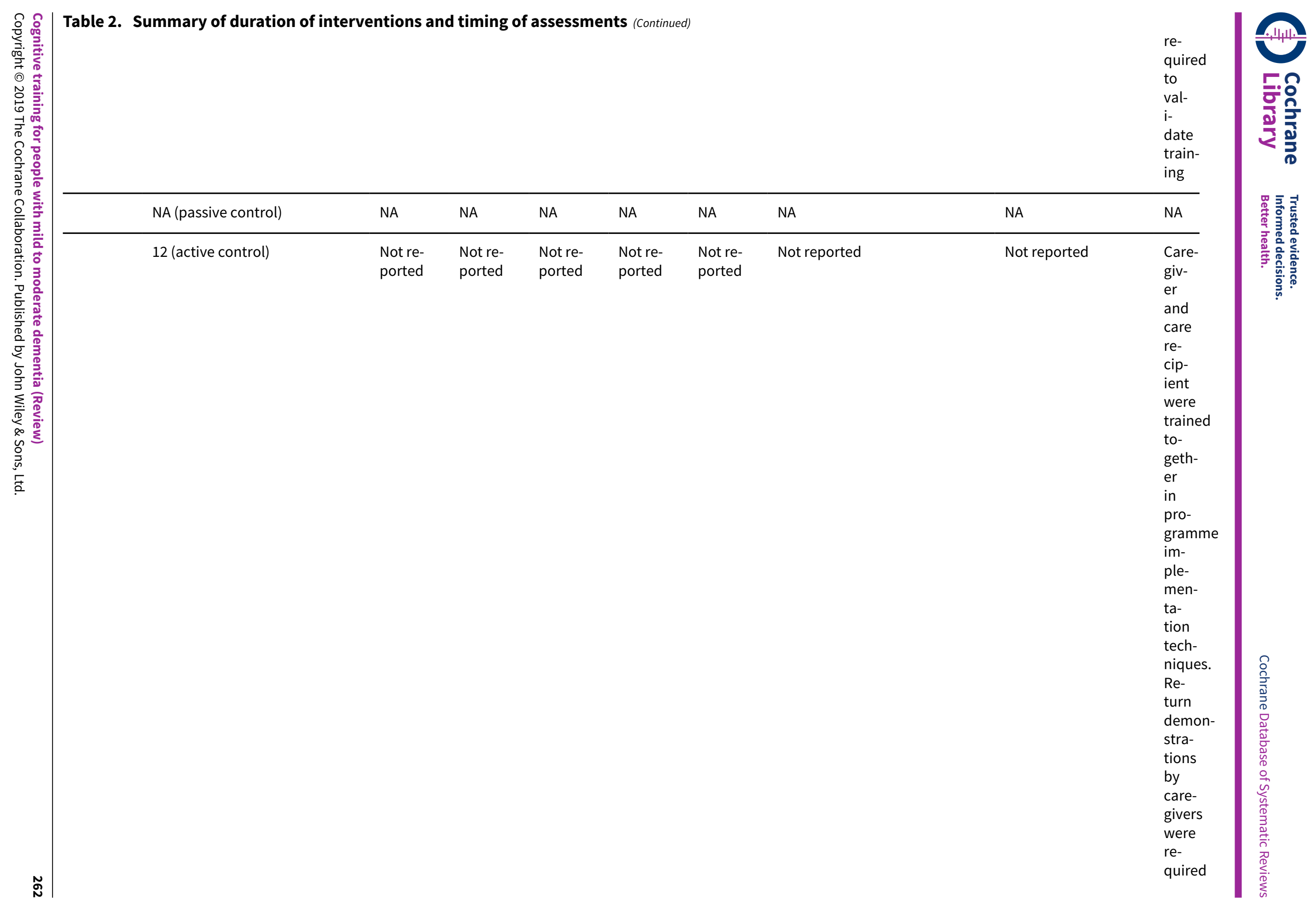




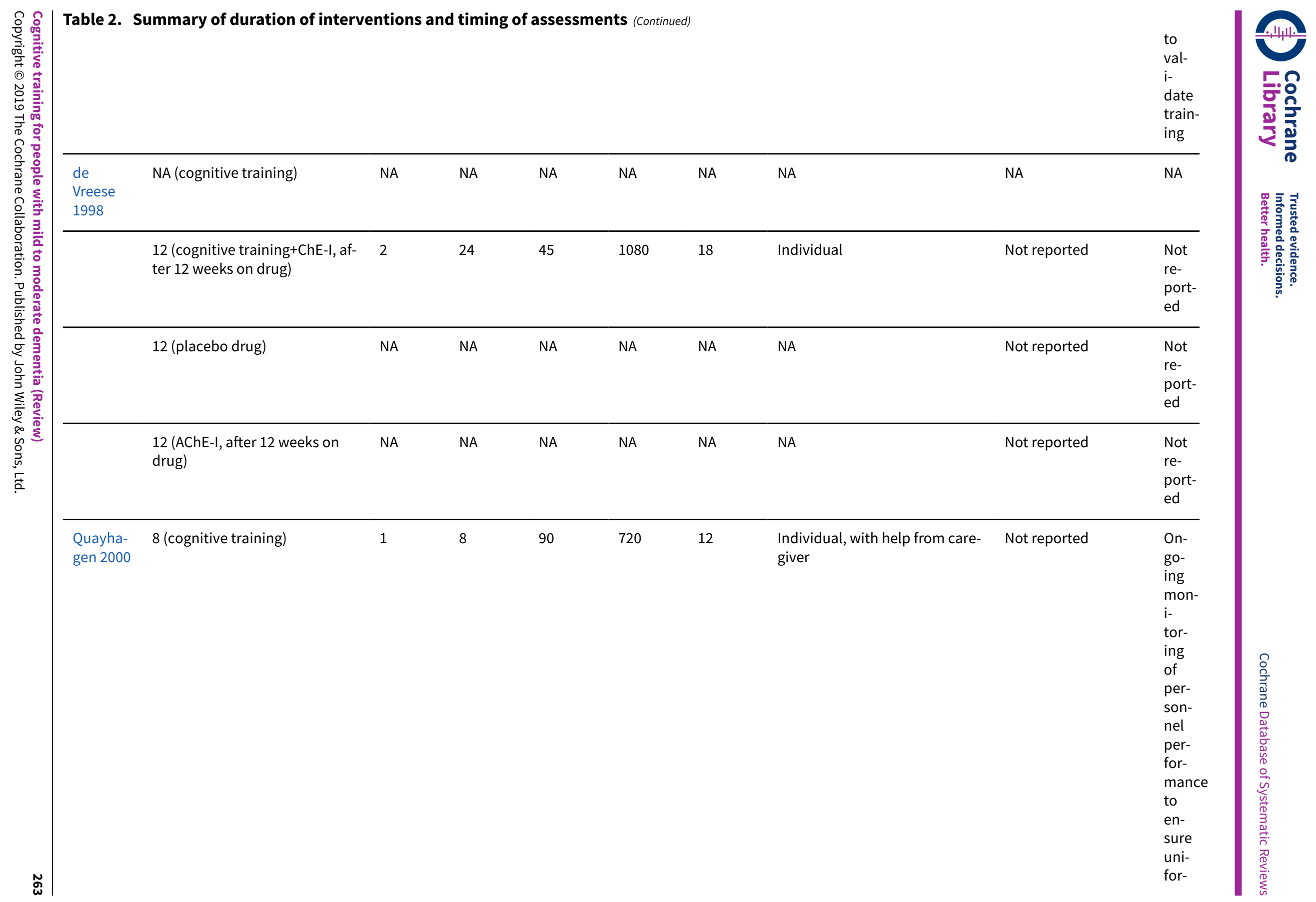




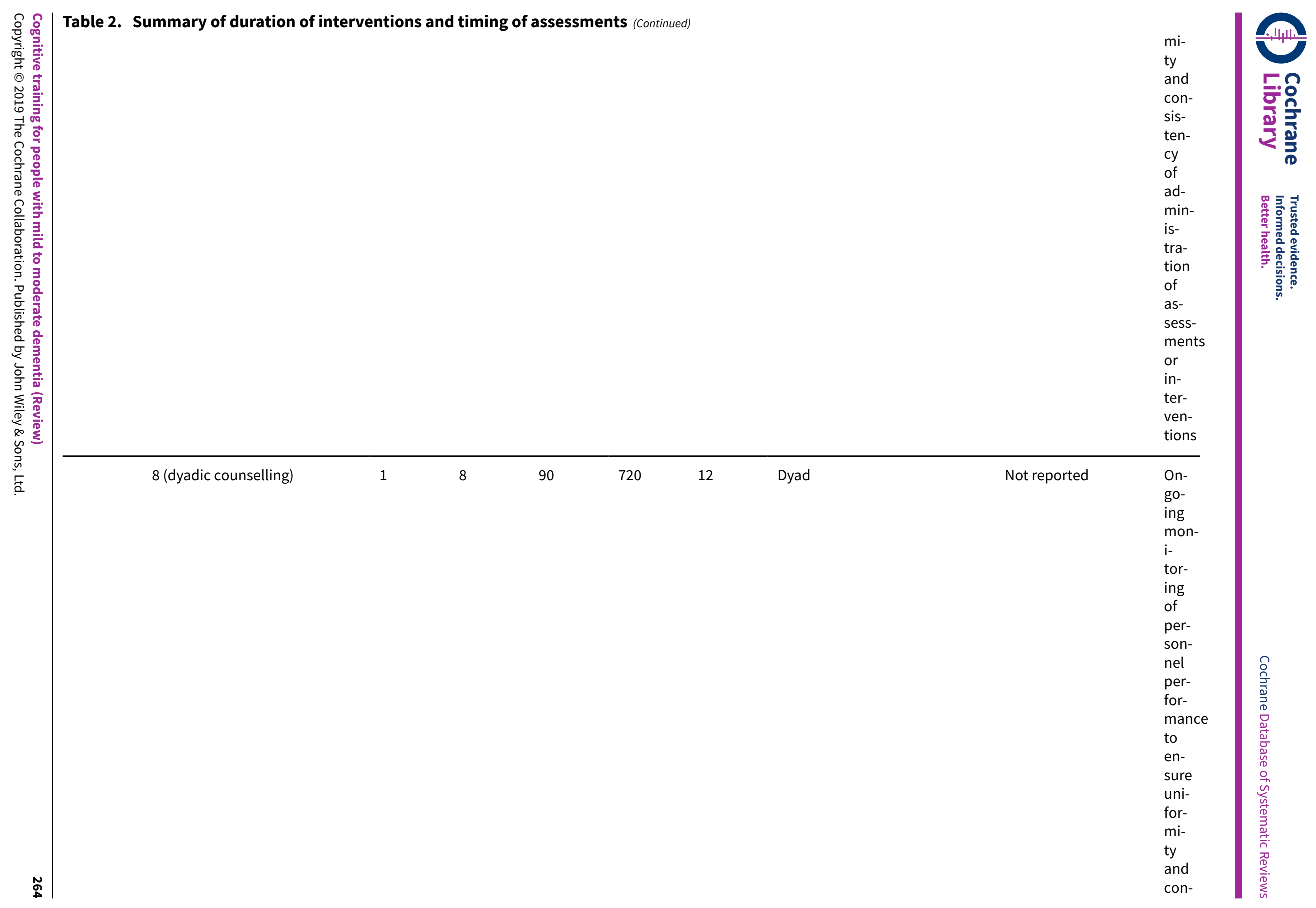




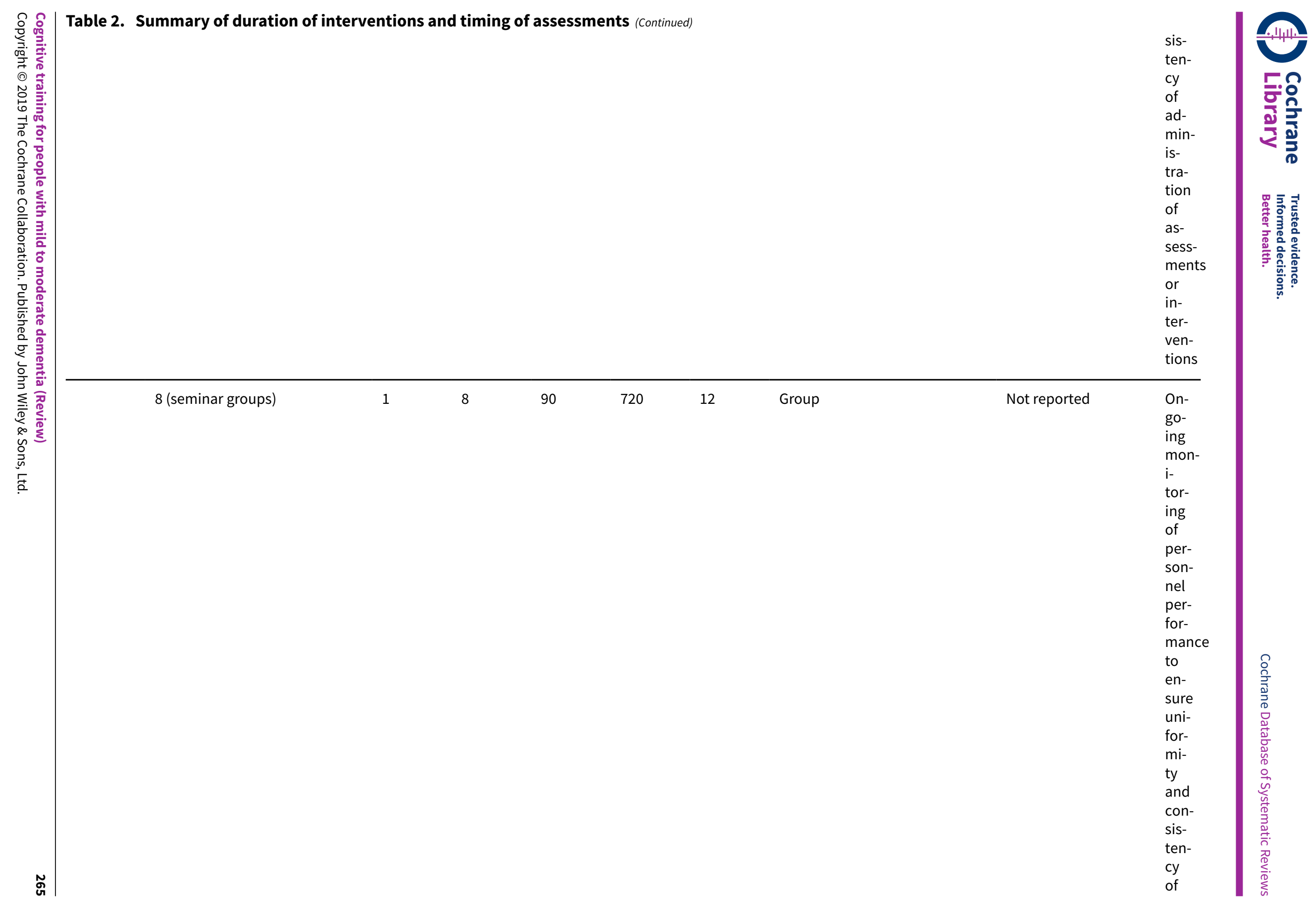




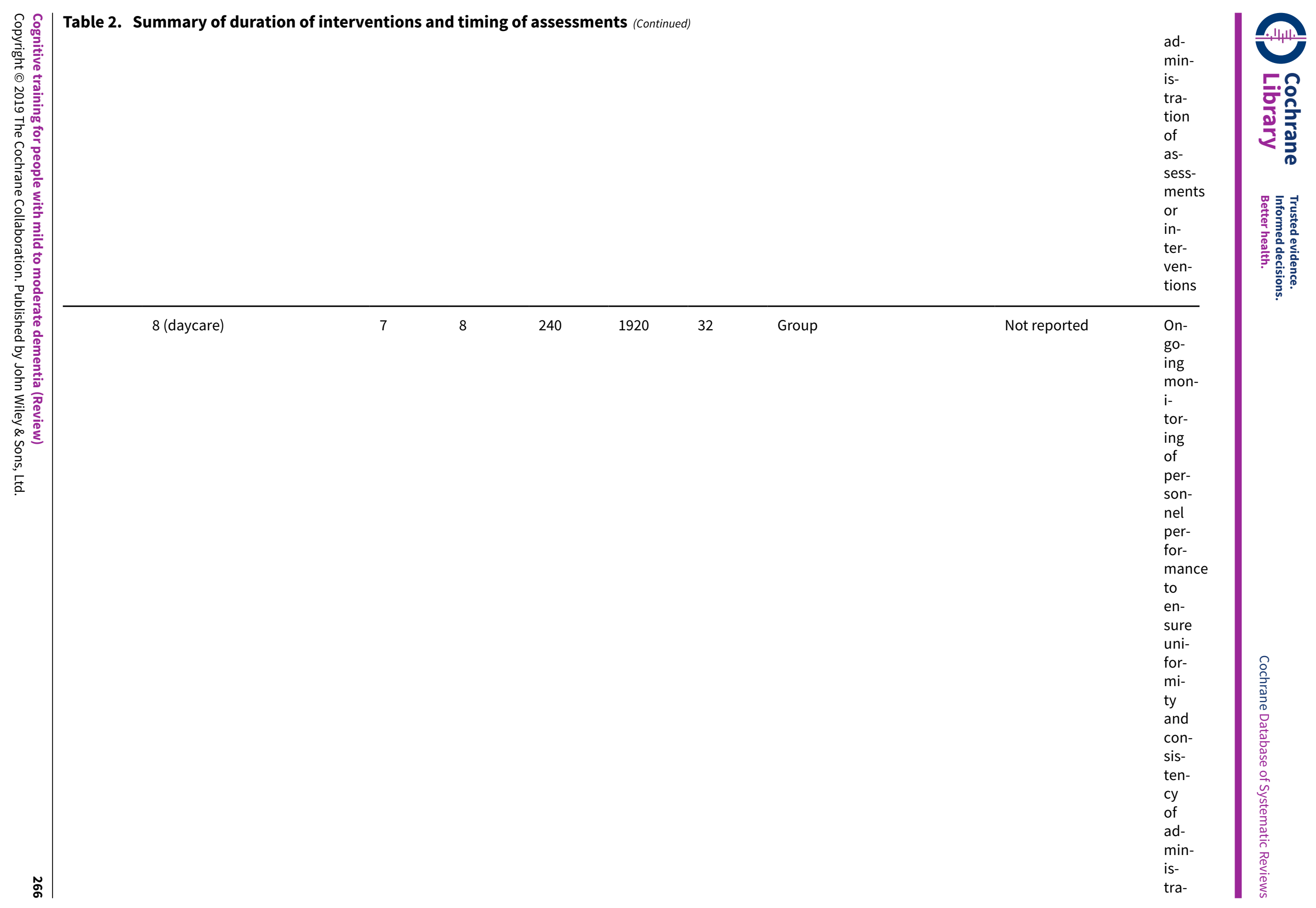




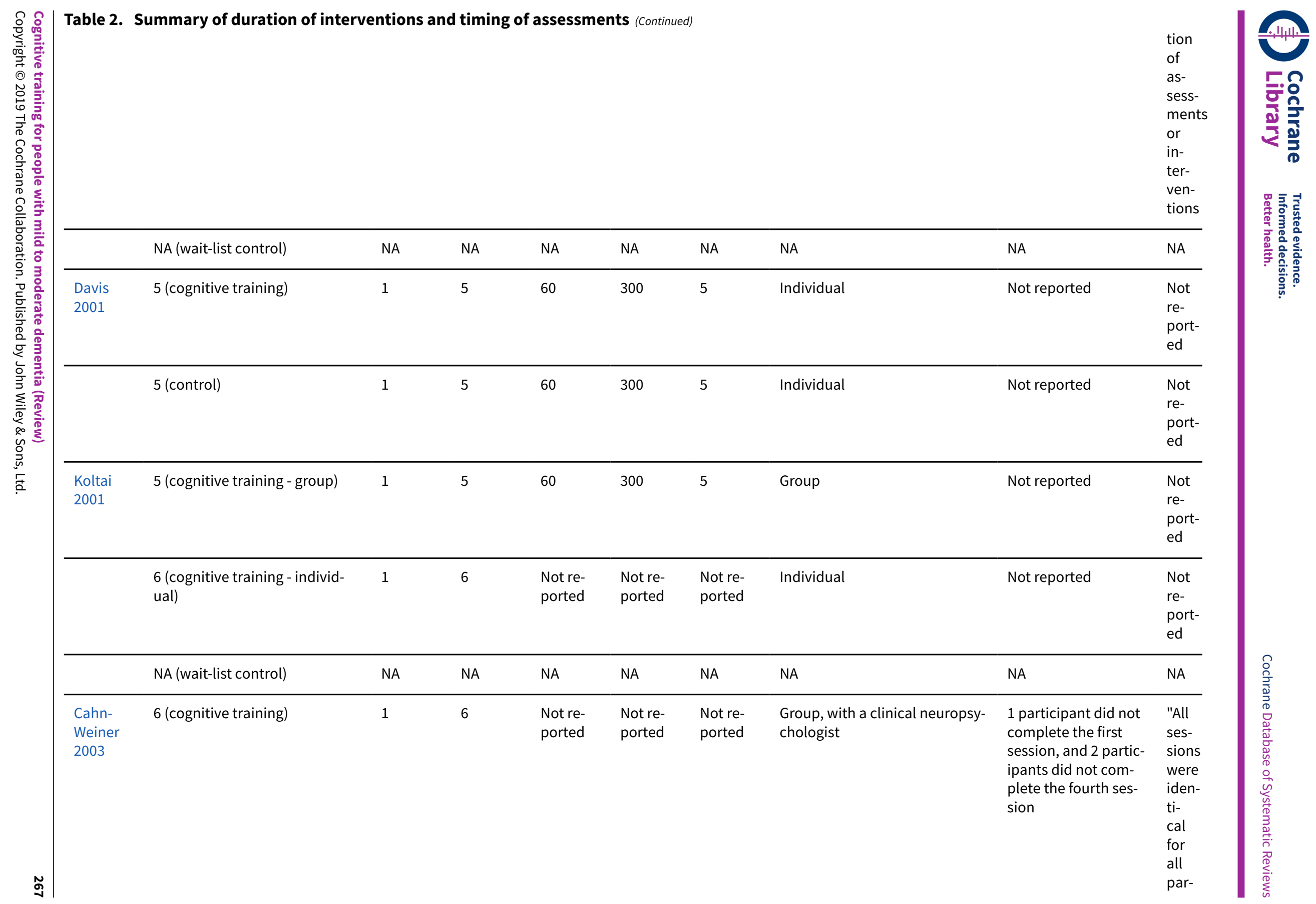




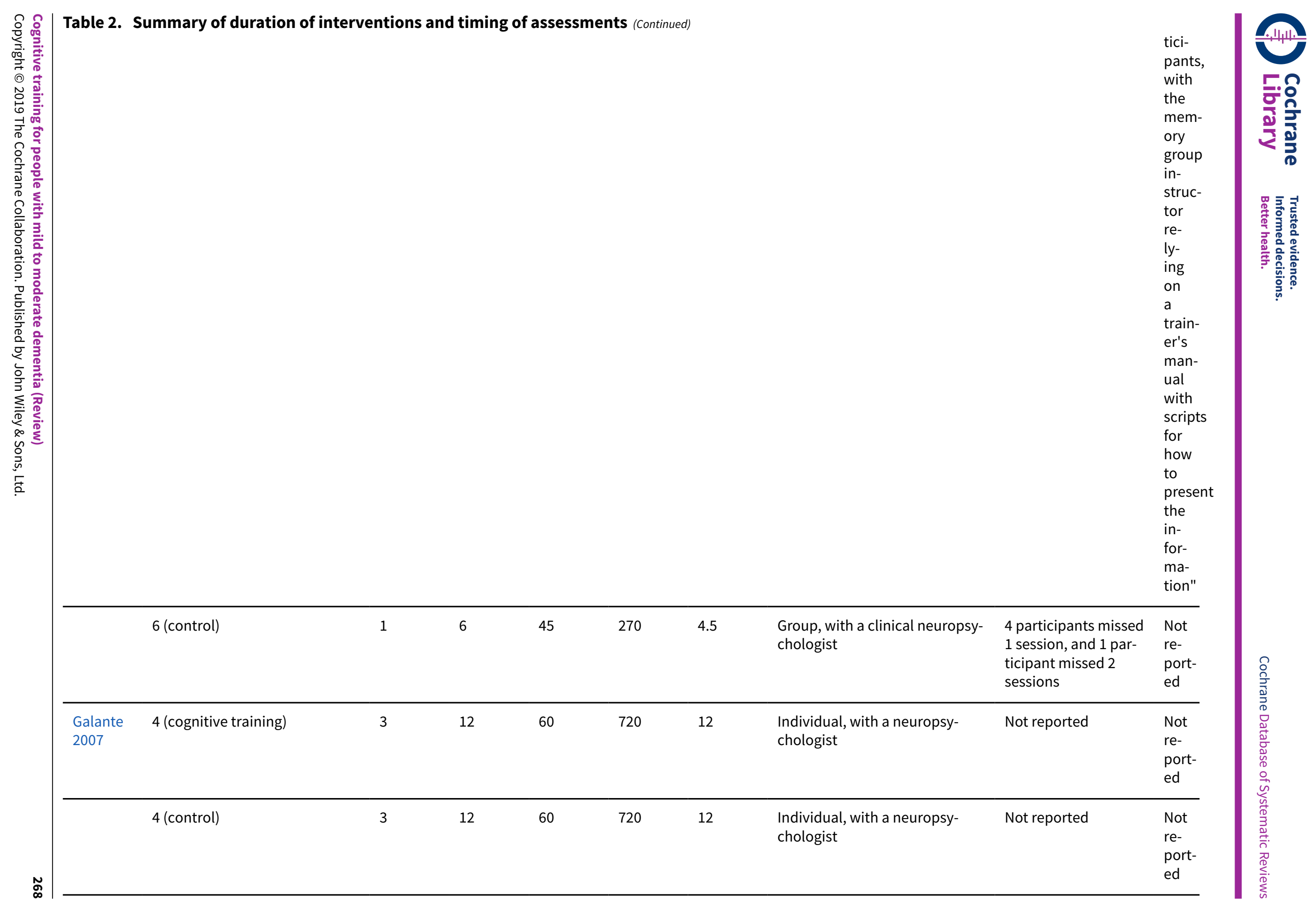




\begin{tabular}{|c|c|c|c|c|c|c|c|c|c|}
\hline $\begin{array}{l}\text { Neely } \\
2009\end{array}$ & $\begin{array}{l}8 \text { (cognitive training - collabo- } \\
\text { rative intervention) }\end{array}$ & 1 & 8 & 30 to 40 & $\begin{array}{l}\text { Approx. } \\
280\end{array}$ & $\begin{array}{l}\text { Approx. } \\
4.5\end{array}$ & Dyads, with a research assistant & Not reported & $\begin{array}{l}\text { Not } \\
\text { re- } \\
\text { port- } \\
\text { ed }\end{array}$ \\
\hline & $\begin{array}{l}8 \text { (cognitive training - individual } \\
\text { intervention) }\end{array}$ & 1 & 8 & 30 to 40 & $\begin{array}{l}\text { Approx. } \\
280\end{array}$ & $\begin{array}{l}\text { Approx. } \\
4.5\end{array}$ & $\begin{array}{l}\text { Individual, with a research as- } \\
\text { sistant }\end{array}$ & Not reported & $\begin{array}{l}\text { Not } \\
\text { re- } \\
\text { port- } \\
\text { ed }\end{array}$ \\
\hline & NA (control group) & NA & NA & NA & NA & NA & NA & NA & NA \\
\hline $\begin{array}{l}\text { Kawashim } \\
2005\end{array}$ & 24 (cognitive training) & 2 to 6 & 48 to 144 & $\begin{array}{l}\text { Approx. } \\
20\end{array}$ & $\begin{array}{l}960 \text { to } \\
2880\end{array}$ & 16 to 48 & $\begin{array}{l}\text { Individual, with the possibility } \\
\text { to ask staff members for advice }\end{array}$ & Not reported & $\begin{array}{l}\text { Not } \\
\text { re- } \\
\text { port- } \\
\text { ed }\end{array}$ \\
\hline & NA (control) & NA & NA & NA & NA & NA & NA & Not reported & $\begin{array}{l}\text { Not } \\
\text { re- } \\
\text { port- } \\
\text { ed }\end{array}$ \\
\hline \multirow[t]{3}{*}{$\begin{array}{l}\text { Boller } \\
2011\end{array}$} & $\begin{array}{l}2 \text { (cognitive training - recollec- } \\
\text { tion) }\end{array}$ & 12 & 24 & 60 & 1440 & 24 & Not reported & Not reported & $\begin{array}{l}\text { Not } \\
\text { re- } \\
\text { port- } \\
\text { ed }\end{array}$ \\
\hline & $\begin{array}{l}2 \text { (cognitive training - recogni- } \\
\text { tion) }\end{array}$ & 12 & 24 & 60 & 1440 & 24 & Not reported & Not reported & $\begin{array}{l}\text { Not } \\
\text { re- } \\
\text { port- } \\
\text { ed }\end{array}$ \\
\hline & NA (control) & NA & NA & NA & NA & NA & NA & NA & NA \\
\hline \multirow[t]{2}{*}{$\begin{array}{l}\text { Fernán- } \\
\text { dez-Cal- } \\
\text { vo } 2011\end{array}$} & 12 (cognitive training - BBA) & 3 & 36 & 60 & 2160 & 36 & $\begin{array}{l}\text { Individual, with an OT and a } \\
\text { psychologist }\end{array}$ & Not reported & $\begin{array}{l}\text { Not } \\
\text { re- } \\
\text { port- } \\
\text { ed }\end{array}$ \\
\hline & 12 (cognitive training - IPP) & 3 & 36 & 60 & 2160 & 36 & $\begin{array}{l}\text { Individual, with an OT and a } \\
\text { psychologist }\end{array}$ & Not reported & $\begin{array}{l}\text { Not } \\
\text { re- } \\
\text { port- } \\
\text { ed }\end{array}$ \\
\hline
\end{tabular}




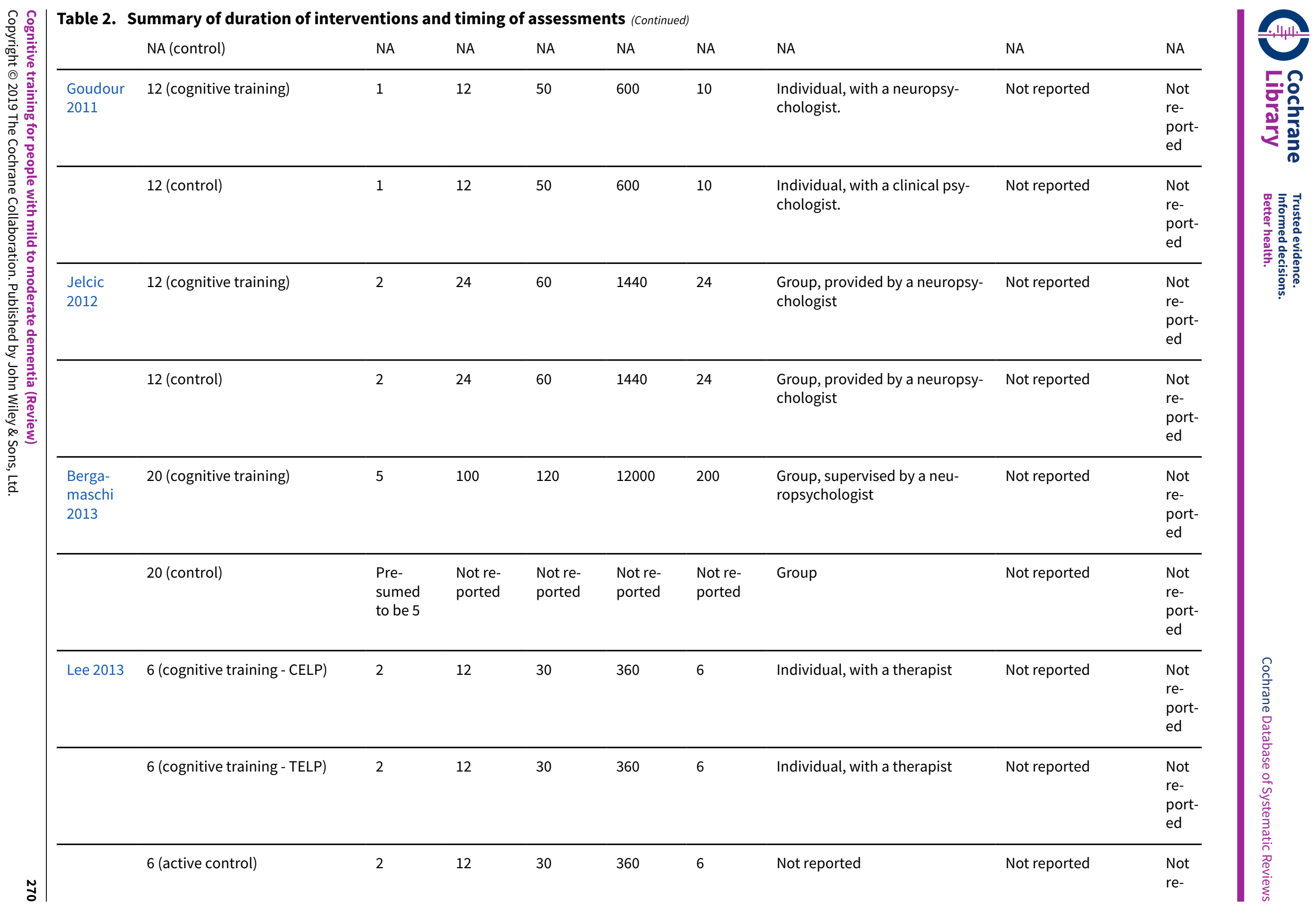




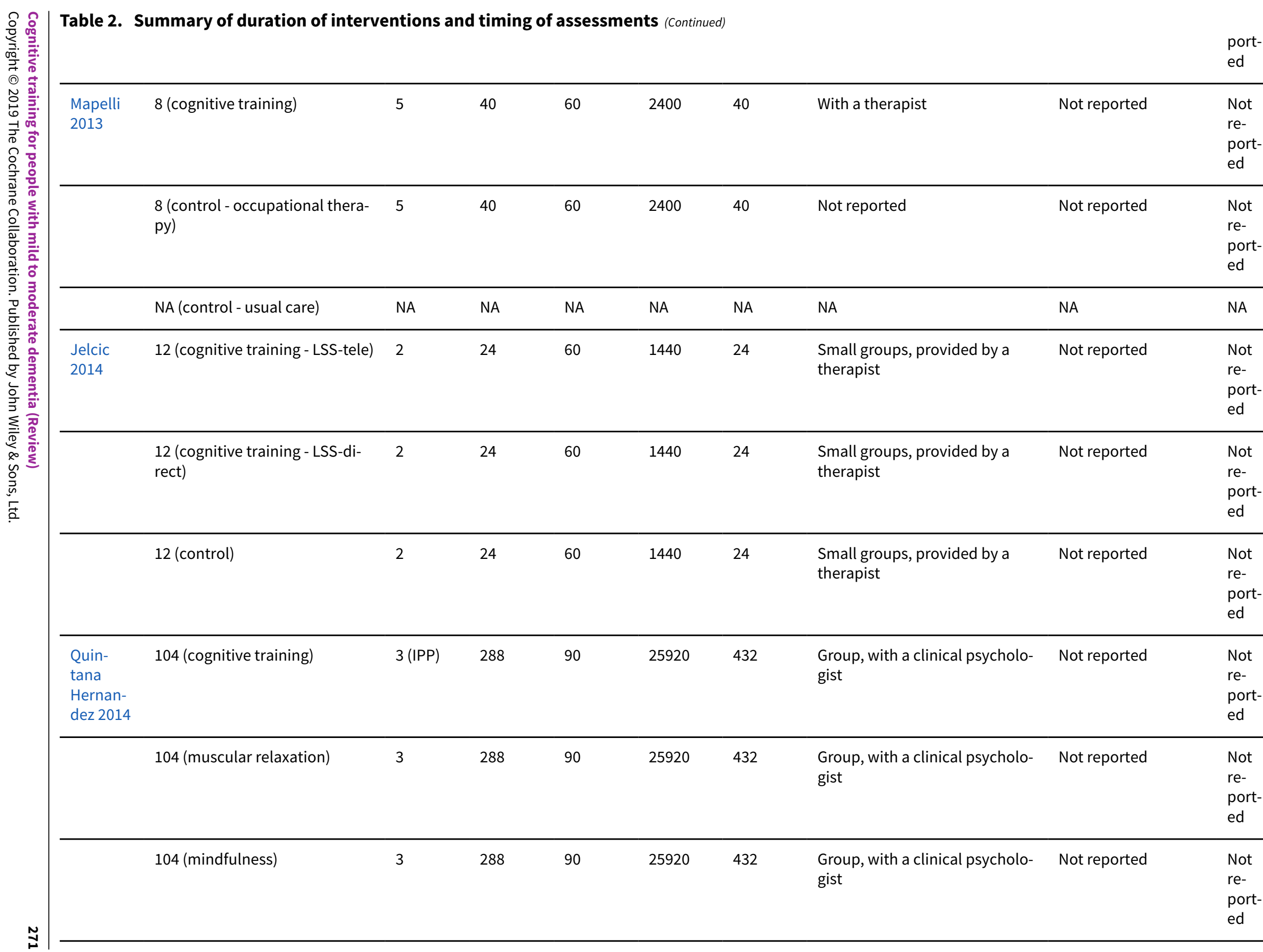




\begin{tabular}{|c|c|c|c|c|c|c|c|c|c|}
\hline & NA (passive control) & NA & NA & NA & NA & NA & NA & Not reported & $\begin{array}{l}\text { Not } \\
\text { re- } \\
\text { port- } \\
\text { ed }\end{array}$ \\
\hline $\begin{array}{l}\text { Kim } \\
2015\end{array}$ & 8 (cognitive training) & 1 & 8 & 60 & 480 & 8 & Individual and in a group & Not reported & $\begin{array}{l}\text { Not } \\
\text { re- } \\
\text { port- } \\
\text { ed }\end{array}$ \\
\hline & 8 (control) & 1 & 8 & 60 & 480 & 8 & Not reported & Not reported & $\begin{array}{l}\text { Not } \\
\text { re- } \\
\text { port- } \\
\text { ed }\end{array}$ \\
\hline $\begin{array}{l}\text { Amieva } \\
2016\end{array}$ & 96 (cognitive training) & $\begin{array}{l}1 \text { (dur- } \\
\text { ing the } \\
\text { first } 3 \\
\text { months); } \\
1 \text { every } \\
6 \text { weeks } \\
\text { (for the } \\
\text { next } 21 \\
\text { months) }\end{array}$ & 15 & 90 & 1350 & 22.5 & Groups & Not reported & $\begin{array}{l}\text { Man- } \\
\text { ual } \\
\text { de- } \\
\text { tail- } \\
\text { ing } \\
\text { the } \\
\text { guide- } \\
\text { lines } \\
\text { of } \\
\text { each } \\
\text { in- } \\
\text { ter- } \\
\text { ven- } \\
\text { tion } \\
\text { was } \\
\text { pro- } \\
\text { vid- } \\
\text { ed. } \\
\text { Stan- } \\
\text { dard- } \\
\text { ised } \\
\text { pro- } \\
\text { ce- } \\
\text { dures } \\
\text { to } \\
\text { guar- } \\
\text { an- } \\
\text { tee } \\
\text { ho- } \\
\text { mo- }\end{array}$ \\
\hline
\end{tabular}




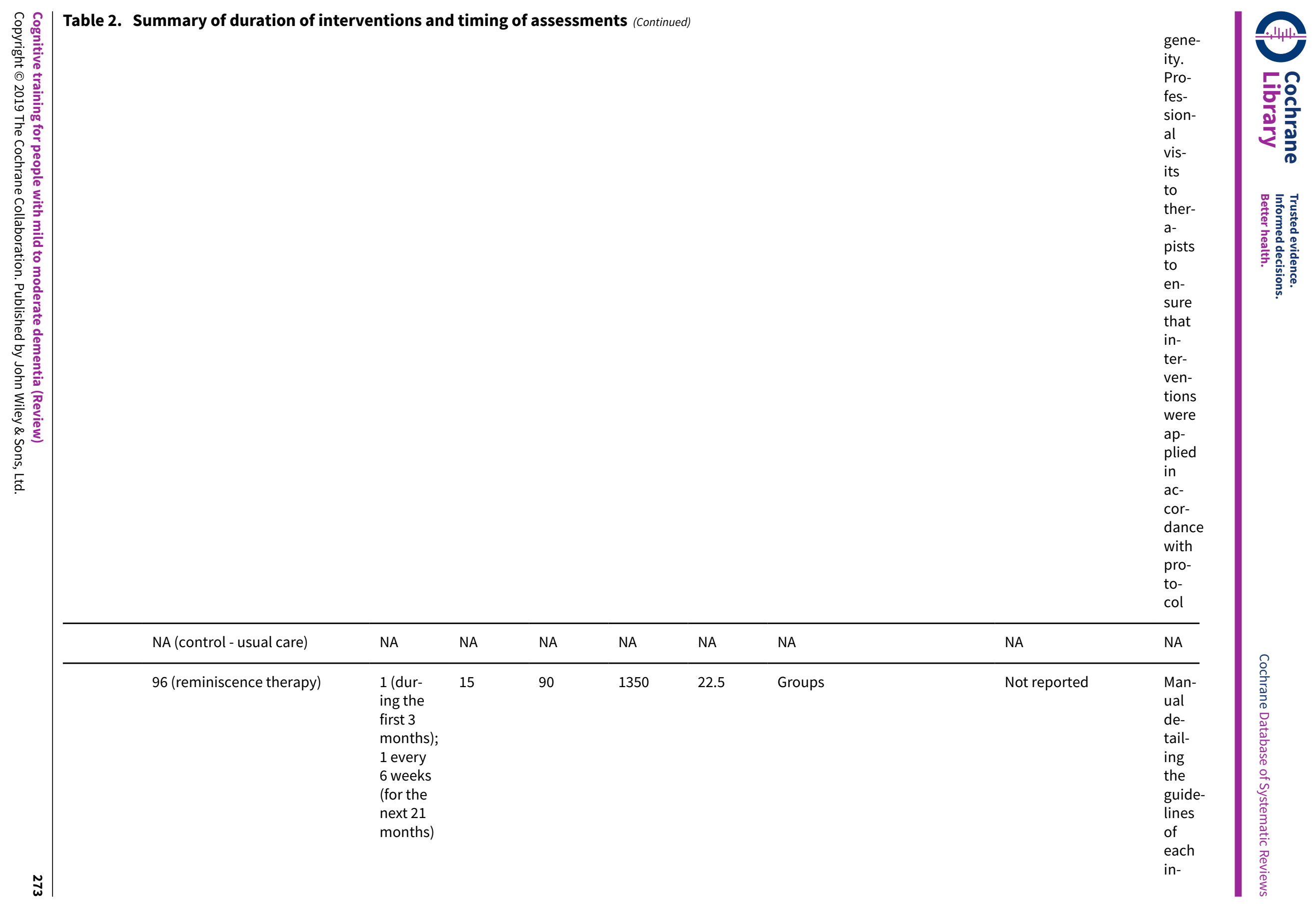


Table 2. Summary of duration of interventions and timing of assessments (Continued)

ce-

fes-

sion-

tions were 


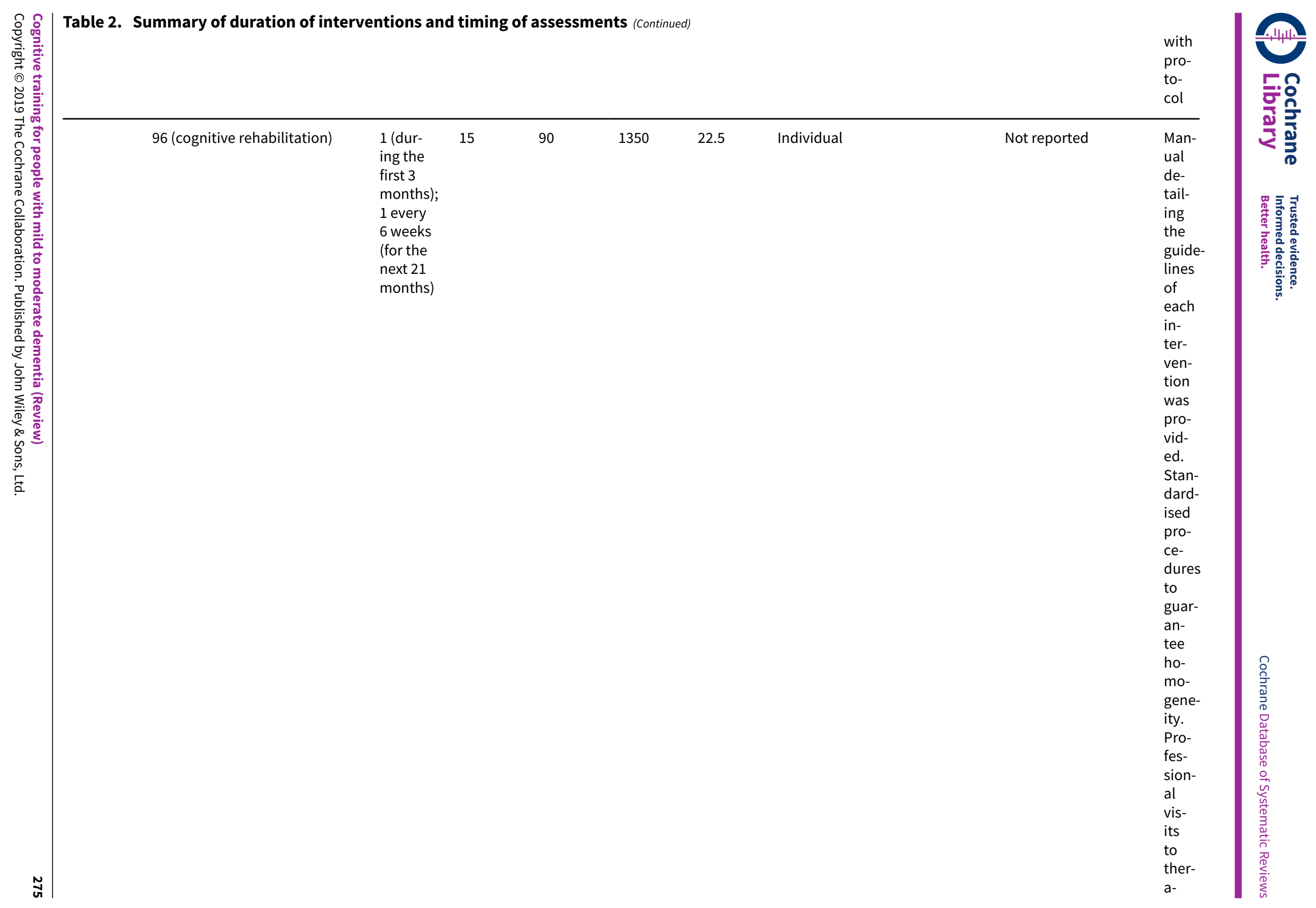




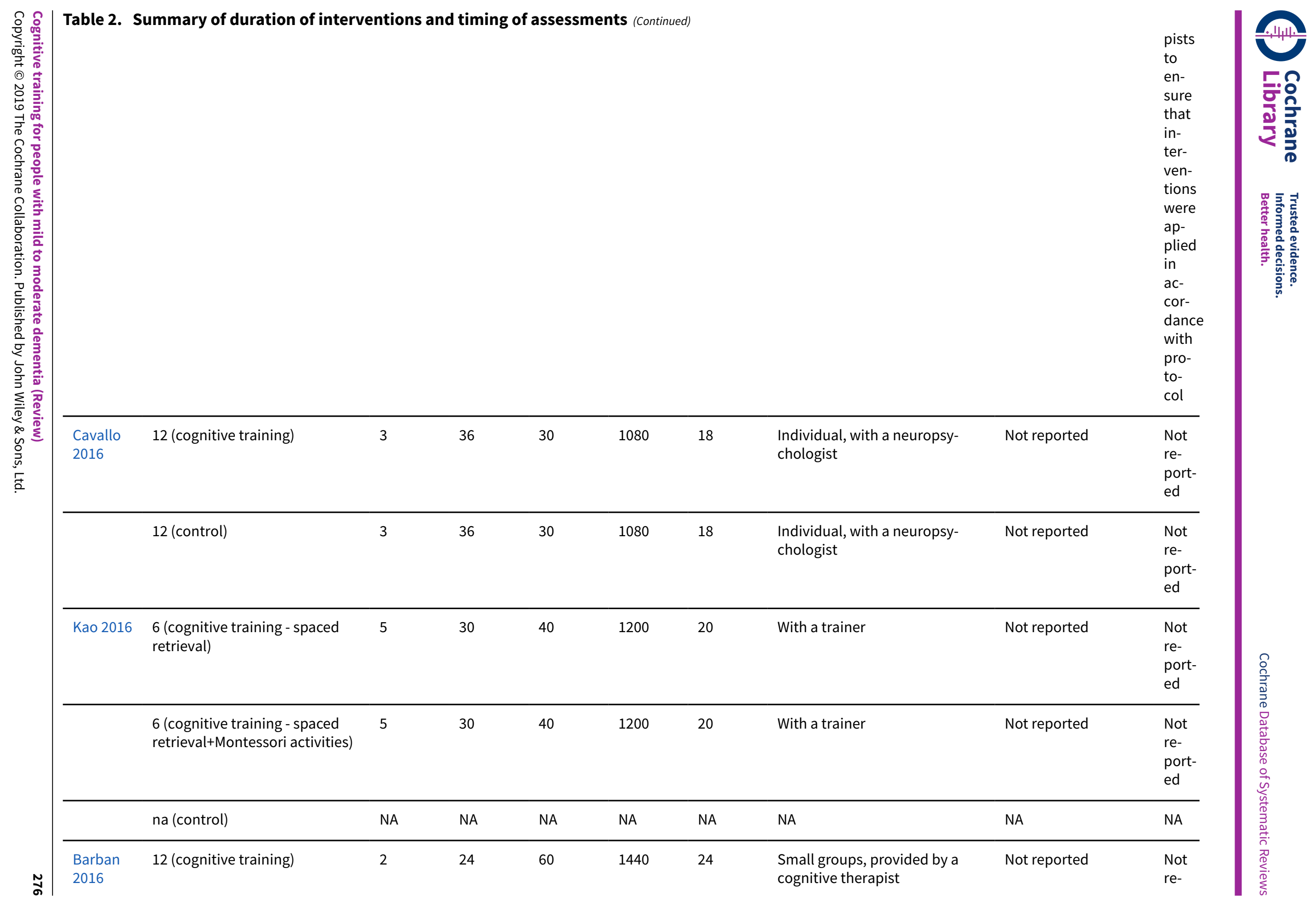




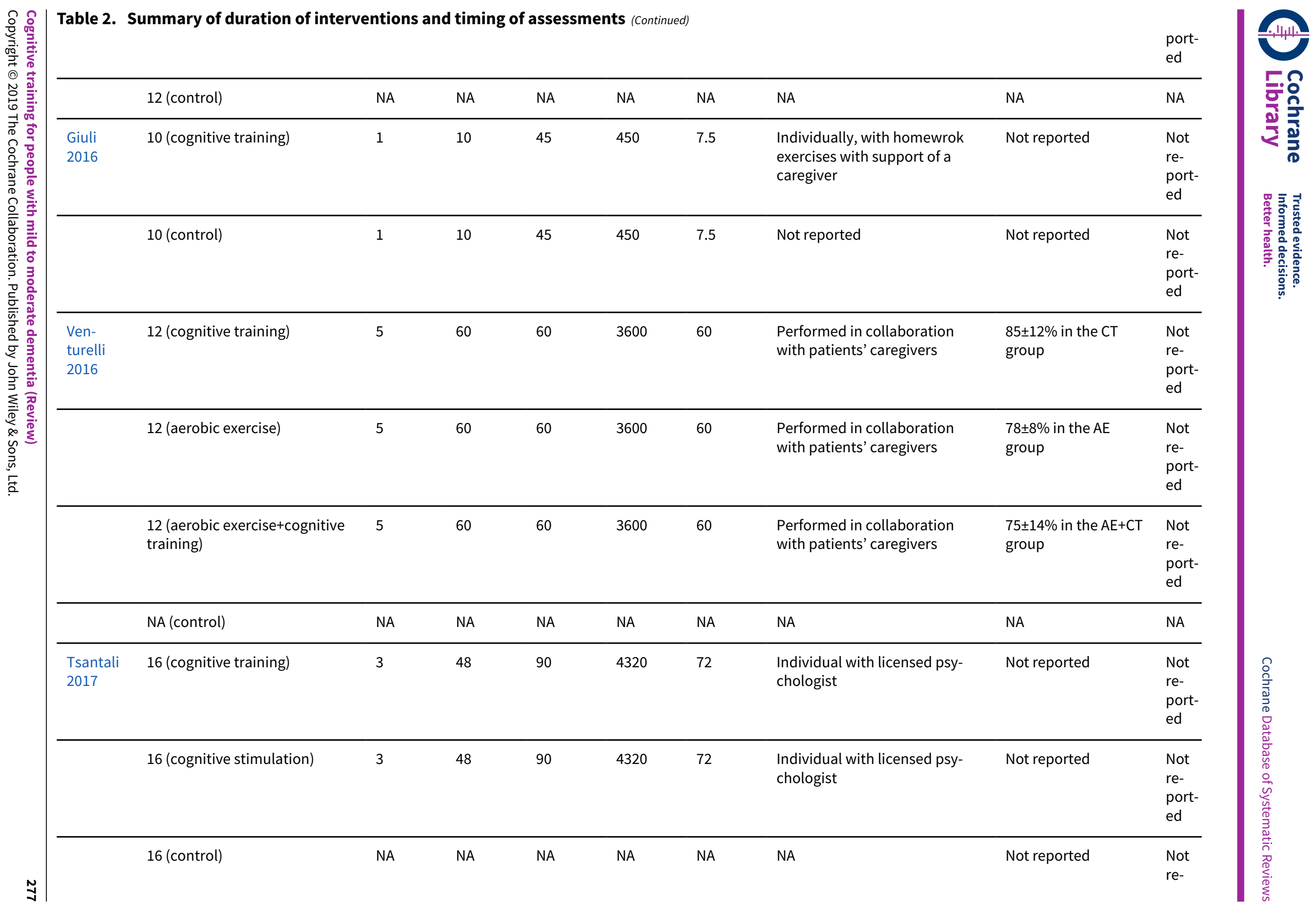




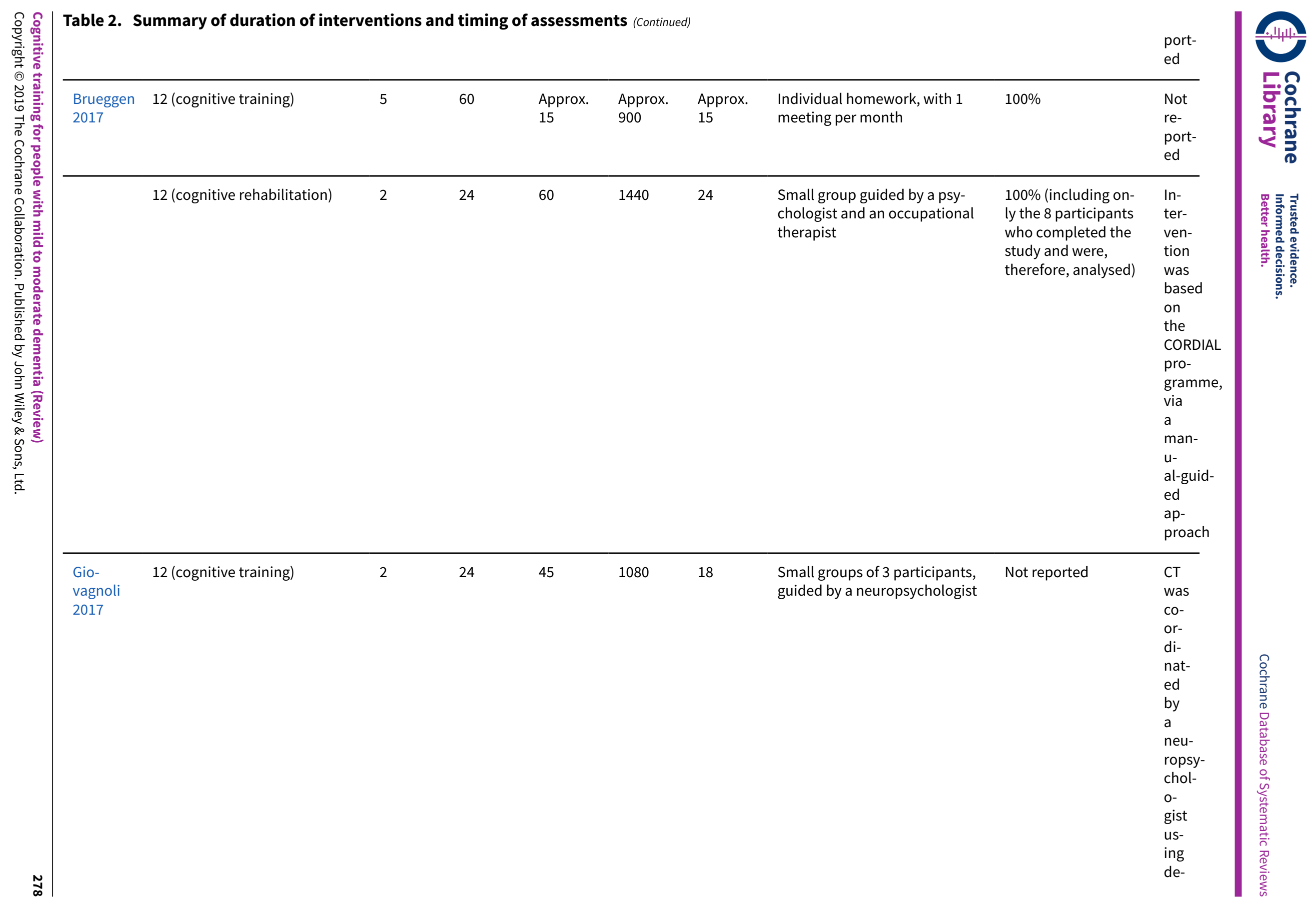




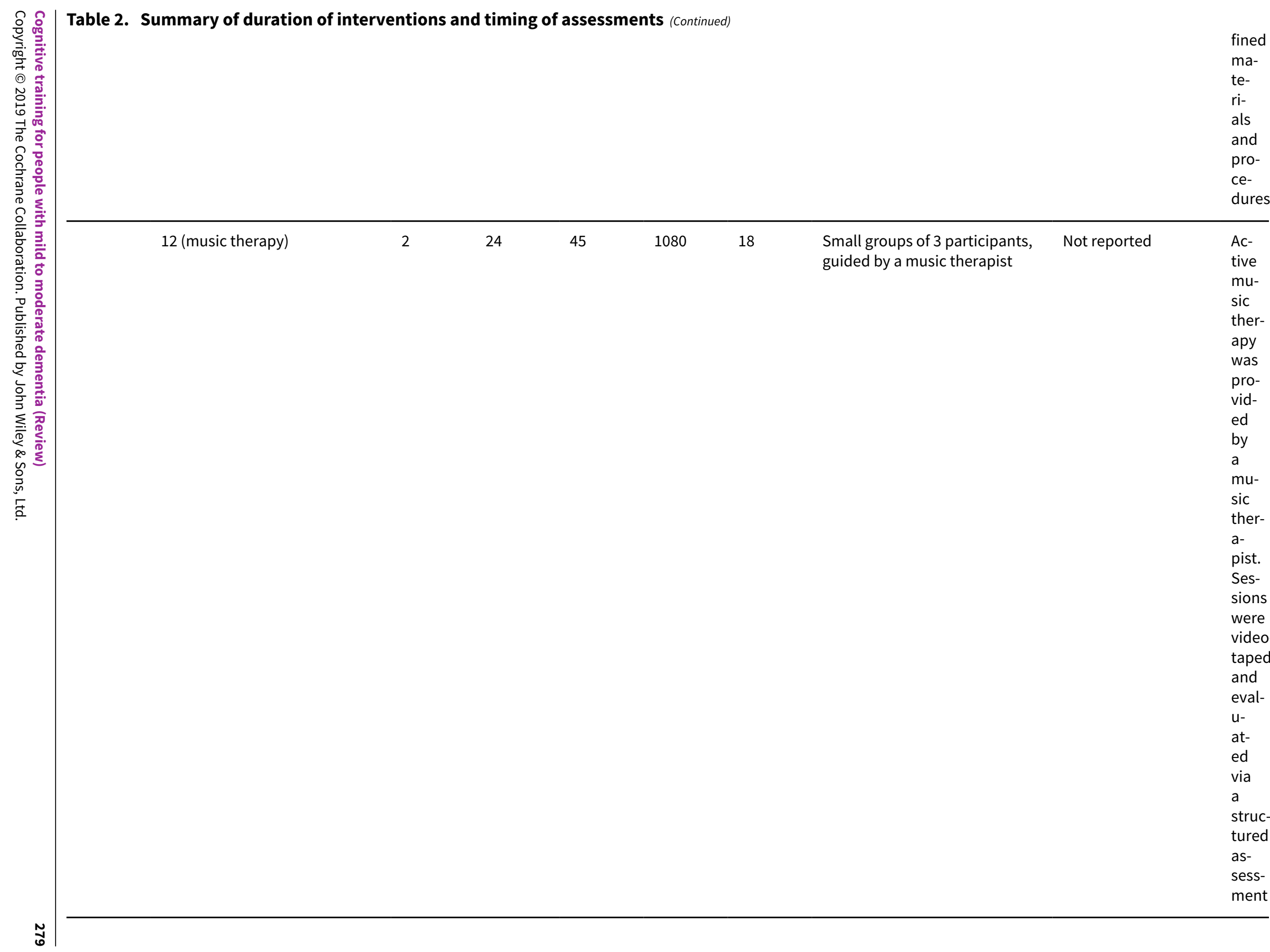




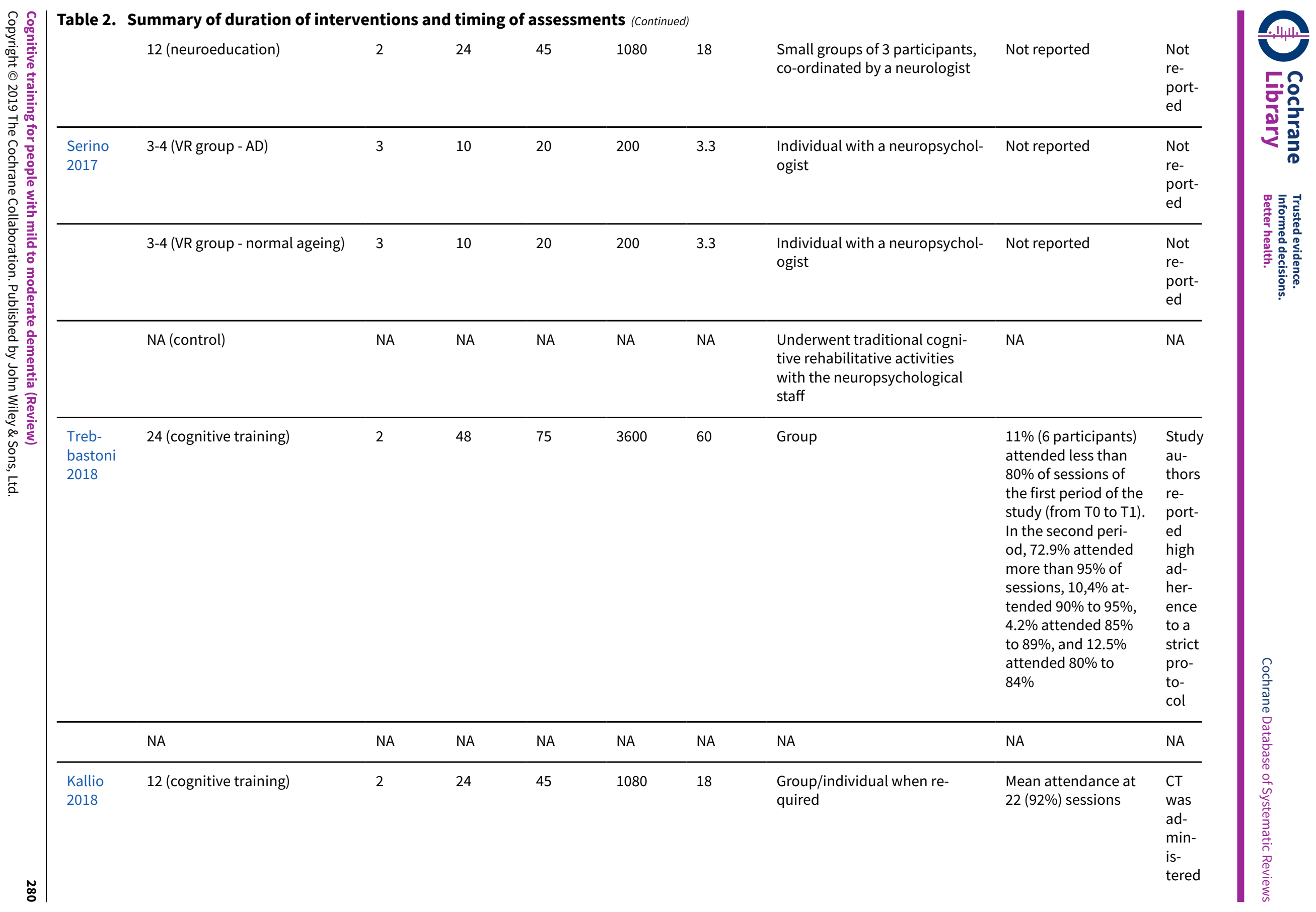




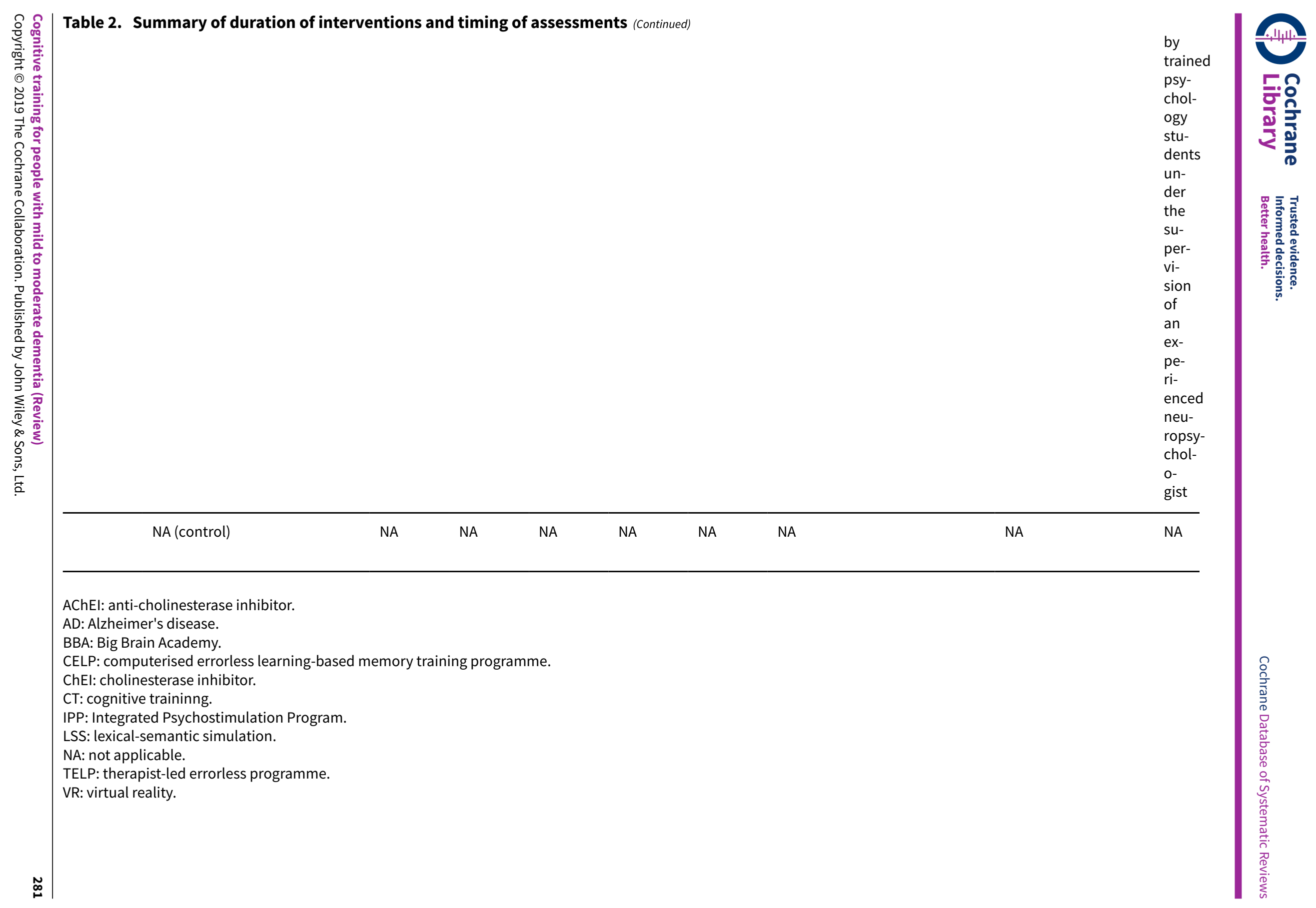




\section{APPENDICES}

\section{Appendix 1. Sources searched and search strategies}

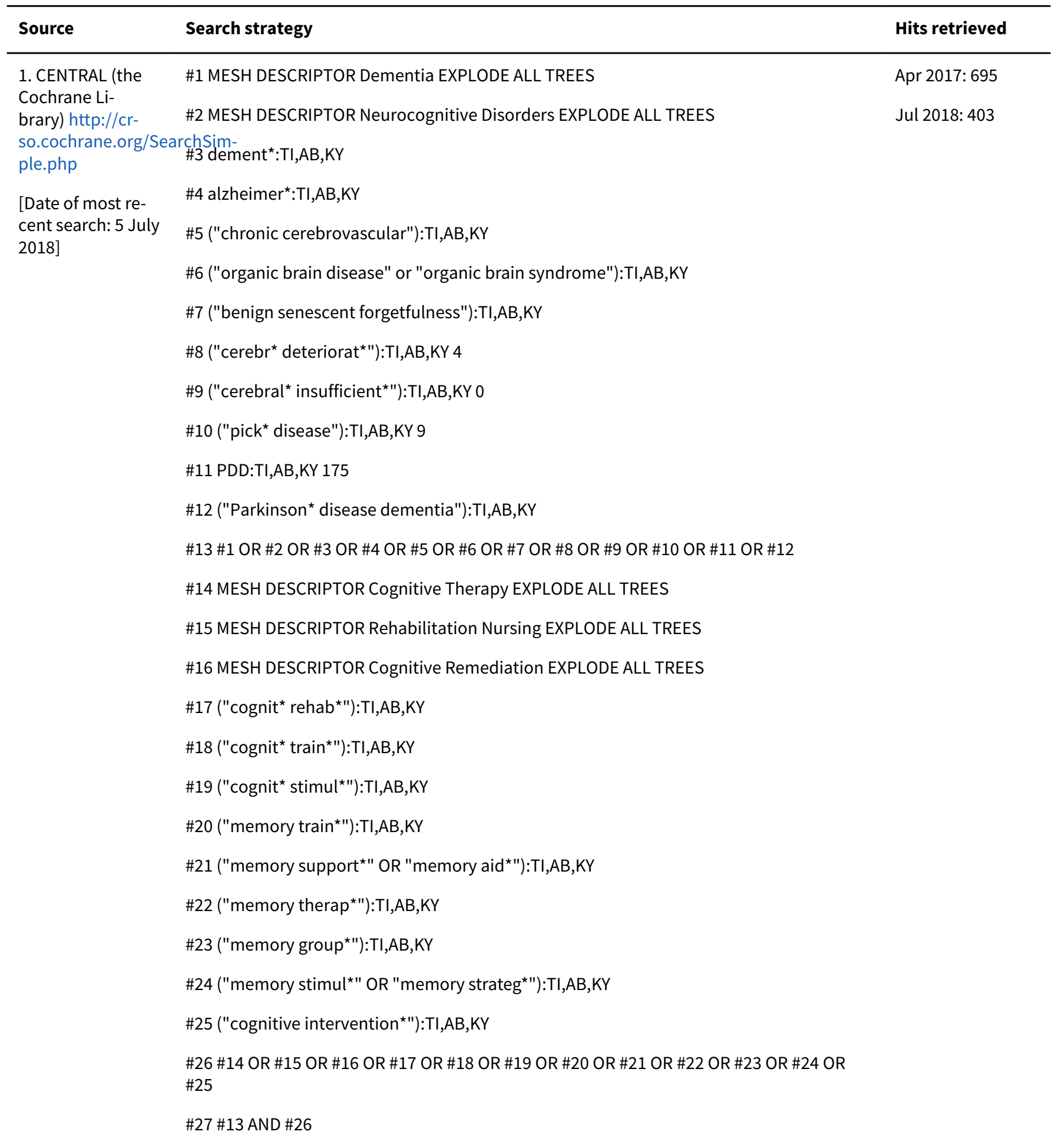

2. MEDLINE Inprocess and other non-indexed
1 exp Dementia/

Apr 2017: 812

2 Delirium, Dementia, Amnestic, Cognitive Disorders/
Jul 2018: 124 
(Continued)

citations and 3 dement $^{\star}$.mp.

MEDLINE 1950-

present (Ovid SP)

4 alzheimer ${ }^{\star} \cdot \mathrm{mp}$.

[Date of most re-

$5\left(\right.$ lewy $^{\star}$ adj2 bod*).mp.

cent search: 5 July

2018]

6 (chronic adj2 cerebrovascular).mp.

7 ("organic brain disease" or "organic brain syndrome").mp.

8 (cerebr* adj2 deteriorat $\left.{ }^{\star}\right) \cdot \mathrm{mp}$.

9 (cerebral $^{\star}$ adj2 insufficient $\left.{ }^{\star}\right)$.mp.

10 (pick* adj2 disease).mp.

11 PDD.mp.

12 "Parkinson* disease dementia".mp.

13 or $/ 1-12$

14 *Cognitive Therapy/

15 Rehabilitation Nursing/

16 Cognitive Remediation/

17 (cognit ${ }^{\star}$ adj2 stimulation).ti,ab.

18 (cognit* adj2 rehabilitation).ti,ab.

19 (cognit* adj2 training).ti,ab.

20 (cognit* $^{\star}$ adj2 retrain $\left.{ }^{\star}\right) . t i, a b$.

21 "cognitive intervention*".ti,ab.

22 "Cognitive skills ADJ2 training".ti,ab.

23 "cognitive support".ti,ab.

24 "Cog* retrain*".ti,ab.

25 "memory function*".ti,ab.

26 (memory adj2 rehabilitation).ti,ab.

27 (memory adj2 therap*).ti,ab.

28 "memory aid*".ti,ab.

29 "memory group*".ti,ab.

30 "Memory rehabilitation".ti,ab.

31 "memory training".ti,ab.

32 "memory retraining".ti,ab.

33 "Memory rehabilitation".ti,ab.

34 "memory re-training".ti,ab.

35 "memory support".ti,ab.

36 "memory stimulation".ti,ab. 
(Continued)

37 "memory strateg*".ti,ab.

38 "memory management".ti,ab.

39 or/14-38

40 randomized controlled trial.pt.

41 controlled clinical trial.pt.

42 randomized.ab.

43 placebo.ab.

44 randomly.ab.

45 trial.ab.

46 groups.ab.

47 or/40-46

48 (animals not (humans and animals)).sh.

4947 not 48

5013 and 39 and 49

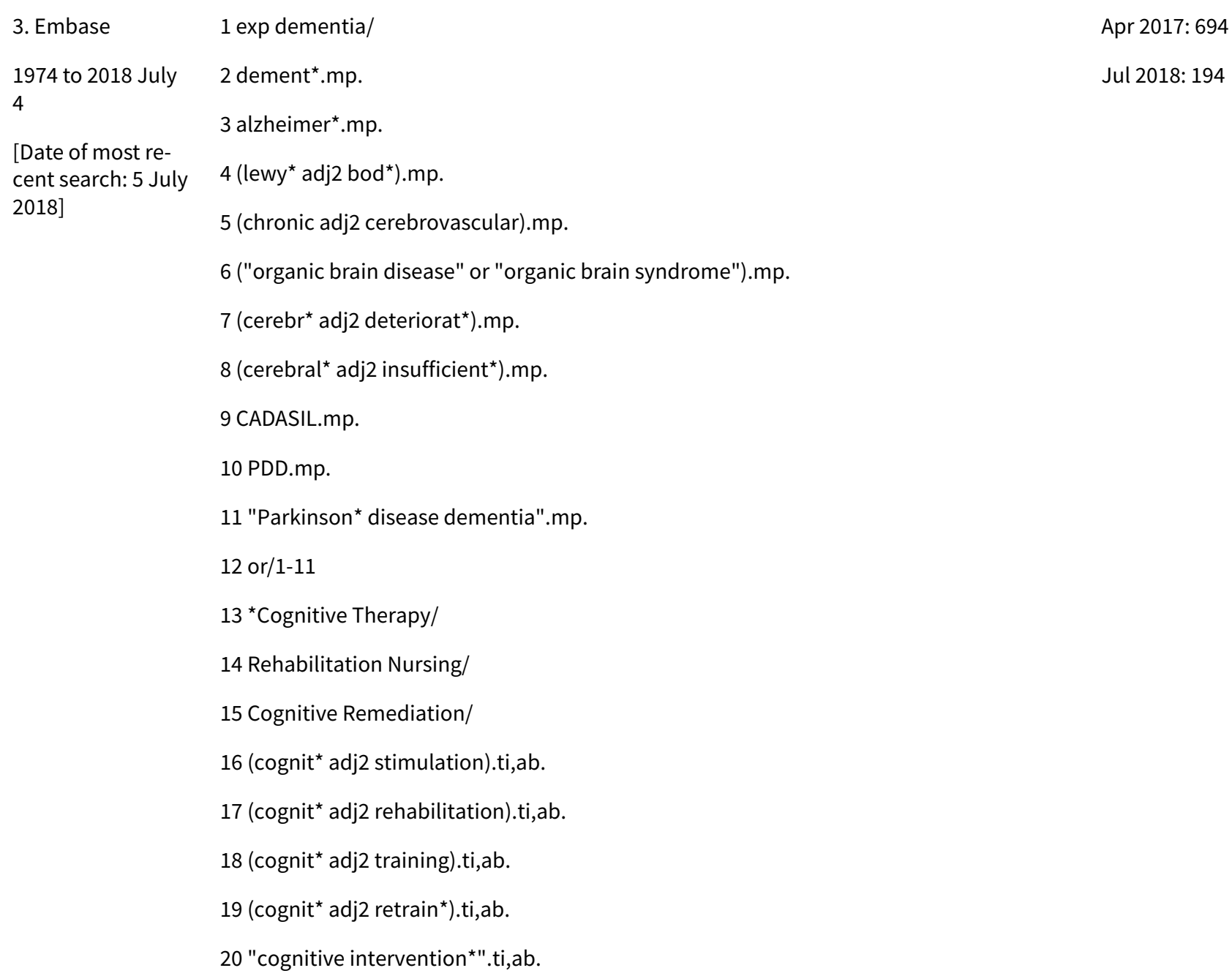

4

3 alzheimer ${ }^{\star} . \mathrm{mp}$.

[Date of most recent search: 5 July 2018]

$4\left(\right.$ lewy $^{\star} \operatorname{adj} 2$ bod $\left.^{\star}\right) \cdot \mathrm{mp}$.

5 (chronic adj2 cerebrovascular).mp.

6 ("organic brain disease" or "organic brain syndrome").mp.

7 (cerebr $^{\star}$ adj2 deteriorat $\left.{ }^{\star}\right) \cdot \mathrm{mp}$.

8 (cerebral $^{\star}$ adj2 insufficient $\left.{ }^{\star}\right) \cdot \mathrm{mp}$.

9 CADASIL.mp.

10 PDD.mp.

11 "Parkinson` disease dementia".mp.

12 or $/ 1-11$

13 * Cognitive Therapy/

14 Rehabilitation Nursing/

15 Cognitive Remediation/

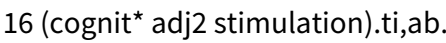

17 (cognit* adj2 rehabilitation).ti,ab.

18 (cognit* adj2 training).ti,ab.

19 (cognit* adj2 retrain $\left.{ }^{\star}\right) . t i, a b$.

20 "cognitive intervention "..ti,ab. 


\section{1 "cognitive support".ti,ab.}

22 "memory function*".ti,ab.

23 (memory adj2 rehabilitation).ti,ab.

24 (memory adj2 therap $\left.{ }^{\star}\right)$.ti,ab.

25 "memory aid*".ti,ab.

26 "memory aid*".ti,ab.

27 "memory group*".ti,ab.

28 "Memory rehabilitation".ti,ab.

29 "memory training".ti,ab.

30 "memory retraining".ti,ab.

31 "memory re-training".ti,ab.

32 "memory support".ti,ab.

33 "memory stimulation".ti,ab.

34 "memory strateg*".ti,ab.

35 "memory management".ti,ab.

36 or/13-25

37 randomly.ab.

38 placebo*.ti,ab.

39 "double-blind *".ti,ab.

40 randomized controlled trial/

41 trial.ti,ab.

42 or/37-41

4312 and 36 and 42

4. PSYCINFO (Ovid
SP)
[Date of most re-
cent search: 5 July
2018]

1 exp Dementia/

2 dement $^{\star} . \mathrm{mp}$.

Jul 2018: 64

3 alzheimer*.mp.

4 (chronic adj2 cerebrovascular).mp.

5 ("organic brain disease" or "organic brain syndrome").mp.

6 (cerebr $^{\star}$ adj2 deteriorat $\left.{ }^{\star}\right) \cdot \mathrm{mp}$.

7 (cerebral $^{\star}$ adj2 insufficient $\left.{ }^{\star}\right) \cdot \mathrm{mp}$.

8 PDD.mp.

9 "Parkinson* disease dementia".mp.

10 or/1-9

11 exp Cognitive Therapy/ 


\section{2 exp Cognitive Rehabilitation/}

13 (cognit* adj2 stimulation).ti,ab.

14 (cognit* adj2 rehabilitation).ti,ab.

15 (cognit* adj2 training).ti,ab.

16 (cognit* adj2 retrain*).ti,ab.

17 "cognitive intervention*".ti,ab.

18 "cognitive support".ti,ab.

19 "memory function*".ti,ab.

20 (memory adj2 rehabilitation).ti,ab.

21 (memory adj2 therap*).ti,ab.

22 "memory aid*".ti,ab.

23 "memory group*".ti,ab.

24 "Memory rehabilitation".ti,ab.

25 "memory training".ti,ab.

26 "memory retraining".ti,ab.

27 "memory re-training".ti,ab.

28 "memory support".ti,ab.

29 "memory stimulation".ti,ab.

30 "memory strateg*".ti,ab.

31 "memory management".ti,ab.

32 or/11-31

33 randomly.ab.

34 randomi?ed.ab.

35 placebo*.ti,ab.

36 trial.ti,ab.

37 RCT.ti,ab.

38 groups.ab.

39 or/33-38

4010 and 32 and 39

5. CINAHL (EBSCO-

$1 \mathrm{MH}$ "Dementia+"

Apr 2017: 448

host)

2 MH "Delirium") or (MH "Delirium, Dementia, Amnestic, Cognitive Disorders"

Jul 2018: 98

[Date of most re-

cent search: 5 July

2018]

3 MH "Wernicke's Encephalopathy"

4 TX dement*

5 TX alzheimer 


\section{TX alzheimer*}

7 TX deliri* $^{\star}$

8 TX chronic N2 cerebrovascular

9 TX "organic brain disease" or "organic brain syndrome"

10 TX "normal pressure hydrocephalus" and "shunt*"

11 TX "benign senescent forgetfulness"

12 TX cerebr* N2 deteriorat*

13 TX cerebral $^{\star}$ N2 insufficient ${ }^{\star}$

14 TX pick* N2 disease

15 TX creutzfeldt or jcd or cjd

16 TX huntington*

17 TX binswanger*

18 TX korsako*

19 TX PDD

20 TX "Parkinson* disease dementia"

21 (S1 OR S2 OR S3 OR S4 OR S5 OR S6 OR S7 OR S8 OR S9 OR S10 OR S11 OR S12 OR S13 OR S14 OR S15 OR S16 OR S17 OR S18 OR S19 OR S20)

$22 \mathrm{MH}$ "Rehabilitation, Cognitive"

23 (MH "Rehabilitation Nursing")

24 (MH "Cognitive Therapy")

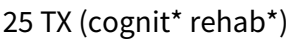

26 TX (cognit* train $\left.^{\star}\right)$

27 TX (memory train*)

28 TX (memory support ${ }^{\star}$ )

29 TX (memory stimul*)

30 TX (cognitive intervention*)

31 (S22 OR S23 OR S24 OR S25 OR S26 OR S27 OR S28 OR S29 OR S30)

32 S21 AND S31

33 MH "Clinical Trials"

34 TX trial

35 TX "single-blind*"

36 TX "double-blind*"

37 TX "treatment as usual"

38 TX randomly 
(Continued)

6. ISI Web of
Science Core Col-
lection - Web of
Science (1945-
present)
[Date of most re-
cent search: 5 July
2018]

(dement* OR VCI OR "vascular cognitive impairment*" OR VaD OR alzheimer ${ }^{\star}$ ) AND

TOPIC: ("cognit* train*" OR "cognit* rehab" OR "memory aid" " OR "memory train*" OR

"memory support"

RCT OR randomized OR randomised)

Timespan: All years.

Search language=Auto memory [Words] and demenc\$ OR dement\$ OR alzheimer\$ [Words] and randomly OR randomised OR randomized OR trial OR ensaio clínico [Words]
Apr 2017: 510

Jul 2018: 125

\begin{tabular}{|c|c|c|}
\hline $\begin{array}{l}\text { 7. LILACS (BIREME) } \\
\text { [Date of most re- } \\
\text { cent search: } 5 \text { July } \\
2018 \text { ] }\end{array}$ & $\begin{array}{l}\text { memory [Words] and demenc\$ OR dement\$ OR alzheimer\$ [Words] and randomly OR } \\
\text { randomised OR randomized OR trial OR ensaio clínico [Words] }\end{array}$ & $\begin{array}{l}\text { Apr 2017: } 22 \\
\text { Jul 2018: } 0\end{array}$ \\
\hline $\begin{array}{l}\text { 8. ClinicalTrial- } \\
\text { s.gov } \\
\text { (www.clinicaltrial- } \\
\text { s.gov) } \\
\text { [Date of most re- } \\
\text { cent search: } 5 \text { July } \\
2018 \text { ] }\end{array}$ & $\begin{array}{l}\text { Interventional Studies | dementia OR alzheimers OR AD OR alzheimer's OR alzheimer | } \\
\text { "cognitive rehabilitation" OR "cognitive training" }\end{array}$ & $\begin{array}{l}\text { Apr 2017: } 50 \\
\text { Jul 2018: } 12\end{array}$ \\
\hline
\end{tabular}

9) ICTRP

[Date of most recent search: 5 July 2018]

\section{dementia OR alzheimers OR AD OR alzheimer's OR alzheimer AND "cognitive rehabilita-} tion" OR "cognitive training"

Apr 2017: 76

Jul 2018: 27

TOTAL before de-duplication

Apr 2017: 3863

Jul 2018: 1059

TOTAL: 4922

\section{CONTRIBUTIONS OF AUTHORS}

Alex Bahar-Fuchs drafted the protocol, screened and selected studies for inclusion, supervised the data extraction process and data entry into RevMan, rated studies for risk of bias, graded the evidence, and wrote the manuscript.

Anthony Martyr screened and selected studies for inclusion, rated studies for risk of bias, conducted the data check, and contributed to writing of the manuscript.

Anita MY Goh screened and selected studies for inclusion, rated studies for risk of bias, extracted data, and contributed to writing of the manuscript.

Linda Clare assisted in resolving issues related to study selection and risk of bias, and contributed to writing of the manuscript.

Julieta Sabates screened and selected studies for inclusion, conducted the data extraction process, completed the relevant tables, rated studies for risk of bias, conducted analyses, graded the evidence, and contributed to writing of the manuscript.

\section{DECLARATIONSOF INTEREST}

Alex Bahar-Fuchs: none known.

Anthony Martyr: none known.

Anita MY Goh: none known. 
Linda Clare: none known.

Julieta Sabates: none known.

\section{SOURCES OF SUPPORT}

\section{Internal sources}

- Cochrane Incentive Award, UK.

\section{External sources}

- NIHR, UK.

This review was supported by the National Institute for Health Research (NIHR), via Cochrane infrastructure funding Cochrane to the Cochrane Dementia and Cognitive Improvement Group. The views and opinions expressed therein are those of the authors and do not necessarily reflect those of the Systematic Reviews Programme, NIHR, the National Health Service, or the Department of Health

- National Health and Medical Research Council of Australia, Australia.

\section{DIFFERENCES BETWEEN PROTOCOLAND REVIEW}

In our protocol, we stated that our primary outcome - global cognition - would be assessed using an available screening measure from each study (e.g. MMSE), and that global cognition would be measured by a composite score computed by us from all cognitive measures in a study serving as a secondary outcome. However, after further consideration, we determined that global cognition as reflected in the composite measure should be our primary outcome. The reasons behind this decision include the fact that a composite score based on all cognitive measures is a more reliable and less biased indicator of global cognition than a single screening measure, the fact that it makes use of all available cognitive data from each trial, and the fact that the composite was calculated for all studies; therefore the meta-analysis is based on larger numbers of studies and participants, increasing statistical power and our confidence in the findings. Global cognition as measured by a screening measure was included as a secondary/sensitivity analysis for this outcome. The findings of the two approaches were comparable.

In addition, in the protocol we stated that we would examine changes in measure of clinical disease severity only in the medium term ( 3 to 12 months post treatment). However, for completeness, we also included results of analysis of this outcome immediately after the intervention as a secondary outcome.

\section{INDEX TERMS}

\section{Medical Subject Headings (MeSH)}

${ }^{\star}$ Cognition; Activities of Daily Living; Cognitive Dysfunction [rehabilitation] [therapy]; Dementia [complications] [rehabilitation] [*therapy]; Randomized Controlled Trials as Topic; Task Performance and Analysis; Therapy, Computer-Assisted [methods]

\section{MeSH check words}

Aged; Aged, 80 and over; Humans; Middle Aged 